\title{
Kinematics of Metal-Poor Stars in the Galaxy. II. Proper Motions for a Large Non-Kinematically Selected Sample
}

\author{
Timothy C. Beers \\ Department of Physics \& Astronomy, Michigan State University, E. Lansing, MI 48824 \\ email: beers@pa.msu.edu \\ Masashi Chiba \\ National Astronomical Observatory, Mitaka, Tokyo 181-8588, Japan \\ email: chibams@gala.mtk.nao.ac.jp \\ Yuzuru Yoshii ${ }^{1}$ \\ Institute of Astronomy, Faculty of Science, University of Tokyo, Mitaka, Tokyo 181-8588, Japan \\ email: yoshii@ioa.s.u-tokyo.ac.jp \\ Imants Platais \\ Department of Astronomy, Yale University, P.O. Box 208101, New Haven, CT 06520-8101 \\ email: imants@astro.yale.edu \\ Robert B. Hanson \\ University of California Observatories / Lick Observatory, University of California, Santa Cruz, \\ CA 95064 \\ email: hanson@ucolick.org \\ Burkhard Fuchs \\ Astronomisches Rechen-Institut Heidelberg, Mönchofstr. 12-14, 69120 Heidelberg, Germany \\ email: fuchs@ari.uni-heidelberg.de \\ Silvia Rossi \\ Instituto Astronômico e Geofísico, Universidade de São Paulo, Brazil \\ email: rossi@orion.iagusp.usp.br
}

\begin{abstract}
We present a revised catalog of 2106 Galactic stars, selected without kinematic bias, and with available radial velocities, distance estimates, and metal abundances in the range $0.0 \leq[\mathrm{Fe} / \mathrm{H}] \leq-4.0$. This update of the Beers \& Sommer-Larsen (1995) catalog includes newly-derived homogeneous photometric distance estimates, revised
\end{abstract}

\footnotetext{
${ }^{1}$ Also RESCEU: Research Center for the Early Universe, Faculty of Science, University of Tokyo, Bunkyo-ku, Tokyo 113-0033, Japan
} 
radial velocities for a number of stars with recently obtained high-resolution spectra, and refined metallicities for stars originally identified in the HK objective-prism survey (which account for nearly half of the catalog) based on a recent re-calibration. A subset of 1258 stars in this catalog have available proper motions, based on measurements obtained with the Hipparcos astrometry satellite, or taken from the updated Astrographic Catalogue (AC 2000; second epoch positions from either the Hubble Space Telescope Guide Star Catalog or the Tycho Catalogue), the Yale/San Juan Southern Proper Motion (SPM) Catalog 2.0, and the Lick Northern Proper Motion (NPM1) Catalog. Our present catalog includes 388 RR Lyrae variables (182 of which are newly added), 38 variables of other types, and 1680 non-variables, with distances in the range 0.1 to $40 \mathrm{kpc}$.

Subject headings: Surveys - Galaxy: halo - Galaxy: abundances - Galaxy: kinematics — Stars: Population II — Stars: Proper Motions

\section{Introduction}

Studies of the kinematics of various stellar populations in the Galaxy, in particular the thick disk and the nascent halo, have long been limited by the availability of large samples of stars with measurements of velocities, distances, and metallicities. Such a database is required in order to constrain plausible scenarios for the formation and evolution of the Milky Way and other large spiral galaxies like it. Current issues which might be addressed with such data include: (a) the rotational character of the thick disk and halo (see, e.g., Beers \& Sommer-Larsen 1995, hereafter BSL, and references therein), (b) the existence and the observed lower limit on the metal abundance of stars in the so-called metal-weak thick disk (MWTD) (Morrison, Flynn, \& Freeman 1990, hereafter MFF; BSL; Carney et al. 1996; Chiba \& Yoshii 1998; Martin \& Morrison 1998) (c) a dual-component (flattened plus spherical) halo population in the Galaxy (Hartwick 1987; Sommer-Larsen \& Zhen 1990; Norris 1994; Kinman, Suntzeff, \& Kraft 1994; Sommer-Larsen et al. 1997), (d) quantitative estimates of the local density of thick disk and halo stars (Yoshii 1982; Preston, Shectman, \& Beers 1991; Morrison 1993), (e) tests for the existence of a putative "counter-rotating" halo component beyond 4-5 kpc from the plane (Majewski 1992; Wilhelm 1995; Carney et al. 1996; Wilhelm et al. 1996; Zinn 1996; Carney 1999), (f) measures of the local halo velocity ellipsoid for comparison with the derived ellipsoid for more distant halo stars (Sommer-Larsen et al. 1997), and (g) derivation of a reliable RR Lyrae absolute magnitude estimate based on statistical parallax analyses (Layden et al. 1996; Fernley et al. 1998; Popowski \& Gould 1998). As more extensive searches are carried out for evidence of past (and present) mergers of smaller galaxies with the Milky Way (e.g., Preston, Beers, \& Shectman 1994; Harding et al. 1998; Helmi \& White 1999; Majewski 1999), it is of equal importance to obtain secure knowledge of the "global" Galactic kinematic properties, so that deviations from the expected 
behavior can be reliably assessed.

To confidently address the above issues (and many others) stars chosen as kinematic tracers should be identified in a manner which does not depend on a kinematic selection criterion itself. Although it may be possible to statistically correct for an input selection bias of this nature (Bahcall \& Casertano 1986; Ryan \& Norris 1991a; Carney et al. 1994; Carney 1999), one is left with lingering doubt concerning the derived kinematic parameters based on post-facto modifications of the results. It is similarly important that the tracer stars cover a wide range of metallicities and distances (over both northern and southern Galactic hemispheres), so that correlations of kinematics as a function of these parameters can be investigated.

Although hints of the impact of a kinematic selection criterion are evident in the work of Yoshii \& Saio (1979), the first large database suitable for exploration of many of these issues was that of Norris (1986), which included some 400 spectroscopically and/or photometrically selected stars with abundances $[\mathrm{Fe} / \mathrm{H}] \leq-0.6$, and with available radial velocities and distance estimates. In Paper I of this series BSL extended the Norris catalog by inclusion of some 900 stars identified from the HK object objective-prism survey of Beers \& colleagues (Beers, Preston, \& Shectman 1985; Beers, Preston, \& Shectman 1992a; Beers et al. 1992b), as well as some 600 additional stars from other smaller samples which appeared in the literature subsequent to the publication of the Norris catalog, obtaining a total sample of 1936 stars.

In this paper we present a revision of the BSL catalog of Paper I, based on additional observational information which has recently become available. In addition to revisions of metallicities for the published HK survey stars, new photometric distance estimates have been made for the entire BSL catalog based on an internally self-consistent methodology, and in some cases, new photometry. Radial velocities have been updated based on recently-obtained high-resolution data for a number of stars. Much more accurate positional information for the stars in our revised catalog has been obtained by comparison with astrometric positions available for many of the brighter stars in our sample, plus improved information from automated scans of wide-field photographic plates, such as compiled in USNO-A V2.0 (Monet et al. 1998), NPM1 (Klemola, Hanson, \& Jones 1993), and SPM 2.0 (Platais et al. 1998). We have also added 182 RR Lyrae variables from the recent work of Layden (1994), Layden et al. (1996), and Fernley et al. (1998), so that useful comparisons of the kinematics of these stars with the non-variables (which presumably sample the same Galactic phase space) can be carried out.

The major difference between the present catalog and the BSL catalog is the addition of proper motions, from a variety of sources, for over half of the stars in our catalog. In $\S 2$ we discuss the assemblage of the present catalog. Revisions of radial velocities and abundances, and in particular, distance estimates, are discussed in $\S 3$. In $\S 4$ we present the new proper motion information, and discuss the averaging we have carried out in order to minimize statistical errors. In $\S 5$ we discuss the observed characteristics of the stars in the revised catalog, and derive estimates of space motions and orbital parameters for the subset of stars with complete 
kinematical information. In the accompanying analysis paper (Chiba \& Beers 2000; Paper III) we use this wealth of new information to consider many of the questions put forth above.

\section{Additions and Subtractions from the Beers \& Sommer-Larsen (1995) Catalog}

The BSL catalog of Paper I contained 1936 stars with available abundances, distances, and radial velocities. As it was our intent to use this catalog as the starting point in searches for stars with measured proper motions, the first requirement was to obtain updated positions for the stars of as high an accuracy as possible. A small number of fainter stars in the BSL catalog with crude positions (and no finding charts) were eliminated entirely. The remaining stars were then compared with the positions listed in the SIMBAD database. A first search was done on the star name, followed by a search on the reported position in the BSL catalog, with the requirement that the magnitude reported in SIMBAD be commensurate with that of the target star. Many of the brighter stars from the BSL catalog now have astrometric positions from Hipparcos, which are accurate at the milli-arcsecond (mas) level. For the stars of intermediate and fainter magnitudes, searches were conducted on the BSL positions by comparison with the USNO-A 2.0 catalog, again with the requirement that the magnitudes be commensurate. The typical accuracy of stellar positions for USNO-A 2.0 is at the 0 '.25 (one-sigma) level (Deutsch 1999). Further searches were conducted within the catalogs we use below to find proper motions - NPM1, SPM 2.0, STARNET, and ACT - which resulted in positional accuracies on the order of $0.2,30$ mas, $00^{\prime \prime} 3$, and 25 mas, respectively. For a small number of stars no improved positional information could be found, hence we retained the positions in the original BSL catalog.

The Hipparcos, Tycho, NPM1, and SPM 2.0 catalogs included a number of "targeted" RR Lyrae stars which were not included in the BSL catalog, some of which have radial velocity and abundance estimates available in the literature. We thus added a total of $182 \mathrm{RR}$ Lyrae stars to our revised catalog, including many with metallicities greater than the nominal cutoff of the BSL catalog. A small number of non-variables which were not included in the BSL catalog, but which have available radial velocities and abundance estimates, were also added. As a result of the revised abundances, described in more detail below, a small number of HK survey stars which were originally assigned metallicities $[\mathrm{Fe} / \mathrm{H}] \leq-0.6$ presently have values above this limit. These stars are noted in the revised catalog.

Column (1) of Table 1A lists the star names. We have endeavored to follow the IAUrecommended nomenclature, but in a few cases had to abbreviate the star name in order to save space. In any case, the positional identification is usually unambiguous. All adopted positions were updated to the ICRS (2000.0) system. Columns (2) and (3) of Table 1A list the right ascension and declination of the adopted positions, respectively. The source of the position is indicated in column (4). 


\section{Revisions of Abundance Estimates, Velocities, and Distances}

\subsection{Revisions of Abundance Estimates}

Roughly $45 \%$ of the stars in the revised catalog were originally identified during the course of the HK objective-prism survey. In the BSL catalog the abundance estimates for these stars were obtained from a calibration of the strength of the CaII $\mathrm{K}$ line as a function of $(B-V)_{o}$ color described in Beers et al. (1990). This calibration is now known to suffer from several deficiencies, the most worrisome of which is the fact that assigned abundances in the range $[\mathrm{Fe} / \mathrm{H}]>-1.5$ are less than optimal as a result of the saturation of the CaII K line, in particular at cooler temperatures. Stars in the metallicity regime $-2.0 \leq[\mathrm{Fe} / \mathrm{H}] \leq-1.0$ represent the transition between the halo and thick disk populations, and in particular, may be members of the MWTD component of the Galaxy, hence it is important that the abundances be correctly estimated. At the low end of the metallicity scale $([\mathrm{Fe} / \mathrm{H}]<-2.5)$, the lack of available calibrators in the Beers et al. (1990) treatment resulted in stellar abundance estimates which were, on the whole, somewhat lower than has proven to be justified on the basis of recent high-resolution spectroscopy, in particular for the hotter stars near the main-sequence turnoff of an old halo population.

The re-calibration of metallicity estimates based on medium resolution spectroscopy described by Beers et al. (1999) avoids, to a great extent, the above difficulties. This re-calibration makes use of an additional metallicity estimator based on the Auto-Correlation Function of metallic lines in a stellar spectrum (originally described by Ratnatunga \& Freeman 1989), which provides a superior estimate of abundance to CaII $\mathrm{K}$ in the regime $[\mathrm{Fe} / \mathrm{H}]>-1.0$. The combination of the CaII K and ACF approaches implemented in Beers et al. (1999) results in metallicity estimates over the interval $-4.0 \leq[\mathrm{Fe} / \mathrm{H}] \leq 0.0$ with typical errors on the order of $0.1-0.2 \mathrm{dex}$, and no significant systematic offsets.

Figure 1 is a comparison of the abundance determinations of the HK survey stars in the revised catalog based on the new and old calibration. Note that the broad sloping region about the one-to-one line, which is dominated by stars with $0.3 \leq(B-V)_{o} \leq 0.5$, indicates that the revised abundances for the hotter HK survey stars are generally higher at the low metallicity end of the scale, and lower at the high metallicity end of the scale, as compared to the old calibration. The abundance estimates for the cooler stars with $0.5<(B-V)_{o} \leq 1.2$ have changed relatively less.

As part of the re-calibration effort, Table 7 of Beers et al. (1999) lists averaged high-resolution abundance determinations from the literature for 551 stars. For stars which also appear in the revised catalog, we adopted these averaged abundance determinations.

The catalog of Cayrel de Strobel et al. (1997) provides detailed information on abundances for stars with determinations based on high-resolution spectroscopy. Suitably averaged abundances for stars which did not appear in Beers et al. (1999) were adopted for the revised catalog listing. A number of stars from MFF have been shown by Ryan \& Lambert (1995) to suffer 
from mis-estimated abundances (generally too low) arising from a faulty calibration of DDO photometry. For MFF stars with high-resolution abundances obtained by Ryan \& Lambert, we simply adopt their values. For other MFF stars which are included in the revised catalog, the abundances should be considered uncertain.

Metallicity estimates for the revised catalog are reported in column (5) of Table 1A. The source of the metallicity estimate is indicated in column (6). Uncertain abundances are indicated with a ':' appended to the reported $[\mathrm{Fe} / \mathrm{H}]$.

\subsection{Revision of Radial Velocities}

Radial velocities of improved accuracy have been reported for a number of stars in our revised catalog based on high-resolution spectroscopic follow-up, in particular for the HK survey stars (McWilliam et al. 1995; Norris, Ryan, \& Beers 1996). The adopted radial velocities are reported in column (7) of Table 1A. Errors on the radial velocity measurements are reported in column (8), and the source of the radial velocity estimate is provided in column (9). For the HK survey stars with only medium-resolution spectroscopy available, we adopt a conservative error estimate of $10 \mathrm{~km} \mathrm{~s}^{-1}$ (based on previous comparisons). For non-variable stars where the original authors did not report an error on the velocity determination, we have adopted an error of $10 \mathrm{~km} \mathrm{~s}^{-1}$. For RR Lyrae variables without reported radial velocity errors, we assign an error of $30 \mathrm{~km} \mathrm{~s}^{-1}$, which accounts for the uncertainty in the systemic velocity arising from limited phase coverage in spectra for a given variable (Smith 1995).

\subsection{Revision of Distance Estimates}

We have determined new photometric estimates of stellar distances for stars in our catalog based on $M_{V}$ vs. $(B-V)_{o}$ relations for non-variable stars, as described below, and an adopted $M_{V}$ vs. $[\mathrm{Fe} / \mathrm{H}]$ relation for $\mathrm{RR}$ Lyrae variables.

\subsubsection{Apparent Magnitudes and Colors}

Our primary source of $V$-band apparent magnitudes and $B-V$ colors for non-variable stars is the photometry obtained during the course of the HK survey, if available (with typical accuracy in $V$ magnitudes and $B-V$ colors in the range 0.005 to 0.01 , respectively). Additional photometric information was taken from the SIMBAD database, the Hipparcos Catalogue, and the calibrated photographic photometry of SPM 2.0, and GSC 1.2.

There are several hundred HK survey stars in our catalog which presently lack measured photometry. For these stars we have estimated the $V$ magnitudes and $B-V$ colors in the 
following manner. An estimate of the $B$ magnitude is obtained from GSC 1.2, where available. An approximate de-reddened $(B-V)_{o}$ color is obtained from available medium resolution spectroscopy, based on the calibration of the Balmer line index HP2 described by Beers et al. (1999). This is then reddened using the listed estimated to reddening in the direction toward the star, and the resulting $B-V$ is obtained. This color is then used to convert the GSC $B$ magnitude estimate to an approximate $V$ magnitude. Given the multiple approximations in these procedures, these estimates should be regarded with appropriate caution - these stars are indicated in Table 1A with parentheses around the $V$ and $B-V$ magnitude estimates. In a few cases, apparent magnitudes for HK survey stars that were not found in the GSC are estimated from the intensity of the spectrum on the original prism plate, and are indicated as such with brackets around the $V$ and $B-V$ magnitudes in the table.

For a number of stars, $V$ magnitudes and $b-y$ colors are available from Schuster et al. (1996), Schuster et al. (2000), or Anthony-Twarog et al. (2000). For these stars, we have listed an approximate $B-V$ color obtained by adopting the transformation $B-V=1.35(b-y)$, and indicate these cases with parentheses about the approximate $B-V$ color in . For RR Lyrae variables, the intensity-averaged $V$ magnitudes, where available, are taken from either Layden (1994), Layden et al. (1996), or Fernley et al. (1998). The adopted photometric quantities are reported in columns (10) and (11) of Table 1A; the source of the photometry is given in column (12); a reddening estimate (as described below) is listed in column (13).

\subsubsection{Estimates of Reddening}

Our primary source of total reddening in the directions of the sample stars is the new map of Schlegel, Finkbeiner, \& Davis (1998, hereafter SFD) based on COBE and IRAS measurements of dust emission in the Galaxy. The SFD map is superior to the widely used map of Burstein \&

Heiles (1982, hereafter BH) in its spatial resolution and its zero point (see also Gould \& Popowski 1998 for arguments in favor of the SFD map). Note, however, that Arce \& Goodman (1999) caution that the SFD map overestimates the reddening values when the color excess $E_{B-V}$ is more than about 0.15 mags, where SFD's calibration from dust column density to reddening may no longer be accurate. For the small number of stars where this large reddening applies, we have adopted estimates from the BH map, where available. The few program stars in such highly reddened directions are generally located at small distances from the Sun, so that the effects of correction for reddening on distance estimates are small.

We adopt a $V$-band absorption $A_{V}=3.1 E_{B-V}$ and assume that the dust layer has a scale height $h=125 \mathrm{pc}$. The reddening to a given star at distance $D$ is reduced compared to the total reddening by a factor $\exp [-|D \sin b| / h]$, where $b$ is the galactic latitude. An iteration procedure is employed to obtain consistent values of $E_{B-V}$ and $D$. The final adopted reddening is listed in column (13) of Table 1A. 


\subsubsection{Classification of Stellar Types}

Column (14) of Table 1A lists the classification of each stellar type. We follow the coding of BSL - D: main-sequence dwarf star; A: main-sequence A-type star; TO: main-sequence turnoff star; SG: subgiant star; G: giant star; AGB: asymptotic giant branch star; FHB: field horizontal-branch star; V: variable stars other than the RR Lyrae type. For the RR Lyraes, we use a code 'RRV' to distinguish them from other types of variable stars. Most of the classifications are taken from the BSL catalog, with the exception of a few stars where we have adopted the classifications from Norris, Bessel, \& Pickles (1995) (HD 13359: G; HD 17233: SG; HD 219221: D; HD 217808: G; CPD-62 394: G) and Anthony-Twarog \& Twarog (1994) (HD 161770: TO; BD-01 1792: G; HD 17072: AGB; HD 41667: G). These revised classifications provide photometric distance estimates which are more consistent with the Hipparcos parallaxes, as described below. Revised classifications are also provided for a number of the HK survey stars, based on more recent observations.

There still remains some uncertainty in the classification of a number of stars with relatively blue colors $\left[0.3 \leq(B-V)_{o} \leq 0.5\right]$. If the corresponding stars have $(U-B)_{o}$ color data from the HK survey, the stars are classified as FHB if $(U-B)_{o}>-0.1$, or TO if $(U-B)_{o} \leq-0.1$ (Wilhelm, Beers, \& Gray 1999). This crude discrimination scheme is adequate for stars with abundances $[\mathrm{Fe} / \mathrm{H}]<-1.5$. For more metal-rich stars, we also consider the alternative classification as possible, and assign a classification of FHB/TO.

\subsubsection{Templates for Distance Estimation}

RR Lyrae distance scales have been reconsidered extensively subsequent to the release of the Hipparcos Catalogue, based on a variety of methods including main-sequence fitting, the Baade-Wesselink method, and statistical parallax. Combining all of the results, Chaboyer (1999) proposed the following $M_{V}$ vs. $[\mathrm{Fe} / \mathrm{H}]$ relation:

$$
M_{V}(R R)=0.23([\mathrm{Fe} / \mathrm{H}]+1.6)+0.56
$$

where $M_{V}(R R)$ denotes the absolute magnitude of RR Lyrae stars. We adopt this relation to estimate the distances of our RR Lyrae sample.

For non-variable stars, we first calibrate fiducial points in the $M_{V}$ vs $(B-V)_{o}$ relation, using published data from various Galactic globular clusters and the Hyades and Pleiades open clusters to cover large ranges of colors and metal abundances. The globular clusters we use include M92 $([\mathrm{Fe} / \mathrm{H}]=-2.24), \mathrm{M} 15(-2.15), \mathrm{M} 3(-1.66), \mathrm{M} 13(-1.65)$, NGC $6752(-1.54)$, NGC 362 $(-1.27)$, and 47 Tuc (-0.71) (Sandage 1970; Buonanno, Corsi, \& Fusi Pecci 1989; Durrell \& Harris 1993; Penny \& Dickens 1986; Harris 1982; Hesser et al. 1987), where metal abundances and extinctions are taken from the compilation of Chaboyer, Demarque, \& Sarajedini (1996). The 
absolute magnitude of the horizontal branch in each cluster is scaled according to Eqn. (1). For the Hyades cluster $([\mathrm{Fe} / \mathrm{H}]=+0.12)$, we adopt the work of Perryman et al. (1998), which utilized the Hipparcos parallaxes to determine the distances to the cluster stars. Using their Figure 21, we read off the fiducial $M_{V}$ vs $(B-V)_{o}$ relation for the stars which are not classified as double or multiple in the Hipparcos Catalogue. For the Pleiades cluster $([\mathrm{Fe} / \mathrm{H}]=+0.11$, essentially identical to the Hyades abundance), we adopt the $M_{V}$ vs $(B-V)_{o}$ relation published by Mermilliod (1981).

Next, polynomial fits to the $M_{V}$ vs $(B-V)_{o}$ relation are made for each type of star; the fitting formula and the coefficients of the fits are provided in Table 2. For G, SG, and AGB stars with abundances $[\mathrm{Fe} / \mathrm{H}]<-2.5$, we use the tabulated relation for $[\mathrm{Fe} / \mathrm{H}]=-2.5$. For $\mathrm{D}$ stars we employ an interpolation/extrapolation procedure among the tabulated relations. Figure 2a demonstrates the adopted $M_{V}$ vs $(B-V)_{o}$ relations of $\mathrm{G}, \mathrm{SG}$, and AGB stars at metal abundances appropriate for M92+M15 $([\mathrm{Fe} / \mathrm{H}]=-2.20)$, M13 (-1.65) and 47 Tuc $(-0.71)$, whereas Figure 2b shows those of D stars for M92+M15, 47 Tuc, and Hyades. Unfortunately, there remains some additional uncertainty for TO stars, due to their positions at the "curved" part of the adopted $M_{V}$ vs $(B-V)_{o}$ relation. We employ the following approximate method. For a given $(B-V)_{o}$ color, $M_{V}$ 's assuming both a D and SG classification are first estimated. If $M_{V}(\mathrm{D})$ is brighter than $M_{V}(\mathrm{SG}), M_{V}(\mathrm{D})$ is adopted as $M_{V}(\mathrm{TO})$. If this is not the case, the intermediate $M_{V}$ which locates between $M_{V}(\mathrm{D})$ and $M_{V}(\mathrm{SG})$ is calibrated using the formula given in Table 2. This formula is designed to reproduce the turnoff point from neighboring points of the isochrones used in Beers et al. (1999).

In order to estimate distances for the hotter main-sequence gravity A-type stars (i.e., those with colors $\left.-0.10 \leq(B-V)_{o} \leq 0.34\right)$ we apply a small vertical shift to the $M_{V}$ vs $(B-V)_{o}$ relation for the Pleiades so that it matches that of the Hyades at $(B-V)_{o}=0.34$. To account for the expected decline in luminosity with decreasing abundance, the adjusted Pleiades line is corrected by applying shifts of the same size as those derived for the cooler stars on the main sequence of the metal-poor globulars as compared to the Hyades. We note that this is only one of several choices we could have made, and it may not be the optimal one, but it should serve for the present purpose.

Taking all of the above information into account, distances are then estimated by means of an iteration procedure which includes modification of the interstellar reddening. These distances, $D_{\text {pho }}$, are indicated in column (2) of Table 1B. In some cases there was no reliable photometry or classification available, and, occasionally, our iteration scheme failed to converge. In these circumstances we made use of the distances provided in the original BSL catalog (who, in such cases, adopted the distance estimates of the authors who provided the abundance and radial velocity information), listed as $D_{B S L}$ in column (5) of Table 1B. For the stars with ambiguous luminosity classifications, we list two alternative distances in column (2).

The small number of stars with uncertain type classifications (either due to the FHB/TO ambiguity noted above, or for the TO stars that appear too blue for their adopted type designation 
when compared with an old isochrone) are noted as such in column 12 of Table 1B, and are ultimately excluded from further analyses.

\subsubsection{Comparison of Photometric and Astrometric Distance Scales}

There are 508 stars in our present catalog which were observed with the Hipparcos astrometric satellite (ESA 1997). To assess the reliability of our photometric distances, $D_{\text {pho }}$, obtained above, we plot in Figure 3 the Hipparcos trigonometric parallaxes, $\pi_{H I P}$, versus our photometric parallaxes, $\pi_{p h o}=1 / D_{p h o}$, for 508 stars with available photometric distances. Plotting parallaxes, rather than distances, in Figure 3 has several advantages. All the trigonometric parallaxes, even small or negative values, can be used in an unbiased way (see the discussion on pp. 27-28 of Arenou \& Luri 1999). Systematic errors in the data can be detected; an incorrect photometric distance scale would be seen as a slope not equal to unity; a zero-point error in the trigonometric parallaxes as a non-zero intercept. For clarity, we have not plotted error bars in Figure 3; the mean error of $\pi_{H I P}$ is $\sim 1.6$ mas; the mean error of $\pi_{p h o}$ is $\leq 20 \%$ of $\pi_{p h o}$.

The general trend of the data in Figure 3 seems to fall slightly below the 45-degree line, indicating that $\pi_{p h o}$ may be $\sim 20 \%$ too large for the nearer stars in the BSL sample. This largely reflects the fact that these nearby stars have the largest errors in $\pi_{p h o}$. The bulk of the data, especially the more distant stars, with smaller $\pi_{p h o}$ errors, falls closer to the 45-degree line. Thus we conclude from Figure 3 that there is not any significant systematic error in our photometric distance scale. It is also worth noting that Figure 3 shows very few outliers, i.e. cases where $\pi_{p h o}$ and $\pi_{H I P}$ badly disagree. The large spread in $\pi_{H I P}$ near $\pi_{p h o}=0$ is due to the large errors in $\pi_{H I P}$ (up to 7 mas) for these faint, distant stars.

For the 424 stars with non-negative Hipparcos parallaxes, Table 1B lists in column (3) the Hipparcos distance estimates $D_{H I P}=1 / \pi_{H I P}$ and in column (4) their relative precisions

$\sigma_{\pi_{H I P}} / \pi_{H I P}$. Because the photometric distances for the nearest stars in our catalog have lower precision than the trigonometric parallaxes, we chose to adopt the Hipparcos distances for 44 stars with $\sigma_{\pi_{H I P}} / \pi_{H I P}<0.12$. The final adopted distance estimates for our program stars are listed as $D_{\text {adopt }}$ in column (6) of Table $1 \mathrm{~B}$.

\section{Proper Motions}

\subsection{Sources of Proper Motions}

\subsubsection{The Hipparcos Catalogue}

The Hipparcos Catalogue is the primary result of the first space mission dedicated to astrometry (ESA 1997), and it provides high accuracy proper motions for 118,218 stars with 
$V<12.5$ mag covering the entire sky. In the present sample, 508 stars are included in this catalog with average accuracies of $1.61 \operatorname{mas~yr}^{-1}$ in $\mu_{\alpha^{*}}\left(=\mu_{\alpha} \cos \delta\right)$ and $1.29 \mathrm{mas} \mathrm{yr}^{-1}$ in $\mu_{\delta}$. Kinematical analyses for a subset of these Hipparcos stars are reported in Chiba \& Yoshii (1998) and Chiba, Yoshii, \& Beers (1999).

\subsubsection{The SPM Catalog 2.0}

The Yale/San Juan Southern Proper Motion (SPM) Catalog 2.0 provides positions, absolute proper motions and photographic $B, V$ magnitudes for 321,608 objects with $5<V<18.5$, mainly in the declination zone from $-37^{\circ}$ to $-27^{\circ}$, except within the Galactic zone of avoidance. In this declination zone an effort was made to measure all HK survey stars from a database provided by T. Beers prior to undertaking measurements of the plates. A total of 505 of our program stars have proper motions extracted from the SPM 2.0 Catalog, using a 10" search radius centered on the catalogued positions, and satisfying the requirement that the apparent magnitudes be within 1 mag in $B$ or $V$ of those adopted in Table 1A. The description of the catalog properties can be find in Platais et al. (1998), however, this paper addresses only the separate catalog at the South Galactic Pole. For additional details of the SPM Catalog 2.0, the reader is referred to the World Wide Web at URL http://www.astro.yale.edu/astrom/. The average accuracy of SPM proper motions for the subset of 508 stars is 2.9 mas $\mathrm{yr}^{-1}$.

\subsubsection{The Lick NPM1 Catalog}

The Lick Northern Proper Motion (NPM) program (Hanson 1997) is a photographic survey measuring absolute proper motions, on an inertial system defined by 50,000 faint galaxies, for over 300,000 stars with $8<B<18$, covering the northern two-thirds of the sky $\left(\delta>-23^{\circ}\right)$. Part I of the NPM program ("NPM1"), outside the disk of the Milky Way ( $|b|>10 \mathrm{deg}$ ), was completed in 1993; the Lick NPM1 Catalog (Klemola, Hanson, and Jones 1993) contains 148,940 stars, with proper motions accurate to 5 mas/yr, and positions accurate to $0^{\prime \prime} 2$ or better. Part II of the NPM program ("NPM2"), in the Milky Way sky, will be completed in roughly three years.

We searched for program stars within a $\pm 10^{\prime \prime} \times 10^{\prime \prime}$ error box, centered on the NPM1 catalog positions (updated to epoch 2000.0 using the NPM1 proper motions). This procedure found matches for 241 stars; $95 \%$ of these comprise a tight core with RMS position difference 0.5 in each coordinate, and can be considered unambiguous matches. The remaining $5 \%$ can be considered highly probable matches; agreement between the cataloged and NPM1 apparent magnitudes (better than $\pm \sim 1 \mathrm{mag}$ ) confirms these.

NPM1 proper motions for an additional 58 RR Lyrae stars, from the list of Layden et al. (1996), were added to our catalogue, making a total of 299 stars with NPM1 proper motion data. 


\subsubsection{The STARNET Catalog}

The proper motions of the STARNET (STN) catalog were determined using the Astrographic Catalogue (AC) as the first epoch positional source, and the astrometrically upgraded Guide Star Catalog (GSC 1.2) as the second epoch source. The AC has been made available in machine readable form by Nesterov et al. (1990). Its reduction to the FK5 system was performed by S. Röser at the Astronomisches Rechen-Institut (ARI). The astrometrical upgrading of the Guide Star Catalog is described in detail by Morrison et al. (1996). GSC 1.2 is presently available at the Astronomical Data Center. The result of combining both catalogs is the STARNET catalog of proper motions (Röser 1996). It contains about 4.3 million stars with an average density of 100 stars per square degree. The median magnitudes of the stars are $B=12.0$ mag in the southern hemisphere and $V=11.5$ in the northern hemisphere, respectively, with the faintest stars reaching $B, V \sim 13.5$. The present rms accuracy of STARNET positions is $0^{\prime \prime} 3$. The accuracy of the proper motions, which have been determined simply from combining the estimated positional errors at both epochs, are about 5 mas $\mathrm{yr}^{-1}$. The catalog is reduced to the FK5 reference system and thus contains some small systematic differences with respect to ICRS (see Vol. 3, Chapter 19 of the Hipparcos and Tycho Catalogues, ESA 1997), in addition to some residual offsets still present in the GSC 1.2 positions. STARNET is as yet unpublished, but is accessible upon request at ARI.

We performed searches for our program stars in STARNET using windows of $40^{\prime \prime}$ diameter centered on the sample stars, and obtained 774 identifications in STARNET, with an average accuracy of the proper motions of 4.7 mas $\mathrm{yr}^{-1}$ in each component, respectively. Since the STARNET catalogue may contain mis-identifications of stars from first epoch to second, proper motions larger than 200 mas $\mathrm{yr}^{-1}$ in one component may be suspect. We therefore inspected such proper motions individually. As it turned out all but one of these stars were listed in the Hipparcos Catalogue; the remaining star could be found in the PPM catalog (Röser and Bastian 1991; Bastian et al. 1993). The agreement between the STARNET motions and the other measurements is usually quite good, with deviations of the order of the expected accuracy of the proper motions.

\subsubsection{The ACT Reference Catalog}

This recently completed catalog by the USNO contains positions and proper motions for 988,758 stars with $V<11 \sim 11.5$ mag covering the entire sky (Urban, Corbin, \& Wycoff 1998a). Accurate proper motions were calculated by combining the positions from the Tycho Catalogue (ESA 1997) with those from new reductions of the Astrographic Catalogue, referred to as AC 2000 (Urban et al. 1998b). We performed a search of our sample stars within a $10^{\prime \prime}$ radius centered at the positions of the cataloged stars, and obtained unambiguous matches for 525 stars. Average

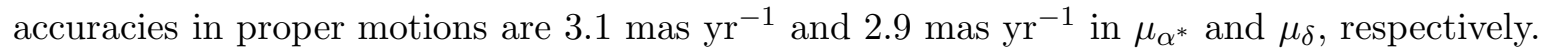




\subsection{Comparison of Proper Motions for Stars with Multiple Measures}

To assess the quality of the adopted proper motions, Figure 4 shows the difference between the Hipparcos Catalogue and other ground-based measurements for each proper-motion component, for the stars in common between the catalogs. While the Hipparcos errors are smallest in general, the figures show that there is an overall agreement with proper motion measurements from the additional sources, without any clearly systematic differences as compared with Hipparcos. It is worth noting that for several stars, the proper motion accuracy in the SPM 2.0 Catalog is better than that in the Hipparcos Catalogue.

For the stars which have been independently measured in two or more catalogs it is possible, by combining all measurements, to reduce the statistical errors as well as minimize any small remaining systematic errors in the individual catalogs, as was argued in Martin \& Morrison (1998). For these stars, we estimate average proper motions, $\langle\mu\rangle$, and their errors, $\left\langle\sigma_{\mu}\right\rangle$, weighted by the inverse variances, as

$$
\begin{aligned}
<\mu> & =\left(\sum_{i=1}^{n} \mu_{i} / \sigma_{\mu_{i}}^{2}\right) /\left(\sum_{i=1}^{n} 1 / \sigma_{\mu_{i}}^{2}\right) \\
<\sigma_{\mu}> & =\left(\sum_{i=1}^{n} 1 / \sigma_{\mu_{i}}^{2}\right)^{-1 / 2}
\end{aligned}
$$

where $n$ denotes the number of measurements. In this manner, the proper motions for 691 stars in the present sample are improved. Our adopted proper motions are listed in columns (7) and (8) of Table 1B; the associated errors are listed in columns (9) and (10). The sources of the proper motions are listed in column (11).

We emphasize the large number of the stars having proper motion data in the present catalog: $N=1258(1214$ with $[\mathrm{Fe} / \mathrm{H}] \leq-0.6)$. This is the largest such dataset among any non-kinematically selected samples, and is substantially larger than a number of recent samples selected on the basis of their high proper motions alone (Ryan \& Norris 1991a: $N=727$ with $[\mathrm{Fe} / \mathrm{H}] \leq-0.6$; Carney et al. 1994: $N=689$ with $[\mathrm{Fe} / \mathrm{H}] \leq-0.6)$. Furthermore, the quality of the proper motion data has been improved in a meaningful manner, compared with that of earlier studies, due to the continuous improvement of astrometric observations from the ground as well as from space. As a consequence the current database allows us to elucidate a great deal of information concerning the space motions of the metal-poor populations of the Milky Way with a lowest-to-date observational error.

\section{The Revised Catalog and its Characteristics}




\subsection{The Catalog}

Tables 1A and 1B present the final revised catalog for 2106 stars. With a constraint of $[\mathrm{Fe} / \mathrm{H}] \leq-0.6$, the present catalog contains 2041 stars, including $356 \mathrm{RR}$ Lyrae variables (168 of which are newly added as compared to the BSL catalog of Paper I), 38 variables of other types, and 1647 non-variables. Proper motions are available for 1214 stars with $[\mathrm{Fe} / \mathrm{H}] \leq-0.6$.

\subsection{Characteristics of the Revised Catalog}

Figure 5a illustrates the distribution of apparent magnitudes for stars in the present catalog. The relatively small number of stars with magnitudes $V>15$, which, for the giants of metal-poor populations, are located at distances in excess of $10 \mathrm{kpc}$ from the Sun, clearly points to the need for future work. The distribution of $(B-V)_{o}$ colors for our catalog stars is shown in Figure 5b. The dominance of $\mathrm{D}$ and MS stars is clear, pointing out the need for additional studies of redder $\mathrm{G}$ and AGB stars of the thick disk and halo, as well as the need for the inclusion of blue FHB stars.

Figure 5c shows the distribution of adopted distances for stars in the present catalog. Stars populating the inner few kpc from the Sun are well represented, with a rather sharp decline beyond $3 \mathrm{kpc}$ from the Sun. The distribution of revised stellar abundances shown in Figure 5d underscores the fact that we have included a large number of stars with abundances below the peak metallicity of the halo metallicity distribution function $([\mathrm{Fe} / \mathrm{H}] \simeq-1.6$, Laird et al. 1988; Ryan \& Norris 1991b). Future work which supplements this sample with additional stars in the intermediate abundance range $-1.5 \leq[\mathrm{Fe} / \mathrm{H}] \leq-0.6$ is clearly needed.

The dashed histograms in Figures 5a-d indicate the distribution of the above observables for the subset of stars with available proper motions. As is evident from inspection of Figure 5a, the great majority of the brighter stars have measured proper motions, but the completeness falls from about $50 \%$ to roughly $30 \%$ for the stars in the magnitude range $12 \leq V \leq 16$. Figure 5 c

shows that the fraction of stars with available proper motions is roughly a constant $\sim 60 \%$ out to distances on the order of $4-5 \mathrm{kpc}$, with no strong dependence on distance.

In Figures $6 \mathrm{a}$ and $6 \mathrm{~b}$ we show the distribution of adopted radial velocities (for all catalog stars with $[\mathrm{Fe} / \mathrm{H}] \leq-0.6$ ) and measured proper motions (where available) as a function of the stellar abundances. The largest radial velocities and proper motions are observed near the center of the abundance distribution, but this is presumably a result of the (presently unavoidable) fact that the relatively rare large observed motions will occur with greatest frequency among the stars which are most commonly represented in the catalog.

The referee has raised the question of whether we have "imported" a kinematic bias into the sample of stars with measured proper motions by the addition of information from the sources we have drawn on. This is demonstrably not the case. None of the five catalogs we have used in our assemblage of proper motion information have lower limits on their proper motions (as 
do, for example, many older catalogs such as those from Giclas and Luyten). The stars in each of these catalogs are chosen in advance of measuring their proper motions, hence a given star is not rejected if its measured proper motion turns out to be small, even if it is consistent with zero. Furthermore, since the proper motions we have included are measured in two independent coordinates, each of which can be individually negative, zero, or positive, errors in the measured proper motions do not result in a net bias away from zero. This bias does exist in many of the older catalogs (where selection was based on the always positive length of a tangential motion vector), but it does not apply to the catalogs we have used for our proper motions.

Since the above biases could lead, in effect, to a distance-related bias in the resulting kinematics, we have performed a simple diagnostic to demonstrate that this is not the case for our sample of stars (other tests are described in Paper III). Figure 6c shows a plot of the estimated tangential velocity of our sample stars as a function of distance from the Sun. Superposed on this figure are lines of constant proper motion, corresponding to $10 \mathrm{mas} / \mathrm{yr}$ and $1 \mathrm{mas} / \mathrm{yr}$, respectively. There does not appear to be any obvious changes in the distribution of predicted tangential velocity $\left(V_{t}=4.74 \mu D\right.$, where $D$ is the distance in $\mathrm{kpc}$, and $\mu$ is in mas/yr) that correlate with increasing distance from the Sun, as might be expected if there were any "hidden" kinematic bias in the sample of stars with measured proper motions.

The spatial distribution of the sample in $(R, Z)$ plane is shown in Figure 7, where filled circles and crosses indicate the stars with and without available proper motions, respectively. Below the Galactic plane there are many remote stars with available proper motions, mainly provided by SPM 2.0.

\subsection{Full Space Motions and Orbital Parameters}

We now obtain estimates of the full space motions for the subsample of stars for which proper motions are available, and derive parameters of their orbital motions in a given gravitational potential. The results are summarized in Table 3. Note that two of the stars in this subsample were judged not to have sufficiently accurate distance estimates to derive space motions, $\mathrm{V}^{*} \mathrm{KR}$ Vir and BPS CS 29512-0032, so these stars are not included in Table 3. Column (1) lists the star name. Column (2) recalls the adopted metallicity from Table 1A. Columns (3) and (4) list the positions of the stars in the meridional plane $(R, Z)$, adopting $R_{\odot}=8.5 \mathrm{kpc}$ as the Galactocentric distance for the Sun. Columns (5)-(7) list the three-dimensional velocities $U, V$, and $W$, directed to the Galactic anticenter, the rotational direction, and the north pole, respectively. These velocity components are corrected for the solar motion $\left(U_{\odot}, V_{\odot}, W_{\odot}\right)=(-9,12,7) \mathrm{km} \mathrm{s}^{-1}$ with respect to the local standard of rest (LSR) (Mihalas \& Binney 1981). Figure 8 is a plot of the $U, V, W$ velocity components for the sample of stars with complete velocity information and $[\mathrm{Fe} / \mathrm{H}] \leq-0.6$, as a function of $[\mathrm{Fe} / \mathrm{H}]$.

Columns (8) and (9) of Table (3) list the velocity components $\left(V_{R}, V_{\phi}\right)$ in the cylindrical rest 
frame $(R, \phi)$, respectively, on the assumption that the rotational speed of the LSR around the Galactic center is $V_{L S R}=220 \mathrm{~km} \mathrm{~s}^{-1}$. Note that for the stars for which accurate distances are not available, columns (2)-(9) contain no data.

To estimate the orbital parameters for these stars, we adopt the analytic Stäckel-type mass model developed by Sommer-Larsen \& Zhen (1990), which consists of a flattened, oblate disk and a nearly spherical massive halo. This model reproduces a flat rotation curve beyond $R=4 \mathrm{kpc}$ and the local mass density at $R_{\odot}$ in a reasonable manner. Its analytic form has the great advantage of maintaining clarity in further analyses (see, e.g., Chiba \& Yoshii 1998). Columns (10) and (11) list the estimated apogalactic distances, $R_{a p}$, and the estimated perigalactic distances, $R_{p r}$ along the Galactic plane, respectively. Column (12) lists the maximum distance above (or below) the plane, $Z_{\text {max }}$, explored by each star in the course of its orbital motion. In column (13) we list the characteristic eccentricities of the orbits, defined as $e=\left(r_{a p}-r_{p r}\right) /\left(r_{a p}+r_{p r}\right)$, where $r_{a p}$ and $r_{p r}$ stand for the apogalactic and perigalactic distances from the Galactic center, respectively. We note that the current mass model of the Galaxy fails to gravitationally bind the 16 stars which lack the data of orbital parameters in columns (10)-(13). These stars probably correspond to those in the "error tail" of overestimated photometric distances, thereby having overestimated transverse velocities.

In the accompanying analysis paper (Chiba \& Beers 2000; Paper III), we make use of the kinematic information in Table 3, and the radial velocity and distance information for the stars presently without available proper motions, to investigate a number of questions concerning the nature of the kinematics of the Galaxy.

\subsection{Future Samples}

Exploration of the kinematic properties of the Galaxy is very much a work in progress. New and extensive samples of several thousand additional non-kinematically selected metal-deficient stars with available velocity, distance, and abundance measurements are expected to become available within the next year (Beers et al. 2000; Cayrel et al. 2000; Norris et al. 2000; Rebolo et al. 2000). Roughly $30 \%$ of these stars already have available proper motions; completing the search for HK survey stars with proper motions awaits the extension of the SPM catalog to other areas of the southern Galactic hemisphere.

Samples of intermediate distance $(2 \leq d \leq 15 \mathrm{kpc})$ FHB and other A-type stars (e.g., Wilhelm et al. 1999), supplemented with observations of more distant metal-poor dwarfs, giants, and FHB and A-type stars from the Hamburg/ESO survey $(10 \leq d \leq 25 \mathrm{kpc}$; Christlieb 1999), and from other sources (e.g., the Sloan Digital Sky Survey, Pier 1999), will provide useful extensions of our catalog.

A project to refine systemic radial velocity measurements for the RR Lyraes with poorly determined values is already underway. Additional $U B V$ and/or Strömgren photometry is required 
in order to resolve ambiguities in stellar classifications which remain in the catalog, and to obtain more precise estimates of distances, especially for the stars with available proper motions.

We are grateful to the referee, Bruce Carney, for a careful reading of this manuscript, and for a number of thoughtful comments.

TCB acknowledges support for this work from grant AST 95-29454 from the National Science Foundation. MC and YY acknowledge partial support from Grants-in-Aid for Scientific Research (09640328) and COE Research (07CE2002) of the Ministry of Education, Science, Sports and Culture of Japan. MC thanks Hideyuki Saio for his help in distance estimates for the current catalog stars. The SPM program is supported by grants from the NSF to Yale University and the Yale Southern Observatory, Inc. IP thanks T. Girard, V. Kozhurina-Platais, and W. van Altena for their expertise and contribution to the SPM program. RBH thanks the National Science Foundation for its long-term support of the Lick Northern Proper Motion program. Current work on the NPM program is supported by NSF grant AST 95-30632. RBH thanks A. Klemola for his help in providing identifications for the Lick NPM1 Catalog stars. BF thanks to S. Röser and S. Frink for help with the STARNET catalog. SR acknowledges partial support for this work from grant 200068/95-4 CNPq, Brazil, and from the Brazilian Agency FAPESP.

This work made use of the SIMBAD database, operated at CDS, Strasbourg, France. 


\section{REFERENCES}

Anthony-Twarog, B.J., \& Twarog, B.A. 1994, AJ, 107, 1577

Anthony-Twarog, B.J., Sarajedini, A., Twarog, B.A., \& Beers, T.C. 2000, AJ, submitted

Arce, H.G., \& Goodman, A.A. 1999, ApJ, 512, L135

Arenou, F., \& Luri, X. 1999, in Harmonizing Cosmic Distance Scales in a Post-Hipparcos Era, eds. D. Egret \& A. Heck (San Francisco: ASP), Vol. 167, p. 13

Bahcall, J.N., \& Casertano, S. 1986, ApJ, 308, 347

Bastian, U. et al. 1993, PPM Star Catalogue, Vols. III and IV (Heidelberg: Spektrum Akademischer Verlag)

Beers, T.C., \& Sommer-Larsen, J. 1995, ApJS, 96, 175 (BSL; Paper I)

Beers, T.C., Preston, G.W., \& Shectman S.A. 1985, AJ, 90, 2089

Beers, T.C., Preston, G.W., \& Shectman, S.A. 1992a, AJ, 103, 1987

Beers, T.C., Preston, G.W., Shectman, S.A., \& Kage, J.A. 1990, AJ, 100, 849

Beers, T.C., Preston, G.W., Shectman, S.A., Doinidis, S.P., \& Griffin, K.E. 1992b, AJ, 103, 267

Beers, T.C., Rossi, S., Norris, J.E., Ryan, S.G., \& Shefler, T. 1999, AJ, 117, 981.

Beers, T.C., Rossi, S., Anthony-Twarog, B., Twarog, B., Hawley, S., Rhee, J., Tourtelot, J., Wilhelm, R., \& Sarajedini, A. 2000, in preparation

Bonifacio, P., Centurion, M., \& Molaro, P. 1999, MNRAS, 309, 533

Buonanno, R., Corsi, C.E., \& Fusi Pecci, F. 1989, A\&A, 216, 80

Burstein, D., \& Heiles, C. 1982, AJ, 87, 1165

Carney, B.W. 1999, in The Third Stromlo Symposium: The Galactic Halo, eds. B.K. Gibson, T.S. Axelrod, \& M.E. Putman, (San Francisco: ASP), Vol. 165, p. 230

Carney, B.W., Laird, J.B., Latham, D.W., \& Aguilar, L.A. 1996, AJ, 112, 668

Carney, B.W., Latham, D.W., Laird, J.B., \& Aguilar, L.A. 1994, AJ, 102, 2240

Cayrel, R., Beers, T.C., Nissen, P.E., Andersen, J., Nördstrom, B., Rossi, S., Spite, M., Spite, F., \& Barbuy, B. 2000, in preparation

Cayrel de Strobel, G., Soubiran, C., Friel, E.D., Ralite, N., \& Francois, P. 1997, A\&AS, 124, 299

Chaboyer, B., Demarque, P., \& Sarajedini, A. 1996, ApJ, 459, 558 
Chaboyer, B. 1999, in Post-Hipparcos Cosmic Candles, eds. A. Heck \& F. Caputo, (Dordrecht: Kluwer), p. 111

Chiba, M., \& Yoshii, Y. 1998, AJ, 115, 168

Chiba, M., Yoshii, Y., \& Beers, T.C. 1999, in The Third Stromlo Symposium: The Galactic Halo, eds. B.K. Gibson, T.S. Axelrod, \& M.E. Putman, (San Francisco: ASP), Vol. 165, p. 273

Chiba, M., \& Beers, T.C. 2000, AJ, submitted (Paper III)

Christlieb, N., Wisotzki, L., Reimers, D., Gehren, T., Reetz, J., \& Beers, T.C. 1999, in The Third Stromlo Symposium: The Galactic Halo, eds. B.K. Gibson, T.S. Axelrod, \& M.E. Putman, (San Francisco: ASP), Vol. 165, p. 259

Deutsch, E.W. 1999, AJ, 118, 1882

Durrell, P.R., \& Harris, W.E. 1993, AJ, 105, 1420

ESA. 1997, The Hipparcos and Tycho Catalogues (ESA SP-1200) (Noordwijk: ESA)

Fernley, J., Barnes, T.G., Skillen, I., Hawley, S.L., Hanley, C.J., Evans, D.W., Solano, E., \& Garrido, R. 1998, A\&A, 330, 515

Gould, A., \& Popowski, P. 1998, ApJ, 508, 844

Hanson, R.B. 1997, in Proper Motions and Galactic Astronomy, ed. R.M. Humphreys, (San Francisco: ASP), Vol. 127, p. 23

Harding, P., Freeman, K.C., Mateo, M., Morrison, H.L., Olszewski, E., \& Norris, J.E. 1998, BAAS, 193, 10507

Harris, W.E. 1982, ApJS, 50, 573

Hartwick, F.D.A. 1987, Mem. S.A.It., 54, 51

Helmi, A., \& White, S.D.M. 1999, MNRAS, 307, 495

Hesser, J.E., Harris, W.E., Vandenberg, D.A., Allwright, J.W.B., \& Stetson, P.B. 1987, PASP, 99, 739

Kinman, T.D., Suntzeff, N.B., \& Kraft, R.P. 1994, AJ, 108, 1722

Klemola, A.R., Hanson, R.B., \& Jones, B.F. 1993, Lick Northern Proper Motions Program: NPM1 Catalog (NSSDC A1199), (Washington: NASA)

Laird, J.B., Carney, B.W., Rupen, M.P., \& Latham, D.W. 1988, AJ, 96, 1908

Layden, A.C. 1994, AJ, 108, 1016 
Layden, A.C., Hanson, R.B., Hawley, S.L., Klemola, A.R., \& Hanley, C.J. 1996, AJ, 112, 2110

Majewski, S.R. 1992, ApJS, 78, 87

Majewski, S.R. 1999, in The Third Stromlo Symposium, eds. B. Gibson, T. Axelrod, \& M. Putman, (San Francisco: ASP), Vol. 165, p. 76

Martin, J.C., \& Morrison, H.L. 1998, AJ, 116, 1724

McWilliam, A., Preston, G.W., Sneden, C., \& Searle, L. 1995, AJ, 109, 2757

Mermilliod, J.C. 1981, A\&A, 97, 235.

Mihalas, D., \& Binney, J. 1981, Galactic Astronomy (2d ed.; San Francisco: Freeman)

Monet, D., Bird, A., Canzian, B., Dahn, C., Guetter, H., Harris, H., Henden, A., Levine, S., Luginbuhl, C., Monet, A.K.B., Rhodes, A., Riepe, B., Sell, S., Stone, R., Vrba, F., \& Walker, R 1998, see http://ftp.nofs.navy.mil/projects/pmm/USNOSA2doc.html

Morrison, H.L. 1993, AJ, 106, 578

Morrison, H.L., Flynn, C., \& Freeman, K.C. 1990, AJ, 100, 1191 (MFF)

Morrison, J.E., Röser, S., Lasker, B.M., Smart, R.L., \& Taff, L.G. 1996, AJ, 111, 1405

Nesterov, V.V., Kislyuk, V.S., \& Potter, Kh.I. 1990, in IAU Symposium No. 141, eds. J.H. Lieske \& V.K. Abalakin, (Dordrecht: Kluwer), p. 482

Norris, J. 1986, ApJS, 61, 667

Norris, J.E. 1994, ApJ, 431, 645

Norris, J., Bessell, M.S., \& Pickles, A.J. 1985, ApJS, 58, 463

Norris, J.E., Ryan, S.G., \& Beers, T.C. 1996, ApJS, 107, 391

Norris, J.E., Beers, T.C., Ryan, S.G., \& Rossi, S. 2000, in preparation

Penny, A.J., \& Dickens, R.J. 1986, MNRAS, 220, 845

Perryman, M.A.C., Brown, A.G.A., Lebreton, Y., Gomez, A., Turon, C., de Strobel, G.C., Mermilliod, J.C., Robichon, N., Kovalevsky, J., \& Crifo, F. 1998, A\&A, 331, 81

Pier, J.R., in The Third Stromlo Symposium: The Galactic Halo, eds. B. Gibson, T. Axelrod, \& M. Putman, (San Francisco: ASP), Vol. 165, p. 274

Platais, I., Girard, T.M., Kozhurina-Platais, V., Van Altena, W.F., Lopez, C.E., Mendez, R.A., Ma, W., Yang, T., MacGillivray, \& Yentis, D.J. 1998, AJ, 116, 2556 
Popowski, P., \& Gould, A. 1998, ApJ, 506, 271

Preston, G.W., Beers, T.C., \& Shectman, S.A. 1994, AJ, 108, 538

Preston, G.W., Shectman, S.A., \& Beers, T.C. 1991, ApJ, 375, 121

Ratnatunga, K.U., \& Freeman, K.C. 1989, ApJ, 339, 126

Rebolo, R., Beers, T.C., Allende Prieto, C., Garcia Lopez, R., Molaro, P., Bonifacio, P., \& Rossi, S. 2000 , in preparation

Röser, S., \& Bastian, U. 1991, PPM Star Catalogue, Vols. I and II (Heidelberg: Spektrum Akademischer Verlag)

Röser, S. 1996, in IAU Symposium No. 172, eds S. Ferraz-Mello et al., (Dordrecht: Kluwer), p. 481

Ryan, S.G., \& Lambert, D.L. 1995, AJ, 109, 2068

Ryan, S.G., Norris, J.E., \& Beers, T.C. 1996, ApJ, 471, 254

Ryan, S.G., \& Norris, J.E. 1991a, AJ, 101, 1835

Ryan, S.G., \& Norris, J.E. 1991b, AJ, 101, 1865

Sandage, A. 1970, ApJ, 162, 841

Schlegel, D.J., Finkbeiner, D.P., \& Davis, M. 1998, ApJ, 500, 525

Schuster, W.J., Nissen, P.E., Parrao, L., Beers, T.C., \& Overgaard, L.P. 1996, A\&AS103, 443

Schuster, W.J., Beers, T.C., Nissen, P.E., Parrao, L., \& Franco, A. 2000, in preparation

Smith, H.A. 1995, "RR Lyrae Stars," Cambridge Astrophysics Series (Cambridge: Cambridge University Press)

Sommer-Larsen, J., \& Zhen, C. 1990, MNRAS, 242, 10

Sommer-Larsen, J., Beers, T.C., Flynn, C., Wilhelm, R., \& Christensen, P.R., 1997 ApJ, 481,775

Urban, S.E., Corbin, T.E., \& Wycoff, G.L. 1998a, ApJ, 115, 2161

Urban, S.E., Corbin, T.E., Wycoff, G.L., Morton, J.C., Jackson, E.S., Zacharias, MI, \& Hall, D.M. 1998b, AJ, 115, 1212

Wilhelm, R. 1995, Ph.D. Dissertation, Michigan State University

Wilhelm, R., Beers, T.C., \& Gray, R. 1999, AJ, 117, 2308 
Wilhelm, R., Beers, T.C., Kriessler, J.R., Pier, J.R., Sommer-Larsen, J., \& Layden, A.C. 1996, in Formation of the Galactic Halo - Inside and Out, eds. H. Morrison \& A. Sarajedini (San Francisco: ASP), Vol. 92, p. 171

Wilhelm, R., Beers, T.C., Sommer-Larsen, J., Pier, J.R., Layden, A.C., Flynn, C., Rossi, S., \& Christensen, P.R. 1999, AJ, 117, 2329

Yoshii, Y. 1982, Pub. Astron. Soc. J., 34, 365

Yoshii, Y., \& Saio, H. 1979, Pub. Astron. Soc. J., 31, 339

Zinn, R. 1996, in Formation of the Galactic Halo - Inside and Out, eds. H. Morrison \& A. Sarajedini (San Francisco: ASP), Vol. 92, p. 211 
Table 1A. Positions, Abundances, Radial Velocities, and Photometry (Abbreviated)

\begin{tabular}{|c|c|c|c|c|c|c|c|c|c|c|c|c|c|}
\hline $\begin{array}{r}\text { Star } \\
(1)\end{array}$ & $\begin{array}{c}\text { RA }(2000.0) \\
(2)\end{array}$ & $\begin{array}{c}\text { DEC } \\
(3) \\
\end{array}$ & $\begin{array}{c}\text { Source }^{\mathrm{a}} \\
(4)\end{array}$ & $\begin{array}{c}{[\mathrm{Fe} / \mathrm{H}]} \\
(\mathrm{dex}) \\
(5)\end{array}$ & $\begin{array}{c}\text { Source }^{b} \\
(6)\end{array}$ & $\begin{array}{c}\mathrm{RV} \\
(\mathrm{km} / \mathrm{s}) \\
(7)\end{array}$ & $\begin{array}{c}\sigma_{\mathrm{RV}} \\
(\mathrm{km} / \mathrm{s}) \\
(8)\end{array}$ & $\begin{array}{c}\text { Source }^{c} \\
(9)\end{array}$ & $\begin{array}{c}V \\
(\mathrm{mag}) \\
(10)\end{array}$ & $\begin{array}{c}B-V \\
(\mathrm{mag}) \\
(11)\end{array}$ & $\begin{array}{c}\text { Source }^{\mathrm{d}} \\
(12)\end{array}$ & $\begin{array}{c}E_{B-V} \\
(\mathrm{mag}) \\
(13)\end{array}$ & $\begin{array}{l}\text { Type } \\
(14)\end{array}$ \\
\hline BPS CS 22957-0026 & 000002.17 & -040708.8 & STN & -1.84 & HK & 14 & 10 & BSL & 13.16 & 0.43 & BPS & 0.04 & $\mathrm{TO}$ \\
\hline V* GV Peg & 000037.00 & +264124.0 & $\mathrm{t}$ & -1.99 & $\mathrm{~L}$ & -335 & 30 & $\mathrm{~L}$ & 13.36 & $\ldots$ & $\mathrm{L}$ & 0.04 & RRV \\
\hline BPS CS 29517-0037 & 000039.51 & -162211.2 & $\mathrm{U}$ & -2.20 & HK & -106 & 10 & BSL & 14.65 & 0.41 & BPS & 0.03 & TO \\
\hline BPS CS 22957-0025 & 000135.35 & -041649.7 & $\mathrm{U}$ & -1.19 & HK & -118 & 10 & BSL & 14.41 & 0.61 & BPS & 0.04 & G \\
\hline BPS CS 22957-0022 & 000145.49 & -054946.6 & STN & -2.44 & HK & 25 & 10 & BSL & 13.34 & 0.61 & BPS & 0.03 & G \\
\hline BPS CS 29517-0029 & 000147.13 & -134725.0 & NPM & -1.77 & HK & -28 & 10 & BSL & 13.99 & 0.43 & BPS & 0.04 & TO \\
\hline BPS CS 29517-0034 & 000147.90 & -155717.7 & $\mathrm{U}$ & -1.76 & HK & -147 & 10 & BSL & 13.85 & 0.42 & BPS & 0.02 & TO \\
\hline $\mathrm{V}^{*} \mathrm{KO}$ Peg & $00 \quad 02 \quad 15.34$ & +300436.6 & $\mathrm{U}$ & -1.87 & BSL & -272 & 30 & BSL & $\ldots$ & $\ldots$ & $\ldots$ & 0.06 & RRV \\
\hline BPS CS 22945-0063 & 000237.00 & -654521.2 & $\mathrm{U}$ & -2.22 & HK & 153 & 10 & BSL & 14.53 & 0.44 & BPS & 0.02 & FHB \\
\hline $\mathrm{V}^{*} \mathrm{RU} \mathrm{Scl}$ & 000248.07 & -245642.9 & HIP & -1.27 & F98 & 34 & 3 & F98 & 10.22 & $\ldots$ & F98 & 0.02 & RRV \\
\hline BPS CS 22957-0031 & $\begin{array}{lll}00 & 03 & 13.17\end{array}$ & -024905.3 & $\mathrm{U}$ & -1.98 & HK & 8 & 10 & BSL & 14.55 & 0.46 & BPS & 0.04 & TO \\
\hline BPS CS 22957-0036 & 000331.24 & -044421.4 & $\mathrm{U}$ & -1.78 & HK & -88 & 10 & BSL & 14.43 & 0.42 & BPS & 0.04 & TO \\
\hline BPS CS 29517-0040 & 000343.02 & -165005.1 & $\mathrm{U}$ & -1.67 & HK & -12 & 10 & BSL & (14.18) & $(0.42)$ & TP & 0.02 & TO \\
\hline $\mathrm{V}^{*} \mathrm{UU}$ Cet & 000405.10 & -165951.5 & HIP & -1.28 & F98 & -114 & 1 & F98 & 12.08 & $\ldots$ & F98 & 0.02 & RRV \\
\hline $\mathrm{V}^{*} \mathrm{AO}$ Tuc & 000406.35 & -592906.2 & $\mathrm{ACT}$ & -1.29 & BSL & 2 & 30 & BSL & 11.13 & $\ldots$ & BSL & 0.01 & RRV \\
\hline [FF93] 513440 & $00 \quad 0414.12$ & -344822.6 & SPM & -0.98 & BSL & 89 & 10 & BSL & 10.71 & 1.29 & SPM & 0.02 & G \\
\hline BPS CS 22876-0040 & 000452.46 & $-3413 \quad 37.2$ & SPM & -2.20 & HK & -191 & 10 & BSL & 15.09 & 0.40 & BPS & 0.01 & TO \\
\hline BPS CS 29503-0010 & 000455.38 & -242419.2 & SPM & -1.88 & HK & -24 & 10 & BSL & 13.74 & 0.54 & SPM & 0.02 & TO \\
\hline BPS CS 29517-0042 & 000503.06 & -162202.1 & $\mathrm{U}$ & -2.17 & HK & -37 & 10 & BSL & 14.10 & 0.39 & BPS & 0.03 & TO \\
\hline BPS CS 29517-0047 & $00 \quad 0514.42$ & -133019.0 & $\mathrm{U}$ & -1.85 & HK & -161 & 10 & BSL & $(13.80)$ & $(0.70)$ & $\mathrm{TP}$ & 0.03 & G \\
\hline HD 20 & $00 \quad 0515.24$ & -271617.8 & HIP & -1.39 & B99 & -57 & 1 & $\mathrm{CY}$ & 9.04 & 0.69 & HIP & 0.01 & G \\
\hline BPS CS 29517-0048 & 000529.86 & -131800.9 & $\mathrm{U}$ & -2.10 & HK & -60 & 10 & BSL & 14.42 & 0.44 & BPS & 0.03 & TO \\
\hline HD 97 & 000546.01 & -194011.3 & HIP & -1.21 & B99 & 76 & 1 & $\mathrm{CY}$ & 9.66 & 0.79 & HIP & 0.02 & G \\
\hline BPS CS 29503-0013 & 000550.64 & -233655.0 & SPM & -1.50 & HK & 22 & 10 & BSL & 14.58 & 0.50 & SPM & 0.02 & TO \\
\hline V* IQ Peg & 000607.64 & +2918 19.7 & $\mathrm{U}$ & -1.54 & BSL & -207 & 30 & BSL & $\ldots$ & $\ldots$ & $\ldots$ & 0.04 & RRV \\
\hline [FF93] 513390 & 000646.28 & -335944.5 & SPM & -0.72 & BSL & 46 & 10 & BSL & 9.78 & 1.14 & SPM & 0.01 & G \\
\hline $\mathrm{V}^{*} \mathrm{NN}$ And & 000655.86 & +312813.3 & $\mathrm{U}$ & -1.79 & BSL & -276 & 10 & BSL & $\ldots$ & $\ldots$ & $\ldots$ & 0.06 & $\mathrm{~V}$ \\
\hline BPS CS 29503-0022 & $00 \quad 0724.91$ & -241822.0 & SPM & -2.06 & HK & 14 & 10 & BSL & 14.58 & 0.71 & SPM & 0.02 & SG \\
\hline BPS CS 22876-0032 & $00 \quad 0737.46$ & -353116.7 & SPM & -3.85 & B99 & -90 & 10 & HK & 12.84 & 0.39 & BPS & 0.01 & TO \\
\hline BPS CS 29503-0017 & 000745.02 & -230704.3 & SPM & -1.98 & HK & 14 & 10 & BSL & 14.31 & 0.49 & SPM & 0.02 & TO \\
\hline
\end{tabular}

a Positions: U: USNO-A 2.0; HIP: Hipparcos; SPM: SPM 2.0; STN: STARNET; ACT: ACT; NPM: NPM1; SBD: SIMBAD; GSC: GSC 1.2; v: rather uncertain; t: uncertain

b Abundances: HK: HK survey; B99: Beers et al. 1999; C97: Cayrel et al. 1997; McW: McWilliam et al. 1995; BCM: Bonifacio, Centurion, \& Molaro 1999; RL: Ryan \& Lambert 1995; BSL: values adopted in the original BSL catalog; MFF: Morrison, Flynn, Freeman 1990; W99: Wilhelm et al. 1999; L: Layden 1994, Layden et al. 1996; F98: Fernley et al. 1998

Radial Velocities: HK: HK survey; BSL: values adopted in the original BSL catalog; L: Layden 1994, Layden et al. 1996; F98: Fernley et al. 1998; CY: values adopted in Chiba \& Yoshii 1998; ATT: Anthony-Twarog \& Twarog 1994

dPhotometry: BPS: HK survey; SBD: SIMBAD; HIP: Hipparcos; GSC: GSC 1.2; SC1: Schuster et al. 1996; SC2: Schuster et al. 2000; SAT: Anthony-Twarog et al. 2000; BSL: values adopted in the original BSL catalog; TP: This paper; L: intensity averaged $\langle V\rangle$ from Layden 1994, Layden et al. 1996; F98: intensity averaged $<V\rangle$ from Fernley et al. 1998 
TABLE 1B. Distance Estimates and Measured Proper Motions (Abbreviated)

\begin{tabular}{|c|c|c|c|c|c|c|c|c|c|c|c|}
\hline $\begin{array}{l}\text { Star } \\
(1)\end{array}$ & $\begin{array}{c}D_{p h o} \\
(\mathrm{kpc}) \\
(2)\end{array}$ & $\begin{array}{c}D_{H I P} \\
(\mathrm{kpc}) \\
(3)\end{array}$ & $\begin{array}{c}\sigma_{\pi_{H I P}} / \pi_{H I P} \\
(4)\end{array}$ & $\begin{array}{c}D_{B S L} \\
(\mathrm{kpc}) \\
(5)\end{array}$ & $\begin{array}{c}D_{\text {adopt }} \\
(\mathrm{kpc}) \\
(6)\end{array}$ & $\begin{array}{c}\mu_{\alpha^{*}} \\
(\mathrm{mas} / \mathrm{yr}) \\
(7)\end{array}$ & $\begin{array}{c}\mu_{\delta} \\
(\mathrm{mas} / \mathrm{yr}) \\
(8)\end{array}$ & $\begin{array}{c}\sigma_{\mu_{\alpha *}} \\
(\mathrm{mas} / \mathrm{yr}) \\
(9)\end{array}$ & $\begin{array}{c}\sigma_{\mu_{\delta}} \\
(\mathrm{mas} / \mathrm{yr}) \\
(10)\end{array}$ & $\begin{array}{c}\text { Source } \\
(11)\end{array}$ & $\begin{array}{c}\text { Comments } \\
\text { (12) }\end{array}$ \\
\hline BPS CS 22957-0026 & 0.601 & $\ldots$ & $\ldots$ & 0.700 & 0.601 & 108. & -53 & 4.6 & 4.6 & STN & \\
\hline V* GV Peg & 3.586 & $\ldots$ & $\ldots$ & $\ldots$ & 3.586 & 6.9 & -30.7 & 5 & 5 & NPM & \\
\hline BPS CS 29517-0037 & 1.325 & $\ldots$ & $\ldots$ & 1.300 & 1.325 & $\ldots$ & $\ldots$ & $\ldots$ & $\ldots$ & $\ldots$ & \\
\hline BPS CS 22957-0025 & 1.337 & $\ldots$ & $\ldots$ & 2.100 & 1.337 & $\ldots$ & $\ldots$ & $\ldots$ & $\ldots$ & $\ldots$ & \\
\hline BPS CS 22957-0022 & 2.407 & $\cdots$ & $\ldots$ & 2.200 & 2.407 & 5. & -29 & 4.3 & 4.3 & STN & \\
\hline BPS CS 29517-0029 & 0.874 & $\ldots$ & $\ldots$ & 0.900 & 0.874 & 6.6 & -38.5 & 5 & 5 & NPM & \\
\hline BPS CS 29517-0034 & 0.855 & $\ldots$ & $\ldots$ & 0.900 & 0.855 & $\ldots$ & $\ldots$ & $\ldots$ & $\ldots$ & $\ldots$ & \\
\hline $\mathrm{V}^{*} \mathrm{KO}$ Peg & $\ldots$ & $\ldots$ & $\ldots$ & 13.182 & 13.182 & $\ldots$ & $\ldots$ & $\ldots$ & $\ldots$ & $\ldots$ & \\
\hline BPS CS 22945-0063 & 6.447 & $\ldots$ & $\ldots$ & 1.700 & 6.447 & $\ldots$ & $\ldots$ & $\ldots$ & $\ldots$ & $\ldots$ & \\
\hline $\mathrm{V}^{*} \mathrm{RU} \mathrm{Scl}$ & 0.801 & 1.587 & 2.65 & 0.855 & 0.801 & 53.7 & -18.4 & 1.2 & 0.9 & HIP,SPM,ACT,STN & \\
\hline BPS CS 22957-0031 & 1.107 & $\ldots$ & $\ldots$ & 1.300 & 1.107 & $\ldots$ & $\ldots$ & $\ldots$ & $\ldots$ & $\ldots$ & \\
\hline BPS CS 22957-0036 & 1.099 & $\ldots$ & $\ldots$ & 1.200 & 1.099 & $\ldots$ & $\ldots$ & $\ldots$ & $\ldots$ & $\ldots$ & \\
\hline BPS CS 29517-0040 & 1.038 & $\ldots$ & $\ldots$ & 1.200 & 1.038 & $\ldots$ & $\ldots$ & $\ldots$ & $\ldots$ & $\ldots$ & \\
\hline $\mathrm{V}^{*} \mathrm{UU}$ Cet & 1.884 & 0.154 & 0.64 & 1.888 & 1.884 & 30.3 & -1.3 & 3.0 & 1.7 & HIP,STN,NPM & \\
\hline $\mathrm{V}^{*} \mathrm{AO}$ Tuc & $\ldots$ & $\ldots$ & $\ldots$ & 1.230 & 1.230 & 15.6 & -49.5 & 2.6 & 3.0 & $\mathrm{ACT}, \mathrm{STN}$ & \\
\hline [FF93] 513440 & 2.467 & $\ldots$ & $\ldots$ & 1.122 & 2.467 & 6.3 & -3.6 & 1.7 & 1.7 & SPM,STN & \\
\hline BPS CS 22876-0040 & 1.682 & $\cdots$ & $\cdots$ & 1.700 & 1.682 & -7.0 & -18.8 & 2.6 & 2.6 & SPM & \\
\hline BPS CS 29503-0010 & 0.697 & $\ldots$ & $\ldots$ & 0.900 & 0.697 & -9.1 & -2.2 & 2.8 & 2.4 & SPM & type uncertain \\
\hline BPS CS 29517-0042 & 1.066 & $\cdots$ & $\ldots$ & 1.300 & 1.066 & $\ldots$ & $\ldots$ & $\cdots$ & $\cdots$ & $\cdots$ & \\
\hline BPS CS 29517-0047 & 2.354 & $\cdots$ & $\cdots$ & 5.700 & 2.354 & $\cdots$ & $\ldots$ & $\cdots$ & $\ldots$ & $\cdots$ & \\
\hline HD 20 & 0.169 & 0.714 & 0.94 & $\ldots$ & 0.169 & 132.7 & -40.7 & 0.9 & 0.7 & HIP,SPM,ACT,STN & \\
\hline BPS CS 29517-0048 & 1.105 & & & 1.200 & 1.105 & $\ldots$ & & $\ldots$ & & & \\
\hline HD 97 & 0.342 & $<0$ & $\ldots$ & 0.500 & 0.342 & -23.3 & -178.5 & 1.2 & 0.7 & HIP,ACT,STN,NPM & \\
\hline BPS CS 29503-0013 & 1.046 & $\cdots$ & $\ldots$ & 1.100 & 1.046 & 5.3 & -16.1 & 5.8 & 6.2 & SPM & \\
\hline V* IQ Peg & $\ldots$ & $\ldots$ & $\ldots$ & 12.941 & 12.941 & $\ldots$ & $\ldots$ & $\ldots$ & $\ldots$ & & \\
\hline [FF93] 513390 & 1.135 & $\ldots$ & $\ldots$ & 1.556 & 1.135 & -10.2 & -4.7 & 1.6 & 1.6 & SPM,ACT,STN & \\
\hline $\mathrm{V}^{*} \mathrm{NN}$ And & $\ldots$ & $\ldots$ & $\ldots$ & 17.060 & 17.060 & $\ldots$ & $\ldots$ & $\ldots$ & $\ldots$ & $\ldots$ & \\
\hline BPS CS $29503-0022$ & $\ldots$ & $\ldots$ & $\ldots$ & 1.500 & 1.500 & 3.3 & -19.5 & 7.2 & 6.6 & SPM & \\
\hline BPS CS 22876-0032 & 0.553 & $\ldots$ & $\cdots$ & 0.509 & 0.553 & 81.2 & -126.5 & 2.2 & 4.9 & SPM,STN & \\
\hline BPS CS 29503-0017 & 0.997 & $\ldots$ & $\ldots$ & 1.200 & 0.997 & -9.7 & -66.1 & 6.9 & 7.1 & SPM & \\
\hline
\end{tabular}


TABle 2. Formulae for Absolute Magnitude Computation

\begin{tabular}{|c|c|c|c|c|}
\hline$a_{0}$ & $a_{1}$ & $a_{2}$ & $a_{3}$ & Abundance and Color Ranges \\
\hline \multicolumn{5}{|c|}{ (1) G \& SG stars: $y=a_{0}+a_{1} x+a_{2} x^{2}+a_{3} x^{3}, y \equiv M_{V}-5.0(B-V)_{o}, x \equiv(B-V)_{o}-0.12([\mathrm{Fe} / \mathrm{H}]+2.0)$} \\
\hline $\begin{array}{r}-1.10 \\
23.5191 \\
4.74\end{array}$ & $\begin{array}{r}-5.86 \\
-61.2122 \\
-8.08\end{array}$ & $\begin{array}{c}\ldots \\
42.0210 \\
\ldots\end{array}$ & $\begin{array}{c}\ldots \\
-10.7516 \\
\ldots\end{array}$ & $\begin{array}{c}1.38 \leq x \\
0.58 \leq x<1.38 \\
0.31 \leq x<0.58\end{array}$ \\
\hline \multicolumn{5}{|c|}{ (2) AGB stars: $y=a_{0}+a_{1} x+a_{2} x^{2}+a_{3} x^{3}, y \equiv M_{V}-5.0(B-V)_{o}, x \equiv(B-V)_{o}-0.12([\mathrm{Fe} / \mathrm{H}]+2.0)$} \\
\hline 0.7814 & -2.2979 & -8.9748 & 3.9225 & $0.4<x<1.3$ \\
\hline \multicolumn{5}{|c|}{$(3)^{a}$ D stars: $M_{V}=a_{0}+a_{1}(B-V)_{o}+a_{2}(B-V)_{o}^{2}+a_{3}(B-V)_{o}^{3}$} \\
\hline $\begin{array}{l}-1.6592 \\
-1.0971 \\
-2.1576 \\
-0.4954\end{array}$ & $\begin{array}{l}19.9889 \\
17.0413 \\
19.9889 \\
11.4212\end{array}$ & $\begin{array}{r}-15.0820 \\
-7.7057 \\
-15.0820 \\
-6.3003\end{array}$ & $\begin{array}{r}4.7913 \\
-0.7223 \\
4.7913 \\
1.8704\end{array}$ & $\begin{array}{c}{[\mathrm{Fe} / \mathrm{H}]=-2.20,0.85<(B-V)_{o}} \\
{[\mathrm{Fe} / \mathrm{H}]=-2.20,0.34 \leq(B-V)_{o} \leq 0.85} \\
{[\mathrm{Fe} / \mathrm{H}]=-0.71,0.34 \leq(B-V)_{o}} \\
{[\mathrm{Fe} / \mathrm{H}]=+0.12,0.34 \leq(B-V)_{o}}\end{array}$ \\
\hline \multicolumn{5}{|c|}{$(4)^{b}$ A stars: $M_{V}=a_{0}+a_{1}(B-V)_{o}+a_{2}(B-V)_{o}^{2}+a_{3}(B-V)_{o}^{3}$} \\
\hline $\begin{array}{l}2.1309 \\
1.4366 \\
1.1261\end{array}$ & $\begin{array}{l}8.6627 \\
8.6627 \\
8.6627\end{array}$ & $\begin{array}{l}-26.7677 \\
-26.7677 \\
-26.7677\end{array}$ & $\begin{array}{l}45.6924 \\
45.6924 \\
45.6924\end{array}$ & $\begin{array}{l}{[\mathrm{Fe} / \mathrm{H}]=-2.20,(B-V)_{o}<0.34} \\
{[\mathrm{Fe} / \mathrm{H}]=-0.71,(B-V)_{o}<0.34} \\
{[\mathrm{Fe} / \mathrm{H}]=+0.12,(B-V)_{o}<0.34}\end{array}$ \\
\hline \multicolumn{5}{|c|}{$(5)^{c}$ TO stars: $y=a_{0}+a_{1}[\mathrm{Fe} / \mathrm{H}]+a_{2}[\mathrm{Fe} / \mathrm{H}]^{2}, y \equiv\left(M_{V}(\mathrm{D})-M_{V}\right) /\left(M_{V}(\mathrm{D})-M_{V}(\mathrm{SG})\right)$} \\
\hline 0.4063 & -0.0141 & 0.0061 & $\cdots$ & \\
\hline
\end{tabular}

${ }^{a}$ Archetypes are M92+M15 for $[\mathrm{Fe} / \mathrm{H}]=-2.20,47$ Tuc for $[\mathrm{Fe} / \mathrm{H}]=-0.71$, and Hyades for $[\mathrm{Fe} / \mathrm{H}]=+0.12$.

${ }^{b}$ An archetype is Pleiades with $[\mathrm{Fe} / \mathrm{H}]=+0.11$. For $[\mathrm{Fe} / \mathrm{H}]=-0.71$ and $[\mathrm{Fe} / \mathrm{H}]=-2.20$, the relation for Pleiades is simply shifted towards fainter absolute magnitudes so as to match the relations for D stars at $(B-V)_{o}=0.34$.

${ }^{\mathrm{c}} M_{V}$ (D) and $M_{V}(\mathrm{SG})$ denote the absolute magnitudes of $\mathrm{D}$ and $\mathrm{SG}$, respectively, calibrated for the given $(B-V)_{o}$ and $[\mathrm{Fe} / \mathrm{H}]$. The tabulated formula is applied to the case $M_{V}(\mathrm{D})>M_{V}(\mathrm{SG})$, unless otherwise $M_{V}(\mathrm{D})$ is adopted. 
TABle 3. Space Motions and Orbital Parameters (Abbreviated)

\begin{tabular}{|c|c|c|c|c|c|c|c|c|c|c|c|c|c|}
\hline $\begin{array}{l}\text { Star } \\
(1)\end{array}$ & $\begin{array}{c}{[\mathrm{Fe} / \mathrm{H}]} \\
(\mathrm{dex}) \\
(2)\end{array}$ & $\begin{array}{c}R \\
(\mathrm{kpc}) \\
(3)\end{array}$ & $\begin{array}{c}Z \\
(\mathrm{kpc}) \\
(4)\end{array}$ & $\begin{array}{c}U \\
(\mathrm{~km} / \mathrm{s}) \\
(5)\end{array}$ & $\begin{array}{c}V \\
(\mathrm{~km} / \mathrm{s}) \\
(6)\end{array}$ & $\begin{array}{c}W \\
(\mathrm{~km} / \mathrm{s}) \\
(7)\end{array}$ & $\begin{array}{c}V_{R} \\
(\mathrm{~km} / \mathrm{s}) \\
(8)\end{array}$ & $\begin{array}{c}V_{\phi} \\
(\mathrm{km} / \mathrm{s}) \\
(9)\end{array}$ & $\begin{array}{c}R_{a p} \\
(\mathrm{kpc}) \\
(10)\end{array}$ & $\begin{array}{c}R_{p r} \\
(\mathrm{kpc}) \\
(11)\end{array}$ & $\begin{array}{c}Z_{\max } \\
(\mathrm{kpc}) \\
(12)\end{array}$ & $\begin{array}{c}e \\
(13)\end{array}$ & $\begin{array}{c}\text { Comments } \\
\text { (14) }\end{array}$ \\
\hline BPS CS 22957-0026 & -1.84 & 8.52 & -0.54 & 186 & -236 & -126 & 185 & -22 & 13.38 & 0.59 & 6.17 & 0.92 & \\
\hline $\mathrm{V}^{*}$ GV Peg & -1.99 & 9.86 & -2.05 & -209 & -533 & -241 & -289 & -241 & 76.34 & 7.82 & 56.49 & 0.81 & \\
\hline BPS CS 22957-0022 & -2.44 & 8.59 & -2.19 & -120 & -266 & -148 & -125 & -31 & 10.56 & 3.26 & 10.68 & 0.55 & \\
\hline BPS CS 29517-0029 & -1.77 & 8.46 & -0.83 & -61 & -143 & -9 & -58 & 79 & 8.83 & 2.02 & 0.92 & 0.63 & \\
\hline $\mathrm{V}^{*} \mathrm{RU} \mathrm{Scl}$ & -1.27 & 8.39 & -0.79 & 131 & -137 & -68 & 132 & 81 & 10.60 & 2.02 & 2.00 & 0.68 & \\
\hline $\mathrm{V}^{*} \mathrm{UU}$ Cet & -1.28 & 8.37 & -1.82 & 230 & -149 & 66 & 233 & 58 & 15.72 & 1.53 & 7.74 & 0.82 & \\
\hline $\mathrm{V}^{*} \mathrm{AO}$ Tuc & -1.29 & 8.05 & -1.03 & -19 & -260 & 139 & -17 & -42 & 8.23 & 1.70 & 5.96 & 0.67 & \\
\hline [FF93] 513440 & -0.98 & 7.96 & -2.41 & 17 & -61 & -88 & 16 & 159 & 8.65 & 5.88 & 3.74 & 0.20 & \\
\hline BPS CS 22876-0040 & -2.20 & 8.14 & -1.64 & -83 & -92 & 221 & -84 & 127 & 14.11 & 5.92 & 11.28 & 0.42 & \\
\hline BPS CS 29503-0010 & -1.88 & 8.41 & -0.69 & -35 & 16 & 36 & -33 & 236 & 10.57 & 8.01 & 1.12 & 0.14 & \\
\hline HD 20 & -1.39 & 8.47 & -0.17 & 77 & -70 & 44 & 78 & 149 & 9.43 & 4.26 & 0.83 & 0.38 & \\
\hline HD 97 & -1.21 & 8.47 & -0.33 & -188 & -206 & -100 & -188 & 16 & 12.68 & 0.40 & 4.81 & 0.94 & \\
\hline BPS CS 29503-0013 & -1.50 & 8.37 & -1.03 & -27 & -67 & -25 & -24 & 154 & 8.51 & 4.80 & 1.21 & 0.28 & \\
\hline [FF93] 513390 & -0.72 & 8.27 & -1.11 & -77 & 14 & -26 & -78 & 234 & 11.88 & 7.16 & 1.81 & 0.25 & \\
\hline BPS CS 29503-0022 & -2.06 & 8.32 & -1.48 & -56 & -119 & -19 & -54 & 103 & 8.73 & 2.86 & 1.70 & 0.51 & \\
\hline BPS CS 22876-0032 & -3.85 & 8.38 & -0.54 & 49 & -376 & 104 & 49 & -156 & 8.89 & 5.60 & 3.14 & 0.23 & \\
\hline BPS CS 29503-0017 & -1.98 & 8.39 & -0.98 & -200 & -238 & -23 & -200 & -15 & 12.76 & 0.32 & 2.07 & 0.95 & \\
\hline BPS CS 29503-0026 & -1.96 & 8.42 & -0.60 & 100 & -63 & 25 & 102 & 156 & 10.12 & 4.33 & 0.98 & 0.40 & \\
\hline BPS CS 29503-0015 & -1.76 & 8.39 & -1.13 & -111 & -183 & 46 & -110 & 39 & 9.76 & 0.94 & 1.62 & 0.82 & \\
\hline $\mathrm{V}^{*} \mathrm{RY}$ Psc & -1.39 & 8.72 & -1.83 & 292 & -227 & -84 & 289 & -38 & 23.20 & 0.76 & 5.11 & 0.94 & \\
\hline HD 868 & -0.79 & 8.37 & -0.82 & 84 & 21 & -30 & 85 & 241 & 12.82 & 7.10 & 1.35 & 0.29 & \\
\hline HD 891 & -0.61 & 8.42 & -0.91 & 4 & -101 & 41 & 6 & 119 & 8.46 & 3.47 & 1.34 & 0.42 & \\
\hline HD 941 & -0.86 & 8.47 & -0.28 & 98 & -167 & 62 & 98 & 53 & 9.43 & 1.33 & 1.58 & 0.75 & \\
\hline BPS CS 29503-0031 & -2.09 & 8.41 & -0.68 & 6 & -62 & -32 & 7 & 158 & 8.45 & 4.97 & 0.93 & 0.26 & \\
\hline HD 991 & -0.88 & 8.39 & -0.55 & 40 & -154 & -31 & 40 & 66 & 8.57 & 1.66 & 0.78 & 0.68 & \\
\hline BPS CS 30339-0002 & -1.54 & 8.36 & -0.70 & -129 & -217 & 76 & -129 & 2 & 10.23 & 0.06 & 2.29 & 0.99 & \\
\hline BPS CS 29503-0042 & -2.17 & 8.43 & -0.68 & -16 & -72 & 23 & -15 & 148 & 8.50 & 4.48 & 0.80 & 0.31 & \\
\hline BPS CS 30339-0019 & -1.77 & 8.32 & -1.07 & -19 & -333 & -40 & -19 & -113 & 8.41 & 3.25 & 1.50 & 0.44 & \\
\hline BPS CS $30339-0007$ & -1.28 & 8.36 & -0.72 & -96 & -243 & 44 & -96 & -23 & 9.31 & 0.56 & 1.20 & 0.89 & \\
\hline CD-23 72 & -1.17 & 8.49 & -0.18 & -9 & -10 & -17 & -9 & 210 & 8.54 & 7.64 & 0.28 & 0.06 & \\
\hline
\end{tabular}


Fig. 4.- Distribution of the differences $\Delta \mu$ between Hipparcos and ground-based measurements for each proper-motion component, plotted against the Hipparcos proper motions $\mu[\mathrm{HIP}]$ (filled circles: $\Delta \mu_{\alpha^{*}}$ vs $\mu_{\alpha^{*}}[\mathrm{HIP}]$, open circles: $\Delta \mu_{\delta}$ vs $\left.\mu_{\delta}[\mathrm{HIP}]\right)$.

Fig. 6.- (a) Heliocentric radial velocities, $V_{\text {rad }}$, for stars with $[\mathrm{Fe} / \mathrm{H}] \leq-0.6$ in the present catalog, as a function of $[\mathrm{Fe} / \mathrm{H}]$. (b) Total proper motions, $\mu=\left(\mu_{\alpha^{*}}^{2}+\mu_{\delta}^{2}\right)^{1 / 2}$, for stars with $[\mathrm{Fe} / \mathrm{H}] \leq-0.6$ as a function of $[\mathrm{Fe} / \mathrm{H}]$. Panel (b) clearly indicates the presence of a substantial number of quite metalpoor stars having small proper motions, stars which have been absent in previous kinematically selected samples (see also the discussion in the text). (c) Distribution of estimated tangential velocities, $V_{t}$, as a function of distance from the Sun. The solid and dashed lines correspond to lines of constant proper motion of magnitude 10 mas/yr and 1 mas/yr, respectively.

Fig. 7.- Distribution of the complete sample in the $(R, Z)$ plane, where a Galactocentric distance of $R_{\odot}=8.5 \mathrm{kpc}$ has been assumed. Filled circles and crosses indicate the stars with and without available proper motions, respectively.

Fig. 8.- Local velocity components, $U, V, W$, for the stars in the revised catalog with complete kinematic information and with abundances $[\mathrm{Fe} / \mathrm{H}] \leq-0.6$, as a function of metallicity. 


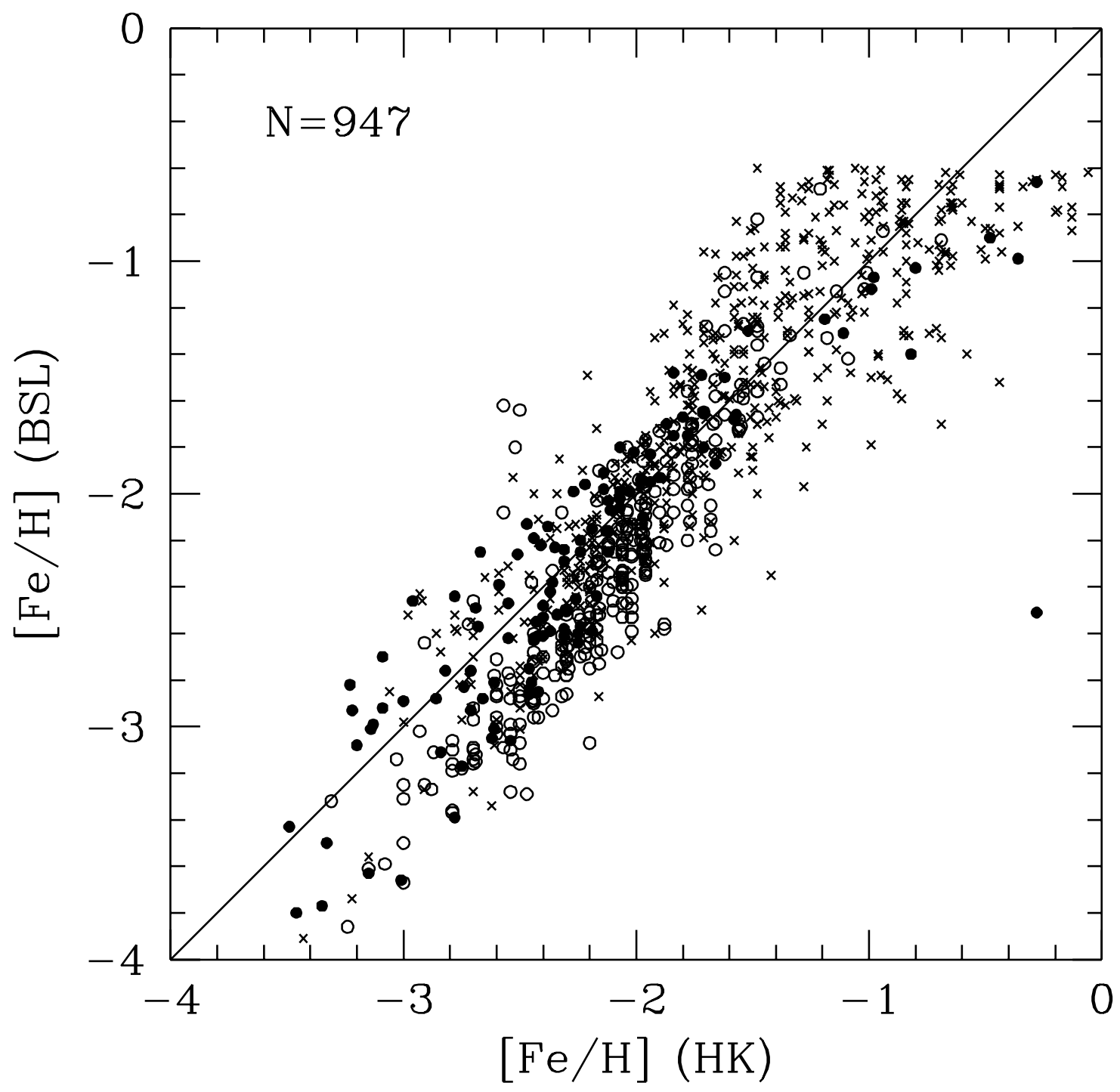

Fig. 1.- Comparison of estimated stellar abundances from the BSL catalog of Paper I with the revised abundances obtained in the HK survey. A one-to-one line is shown. Stars with $0.3 \leq(B-V)_{o} \leq 0.5$ are indicated with open circles, while cooler stars are indicated with filled circles, respectively. Stars without available photometry, where abundance estimates rely on a revised correlation of the Balmer-line index $H P 2$ with de-reddened $(B-V)_{o}$ color (see Beers et al. 1999) are indicated with crosses. 

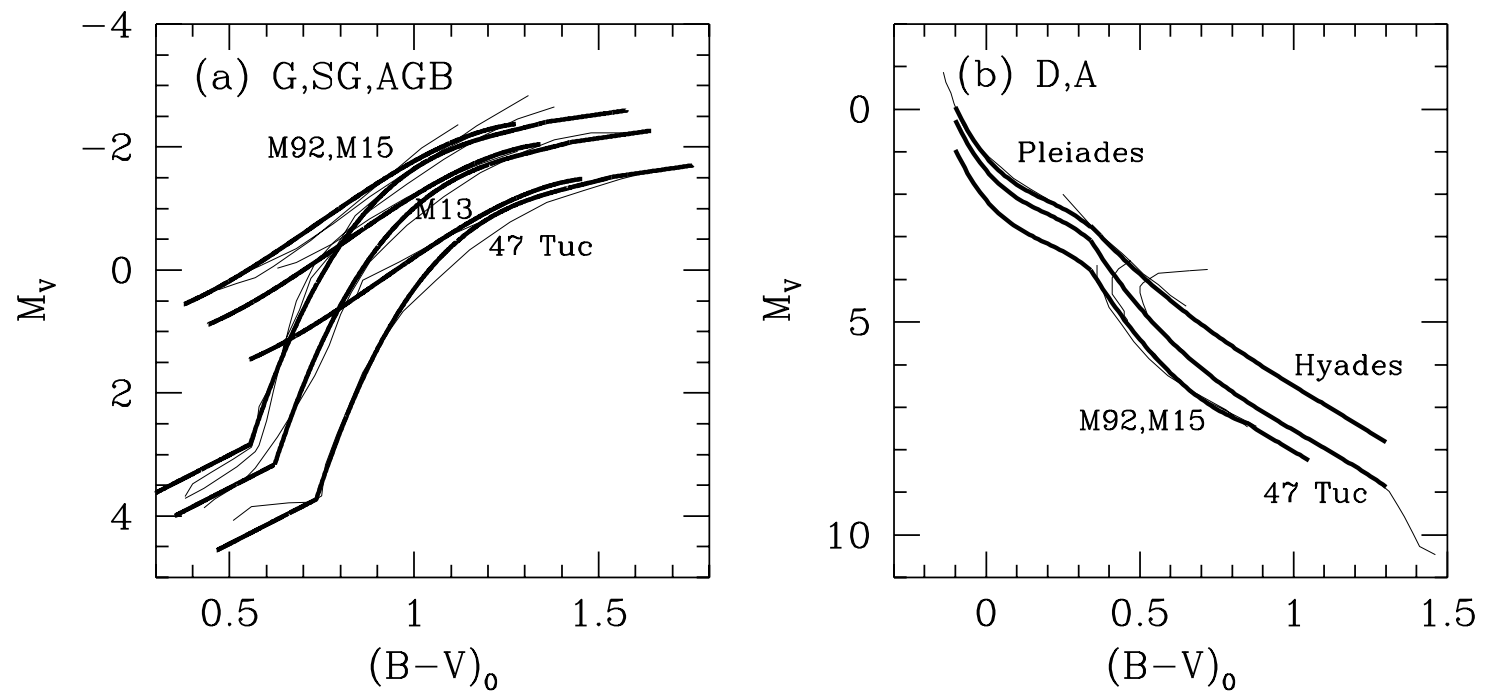

Fig. 2.- (a) $M_{V}$ vs $(B-V)_{o}$ relations for $\mathrm{G}, \mathrm{SG}$, and AGB stars in the clusters M92 $([\mathrm{Fe} / \mathrm{H}]=-2.24), \mathrm{M} 15(-2.15), \mathrm{M} 13(-1.65)$, and 47 Tuc $(-0.71)$ (thin solid lines), together with the adopted $M_{V}$ vs $(B-V)_{o}$ relations at $[\mathrm{Fe} / \mathrm{H}]=-2.20,-1.65$, and -0.71 (thick solid lines). (b) The same as in panel (a) but for D stars of M92, M15, 47 Tuc, and Hyades $([\mathrm{Fe} / \mathrm{H}]=+0.12)$. 


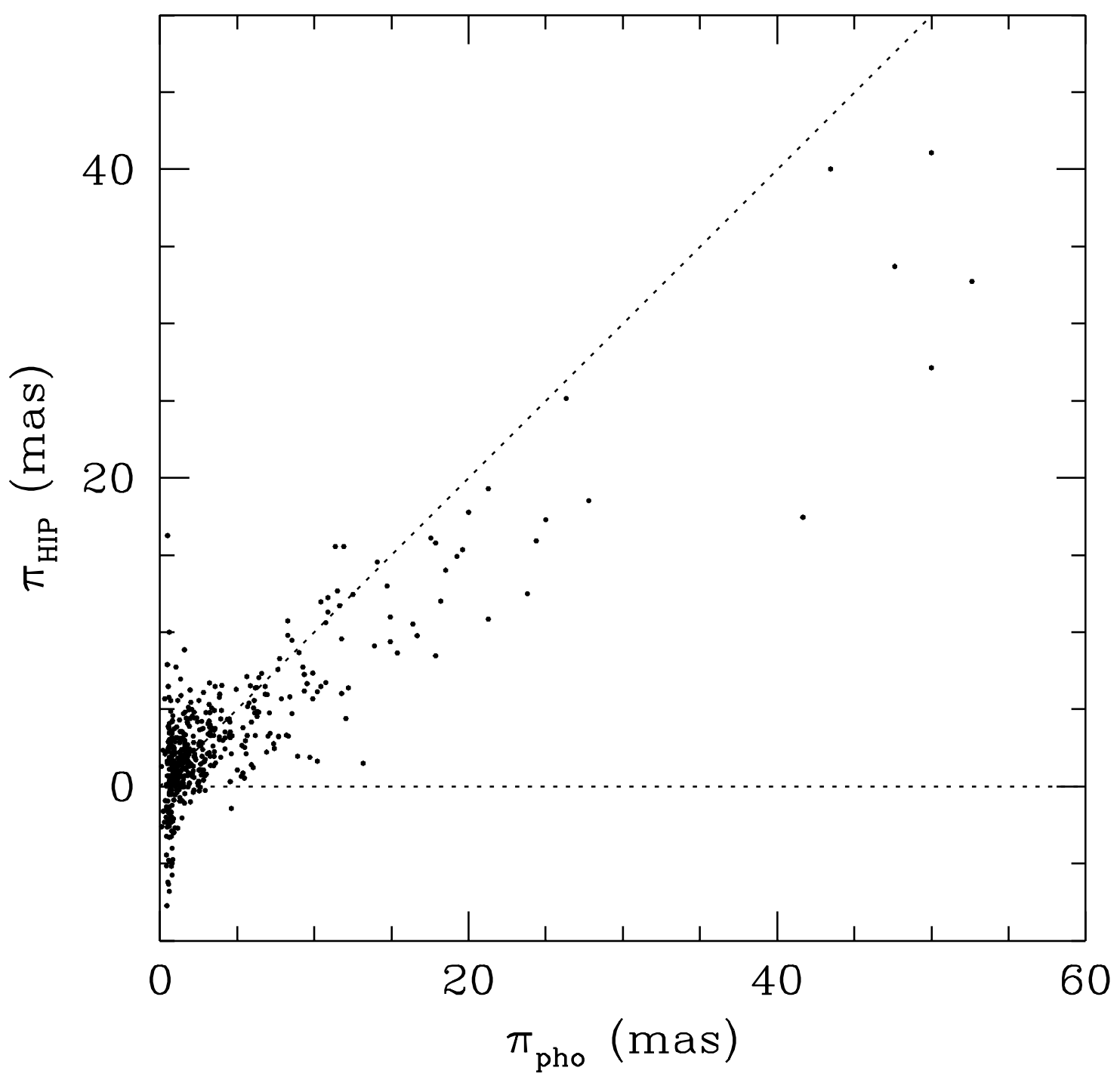

Fig. 3.- Hipparcos trigonometric parallax $\pi_{H I P}$ versus photometric parallax $\pi_{\text {pho }}$ for 508 Hipparcos stars having photometric distance estimates in our catalog. Units are milli-arc-seconds (mas). The horizontal dashed line indicates $\pi_{H I P}=0$. The 45-degree dashed line indicates $\pi_{H I P}=\pi_{p h o}$. 

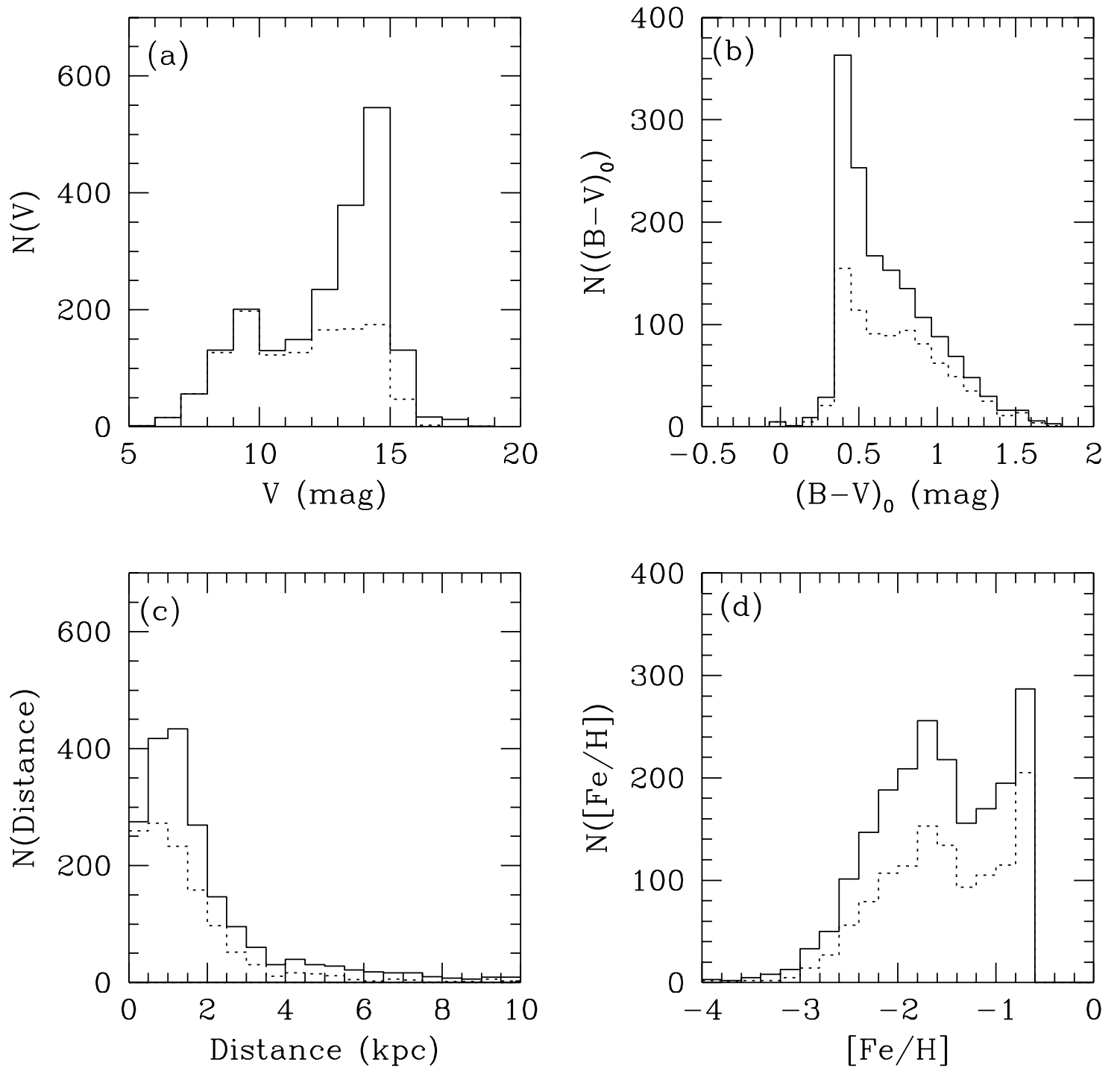

Fig. 5.- Distributions of (a) $V$ magnitudes, (b) $(B-V)_{o}$ colors, (c) adopted distance estimates, and $(\mathrm{d})$ metal abundances for stars with $[\mathrm{Fe} / \mathrm{H}] \leq-0.6$ in the present catalog. The solid lines apply for the full sample of stars, while the dashed lines apply for the subsample of stars with available proper motions. 
TABle 1A. Positions, Abundances, Radial Velocities, and Photometry

\begin{tabular}{|c|c|c|c|c|c|c|c|c|c|c|}
\hline $\begin{array}{l}\text { Star } \\
\text { (1) }\end{array}$ & (2) & DEC & Source ${ }^{a}$ & $\begin{array}{c}{[\mathrm{Fe} / \mathrm{H}]} \\
(\mathrm{dex}) \\
(5)\end{array}$ & $\begin{array}{c}\text { Source }^{b} \\
(6)\end{array}$ & $\begin{array}{c}\mathrm{RV} \\
(\mathrm{km} / \mathrm{s}) \\
(7)\end{array}$ & $\begin{array}{c}\sigma_{\mathrm{RV}} \\
(\mathrm{km} / \mathrm{s}) \\
(8)\end{array}$ & $\begin{array}{c}\text { Source }^{c} \\
(9)\end{array}$ & $\begin{array}{c}V \\
(\mathrm{mag}) \\
(10)\end{array}$ & $\begin{array}{c}B-V \\
(\mathrm{mag}) \\
(11)\end{array}$ \\
\hline BPS CS 22957-0026 & 000002.17 & -040708.8 & STN & -1.84 & HK & 14 & 10 & BSL & 13.16 & 0.43 \\
\hline $\mathrm{V}^{*}$ GV Peg & 000037.00 & +264124.0 & $\mathrm{t}$ & -1.99 & L & -335 & 30 & L & 13.36 & $\ldots$ \\
\hline BPS CS 29517-0037 & 000039.51 & -162211.2 & $\mathrm{U}$ & -2.20 & HK & -106 & 10 & BSL & 14.65 & 0.41 \\
\hline BPS CS 22957-0025 & 000135.35 & -041649.7 & $\mathrm{U}$ & -1.19 & HK & -118 & 10 & BSL & 14.41 & 0.61 \\
\hline BPS CS 22957-0022 & 000145.49 & -054946.6 & STN & -2.44 & HK & 25 & 10 & BSL & 13.34 & 0.61 \\
\hline BPS CS 29517-0029 & 000147.13 & -134725.0 & NPM & -1.77 & HK & -28 & 10 & BSL & 13.99 & 0.43 \\
\hline BPS CS 29517-0034 & 000147.90 & $-1557 \quad 17.7$ & $\mathrm{U}$ & -1.76 & HK & -147 & 10 & BSL & 13.85 & 0.42 \\
\hline $\mathrm{V}^{*} \mathrm{KO}$ Peg & $00 \quad 0215.34$ & +300436.6 & $\mathrm{U}$ & -1.87 & BSL & -272 & 30 & BSL & $\ldots$ & $\ldots$ \\
\hline BPS CS 22945-0063 & 000237.00 & -654521.2 & $\mathrm{U}$ & -2.22 & HK & 153 & 10 & BSL & 14.53 & 0.44 \\
\hline $\mathrm{V}^{*} \mathrm{RU} \mathrm{Scl}$ & 000248.07 & -245642.9 & HIP & -1.27 & F98 & 34 & 3 & F98 & 10.22 & $\cdots$ \\
\hline BPS CS 22957-0031 & 000313.17 & -024905.3 & $\mathrm{U}$ & -1.98 & HK & 8 & 10 & BSL & 14.55 & 0.46 \\
\hline BPS CS 22957-0036 & 000331.24 & -044421.4 & $\mathrm{U}$ & -1.78 & HK & -88 & 10 & BSL & 14.43 & 0.42 \\
\hline BPS CS 29517-0040 & 000343.02 & -165005.1 & $\mathrm{U}$ & -1.67 & HK & -12 & 10 & BSL & $(14.18)$ & $(0.42)$ \\
\hline $\mathrm{V}^{*} \mathrm{UU}$ Cet & 000405.10 & -165951.5 & HIP & -1.28 & F98 & -114 & 1 & F98 & 12.08 & $\ldots$ \\
\hline $\mathrm{V}^{*} \mathrm{AO} \mathrm{Tuc}$ & 000406.35 & -592906.2 & $\mathrm{ACT}$ & -1.29 & BSL & 2 & 30 & BSL & 11.13 & $\ldots$ \\
\hline [FF93] 513440 & $00 \quad 0414.12$ & -344822.6 & SPM & -0.98 & BSL & 89 & 10 & BSL & 10.71 & 1.29 \\
\hline BPS CS 22876-0040 & 000452.46 & -341337.2 & SPM & -2.20 & HK & -191 & 10 & BSL & 15.09 & 0.40 \\
\hline BPS CS 29503-0010 & 000455.38 & -242419.2 & SPM & -1.88 & HK & -24 & 10 & BSL & 13.74 & 0.54 \\
\hline BPS CS 29517-0042 & 000503.06 & -162202.1 & $\mathrm{U}$ & -2.17 & HK & -37 & 10 & BSL & 14.10 & 0.39 \\
\hline BPS CS 29517-0047 & 000514.42 & -133019.0 & $\mathrm{U}$ & -1.85 & HK & -161 & 10 & BSL & $(13.80)$ & $(0.70)$ \\
\hline HD 20 & 000515.24 & -271617.8 & HIP & -1.39 & B99 & -57 & 1 & CY & 9.04 & 0.69 \\
\hline BPS CS 29517-0048 & 000529.86 & -131800.9 & $\mathrm{U}$ & -2.10 & HK & -60 & 10 & BSL & 14.42 & 0.44 \\
\hline HD 97 & 000546.01 & -194011.3 & HIP & -1.21 & B99 & 76 & 1 & $\mathrm{CY}$ & 9.66 & 0.79 \\
\hline BPS CS 29503-0013 & 000550.64 & -233655.0 & SPM & -1.50 & HK & 22 & 10 & BSL & 14.58 & 0.50 \\
\hline $\mathrm{V}^{*}$ IQ Peg & 000607.64 & +2918 19.7 & $\mathrm{U}$ & -1.54 & BSL & -207 & 30 & BSL & $\ldots$ & $\ldots$ \\
\hline [FF93] 513390 & 000646.28 & -335944.5 & SPM & -0.72 & BSL & 46 & 10 & BSL & 9.78 & 1.14 \\
\hline $\mathrm{V}^{*} \mathrm{NN}$ And & 000655.86 & +312813.3 & $\mathrm{U}$ & -1.79 & BSL & -276 & 10 & BSL & $\ldots$ & $\ldots$ \\
\hline BPS CS 29503-0022 & 000724.91 & -241822.0 & SPM & -2.06 & HK & 14 & 10 & BSL & 14.58 & 0.71 \\
\hline BPS CS 22876-0032 & $00 \quad 0737.46$ & -353116.7 & SPM & -3.85 & B99 & -90 & 10 & HK & 12.84 & 0.39 \\
\hline BPS CS 29503-0017 & 000745.02 & -230704.3 & SPM & -1.98 & HK & 14 & 10 & BSL & 14.31 & 0.49 \\
\hline BPS CS 29503-0026 & 000840.76 & -260705.1 & SPM & -1.96 & HK & -40 & 10 & BSL & 13.48 & 0.56 \\
\hline BPS CS 29503-0015 & 001032.12 & -224538.2 & SPM & -1.76 & HK & -57 & 10 & BSL & 14.75 & 0.51 \\
\hline $\mathrm{V}^{*} \mathrm{NQ}$ And & 001132.69 & +305141.0 & U & -1.77 & BSL & -235 & 30 & BSL & $\ldots$ & $\ldots$ \\
\hline $\mathrm{V}^{*} \mathrm{RY}$ Psc & 001141.06 & -014455.4 & GSC & -1.39 & $\mathrm{~L}$ & -1 & 9 & L & 12.28 & $\cdots$ \\
\hline HD 868 & 001258.87 & -300328.5 & SPM & -0.79 & BSL & 23 & 10 & BSL & 8.40 & 1.33 \\
\hline HD 891 & 001308.86 & -225607.6 & HIP & -0.61 & BSL & -50 & 10 & BSL & 9.03 & 1.26 \\
\hline HD 941 & 001345.42 & -231252.6 & HIP & -0.86 & BSL & -88 & 10 & BSL & 6.86 & 1.10 \\
\hline BPS CS 29503-0031 & 001354.85 & -273638.8 & SPM & -2.09 & HK & 32 & 10 & BSL & 13.71 & 0.57 \\
\hline HD 991 & $0014 \quad 13.20$ & -354829.9 & HIP & -0.88 & BSL & 37 & 10 & BSL & 7.84 & 1.20 \\
\hline BPS CS 30339-0002 & 001430.08 & -363834.2 & SPM & -1.54 & HK & -27 & 10 & BSL & 13.86 & 0.53 \\
\hline
\end{tabular}


TABLE 1A. (continued)

\begin{tabular}{|c|c|c|c|c|c|c|c|c|c|c|}
\hline $\begin{array}{l}\text { Star } \\
(1)\end{array}$ & $\begin{array}{c}\text { RA (2000.0) } \\
(2)\end{array}$ & DEC & $\begin{array}{c}\text { Source }^{\mathrm{a}} \\
(4)\end{array}$ & $\begin{array}{c}{[\mathrm{Fe} / \mathrm{H}]} \\
(\mathrm{dex}) \\
(5)\end{array}$ & $\begin{array}{c}\text { Source }^{b} \\
(6)\end{array}$ & $\begin{array}{c}\mathrm{RV} \\
(\mathrm{km} / \mathrm{s}) \\
(7)\end{array}$ & $\begin{array}{c}\sigma_{\mathrm{RV}} \\
(\mathrm{km} / \mathrm{s}) \\
(8)\end{array}$ & $\begin{array}{c}\text { Source }^{\mathrm{c}} \\
(9)\end{array}$ & $\begin{array}{c}V \\
(\mathrm{mag}) \\
(10)\end{array}$ & $\begin{array}{c}B-V \\
(\mathrm{mag}) \\
(11)\end{array}$ \\
\hline BPS CS 29503-0042 & 001535.60 & -242912.1 & SPM & -2.17 & HK & -24 & 10 & BSL & 13.91 & 0.66 \\
\hline BPS CS 30339-0019 & 001540.78 & -323317.9 & SPM & -1.77 & HK & 52 & 10 & BSL & 13.70 & 0.34 \\
\hline BPS CS 30339-0007 & 001544.58 & -353446.6 & SPM & -1.28 & HK & -5 & 10 & BSL & 14.28 & 0.63 \\
\hline CD-23 72 & 001616.54 & -223440.6 & HIP & -1.17 & B99 & 20 & 1 & CY & 9.58 & 0.71 \\
\hline BPS CS 30339-0010 & 001639.90 & -345054.9 & SPM & -1.70 & HK & 37 & 10 & BSL & 14.80 & 0.58 \\
\hline BPS CS 29503-0043 & 001653.76 & -244204.3 & SPM & -1.33 & HK & -19 & 10 & BSL & 14.68 & 0.31 \\
\hline BPS CS 30339-0015 & 001658.10 & $-3348 \quad 17.3$ & SPM & -2.20 & HK & 111 & 10 & BSL & 15.28 & 0.40 \\
\hline BPS CS 30339-0009 & 001704.83 & -351139.0 & SPM & -1.78 & HK & -37 & 10 & BSL & 13.81 & 0.54 \\
\hline BPS CS 30339-0005 & 001742.01 & -363435.2 & SPM & -1.50 & HK & -68 & 10 & BSL & 14.02 & 0.62 \\
\hline [FF93] 513135 & 001838.07 & -371211.9 & SPM & -0.70 & BSL & 60 & 10 & BSL & 9.89 & 1.50 \\
\hline CD-35 87 & 001906.48 & -344632.8 & SPM & -0.67 & BSL & -73 & 10 & BSL & 10.63 & 1.11 \\
\hline BPS CS 29503-0037 & 001916.86 & $\begin{array}{lll}-23 & 07 & 01.7\end{array}$ & SPM & -0.86 & HK & 0 & 10 & BSL & 13.54 & 0.39 \\
\hline BPS CS 22882-0005 & 002018.72 & -293031.0 & SPM & -2.31 & HK & 69 & 10 & BSL & 14.29 & 0.58 \\
\hline BPS CS 22882-0001 & 002025.29 & -313904.0 & SPM & -2.30 & HK & 28 & 10 & BSL & 14.82 & 0.40 \\
\hline BPS CS 30339-0040 & 002026.16 & -363019.9 & SPM & -2.30 & HK & 361 & 10 & BSL & 13.00 & 0.60 \\
\hline HD 1638 & 002028.30 & -303319.8 & HIP & -0.88 & BSL & 26 & 10 & BSL & 8.78 & 1.32 \\
\hline BPS CS 30339-0037 & 002028.90 & $\begin{array}{lll}-36 & 12 & 00.7\end{array}$ & SPM & -2.13 & HK & -245 & 10 & BSL & 13.61 & 0.44 \\
\hline BPS CS 30339-0028 & 002100.54 & -341715.6 & SPM & -1.92 & HK & -23 & 10 & BSL & 14.72 & 0.40 \\
\hline NGC 104 LEE 3516 & 002124.02 & -715750.3 & U & -1.70 & BSL & 425 & 10 & BSL & 13.20 & 1.18 \\
\hline BPS CS 22882-0006 & 002159.85 & -292926.0 & SPM & -2.30 & HK & -72 & 10 & BSL & 14.14 & 0.41 \\
\hline BPS CS 29503-0049 & 002219.12 & -270203.9 & SPM & -2.08 & HK & -84 & 10 & BSL & 14.01 & 0.61 \\
\hline BPS CS 30339-0052 & 002250.14 & -340720.8 & SPM & -2.35 & HK & 33 & 10 & BSL & 15.30 & 0.49 \\
\hline BPS CS 30339-0041 & 002312.93 & -370126.7 & SPM & -2.59 & HK & -32 & 10 & BSL & 13.87 & 0.55 \\
\hline BPS CS 29503-0054 & 002314.08 & -232440.8 & SPM & -0.67 & HK & -11 & 10 & BSL & 14.05 & 0.34 \\
\hline BPS CS 22882-0009 & 002316.89 & -283129.0 & SPM & -2.06 & HK & -39 & 10 & BSL & 15.50 & 0.40 \\
\hline BPS CS 30339-0053 & 002321.40 & -335512.0 & SPM & -1.48 & HK & 78 & 10 & BSL & 15.12 & 0.39 \\
\hline BPS CS 22882-0036 & 002324.56 & -311249.9 & $\mathrm{SPM}$ & -1.96 & HK & 15 & 10 & BSL & 14.53 & 0.38 \\
\hline $\mathrm{V}^{*} \mathrm{SW}$ And & 002343.09 & +292403.8 & HIP & -0.24 & F98 & -21 & 1 & F98 & 9.71 & $\cdots$ \\
\hline BPS CS 30339-0046 & 002521.67 & -363024.5 & SPM & -2.79 & HK & 14 & 10 & BSL & 15.31 & 0.39 \\
\hline BPS CS 30339-0061 & 002810.73 & -325238.5 & SPM & -1.57 & HK & 36 & 10 & BSL & 12.91 & 0.51 \\
\hline BPS CS 30339-0062 & 002822.84 & -333908.4 & SPM & -2.44 & HK & 60 & 10 & BSL & 14.16 & 0.69 \\
\hline BPS CS 30339-0057 & 002857.16 & $-3241 \quad 16.9$ & $\mathrm{SPM}$ & -2.21 & HK & -70 & 10 & BSL & 14.75 & 0.65 \\
\hline [FF93] 514765 & 002902.99 & -365045.4 & SPM & -0.62 & BSL & -20 & 10 & BSL & 10.35 & 1.19 \\
\hline BPS CS 30339-0069 & 003015.94 & -355651.0 & SPM & -3.15 & HK & 12 & 10 & BSL & 14.75 & 0.36 \\
\hline BPS CS 22882-0030 & 003020.05 & -302058.3 & SPM & -2.60 & HK & 25 & 10 & BSL & 14.83 & 0.38 \\
\hline BPS CS 22882-0012 & 003022.49 & -273527.1 & SPM & -2.50 & HK & 10 & 10 & BSL & 15.26 & 0.38 \\
\hline BPS CS 30339-0064 & 003034.75 & -341840.2 & $\mathrm{SPM}$ & -1.36 & HK & 108 & 10 & BSL & 13.95 & 0.59 \\
\hline HD 2665 & 003045.40 & +570354.2 & HIP & -1.92 & C97 & -379 & 2 & $\mathrm{CY}$ & 7.72 & 0.75 \\
\hline HD 2743 & 003049.93 & -302125.9 & SPM & -0.62 & BSL & -32 & 10 & BSL & 10.02 & 1.14 \\
\hline BPS CS 30339-0055 & 003102.91 & -321856.7 & SPM & -0.84 & HK & 28 & 10 & BSL & 14.56 & 0.63 \\
\hline
\end{tabular}


TABLE 1A. (continued)

\begin{tabular}{|c|c|c|c|c|c|c|c|c|c|c|c|}
\hline $\begin{array}{l}\text { Star } \\
\text { (1) }\end{array}$ & RA (2000.0) & DEC & $\begin{array}{c}\text { Source }^{\mathrm{a}} \\
(4)\end{array}$ & $\begin{array}{c}{[\mathrm{Fe} / \mathrm{H}]} \\
(\operatorname{dex}) \\
(5)\end{array}$ & $\begin{array}{c}\text { Source }^{b} \\
(6)\end{array}$ & $\begin{array}{c}\mathrm{RV} \\
(\mathrm{km} / \mathrm{s}) \\
(7)\end{array}$ & $\begin{array}{c}\sigma_{\mathrm{RV}} \\
(\mathrm{km} / \mathrm{s}) \\
(8)\end{array}$ & $\begin{array}{c}\text { Source }{ }^{\mathrm{c}} \\
(9)\end{array}$ & $\begin{array}{c}V \\
(\mathrm{mag}) \\
(10)\end{array}$ & $\begin{array}{c}B-V \\
(\mathrm{mag}) \\
(11)\end{array}$ & Soul \\
\hline BPS CS 22882-0016 & 003113.65 & $-2828 \quad 32.2$ & SPM & -1.94 & HK & -35 & 10 & BSL & 14.12 & 0.63 & \\
\hline HD 2796 & 003116.92 & -164740.3 & HIP & -2.31 & B99 & -61 & 1 & $\mathrm{CY}$ & 8.48 & 0.71 & \\
\hline HD 2857 & 003153.80 & $-05 \quad 1542.3$ & HIP & -1.51 & C97 & -145 & 10 & BSL & 9.98 & 0.22 & \\
\hline HD 3010 & 003313.54 & -282232.0 & SPM & -0.60 & BSL & 76 & 10 & BSL & 9.04 & 1.19 & \\
\hline HD 3008 & 003314.30 & -104342.7 & HIP & -1.90 & B99 & -81 & 1 & $\mathrm{CY}$ & 9.49 & 1.27 & \\
\hline $\mathrm{V}^{*} \mathrm{RX}$ Cet & 003338.30 & -152914.3 & HIP & -1.28 & F98 & -57 & 2 & F98 & 11.44 & $\cdots$ & \\
\hline BPS CS 22882-0029 & 003405.63 & -305201.1 & SPM & -2.17 & HK & 0 & 10 & BSL & 14.79 & 0.39 & \\
\hline BPS CS 30339-0080 & 003421.31 & -330417.2 & SPM & -2.02 & HK & -78 & 10 & BSL & 14.72 & 0.37 & \\
\hline HD 3179 & 003450.57 & -215255.5 & HIP & -0.92 & $\mathrm{ATT}$ & -75 & 1 & $\mathrm{CY}$ & 9.75 & 0.74 & \\
\hline BPS CS 30339-0073 & 003452.21 & -365529.3 & GSC & -2.78 & HK & 171 & 10 & BSL & 14.75 & 0.54 & \\
\hline [FF93] 514560 & 003456.10 & -371207.0 & SPM & -0.77 & BSL & 6 & 10 & BSL & 10.16 & 1.03 & s \\
\hline HD 3232 & 003519.44 & -183350.9 & HIP & -0.76 & BSL & -36 & 10 & BSL & 7.78 & 1.37 & \\
\hline HD 3276 & 003540.46 & -282925.0 & SPM & -0.62 & BSL & -17 & 10 & BSL & 10.04 & 1.23 & \\
\hline BPS CS 29527-0031 & 003633.43 & -222431.6 & $\mathrm{U}$ & -2.10 & W99 & 6 & 10 & BSL & 13.69 & 0.15 & \\
\hline BPS CS 22882-0024 & 003643.23 & $-3024 \quad 42.8$ & SPM & -1.87 & HK & 81 & 10 & BSL & 14.47 & 0.39 & \\
\hline BPS CS 30339-0076 & 003721.77 & -355357.0 & SPM & -2.18 & HK & 42 & 10 & BSL & 13.03 & 0.64 & $\mathrm{~S}$ \\
\hline BPS CS 22882-0026 & 003725.37 & -314622.5 & SPM & -2.04 & HK & -75 & 10 & BSL & 14.44 & 0.43 & $\mathrm{E}$ \\
\hline BPS CS 22882-0021 & 003732.94 & -295103.2 & SPM & -2.06 & HK & 59 & 10 & BSL & 13.85 & 0.39 & E \\
\hline [FF93] 514450 & 003809.93 & -342126.0 & SPM & -0.87 & BSL & -27 & 10 & BSL & 10.78 & 1.21 & $\mathrm{~S}$ \\
\hline HD 3567 & 003831.94 & -081828.6 & HIP & -1.29 & B99 & -53 & 10 & HK & 9.25 & 0.47 & \\
\hline HD 3582 & 003834.09 & -324043.7 & SPM & -0.60 & BSL & -33 & 10 & BSL & 9.76 & 1.35 & \\
\hline HD 3633 & 003850.41 & -362726.0 & SPM & -0.97 & BSL & 41 & 10 & BSL & 9.44 & 1.21 & $\mathrm{~S}$ \\
\hline BPS CS 29497-0033 & 004108.44 & -255629.2 & SPM & -1.90 & HK & 73 & 10 & HK & 13.86 & 0.14 & $\mathrm{E}$ \\
\hline BPS CS 22170-0021 & 004225.88 & -113701.4 & $\mathrm{U}$ & -2.02 & HK & -114 & 10 & BSL & 14.16 & 0.40 & $\mathrm{E}$ \\
\hline$-29201 \mathrm{~W} 1$ & 004230.74 & -283433.6 & SPM & -1.20 & BSL & 42 & 10 & BSL & 11.31 & -0.01 & $\mathrm{~S}$ \\
\hline HD 4024 & 004232.97 & -275408.3 & SPM & -0.67 & BSL & -1 & 10 & BSL & 9.60 & 1.12 & \\
\hline [FF93] 514210 & 004437.01 & -365637.4 & SPM & -0.95 & BSL & 24 & 10 & BSL & 9.98 & 1.61 & \\
\hline HD 4261 & 004445.69 & -312321.9 & HIP & -0.70 & BSL & -2 & 10 & BSL & 7.66 & 1.03 & \\
\hline HD 4306 & 004527.13 & -093240.0 & HIP & -2.71 & B99 & -67 & 1 & $\mathrm{CY}$ & 9.02 & 0.75 & \\
\hline BPS CS 22942-0003 & 004549.94 & -243247.2 & SPM & -1.67 & HK & 5 & 10 & BSL & 15.12 & 0.42 & \\
\hline [FF93] 514133 & 004622.98 & $\begin{array}{llll}-35 & 01 & 41.4\end{array}$ & SPM & -0.65 & BSL & 20 & 10 & BSL & 11.09 & 1.03 & $\mathrm{~S}$ \\
\hline BPS CS 22942-0002 & 004635.86 & -244302.2 & SPM & -2.62 & HK & -155 & 10 & BSL & 13.86 & 0.58 & $\mathrm{E}$ \\
\hline CD-38 245 & 004636.18 & -373933.5 & HIP & -3.92 & B99 & 47 & 1 & $\mathrm{CY}$ & 11.97 & 0.82 & \\
\hline [FF93] 514104 & 004703.72 & -342421.8 & SPM & -0.60 & BSL & 29 & 10 & BSL & 10.90 & 1.01 & $\mathrm{~S}$ \\
\hline CD-29 222 & 004739.52 & $\begin{array}{lll}-29 & 01 & 02.3\end{array}$ & SPM & -0.72 & BSL & 11 & 10 & BSL & 10.53 & 1.36 & $\mathrm{~S}$ \\
\hline$-33302 \mathrm{~S} 1$ & 004754.08 & -325348.5 & SPM & -2.20 & BSL & -61 & 10 & BSL & 12.91 & 0.35 & $\mathrm{~S}$ \\
\hline BD-11 145 & 004824.32 & -104130.7 & HIP & -2.02 & ATT & -93 & 1 & $\mathrm{CY}$ & 10.81 & 0.91 & \\
\hline [FF93] 514033 & 004900.34 & -370530.5 & SPM & -0.60 & BSL & 17 & 10 & BSL & 10.09 & 1.42 & $\mathrm{~S}$ \\
\hline BPS CS 22170-0040 & 004931.82 & -075645.0 & $\mathrm{U}$ & -1.48 & HK & -150 & 10 & BSL & 13.86 & 0.48 & \\
\hline $\mathrm{V}^{*} \mathrm{ZZ}$ And & 004934.86 & +270124.0 & GSC & -1.58 & L & -13 & 53 & L & 13.01 & $\cdots$ & \\
\hline
\end{tabular}


TABLE 1A. (continued)

\begin{tabular}{|c|c|c|c|c|c|c|c|c|c|c|}
\hline Star & RA (2000.0) & DEC & Source $^{\mathrm{a}}$ & $\begin{array}{c}{[\mathrm{Fe} / \mathrm{H}]} \\
(\mathrm{dex}) \\
(5)\end{array}$ & $\begin{array}{c}\text { Source } \\
\text { (6) }\end{array}$ & $\begin{array}{c}\mathrm{RV} \\
(\mathrm{km} / \mathrm{s}) \\
(7)\end{array}$ & $\begin{array}{c}\sigma_{\mathrm{RV}} \\
(\mathrm{km} / \mathrm{s}) \\
(8)\end{array}$ & $\begin{array}{c}\text { Source }^{\mathrm{c}} \\
(9)\end{array}$ & $\begin{array}{c}V \\
(\mathrm{mag}) \\
(10)\end{array}$ & $\begin{array}{c}B-V \\
(\mathrm{mag}) \\
(11)\end{array}$ \\
\hline $\mathrm{V}^{*}$ AE Tuc & 005000.60 & -623807.5 & $\mathrm{U}$ & -0.80 & $\mathrm{~L}$ & 76 & 10 & $\mathrm{~L}$ & 12.10 & $\cdots$ \\
\hline HD 5098 & 005240.60 & -240021.3 & HIP & -0.39 & C97 & 37 & 10 & BSL & 5.47 & 1.27 \\
\hline BPS CS 22942-0010 & 005243.69 & -235706.0 & SPM & -1.54 & HK & 3 & 10 & BSL & 14.53 & 0.49 \\
\hline E-29.0.069 & 005314.83 & -284401.9 & SPM & -0.71 & BSL & -9 & 10 & BSL & 12.03 & 0.96 \\
\hline E-29.3.052 & 005335.48 & -291925.5 & SPM & -0.69 & BSL & 30 & 10 & BSL & 18.43 & 0.29 \\
\hline BPS CS 22942-0011 & 005413.01 & -250433.8 & SPM & -2.40 & HK & 20 & 10 & BSL & 12.94 & 0.63 \\
\hline$-28285 \mathrm{~S} 1$ & 005441.44 & -281355.3 & GSC & -1.20 & BSL & 106 & 10 & BSL & 13.78 & 0.19 \\
\hline E-30.3.078 & 005441.46 & -281355.1 & SPM & -0.60 & BSL & -42 & 10 & BSL & 13.57 & 0.40 \\
\hline $\mathrm{V}^{*}$ AG Tuc & 005453.27 & -664228.9 & $\mathrm{v}$ & -1.95 & L & 196 & 9 & L & 12.88 & $\ldots$ \\
\hline BPS CS 22166-0007 & 005505.41 & -120723.6 & $\mathrm{U}$ & -2.50 & HK & 41 & 10 & BSL & $(15.12)$ & $(0.38)$ \\
\hline E-30.5.084 & 005535.72 & -303808.9 & SPM & -0.84 & BSL & -48 & 10 & BSL & 12.44 & 1.09 \\
\hline-24 396E1 & 005535.75 & -232830.1 & GSC & -1.70 & BSL & -35 & 10 & BSL & 12.25 & 0.19 \\
\hline HD 5426 & 005541.03 & -334511.0 & HIP & -2.25 & $\mathrm{C} 97$ & 28 & 1 & $\mathrm{CY}$ & 9.62 & 0.72 \\
\hline HD 5422 & 005553.30 & -184550.2 & HIP & -0.61 & BSL & -10 & 10 & BSL & 8.44 & 1.22 \\
\hline BPS CS 22942-0021 & 005606.65 & $-23 \quad 1753.0$ & GSC & -1.14 & HK & 110 & 10 & BSL & 14.34 & 0.37 \\
\hline E-30.1.080 & 005612.89 & -295724.5 & SPM & -0.70 & BSL & 105 & 10 & BSL & 12.69 & 1.03 \\
\hline CD-28 292 & 005618.51 & -272748.6 & SPM & -0.89 & BSL & 54 & 10 & BSL & 10.97 & 1.39 \\
\hline E-29.1.079 & 005635.33 & -285352.6 & SPM & -1.72 & BSL & -4 & 10 & BSL & 12.79 & 1.01 \\
\hline BD-20 170 & $0057 \quad 10.21$ & -194958.2 & HIP & -1.31 & ATT & -5 & 1 & CY & 10.25 & 0.75 \\
\hline CD-30 288 & 005732.87 & -300000.8 & SPM & -0.64 & BSL & 45 & 10 & BSL & 9.93 & 0.87 \\
\hline $\mathrm{V}^{*} \mathrm{~W}$ Tuc & 005809.70 & -632344.2 & HIP & -1.57 & F98 & 65 & 1 & F98 & 11.41 & $\cdots$ \\
\hline Bok II 274 & 005811.94 & -271506.7 & STN & -0.67 & BSL & 43 & 10 & BSL & 11.81 & 1.02 \\
\hline Bok III 233 & 005812.69 & $\begin{array}{lll}-30 & 02 & 44.3\end{array}$ & GSC & -1.54 & BSL & 0 & 10 & BSL & 12.43 & 0.81 \\
\hline Bok II 284 & 005822.98 & -274014.1 & SPM & -0.92 & BSL & 41 & 10 & BSL & 12.45 & 0.86 \\
\hline BPS CS 22166-0016 & 005823.87 & -144707.3 & $\mathrm{STN}$ & -2.37 & HK & -215 & 10 & BSL & 12.73 & 0.61 \\
\hline CD-30 298 & 005843.83 & -300557.9 & HIP & -2.98 & B99 & 30 & 1 & $\mathrm{CY}$ & 10.80 & 0.67 \\
\hline E-29.3.099 & 005927.48 & -291703.3 & SPM & -0.63 & BSL & 49 & 10 & BSL & 12.49 & 1.02 \\
\hline Bok III 281 & 005941.14 & -311908.5 & SPM & -0.65 & BSL & -35 & 10 & BSL & 11.88 & 0.69 \\
\hline BPS CS 22942-0024 & 005955.34 & -233038.5 & SPM & -2.60 & HK & -45 & 10 & BSL & 14.16 & 0.40 \\
\hline Bok III 167 & 010008.05 & -304232.4 & GSC & -0.64 & BSL & 30 & 10 & BSL & 10.57 & 1.44 \\
\hline CD-31 394 & $0100 \quad 08.11$ & -304232.2 & SPM & -0.78 & BSL & 22 & 10 & BSL & 10.70 & 1.44 \\
\hline $\mathrm{V}^{*} \mathrm{WY} \mathrm{Scl}$ & $\begin{array}{lll}01 & 00 & 27.77\end{array}$ & $-28 \quad 12 \quad 20.4$ & SPM & -1.51 & L & 115 & 9 & L & 13.07 & $\ldots$ \\
\hline Bok II 256 & 010030.39 & -282328.8 & GSC & -0.75 & BSL & 38 & 10 & BSL & 11.88 & 1.00 \\
\hline Bok III 179 & 010032.58 & -305435.2 & SPM & -2.27 & BSL & -7 & 10 & BSL & 11.43 & 0.89 \\
\hline $\mathrm{V}^{*} \mathrm{RU}$ Cet & 010040.29 & -155727.4 & HIP & -1.66 & F98 & 46 & 10 & F98 & 11.68 & $\ldots$ \\
\hline$-28308 \mathrm{~S} 1$ & 010052.28 & -280528.9 & SPM & -1.80 & BSL & -14 & 10 & BSL & 14.18 & 0.24 \\
\hline$-290055 \mathrm{D}$ & 010056.32 & -293217.0 & SPM & -1.50 & BSL & 0 & 10 & BSL & 13.98 & 1.09 \\
\hline E-29.0.138 & 010115.69 & -285304.4 & HIP & -0.69 & BSL & -101 & 10 & BSL & 9.34 & 0.86 \\
\hline HD 6054 & 010129.84 & -272702.7 & SPM & -0.77 & BSL & 13 & 10 & BSL & 9.16 & 1.55 \\
\hline$-290055 \mathrm{E}$ & 010131.41 & -292418.9 & SPM & -0.80 & BSL & 27 & 10 & BSL & 14.06 & 0.99 \\
\hline
\end{tabular}


TABLE 1A. (continued)

\begin{tabular}{|c|c|c|c|c|c|c|c|c|c|c|}
\hline $\begin{array}{l}\text { Star } \\
(1)\end{array}$ & $\begin{array}{c}\text { RA (2000.0) } \\
(2)\end{array}$ & DEC & $\begin{array}{c}\text { Source }^{\mathrm{a}} \\
(4)\end{array}$ & $\begin{array}{c}{[\mathrm{Fe} / \mathrm{H}]} \\
(\mathrm{dex}) \\
(5)\end{array}$ & $\begin{array}{c}\text { Source }^{b} \\
(6)\end{array}$ & $\begin{array}{c}\mathrm{RV} \\
(\mathrm{km} / \mathrm{s}) \\
(7)\end{array}$ & $\begin{array}{c}\sigma_{\mathrm{RV}} \\
(\mathrm{km} / \mathrm{s}) \\
(8)\end{array}$ & $\begin{array}{c}\text { Source }^{c} \\
(9)\end{array}$ & $\begin{array}{c}V \\
(\mathrm{mag}) \\
(10)\end{array}$ & $\begin{array}{c}B-V \\
(\mathrm{mag}) \\
(11)\end{array}$ \\
\hline$-300055 \mathrm{~A}$ & 010140.30 & $\begin{array}{lll}-30 & 00 & 03.9\end{array}$ & SPM & -1.50 & BSL & 8 & 10 & BSL & 14.34 & 1.59 \\
\hline BPS CS 22166-0022 & 010141.10 & -121904.5 & $\mathrm{U}$ & -2.19 & HK & -62 & 10 & BSL & (14.13) & $(0.47)$ \\
\hline BPS CS 22942-0029 & 010152.84 & -265622.9 & SPM & -1.96 & HK & 86 & 10 & BSL & 14.84 & 0.40 \\
\hline$-290055 \mathrm{C}$ & 010157.65 & -292120.5 & SPM & -1.60 & BSL & 18 & 10 & BSL & 14.36 & 1.09 \\
\hline CD-30 314 & 010201.22 & -293116.1 & $\mathrm{STN}$ & -1.22 & BSL & 56 & 10 & BSL & 12.85 & 0.29 \\
\hline Bok III 82 & 010203.89 & $-3007 \quad 17.4$ & GSC & -0.70 & BSL & 44 & 10 & BSL & 11.73 & 1.06 \\
\hline BPS CS 22953-0003 & 010215.85 & -614345.6 & $\mathrm{U}$ & -2.81 & B99 & 208 & 10 & HK & 13.72 & 0.67 \\
\hline HD 6446 & 010236.79 & -773303.3 & HIP & -1.60 & BSL & 62 & 10 & BSL & 7.24 & 1.39 \\
\hline$-310100 \mathrm{C}$ & 010251.93 & -305712.7 & SPM & -1.30 & BSL & 18 & 10 & BSL & 15.20 & 0.84 \\
\hline$-320100 \mathrm{~A}$ & 010252.07 & -314524.6 & SPM & -1.70 & BSL & -63 & 10 & BSL & 15.73 & 1.25 \\
\hline$-290100 \mathrm{C}$ & 010256.07 & -291028.0 & SPM & -1.30 & BSL & -67 & 10 & BSL & 15.26 & 0.99 \\
\hline [FF93] 515282 & 010304.88 & -365659.0 & SPM & -0.77 & BSL & 82 & 10 & BSL & 10.82 & 1.09 \\
\hline CD-29 308 & 010308.11 & -285505.2 & SPM & -0.60 & BSL & 2 & 10 & BSL & 10.97 & 0.53 \\
\hline BPS CS 22942-0033 & $\begin{array}{lll}01 & 0308.33\end{array}$ & -253017.3 & SPM & -1.63 & BSL & 178 & 10 & BSL & 13.97 & 0.39 \\
\hline HD 6255 & 010312.24 & -270629.4 & HIP & -0.70 & BSL & -35 & 10 & BSL & 8.98 & 1.60 \\
\hline HD 6268 & 010318.19 & -275249.7 & HIP & -2.48 & B99 & 40 & 1 & $\mathrm{CY}$ & 8.09 & 0.82 \\
\hline$-280100 \mathrm{~A}$ & 010332.69 & -275922.3 & SPM & -1.10 & BSL & 116 & 10 & BSL & 14.37 & 1.13 \\
\hline$-300100 \mathrm{~F}$ & 010334.34 & -301150.0 & SPM & -1.10 & BSL & 1 & 10 & BSL & 15.20 & 1.15 \\
\hline HD 6229 & 010336.45 & +234606.6 & HIP & -1.09 & ATT & -96 & 10 & BSL & 8.60 & 0.74 \\
\hline BPS CS 22166-0024 & 010355.04 & -124151.4 & $\mathrm{U}$ & -2.08 & HK & -373 & 10 & BSL & 13.86 & 0.41 \\
\hline BPS CS 22942-0035 & 010357.47 & -235245.2 & SPM & -2.44 & HK & -103 & 10 & BSL & 14.56 & 0.61 \\
\hline [FF93] 507215 & 010454.94 & -262653.9 & SPM & -0.74 & BSL & -76 & 10 & BSL & 10.85 & 1.24 \\
\hline V* DR And & $\begin{array}{lll}01 & 05 & 10.74\end{array}$ & +341306.6 & STN & -1.48 & L & -81 & 30 & L & 12.34 & $\ldots$ \\
\hline$-300100 \mathrm{~A}$ & $\begin{array}{lll}01 & 05 & 17.97\end{array}$ & -295633.9 & SPM & -1.80 & BSL & -24 & 10 & BSL & 14.48 & 1.27 \\
\hline HD 6461 & 010525.41 & -125412.5 & HIP & -0.93 & B99 & 1 & 10 & HK & 7.65 & 0.79 \\
\hline CD-28 337 & 010531.88 & -272352.2 & GSC & -0.62 & BSL & -32 & 10 & BSL & 10.87 & 1.08 \\
\hline CD-29 324 & 010533.48 & -284342.9 & GSC & -0.65 & BSL & 66 & 10 & BSL & 11.62 & 0.94 \\
\hline Bok III 45 & 010534.90 & $\begin{array}{llll}-31 & 07 & 24.7\end{array}$ & STN & -0.69 & BSL & -7 & 10 & BSL & 12.29 & 1.24 \\
\hline CD-31 434 & 010537.35 & -310537.5 & SPM & -0.71 & BSL & -16 & 10 & BSL & 10.12 & 0.98 \\
\hline-310100 A & 010556.77 & -304637.4 & SPM & -1.70 & BSL & 35 & 10 & BSL & 13.21 & 1.10 \\
\hline CD-30 339 & 010557.72 & -292835.2 & GSC & -0.89 & BSL & -23 & 10 & BSL & 11.68 & 1.18 \\
\hline BPS CS 29514-0004 & 010560.00 & -261241.2 & SPM & -2.24 & HK & -12 & 10 & BSL & 14.55 & 0.60 \\
\hline Bok III 43 & 010625.17 & -305435.0 & $\mathrm{~V}$ & -0.75 & BSL & -21 & 10 & BSL & 10.05 & 1.02 \\
\hline [FF93] 507267 & 010636.94 & -260912.6 & SPM & -0.69 & BSL & 33 & 10 & BSL & 10.93 & 1.13 \\
\hline BPS CS 29514-0007 & 010640.56 & -245841.3 & SPM & -2.91 & HK & 31 & 10 & BSL & 13.95 & 0.41 \\
\hline$-300100 \mathrm{~B}$ & 010647.94 & -294551.1 & SPM & -1.20 & BSL & -18 & 10 & BSL & 15.77 & 0.91 \\
\hline$-28336 \mathrm{~N} 1$ & 010658.16 & -280744.9 & SPM & -1.90 & BSL & 82 & 10 & BSL & 13.17 & 0.27 \\
\hline$-290100 \mathrm{E}$ & $\begin{array}{lll}01 & 07 & 07.13\end{array}$ & -292527.4 & SPM & -0.70 & BSL & -65 & 10 & BSL & 13.43 & 0.88 \\
\hline $\mathrm{V}^{*} \mathrm{AE} \mathrm{Scl}$ & 010719.92 & -321749.6 & GSC & -1.89 & $\mathrm{~L}$ & 38 & 11 & L & 12.45 & $\cdots$ \\
\hline BPS CS 22953-0005 & 010737.81 & -612517.5 & $\mathrm{U}$ & -2.31 & HK & -2 & 10 & BSL & 13.64 & 0.58 \\
\hline
\end{tabular}


TABLE 1A. (continued)

\begin{tabular}{|c|c|c|c|c|c|c|c|c|c|c|}
\hline $\begin{array}{l}\text { Star } \\
(1)\end{array}$ & $\begin{array}{c}\text { RA (2000.0) } \\
(2)\end{array}$ & DEC & $\begin{array}{c}\text { Source }^{\mathrm{a}} \\
(4)\end{array}$ & $\begin{array}{c}{[\mathrm{Fe} / \mathrm{H}]} \\
(\mathrm{dex}) \\
(5)\end{array}$ & $\begin{array}{c}\text { Source }^{b} \\
(6)\end{array}$ & $\begin{array}{c}\mathrm{RV} \\
(\mathrm{km} / \mathrm{s}) \\
(7)\end{array}$ & $\begin{array}{c}\sigma_{\mathrm{RV}} \\
(\mathrm{km} / \mathrm{s}) \\
(8)\end{array}$ & $\begin{array}{c}\text { Source }^{c} \\
(9)\end{array}$ & $\begin{array}{c}V \\
(\mathrm{mag}) \\
(10)\end{array}$ & $\begin{array}{c}B-V \\
(\mathrm{mag}) \\
(11)\end{array}$ \\
\hline BPS CS 29514-0001 & 010741.44 & -270548.2 & SPM & -2.02 & HK & 66 & 10 & BSL & 14.48 & 0.57 \\
\hline CD-33 410 & 010752.48 & -331358.7 & SPM & -0.77 & BSL & 5 & 10 & BSL & 9.50 & 1.26 \\
\hline BPS CS 22953-0011 & $\begin{array}{lll}01 & 08 & 10.82\end{array}$ & -591100.2 & U & -1.58 & HK & 174 & 10 & BSL & $(15.00)$ & $(0.40)$ \\
\hline$-290105 \mathrm{H}$ & 010842.60 & -291829.1 & SPM & -0.70 & BSL & 0 & 10 & BSL & 13.75 & 0.94 \\
\hline [FF93] 515144 & 010844.11 & -345554.6 & SPM & -0.79 & BSL & -34 & 10 & BSL & 10.56 & 1.03 \\
\hline [FF93] 507329 & 010852.40 & -252954.0 & SPM & -0.70 & BSL & -23 & 10 & BSL & 10.60 & 1.10 \\
\hline BPS CS 22183-0031 & 010905.02 & -044320.9 & U & -2.46 & HK & 8 & 10 & BSL & 13.62 & 0.62 \\
\hline$-290105 \mathrm{G}$ & 010914.43 & -291138.5 & SPM & -0.80 & BSL & 81 & 10 & BSL & 13.89 & 0.96 \\
\hline-290105 B & 010927.28 & -284405.7 & SPM & -0.80 & BSL & 63 & 10 & BSL & 13.55 & 0.87 \\
\hline BPS CS 29514-0018 & 010928.59 & -263948.7 & SPM & -2.32 & HK & 74 & 10 & BSL & 13.34 & 0.50 \\
\hline [FF93] 507335 & 010929.07 & -232715.8 & SPM & -0.63 & BSL & 118 & 10 & BSL & 10.80 & 1.08 \\
\hline-23 418W1 & 010935.07 & -224037.7 & GSC & -1.20 & BSL & 26 & 10 & BSL & 12.88 & 0.28 \\
\hline HD 6755 & 010942.30 & +613249.5 & HIP & -1.49 & B99 & -329 & 1 & $\mathrm{CY}$ & 7.72 & 0.70 \\
\hline BPS CS 29514-0017 & 010944.76 & -261654.8 & SPM & -2.31 & HK & -10 & 10 & BSL & 13.55 & 0.62 \\
\hline HD 7041 & 010949.33 & -562121.8 & HIP & -1.08 & ATT & 99 & 10 & BSL & 9.02 & 0.80 \\
\hline BPS CS 22953-0016 & 010951.18 & -584454.9 & $\mathrm{U}$ & -2.07 & HK & 110 & 10 & BSL & 14.69 & 0.58 \\
\hline HD 6833 & 010952.24 & +544420.0 & HIP & -0.93 & B99 & -245 & 5 & $\mathrm{CY}$ & 6.75 & 1.14 \\
\hline [FF93] 515107 & 010952.63 & -370918.1 & SPM & -0.63 & BSL & 70 & 10 & BSL & 9.58 & 1.08 \\
\hline CD-33 426 & 010958.13 & -323925.0 & SPM & -0.71 & BSL & -23 & 10 & BSL & 9.84 & 1.17 \\
\hline $\mathrm{V}^{*} \mathrm{TZ}$ Phe & 010958.35 & $\begin{array}{lll}-42 & 07 & 43.9\end{array}$ & SPM & -1.29 & L & 92 & 3 & L & 12.53 & $\cdots$ \\
\hline CD-27 379 & $01 \quad 1024.26$ & -271100.6 & SPM & -0.62 & BSL & 70 & 10 & BSL & 10.82 & 0.94 \\
\hline E-30.0.203 & 011028.03 & -294729.7 & SPM & -0.65 & BSL & 31 & 10 & BSL & 12.39 & 0.93 \\
\hline$-290105 \mathrm{E}$ & 011107.48 & -293804.0 & SPM & -0.90 & BSL & -27 & 10 & BSL & 13.55 & 1.01 \\
\hline BPS CS 29514-0012 & 011128.31 & -250456.8 & SPM & -0.34 & HK & 18 & 10 & BSL & 14.33 & 0.61 \\
\hline HD 7150 & 011128.55 & -270730.5 & SPM & -0.97 & BSL & -33 & 10 & BSL & 9.54 & 1.20 \\
\hline BPS CS 29514-0014 & 011211.73 & -260539.4 & SPM & -2.17 & HK & -12 & 10 & BSL & 13.75 & 0.59 \\
\hline$-290105 \mathrm{~N}$ & 011222.15 & -291252.9 & $\mathrm{SPM}$ & -0.80 & BSL & -6 & 10 & BSL & 14.38 & 1.17 \\
\hline BPS CS 22946-0003 & 011223.66 & -204224.5 & U & -2.15 & HK & 54 & 10 & BSL & 14.82 & 0.49 \\
\hline-290110 A & 011233.18 & -290934.4 & SPM & -1.20 & BSL & 10 & 10 & BSL & 13.99 & 0.89 \\
\hline BPS CS 22946-0002 & 011315.54 & -213607.1 & $\mathrm{U}$ & -2.36 & HK & -62 & 10 & BSL & 14.60 & 0.34 \\
\hline BPS CS 22953-0023 & 011323.86 & -611330.5 & U & -1.71 & HK & 145 & 10 & BSL & 13.71 & 0.51 \\
\hline [FF93] 507458 & 011355.33 & -253326.0 & SPM & -0.67 & BSL & -18 & 10 & BSL & 11.10 & 1.29 \\
\hline [FF93] 507463 & 011408.73 & -242412.4 & SPM & -0.60 & BSL & -16 & 10 & BSL & 9.96 & 1.31 \\
\hline$-310110 \mathrm{C}$ & 011414.56 & $\begin{array}{lll}-31 & 07 & 14.0\end{array}$ & SPM & -0.70 & BSL & 89 & 10 & BSL & 13.32 & 1.12 \\
\hline BPS CS 22174-0012 & 011416.12 & -082945.8 & $\mathrm{U}$ & -1.48 & HK & -232 & 10 & BSL & 14.98 & $(0.74)$ \\
\hline$-300110 \mathrm{C}$ & 011416.66 & -301043.5 & SPM & -1.10 & BSL & -20 & 10 & BSL & 15.60 & 1.07 \\
\hline Bok IV 43 & 011422.03 & -284038.2 & GSC & -1.05 & BSL & 22 & 10 & BSL & 11.74 & 0.62 \\
\hline $\mathrm{V}^{*} \mathrm{RU}$ Psc & 011425.98 & +242456.7 & HIP & -1.75 & F98 & -131 & 1 & F98 & 10.19 & $\cdots$ \\
\hline$-290110 \mathrm{C}$ & 011442.53 & -294621.7 & SPM & -1.60 & BSL & -147 & 10 & BSL & 13.24 & 1.38 \\
\hline HD 7595 & 011529.53 & -281553.4 & $\mathrm{SPM}$ & -0.80 & $\mathrm{C} 97$ & -35 & 10 & BSL & 9.75 & 1.18 \\
\hline
\end{tabular}


TABLE 1A. (continued)

\begin{tabular}{|c|c|c|c|c|c|c|c|c|c|c|}
\hline $\begin{array}{l}\text { Star } \\
(1)\end{array}$ & $\begin{array}{c}\text { RA (2000.0) } \\
(2)\end{array}$ & DEC & $\begin{array}{c}\text { Source }^{\mathrm{a}} \\
(4)\end{array}$ & $\begin{array}{c}{[\mathrm{Fe} / \mathrm{H}]} \\
(\mathrm{dex}) \\
(5)\end{array}$ & $\begin{array}{c}\text { Source }^{b} \\
(6)\end{array}$ & $\begin{array}{c}\mathrm{RV} \\
(\mathrm{km} / \mathrm{s}) \\
(7)\end{array}$ & $\begin{array}{c}\sigma_{\mathrm{RV}} \\
(\mathrm{km} / \mathrm{s}) \\
(8)\end{array}$ & $\begin{array}{c}\text { Source }^{c} \\
(9)\end{array}$ & $\begin{array}{c}V \\
(\mathrm{mag}) \\
(10)\end{array}$ & $\begin{array}{c}B-V \\
(\mathrm{mag}) \\
(11)\end{array}$ \\
\hline-310110 B & $01 \quad 1543.38$ & $\begin{array}{lll}-31 & 40 & 20.2\end{array}$ & SPM & -0.70 & BSL & -7 & 10 & BSL & 13.38 & 0.98 \\
\hline [FF93] 516565 & $01 \quad 1543.56$ & -333058.6 & SPM & -0.73 & BSL & 6 & 10 & BSL & 10.67 & 1.52 \\
\hline$-310110 \mathrm{~A}$ & 011550.35 & -310011.0 & SPM & -0.60 & BSL & 60 & 10 & BSL & 14.16 & 0.96 \\
\hline CD-27 412 & 011601.60 & $-27 \quad 1807.1$ & SPM & -0.72 & $\ldots$ & 24 & 10 & BSL & 10.35 & 0.88 \\
\hline$-290110 \mathrm{D}$ & 011647.60 & -29 1929.6 & SPM & -1.00 & BSL & 169 & 10 & BSL & 15.43 & 1.09 \\
\hline $\mathrm{V}^{*} \mathrm{XX}$ And & 011727.37 & +385702.3 & HIP & -1.94 & F98 & 0 & 1 & F98 & 10.68 & $\cdots$ \\
\hline-300115 B & 011740.14 & -303225.6 & SPM & -2.20 & BSL & 91 & 10 & BSL & 13.16 & 1.46 \\
\hline$-300115 \mathrm{C}$ & 011746.18 & -294614.1 & SPM & -0.80 & BSL & 37 & 10 & BSL & 13.48 & 1.11 \\
\hline$-270115 \mathrm{~A}$ & 011806.39 & -274307.1 & SPM & -1.30 & BSL & -19 & 10 & BSL & 15.59 & 1.03 \\
\hline BPS CS 29514-0031 & 011809.77 & -232256.3 & SPM & -0.86 & HK & -57 & 10 & BSL & 14.12 & 0.51 \\
\hline $\mathrm{V}^{*} \mathrm{VW} \mathrm{Scl}$ & 011814.95 & -391244.5 & HIP & -0.84 & F98 & 53 & 10 & F98 & 11.03 & $\ldots$ \\
\hline $\mathrm{V}^{*} \mathrm{AM}$ Tuc & 011830.61 & -675504.7 & HIP & -1.49 & F98 & 130 & 10 & F98 & 11.67 & $\cdots$ \\
\hline BPS CS 29514-0034 & $01 \quad 1835.77$ & -251243.2 & SPM & -1.75 & HK & 42 & 10 & BSL & 14.53 & 0.66 \\
\hline Bok IV 16 & 011853.62 & -281143.9 & $\mathrm{STN}$ & -0.61 & BSL & 28 & 10 & BSL & 11.86 & 1.00 \\
\hline HD 7983 & 011900.12 & -085618.2 & HIP & -0.68 & B99 & -7 & 10 & HK & 8.90 & 0.60 \\
\hline BPS CS 22174-0020 & 012027.22 & -084715.1 & $\mathrm{U}$ & -2.70 & HK & 117 & 10 & BSL & 15.06 & 0.40 \\
\hline $\mathrm{V}^{*} \mathrm{SS}$ Psc & 012052.37 & +214343.2 & $\mathrm{ACT}$ & -0.89 & BSL & 5 & 30 & BSL & 10.98 & $\ldots$ \\
\hline$-310115 \mathrm{~A}$ & 012108.87 & -313019.8 & SPM & -1.40 & BSL & -40 & 10 & BSL & 16.05 & 1.08 \\
\hline HD 8186 & 012124.95 & +135522.0 & $\mathrm{ACT}$ & -0.67 & BSL & -37 & 10 & BSL & 8.98 & 1.00 \\
\hline BPS CS 22946-0017 & 012137.51 & -172552.9 & $\mathrm{U}$ & -1.90 & HK & 28 & 10 & BSL & 14.85 & 0.42 \\
\hline BPS CS 29514-0037 & 012141.67 & -270331.0 & SPM & -2.16 & HK & -31 & 10 & BSL & 13.95 & 0.50 \\
\hline-300115 A & 012146.21 & -301615.5 & $\mathrm{SPM}$ & -1.10 & BSL & 1 & 10 & BSL & 15.69 & 1.13 \\
\hline$-290115 \mathrm{~A}$ & 012153.90 & -291526.5 & SPM & -1.50 & BSL & -205 & 10 & BSL & 13.85 & 1.42 \\
\hline BPS CS 22946-0012 & 012336.31 & -192555.0 & U & -1.71 & HK & 88 & 10 & BSL & 14.65 & 0.46 \\
\hline BPS CS 22946-0014 & 012405.57 & -175121.5 & $\mathrm{U}$ & -2.06 & HK & 95 & 10 & BSL & 14.02 & 0.39 \\
\hline BPS CS 22953-0037 & 012506.51 & $\begin{array}{lll}-59 & 16 & 01.3\end{array}$ & $\mathrm{U}$ & -2.94 & B99 & -153 & 10 & HK & 13.64 & 0.37 \\
\hline BPS CS 29504-0012 & 012537.68 & -331048.1 & $\mathrm{SPM}$ & -2.20 & HK & -123 & 10 & BSL & 15.19 & 0.41 \\
\hline HD 8724 & 012617.56 & +170735.8 & HIP & -1.64 & B99 & -110 & 1 & CY & 8.30 & 0.96 \\
\hline [FF93] 516243 & 012639.16 & -365446.4 & SPM & -0.70 & BSL & 76 & 10 & BSL & 10.46 & 1.30 \\
\hline V* XY And & 012647.87 & +340239.7 & $\mathrm{v}$ & -0.92 & $\mathrm{~L}$ & -64 & 53 & L & 13.63 & $\cdots$ \\
\hline BPS CS 29504-0002 & 012822.67 & -365557.3 & SPM & -0.86 & HK & -25 & 10 & BSL & 14.72 & 0.61 \\
\hline HD 9051 & 012846.47 & -242025.3 & HIP & -1.50 & ATT & -73 & 1 & $\mathrm{CY}$ & 8.93 & 0.82 \\
\hline BPS CS 29504-0009 & 012914.56 & -352535.4 & $\mathrm{SPM}$ & -1.56 & HK & 100 & 10 & BSL & 15.16 & 0.54 \\
\hline HD 9360 & 013141.01 & -231358.4 & SPM & -0.62 & BSL & -18 & 10 & BSL & 9.39 & 1.19 \\
\hline BPS CS 29504-0006 & 013204.96 & -362243.0 & SPM & -2.93 & HK & -90 & 10 & BSL & 14.49 & 0.38 \\
\hline $\mathrm{V}^{*} \mathrm{RR}$ Cet & 013208.17 & +012030.6 & HIP & -1.45 & F98 & -74 & 1 & F98 & 9.73 & $\ldots$ \\
\hline BPS CS 22180-0013 & 013233.07 & -120410.6 & $\mathrm{STN}$ & -0.60 & HK & 12 & 10 & BSL & $(11.63)$ & $(0.67)$ \\
\hline BPS CS 22953-0031 & 013327.28 & -62 1331.4 & $\mathrm{U}$ & -1.51 & HK & 199 & 10 & BSL & $(14.01)$ & $(0.49)$ \\
\hline BPS CS 22180-0014 & 013402.12 & -124149.0 & $\mathrm{U}$ & -2.30 & HK & 32 & 10 & BSL & 13.58 & 0.43 \\
\hline BPS CS 22180-0005 & 013427.77 & -101619.8 & $\mathrm{U}$ & -2.19 & HK & 38 & 10 & BSL & 14.02 & 0.50 \\
\hline
\end{tabular}


TABLE 1A. (continued)

\begin{tabular}{|c|c|c|c|c|c|c|c|c|c|c|}
\hline $\begin{array}{l}\text { Star } \\
\text { (1) }\end{array}$ & RA (2000.0) & DEC & Source $^{\mathrm{a}}$ & $\begin{array}{c}{[\mathrm{Fe} / \mathrm{H}]} \\
(\mathrm{dex}) \\
(5)\end{array}$ & $\begin{array}{c}\text { Source }^{b} \\
(6)\end{array}$ & $\begin{array}{c}\mathrm{RV} \\
(\mathrm{km} / \mathrm{s}) \\
(7)\end{array}$ & $\begin{array}{c}\sigma_{\mathrm{RV}} \\
(\mathrm{km} / \mathrm{s}) \\
(8)\end{array}$ & $\begin{array}{c}\text { Source }^{c} \\
(9)\end{array}$ & $\begin{array}{c}V \\
(\mathrm{mag}) \\
(10)\end{array}$ & $\begin{array}{c}B-V \\
(\mathrm{mag}) \\
(11)\end{array}$ \\
\hline BPS CS 22180-0010 & 013430.22 & -110331.8 & $\mathrm{U}$ & -0.97 & HK & -37 & 10 & BSL & $(14.60)$ & $(0.40)$ \\
\hline HD 9731 & 013451.55 & -234159.0 & HIP & -0.65 & BSL & 38 & 10 & BSL & 6.41 & 1.31 \\
\hline $\mathrm{V}^{*} \mathrm{VX} \mathrm{Scl}$ & 013523.64 & -350742.4 & HIP & -2.25 & F98 & -47 & 11 & F98 & 12.02 & $\ldots$ \\
\hline BPS CS 22180-0034 & 013631.11 & -092313.5 & $\mathrm{U}$ & -2.06 & HK & -17 & 10 & BSL & 14.31 & 0.42 \\
\hline BD-18 271 & 013718.88 & -172903.7 & HIP & -2.17 & B99 & -209 & 1 & $\mathrm{CY}$ & 9.81 & 1.22 \\
\hline HD 10141 & 013832.63 & -321458.4 & SPM & -0.92 & BSL & 87 & 10 & BSL & 9.47 & 1.04 \\
\hline BPS CS 29504-0032 & 013922.40 & $\begin{array}{lll}-33 & 04 & 22.1\end{array}$ & SPM & -1.48 & HK & -29 & 10 & BSL & 12.86 & 0.52 \\
\hline HD 10413 & 014058.06 & -275548.2 & SPM & -0.66 & BSL & 26 & 10 & BSL & 9.25 & 1.20 \\
\hline HD 10607 & $0141 \quad 14.20$ & -674033.1 & HIP & -1.14 & $\mathrm{C} 97$ & 0 & 10 & BSL & 8.33 & 0.54 \\
\hline BPS CS 22958-0008 & 014440.15 & -550027.0 & $\mathrm{U}$ & -2.29 & HK & 136 & 10 & BSL & $(12.84)$ & $(0.56)$ \\
\hline BPS CS 22180-0030 & 014440.27 & -102108.5 & $\mathrm{U}$ & -2.14 & HK & -88 & 10 & BSL & 13.78 & 0.65 \\
\hline $\mathrm{V}^{*} \mathrm{SV} \mathrm{Scl}$ & 014459.66 & -300333.1 & HIP & -1.77 & F98 & -10 & 10 & F98 & 11.38 & $\cdots$ \\
\hline BPS CS 22958-0014 & 014637.52 & -534655.4 & U & -2.10 & HK & -31 & 10 & BSL & {$[15.10]$} & {$[0.44]$} \\
\hline BPS CS 29504-0044 & 014647.84 & -341743.5 & SPM & -2.04 & HK & 262 & 10 & BSL & 13.57 & 0.50 \\
\hline BPS CS 22958-0004 & 014728.36 & -564723.1 & $\mathrm{U}$ & -1.66 & HK & 27 & 10 & BSL & $(14.11)$ & $(0.49)$ \\
\hline BPS CS 22958-0002 & 014816.48 & $\begin{array}{lll}-57 & 1702.8\end{array}$ & STN & -2.37 & HK & -83 & 10 & BSL & 12.70 & 0.67 \\
\hline BPS CS 22958-0026 & 015014.28 & -545331.9 & U & -2.17 & HK & 100 & 10 & BSL & $(13.00)$ & $(0.50)$ \\
\hline HD 11569 & 015024.28 & -715656.4 & HIP & -1.65 & BSL & -18 & 10 & BSL & 9.01 & 0.37 \\
\hline BD-10 388 & 015032.50 & -092103.7 & HIP & -2.44 & B99 & 33 & 10 & HK & 10.34 & 0.43 \\
\hline BPS CS 22958-0025 & 015111.90 & -543302.5 & $\mathrm{U}$ & -2.16 & HK & 132 & 10 & BSL & $(14.73)$ & $(0.37)$ \\
\hline BPS CS 22958-0019 & 015239.30 & $-5402 \quad 11.6$ & $\mathrm{U}$ & -1.81 & HK & -74 & 10 & BSL & $(14.75)$ & $(0.55)$ \\
\hline HD 11582 & 015300.23 & -341734.5 & HIP & -1.57 & ATT & 8 & 10 & BSL & 9.57 & 0.67 \\
\hline $\mathrm{V}^{*} \mathrm{CI}$ And & 015508.29 & +434556.5 & HIP & -0.69 & F98 & 24 & 5 & F98 & 12.28 & $\ldots$ \\
\hline BPS CS 22958-0068 & 015526.87 & -525931.5 & $\mathrm{U}$ & -0.50 & HK & 54 & 10 & BSL & $(14.48)$ & $(0.62)$ \\
\hline $\mathrm{V}^{*} \mathrm{U}$ Tri & 015531.44 & +334607.6 & STN & -0.79 & $\mathrm{~L}$ & 6 & 23 & L & 12.60 & $\ldots$ \\
\hline BPS CS 22958-0036 & 015804.72 & -570325.9 & GSC & -1.45 & HK & 64 & 10 & BSL & {$[13.70]$} & {$[0.38]$} \\
\hline BPS CS 22958-0037 & 015839.68 & -570326.7 & GSC & -2.42 & HK & 203 & 10 & BSL & 14.91 & $(0.53)$ \\
\hline BPS CS 22958-0061 & 015917.71 & $\begin{array}{lll}-55 & 0923.8\end{array}$ & U & -2.29 & HK & 109 & 10 & BSL & $(14.33)$ & $(0.47)$ \\
\hline BPS CS 22958-0038 & 015924.15 & -565221.9 & STN & -2.04 & HK & 72 & 10 & BSL & (13.28) & $(0.42)$ \\
\hline BPS CS 22958-0042 & 020107.32 & -571658.5 & U & -2.62 & HK & 151 & 10 & BSL & 14.54 & $(0.46)$ \\
\hline BPS CS 22958-0041 & 020144.50 & -564726.9 & $\mathrm{U}$ & -2.70 & HK & -52 & 10 & BSL & 15.07 & $(0.42)$ \\
\hline BPS CS 22958-0065 & 020253.53 & -533840.3 & $\mathrm{U}$ & -2.30 & HK & 112 & 10 & BSL & 14.48 & $(0.46)$ \\
\hline BPS CS 22958-0073 & 020620.05 & -531107.8 & $\mathrm{U}$ & -1.88 & HK & 47 & 10 & BSL & $(14.43)$ & $(0.37)$ \\
\hline $\mathrm{V}^{*} \mathrm{SS}$ For & 020751.96 & -265157.1 & HIP & -0.94 & F98 & -113 & 1 & F98 & 10.19 & $\cdots$ \\
\hline BPS CS 22171-0037 & 020800.55 & -090346.6 & $\mathrm{U}$ & -3.31 & HK & -234 & 10 & BSL & 14.93 & 0.35 \\
\hline BPS CS 22958-0079 & 020826.75 & -540106.4 & $\mathrm{U}$ & -1.88 & HK & -1 & 10 & BSL & $(15.14)$ & $(0.46)$ \\
\hline BPS CS 22171-0031 & 020924.40 & -103415.2 & U & -2.06 & HK & 36 & 10 & BSL & 13.93 & 0.39 \\
\hline BPS CS 22171-0034 & 021002.65 & -100021.6 & STN & -2.12 & HK & -92 & 10 & BSL & 13.29 & 0.41 \\
\hline HD 13359 & 021007.03 & -204551.5 & HIP & -1.66 & BSL & 31 & 10 & BSL & 9.70 & 0.70 \\
\hline BPS CS 22958-0049 & 021011.78 & $\begin{array}{lll}-56 & 07 & 17.1\end{array}$ & $\mathrm{U}$ & -1.80 & HK & 292 & 10 & BSL & $(14.42)$ & $(0.48)$ \\
\hline
\end{tabular}


TABLE 1A. (continued)

\begin{tabular}{|c|c|c|c|c|c|c|c|c|c|c|}
\hline $\begin{array}{l}\text { Star } \\
(1)\end{array}$ & $\begin{array}{c}\text { RA (2000.0) } \\
(2)\end{array}$ & DEC & $\begin{array}{c}\text { Source }^{\mathrm{a}} \\
(4)\end{array}$ & $\begin{array}{c}{[\mathrm{Fe} / \mathrm{H}]} \\
(\mathrm{dex}) \\
(5)\end{array}$ & $\begin{array}{c}\text { Source }^{b} \\
(6)\end{array}$ & $\begin{array}{c}\mathrm{RV} \\
(\mathrm{km} / \mathrm{s}) \\
(7)\end{array}$ & $\begin{array}{c}\sigma_{\mathrm{RV}} \\
(\mathrm{km} / \mathrm{s}) \\
(8)\end{array}$ & $\begin{array}{c}\text { Source }^{c} \\
(9)\end{array}$ & $\begin{array}{c}V \\
(\mathrm{mag}) \\
(10)\end{array}$ & $\begin{array}{c}B-V \\
(\mathrm{mag}) \\
(11)\end{array}$ \\
\hline BPS CS 22958-0074 & $02 \quad 1034.19$ & -532242.6 & $\mathrm{U}$ & -2.30 & HK & 321 & 10 & BSL & 14.80 & $(0.49)$ \\
\hline BPS CS 22958-0053 & 021237.75 & -553350.1 & $\mathrm{U}$ & -1.81 & HK & 40 & 10 & BSL & $(14.62)$ & $(0.58)$ \\
\hline BPS CS 22958-0052 & $02 \quad 12 \quad 48.13$ & -562908.8 & $\mathrm{U}$ & -2.16 & HK & 99 & 10 & BSL & 14.22 & $(0.43)$ \\
\hline HD 13889 & 021410.69 & $-41 \quad 42 \quad 45.4$ & HIP & -1.17 & BSL & 61 & 10 & BSL & 9.55 & 0.55 \\
\hline BPS CS 22958-0082 & 021501.63 & -541738.4 & $\mathrm{U}$ & -2.12 & HK & -24 & 10 & BSL & $(14.38)$ & $(0.42)$ \\
\hline $\mathrm{V}^{*} \mathrm{RV}$ Cet & 021514.89 & -104800.5 & HIP & -1.60 & F98 & -94 & 3 & F98 & 10.92 & $\ldots$ \\
\hline HD 13979 & 021520.83 & -255454.4 & HIP & -2.61 & B99 & 54 & 1 & $\mathrm{CY}$ & 9.17 & 0.65 \\
\hline BPS CS 22958-0083 & 021542.73 & -535956.1 & $\mathrm{U}$ & -2.53 & HK & 180 & 10 & BSL & 14.44 & 0.65 \\
\hline $\mathrm{V}^{*} \mathrm{RW}$ Ari & 021603.68 & +173158.4 & STN & -1.16 & BSL & -60 & 30 & BSL & 12.39 & $\cdots$ \\
\hline $\mathrm{V}^{*} \mathrm{TW}$ Hyi & 021930.63 & -733357.0 & GSC & -1.66 & L & 227 & 9 & L & 13.04 & $\cdots$ \\
\hline BD-22 395 & $02 \quad 2157.91$ & -213042.7 & HIP & -2.14 & ATT & 103 & 1 & $\mathrm{CY}$ & 10.52 & 0.80 \\
\hline HD 14829 & 022309.23 & -104038.9 & HIP & -1.25 & C97 & -180 & 10 & BSL & 10.29 & 0.02 \\
\hline HD 15061 & 022602.28 & +175913.8 & $\mathrm{ACT}$ & -0.91 & BSL & 10 & 10 & BSL & 9.50 & 1.50 \\
\hline HD 15395 & $02 \quad 2644.72$ & -543233.9 & HIP & -1.14 & BSL & 41 & 10 & BSL & 9.43 & 0.56 \\
\hline $\mathrm{BD}+44493$ & 022649.64 & +445746.8 & HIP & -2.90 & BSL & -151 & 10 & BSL & 9.04 & 0.72 \\
\hline V* RZ Cet & 022832.43 & -082130.1 & HIP & -1.36 & F98 & -7 & 3 & F98 & 11.85 & $\cdots$ \\
\hline BD-17 484 & 023125.52 & -165904.5 & HIP & -1.68 & BSL & 223 & 10 & BSL & 10.46 & 0.45 \\
\hline BPS CS 22184-0001 & 023217.05 & -103251.7 & $\mathrm{U}$ & -1.58 & HK & 161 & 10 & BSL & $(14.50)$ & $(0.40)$ \\
\hline BPS CS 22189-0004 & 023226.53 & -143833.7 & $\mathrm{U}$ & -1.62 & HK & 168 & 10 & BSL & 13.43 & 0.39 \\
\hline HD 16031 & 023411.01 & -122301.8 & HIP & -1.71 & B99 & 19 & 10 & HK & 9.77 & 0.44 \\
\hline V* CS Eri & 023705.68 & -425748.1 & HIP & -1.41 & F98 & -147 & 10 & F98 & 9.00 & $\cdots$ \\
\hline BPS CS 22189-0007 & 023734.12 & -125622.0 & STN & -2.12 & HK & -281 & 10 & BSL & 13.21 & 0.58 \\
\hline BPS CS 22954-0004 & 023815.17 & -054315.6 & U & -2.32 & HK & 28 & 10 & BSL & 14.27 & $(0.47)$ \\
\hline CD-50 776 & 023902.42 & -492746.3 & HIP & -0.92 & BSL & 18 & 10 & BSL & 10.11 & 0.78 \\
\hline BPS CS 22954-0015 & 023921.64 & -022937.6 & STN & -1.67 & HK & -30 & 10 & BSL & 13.02 & $(0.46)$ \\
\hline HD 17072 & 024039.99 & -691358.7 & HIP & -1.00 & ATT & 63 & 10 & BSL & 6.58 & 0.66 \\
\hline BPS CS 22189-0009 & 024142.34 & -132810.0 & $\mathrm{U}$ & -3.44 & B99 & -26 & 10 & HK & 14.04 & 0.72 \\
\hline HD 17233 & 024352.03 & -544728.3 & HIP & -0.60 & BSL & -18 & 10 & BSL & 9.03 & 0.79 \\
\hline BPS CS 22189-0018 & 024412.75 & -154723.9 & $\mathrm{U}$ & -2.91 & HK & -192 & 10 & BSL & 15.33 & 0.36 \\
\hline BPS CS 22189-0027 & 024433.88 & -140254.3 & $\mathrm{U}$ & -1.84 & HK & -7 & 10 & BSL & 14.11 & 0.42 \\
\hline BPS CS 22189-0029 & 024629.03 & -135702.7 & $\mathrm{U}$ & -1.96 & HK & -79 & 10 & BSL & 14.20 & 0.38 \\
\hline BD-10 548 & 024655.72 & -100735.0 & HIP & -1.07 & B99 & 239 & 1 & $\mathrm{CY}$ & 10.41 & 0.77 \\
\hline CD-36 1052 & 024737.44 & -360627.1 & HIP & -2.19 & ATT & 306 & 5 & $\mathrm{CY}$ & 9.94 & 0.47 \\
\hline BPS CS 22184-0040 & 024747.23 & -09 1216.6 & STN & -1.72 & HK & 177 & 10 & BSL & 13.63 & 0.60 \\
\hline BPS CS 22189-0036 & 024944.28 & -130734.9 & U & -2.12 & HK & 171 & 10 & BSL & 14.38 & 0.43 \\
\hline $\mathrm{V}^{*} \mathrm{BK}$ Eri & 024955.86 & -012512.2 & STN & -1.64 & $\mathrm{~L}$ & 141 & 10 & L & 12.67 & $\ldots$ \\
\hline BPS CS 22954-0011 & 025034.28 & -052217.2 & U & -1.71 & HK & -112 & 10 & BSL & $(14.15)$ & $(0.55)$ \\
\hline$-610250 \mathrm{G}$ & 025109.56 & $\begin{array}{lll}-61 & 40 & 15.1\end{array}$ & U & -1.30 & BSL & 156 & 10 & BSL & 15.97 & 1.06 \\
\hline $\mathrm{V}^{*} \mathrm{BY}$ Eri & 025134.16 & -474804.8 & STN & -1.16 & BSL & 140 & 30 & BSL & 12.16 & $\cdots$ \\
\hline$-600250 \mathrm{M}$ & 025533.42 & -595043.4 & U & -1.10 & BSL & 73 & 10 & BSL & 15.56 & 1.29 \\
\hline
\end{tabular}


TABLE 1A. (continued)

\begin{tabular}{|c|c|c|c|c|c|c|c|c|c|c|}
\hline $\begin{array}{l}\text { Star } \\
(1)\end{array}$ & $\begin{array}{c}\text { RA (2000.0) } \\
(2)\end{array}$ & DEC & $\begin{array}{c}\text { Source }^{\mathrm{a}} \\
(4)\end{array}$ & $\begin{array}{c}{[\mathrm{Fe} / \mathrm{H}]} \\
(\mathrm{dex}) \\
(5)\end{array}$ & $\begin{array}{c}\text { Source }^{b} \\
(6)\end{array}$ & $\begin{array}{c}\mathrm{RV} \\
(\mathrm{km} / \mathrm{s}) \\
(7)\end{array}$ & $\begin{array}{c}\sigma_{\mathrm{RV}} \\
(\mathrm{km} / \mathrm{s}) \\
(8)\end{array}$ & $\begin{array}{c}\text { Source }^{c} \\
(9)\end{array}$ & $\begin{array}{c}V \\
(\mathrm{mag}) \\
(10)\end{array}$ & $\begin{array}{c}B-V \\
(\mathrm{mag}) \\
(11)\end{array}$ \\
\hline BPS CS 22963-0004 & 025646.50 & $-0451 \quad 17.2$ & $\mathrm{U}$ & -3.01 & HK & 299 & 10 & BSL & 14.98 & 0.53 \\
\hline CD-30 1121 & 025700.27 & -302437.2 & HIP & -1.62 & $\mathrm{C} 97$ & 104 & 1 & $\mathrm{CY}$ & 10.31 & 0.77 \\
\hline$-580250 \mathrm{G}$ & 025720.85 & -575732.5 & $\mathrm{U}$ & -1.60 & BSL & 46 & 10 & BSL & 15.74 & 0.93 \\
\hline BPS CS 22968-0009 & $02 \quad 5727.99$ & -532535.7 & $\mathrm{U}$ & -1.62 & HK & 28 & 10 & BSL & 14.82 & 0.39 \\
\hline BPS CS 22963-0009 & 025740.82 & -053843.0 & $\mathrm{U}$ & -1.92 & HK & 32 & 10 & BSL & 13.54 & $(0.51)$ \\
\hline BPS CS 22968-0001 & 025750.91 & -565115.9 & $\mathrm{U}$ & -2.60 & HK & 117 & 10 & BSL & 14.74 & 0.39 \\
\hline BPS CS 22968-0007 & 025757.72 & -534329.4 & $\mathrm{U}$ & -1.66 & HK & 44 & 10 & BSL & 14.94 & 0.37 \\
\hline HD 18710 & 025842.59 & -484356.6 & HIP & -1.05 & BSL & -17 & 10 & BSL & 9.68 & 0.94 \\
\hline $\mathrm{V}^{*} \mathrm{SW}$ For & 030108.73 & $\begin{array}{lll}-38 & 07 & 43.4\end{array}$ & GSC & -1.95 & $\mathrm{~L}$ & 174 & 18 & L & 12.34 & $\cdots$ \\
\hline$-600250 \mathrm{~A}$ & 030109.55 & -601345.5 & $\mathrm{U}$ & -1.30 & BSL & 64 & 10 & BSL & 13.73 & 1.17 \\
\hline BPS CS 22963-0005 & 030131.34 & -050015.9 & $\mathrm{U}$ & -0.85 & HK & 135 & 10 & BSL & {$[14.20]$} & [0.63] \\
\hline$-610300 \mathrm{C}$ & 030301.21 & -612119.6 & $\mathrm{U}$ & -0.70 & BSL & 107 & 10 & BSL & 15.06 & 1.19 \\
\hline BPS CS 22963-0023 & 030558.82 & -041509.3 & $\mathrm{U}$ & -0.64 & HK & 47 & 10 & BSL & $(14.19)$ & $(0.41)$ \\
\hline BPS CS 22968-0014 & 030629.48 & -543032.5 & $\mathrm{U}$ & -3.43 & B99 & 158 & 10 & HK & 13.72 & 0.72 \\
\hline $\mathrm{V}^{*} \mathrm{X}$ Ari & 030830.85 & +102646.0 & HIP & -2.43 & F98 & -36 & 1 & F98 & 9.57 & $\cdots$ \\
\hline$-580300 \mathrm{D}$ & 030846.65 & $\begin{array}{lll}-58 & 03 & 25.1\end{array}$ & $\mathrm{U}$ & -1.90 & BSL & 36 & 10 & BSL & 15.16 & 0.99 \\
\hline $\mathrm{V}^{*} \mathrm{TU}$ Per & 030904.74 & +531136.4 & $\mathrm{U}$ & -1.50 & L & -377 & 11 & L & 12.53 & $\cdots$ \\
\hline HD 20038 & 031026.81 & -584940.5 & HIP & -0.87 & B99 & 27 & 10 & HK & 8.90 & 0.84 \\
\hline HD 19697 & 031046.07 & +142035.4 & $\mathrm{ACT}$ & -0.65 & BSL & 10 & 10 & BSL & 8.89 & 1.68 \\
\hline $\mathrm{V}^{*} \mathrm{RX}$ For & 031113.18 & -262858.9 & GSC & -1.26 & L & 22 & 9 & L & 11.90 & $\cdots$ \\
\hline $\mathrm{V}^{*} \mathrm{SV}$ Eri & $\begin{array}{lll}03 & 11 & 52.10\end{array}$ & -112113.6 & HIP & -1.70 & F98 & -14 & 4 & F98 & 9.96 & $\cdots$ \\
\hline BPS CS 22968-0043 & 031317.57 & -531258.0 & $\mathrm{U}$ & -2.25 & HK & -83 & 10 & BSL & 14.64 & 0.49 \\
\hline $\mathrm{V}^{*} \mathrm{UY}$ Eri & 031339.12 & -102632.4 & HIP & -1.60 & BSL & 171 & 1 & BSL & 11.46 & 0.50 \\
\hline HD 20305 & 031625.45 & +135105.4 & HIP & -0.74 & BSL & 31 & 10 & BSL & 8.47 & 1.54 \\
\hline BPS CS 22968-0029 & 031627.67 & -550459.9 & $\mathrm{U}$ & -2.87 & HK & 84 & 10 & BSL & 14.29 & 0.41 \\
\hline BPS CS 22185-0007 & 031736.01 & -151722.2 & $\mathrm{U}$ & -2.59 & HK & 105 & 10 & BSL & 13.34 & 0.71 \\
\hline BPS CS 22968-0030 & 031736.13 & -544422.1 & $\mathrm{U}$ & -2.10 & HK & 95 & 10 & BSL & 15.49 & 0.43 \\
\hline BPS CS 22968-0039 & 031749.88 & $\begin{array}{lll}-53 & 07 & 02.9\end{array}$ & $\mathrm{U}$ & -1.77 & HK & 47 & 10 & BSL & $(14.06)$ & $(0.64)$ \\
\hline BPS CS 22185-0006 & 031811.10 & -153134.6 & $\mathrm{U}$ & -2.04 & HK & 103 & 10 & BSL & 14.21 & 0.45 \\
\hline V* UU Hor & 031825.41 & -493601.8 & STN & -1.80 & $\mathrm{~L}$ & 220 & 20 & L & 12.44 & $\cdots$ \\
\hline$-580310 \mathrm{~A}$ & 032033.01 & -583505.8 & $\mathrm{U}$ & -0.60 & BSL & 99 & 10 & BSL & 15.03 & 1.01 \\
\hline BPS CS 22968-0026 & 032048.84 & -553815.2 & $\mathrm{U}$ & -2.20 & HK & 102 & 10 & BSL & 14.24 & 0.43 \\
\hline$-610320 \mathrm{C}$ & 032210.51 & -605727.9 & $\mathrm{U}$ & -1.30 & BSL & 114 & 10 & BSL & 13.42 & 1.18 \\
\hline BPS CS 22185-0002 & 032210.52 & -165540.4 & $\mathrm{U}$ & -1.78 & HK & -12 & 10 & BSL & 13.77 & 0.41 \\
\hline HD 21022 & 032221.59 & -325939.7 & HIP & -1.99 & ATT & 110 & 3 & $\mathrm{CY}$ & 9.20 & 0.95 \\
\hline $\mathrm{V}^{*} \mathrm{RX}$ Men & 032227.66 & -812208.8 & $\mathrm{U}$ & -1.54 & $\mathrm{~L}$ & -18 & 40 & L & 14.18 & $\cdots$ \\
\hline BPS CS 22172-0019 & 032251.58 & -112351.3 & $\mathrm{U}$ & -1.87 & HK & 220 & 10 & BSL & 14.29 & 0.42 \\
\hline BPS CS 22968-0033 & 032300.60 & -543624.0 & U & -0.36 & HK & 8 & 10 & BSL & 15.28 & 0.57 \\
\hline $\mathrm{V}^{*} \mathrm{~V}$ Ret & 032310.78 & -611826.6 & GSC & -1.79 & L & 98 & 40 & L & 13.86 & $\ldots$ \\
\hline BPS CS 22968-0032 & 032337.76 & -543906.6 & $\mathrm{U}$ & -0.70 & HK & 84 & 10 & BSL & {$[15.70]$} & {$[0.62]$} \\
\hline
\end{tabular}


TABLE 1A. (continued)

\begin{tabular}{|c|c|c|c|c|c|c|c|c|c|c|}
\hline Star & $\begin{array}{c}\text { RA (2000.0) } \\
(2)\end{array}$ & DEC & $\begin{array}{c}\text { Source }^{\mathrm{a}} \\
(4)\end{array}$ & $\begin{array}{c}{[\mathrm{Fe} / \mathrm{H}]} \\
(\mathrm{dex}) \\
(5)\end{array}$ & $\begin{array}{c}\text { Source }^{b} \\
(6)\end{array}$ & $\begin{array}{c}\mathrm{RV} \\
(\mathrm{km} / \mathrm{s}) \\
(7)\end{array}$ & $\begin{array}{c}\sigma_{\mathrm{RV}} \\
(\mathrm{km} / \mathrm{s}) \\
(8)\end{array}$ & $\begin{array}{c}\text { Source }{ }^{\mathrm{c}} \\
(9)\end{array}$ & $\begin{array}{c}V \\
(\mathrm{mag}) \\
(10)\end{array}$ & $\begin{array}{c}B-V \\
(\mathrm{mag}) \\
(11)\end{array}$ \\
\hline $\mathrm{V}^{*} \mathrm{X}$ Ret & 032520.00 & $\begin{array}{lll}-65 & 03 & 18.7\end{array}$ & GSC & -1.32 & L & 144 & 13 & L & 11.72 & \\
\hline BPS CS 22185-0018 & 032757.43 & -131532.4 & U & -0.44 & HK & 23 & 10 & BSL & $(13.86)$ & $(0.64)$ \\
\hline$-600320 \mathrm{~L}$ & 032832.56 & -595608.0 & $\mathrm{U}$ & -1.50 & BSL & 328 & 10 & BSL & 15.30 & 1.01 \\
\hline HD 21581 & 032854.45 & -002502.4 & HIP & -1.63 & B99 & 154 & 1 & $\mathrm{CY}$ & 8.70 & 0.79 \\
\hline $\mathrm{V}^{*} \mathrm{SX}$ For & 033022.32 & $\begin{array}{lll}-36 & 03 & 13.4\end{array}$ & HIP & -1.66 & F98 & 230 & 3 & F98 & 11.12 & $\cdots$ \\
\hline BPS CS 22172-0029 & 033045.33 & -103709.0 & $\mathrm{U}$ & -2.07 & HK & -58 & 10 & BSL & 14.36 & 0.60 \\
\hline BPS CS 22172-0025 & 033232.84 & -112837.7 & STN & -1.17 & HK & 23 & 10 & BSL & $(12.86)$ & $(0.44)$ \\
\hline $\mathrm{V}^{*} \mathrm{SS} \mathrm{Tau}$ & 033641.83 & +052140.9 & GSC & -0.28 & $\mathrm{~L}$ & -11 & 10 & L & 12.50 & $\ldots$ \\
\hline $\mathrm{V}^{*} \mathrm{TV}$ For & 033653.40 & $-31 \quad 1548.3$ & U & -0.93 & $\mathrm{~L}$ & 193 & 40 & $\mathrm{~L}$ & 14.15 & $\cdots$ \\
\hline CD-24 1782 & 033841.43 & -240249.2 & HIP & -2.46 & B99 & 119 & 4 & $\mathrm{CY}$ & 9.92 & 0.65 \\
\hline HD 22879 & 034021.66 & -031259.3 & HIP & -0.78 & B99 & 118 & 10 & HK & 6.68 & 0.55 \\
\hline HD 23332 & 034136.58 & -554029.7 & HIP & -0.90 & BSL & -33 & 10 & BSL & 8.90 & 0.90 \\
\hline $\mathrm{V}^{*} \mathrm{TW}$ For & 034242.88 & -314350.6 & $\mathrm{U}$ & -1.95 & L & 117 & 35 & L & 14.31 & $\ldots$ \\
\hline BPS CS 29529-0004 & 034308.95 & -614448.8 & $\mathrm{U}$ & -0.95 & HK & 25 & 10 & BSL & $(13.66)$ & $(0.44)$ \\
\hline BPS CS 29529-0012 & 034326.10 & -602018.3 & $\mathrm{U}$ & -2.27 & $\mathrm{BCM}$ & 94 & 10 & BSL & $(14.80)$ & $(0.40)$ \\
\hline HD 23592 & 034354.99 & $\begin{array}{lll}-52 & 02 & 48.7\end{array}$ & HIP & -1.50 & BSL & -54 & 10 & BSL & 9.45 & 0.86 \\
\hline BPS CS 29529-0028 & 034603.60 & -581150.6 & $\mathrm{U}$ & -2.08 & HK & 220 & 10 & BSL & $(13.46)$ & $(0.54)$ \\
\hline BPS CS 29529-0025 & $0346 \quad 15.34$ & $-58 \quad 1852.1$ & U & -0.69 & HK & -6 & 10 & HK & $(12.32)$ & $(0.48)$ \\
\hline BPS CS 29529-0024 & 034616.52 & -582155.4 & $\mathrm{U}$ & -0.65 & HK & 49 & 10 & HK & $(12.95)$ & $(0.55)$ \\
\hline BPS CS 29529-0026 & 034620.77 & -581914.5 & $\mathrm{U}$ & -1.18 & HK & 30 & 10 & BSL & $(13.86)$ & $(0.44)$ \\
\hline HD 23798 & 034645.71 & $-3051 \quad 13.3$ & HIP & -2.20 & ATT & 90 & 1 & $\mathrm{CY}$ & 8.28 & 1.03 \\
\hline BPS CS 29529-0005 & 034728.54 & -612817.0 & $\mathrm{U}$ & -1.18 & HK & 13 & 10 & BSL & $(13.78)$ & $(0.72)$ \\
\hline BPS CS 29529-0002 & 034836.53 & $-62 \quad 1630.2$ & U & -0.84 & HK & 50 & 10 & BSL & $(12.90)$ & $(0.60)$ \\
\hline BPS CS 29529-0021 & 034839.18 & -585220.8 & U & -0.44 & HK & -1 & 10 & BSL & $(13.77)$ & $(0.53)$ \\
\hline $8578 \mathrm{~N} 1$ & 035035.20 & +092553.1 & $\mathrm{v}$ & -1.41 & BSL & 79 & 10 & BSL & 9.40 & $\ldots$ \\
\hline BPS CS 29529-0042 & 035201.50 & -602759.2 & U & -1.65 & HK & 138 & 10 & BSL & $(14.10)$ & $(0.50)$ \\
\hline BPS CS 22190-0007 & 035221.79 & -162432.1 & U & -2.29 & HK & -132 & 10 & BSL & 14.20 & 0.46 \\
\hline BPS CS 29529-0039 & 035246.78 & -594232.8 & $\mathrm{U}$ & -0.65 & HK & 11 & 10 & BSL & $(14.15)$ & $(0.55)$ \\
\hline BPS CS 29529-0034 & 035353.42 & -590629.2 & $\mathrm{U}$ & -2.24 & HK & 94 & 10 & BSL & $(14.94)$ & $(0.46)$ \\
\hline BPS CS 29529-0040 & 035458.49 & -594453.8 & $\mathrm{U}$ & -2.07 & HK & -3 & 10 & BSL & 13.41 & 0.71 \\
\hline BPS CS 29529-0045 & 035848.03 & $\begin{array}{lll}-60 & 40 & 31.9\end{array}$ & $\mathrm{U}$ & -1.51 & HK & 67 & 10 & BSL & $(13.61)$ & $(0.49)$ \\
\hline BPS CS 30494-0003 & 035910.00 & -210647.8 & STN & -2.37 & HK & -49 & 10 & BSL & 12.40 & $(0.65)$ \\
\hline BPS CS 22173-0002 & 035913.20 & -204225.8 & U & -1.96 & HK & -4 & 10 & BSL & 13.47 & 0.40 \\
\hline BPS CS 29529-0054 & 035923.76 & $\begin{array}{lll}-62 & 10 & 08.3\end{array}$ & U & -2.71 & HK & 111 & 10 & BSL & 14.87 & 0.54 \\
\hline BPS CS 29529-0055 & 035944.21 & $-62 \quad 1120.3$ & GSC & -0.75 & HK & 64 & 10 & HK & 14.87 & 0.39 \\
\hline HD 26169 & 040052.12 & -753612.1 & HIP & -2.47 & B99 & -22 & 10 & HK & 8.78 & 0.71 \\
\hline BPS CS 22173-0007 & 040105.84 & -183351.5 & $\mathrm{U}$ & -2.17 & HK & 260 & 10 & BSL & 13.77 & 0.52 \\
\hline BPS CS 22169-0008 & 040336.28 & -124938.9 & U & -2.40 & HK & 178 & 10 & BSL & 14.99 & 0.43 \\
\hline BPS CS 22173-0025 & 040342.84 & -204345.2 & STN & -0.97 & HK & 65 & 10 & BSL & $(12.32)$ & $(0.38)$ \\
\hline HD 25532 & 040410.95 & +232428.1 & HIP & -1.28 & B99 & -113 & 1 & $\mathrm{CY}$ & 8.20 & 0.66 \\
\hline
\end{tabular}


TABLE 1A. (continued)

\begin{tabular}{|c|c|c|c|c|c|c|c|c|c|c|}
\hline $\begin{array}{l}\text { Star } \\
(1)\end{array}$ & $\begin{array}{c}\text { RA (2000.0) } \\
(2)\end{array}$ & $\begin{array}{c}\text { DEC } \\
(3)\end{array}$ & $\begin{array}{c}\text { Source }^{\mathrm{a}} \\
(4)\end{array}$ & $\begin{array}{c}{[\mathrm{Fe} / \mathrm{H}]} \\
(\mathrm{dex}) \\
(5)\end{array}$ & $\begin{array}{c}\text { Source }^{b} \\
(6)\end{array}$ & $\begin{array}{c}\mathrm{RV} \\
(\mathrm{km} / \mathrm{s}) \\
(7)\end{array}$ & $\begin{array}{c}\sigma_{\mathrm{RV}} \\
(\mathrm{km} / \mathrm{s}) \\
(8)\end{array}$ & $\begin{array}{c}\text { Source }^{c} \\
(9)\end{array}$ & $\begin{array}{c}V \\
(\mathrm{mag}) \\
(10)\end{array}$ & $\begin{array}{c}B-V \\
(\mathrm{mag}) \\
(11)\end{array}$ \\
\hline BPS CS 30494-0016 & 040428.08 & -172700.4 & $\mathrm{U}$ & -2.06 & HK & 113 & 10 & BSL & 14.75 & $(0.46)$ \\
\hline BPS CS 22173-0014 & 040439.34 & -172106.3 & $\mathrm{U}$ & -2.02 & HK & 191 & 10 & BSL & 13.73 & 0.37 \\
\hline BPS CS 22173-0015 & 040547.77 & -172103.8 & $\mathrm{U}$ & -1.90 & HK & -226 & 10 & BSL & 13.22 & 0.42 \\
\hline BPS CS 22173-0021 & 040603.02 & -183609.8 & $\mathrm{U}$ & -1.76 & HK & -19 & 10 & BSL & 14.00 & 0.42 \\
\hline BPS CS 22169-0012 & 040644.97 & $-12 \quad 1821.3$ & $\mathrm{U}$ & -1.26 & HK & 16 & 10 & BSL & $(14.11)$ & $(0.49)$ \\
\hline BPS CS 22169-0014 & 040658.93 & -141101.3 & $\mathrm{U}$ & -1.00 & HK & 90 & 10 & BSL & $(14.58)$ & $(0.52)$ \\
\hline BPS CS 29529-0075 & 040701.98 & -575116.5 & $\mathrm{U}$ & -2.26 & HK & 280 & 10 & BSL & (14.18) & $(0.52)$ \\
\hline BPS CS 22169-0017 & 040703.87 & -150149.1 & $\mathrm{U}$ & -1.39 & HK & 149 & 10 & BSL & (13.08) & $(0.52)$ \\
\hline BPS CS 22177-0009 & 040740.61 & -250242.8 & $\mathrm{U}$ & -3.00 & HK & -193 & 10 & BSL & 14.27 & 0.40 \\
\hline BPS CS 29529-0093 & 040743.47 & -613243.3 & $\mathrm{U}$ & -0.48 & HK & 52 & 10 & BSL & (14.07) & $(0.63)$ \\
\hline BPS CS 30494-0038 & 040832.97 & -180030.1 & $\mathrm{U}$ & -1.18 & HK & 52 & 10 & BSL & $(13.28)$ & $(0.62)$ \\
\hline HD 26297 & 040903.39 & -155327.0 & HIP & -1.78 & B99 & 14 & 1 & $\mathrm{CY}$ & 7.46 & 1.09 \\
\hline BPS CS 29529-0092 & 040914.79 & -611147.5 & GSC & -2.04 & HK & 148 & 10 & BSL & {$[14.60]$} & {$[0.42]$} \\
\hline BPS CS 22177-0010 & 041000.46 & -254438.5 & U & -2.50 & HK & 93 & 10 & BSL & 14.31 & 0.40 \\
\hline BPS CS 30494-0049 & $0410 \quad 07.42$ & -193541.7 & $\mathrm{U}$ & -0.84 & HK & -1 & 10 & BSL & $(14.40)$ & $(0.60)$ \\
\hline $\mathrm{V}^{*} \mathrm{XY}$ Eri & 041121.00 & -135124.0 & $\mathrm{t}$ & -2.08 & $\mathrm{~L}$ & 221 & 11 & L & 13.02 & $\ldots$ \\
\hline BPS CS 30494-0045 & 041154.47 & -183534.6 & U & -1.36 & HK & -27 & 10 & BSL & $(13.83)$ & $(0.47)$ \\
\hline BPS CS 22169-0035 & $04 \quad 12 \quad 13.86$ & -120505.0 & STN & -3.23 & HK & 2 & 10 & BSL & 12.88 & 0.89 \\
\hline BPS CS 22186-0005 & 041309.04 & -355038.9 & SPM & -2.77 & B99 & 192 & 10 & HK & 12.96 & 0.37 \\
\hline $\mathrm{BD}+06648$ & 041313.10 & +063602.1 & HIP & -2.09 & B99 & -141 & 1 & $\mathrm{CY}$ & 9.09 & 1.28 \\
\hline BPS CS 22186-0002 & 041357.18 & -364625.3 & SPM & -2.22 & HK & 352 & 10 & BSL & 13.22 & 0.45 \\
\hline BPS CS 22169-0019 & 041400.04 & -160541.4 & $\mathrm{U}$ & -2.06 & HK & 183 & 10 & BSL & 13.76 & 0.39 \\
\hline BPS CS 22186-0017 & 041622.20 & -341757.9 & SPM & -2.53 & HK & 293 & 10 & BSL & 13.53 & 0.43 \\
\hline BPS CS 22182-0022 & 041658.22 & -314434.7 & SPM & -1.96 & HK & 317 & 10 & BSL & 13.02 & 0.40 \\
\hline $\mathrm{V}^{*}$ AR Per & $0417 \quad 17.20$ & +472400.7 & HIP & -0.30 & F98 & 5 & 1 & F98 & 10.51 & $\ldots$ \\
\hline HD 27145 & 041742.99 & +135011.3 & HIP & -0.70 & BSL & 55 & 10 & BSL & 6.90 & 0.99 \\
\hline BPS CS 22182-0021 & 041814.53 & -312952.6 & SPM & -1.26 & HK & 23 & 10 & BSL & 14.67 & 0.57 \\
\hline BPS CS 22186-0023 & 041945.53 & -365136.0 & SPM & -2.55 & HK & 51 & 10 & BSL & 12.92 & 0.65 \\
\hline BPS CS 22182-0024 & 042000.85 & -312150.5 & SPM & -1.56 & HK & 82 & 10 & BSL & 12.88 & 0.38 \\
\hline BPS CS 22186-0022 & 042101.53 & -363502.6 & SPM & -1.97 & HK & 261 & 10 & BSL & 13.80 & 0.40 \\
\hline BPS CS 22182-0047 & 042230.10 & -273449.9 & SPM & -1.96 & HK & 26 & 10 & BSL & 13.24 & 0.48 \\
\hline HD 27686 & 042237.94 & +145435.0 & $\mathrm{ACT}$ & -0.79 & BSL & 5 & 10 & BSL & 8.13 & 1.54 \\
\hline HD 27928 & 042255.12 & -371548.7 & HIP & -2.25 & ATT & 15 & 1 & $\mathrm{CY}$ & 9.54 & 0.71 \\
\hline BPS CS 22182-0033 & 042310.46 & -303704.1 & SPM & -2.30 & HK & 237 & 10 & BSL & 14.67 & 0.44 \\
\hline BPS CS 22182-0025 & 042347.76 & -321725.5 & SPM & -1.96 & HK & 205 & 10 & BSL & 14.57 & 0.39 \\
\hline HD 28085 & 042631.29 & +1709 18.1 & $\mathrm{ACT}$ & -0.63 & BSL & -22 & 10 & BSL & 7.51 & 1.22 \\
\hline BPS CS 22186-0050 & 043208.03 & -370315.8 & SPM & -1.98 & HK & 53 & 10 & BSL & 13.64 & 0.43 \\
\hline BPS CS 22191-0017 & 043630.75 & -401444.2 & $\mathrm{U}$ & -1.96 & $\mathrm{HK}$ & 58 & 10 & BSL & 13.86 & 0.39 \\
\hline CD-57 949 & 043640.36 & -573240.7 & HIP & -0.69 & B99 & 18 & 10 & HK & 9.26 & 0.91 \\
\hline $\mathrm{V}^{*}$ HP Eri & 043855.74 & -132047.7 & HIP & -1.96 & ATT & 24 & 1 & $\mathrm{CY}$ & 8.38 & 1.32 \\
\hline
\end{tabular}


TABLE 1A. (continued)

\begin{tabular}{|c|c|c|c|c|c|c|c|c|c|c|}
\hline $\begin{array}{l}\text { Star } \\
(1)\end{array}$ & $\begin{array}{c}\text { RA (2000.0) } \\
\text { (2) }\end{array}$ & $\begin{array}{c}\text { DEC } \\
(3)\end{array}$ & $\begin{array}{c}\text { Source }^{\mathrm{a}} \\
(4)\end{array}$ & $\begin{array}{c}{[\mathrm{Fe} / \mathrm{H}]} \\
(\mathrm{dex}) \\
(5)\end{array}$ & $\begin{array}{c}\text { Source }^{b} \\
(6)\end{array}$ & $\begin{array}{c}\mathrm{RV} \\
(\mathrm{km} / \mathrm{s}) \\
(7)\end{array}$ & $\begin{array}{c}\sigma_{\mathrm{RV}} \\
(\mathrm{km} / \mathrm{s}) \\
(8)\end{array}$ & $\begin{array}{c}\text { Source }^{\mathrm{c}} \\
(9)\end{array}$ & $\begin{array}{c}V \\
(\mathrm{mag}) \\
(10)\end{array}$ & $\begin{array}{c}B-V \\
(\mathrm{mag}) \\
(11)\end{array}$ \\
\hline HD 30229 & $0441 \quad 12.39$ & -651843.5 & HIP & -2.32 & ATT & 304 & 3 & CY & 9.40 & 0.66 \\
\hline BPS CS 22191-0024 & 044201.04 & -393703.8 & $\mathrm{U}$ & -2.22 & HK & 282 & 10 & BSL & 13.57 & 0.69 \\
\hline $\mathrm{V}^{*} \mathrm{BC}$ Eri & 044659.00 & -143723.3 & HIP & -1.16 & BSL & 65 & 1 & BSL & 10.90 & 0.44 \\
\hline BPS CS 22191-0029 & 044742.45 & -390726.5 & $\mathrm{U}$ & -2.54 & HK & 70 & 10 & BSL & 14.05 & 0.41 \\
\hline $\mathrm{V}^{*} \mathrm{RX}$ Eri & 044944.30 & -154428.2 & HIP & -1.33 & F98 & 67 & 1 & F98 & 9.69 & $\ldots$ \\
\hline BD-22 928 & 044958.13 & -221450.2 & $\mathrm{ACT}$ & -0.97 & BSL & 11 & 10 & BSL & 9.68 & 0.99 \\
\hline $\mathrm{V}^{*} \mathrm{U} \mathrm{Pic}$ & 045006.63 & -503925.3 & HIP & -0.72 & F98 & 21 & 6 & F98 & 11.38 & $\ldots$ \\
\hline CPD-62 394 & 045209.06 & -620912.3 & HIP & -2.38 & BSL & 168 & 10 & BSL & 10.13 & 0.83 \\
\hline HD 31128 & 045209.80 & -270350.7 & HIP & -1.82 & B99 & 105 & 10 & HK & 9.13 & 0.50 \\
\hline $\mathrm{V}^{*} \mathrm{U}$ Cae & 045314.40 & -374916.0 & SPM & -1.11 & L & -1 & 20 & $\mathrm{~L}$ & 12.16 & $\cdots$ \\
\hline $\mathrm{V}^{*} \mathrm{BB}$ Eri & 045337.50 & -19 2601.1 & HIP & -1.32 & F98 & 247 & 2 & F98 & 11.52 & $\cdots$ \\
\hline $\mathrm{V}^{*} \mathrm{U}$ Lep & 045617.96 & -211301.5 & HIP & -1.78 & F98 & 125 & 2 & F98 & 10.57 & $\ldots$ \\
\hline HD 32546 & 045848.63 & $\begin{array}{lll}-67 & 06 & 06.1\end{array}$ & HIP & -1.30 & ATT & 157 & 10 & BSL & 9.35 & 1.06 \\
\hline $\mathrm{V}^{*} \mathrm{~V}$ Cae & 050137.67 & -390804.9 & SPM & -2.00 & $\mathrm{~L}$ & -31 & 10 & L & 12.87 & $\ldots$ \\
\hline HD 268957 & 050144.38 & -700254.8 & HIP & -1.63 & $\mathrm{ATT}$ & 172 & 1 & $\mathrm{CY}$ & 9.78 & 0.49 \\
\hline $\mathrm{V}^{*} \mathrm{SW}$ Dor & 050204.79 & -671737.7 & $\mathrm{U}$ & -1.60 & L & 358 & 40 & L & 13.80 & $\ldots$ \\
\hline V* SX Dor & 050332.06 & -654258.2 & $\mathrm{U}$ & -2.00 & $\mathrm{~L}$ & 105 & 40 & $\mathrm{~L}$ & 13.97 & $\ldots$ \\
\hline $\mathrm{V}^{*}$ V964 Ori & 050523.22 & $\begin{array}{lll}-02 & 1239.1\end{array}$ & SBD & -1.89 & $\mathrm{~L}$ & 178 & 11 & $\mathrm{~L}$ & 12.95 & $\ldots$ \\
\hline HD 33073 & 050632.00 & -283336.9 & HIP & -1.30 & BSL & 78 & 10 & BSL & 9.52 & 0.69 \\
\hline $\mathrm{V}^{*} \mathrm{TX}$ Men & 050746.77 & -790303.0 & $\mathrm{U}$ & -2.48 & L & 250 & 35 & L & 13.82 & $\cdots$ \\
\hline $\mathrm{V}^{*} \mathrm{~T}$ Men & 050956.22 & -713840.7 & $\mathrm{U}$ & -0.85 & L & 92 & 35 & L & 13.53 & $\cdots$ \\
\hline CPD-57 752 & 051011.27 & -573512.0 & HIP & -0.77 & BSL & 8 & 10 & BSL & 9.82 & 0.87 \\
\hline HD 34048 & 051019.42 & -624120.2 & HIP & -1.50 & ATT & 260 & 10 & BSL & 9.97 & 0.55 \\
\hline HD 33771 & 051049.56 & -374902.9 & HIP & -1.93 & $\mathrm{ATT}$ & 18 & 10 & BSL & 9.45 & 0.85 \\
\hline HD 33605 & 051234.10 & +273615.4 & HIP & -1.51 & BSL & 25 & 10 & BSL & 8.58 & 1.35 \\
\hline HD 34328 & $\begin{array}{lll}05 & 1304.20\end{array}$ & -593848.8 & HIP & -1.61 & B99 & 234 & 10 & HK & 9.43 & 0.50 \\
\hline $\mathrm{V}^{*} \mathrm{RY} \mathrm{Col}$ & 051507.75 & -413741.9 & HIP & -0.91 & F98 & 474 & 4 & F98 & 10.90 & $\ldots$ \\
\hline HD 241894 & $05 \quad 1516.14$ & +291702.9 & $\mathrm{ACT}$ & -1.22 & BSL & 30 & 10 & BSL & 10.00 & 1.10 \\
\hline HD 242106 & 051625.34 & +270629.0 & HIP & -0.75 & BSL & -33 & 10 & BSL & 8.89 & 1.15 \\
\hline $\mathrm{V}^{*} \mathrm{RT} \mathrm{Col}$ & 051648.05 & $\begin{array}{lll}-27 & 28 & 04.3\end{array}$ & SPM & -1.43 & L & 276 & 21 & L & 12.67 & $\cdots$ \\
\hline HD 273975 & 051819.52 & -485210.9 & HIP & -1.40 & $\mathrm{C} 97$ & 312 & 10 & BSL & 10.67 & 0.53 \\
\hline HD 34414 & 051828.67 & +302239.5 & HIP & -0.84 & BSL & -65 & 10 & BSL & 8.34 & 1.06 \\
\hline HD 35179 & 052221.01 & -142349.9 & HIP & -0.67 & B99 & 15 & 10 & HK & 9.49 & 0.96 \\
\hline HD 35238 & 052438.44 & +311326.2 & HIP & -0.69 & BSL & 37 & 10 & BSL & 6.30 & 1.25 \\
\hline $\mathrm{V}^{*} \mathrm{RT}$ Dor & 052944.70 & -641712.7 & GSC & -1.52 & L & 320 & 20 & L & 12.48 & $\ldots$ \\
\hline HD 36054 & $\begin{array}{lll}05 & 30 & 03.22\end{array}$ & +234531.5 & HIP & -1.38 & BSL & 4 & 10 & BSL & 8.12 & 1.10 \\
\hline HD 36702 & 053152.23 & -383323.9 & HIP & -2.06 & B99 & 122 & 1 & CY & 8.33 & 1.15 \\
\hline HD 274939 & 053317.95 & -475612.8 & HIP & -1.76 & B99 & 190 & 12 & CY & 9.43 & 0.74 \\
\hline HD 244759 & 053339.03 & +263205.5 & $\mathrm{ACT}$ & -0.96 & BSL & -58 & 10 & BSL & 9.30 & 1.20 \\
\hline HD 36931 & 053626.16 & +303526.2 & $\mathrm{ACT}$ & -2.09 & BSL & 2 & 10 & BSL & 7.92 & 1.10 \\
\hline
\end{tabular}


TABLE 1A. (continued)

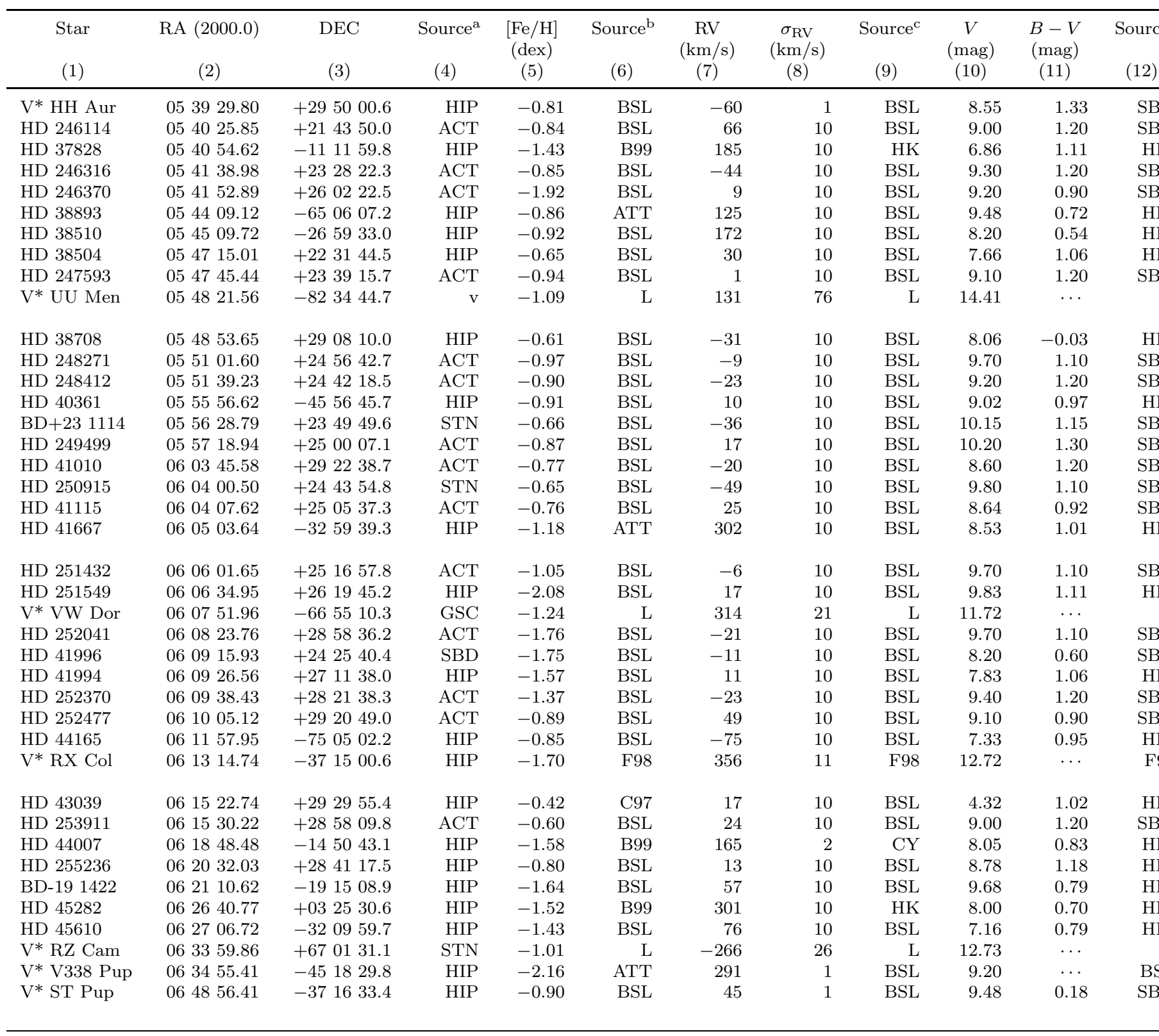


TABLE 1A. (continued)

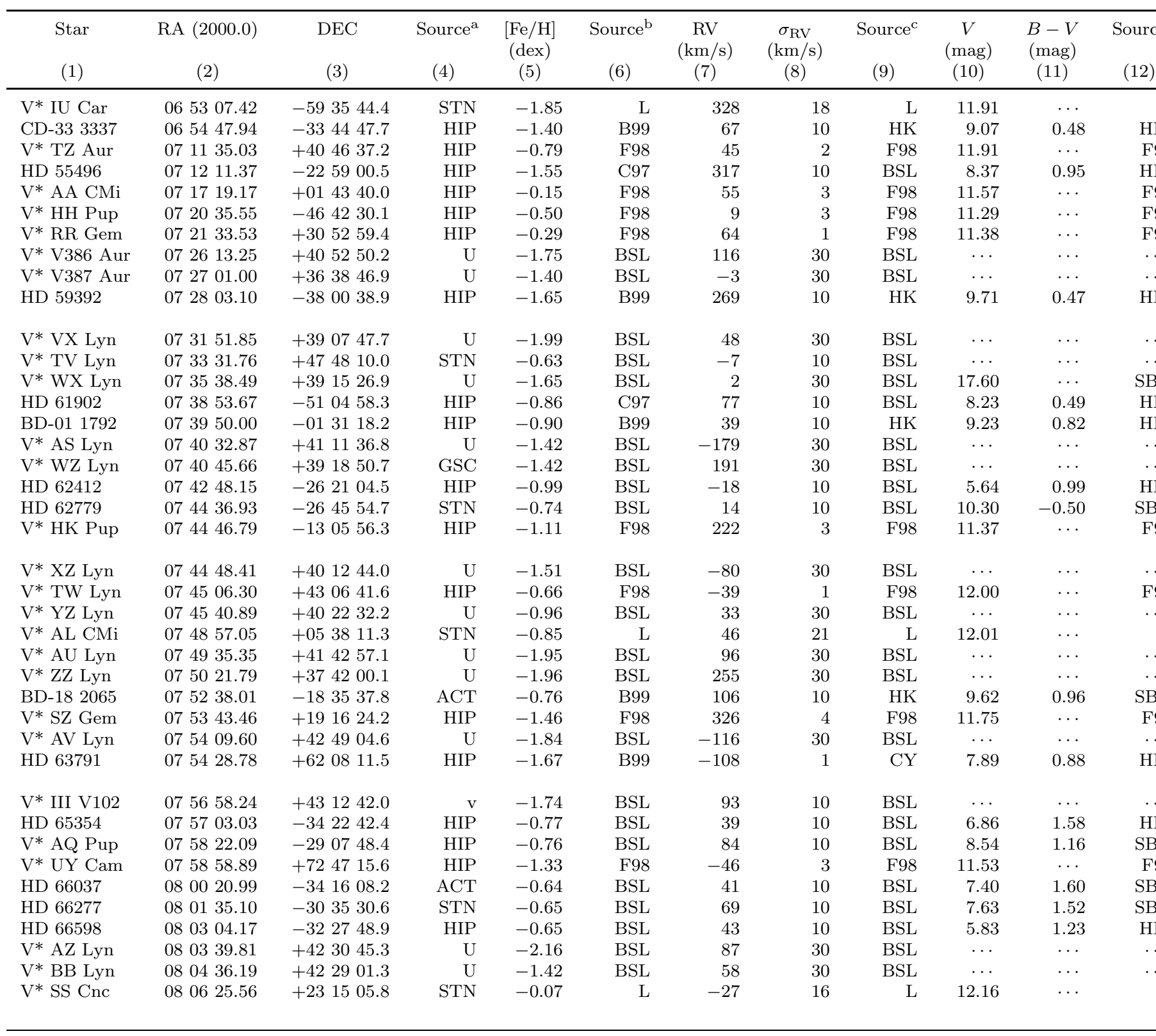


TABLE 1A. (continued)

\begin{tabular}{|c|c|c|c|c|c|c|c|c|c|c|c|}
\hline $\begin{array}{l}\text { Star } \\
(1)\end{array}$ & $\begin{array}{c}\text { RA (2000.0) } \\
(2)\end{array}$ & $\begin{array}{c}\text { DEC } \\
(3)\end{array}$ & $\begin{array}{c}\text { Source }^{\mathrm{a}} \\
\text { (4) }\end{array}$ & $\begin{array}{c}{[\mathrm{Fe} / \mathrm{H}]} \\
(\mathrm{dex}) \\
(5)\end{array}$ & $\begin{array}{c}\text { Source }^{b} \\
(6)\end{array}$ & $\begin{array}{c}\mathrm{RV} \\
(\mathrm{km} / \mathrm{s}) \\
(7)\end{array}$ & $\begin{array}{c}\sigma_{\mathrm{RV}} \\
(\mathrm{km} / \mathrm{s}) \\
(8)\end{array}$ & $\begin{array}{c}\text { Source }^{\mathrm{c}} \\
(9)\end{array}$ & $\begin{array}{c}V \\
(\mathrm{mag}) \\
(10)\end{array}$ & $\begin{array}{c}B-V \\
(\mathrm{mag}) \\
(11)\end{array}$ & $\begin{array}{l}\text { Source } \\
\text { (12) }\end{array}$ \\
\hline HD 67380 & 080631.93 & -304837.7 & HIP & -0.60 & BSL & 28 & 10 & BSL & 8.92 & 1.09 & \\
\hline $\mathrm{V}^{*}$ SZ Lyn & 080935.76 & +442817.8 & HIP & -1.16 & BSL & 32 & 1 & BSL & $\ldots$ & $\cdots$ & \\
\hline $\mathrm{V}^{*}$ BC Lyn & 080937.41 & +423331.1 & $\mathrm{U}$ & -1.75 & BSL & 239 & 30 & BSL & $\cdots$ & $\ldots$ & \\
\hline HD 68298 & $08 \quad 1023.72$ & -361356.2 & $\mathrm{ACT}$ & -0.60 & BSL & -3 & 10 & BSL & 7.60 & 1.40 & \\
\hline HD 69083 & 081355.89 & -374042.5 & HIP & -0.85 & BSL & 24 & 10 & BSL & 8.16 & 0.72 & \\
\hline CD-44 4311 & 082035.67 & -450857.3 & HIP & -0.85 & BSL & -75 & 10 & BSL & 9.75 & 0.99 & \\
\hline HD 70765 & $\begin{array}{lll}08 & 22 & 07.47\end{array}$ & $\begin{array}{lll}-41 & 4134.3\end{array}$ & $\mathrm{ACT}$ & -0.70 & BSL & 31 & 10 & BSL & 7.90 & 2.10 & $\mathrm{SBI}$ \\
\hline HD 70714 & 082215.65 & -315516.6 & $\mathrm{ACT}$ & -0.73 & BSL & 9 & 10 & BSL & 7.90 & 1.20 & SB \\
\hline V* BB Pup & 082424.17 & -193326.1 & $\mathrm{U}$ & -0.57 & L & 98 & 9 & $\mathrm{~L}$ & 12.17 & $\cdots$ & \\
\hline HD 72348 & 083046.45 & -413054.2 & HIP & -0.60 & BSL & 18 & 10 & BSL & 6.62 & 0.90 & \\
\hline BD-20 2583 & 083214.16 & -204425.8 & HIP & -1.66 & BSL & 261 & 10 & BSL & 10.01 & 0.63 & II] \\
\hline V* TT Cnc & 083255.21 & +131128.7 & HIP & -1.57 & F98 & 49 & 1 & F98 & 11.35 & $\ldots$ & \\
\hline HD 73075 & 083435.69 & $\begin{array}{llll}-44 & 16 & 12.8\end{array}$ & HIP & -0.65 & BSL & -8 & 10 & BSL & 7.31 & 1.00 & [I] \\
\hline HD 73676 & 083752.76 & -423333.3 & HIP & -0.70 & BSL & 3 & 10 & BSL & 8.97 & 1.04 & \\
\hline HD 73700 & 083755.93 & -442909.0 & HIP & -0.71 & BSL & 18 & 10 & BSL & 8.57 & 1.13 & \\
\hline HD 73808 & 083844.86 & -400359.8 & $\mathrm{ACT}$ & -0.68 & BSL & 38 & 10 & BSL & 8.38 & 1.02 & \\
\hline$V^{*}$ GL Hya & 084049.94 & +023743.3 & $\mathrm{U}$ & -1.45 & L & 223 & 21 & L & 12.95 & $\ldots$ & \\
\hline HD 74000 & 084050.59 & -162038.3 & HIP & -1.82 & B99 & 204 & 10 & HK & 9.67 & 0.43 & \\
\hline HD 74617 & 084332.24 & -393559.7 & HIP & -0.76 & BSL & 54 & 10 & BSL & 7.78 & 1.55 & $\mathrm{HI}$ \\
\hline HD 74721 & $08 \quad 45 \quad 59.29$ & +131549.6 & HIP & -1.42 & C97 & 21 & 10 & BSL & 8.70 & 0.04 & $\mathrm{HI}$ \\
\hline BD-12 2669 & 084639.78 & -132124.0 & HIP & -1.40 & B99 & 28 & 10 & HK & 10.24 & 0.31 & $\mathrm{HI}$ \\
\hline HD 77142 & 085844.56 & -500005.0 & HIP & -0.70 & BSL & 37 & 10 & BSL & 9.17 & 0.96 & \\
\hline $\mathrm{V}^{*} \mathrm{DH}$ Hya & 090017.72 & -094656.9 & $\mathrm{U}$ & -1.55 & L & 355 & 8 & L & 12.13 & $\ldots$ & \\
\hline HD 77489 & 090053.82 & -502608.7 & HIP & -0.61 & BSL & -5 & 10 & BSL & 7.93 & 1.01 & \\
\hline
\end{tabular}


TABLE 1A. (continued)

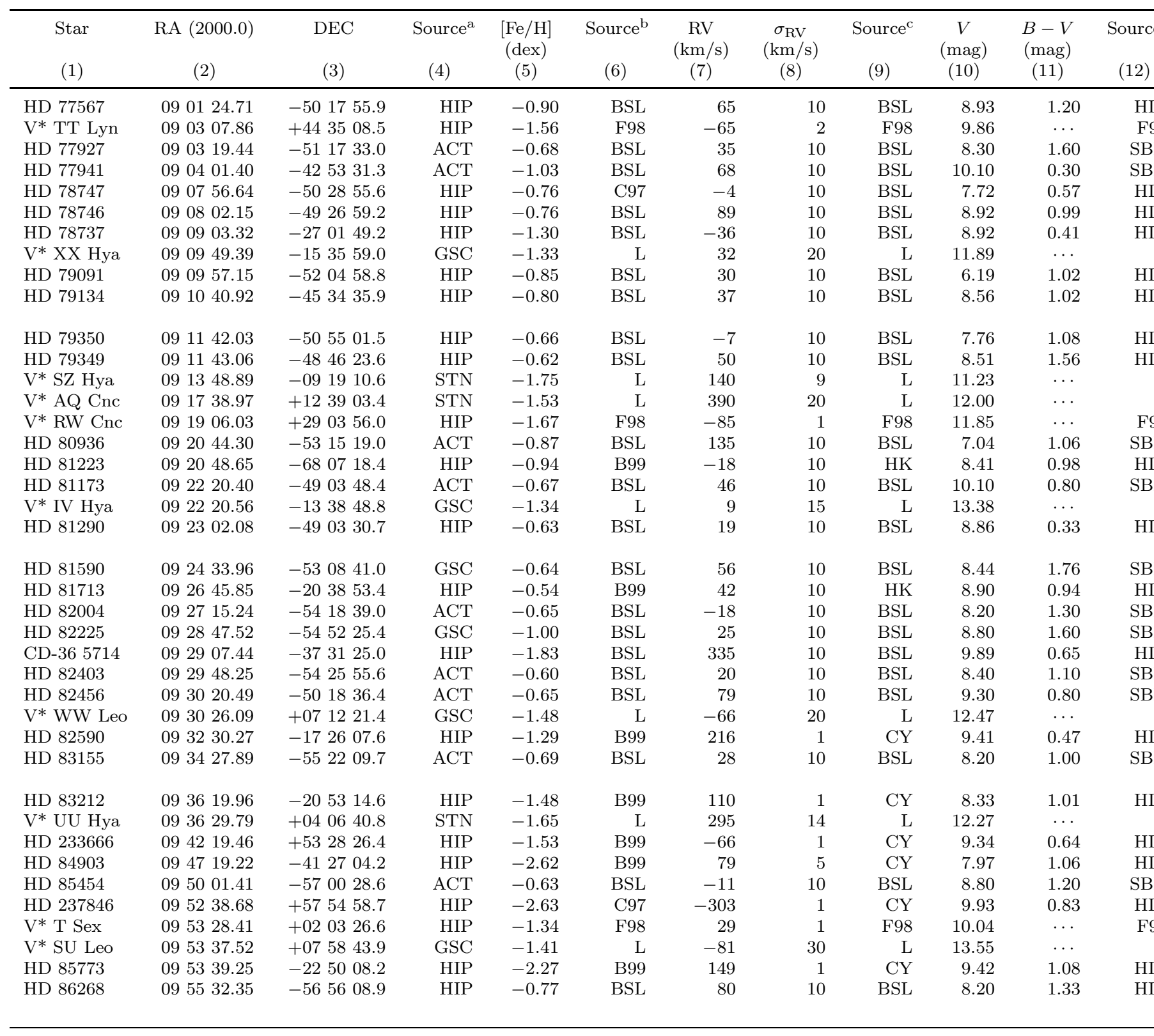


TABLE 1A. (continued)

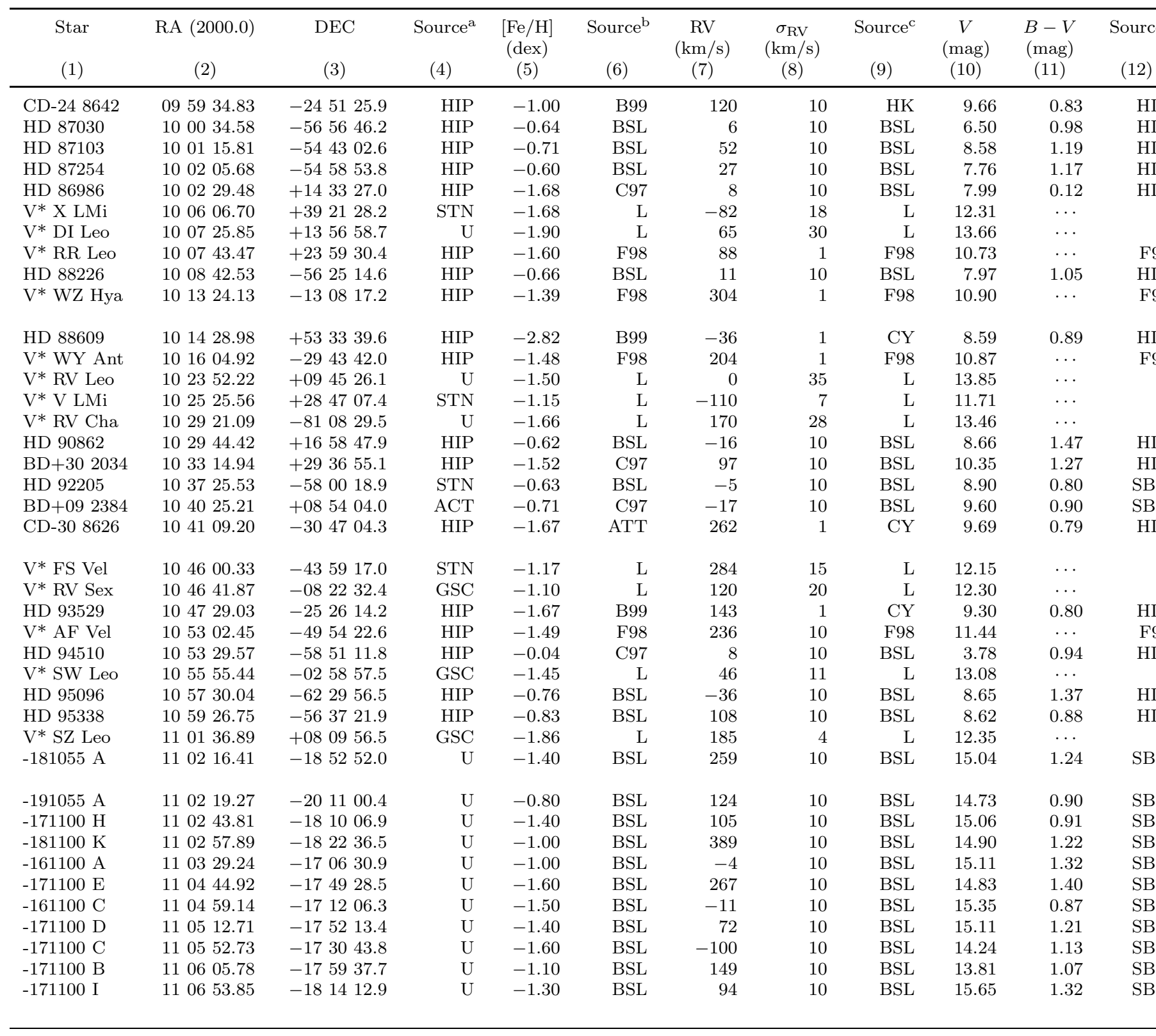


TABLE 1A. (continued)

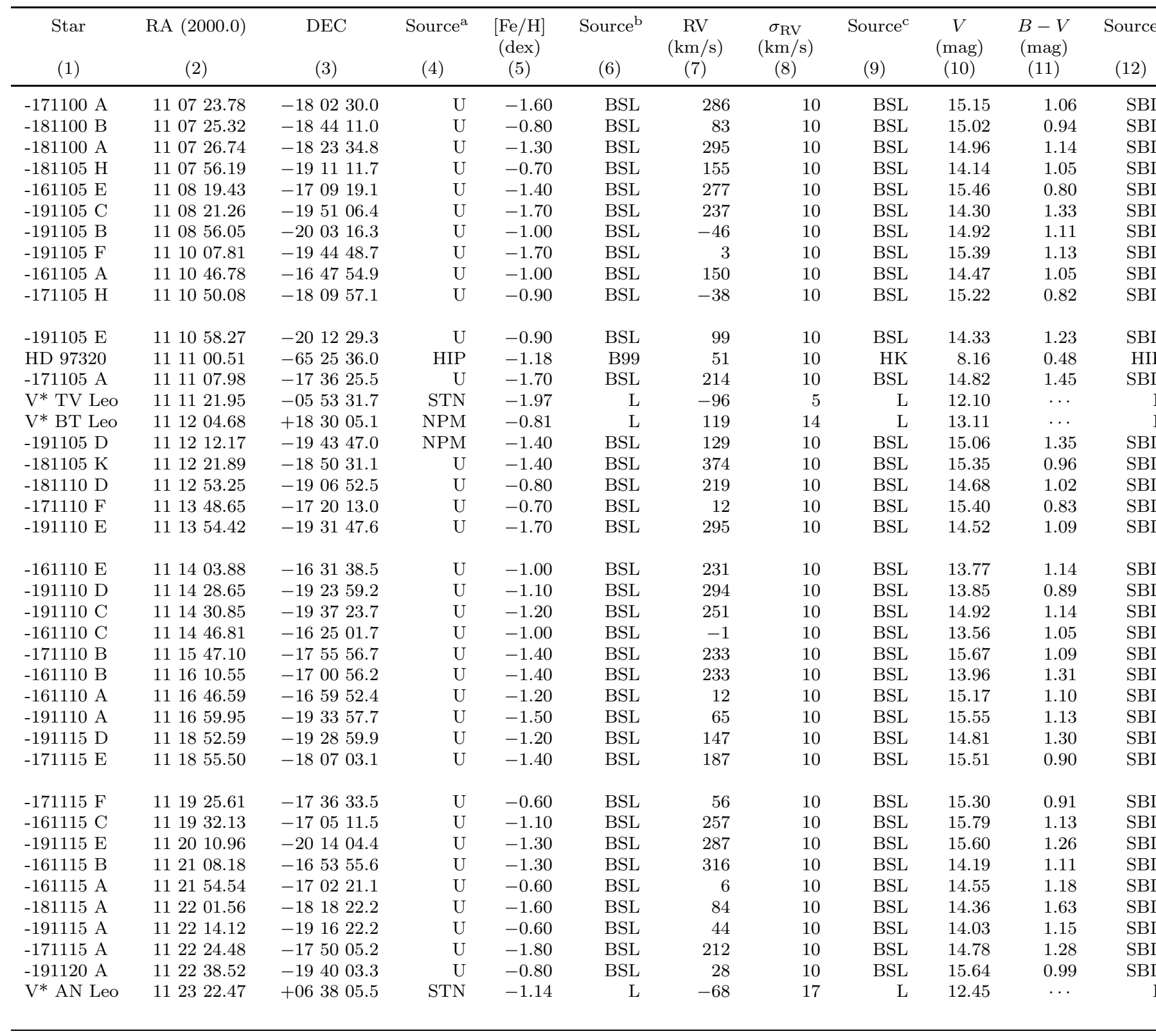


TABLE 1A. (continued)

\begin{tabular}{|c|c|c|c|c|c|c|c|c|c|c|}
\hline $\begin{array}{l}\text { Star } \\
(1)\end{array}$ & $\begin{array}{c}\text { RA (2000.0) } \\
\text { (2) }\end{array}$ & $\begin{array}{c}\text { DEC } \\
(3)\end{array}$ & $\begin{array}{c}\text { Source }^{\mathrm{a}} \\
(4)\end{array}$ & $\begin{array}{c}{[\mathrm{Fe} / \mathrm{H}]} \\
(\mathrm{dex}) \\
(5)\end{array}$ & $\begin{array}{c}\text { Source }^{b} \\
(6)\end{array}$ & $\begin{array}{c}\mathrm{RV} \\
(\mathrm{km} / \mathrm{s}) \\
(7)\end{array}$ & $\begin{array}{c}\sigma_{\mathrm{RV}} \\
(\mathrm{km} / \mathrm{s}) \\
(8)\end{array}$ & $\begin{array}{c}\text { Source }^{\mathrm{c}} \\
(9)\end{array}$ & $\begin{array}{c}V \\
(\mathrm{mag}) \\
(10)\end{array}$ & $\begin{array}{c}B-V \\
(\mathrm{mag}) \\
(11)\end{array}$ \\
\hline $\mathrm{V}^{*} \mathrm{RX}$ Leo & 112358.36 & +263654.1 & STN & -1.38 & L & -121 & 6 & L & 11.90 & $\cdots$ \\
\hline HD 99383 & 112549.93 & -385218.5 & HIP & -1.65 & B99 & 227 & 10 & HK & 9.07 & 0.48 \\
\hline $\mathrm{V}^{*}$ AE Leo & 112612.18 & +173939.7 & GSC & -1.71 & L & -53 & 10 & L & 12.52 & $\ldots$ \\
\hline HD 99513 & 112624.31 & $-5916 \quad 56.2$ & $\mathrm{ACT}$ & -0.60 & BSL & 62 & 10 & BSL & 7.90 & 1.10 \\
\hline $\mathrm{V}^{*} \mathrm{~W}$ Crt & 112629.66 & -175451.6 & HIP & -0.54 & F98 & 60 & 1 & F98 & 11.54 & $\ldots$ \\
\hline $\mathrm{BD}+042466$ & 112649.20 & +035152.2 & HIP & -1.88 & B99 & 38 & 1 & $\mathrm{CY}$ & 10.53 & 0.82 \\
\hline HD 99833 & 112909.83 & +162245.1 & HIP & -0.70 & BSL & 7 & 10 & BSL & 7.71 & 0.81 \\
\hline HD 99978 & 112937.28 & -555344.5 & HIP & -1.12 & $\mathrm{RL}$ & 68 & 10 & BSL & 8.65 & 0.95 \\
\hline $\mathrm{V}^{*} \mathrm{TU}$ UMa & 112948.54 & +300402.9 & HIP & -1.51 & F98 & 89 & 2 & F98 & 9.82 & $\ldots$ \\
\hline $\mathrm{V}^{*}$ AX Leo & 113303.76 & +120914.1 & HIP & -1.72 & F98 & 193 & 6 & F98 & 12.26 & $\cdots$ \\
\hline $\mathrm{V}^{*} \mathrm{SS}$ Leo & 113354.51 & -00 0159.8 & HIP & -1.79 & F98 & 163 & 2 & F98 & 11.03 & $\ldots$ \\
\hline HD 100906 & 113642.42 & -185810.7 & HIP & -0.43 & B99 & -19 & 10 & HK & 9.65 & 0.83 \\
\hline HD 101063 & 113740.61 & -285104.7 & HIP & -1.15 & B99 & 178 & 10 & HK & 9.43 & 0.75 \\
\hline $\mathrm{V}^{*} \mathrm{SU}$ Dra & 113756.68 & +671947.7 & HIP & -1.80 & F98 & -167 & 1 & F98 & 9.78 & $\ldots$ \\
\hline $\mathrm{V}^{*} \mathrm{BX}$ Leo & 113802.08 & +163236.3 & HIP & -1.28 & F98 & 26 & 5 & F98 & 11.61 & $\ldots$ \\
\hline $\mathrm{V}^{*} \mathrm{ST}$ Leo & 113832.68 & +103342.1 & HIP & -1.17 & F98 & 168 & 8 & F98 & 11.49 & $\ldots$ \\
\hline $\mathrm{V}^{*}$ AA Leo & 113914.28 & +101939.1 & GSC & -1.47 & L & 32 & 24 & L & 12.27 & $\cdots$ \\
\hline HD 102200 & 114534.18 & -460345.4 & HIP & -1.20 & B99 & 165 & 10 & HK & 8.75 & 0.43 \\
\hline $\mathrm{V}^{*} \mathrm{BI}$ Cen & 114554.60 & -592240.4 & GSC & -0.83 & L & 210 & 30 & L & 11.86 & $\cdots$ \\
\hline $\mathrm{V}^{*} \mathrm{KS}$ Cen & 114710.57 & -413144.8 & GSC & -0.80 & $\mathrm{~L}$ & -55 & 18 & $\mathrm{~L}$ & 13.39 & $\cdots$ \\
\hline V* X Crt & 114856.22 & -102628.2 & HIP & -2.00 & F98 & 80 & 2 & F98 & 11.48 & $\cdots$ \\
\hline HD 102780 & 115008.04 & +201713.4 & HIP & -0.63 & BSL & 75 & 10 & BSL & 8.18 & 1.59 \\
\hline HD 102866 & 115021.41 & -610158.4 & SBD & -0.92 & BSL & 34 & 10 & BSL & 9.43 & 1.01 \\
\hline CD-70 889 & 115059.76 & -704844.1 & HIP & -2.57 & B99 & 300 & 5 & CY & 9.26 & 0.86 \\
\hline $\mathrm{BD}+222411$ & 115133.54 & +220652.3 & HIP & -1.95 & $\mathrm{ATT}$ & 35 & 2 & $\mathrm{CY}$ & 10.06 & 1.25 \\
\hline $\mathrm{V}^{*} \mathrm{TY}$ Vir & 115150.13 & -054543.8 & HIP & -1.78 & B99 & 229 & 2 & $\mathrm{CY}$ & 8.18 & 1.29 \\
\hline HD 103295 & 115337.06 & -283813.1 & HIP & -1.04 & B99 & -7 & 10 & HK & 9.58 & 0.79 \\
\hline BD-01 2582 & 115337.33 & -020035.7 & HIP & -2.23 & B99 & 0 & 2 & $\mathrm{CY}$ & 9.59 & 0.73 \\
\hline [OM87] 115221.21-0 & 115454.10 & -261713.2 & STN & -2.21 & BSL & 160 & 10 & BSL & 11.08 & $\ldots$ \\
\hline HD 103545 & 115527.19 & +090745.4 & HIP & -2.14 & B99 & 180 & 1 & $\mathrm{CY}$ & 9.42 & 0.84 \\
\hline CD-35 7576 & 115814.59 & -361144.0 & HIP & -1.88 & BSL & 102 & 10 & BSL & 10.10 & 0.53 \\
\hline HD 104053 & 115905.95 & +235421.6 & $\mathrm{ACT}$ & -1.10 & BSL & -56 & 10 & BSL & 9.04 & 1.21 \\
\hline $\mathrm{V}^{*} \mathrm{SW} \mathrm{Cru}$ & 115907.26 & -601938.1 & GSC & -0.54 & L & -23 & 30 & L & 12.33 & $\ldots$ \\
\hline HD 233891 & 115959.08 & +514617.5 & HIP & -1.54 & B99 & -45 & 2 & $\mathrm{CY}$ & 8.80 & 0.80 \\
\hline $\mathrm{V}^{*}$ GK Com & 120004.76 & +192510.0 & HIP & -1.93 & BSL & 38 & 1 & BSL & 6.99 & 1.46 \\
\hline $\mathrm{BD}+092574$ & 120203.93 & +090042.0 & HIP & -1.95 & ATT & -49 & 1 & $\mathrm{CY}$ & 10.52 & 0.79 \\
\hline HD 104785 & 120401.07 & +184853.2 & HIP & -0.78 & BSL & -41 & 10 & BSL & 7.70 & 1.23 \\
\hline HD 104893 & 120443.15 & -291105.1 & HIP & -1.99 & B99 & 24 & 1 & $\mathrm{CY}$ & 9.26 & 1.22 \\
\hline V* IK Hya & 120447.31 & $-27 \quad 4042.8$ & HIP & -1.24 & F98 & 258 & 15 & F98 & 10.11 & $\cdots$ \\
\hline CD-37 7677 & 120753.16 & -384024.8 & HIP & -1.53 & BSL & 205 & 10 & BSL & 9.95 & 0.86 \\
\hline
\end{tabular}


TABLE 1A. (continued)

\begin{tabular}{|c|c|c|c|c|c|c|c|c|c|c|}
\hline $\begin{array}{l}\text { Star } \\
(1)\end{array}$ & $\begin{array}{c}\text { RA (2000.0) } \\
(2)\end{array}$ & $\begin{array}{c}\text { DEC } \\
(3)\end{array}$ & $\begin{array}{c}\text { Source }^{\mathrm{a}} \\
(4)\end{array}$ & $\begin{array}{c}{[\mathrm{Fe} / \mathrm{H}]} \\
(\mathrm{dex}) \\
(5)\end{array}$ & $\begin{array}{c}\text { Source }^{b} \\
(6)\end{array}$ & $\begin{array}{c}\mathrm{RV} \\
(\mathrm{km} / \mathrm{s}) \\
(7)\end{array}$ & $\begin{array}{c}\sigma_{\mathrm{RV}} \\
(\mathrm{km} / \mathrm{s}) \\
(8)\end{array}$ & $\begin{array}{c}\text { Source }^{c} \\
(9)\end{array}$ & $\begin{array}{c}V \\
(\mathrm{mag}) \\
(10)\end{array}$ & $\begin{array}{c}B-V \\
(\mathrm{mag}) \\
(11)\end{array}$ \\
\hline $\mathrm{V}^{*} \mathrm{UU}$ Vir & 120835.10 & -002724.3 & HIP & -0.87 & F98 & -8 & 1 & F98 & 10.56 & $\cdots$ \\
\hline HD 105546 & 120902.76 & +590105.7 & HIP & -1.40 & B99 & 19 & 1 & $\mathrm{CY}$ & 8.61 & 0.66 \\
\hline CD-28 9325 & $1209 \quad 19.29$ & -293232.0 & SPM & -1.83 & BSL & 145 & 10 & BSL & 10.60 & 1.15 \\
\hline V* V Com & $1210 \quad 15.92$ & +272554.2 & STN & -1.75 & $\mathrm{~L}$ & 23 & 28 & L & 13.16 & $\ldots$ \\
\hline HD 105740 & 121016.98 & +162214.2 & HIP & -0.82 & BSL & 1 & 10 & BSL & 8.37 & 0.97 \\
\hline $\mathrm{V}^{*} \mathrm{AB} \mathrm{UMa}$ & 121114.60 & +474943.9 & HIP & -0.49 & F98 & -50 & 1 & F98 & 10.94 & $\cdots$ \\
\hline HD 105976 & 121150.97 & $\begin{array}{lll}-62 & 00 & 48.7\end{array}$ & GSC & -0.74 & BSL & -36 & 10 & BSL & 8.60 & 1.30 \\
\hline HD 106373 & 121413.36 & $-28 \quad 1506.7$ & HIP & -2.48 & $\mathrm{ATT}$ & 96 & 12 & $\mathrm{CY}$ & 8.91 & 0.44 \\
\hline HD 106383 & 121414.03 & +332700.5 & HIP & -0.61 & BSL & -26 & 10 & BSL & 8.04 & 0.86 \\
\hline CD-28 9374 & 121429.76 & -293554.6 & HIP & -0.79 & B99 & 59 & 10 & HK & 10.25 & 0.83 \\
\hline HD 106411 & 121432.24 & -440619.9 & HIP & -0.75 & $\mathrm{C} 97$ & 72 & 10 & BSL & 9.28 & 0.57 \\
\hline HD 106670 & 121611.96 & -495426.3 & HIP & -1.06 & B99 & -36 & 10 & HK & 8.86 & 0.81 \\
\hline $\mathrm{V}^{*} \mathrm{LN}$ Cen & 121655.88 & -475827.6 & GSC & -1.89 & L & 148 & 20 & L & 14.11 & $\cdots$ \\
\hline $\mathrm{V}^{*}$ IW Com & 121656.61 & +274423.1 & HIP & -1.47 & BSL & -21 & 1 & BSL & 8.06 & 1.52 \\
\hline $\mathrm{V}^{*} \mathrm{SW}$ Dra & 121746.67 & +693038.3 & HIP & -1.12 & F98 & -29 & 1 & F98 & 10.48 & $\cdots$ \\
\hline HD 107363 & 122033.72 & +134504.5 & HIP & -0.72 & BSL & -14 & 10 & BSL & 9.88 & 1.00 \\
\hline$V^{*}$ UV Vir & $1221 \quad 16.74$ & +002202.7 & STN & -1.19 & $\mathrm{~L}$ & 99 & 11 & $\mathrm{~L}$ & 11.83 & $\ldots$ \\
\hline HD 107752 & 122252.75 & +113625.8 & HIP & -2.74 & B99 & 220 & 1 & CY & 10.01 & 0.81 \\
\hline [MFF90] PHI 2/2 8 & 122414.42 & -314935.1 & $\mathrm{U}$ & $-0.69:$ & MFF & -26 & 10 & BSL & 13.43 & $\cdots$ \\
\hline $\mathrm{BD}+312360$ & 122417.41 & +304648.4 & GSC & -0.63 & BSL & 32 & 10 & BSL & 11.28 & 0.92 \\
\hline [MFF90] PHI 2/2 7 & 122437.52 & -315417.8 & $\mathrm{U}$ & $-1.37:$ & MFF & 105 & 10 & BSL & 13.37 & $\cdots$ \\
\hline [MFF90] PHI 2/2 4 & 122448.35 & -322625.1 & $\mathrm{U}$ & $-0.83:$ & MFF & 8 & 10 & BSL & 13.37 & $\ldots$ \\
\hline $\mathrm{BD}+292287$ & 122523.79 & +283820.3 & $\mathrm{ACT}$ & -0.66 & BSL & -35 & 10 & BSL & 10.13 & 0.97 \\
\hline MA 300141 & 122544.10 & +294953.0 & $\mathrm{U}$ & -2.56 & BSL & -2 & 10 & BSL & 13.44 & $\cdots$ \\
\hline [MFF90] PHI 2/1 66 & 122546.36 & -320911.5 & $\mathrm{U}$ & $-1.35:$ & $\mathrm{MFF}$ & -6 & 10 & BSL & 13.32 & $\cdots$ \\
\hline MA 300142 & 122550.56 & +294901.0 & STN & -0.81 & BSL & 49 & 10 & BSL & 12.60 & $\cdots$ \\
\hline HD 108317 & 122636.93 & +05 1809.2 & HIP & -2.27 & B99 & 7 & 1 & $\mathrm{CY}$ & 8.03 & 0.61 \\
\hline MA 300157 & 122712.19 & +295620.6 & STN & -0.61 & BSL & -29 & 10 & BSL & 13.14 & $\cdots$ \\
\hline HD 108405 & 122728.72 & -480750.9 & HIP & -0.60 & B99 & 7 & 10 & HK & 9.28 & 0.58 \\
\hline MA 300162 & 122729.90 & +295453.5 & $\mathrm{U}$ & -1.30 & BSL & 0 & 10 & BSL & 13.07 & $\cdots$ \\
\hline [MFF90] PHI 2/2 97 & 122740.53 & -310459.4 & SPM & -1.10 & MFF & 12 & 10 & BSL & 12.89 & 0.95 \\
\hline [MFF90] PHI 2/1 52 & 122746.78 & $-32 \quad 1512.1$ & $\mathrm{U}$ & $-2.11:$ & MFF & 227 & 10 & BSL & 13.06 & $\cdots$ \\
\hline MA 300168 & 122758.52 & +302320.5 & $\mathrm{U}$ & -1.08 & BSL & -44 & 10 & BSL & 13.57 & $\cdots$ \\
\hline [MFF90] PHI 2/1 49 & 122811.08 & -314325.4 & $\mathrm{U}$ & $-0.68:$ & $\mathrm{MFF}$ & -3 & 10 & BSL & 12.59 & $\cdots$ \\
\hline HD 108577 & 122816.91 & +122041.5 & HIP & -2.56 & B99 & -112 & 1 & $\mathrm{CY}$ & 9.57 & 0.70 \\
\hline $\mathrm{BD}+422309$ & 122822.18 & +413852.7 & HIP & -1.24 & BSL & -137 & 10 & BSL & 10.76 & 0.04 \\
\hline $\mathrm{V}^{*} \mathrm{IX}$ Com & 122832.71 & +293523.7 & HIP & -1.08 & BSL & -20 & 1 & BSL & 8.61 & 1.65 \\
\hline $\mathrm{BD}+042621$ & 122844.65 & +040127.0 & HIP & -2.41 & B99 & -42 & 1 & $\mathrm{CY}$ & 9.92 & 0.88 \\
\hline $\mathrm{V}^{*} \mathrm{RR} \mathrm{CVn}$ & 122907.51 & +343850.2 & GSC & -1.08 & L & -5 & 21 & L & 12.55 & $\cdots$ \\
\hline $\mathrm{BD}+302282$ & 122933.92 & +300451.4 & STN & -1.12 & BSL & -2 & 10 & BSL & 10.22 & 0.93 \\
\hline
\end{tabular}


TABLE 1A. (continued)

\begin{tabular}{|c|c|c|c|c|c|c|c|c|c|c|}
\hline $\begin{array}{l}\text { Star } \\
(1)\end{array}$ & $\begin{array}{c}\text { RA (2000.0) } \\
(2)\end{array}$ & DEC & $\begin{array}{c}\text { Source }^{\mathrm{a}} \\
(4)\end{array}$ & $\begin{array}{c}{[\mathrm{Fe} / \mathrm{H}]} \\
(\mathrm{dex}) \\
(5)\end{array}$ & $\begin{array}{c}\text { Source }^{b} \\
(6)\end{array}$ & $\begin{array}{c}\mathrm{RV} \\
(\mathrm{km} / \mathrm{s}) \\
(7)\end{array}$ & $\begin{array}{c}\sigma_{\mathrm{RV}} \\
(\mathrm{km} / \mathrm{s}) \\
(8)\end{array}$ & $\begin{array}{c}\text { Source }^{c} \\
(9)\end{array}$ & $\begin{array}{c}V \\
(\mathrm{mag}) \\
(10)\end{array}$ & $\begin{array}{c}B-V \\
(\mathrm{mag}) \\
(11)\end{array}$ \\
\hline [MFF90] PHI 2/1 43 & 122934.54 & -323307.2 & U & $-1.60:$ & $\mathrm{MFF}$ & 245 & 10 & BSL & 12.61 & $\cdots$ \\
\hline $\mathrm{V}^{*} \mathrm{UZ}$ CVn & 123027.71 & +403032.3 & HIP & -1.89 & F98 & -28 & 3 & F98 & 12.12 & $\ldots$ \\
\hline V* SV Hya & 123030.53 & -260251.2 & HIP & -1.50 & F98 & 107 & 2 & F98 & 10.53 & $\ldots$ \\
\hline HD 108976 & 123103.11 & +274349.3 & HIP & -1.43 & BSL & -2 & 10 & BSL & 8.56 & 0.48 \\
\hline MA 30210 & 123136.36 & +301253.2 & STN & -1.04 & BSL & 1 & 10 & BSL & 12.26 & 0.68 \\
\hline [MFF90] PHI 2/2 49 & 123207.20 & -301636.1 & $\mathrm{U}$ & $-1.09:$ & $\mathrm{MFF}$ & 137 & 10 & BSL & 12.03 & $\cdots$ \\
\hline [MFF90] PHI 2/2 46 & 123234.26 & -313226.0 & SPM & $-1.34:$ & $\mathrm{MFF}$ & 296 & 10 & BSL & 12.66 & 1.58 \\
\hline $\mathrm{V}^{*} \mathrm{~S}$ Com & 123245.64 & +270145.6 & HIP & -1.91 & F98 & -55 & 1 & F98 & 11.63 & $\cdots$ \\
\hline MA 30225 & 123304.35 & +293728.5 & $\mathrm{U}$ & -2.59 & BSL & -39 & 10 & BSL & 14.34 & 0.54 \\
\hline [MFF90] PHI 2/2 40 & 123332.04 & -305753.5 & SPM & $-0.75:$ & $\mathrm{MFF}$ & 28 & 10 & BSL & 12.72 & 1.03 \\
\hline CD-33 8486 & 123337.09 & -343318.1 & HIP & -1.87 & BSL & 149 & 10 & BSL & 11.60 & 0.79 \\
\hline [OM87] 123129.29-0 & 123409.65 & -345343.7 & SPM & -2.35 & BSL & 104 & 10 & BSL & 11.99 & 1.02 \\
\hline $\mathrm{BD}+302294$ & 123422.42 & +300317.5 & $\mathrm{ACT}$ & -1.09 & BSL & 56 & 10 & BSL & 10.20 & 1.20 \\
\hline BD+30 2297 & 123529.55 & +295043.8 & STN & -1.17 & BSL & 47 & 10 & BSL & 11.00 & $\cdots$ \\
\hline [MFF90] PHI 2/2 23 & 123553.43 & -310760.0 & $\mathrm{U}$ & -2.26 : & $\mathrm{MFF}$ & 322 & 10 & BSL & 12.75 & $\cdots$ \\
\hline $\mathrm{V}^{*}$ EM Mus & 123556.02 & -725200.0 & $\mathrm{U}$ & -1.55 & L & 99 & 20 & $\mathrm{~L}$ & 12.43 & $\ldots$ \\
\hline $\mathrm{V}^{*} \mathrm{SV}$ CVn & 123556.04 & +371224.1 & NPM & -2.20 & $\mathrm{~L}$ & 29 & 28 & $\mathrm{~L}$ & 12.59 & $\ldots$ \\
\hline $\mathrm{BD}+262368$ & 123600.82 & +254519.2 & $\mathrm{ACT}$ & -1.08 & BSL & 100 & 10 & BSL & 10.07 & 1.05 \\
\hline V* BQ Vir & 123627.29 & -022533.6 & GSC & -1.32 & L & 129 & 9 & $\mathrm{~L}$ & 12.48 & $\cdots$ \\
\hline MA 300285 & 123735.20 & +30 1513.1 & $\mathrm{U}$ & -0.80 & BSL & -8 & 10 & BSL & 13.60 & $\cdots$ \\
\hline HD 109823 & 123738.31 & +283745.9 & HIP & -1.68 & $\cdots$ & 8 & 10 & BSL & 8.07 & 1.15 \\
\hline MA 300289 & 123750.38 & +302239.6 & $\mathrm{U}$ & -0.80 & BSL & 43 & 10 & BSL & 13.35 & $\ldots$ \\
\hline MA 300290 & 123751.92 & +300648.7 & $\mathrm{U}$ & -0.82 & BSL & -26 & 10 & BSL & 13.70 & $\ldots$ \\
\hline $\mathrm{V}^{*} \mathrm{FU}$ Vir & 123822.20 & +130319.0 & $\mathrm{t}$ & -1.17 & $\mathrm{~L}$ & -90 & 8 & $\mathrm{~L}$ & 12.63 & $\ldots$ \\
\hline MA 300293 & 123828.16 & +300851.5 & GSC & -0.76 & BSL & -6 & 10 & BSL & 13.29 & $\cdots$ \\
\hline [MFF90] PHI 4/2 34 & 123829.02 & -295306.0 & $\mathrm{U}$ & $-0.93:$ & MFF & -35 & 10 & BSL & 13.56 & $\cdots$ \\
\hline HD 109995 & 123847.69 & +3918 32.9 & HIP & -1.66 & C97 & -131 & 10 & BSL & 7.60 & 0.05 \\
\hline [MFF90] PHI 4/2 15 & 123925.05 & -295511.1 & $\mathrm{U}$ & $-0.62:$ & $\mathrm{MFF}$ & -19 & 10 & BSL & 13.20 & $\cdots$ \\
\hline [MFF90] PHI 4/3 58 & 123928.32 & -293713.6 & $\mathrm{U}$ & $-0.78:$ & $\mathrm{MFF}$ & 72 & 10 & BSL & 13.42 & $\cdots$ \\
\hline $\mathrm{V}^{*} \mathrm{U}$ Com & 124003.23 & +272956.3 & HIP & -1.25 & F98 & -22 & 3 & F98 & 11.74 & $\cdots$ \\
\hline HD 110184 & 124014.09 & +083138.1 & HIP & -2.46 & B99 & 140 & 1 & $\mathrm{CY}$ & 8.27 & 1.15 \\
\hline $\mathrm{V}^{*} \mathrm{SW} \mathrm{CVn}$ & 124050.80 & +370533.9 & $\mathrm{U}$ & -1.53 & L & -18 & 21 & L & 12.74 & $\cdots$ \\
\hline $\mathrm{V}^{*} \mathrm{KR}$ Vir & 124103.62 & -003713.5 & HIP & -1.59 & ATT & 142 & 1 & $\mathrm{CY}$ & 9.39 & 1.71 \\
\hline$[\mathrm{M} 60 \mathrm{~b}]+31351$ & 124117.10 & +304107.8 & $\mathrm{ACT}$ & -1.33 & BSL & 38 & 10 & BSL & 11.11 & 0.89 \\
\hline [MFF90] PHI 4/3 20 & 124144.21 & -282123.0 & STN & $-0.68:$ & $\mathrm{MFF}$ & -27 & 10 & BSL & 13.34 & $\cdots$ \\
\hline [MFF90] PHI 4/3 15 & 124210.66 & -282956.8 & $\mathrm{U}$ & $-0.74:$ & $\mathrm{MFF}$ & 14 & 10 & BSL & 13.53 & $\cdots$ \\
\hline [MFF90] PHI 4/3 13 & 124212.66 & -283112.7 & STN & $-0.72:$ & MFF & 53 & 10 & BSL & 13.00 & $\cdots$ \\
\hline $\mathrm{BD}+282144$ & $1242 \quad 30.25$ & +272641.6 & STN & -0.82 & BSL & 84 & 10 & BSL & 10.88 & 1.01 \\
\hline [MFF90] PHI 4/3 4 & 124314.45 & -285625.6 & GSC & -0.67 & B99 & 2 & 10 & HK & 11.63 & 0.93 \\
\hline [MFF90] PHI 4/3 1 & 124320.83 & -282720.7 & STN & $-1.67:$ & MFF & 215 & 10 & BSL & 12.74 & $\cdots$ \\
\hline
\end{tabular}


TABLE 1A. (continued)

\begin{tabular}{|c|c|c|c|c|c|c|c|c|c|c|}
\hline $\begin{array}{l}\text { Star } \\
(1)\end{array}$ & $\begin{array}{c}\text { RA (2000.0) } \\
(2)\end{array}$ & $\begin{array}{l}\text { DEC } \\
(3)\end{array}$ & $\begin{array}{c}\text { Source }^{\mathrm{a}} \\
\text { (4) }\end{array}$ & $\begin{array}{c}{[\mathrm{Fe} / \mathrm{H}]} \\
(\mathrm{dex}) \\
(5)\end{array}$ & $\begin{array}{c}\text { Source }^{b} \\
(6)\end{array}$ & $\begin{array}{c}\mathrm{RV} \\
(\mathrm{km} / \mathrm{s}) \\
(7)\end{array}$ & $\begin{array}{c}\sigma_{\mathrm{RV}} \\
(\mathrm{km} / \mathrm{s}) \\
(8)\end{array}$ & $\begin{array}{c}\text { Source }^{\mathrm{c}} \\
(9)\end{array}$ & $\begin{array}{c}V \\
(\mathrm{mag}) \\
(10)\end{array}$ & $\begin{array}{c}B-V \\
(\mathrm{mag}) \\
(11)\end{array}$ \\
\hline HD 110621 & 124343.40 & -444031.4 & HIP & -1.86 & BSL & 219 & 10 & BSL & 9.91 & 0.40 \\
\hline [OM87] 124102.16-0 & 124343.89 & -330343.9 & SPM & -0.99 & RL & 74 & 10 & BSL & 11.82 & 0.88 \\
\hline [MFF90] PHI 4/1 178 & 124355.28 & -295433.0 & $\mathrm{U}$ & $-0.79:$ & MFF & 231 & 10 & BSL & 12.78 & $\ldots$ \\
\hline [MFF90] PHI 4/1 170 & 124408.05 & $-3001 \quad 47.3$ & STN & $-1.41:$ & $\mathrm{MFF}$ & 168 & 10 & BSL & 12.36 & $\ldots$ \\
\hline $\mathrm{BD}+262386$ & 124416.07 & +255754.9 & GSC & -0.73 & BSL & -18 & 10 & BSL & 10.72 & 1.04 \\
\hline [MFF90] PHI 4/1 167 & 124419.76 & -295520.0 & $\mathrm{U}$ & $-1.71:$ & MFF & 205 & 10 & BSL & 13.30 & $\ldots$ \\
\hline UP 25091 & 124424.02 & +245717.7 & STN & -0.65 & BSL & 47 & 10 & BSL & 11.70 & 1.00 \\
\hline MA 34370 & 124442.29 & +335258.5 & STN & -0.86 & BSL & -32 & 10 & BSL & 11.78 & 1.54 \\
\hline [M60b]+34 382 & 124618.29 & +334904.7 & STN & -0.77 & BSL & -36 & 10 & BSL & 10.94 & 1.06 \\
\hline [MFF90] PHI 4/2 121 & 124659.10 & -292843.2 & $\mathrm{U}$ & $-0.66:$ & $\mathrm{MFF}$ & 86 & 10 & BSL & 12.18 & $\cdots$ \\
\hline [MFF90] PHI 4/1 99 & $1247 \quad 17.77$ & -304133.8 & SPM & $-2.01:$ & $\mathrm{MFF}$ & 183 & 10 & BSL & 13.23 & 1.34 \\
\hline CD-27 8864 & 124722.92 & -282259.7 & SPM & -1.71 & BSL & 247 & 10 & BSL & 10.80 & 1.00 \\
\hline $\mathrm{BD}+332273$ & 124755.05 & +3303 18.9 & STN & -1.05 & BSL & 41 & 10 & BSL & 10.90 & 0.98 \\
\hline [MFF90] PHI 4/2 105 & 124802.02 & -284902.1 & $\mathrm{U}$ & $-0.61:$ & MFF & -8 & 10 & BSL & 13.61 & $\ldots$ \\
\hline UP 29143 & 124906.00 & +285340.0 & $\mathrm{t}$ & -0.73 & BSL & 89 & 10 & BSL & 11.50 & $\cdots$ \\
\hline [MFF90] PHI 4/1 65 & 124908.65 & $\begin{array}{llll}-31 & 14 & 34.1\end{array}$ & $\mathrm{U}$ & $-1.14:$ & $\mathrm{MFF}$ & 93 & 10 & BSL & 13.27 & $\ldots$ \\
\hline [MFF90] PHI 4/1 62 & 124924.70 & -311400.6 & $\mathrm{U}$ & $-0.91:$ & MFF & 27 & 10 & BSL & 13.08 & $\cdots$ \\
\hline $\mathrm{V}^{*} \mathrm{Z} \mathrm{CVn}$ & 124945.50 & +434626.3 & GSC & -1.98 & L & 14 & 10 & L & 11.93 & $\ldots$ \\
\hline HD 111464 & 125014.05 & -623828.8 & HIP & -0.81 & BSL & 28 & 10 & BSL & 6.61 & 1.40 \\
\hline [MFF90] PHI 4/2 73 & 125018.71 & -284552.9 & $\mathrm{U}$ & $-1.82:$ & $\mathrm{MFF}$ & 121 & 10 & BSL & 12.61 & $\cdots$ \\
\hline [MFF90] PHI 4/2 68 & 125045.90 & -282656.2 & STN & $-2.01:$ & $\mathrm{MFF}$ & 57 & 10 & BSL & 12.46 & $\cdots$ \\
\hline HD 111721 & 125125.36 & -132925.4 & HIP & -1.26 & B99 & 27 & 10 & HK & 7.97 & 0.80 \\
\hline [MFF90] PHI 4/1 21 & 125143.51 & -304602.3 & $\mathrm{U}$ & $-1.86:$ & MFF & 202 & 10 & BSL & 12.80 & $\ldots$ \\
\hline $\mathrm{BD}+272181$ & 125154.05 & +264707.9 & STN & -0.70 & BSL & -49 & 10 & BSL & 11.27 & 1.02 \\
\hline [MFF90] PHI 4/1 7 & 125216.87 & -300154.0 & STN & -1.01 & B99 & 14 & 10 & HK & 11.91 & $\ldots$ \\
\hline $\mathrm{V}^{*}$ AS Vir & 125245.84 & -101535.7 & GSC & -1.49 & L & 70 & 23 & $\mathrm{~L}$ & 11.90 & $\ldots$ \\
\hline HD 111980 & 125314.87 & $-1831 \quad 13.0$ & HIP & -0.99 & B99 & 154 & 10 & HK & 8.36 & 0.55 \\
\hline HD 111971 & 125342.51 & -573923.8 & HIP & -0.55 & B99 & -16 & 10 & HK & 8.02 & 0.51 \\
\hline HD 112126 & 125349.20 & +323027.9 & HIP & -1.52 & BSL & -62 & 10 & BSL & 8.74 & 1.37 \\
\hline $\mathrm{BD}+282162$ & 125410.15 & +271756.4 & STN & -0.95 & BSL & 14 & 10 & BSL & 11.05 & 1.12 \\
\hline $\mathrm{V}^{*}$ AT Vir & 125510.58 & -052732.1 & HIP & -1.60 & F98 & 336 & 3 & F98 & 11.34 & $\ldots$ \\
\hline $\mathrm{BD}+272191$ & 125519.11 & +262201.4 & $\mathrm{ACT}$ & -0.77 & BSL & 52 & 10 & BSL & 10.27 & 0.75 \\
\hline $\mathrm{BD}+272196$ & 125632.73 & +262341.6 & $\mathrm{v}$ & -0.76 & BSL & 48 & 10 & BSL & 10.91 & 1.18 \\
\hline $\mathrm{BD}+102495$ & 125919.96 & +09 1435.4 & HIP & -1.83 & B99 & 253 & 1 & $\mathrm{CY}$ & 9.72 & 0.75 \\
\hline Weis 60529 & 125953.24 & +285228.5 & STN & -0.77 & BSL & 68 & 10 & BSL & 12.94 & 1.01 \\
\hline Weis 62013 & 130004.53 & +282410.5 & $\mathrm{U}$ & -0.73 & BSL & 5 & 10 & BSL & 12.95 & 1.73 \\
\hline Weis 60443 & 130013.22 & +285406.3 & $\mathrm{U}$ & -1.58 & BSL & -71 & 10 & BSL & 14.97 & 0.63 \\
\hline Weis 62339 & 130019.00 & +281506.6 & STN & -0.78 & BSL & -41 & 10 & BSL & 13.76 & 0.65 \\
\hline Weis 62021 & 130020.34 & +282128.2 & $\mathrm{U}$ & -0.69 & BSL & 10 & 10 & BSL & 14.10 & 1.75 \\
\hline HD 113002 & 130028.85 & +193756.1 & HIP & -1.08 & BSL & -96 & 10 & BSL & 8.73 & 0.74 \\
\hline
\end{tabular}


TABLE 1A. (continued)

\begin{tabular}{|c|c|c|c|c|c|c|c|c|c|c|}
\hline $\begin{array}{l}\text { Star } \\
(1)\end{array}$ & $\begin{array}{c}\text { RA (2000.0) } \\
(2)\end{array}$ & DEC & Source ${ }^{\mathrm{a}}$ & $\begin{array}{c}{[\mathrm{Fe} / \mathrm{H}]} \\
(\mathrm{dex}) \\
(5)\end{array}$ & $\begin{array}{c}\text { Source }^{b} \\
(6)\end{array}$ & $\begin{array}{c}\mathrm{RV} \\
(\mathrm{km} / \mathrm{s}) \\
(7)\end{array}$ & $\begin{array}{c}\sigma_{\mathrm{RV}} \\
(\mathrm{km} / \mathrm{s}) \\
(8)\end{array}$ & $\begin{array}{c}\text { Source }^{\mathrm{c}} \\
(9)\end{array}$ & $\begin{array}{c}V \\
(\mathrm{mag}) \\
(10)\end{array}$ & $\begin{array}{c}B-V \\
(\mathrm{mag}) \\
(11)\end{array}$ \\
\hline Weis 33496 & 130039.00 & +300734.2 & GSC & -0.77 & BSL & -2 & 10 & BSL & 11.59 & 0.82 \\
\hline $\mathrm{BD}+292354$ & 130041.88 & +283036.3 & $\mathrm{ACT}$ & -1.85 & BSL & 13 & 10 & BSL & 9.83 & 0.58 \\
\hline Weis 64018 & 130106.56 & +273506.1 & GSC & -1.47 & BSL & 63 & 10 & BSL & 14.01 & 1.51 \\
\hline HD 112911 & 130110.12 & -630123.4 & HIP & -0.76 & BSL & 32 & 10 & BSL & 7.43 & 1.71 \\
\hline Weis 63200 & 130122.78 & +280520.1 & GSC & -1.31 & BSL & 35 & 10 & BSL & 13.84 & 0.78 \\
\hline HD 113083 & 130126.58 & -272226.5 & HIP & -1.04 & B99 & 225 & 10 & HK & 8.04 & 0.55 \\
\hline Weis 63150 & 130145.80 & +275740.4 & GSC & -1.96 & BSL & -19 & 10 & BSL & 11.45 & 1.25 \\
\hline $\mathrm{BD}+292356$ & 130152.42 & +291117.8 & STN & -1.06 & BSL & -201 & 10 & BSL & 11.50 & 0.77 \\
\hline CD-32 9096 & 130200.55 & -330911.1 & HIP & -2.09 & BSL & 421 & 10 & BSL & 10.42 & 1.45 \\
\hline Weis 63059 & 130203.20 & +280523.7 & STN & -1.53 & BSL & 5 & 10 & BSL & 12.43 & 0.92 \\
\hline Weis 31775 & 130205.18 & +305645.0 & GSC & -0.61 & BSL & 4 & 10 & BSL & 11.41 & 1.01 \\
\hline Weis 35550 & 130212.08 & +292411.4 & $\mathrm{STN}$ & -0.62 & BSL & 4 & 10 & BSL & 11.99 & 0.66 \\
\hline CD-33 8772 & 130218.09 & -342134.1 & SPM & -2.03 & BSL & 59 & 10 & BSL & 11.06 & 0.87 \\
\hline Weis 62864 & 130221.50 & +281350.4 & GSC & -1.91 & BSL & -1 & 10 & BSL & 13.52 & 0.50 \\
\hline Weis 62467 & 130222.25 & +281914.8 & STN & -0.99 & BSL & -3 & 10 & BSL & 13.81 & 0.73 \\
\hline Weis 61345 & 130243.72 & +284737.1 & GSC & -1.31 & BSL & -57 & 10 & BSL & 14.35 & 1.00 \\
\hline Weis 62990 & 130316.45 & +275425.5 & $\mathrm{U}$ & -1.49 & BSL & 9 & 10 & BSL & 14.46 & 0.51 \\
\hline Weis 62981 & 130333.22 & +275511.1 & STN & -1.50 & BSL & 88 & 10 & BSL & 13.17 & 0.73 \\
\hline $\mathrm{BD}+282176$ & 130337.50 & +273530.6 & $\mathrm{ACT}$ & -1.49 & BSL & -6 & 10 & BSL & 9.79 & 0.99 \\
\hline Weis 62561 & 130354.37 & +281640.3 & STN & -0.63 & BSL & 100 & 10 & BSL & 13.15 & 0.83 \\
\hline Weis 61803 & 130355.54 & +28 2951.9 & GSC & -1.13 & BSL & 13 & 10 & BSL & 15.00 & 0.53 \\
\hline Weis 61514 & 130355.70 & +284957.1 & $\mathrm{U}$ & -2.85 & BSL & -161 & 10 & BSL & 15.36 & 0.64 \\
\hline Weis 61766 & 130401.16 & +282325.5 & $\mathrm{U}$ & -1.07 & BSL & 47 & 10 & BSL & 14.31 & 0.81 \\
\hline $\mathrm{BD}+122547$ & 130406.59 & +112616.3 & HIP & -2.07 & ATT & 6 & 1 & CY & 9.92 & 1.03 \\
\hline Weis 61574 & 130432.75 & +283918.4 & GSC & -0.91 & BSL & -67 & 10 & BSL & 14.57 & 0.93 \\
\hline Weis 61730 & 130502.33 & +282502.9 & $\mathrm{U}$ & -0.60 & BSL & 51 & 10 & BSL & 14.44 & 0.85 \\
\hline $\mathrm{V}^{*} \mathrm{RY}$ Com & 130507.99 & +231642.6 & GSC & -1.65 & L & -31 & 8 & $\mathrm{~L}$ & 12.30 & $\cdots$ \\
\hline BD+30 2364 & 130518.45 & +301047.5 & GSC & -0.63 & BSL & -16 & 10 & BSL & 10.67 & 1.38 \\
\hline Basel SA 57-1416 & 130626.38 & +293245.2 & $\mathrm{U}$ & -1.13 & BSL & -14 & 10 & BSL & 15.17 & 0.58 \\
\hline Basel SA 57-1372 & 130636.46 & +291656.9 & $\mathrm{U}$ & -1.00 & BSL & 30 & 10 & BSL & 17.34 & 0.68 \\
\hline Basel SA 57-1284 & 130638.18 & +285542.0 & $\mathrm{U}$ & -1.04 & BSL & 45 & 10 & BSL & 14.71 & 0.59 \\
\hline Basel SA 57-1378 & 130638.49 & +292035.0 & $\mathrm{U}$ & -0.63 & BSL & -11 & 10 & BSL & 15.04 & 0.79 \\
\hline Basel SA 57-1373 & 130638.60 & +291656.1 & $\mathrm{U}$ & -3.00 & BSL & 48 & 10 & BSL & 17.70 & 0.56 \\
\hline Basel SA 57-1374 & 130644.83 & +291735.9 & $\mathrm{U}$ & -0.96 & BSL & -19 & 10 & BSL & 16.16 & 0.69 \\
\hline Basel SA 57-1302 & 130645.73 & +2907 13.9 & $\mathrm{U}$ & -0.78 & BSL & 21 & 10 & BSL & 14.04 & 0.64 \\
\hline Basel SA 57-1383 & 130647.98 & +292124.5 & $\mathrm{U}$ & -0.90 & BSL & 12 & 10 & BSL & 16.44 & 0.70 \\
\hline Basel SA 57-1375 & 130650.75 & +2918 26.9 & $\mathrm{U}$ & -1.08 & BSL & -32 & 10 & BSL & 14.93 & 0.79 \\
\hline Basel SA 57-1299 & 130651.05 & +290348.7 & $\mathrm{U}$ & -1.58 & BSL & -45 & 10 & BSL & 14.98 & 0.47 \\
\hline Basel SA 57-1148 & 130657.39 & +284941.0 & $\mathrm{U}$ & -1.45 & BSL & -31 & 10 & BSL & 16.77 & 0.69 \\
\hline Basel SA 57-1110 & 130659.57 & +285713.4 & $\mathrm{U}$ & -1.15 & BSL & -38 & 10 & BSL & 15.13 & 0.75 \\
\hline
\end{tabular}


TABLE 1A. (continued)

\begin{tabular}{|c|c|c|c|c|c|c|c|c|c|c|}
\hline Star & RA (2000.0) & DEC & Source ${ }^{a}$ & $\begin{array}{c}{[\mathrm{Fe} / \mathrm{H}]} \\
(\mathrm{dex}) \\
(5)\end{array}$ & $\begin{array}{c}\text { Source }^{b} \\
(6)\end{array}$ & $\begin{array}{c}\mathrm{RV} \\
(\mathrm{km} / \mathrm{s}) \\
(7)\end{array}$ & $\begin{array}{c}\sigma_{\mathrm{RV}} \\
(\mathrm{km} / \mathrm{s}) \\
(8)\end{array}$ & $\begin{array}{c}\text { Source }{ }^{\mathrm{c}} \\
(9)\end{array}$ & $\begin{array}{c}V \\
(\mathrm{mag}) \\
(10)\end{array}$ & $\begin{array}{c}B-V \\
(\mathrm{mag}) \\
(11)\end{array}$ \\
\hline $\mathrm{BD}+312450$ & 130659.64 & +310620.8 & GSC & -0.63 & BSL & -34 & 10 & BSL & 9.68 & 1.60 \\
\hline Basel SA 57-1144 & 130703.15 & +285209.1 & $\mathrm{U}$ & -3.00 & BSL & 79 & 10 & BSL & 16.21 & 0.71 \\
\hline Basel SA 57-1147 & 130704.72 & +285035.8 & $\mathrm{U}$ & -0.76 & BSL & -2 & 10 & BSL & 17.25 & 0.93 \\
\hline Basel SA 57-1146 & 130707.76 & +285111.0 & $\mathrm{U}$ & -3.00 & BSL & -45 & 10 & BSL & 17.83 & 0.50 \\
\hline Basel SA 57-1142 & 130709.45 & +285239.6 & $\mathrm{U}$ & -0.96 & BSL & 3 & 10 & BSL & 17.15 & 0.69 \\
\hline Weis 51212 & 130709.71 & +282744.2 & GSC & -1.31 & BSL & 18 & 10 & BSL & 11.25 & 1.20 \\
\hline Basel SA 57-1141 & $1307 \quad 11.81$ & +285254.7 & $\mathrm{U}$ & -3.00 & BSL & 28 & 10 & BSL & 17.47 & 0.68 \\
\hline Basel SA 57-1152 & 130711.94 & +284757.6 & $\mathrm{U}$ & -0.72 & BSL & -61 & 10 & BSL & 16.64 & 0.64 \\
\hline Basel SA 57-1140 & 130716.17 & +285310.3 & $\mathrm{U}$ & -0.88 & BSL & -18 & 10 & BSL & 14.07 & 0.66 \\
\hline Basel SA 57-0996 & 130718.95 & +293444.2 & $\mathrm{U}$ & -0.84 & BSL & -19 & 10 & BSL & 14.73 & 0.57 \\
\hline Basel SA 57-1060 & 130719.99 & +291413.0 & $\mathrm{U}$ & -0.84 & BSL & 28 & 10 & BSL & 14.15 & 0.59 \\
\hline Basel SA 57-0993 & 130727.51 & +293836.1 & $\mathrm{U}$ & -0.76 & BSL & 16 & 10 & BSL & 14.34 & 0.61 \\
\hline Basel SA 57-1003 & 130727.80 & +292621.2 & $\mathrm{U}$ & -0.76 & BSL & -26 & 10 & BSL & 15.00 & 0.69 \\
\hline Basel SA 57-0987 & $1307 \quad 27.86$ & +294345.3 & $\mathrm{U}$ & -0.93 & BSL & 22 & 10 & BSL & 14.47 & 0.60 \\
\hline Basel SA 57-1132 & 130729.45 & +285457.9 & $\mathrm{U}$ & -1.73 & BSL & 53 & 10 & BSL & 16.58 & 0.61 \\
\hline Basel SA 57-0982 & 130736.00 & +294125.8 & $\mathrm{U}$ & -1.83 & BSL & 39 & 10 & BSL & 17.51 & 0.48 \\
\hline Basel SA 57-0983 & 130736.46 & +294205.4 & $\mathrm{U}$ & -0.78 & BSL & 1 & 10 & BSL & 13.75 & 0.72 \\
\hline Basel SA 57-1156 & 130737.18 & +284838.5 & $\mathrm{U}$ & -1.04 & BSL & 11 & 10 & BSL & 13.98 & 0.41 \\
\hline Basel SA 57-1157 & 130737.26 & +284827.5 & $\mathrm{U}$ & -1.92 & BSL & -37 & 10 & BSL & 17.70 & 0.74 \\
\hline Basel SA 57-1131 & 130737.92 & +285531.2 & $\mathrm{U}$ & -1.15 & BSL & 1 & 10 & BSL & 16.95 & 0.68 \\
\hline Basel SA 57-0978 & 130751.78 & +294039.1 & $\mathrm{U}$ & -0.99 & BSL & 36 & 10 & BSL & 15.12 & 0.65 \\
\hline Basel SA 57-1012 & 130758.44 & +292733.0 & $\mathrm{U}$ & -0.75 & BSL & -2 & 10 & BSL & 14.72 & 0.60 \\
\hline Basel SA 57-0977 & 130759.30 & +2938 11.9 & $\mathrm{U}$ & -1.83 & BSL & -10 & 10 & BSL & 15.38 & 0.78 \\
\hline Basel SA 57-1117 & 130802.40 & +285701.1 & $\mathrm{U}$ & -0.67 & BSL & -37 & 10 & BSL & 15.02 & 0.68 \\
\hline Basel SA 57-0966 & 130809.79 & +294053.8 & U & -1.93 & BSL & 144 & 10 & BSL & 14.76 & 0.88 \\
\hline Basel SA 57-1016 & 130818.09 & +292739.6 & NPM & -1.04 & BSL & -12 & 10 & BSL & 15.03 & 0.68 \\
\hline $\mathrm{V}^{*} \mathrm{Z}$ Com & 130819.68 & +183310.3 & $\mathrm{U}$ & -1.50 & L & -50 & 35 & $\mathrm{~L}$ & 13.73 & $\cdots$ \\
\hline Basel SA 57-0692 & 130820.16 & +285438.7 & $\mathrm{U}$ & -3.00 & BSL & 24 & 10 & BSL & 14.62 & 0.74 \\
\hline Basel SA 57-0715 & 130820.68 & +290938.8 & U & -1.23 & BSL & -33 & 10 & BSL & 14.69 & 0.60 \\
\hline Basel SA 57-0789 & 130827.61 & +292804.5 & NPM & -1.74 & BSL & -30 & 10 & BSL & 14.94 & 0.46 \\
\hline Basel SA 57-0792 & 130834.10 & +293231.1 & $\mathrm{U}$ & -0.78 & BSL & -16 & 10 & BSL & 13.50 & 0.77 \\
\hline Basel SA 57-0827 & 130837.61 & +293943.0 & $\mathrm{U}$ & -1.18 & BSL & -2 & 10 & BSL & 15.43 & 0.64 \\
\hline Basel SA 57-0786 & 130838.17 & +292605.7 & $\mathrm{U}$ & -1.13 & BSL & 11 & 10 & BSL & 13.99 & 0.83 \\
\hline Basel SA 57-0758 & 130838.47 & +291757.5 & NPM & -1.26 & BSL & -23 & 10 & BSL & 16.96 & 0.63 \\
\hline Basel SA 57-0760 & 130839.82 & +291628.6 & NPM & -0.70 & BSL & -15 & 10 & BSL & 15.91 & 0.77 \\
\hline Basel SA 57-0712 & 130840.42 & +290234.4 & $\mathrm{U}$ & -0.97 & BSL & -35 & 10 & BSL & 15.34 & 0.90 \\
\hline Basel SA 57-0709 & 130842.08 & +290638.4 & $\mathrm{U}$ & -0.63 & BSL & 26 & 10 & BSL & 16.87 & 0.85 \\
\hline Basel SA 57-0699 & $1308 \quad 42.13$ & +285934.9 & $\mathrm{U}$ & -0.72 & BSL & 43 & 10 & BSL & 14.29 & 0.51 \\
\hline Basel SA 57-0825 & 130844.07 & +293833.7 & $\mathrm{U}$ & -0.85 & BSL & -38 & 10 & BSL & 17.57 & 0.51 \\
\hline Basel SA 57-0718 & 130845.10 & +291029.5 & $\mathrm{U}$ & -1.21 & BSL & -104 & 10 & BSL & 15.94 & 0.82 \\
\hline
\end{tabular}


TABLE 1A. (continued)

\begin{tabular}{|c|c|c|c|c|c|c|c|c|c|c|}
\hline Star & RA (2000.0) & DEC & $\begin{array}{c}\text { Source }^{\mathrm{a}} \\
(4)\end{array}$ & $\begin{array}{c}{[\mathrm{Fe} / \mathrm{H}]} \\
(\mathrm{dex}) \\
(5)\end{array}$ & $\begin{array}{c}\text { Source }^{b} \\
(6)\end{array}$ & $\begin{array}{c}\mathrm{RV} \\
(\mathrm{km} / \mathrm{s}) \\
(7)\end{array}$ & $\begin{array}{c}\sigma_{\mathrm{RV}} \\
(\mathrm{km} / \mathrm{s}) \\
(8)\end{array}$ & $\begin{array}{c}\text { Source }^{c} \\
(9)\end{array}$ & $\begin{array}{c}V \\
(\mathrm{mag}) \\
(10)\end{array}$ & $\begin{array}{c}B-V \\
(\mathrm{mag}) \\
(11)\end{array}$ \\
\hline Basel SA 57-0650 & 130846.69 & +284653.3 & $\mathrm{U}$ & -1.35 & BSL & 127 & 10 & BSL & 15.23 & 0.54 \\
\hline Basel SA 57-0719 & 130848.75 & +290909.9 & $\mathrm{U}$ & -2.27 & BSL & -38 & 10 & BSL & 16.57 & 0.64 \\
\hline Basel SA 57-0708 & 130849.90 & +290640.3 & $\mathrm{U}$ & -1.62 & BSL & -48 & 10 & BSL & 17.57 & 0.64 \\
\hline Basel SA 57-0799 & 130850.32 & +293128.5 & $\mathrm{U}$ & -1.77 & BSL & -208 & 10 & BSL & 16.98 & 0.91 \\
\hline Basel SA 57-0720 & 130856.85 & +290916.5 & $\mathrm{U}$ & -0.93 & BSL & -61 & 10 & BSL & 17.38 & 0.58 \\
\hline Basel SA 57-0739 & 130858.20 & +291624.7 & NPM & -3.00 & BSL & 17 & 10 & BSL & 15.21 & 0.35 \\
\hline Basel SA 57-0802 & 130858.74 & +293027.5 & $\mathrm{U}$ & -1.02 & BSL & -84 & 10 & BSL & 16.02 & 0.57 \\
\hline Weis 50158 & 130907.04 & +285950.4 & STN & -0.48 & HK & 36 & 10 & BSL & 12.81 & 0.64 \\
\hline Basel SA 57-0803 & 130907.47 & +293130.0 & $\mathrm{U}$ & -1.08 & BSL & -8 & 10 & BSL & 16.94 & 0.95 \\
\hline Basel SA 57-0677 & 130921.20 & +285345.7 & $\mathrm{U}$ & -1.51 & BSL & -50 & 10 & BSL & 14.58 & 0.63 \\
\hline Basel SA 57-0659 & 130927.44 & +285213.8 & $\mathrm{U}$ & -1.33 & BSL & 34 & 10 & BSL & 15.82 & 0.62 \\
\hline Basel SA 57-0608 & 130929.71 & +284543.0 & $\mathrm{U}$ & -0.97 & BSL & -31 & 10 & BSL & 14.46 & 0.74 \\
\hline Basel SA 57-0808 & 130930.11 & +293130.7 & $\mathrm{U}$ & -1.27 & BSL & 9 & 10 & BSL & 14.91 & 0.73 \\
\hline Basel SA 57-0664 & 130933.90 & +285756.2 & $\mathrm{U}$ & -1.05 & BSL & 11 & 10 & BSL & 14.17 & 0.64 \\
\hline Basel SA 57-0671 & 130938.09 & +290159.7 & $\mathrm{U}$ & -0.68 & BSL & -32 & 10 & BSL & 13.72 & 0.63 \\
\hline Basel SA 57-0488 & 130952.20 & +290616.2 & $\mathrm{U}$ & -1.09 & BSL & 94 & 10 & BSL & 15.52 & 0.68 \\
\hline Basel SA 57-0564 & 130952.67 & +284418.6 & $\mathrm{U}$ & -0.87 & BSL & -3 & 10 & BSL & 14.58 & 0.68 \\
\hline Basel SA 57-0559 & 130953.75 & +284717.3 & $\mathrm{U}$ & -1.47 & BSL & -15 & 10 & BSL & 15.40 & 0.83 \\
\hline Basel SA 57-0467 & 130957.35 & +29 1635.2 & $\mathrm{U}$ & -1.09 & BSL & -68 & 10 & BSL & 14.88 & 0.62 \\
\hline Basel SA 57-0548 & 130958.35 & +285249.1 & $\mathrm{U}$ & -0.76 & BSL & 2 & 10 & BSL & 16.58 & 1.00 \\
\hline Basel SA 57-0546 & 131001.62 & +285432.4 & U & -0.63 & BSL & 17 & 10 & BSL & 14.70 & 0.58 \\
\hline Weis 50001 & 131001.66 & +290809.8 & STN & -0.78 & BSL & -70 & 10 & BSL & 11.75 & 1.19 \\
\hline CD-33 8865 & 131013.06 & -334236.9 & HIP & -1.93 & BSL & 48 & 10 & BSL & 10.89 & 1.12 \\
\hline Basel SA 57-0455 & 131013.57 & +292427.5 & $\mathrm{U}$ & -0.71 & BSL & -14 & 10 & BSL & 15.07 & 0.85 \\
\hline Basel SA 57-0505 & 131018.32 & +290237.9 & $\mathrm{U}$ & -0.99 & BSL & -67 & 10 & BSL & 17.40 & 0.55 \\
\hline Basel SA 57-0735 & 131022.00 & +291651.0 & $\mathrm{t}$ & -1.88 & BSL & -20 & 10 & BSL & 14.46 & $\cdots$ \\
\hline BPS CS 22877-0013 & 131123.97 & -092335.8 & U & -2.31 & HK & 192 & 10 & BSL & 14.64 & 0.51 \\
\hline CD-31 10167 & 131338.16 & -315523.7 & GSC & -1.61 & BSL & 290 & 10 & BSL & 11.31 & 0.49 \\
\hline BPS CS 22877-0016 & 131344.57 & -084953.9 & $\mathrm{U}$ & -1.20 & HK & 5 & 10 & BSL & $(13.49)$ & $(0.61)$ \\
\hline BPS CS 22877-0015 & 131345.29 & -091542.8 & GSC & -1.74 & HK & 97 & 10 & BSL & 13.23 & 0.40 \\
\hline BPS CS 22877-0001 & 131355.35 & $-12 \quad 1141.5$ & STN & -2.76 & HK & 169 & 10 & BSL & $(11.78)$ & $(0.72)$ \\
\hline BPS CS 22877-0011 & 131440.16 & -093547.1 & GSC & -2.92 & B99 & 222 & 10 & HK & 13.86 & 0.60 \\
\hline HD 115338 & 131611.97 & +305338.9 & $\mathrm{ACT}$ & -0.63 & BSL & 23 & 10 & BSL & 8.69 & 1.19 \\
\hline HD 115444 & 131642.46 & +362253.2 & HIP & -2.73 & B99 & -28 & 5 & $\mathrm{CY}$ & 8.96 & 0.75 \\
\hline $\mathrm{V}^{*} \mathrm{YZ}$ Vir & 131712.48 & -050452.2 & $\mathrm{U}$ & -0.90 & BSL & 192 & 10 & BSL & 12.90 & $\cdots$ \\
\hline $\mathrm{V}^{*} \mathrm{ST}$ Com & 131751.36 & +204651.1 & HIP & -1.10 & F98 & -68 & 10 & F98 & 11.46 & $\cdots$ \\
\hline BPS CS 22877-0029 & 131824.04 & -090645.0 & $\mathrm{U}$ & -0.84 & HK & -48 & 10 & BSL & {$[13.60]$} & {$[0.60]$} \\
\hline HD 115772 & 132000.40 & -395620.0 & HIP & -0.70 & B99 & -38 & 10 & HK & 9.66 & 0.84 \\
\hline $\mathrm{V}^{*}$ AV Vir & 132011.56 & +09 1116.7 & HIP & -1.25 & F98 & 153 & 1 & F98 & 11.82 & $\cdots$ \\
\hline HD 116064 & 132144.36 & -391840.8 & HIP & -1.91 & B99 & 146 & 10 & HK & 8.80 & 0.45 \\
\hline
\end{tabular}


TABLE 1A. (continued)

\begin{tabular}{|c|c|c|c|c|c|c|c|c|c|c|}
\hline Star & $\begin{array}{c}\text { RA (2000.0) } \\
(2)\end{array}$ & DEC & $\begin{array}{c}\text { Source }^{\mathrm{a}} \\
(4)\end{array}$ & $\begin{array}{c}{[\mathrm{Fe} / \mathrm{H}]} \\
(\mathrm{dex}) \\
(5)\end{array}$ & $\begin{array}{c}\text { Source }^{b} \\
(6)\end{array}$ & $\begin{array}{c}\mathrm{RV} \\
(\mathrm{km} / \mathrm{s}) \\
(7)\end{array}$ & $\begin{array}{c}\sigma_{\mathrm{RV}} \\
(\mathrm{km} / \mathrm{s}) \\
(8)\end{array}$ & $\begin{array}{c}\text { Source }^{c} \\
(9)\end{array}$ & $\begin{array}{c}V \\
(\mathrm{mag}) \\
(10)\end{array}$ & $\begin{array}{c}B-V \\
(\mathrm{mag}) \\
(11)\end{array}$ \\
\hline $\mathrm{V}^{*} \mathrm{BC}$ Vir & 132221.15 & +055311.1 & STN & -1.50 & L & 4 & 13 & L & 12.21 & \\
\hline BPS CS 22877-0051 & 132331.27 & -123512.3 & U & -2.50 & HK & 276 & 10 & BSL & 14.22 & $(0.44)$ \\
\hline $\mathrm{V}^{*}$ AM Vir & 132333.33 & -163957.5 & HIP & -1.37 & F98 & 98 & 1 & F98 & 11.52 & $\ldots$ \\
\hline BPS CS 22877-0024 & 132433.22 & -083233.4 & $\mathrm{U}$ & -1.54 & HK & 106 & 10 & BSL & {$[14.60]$} & {$[0.40]$} \\
\hline $\mathrm{V}^{*}$ AU Vir & 132448.01 & -065845.3 & HIP & -1.50 & F98 & 119 & 2 & F98 & 11.59 & $\ldots$ \\
\hline $\mathrm{V}^{*} \mathrm{~W}$ Vir & 132602.00 & -032243.4 & HIP & -0.60 & BSL & -65 & 1 & BSL & 9.69 & 0.64 \\
\hline $\mathrm{V}^{*} \mathrm{SX}$ UMa & 132613.53 & +561525.0 & HIP & -1.81 & F98 & -154 & 1 & F98 & 10.84 & $\ldots$ \\
\hline V* FX Hya & 132800.70 & -270610.1 & SPM & -1.08 & $\mathrm{~L}$ & 50 & 23 & $\mathrm{~L}$ & 12.64 & $\cdots$ \\
\hline $\mathrm{V}^{*} \mathrm{WW}$ Vir & 132823.86 & -05 1709.2 & GSC & -2.13 & $\mathrm{~L}$ & 129 & 10 & $\mathrm{~L}$ & 12.77 & $\cdots$ \\
\hline [OM87] 132605.43-0 & 132854.31 & -312043.5 & SPM & -0.66 & $\mathrm{RL}$ & -57 & 10 & BSL & 11.28 & 0.79 \\
\hline BPS CS 22877-0060 & 132907.43 & -085944.7 & $\mathrm{U}$ & -1.45 & HK & 262 & 10 & BSL & $(14.62)$ & $(0.48)$ \\
\hline HD 117220 & 132935.61 & -373335.6 & HIP & -0.86 & B99 & -19 & 10 & HK & 9.01 & 0.84 \\
\hline $\mathrm{BD}+032782$ & 132955.93 & +024526.8 & HIP & -2.02 & B99 & 32 & 1 & CY & 9.70 & 1.09 \\
\hline $\mathrm{V}^{*} \mathrm{RV} \mathrm{UMa}$ & 133318.11 & +535915.0 & HIP & -1.20 & F98 & -185 & 1 & F98 & 10.77 & $\ldots$ \\
\hline HD 117880 & 133329.86 & -183053.1 & HIP & -1.73 & BSL & 156 & 10 & BSL & 9.06 & 0.08 \\
\hline BPS CS 22889-0006 & 133420.44 & -095525.9 & $\mathrm{U}$ & -1.77 & HK & 41 & 10 & BSL & 14.65 & 0.39 \\
\hline HD 118055 & 133439.93 & -161922.6 & HIP & -1.81 & B99 & -101 & 1 & $\mathrm{CY}$ & 8.86 & 1.27 \\
\hline $\mathrm{V}^{*} \mathrm{WY}$ Vir & 133516.16 & -065822.7 & GSC & -2.84 & L & 181 & 10 & L & 13.38 & $\cdots$ \\
\hline $\mathrm{BD}+182757$ & 133631.98 & +18 0902.4 & HIP & -2.19 & B99 & -22 & 5 & CY & 9.80 & 0.77 \\
\hline V* V671 Cen & 134009.71 & -372627.8 & SPM & -1.40 & $\mathrm{~L}$ & 108 & 14 & L & 12.43 & $\cdots$ \\
\hline BPS CS 22889-0029 & 134208.04 & -115701.0 & $\mathrm{U}$ & -2.22 & HK & 15 & 10 & BSL & $(13.94)$ & $(0.56)$ \\
\hline BPS CS 22889-0038 & 134246.69 & -084654.4 & $\mathrm{U}$ & -2.40 & HK & -53 & 10 & BSL & $(14.56)$ & $(0.54)$ \\
\hline HD 119516 & 134326.76 & +153431.2 & HIP & -2.49 & ATT & -287 & 1 & $\mathrm{CY}$ & 9.05 & 0.66 \\
\hline BPS CS 22889-0045 & 134345.90 & -084026.8 & $\mathrm{U}$ & -2.13 & HK & 2 & 10 & BSL & 13.31 & 0.46 \\
\hline $\mathrm{V}^{*} \mathrm{RZ} \mathrm{CVn}$ & 134503.05 & +323916.7 & HIP & -1.84 & F98 & -12 & 1 & F98 & 11.57 & $\cdots$ \\
\hline BPS CS 22889-0050 & 134530.24 & -094711.8 & $\mathrm{U}$ & -2.75 & HK & 233 & 10 & BSL & $(14.14)$ & $(0.46)$ \\
\hline CD-33 9314 & 134603.47 & -335626.8 & HIP & -2.10 & BSL & 407 & 10 & BSL & 9.97 & 0.79 \\
\hline $\mathrm{V}^{*} \mathrm{RV}$ Oct & 134631.95 & -842406.5 & HIP & -1.71 & F98 & 136 & 1 & F98 & 10.98 & $\cdots$ \\
\hline HD 120183 & 134712.60 & +331826.0 & HIP & -0.65 & BSL & -27 & 10 & BSL & 8.75 & 0.91 \\
\hline BPS CS 22889-0064 & 134724.95 & -075429.2 & $\mathrm{U}$ & -1.84 & HK & 61 & 10 & BSL & $(14.14)$ & $(0.46)$ \\
\hline BPS CS 22889-0047 & 134728.24 & -09 1539.2 & $\mathrm{U}$ & -2.04 & HK & -11 & 10 & BSL & $(14.49)$ & $(0.51)$ \\
\hline $\mathrm{V}^{*} \mathrm{SS}$ CVn & 134815.94 & +395403.4 & HIP & -1.37 & F98 & -40 & 3 & F98 & 11.84 & $\ldots$ \\
\hline $\mathrm{V}^{*} \mathrm{RX} \mathrm{CVn}$ & 134842.65 & +412306.8 & STN & -1.31 & L & -158 & 28 & L & 12.57 & $\cdots$ \\
\hline $\mathrm{V}^{*} \mathrm{BB}$ Vir & 135140.78 & +062551.5 & $\mathrm{ACT}$ & -1.61 & L & -38 & 13 & L & 11.07 & $\cdots$ \\
\hline HD 120559 & 135140.79 & -572604.7 & HIP & -1.13 & B99 & 13 & 10 & HK & 7.97 & 0.66 \\
\hline HD 121135 & 135333.56 & +024141.4 & HIP & -1.57 & B99 & 126 & 1 & $\mathrm{CY}$ & 9.36 & 0.80 \\
\hline $\mathrm{V}^{*}$ FY Hya & 135335.46 & -293447.2 & GSC & -2.33 & L & 82 & 24 & L & 12.46 & $\cdots$ \\
\hline HD 121261 & 135505.04 & -360818.6 & HIP & -1.52 & ATT & 99 & 1 & CY & 9.25 & 1.24 \\
\hline$V^{*}$ V499 Cen & 135514.76 & -431424.6 & HIP & -1.43 & F98 & 331 & 3 & F98 & 11.12 & $\cdots$ \\
\hline $\mathrm{V}^{*} \mathrm{ST}$ CVn & 135734.07 & +295128.8 & HIP & -1.07 & F98 & -129 & 1 & F98 & 11.37 & $\cdots$ \\
\hline
\end{tabular}


TABLE 1A. (continued)

\begin{tabular}{|c|c|c|c|c|c|c|c|c|c|c|}
\hline $\begin{array}{l}\text { Star } \\
(1)\end{array}$ & $\begin{array}{c}\text { RA (2000.0) } \\
(2)\end{array}$ & DEC & $\begin{array}{c}\text { Source }^{\mathrm{a}} \\
(4)\end{array}$ & $\begin{array}{c}{[\mathrm{Fe} / \mathrm{H}]} \\
(\mathrm{dex}) \\
(5)\end{array}$ & $\begin{array}{c}\text { Source }^{b} \\
(6)\end{array}$ & $\begin{array}{c}\mathrm{RV} \\
(\mathrm{km} / \mathrm{s}) \\
(7)\end{array}$ & $\begin{array}{c}\sigma_{\mathrm{RV}} \\
(\mathrm{km} / \mathrm{s}) \\
(8)\end{array}$ & $\begin{array}{c}\text { Source }^{c} \\
(9)\end{array}$ & $\begin{array}{c}V \\
(\mathrm{mag}) \\
(10)\end{array}$ & $\begin{array}{c}B-V \\
(\mathrm{mag}) \\
(11)\end{array}$ \\
\hline V* UY Boo & 135846.34 & +125706.9 & HIP & -2.56 & F98 & 145 & 2 & F98 & 10.94 & $\cdots$ \\
\hline $\mathrm{V}^{*} \mathrm{RU} \mathrm{CVn}$ & 135933.27 & +313904.1 & STN & -1.37 & $\mathrm{~L}$ & -27 & 21 & $\mathrm{~L}$ & 11.96 & $\ldots$ \\
\hline HD 122196 & 140102.45 & -380302.5 & HIP & -1.89 & B99 & -23 & 10 & HK & 8.73 & 0.46 \\
\hline HD 122563 & 140231.96 & +094110.6 & HIP & -2.62 & B99 & -25 & 1 & CY & 6.18 & 0.85 \\
\hline$V^{*}$ V674 Cen & 140324.11 & -362420.0 & SPM & -1.53 & $\mathrm{~L}$ & 138 & 8 & L & 11.65 & $\cdots$ \\
\hline HD 122956 & 140513.08 & -145125.1 & HIP & -1.75 & B99 & 166 & 1 & $\mathrm{CY}$ & 7.22 & 0.94 \\
\hline $\mathrm{V}^{*} \mathrm{AD}$ Vir & 140537.08 & $-07 \quad 1450.9$ & GSC & -1.15 & L & 134 & 14 & $\mathrm{~L}$ & 13.04 & $\cdots$ \\
\hline $\mathrm{V}^{*} \mathrm{~W} \mathrm{CVn}$ & 140627.99 & +374941.6 & HIP & -1.22 & F98 & 42 & 3 & F98 & 10.55 & $\cdots$ \\
\hline $\mathrm{V}^{*} \mathrm{AL}$ Vir & 141109.05 & -131838.4 & HIP & -0.70 & BSL & 23 & 1 & BSL & 9.68 & 0.45 \\
\hline $\mathrm{BD}+092860$ & 141319.74 & +083640.4 & HIP & -1.67 & ATT & -19 & 1 & $\mathrm{CY}$ & 10.83 & 0.71 \\
\hline HD 124358 & 141321.38 & -120923.9 & HIP & -1.98 & ATT & 325 & 1 & CY & 9.50 & 0.92 \\
\hline BPS CS 22883-0004 & 141419.78 & +093146.5 & $\mathrm{U}$ & -2.04 & HK & -188 & 10 & BSL & 14.22 & 0.43 \\
\hline $\mathrm{V}^{*}$ GS Hya & 141539.56 & -253957.7 & GSC & -1.76 & L & 187 & 10 & L & 12.78 & $\cdots$ \\
\hline HD 124884 & 141546.80 & +124501.4 & $\mathrm{ACT}$ & -0.84 & BSL & 8 & 10 & BSL & 8.81 & 1.59 \\
\hline $\mathrm{V}^{*} \mathrm{CM}$ Boo & 141603.15 & +200336.1 & GSC & -1.48 & $\mathrm{~L}$ & -91 & 48 & L & 12.09 & $\ldots$ \\
\hline $\mathrm{BD}+092870$ & 141629.98 & +082752.9 & HIP & -2.39 & B99 & -121 & 1 & $\mathrm{CY}$ & 9.40 & 1.04 \\
\hline $\mathrm{V}^{*} \mathrm{TV}$ Boo & 141636.58 & +422136.0 & HIP & -2.44 & F98 & -98 & 10 & F98 & 10.97 & $\cdots$ \\
\hline$V^{*}$ XX Vir & 141648.61 & $-0617 \quad 15.2$ & STN & -2.40 & BSL & -55 & 30 & BSL & 12.20 & $\ldots$ \\
\hline BPS CS 22883-0021 & 141754.49 & +085643.9 & U & -0.70 & HK & -131 & 10 & BSL & 14.30 & 0.62 \\
\hline BD+01 2916 & 142145.28 & +004659.3 & HIP & -1.82 & B99 & -13 & 1 & CY & 9.66 & 1.34 \\
\hline BPS CS 22883-0030 & 142201.15 & +110000.0 & $\mathrm{U}$ & -1.84 & HK & 117 & 10 & BSL & 14.51 & 0.40 \\
\hline $\mathrm{BD}+082856$ & 142358.16 & +08 0133.5 & HIP & -2.03 & B99 & 65 & 1 & $\mathrm{CY}$ & 9.99 & 0.98 \\
\hline BPS CS 22883-0037 & 142419.42 & +112926.4 & $\mathrm{U}$ & -2.32 & BSL & -284 & 10 & BSL & 14.73 & 0.55 \\
\hline $\mathrm{V}^{*} \mathrm{Y}$ Oct & 142441.28 & -883701.3 & $\mathrm{U}$ & -1.52 & L & 144 & 24 & L & 12.18 & $\ldots$ \\
\hline HD 126238 & 142529.94 & -433836.0 & HIP & -1.65 & B99 & 247 & 5 & $\mathrm{CY}$ & 7.66 & 0.79 \\
\hline HD 126587 & 142700.37 & -221438.5 & HIP & -2.62 & B99 & 149 & 1 & $\mathrm{CY}$ & 9.11 & 0.77 \\
\hline $\mathrm{V}^{*} \mathrm{AE}$ Vir & 142729.38 & +034641.0 & NPM & -1.16 & L & 208 & 10 & L & 13.26 & $\cdots$ \\
\hline $\mathrm{V}^{*} \mathrm{RY} \mathrm{Lib}$ & 142730.03 & -213127.4 & GSC & -1.48 & $\mathrm{~L}$ & 33 & 11 & $\mathrm{~L}$ & 13.15 & $\cdots$ \\
\hline $\mathrm{V}^{*} \mathrm{SW}$ Boo & 142734.98 & +360244.1 & GSC & -1.12 & $\mathrm{~L}$ & -18 & 18 & $\mathrm{~L}$ & 12.34 & $\cdots$ \\
\hline $\mathrm{V}^{*} \mathrm{ST}$ Vir & 142739.08 & -005405.6 & HIP & -0.67 & F98 & 1 & 4 & F98 & 11.52 & $\cdots$ \\
\hline $\mathrm{V}^{*}$ AF Vir & 142809.85 & +06 3243.9 & HIP & -1.33 & F98 & -20 & 8 & F98 & 11.80 & $\cdots$ \\
\hline $\mathrm{BD}+182890$ & 143213.49 & +172525.2 & HIP & -1.61 & $\mathrm{C} 97$ & -23 & 5 & $\mathrm{CY}$ & 9.84 & 0.82 \\
\hline $\mathrm{V}^{*} \mathrm{KS}$ Hya & 143243.87 & -264540.9 & $\mathrm{STN}$ & -1.61 & BSL & -36 & 10 & BSL & 13.49 & 0.46 \\
\hline BPS CS 22874-0018 & 143302.86 & -245023.6 & $\mathrm{U}$ & -0.86 & HK & 7 & 10 & BSL & $(13.61)$ & $(0.49)$ \\
\hline $\mathrm{V}^{*} \mathrm{RS}$ Boo & 143333.21 & +314516.6 & HIP & -0.36 & F98 & -7 & 1 & F98 & 10.37 & $\cdots$ \\
\hline $\mathrm{V}^{*} \mathrm{SV}$ Boo & 143406.36 & +390632.9 & GSC & -1.55 & $\mathrm{~L}$ & -131 & 22 & L & 13.12 & $\cdots$ \\
\hline BPS CS 22871-0012 & 143449.42 & -194214.5 & $\mathrm{U}$ & -1.68 & HK & 363 & 10 & BSL & 15.09 & 0.41 \\
\hline HD 128188 & 143546.83 & -112412.2 & HIP & -1.37 & B99 & -37 & 10 & HK & 10.02 & 0.99 \\
\hline BPS CS 22874-0038 & 143601.15 & -243909.3 & $\mathrm{U}$ & -2.41 & HK & 30 & 10 & BSL & $(13.21)$ & $(0.79)$ \\
\hline BPS CS 22874-0049 & 143648.23 & -255825.4 & $\mathrm{U}$ & -2.06 & HK & 78 & 10 & BSL & $(14.19)$ & $(0.61)$ \\
\hline
\end{tabular}


TABLE 1A. (continued)

\begin{tabular}{|c|c|c|c|c|c|c|c|c|c|c|}
\hline $\begin{array}{l}\text { Star } \\
(1)\end{array}$ & $\begin{array}{c}\text { RA (2000.0) } \\
(2)\end{array}$ & $\begin{array}{c}\text { DEC } \\
(3)\end{array}$ & $\begin{array}{c}\text { Source }^{\mathrm{a}} \\
(4)\end{array}$ & $\begin{array}{c}{[\mathrm{Fe} / \mathrm{H}]} \\
(\mathrm{dex}) \\
(5)\end{array}$ & $\begin{array}{c}\text { Source }^{b} \\
(6)\end{array}$ & $\begin{array}{c}\mathrm{RV} \\
(\mathrm{km} / \mathrm{s}) \\
(7)\end{array}$ & $\begin{array}{c}\sigma_{\mathrm{RV}} \\
(\mathrm{km} / \mathrm{s}) \\
(8)\end{array}$ & $\begin{array}{c}\text { Source }^{\mathrm{c}} \\
(9)\end{array}$ & $\begin{array}{c}V \\
(\mathrm{mag}) \\
(10)\end{array}$ & $\begin{array}{c}B-V \\
(\mathrm{mag}) \\
(11)\end{array}$ \\
\hline HD 128279 & 143648.47 & -290643.6 & HIP & -2.20 & B99 & -82 & 4 & $\mathrm{CY}$ & 8.02 & 0.64 \\
\hline $\mathrm{V}^{*}$ DO Vir & 143846.10 & -051931.3 & NPM & -0.80 & L & 24 & 36 & L & 14.14 & $\ldots$ \\
\hline BPS CS 22871-0035 & 143937.53 & -195306.8 & $\mathrm{U}$ & -1.26 & HK & -62 & 10 & BSL & $(14.35)$ & $(0.75)$ \\
\hline BPS CS 22871-0070 & 144116.34 & -183641.1 & $\mathrm{U}$ & -1.77 & HK & -250 & 10 & BSL & 14.76 & 0.45 \\
\hline BPS CS 22871-0060 & 144143.20 & -205025.7 & STN & -0.69 & HK & 23 & 10 & BSL & 14.17 & 0.40 \\
\hline $\mathrm{V}^{*} \mathrm{SZ} \mathrm{Boo}$ & 144213.38 & +281223.3 & GSC & -1.68 & $\mathrm{~L}$ & -38 & 21 & $\mathrm{~L}$ & 12.60 & $\ldots$ \\
\hline BPS CS 22871-0095 & 144445.63 & -213020.3 & $\mathrm{U}$ & -1.97 & HK & 120 & 10 & BSL & $(14.73)$ & $(0.47)$ \\
\hline $\mathrm{V}^{*} \mathrm{TW}$ Boo & 144505.95 & +410144.6 & HIP & -1.46 & F98 & -99 & 1 & F98 & 11.29 & s \\
\hline BPS CS 22874-0091 & 144515.99 & $-24 \quad 4253.7$ & $\mathrm{U}$ & -1.33 & HK & 78 & 10 & BSL & $(14.71)$ & $(0.59)$ \\
\hline $\mathrm{V}^{*} \mathrm{RU}$ Boo & 144600.31 & +231844.3 & GSC & -1.50 & $\mathrm{~L}$ & -60 & 35 & L & 13.60 & $\ldots$ \\
\hline BPS CS 22874-0094 & 144608.33 & -245856.7 & $\mathrm{U}$ & -2.30 & HK & 57 & 10 & BSL & (13.93) & $(0.57)$ \\
\hline $\mathrm{V}^{*}$ AE Boo & 144735.28 & +165043.9 & HIP & -1.39 & F98 & 79 & 1 & F98 & 10.65 & $\ldots$ \\
\hline $\mathrm{V}^{*} \mathrm{TY}$ Aps & 144850.08 & $-71 \quad 1942.0$ & HIP & -0.95 & F98 & 88 & 5 & F98 & 11.85 & $\ldots$ \\
\hline BPS CS 22874-0123 & 144857.75 & -242115.0 & $\mathrm{U}$ & -2.36 & HK & 95 & 10 & BSL & 14.70 & 0.45 \\
\hline BPS CS 22871-0105 & 144920.78 & -205317.3 & $\mathrm{U}$ & -0.94 & BSL & 223 & 10 & BSL & {$[15.60]$} & $\ldots$ \\
\hline BPS CS 22874-0119 & 145038.23 & -252253.2 & $\mathrm{U}$ & -0.64 & HK & -31 & 10 & BSL & $(14.25)$ & $(0.75)$ \\
\hline $\mathrm{V}^{*} \mathrm{BT}$ Dra & 145144.64 & +600409.3 & HIP & -1.75 & F98 & -164 & 2 & F98 & 11.64 & $\ldots$ \\
\hline $\mathrm{V}^{*} \mathrm{XZ}$ Aps & 145205.48 & -794046.7 & HIP & -1.06 & F98 & 202 & 1 & F98 & 12.38 & $\ldots$ \\
\hline BPS CS 22871-0102 & 145230.52 & -210049.3 & $\mathrm{U}$ & -1.71 & BSL & 38 & 10 & BSL & 13.37 & 0.32 \\
\hline $\mathrm{V}^{*} \mathrm{UY}$ Aps & 145936.95 & -714749.9 & $\mathrm{U}$ & -0.68 & L & 130 & 39 & L & 13.55 & $\cdots$ \\
\hline HD 132475 & 145950.12 & -220041.4 & HIP & -1.70 & B99 & 174 & 10 & HK & 8.55 & 0.51 \\
\hline BD-11 3853 & 150031.02 & -122656.3 & HIP & -0.81 & $\mathrm{C} 97$ & 18 & 10 & BSL & 10.26 & 0.53 \\
\hline HD 133461 & 150410.79 & +144529.1 & HIP & -0.61 & BSL & 45 & 10 & BSL & 8.95 & 1.16 \\
\hline BD-08 3901 & 150451.25 & -084856.5 & HIP & -1.43 & BSL & -109 & 10 & BSL & 9.47 & 0.91 \\
\hline $\mathrm{BD}+302611$ & 150653.82 & +300037.0 & HIP & -1.32 & B99 & -278 & 1 & $\mathrm{CY}$ & 9.13 & 1.25 \\
\hline $\mathrm{V}^{*} \mathrm{XX} \mathrm{Lib}$ & $1507 \quad 10.06$ & -255951.3 & GSC & -1.47 & L & 92 & 10 & L & 12.18 & $\ldots$ \\
\hline $\mathrm{BD}+182976$ & 150724.30 & +172658.6 & HIP & -2.42 & ATT & -173 & 2 & $\mathrm{CY}$ & 9.82 & 1.05 \\
\hline HD 135148 & $1513 \quad 17.47$ & +122725.5 & HIP & -1.90 & B99 & -92 & 4 & $\mathrm{CY}$ & 9.49 & 1.39 \\
\hline BPS CS 22890-0011 & 151321.25 & +021501.0 & $\mathrm{U}$ & -2.32 & HK & -121 & 10 & BSL & 14.61 & 0.38 \\
\hline $\mathrm{V}^{*}$ AP Ser & 151400.95 & +095852.2 & HIP & -1.58 & F98 & -88 & 2 & F98 & 11.11 & $\cdots$ \\
\hline $\mathrm{V}^{*} \mathrm{BH}$ Ser & 151501.09 & +192635.3 & GSC & -1.59 & $\mathrm{~L}$ & -113 & 11 & $\mathrm{~L}$ & 12.85 & $\ldots$ \\
\hline$V^{*}$ DF Ser & 151520.34 & +183920.9 & GSC & -0.74 & $\mathrm{~L}$ & -10 & 14 & $\mathrm{~L}$ & 12.69 & $\ldots$ \\
\hline BPS CS 22890-0024 & 151605.45 & -000356.4 & $\mathrm{U}$ & -2.30 & HK & 226 & 10 & BSL & 13.41 & 0.57 \\
\hline HD 135449 & $15 \quad 16 \quad 10.48$ & -325332.7 & HIP & -1.47 & ATT & -23 & 12 & $\mathrm{CY}$ & 9.44 & 0.36 \\
\hline $\mathrm{V}^{*} \mathrm{BF}$ Ser & 151628.51 & +162639.8 & HIP & -2.50 & BSL & -155 & 1 & BSL & $\ldots$ & $\ldots$ \\
\hline $\mathrm{V}^{*} \mathrm{UU}$ Boo & 151705.32 & +350657.7 & GSC & -1.92 & $\mathrm{~L}$ & 10 & 28 & L & 12.22 & $\ldots$ \\
\hline $\mathrm{BD}+172844$ & $15 \quad 17 \quad 06.17$ & +170400.0 & HIP & -0.64 & BSL & -12 & 10 & BSL & 8.41 & 1.54 \\
\hline BPS CS 22890-0064 & $1517 \quad 34.04$ & +024528.4 & $\mathrm{U}$ & -2.60 & HK & -51 & 10 & BSL & 14.70 & 0.39 \\
\hline $\mathrm{V}^{*} \mathrm{TV}$ Lib & 151821.90 & -082743.0 & GSC & -0.27 & $\mathrm{~L}$ & -61 & 10 & L & 11.94 & $\ldots$ \\
\hline BPS CS 22890-0029 & 151823.03 & -004501.6 & $\mathrm{U}$ & -1.34 & HK & 111 & 10 & BSL & $(13.48)$ & $(0.42)$ \\
\hline
\end{tabular}


TABLE 1A. (continued)

\begin{tabular}{|c|c|c|c|c|c|c|c|c|c|c|}
\hline $\begin{array}{l}\text { Star } \\
(1)\end{array}$ & $\begin{array}{c}\text { RA (2000.0) } \\
(2)\end{array}$ & DEC & $\begin{array}{c}\text { Source }^{\mathrm{a}} \\
(4)\end{array}$ & $\begin{array}{c}{[\mathrm{Fe} / \mathrm{H}]} \\
(\mathrm{dex}) \\
(5)\end{array}$ & $\begin{array}{c}\text { Source }^{b} \\
(6)\end{array}$ & $\begin{array}{c}\mathrm{RV} \\
(\mathrm{km} / \mathrm{s}) \\
(7)\end{array}$ & $\begin{array}{c}\sigma_{\mathrm{RV}} \\
(\mathrm{km} / \mathrm{s}) \\
(8)\end{array}$ & $\begin{array}{c}\text { Source }^{c} \\
(9)\end{array}$ & $\begin{array}{c}V \\
(\mathrm{mag}) \\
(10)\end{array}$ & $\begin{array}{c}B-V \\
(\operatorname{mag}) \\
(11)\end{array}$ \\
\hline $\mathrm{V}^{*}$ AB Aps & 151930.42 & -784101.5 & $\mathrm{U}$ & -1.30 & L & 44 & 15 & L & 13.20 & $\cdots$ \\
\hline BPS CS 22890-0063 & 151944.92 & +023600.6 & GSC & -2.17 & HK & 14 & 10 & BSL & 13.84 & 0.40 \\
\hline HD 136316 & 152217.97 & -531414.6 & HIP & -1.92 & B99 & -45 & 12 & CY & 7.60 & 1.12 \\
\hline $\mathrm{V}^{*} \mathrm{TV} \mathrm{CrB}$ & 152221.44 & +265237.7 & HIP & -2.33 & F98 & -126 & 7 & F98 & 11.87 & $\ldots$ \\
\hline $\mathrm{V}^{*} \mathrm{FW}$ Lup & 152225.38 & -405536.1 & HIP & -0.20 & F98 & 3 & 3 & F98 & 9.06 & $\cdots$ \\
\hline $\mathrm{BD}+013070$ & 152240.08 & +011552.9 & HIP & -1.85 & ATT & -329 & 3 & CY & 10.06 & 0.77 \\
\hline BPS CS 22890-0095 & 152755.20 & +022500.7 & $\mathrm{U}$ & -1.80 & HK & -127 & 10 & BSL & 14.20 & 0.46 \\
\hline $\mathrm{V}^{*}$ CS Ser & 152836.34 & +030523.6 & STN & -1.57 & $\mathrm{~L}$ & 2 & 14 & L & 12.39 & $\ldots$ \\
\hline $\mathrm{V}^{*} \mathrm{ST}$ Boo & 153039.24 & +354704.4 & HIP & -1.76 & F98 & 13 & 1 & F98 & 11.01 & $\cdots$ \\
\hline V* VY Ser & 153101.99 & +014101.8 & HIP & -1.79 & F98 & -146 & 1 & F98 & 10.13 & $\cdots$ \\
\hline BPS CS 22884-0005 & 153244.01 & -115102.7 & $\mathrm{U}$ & -1.57 & HK & 79 & 10 & BSL & 13.75 & 0.67 \\
\hline BPS CS 30312-0006 & 153257.77 & -015321.5 & $\mathrm{U}$ & -1.87 & HK & -363 & 10 & BSL & 13.65 & 0.62 \\
\hline BPS CS 30312-0002 & 153259.06 & -022038.6 & U & -1.02 & HK & 49 & 10 & BSL & $(14.17)$ & $(0.63)$ \\
\hline $\mathrm{V}^{*} \mathrm{AR}$ Ser & 153330.83 & +024637.5 & STN & -1.78 & L & 132 & 4 & L & 11.85 & $\ldots$ \\
\hline CD-33 10593 & 153416.23 & -335625.3 & HIP & -0.85 & BSL & 22 & 10 & BSL & 9.55 & 0.50 \\
\hline BPS CS 30312-0059 & 153448.92 & -012336.8 & $\mathrm{U}$ & -2.71 & HK & -153 & 10 & BSL & 13.14 & 0.71 \\
\hline $\mathrm{V}^{*} \mathrm{CG} \mathrm{Lib}$ & 153516.84 & -242012.3 & HIP & -1.19 & F98 & -179 & 10 & F98 & 11.55 & $\cdots$ \\
\hline BPS CS 30312-0067 & 153536.07 & $-02 \quad 12 \quad 11.5$ & $\mathrm{U}$ & -2.26 & HK & 237 & 10 & BSL & $(14.46)$ & $(0.64)$ \\
\hline BPS CS 22884-0033 & 153537.46 & -101506.9 & $\mathrm{U}$ & -2.79 & HK & -117 & 10 & BSL & 14.52 & 0.50 \\
\hline BPS CS 30312-0044 & 153707.59 & +00 3253.9 & $\mathrm{U}$ & -2.59 & HK & 94 & 10 & BSL & 14.00 & 0.70 \\
\hline BPS CS 30312-0062 & 153732.86 & -014353.5 & STN & -2.63 & BSL & 52 & 10 & BSL & 12.58 & 0.33 \\
\hline BPS CS 22884-0065 & 153929.20 & -075124.9 & $\mathrm{U}$ & -1.55 & HK & -34 & 10 & BSL & 13.83 & 0.48 \\
\hline HD 140283 & 154303.76 & -105557.9 & HIP & -2.47 & B99 & -172 & 10 & HK & 7.20 & 0.48 \\
\hline BPS CS 30312-0100 & 154331.61 & +020118.5 & $\mathrm{U}$ & -2.34 & HK & -124 & 10 & BSL & 13.05 & 0.65 \\
\hline $\mathrm{V}^{*}$ AR Aps & 154418.70 & $-72 \quad 2518.7$ & $\mathrm{U}$ & -1.77 & $\mathrm{~L}$ & 104 & 39 & L & 13.83 & $\cdots$ \\
\hline BPS CS 22884-0097 & 154722.94 & $\begin{array}{lll}-11 & 07 & 02.7\end{array}$ & $\mathrm{U}$ & -2.45 & HK & -100 & 10 & BSL & 14.86 & 0.60 \\
\hline HD 141531 & 154916.51 & +093642.9 & HIP & -1.57 & ATT & 3 & 1 & $\mathrm{CY}$ & 9.13 & 1.24 \\
\hline BPS CS 22884-0108 & 154957.63 & -091408.8 & U & -3.10 & B99 & -27 & 10 & HK & 14.24 & 0.50 \\
\hline V* V765 Sco & 155054.91 & -245150.3 & GSC & -1.18 & $\mathrm{~L}$ & 29 & 10 & $\mathrm{~L}$ & 13.36 & $\cdots$ \\
\hline V* VY Lib & 155116.99 & $-15 \quad 4502.9$ & HIP & -1.34 & F98 & 151 & 6 & F98 & 11.73 & $\cdots$ \\
\hline $\mathrm{BD}+053098$ & 155217.28 & +051744.8 & HIP & -2.40 & ATT & -161 & 1 & $\mathrm{CY}$ & 10.53 & 0.78 \\
\hline$V^{*}$ AN Ser & 155331.05 & +125740.2 & HIP & -0.07 & F98 & -47 & 1 & F98 & 10.94 & $\ldots$ \\
\hline$V^{*}$ AT Ser & 155540.37 & +075919.7 & HIP & -2.03 & F98 & -67 & 3 & F98 & 11.48 & $\cdots$ \\
\hline HD 141864 & 155906.02 & -763704.0 & HIP & -2.27 & BSL & 311 & 10 & BSL & 8.99 & 0.66 \\
\hline $\mathrm{V}^{*} \mathrm{VX}$ Aps & 155935.37 & $\begin{array}{lll}-75 & 12 & 25.0\end{array}$ & GSC & -1.44 & L & 327 & 26 & L & 12.09 & $\cdots$ \\
\hline HD 142948 & 160002.43 & $\begin{array}{lll}-53 & 51 & 05.1\end{array}$ & HIP & -0.89 & B99 & 25 & 10 & HK & 8.02 & 0.97 \\
\hline $\mathrm{V}^{*}$ AR Her & 160032.28 & +465525.6 & HIP & -1.30 & F98 & -350 & 10 & F98 & 11.24 & $\cdots$ \\
\hline V* AV Ser & 160120.75 & +004425.1 & $\mathrm{v}$ & -1.20 & $\mathrm{~L}$ & -45 & 13 & $\mathrm{~L}$ & 11.40 & $\cdots$ \\
\hline$V^{*}$ AW Ser & 160628.81 & +152205.7 & STN & -1.67 & $\mathrm{~L}$ & -126 & 15 & L & 12.79 & $\cdots$ \\
\hline HD 145293 & 161044.06 & -133046.4 & HIP & -1.10 & BSL & 74 & 10 & BSL & 10.02 & 0.67 \\
\hline
\end{tabular}


TABLE 1A. (continued)

\begin{tabular}{|c|c|c|c|c|c|c|c|c|c|c|}
\hline Star & $\begin{array}{c}\text { RA (2000.0) } \\
(2)\end{array}$ & DEC & $\begin{array}{c}\text { Source }^{\mathrm{a}} \\
(4)\end{array}$ & $\begin{array}{c}{[\mathrm{Fe} / \mathrm{H}]} \\
(\mathrm{dex}) \\
(5)\end{array}$ & $\begin{array}{c}\text { Source }^{b} \\
(6)\end{array}$ & $\begin{array}{c}\mathrm{RV} \\
(\mathrm{km} / \mathrm{s}) \\
(7)\end{array}$ & $\begin{array}{c}\sigma_{\mathrm{RV}} \\
(\mathrm{km} / \mathrm{s}) \\
(8)\end{array}$ & $\begin{array}{c}\text { Source }{ }^{\mathrm{c}} \\
(9)\end{array}$ & $\begin{array}{c}V \\
(\mathrm{mag}) \\
(10)\end{array}$ & $\begin{array}{c}B-V \\
(\mathrm{mag}) \\
(11)\end{array}$ \\
\hline BPS CS 22872-0027 & 161853.12 & -020308.4 & $\mathrm{U}$ & -2.14 & HK & -138 & 10 & BSL & 13.82 & 0.74 \\
\hline $\mathrm{V}^{*} \mathrm{RV} \mathrm{CrB}$ & 161925.87 & +294247.9 & HIP & -1.69 & F98 & -125 & 5 & F98 & 11.41 & $\cdots$ \\
\hline $\mathrm{V}^{*}$ BS Aps & 162051.48 & -714015.7 & STN & -1.33 & $\mathrm{~L}$ & -82 & 18 & $\mathrm{~L}$ & 12.38 & $\ldots$ \\
\hline $\mathrm{V}^{*} \mathrm{~V} 1023 \mathrm{Oph}$ & 162147.24 & -040553.9 & STN & -1.21 & $\mathrm{~L}$ & -29 & 10 & $\mathrm{~L}$ & 12.88 & $\cdots$ \\
\hline BPS CS 22872-0036 & 162245.01 & -022351.4 & U & -2.61 & HK & -4 & 10 & BSL & $(15.03)$ & $(0.57)$ \\
\hline BPS CS 22872-0054 & 162249.96 & -045437.0 & $\mathrm{U}$ & -1.03 & HK & -50 & 10 & BSL & $(13.76)$ & $(0.64)$ \\
\hline V* V445 Oph & 162441.22 & -063230.0 & HIP & -0.19 & F98 & -20 & 1 & F98 & 11.05 & $\cdots$ \\
\hline $\mathrm{V}^{*} \mathrm{~V} 413 \mathrm{Oph}$ & 162511.12 & -103125.2 & GSC & -1.00 & L & -39 & 30 & $\mathrm{~L}$ & 12.08 & $\cdots$ \\
\hline BPS CS 22872-0079 & 162611.49 & -014639.8 & U & -2.52 & HK & 134 & 10 & BSL & 15.00 & 0.49 \\
\hline BPS CS 22872-0102 & 162920.76 & -030452.0 & $\mathrm{U}$ & -2.61 & HK & -42 & 10 & BSL & 13.65 & 0.59 \\
\hline BD+11 2998 & 163016.85 & +105952.2 & HIP & -1.14 & C97 & 50 & 1 & $\mathrm{CY}$ & 9.06 & 0.67 \\
\hline $\mathrm{V}^{*} \mathrm{VX}$ Her & 163040.83 & +182200.4 & HIP & -1.58 & F98 & -377 & 3 & F98 & 10.69 & $\cdots$ \\
\hline V* V716 Oph & 163049.47 & -053019.9 & STN & -1.55 & BSL & -230 & 30 & BSL & 12.50 & $\cdots$ \\
\hline BPS CS 22878-0017 & 163117.49 & +110051.6 & $\mathrm{U}$ & -0.94 & HK & -144 & 10 & BSL & 14.87 & 0.47 \\
\hline BPS CS 22878-0013 & 163156.21 & +090858.2 & STN & -2.16 & HK & 19 & 10 & BSL & 13.27 & 0.47 \\
\hline $\mathrm{V}^{*} \mathrm{UV}$ Oct & 163225.85 & -835409.4 & HIP & -1.74 & F98 & 116 & 6 & F98 & 9.50 & $\cdots$ \\
\hline BD+09 3223 & 163335.60 & +090617.2 & HIP & -2.41 & ATT & 67 & 1 & $\mathrm{CY}$ & 9.25 & 0.62 \\
\hline BPS CS 22878-0003 & 163345.47 & +075523.7 & $\mathrm{U}$ & -1.66 & HK & -73 & 10 & BSL & 14.42 & 0.46 \\
\hline BPS CS 22878-0002 & 163349.17 & +075340.7 & $\mathrm{U}$ & -2.16 & HK & -203 & 10 & BSL & 14.36 & 0.45 \\
\hline V* RW Dra & 163531.61 & +575023.2 & HIP & -1.55 & F98 & -112 & 1 & F98 & 11.71 & $\cdots$ \\
\hline BPS CS 22878-0027 & 163735.86 & +102209.8 & $\mathrm{U}$ & -2.60 & HK & -116 & 10 & BSL & 14.41 & 0.44 \\
\hline $\mathrm{V}^{*}$ GY Her & 163818.09 & +374804.6 & STN & -1.92 & L & -157 & 53 & L & 12.57 & $\cdots$ \\
\hline BPS CS 22878-0073 & 163830.72 & +114351.3 & $\mathrm{U}$ & -2.06 & HK & -254 & 10 & BSL & 14.62 & 0.44 \\
\hline $\mathrm{V}^{*}$ AF Her & 163938.28 & +410645.1 & NPM & -1.94 & L & -268 & 9 & $\mathrm{~L}$ & 12.82 & $\cdots$ \\
\hline BPS CS 22878-0076 & 163948.48 & +114341.0 & $\mathrm{U}$ & -1.78 & HK & -214 & 10 & BSL & 14.74 & 0.55 \\
\hline $\mathrm{V}^{*}$ AG Her & 164032.85 & +403706.0 & STN & -2.01 & L & -103 & 21 & L & 12.66 & $\cdots$ \\
\hline $\mathrm{V}^{*}$ CK Aps & 164206.43 & -741302.4 & U & -1.49 & L & 91 & 24 & L & 12.81 & $\cdots$ \\
\hline BPS CS 22878-0101 & 164531.50 & +081445.2 & $\mathrm{U}$ & -3.13 & B99 & -135 & 10 & HK & 13.79 & 0.80 \\
\hline BPS CS 22878-0121 & 164750.06 & +113912.2 & $\mathrm{U}$ & -1.97 & HK & -377 & 10 & BSL & 13.99 & 0.54 \\
\hline BPS CS 22878-0116 & $1648 \quad 09.87$ & +103103.3 & $\mathrm{U}$ & -1.48 & HK & -296 & 10 & BSL & 14.65 & 0.49 \\
\hline $\mathrm{V}^{*} \mathrm{CW}$ Her & 165038.27 & +352704.8 & STN & -2.09 & $\mathrm{~L}$ & -285 & 30 & $\mathrm{~L}$ & 12.47 & $\cdots$ \\
\hline $\mathrm{V}^{*} \mathrm{SW}$ Her & 165827.64 & +213251.2 & NPM & -1.50 & L & -130 & 35 & $\mathrm{~L}$ & 14.14 & $\cdots$ \\
\hline $\mathrm{V}^{*} \mathrm{RW} \operatorname{Tr} \mathrm{A}$ & 170046.70 & -663949.9 & HIP & -0.13 & F98 & -56 & 17 & F98 & 11.40 & $\cdots$ \\
\hline $\mathrm{V}^{*} \mathrm{DD}$ Aps & 170353.58 & -743649.2 & $\mathrm{U}$ & -1.06 & L & -17 & 30 & L & 12.72 & $\cdots$ \\
\hline $\mathrm{V}^{*} \mathrm{DI}$ Aps & 170948.58 & -732541.9 & $\mathrm{U}$ & -1.60 & $\mathrm{~L}$ & 70 & 11 & $\mathrm{~L}$ & 12.98 & $\cdots$ \\
\hline $\mathrm{V}^{*} \mathrm{VZ}$ Her & 171304.02 & +355843.2 & HIP & -1.02 & F98 & -115 & 1 & F98 & 11.48 & $\cdots$ \\
\hline $\mathrm{V}^{*} \mathrm{~V} 452 \mathrm{Oph}$ & 171759.68 & +110427.6 & GSC & -1.72 & L & -375 & 30 & L & 12.18 & $\cdots$ \\
\hline $\mathrm{V}^{*} \mathrm{DL}$ Her & 172031.38 & +142856.8 & U & -1.32 & $\mathrm{~L}$ & -61 & 14 & L & 12.37 & $\ldots$ \\
\hline$V^{*}$ V394 Her & 172227.75 & +175328.9 & $\mathrm{U}$ & -1.48 & $\mathrm{~L}$ & -74 & 10 & L & 12.87 & $\cdots$ \\
\hline $\mathrm{BD}+173248$ & 172814.50 & +173036.0 & HIP & -2.03 & B99 & -146 & 1 & $\mathrm{CY}$ & 9.34 & 0.67 \\
\hline
\end{tabular}


TABLE 1A. (continued)

\begin{tabular}{|c|c|c|c|c|c|c|c|c|c|c|c|}
\hline Star & $\begin{array}{c}\text { RA (2000.0) } \\
(2)\end{array}$ & DEC & $\begin{array}{c}\text { Source }^{\mathrm{a}} \\
(4)\end{array}$ & $\begin{array}{c}{[\mathrm{Fe} / \mathrm{H}]} \\
(\mathrm{dex}) \\
(5)\end{array}$ & $\begin{array}{c}\text { Source }^{b} \\
(6)\end{array}$ & $\begin{array}{c}\mathrm{RV} \\
(\mathrm{km} / \mathrm{s}) \\
(7)\end{array}$ & $\begin{array}{c}\sigma_{\mathrm{RV}} \\
(\mathrm{km} / \mathrm{s}) \\
(8)\end{array}$ & $\begin{array}{c}\text { Source }{ }^{\mathrm{c}} \\
(9)\end{array}$ & $\begin{array}{c}V \\
(\mathrm{mag}) \\
(10)\end{array}$ & $\begin{array}{c}B-V \\
(\mathrm{mag}) \\
(11)\end{array}$ & $\begin{array}{r}\text { Sourc } \\
\text { (12) }\end{array}$ \\
\hline V* ST Oph & 173359.38 & -010451.6 & STN & -1.30 & $\mathrm{~L}$ & 12 & 7 & $\mathrm{~L}$ & 12.05 & $\cdots$ & \\
\hline$V^{*}$ V784 Oph & 173525.23 & +074521.4 & GSC & -1.80 & $\mathrm{~L}$ & -167 & 10 & $\mathrm{~L}$ & 12.48 & $\ldots$ & \\
\hline $\mathrm{V}^{*} \mathrm{EL} \mathrm{Aps}$ & 173541.24 & $\begin{array}{lll}-76 & 13 & 16.0\end{array}$ & STN & -1.56 & $\mathrm{~L}$ & 69 & 10 & $\mathrm{~L}$ & 12.90 & $\ldots$ & \\
\hline$V^{*}$ V494 Sco & 174047.36 & -313243.3 & STN & -1.01 & $\mathrm{~L}$ & 26 & 30 & L & 11.27 & $\ldots$ & \\
\hline $\mathrm{V}^{*} \mathrm{CE}$ Her & 174156.54 & +150431.3 & GSC & -1.80 & BSL & -258 & 30 & BSL & 11.90 & $\cdots$ & \\
\hline V* V816 Oph & 174237.67 & +045729.8 & STN & -0.96 & L & -28 & 10 & L & 12.48 & $\ldots$ & \\
\hline HD 160617 & 174249.37 & -401912.1 & HIP & -1.78 & B99 & 95 & 10 & HK & 8.72 & 0.46 & $\mathrm{H}$ \\
\hline $\mathrm{V}^{*} \mathrm{~V} 554 \mathrm{Oph}$ & 174251.37 & -213011.7 & GSC & -1.10 & BSL & 110 & 10 & BSL & 13.60 & $\ldots$ & \\
\hline $\mathrm{V}^{*} \mathrm{LU}$ Aps & 174310.52 & -702345.6 & STN & -2.06 & L & 122 & 33 & $\mathrm{~L}$ & 12.99 & $\cdots$ & \\
\hline $\mathrm{V}^{*}$ ER Aps & 174359.06 & -761440.9 & GSC & -1.39 & $\mathrm{~L}$ & 302 & 35 & $\mathrm{~L}$ & 13.38 & $\cdots$ & \\
\hline PSD 157-558 & 174525.81 & -285627.6 & GSC & -0.95 & BSL & -44 & 10 & BSL & 10.10 & $\ldots$ & \\
\hline $\mathrm{V}^{*} \mathrm{TX}$ Pav & 174745.27 & -600503.3 & GSC & -0.37 & L & -71 & 10 & $\mathrm{~L}$ & 12.89 & $\ldots$ & \\
\hline HD 161770 & 174746.16 & -093616.6 & HIP & -2.12 & B99 & -129 & 10 & HK & 9.68 & 0.67 & $\mathrm{H}$ \\
\hline $\mathrm{V}^{*}$ TY Pav & 174842.97 & -623558.3 & GSC & -2.31 & $\mathrm{~L}$ & 245 & 9 & L & 12.58 & $\ldots$ & \\
\hline $\mathrm{V}^{*} \mathrm{CQ}$ Sco & 174952.59 & -401940.5 & $\mathrm{U}$ & -1.40 & BSL & -73 & 10 & BSL & 13.90 & $\cdots$ & B \\
\hline V* V709 Sco & 175101.44 & -345041.8 & $\mathrm{U}$ & -0.60 & BSL & 41 & 10 & BSL & 13.60 & $\ldots$ & $\mathrm{B}$ \\
\hline $\mathrm{V}^{*} \mathrm{EX}$ Aps & 175148.96 & -691937.7 & $\mathrm{U}$ & -0.81 & L & -59 & 32 & L & 12.27 & $\cdots$ & \\
\hline CD-68 1881 & 175235.88 & -690145.0 & STN & -1.81 & BSL & 134 & 10 & BSL & 9.59 & 1.04 & B \\
\hline $\mathrm{V}^{*} \mathrm{TW}$ Her & 175431.20 & +302437.7 & HIP & -0.69 & F98 & -5 & 1 & F98 & 11.28 & $\cdots$ & $\mathrm{F}$ \\
\hline $\mathrm{V}^{*}$ WY Pav & 175612.39 & -570943.1 & HIP & -0.98 & F98 & -76 & 14 & F98 & 12.18 & $\cdots$ & $\mathrm{F}$ \\
\hline $\mathrm{V}^{*} \mathrm{MR}$ Ara & 175641.02 & -454624.6 & STN & -1.00 & BSL & -68 & 10 & BSL & 12.30 & $\cdots$ & SB \\
\hline $\mathrm{V}^{*}$ V690 Sco & 175738.67 & -403326.2 & STN & -1.16 & BSL & -63 & 30 & BSL & 11.60 & $\cdots$ & \\
\hline $\mathrm{V}^{*} \mathrm{~V} 446 \mathrm{Sco}$ & 175821.13 & -361548.3 & $\mathrm{U}$ & -1.00 & BSL & -123 & 10 & BSL & $\ldots$ & $\cdots$ & \\
\hline $\mathrm{V}^{*} \mathrm{~S}$ Ara & 175910.75 & $\begin{array}{lll}-49 & 26 & 00.4\end{array}$ & HIP & -0.71 & F98 & 185 & 10 & F98 & 10.78 & $\cdots$ & $\mathrm{F}$ \\
\hline $\mathrm{V}^{*} \mathrm{KQ} \mathrm{CrA}$ & 175934.47 & -393507.0 & STN & -0.70 & BSL & 14 & 10 & BSL & $\ldots$ & $\cdots$ & \\
\hline $\mathrm{V}^{*} \mathrm{~V} 478$ Oph & 175939.00 & +004709.2 & $\mathrm{U}$ & -0.70 & BSL & -302 & 10 & BSL & 13.00 & $\cdots$ & B \\
\hline $\mathrm{V}^{*} \mathrm{MS}$ Ara & 180304.30 & -524320.3 & HIP & -1.48 & F98 & 101 & 14 & F98 & 12.07 & $\cdots$ & $F$ \\
\hline $\mathrm{V}^{*}$ V1438 Sgr & 180333.97 & $\begin{array}{lll}-30 & 03 & 00.8\end{array}$ & $\mathrm{v}$ & -0.76 & BSL & 113 & 30 & BSL & 13.60 & $\cdots$ & $B$ \\
\hline HD 165195 & 180440.09 & +034645.4 & HIP & -2.14 & B99 & 0 & 5 & $\mathrm{CY}$ & 7.31 & 1.23 & \\
\hline $\mathrm{V}^{*} \mathrm{WW}$ CrA & 180536.55 & -435146.3 & GSC & -1.38 & $\mathrm{~L}$ & 38 & 14 & $\mathrm{~L}$ & 12.11 & $\cdots$ & \\
\hline $\mathrm{V}^{*}$ IN Ara & 180740.18 & -543628.9 & STN & -1.75 & L & 115 & 33 & L & 12.09 & $\cdots$ & \\
\hline HD 166161 & 180940.74 & -084643.9 & HIP & -1.22 & B99 & 68 & 1 & $\mathrm{CY}$ & 8.12 & 0.88 & $\mathrm{H}$ \\
\hline $\mathrm{V}^{*} \mathrm{~V} 675 \mathrm{Sgr}$ & 181335.39 & -341901.7 & HIP & -2.28 & F98 & -105 & 10 & F98 & 10.33 & $\cdots$ & $\mathrm{F}$ \\
\hline V* BC Dra & 181419.11 & +764108.1 & HIP & -2.00 & F98 & -141 & 2 & F98 & 11.60 & $\cdots$ & $\mathrm{F}$ \\
\hline $\mathrm{V}^{*}$ V455 Oph & $1815 \quad 18.54$ & +122108.7 & HIP & -1.07 & F98 & -32 & 1 & F98 & 12.36 & $\cdots$ & $\mathrm{F}$ \\
\hline HD 166913 & 181625.98 & -592410.1 & HIP & -1.52 & B99 & -54 & 10 & HK & 8.22 & 0.44 & $\mathrm{H}$ \\
\hline $\mathrm{V}^{*} \mathrm{BI}$ Tel & 181817.48 & -532220.1 & GSC & -1.96 & L & 0 & 14 & L & 12.55 & $\cdots$ & \\
\hline $\mathrm{V}^{*}$ BD Dra & 181821.21 & +771743.2 & $\mathrm{v}$ & -1.74 & L & -253 & 30 & L & 12.69 & $\cdots$ & \\
\hline $\mathrm{V}^{*} \mathrm{~V} 1290 \mathrm{Sgr}$ & 182137.31 & -323801.2 & STN & -1.20 & BSL & -15 & 10 & BSL & $\ldots$ & $\cdots$ & \\
\hline $\mathrm{V}^{*} \mathrm{IO} \mathrm{Lyr}$ & 182238.01 & +325732.3 & HIP & -1.14 & F98 & -188 & 2 & F98 & 11.85 & $\cdots$ & $\mathrm{F}$ \\
\hline
\end{tabular}


TABLE 1A. (continued)

\begin{tabular}{|c|c|c|c|c|c|c|c|c|c|c|}
\hline $\begin{array}{l}\text { Star } \\
\text { (1) }\end{array}$ & RA (2000.0) & DEC & $\begin{array}{c}\text { Source }^{\mathrm{a}} \\
(4)\end{array}$ & $\begin{array}{c}{[\mathrm{Fe} / \mathrm{H}]} \\
(\mathrm{dex}) \\
(5)\end{array}$ & $\begin{array}{c}\text { Source }^{b} \\
(6)\end{array}$ & $\begin{array}{c}\mathrm{RV} \\
(\mathrm{km} / \mathrm{s}) \\
(7)\end{array}$ & $\begin{array}{c}\sigma_{\mathrm{RV}} \\
(\mathrm{km} / \mathrm{s}) \\
(8)\end{array}$ & $\begin{array}{c}\text { Source }^{c} \\
(9)\end{array}$ & $\begin{array}{c}V \\
(\mathrm{mag}) \\
(10)\end{array}$ & $\begin{array}{c}B-V \\
(\mathrm{mag}) \\
(11)\end{array}$ \\
\hline V* V1189 Sgr & 182336.07 & -354540.3 & $\mathrm{U}$ & -1.10 & BSL & -48 & 10 & BSL & $\cdots$ & $\cdots$ \\
\hline $\mathrm{V}^{*} \mathrm{~V} 592 \mathrm{CrA}$ & 182620.76 & -445948.5 & GSC & -1.82 & $\mathrm{~L}$ & -228 & 3 & L & 12.16 & $\ldots$ \\
\hline $\mathrm{V}^{*}$ AE Dra & 182706.74 & +552932.7 & GSC & -1.54 & $\mathrm{~L}$ & -243 & 30 & $\mathrm{~L}$ & 12.65 & $\ldots$ \\
\hline$V^{*}$ V1303 Sgr & 182852.81 & -264524.5 & $\mathrm{U}$ & -0.80 & BSL & 132 & 10 & BSL & $\ldots$ & $\cdots$ \\
\hline HD 170737 & 182954.05 & +263926.7 & HIP & -0.91 & ATT & -137 & 10 & BSL & 8.13 & 0.80 \\
\hline $\mathrm{V}^{*}$ AC Her & 183016.24 & +215200.6 & HIP & -1.20 & BSL & 0 & 1 & BSL & 7.63 & 0.63 \\
\hline $\mathrm{V}^{*} \mathrm{KX}$ Lyr & 183322.44 & +401052.5 & $\mathrm{U}$ & -0.46 & L & -50 & 21 & L & 11.00 & $\ldots$ \\
\hline $\mathrm{V}^{*} \mathrm{CC}$ Lyr & 183357.41 & +313824.2 & HIP & -2.20 & BSL & -25 & 1 & BSL & 12.10 & $\cdots$ \\
\hline $\mathrm{V}^{*} \mathrm{BH}$ Pav & 183440.62 & -652702.5 & GSC & -1.60 & $\mathrm{~L}$ & 363 & 10 & L & 12.41 & $\cdots$ \\
\hline HD 171496 & 183607.50 & -242611.3 & HIP & -1.12 & C97 & -44 & 10 & BSL & 8.49 & 1.08 \\
\hline V* Y Lyr & 183713.31 & +435713.6 & NPM & -1.03 & $\mathrm{~L}$ & -65 & 23 & $\mathrm{~L}$ & 13.28 & $\cdots$ \\
\hline $\mathrm{V}^{*} \mathrm{LX}$ Lyr & 184023.33 & +410222.7 & $\mathrm{STN}$ & -1.42 & $\mathrm{~L}$ & -96 & 30 & L & 13.11 & $\cdots$ \\
\hline BPS CS 22959-0035 & 184047.76 & -650618.0 & $\mathrm{U}$ & -0.30 & HK & -72 & 10 & BSL & {$[14.70]$} & {$[0.69]$} \\
\hline BPS CS 22959-0007 & 184113.30 & $-67 \quad 1523.1$ & $\mathrm{U}$ & -1.56 & HK & 51 & 10 & BSL & $(14.07)$ & $(0.43)$ \\
\hline $\mathrm{V}^{*}$ CN Lyr & 184115.95 & +284321.2 & HIP & -0.58 & F98 & 27 & 3 & F98 & 11.48 & $\ldots$ \\
\hline $\mathrm{V}^{*} \mathrm{~V} 1640 \mathrm{Sgr}$ & 184335.47 & -345213.5 & $\mathrm{STN}$ & -0.54 & $\mathrm{~L}$ & 41 & 10 & $\mathrm{~L}$ & 12.68 & $\cdots$ \\
\hline $\mathrm{V}^{*} \mathrm{RZ} \mathrm{Lyr}$ & 184337.88 & +324753.8 & GSC & -2.13 & $\mathrm{~L}$ & -233 & 23 & L & 11.51 & $\cdots$ \\
\hline BPS CS 22959-0072 & 184644.95 & -641549.6 & $\mathrm{U}$ & -1.71 & HK & -26 & 10 & BSL & $(14.16)$ & $(0.54)$ \\
\hline HD 175305 & 184705.73 & +744330.8 & HIP & -1.45 & C97 & -181 & 3 & CY & 7.18 & 0.76 \\
\hline $\mathrm{V}^{*} \mathrm{EZ} \mathrm{Lyr}$ & 184740.32 & +35 5934.0 & HIP & -1.22 & F98 & -74 & 10 & F98 & 11.57 & $\cdots$ \\
\hline V* V413 CrA & 184757.65 & -374422.3 & HIP & -1.26 & F98 & -89 & 10 & F98 & 10.60 & $\cdots$ \\
\hline $\mathrm{V}^{*}$ AP Her & 185027.73 & +155624.7 & HIP & -0.80 & BSL & -26 & 1 & BSL & 11.00 & $\ldots$ \\
\hline BPS CS 22959-0090 & $1851 \quad 14.71$ & $-66 \quad 1542.4$ & $\mathrm{U}$ & -1.71 & HK & -13 & 10 & BSL & {$[15.10]$} & {$[0.55]$} \\
\hline V* CX Lyr & $1851 \quad 17.27$ & +284747.8 & GSC & -1.79 & $\mathrm{~L}$ & -203 & 30 & L & 12.83 & $\ldots$ \\
\hline BPS CS 22959-0074 & 185147.55 & -642760.0 & $\mathrm{U}$ & -2.40 & HK & -139 & 10 & BSL & 14.22 & 0.66 \\
\hline HD 174578 & 185213.26 & -252058.7 & HIP & -1.69 & BSL & -4 & 10 & BSL & 9.47 & 1.22 \\
\hline BPS CS 22959-0092 & 185440.04 & -663308.1 & $\mathrm{U}$ & -0.64 & HK & -12 & 10 & BSL & $(13.66)$ & $(0.64)$ \\
\hline BPS CS 22959-0095 & 185456.36 & -670050.1 & $\mathrm{U}$ & -0.28 & HK & -50 & 10 & BSL & $(14.02)$ & $(0.58)$ \\
\hline BPS CS 22959-0097 & 185537.13 & -671745.4 & $\mathrm{U}$ & -0.67 & HK & -92 & 10 & BSL & $(13.51)$ & $(0.89)$ \\
\hline BPS CS 22959-0139 & 185553.85 & -630616.4 & $\mathrm{U}$ & -2.17 & HK & -28 & 10 & BSL & 14.29 & 0.40 \\
\hline HD 175606 & 185851.32 & -512755.1 & HIP & -2.45 & BSL & 148 & 10 & BSL & 9.79 & 0.45 \\
\hline HD 176203 & 190004.17 & $\begin{array}{lll}-23 & 03 & 46.7\end{array}$ & HIP & -1.61 & BSL & 68 & 10 & BSL & 8.78 & 0.73 \\
\hline $\mathrm{V}^{*} \mathrm{MT}$ Tel & 190212.40 & -463911.1 & HIP & -1.85 & F98 & 56 & 10 & F98 & 8.98 & $\cdots$ \\
\hline $\mathrm{V}^{*} \mathrm{NR}$ Lyr & 190817.71 & +384742.8 & STN & -2.42 & L & -84 & 30 & L & 12.50 & $\cdots$ \\
\hline BPS CS 22947-0049 & 190841.31 & -483250.9 & $\mathrm{U}$ & -0.28 & HK & 74 & 10 & BSL & 14.47 & 0.60 \\
\hline $\mathrm{V}^{*} \mathrm{XZ}$ Dra & 190942.59 & +645132.1 & HIP & -0.79 & F98 & -30 & 1 & F98 & 10.25 & $\ldots$ \\
\hline BPS CS 22959-0192 & 190945.20 & -664442.0 & $\mathrm{U}$ & -0.84 & HK & -56 & 10 & BSL & $(13.45)$ & $(0.65)$ \\
\hline $\mathrm{V}^{*} \mathrm{FN}$ Lyr & 191022.25 & +422731.6 & GSC & -2.01 & $\mathrm{~L}$ & -194 & 30 & L & 12.68 & $\cdots$ \\
\hline HD 178443 & 191036.86 & -431635.7 & HIP & -2.07 & B99 & 351 & 10 & HK & 9.99 & 0.70 \\
\hline BPS CS 22947-0101 & 191355.05 & -484839.7 & $\mathrm{U}$ & -1.90 & HK & -62 & 10 & BSL & 13.21 & 0.45 \\
\hline
\end{tabular}


TABLE 1A. (continued)

\begin{tabular}{|c|c|c|c|c|c|c|c|c|c|c|}
\hline $\begin{array}{l}\text { Star } \\
\text { (1) }\end{array}$ & RA (2000.0) & DEC & $\begin{array}{c}\text { Source }^{\mathrm{a}} \\
(4)\end{array}$ & $\begin{array}{c}{[\mathrm{Fe} / \mathrm{H}]} \\
(\mathrm{dex}) \\
(5)\end{array}$ & $\begin{array}{c}\text { Source }^{b} \\
(6)\end{array}$ & $\begin{array}{c}\mathrm{RV} \\
(\mathrm{km} / \mathrm{s}) \\
(7)\end{array}$ & $\begin{array}{c}\sigma_{\mathrm{RV}} \\
(\mathrm{km} / \mathrm{s}) \\
(8)\end{array}$ & $\begin{array}{c}\text { Source }^{c} \\
(9)\end{array}$ & $\begin{array}{c}V \\
(\mathrm{mag}) \\
(10)\end{array}$ & $\begin{array}{c}B-V \\
(\mathrm{mag}) \\
(11)\end{array}$ \\
\hline BPS CS 22959-0225 & 191425.98 & -645356.5 & $\mathrm{U}$ & -1.54 & HK & -180 & 10 & BSL & $(14.19)$ & $(0.51)$ \\
\hline BPS CS 22959-0231 & 191521.00 & -640944.4 & $\mathrm{U}$ & -2.29 & HK & 85 & 10 & BSL & {$[15.10]$} & {$[0.49]$} \\
\hline BPS CS 22947-0322 & 191541.28 & -482144.0 & $\mathrm{U}$ & -0.80 & HK & -69 & 10 & BSL & 13.98 & 0.79 \\
\hline BPS CS 22891-0089 & 191555.91 & -612221.0 & $\mathrm{U}$ & -1.78 & HK & 24 & 10 & BSL & {$[14.80]$} & {$[0.43]$} \\
\hline BPS CS 22959-0219 & 191609.38 & -654525.2 & $\mathrm{U}$ & -2.04 & HK & 81 & 10 & BSL & $(13.14)$ & $(0.46)$ \\
\hline $\mathrm{V}^{*} \mathrm{BK}$ Dra & 191820.69 & +662447.7 & HIP & -1.95 & F98 & -77 & 12 & F98 & 11.19 & $\ldots$ \\
\hline BPS CS 22947-0187 & 191831.58 & $-47 \quad 1331.1$ & STN & -2.49 & B99 & -256 & 10 & HK & 12.95 & 0.65 \\
\hline BPS CS 22896-0015 & 191845.40 & -552444.4 & $\mathrm{U}$ & -2.26 & HK & 50 & 10 & BSL & 14.26 & 0.59 \\
\hline HD 181007 & 191928.51 & -202538.1 & HIP & -1.64 & BSL & -2 & 10 & BSL & 9.57 & 0.80 \\
\hline $\mathrm{V}^{*}$ V1130 Sgr & 192032.51 & -203220.2 & STN & -1.70 & L & -54 & 10 & L & 12.53 & $\cdots$ \\
\hline BPS CS 22891-0115 & 192110.87 & -60 1347.2 & $\mathrm{U}$ & -1.56 & HK & 297 & 10 & BSL & 15.16 & 0.40 \\
\hline BPS CS 22896-0028 & 192221.11 & -540843.0 & $\mathrm{U}$ & -1.77 & HK & 39 & 10 & BSL & 14.13 & 0.42 \\
\hline HD 181743 & 192342.80 & -450449.8 & HIP & -1.79 & B99 & 26 & 10 & HK & 9.68 & 0.47 \\
\hline BPS CS 22896-0072 & 192439.55 & -555238.0 & $\mathrm{U}$ & -0.88 & HK & -20 & 10 & BSL & $(14.50)$ & $(0.70)$ \\
\hline $\mathrm{V}^{*} \mathrm{RR}$ Lyr & 192528.00 & +424705.4 & HIP & -1.39 & F98 & -63 & 8 & F98 & 7.76 & \\
\hline BPS CS 22896-0044 & 192529.68 & -533445.2 & $\mathrm{U}$ & -1.84 & HK & 26 & 10 & BSL & $(13.91)$ & $(0.49)$ \\
\hline BPS CS 22891-0184 & 192612.34 & -603406.6 & $\mathrm{U}$ & -2.29 & HK & 28 & 10 & BSL & 13.83 & 0.50 \\
\hline BPS CS 22891-0155 & 192619.47 & -580014.0 & $\mathrm{U}$ & -0.84 & HK & -11 & 10 & BSL & {$[15.10]$} & {$[0.59]$} \\
\hline $\mathrm{V}^{*} \mathrm{BN}$ Vul & 192756.09 & +242050.8 & HIP & -1.61 & F98 & -267 & 5 & F98 & 11.02 & $\ldots$ \\
\hline BPS CS 22896-0055 & 192943.87 & -535928.7 & $\mathrm{U}$ & -2.06 & HK & -84 & 10 & BSL & 13.53 & 0.43 \\
\hline BPS CS 22891-0194 & 193040.48 & -622036.3 & $\mathrm{U}$ & -1.68 & HK & -85 & 10 & BSL & 14.49 & 0.42 \\
\hline $\mathrm{V}^{*} \mathrm{~V} 440 \mathrm{Sgr}$ & 193220.79 & -235112.3 & HIP & -1.40 & F98 & -62 & 1 & F98 & 10.34 & $\cdots$ \\
\hline $\mathrm{V}^{*} \mathrm{XZ}$ Cyg & 193229.22 & +562317.7 & HIP & -1.44 & F98 & -162 & 7 & F98 & 9.68 & $\cdots$ \\
\hline$V^{*}$ V894 Cyg & 193257.76 & +461348.8 & U & -1.71 & L & -177 & 30 & L & 12.72 & $\cdots$ \\
\hline $\mathrm{V}^{*} \mathrm{HH}$ Tel & 193320.27 & -453949.4 & STN & -1.02 & $\mathrm{~L}$ & 81 & 14 & $\mathrm{~L}$ & 12.31 & $\cdots$ \\
\hline $\mathrm{V}^{*}$ WY Dra & 193320.92 & +805542.7 & GSC & -1.66 & $\mathrm{~L}$ & -6 & 30 & L & 12.67 & $\cdots$ \\
\hline BPS CS 22896-0089 & 193325.78 & -564718.6 & $\mathrm{U}$ & -1.98 & HK & 279 & 10 & BSL & 14.26 & 0.47 \\
\hline HD 184266 & 193415.31 & -161858.5 & HIP & -1.63 & B99 & -348 & 1 & $\mathrm{CY}$ & 7.59 & 0.55 \\
\hline BPS CS 22896-0115 & 193505.17 & $\begin{array}{lll}-52 & 4709.8\end{array}$ & $\mathrm{U}$ & -2.44 & HK & 1 & 10 & BSL & 14.13 & 0.42 \\
\hline BPS CS 22891-0221 & 193510.52 & -594157.1 & $\mathrm{U}$ & -2.31 & HK & 27 & 10 & BSL & 14.43 & 0.56 \\
\hline BPS CS 22891-0200 & 193519.09 & $\begin{array}{lll}-61 & 42 & 24.7\end{array}$ & $\mathrm{U}$ & -3.49 & B99 & 131 & 10 & HK & 13.93 & 0.86 \\
\hline $\mathrm{V}^{*} \mathrm{XZ}$ Oct & 193547.73 & -753027.5 & GSC & -1.76 & L & 154 & 35 & L & 13.28 & $\cdots$ \\
\hline BPS CS 22896-0110 & 193547.99 & -532616.2 & $\mathrm{U}$ & -2.25 & HK & 77 & 10 & BSL & 13.56 & 0.53 \\
\hline HD 184711 & 193711.92 & -394436.6 & HIP & -2.51 & B99 & 102 & 1 & $\mathrm{CY}$ & 7.97 & 1.24 \\
\hline BPS CS 22891-0206 & 193733.08 & $\begin{array}{lll}-61 & 06 & 04.7\end{array}$ & $\mathrm{U}$ & -1.97 & HK & 9 & 10 & BSL & 14.88 & 0.46 \\
\hline $\mathrm{V}^{*} \mathrm{BN}$ Pav & 193803.38 & -603639.6 & HIP & -1.32 & F98 & 114 & 14 & F98 & 12.60 & $\cdots$ \\
\hline HD 232078 & 193812.06 & +164825.9 & HIP & -1.61 & C97 & -391 & 10 & BSL & 8.57 & 2.00 \\
\hline BPS CS 22896-0152 & 193900.84 & -564234.8 & GSC & -0.99 & HK & -57 & 10 & BSL & 13.32 & 0.65 \\
\hline BPS CS 22896-0136 & 193953.16 & -541023.2 & $\mathrm{U}$ & -2.24 & HK & -22 & 10 & BSL & 14.71 & 0.41 \\
\hline BPS CS 22891-0209 & 194202.14 & -610344.6 & $\mathrm{U}$ & -3.18 & B99 & 75 & 10 & HK & 12.17 & 0.82 \\
\hline
\end{tabular}


TABLE 1A. (continued)

\begin{tabular}{|c|c|c|c|c|c|c|c|c|c|c|}
\hline $\begin{array}{l}\text { Star } \\
\text { (1) }\end{array}$ & RA (2000.0) & DEC & $\begin{array}{c}\text { Source }^{\mathrm{a}} \\
(4)\end{array}$ & $\begin{array}{c}{[\mathrm{Fe} / \mathrm{H}]} \\
(\mathrm{dex}) \\
(5)\end{array}$ & $\begin{array}{c}\text { Source }^{b} \\
(6)\end{array}$ & $\begin{array}{c}\mathrm{RV} \\
(\mathrm{km} / \mathrm{s}) \\
(7)\end{array}$ & $\begin{array}{c}\sigma_{\mathrm{RV}} \\
(\mathrm{km} / \mathrm{s}) \\
(8)\end{array}$ & $\begin{array}{c}\text { Source }^{c} \\
(9)\end{array}$ & $\begin{array}{c}V \\
(\mathrm{mag}) \\
(10)\end{array}$ & $\begin{array}{c}B-V \\
(\operatorname{mag}) \\
(11)\end{array}$ \\
\hline BPS CS 22896-0150 & 194203.99 & $-5641 \quad 19.6$ & $\mathrm{U}$ & -1.46 & HK & 116 & 10 & BSL & $(14.09)$ & $(0.41)$ \\
\hline BPS CS 22896-0154 & 194226.89 & -565833.9 & $\mathrm{U}$ & -2.73 & B99 & 139 & 10 & HK & 13.64 & 0.62 \\
\hline HD 186478 & 194514.15 & -172926.3 & HIP & -2.58 & B99 & 31 & 2 & $\mathrm{CY}$ & 9.14 & 0.97 \\
\hline BPS CS 22873-0015 & 194638.48 & -594309.7 & $\mathrm{U}$ & -2.04 & HK & 68 & 10 & BSL & 14.08 & 0.46 \\
\hline V* V796 Sgr & 194639.91 & -402722.5 & SPM & -1.87 & $\mathrm{~L}$ & -158 & 14 & L & 12.13 & $\cdots$ \\
\hline BPS CS 22964-0024 & 194724.24 & -392260.0 & SPM & -1.76 & HK & -120 & 10 & BSL & 13.87 & 0.46 \\
\hline BPS CS 22964-0019 & 194730.38 & -394620.0 & SPM & -2.44 & HK & -317 & 10 & BSL & 13.27 & 0.81 \\
\hline V* V2194 Sgr & 194818.17 & -383032.9 & SPM & -1.62 & BSL & -16 & 10 & BSL & 14.80 & 0.42 \\
\hline HD 187111 & 194839.55 & -120719.2 & HIP & -1.78 & B99 & -181 & 3 & $\mathrm{CY}$ & 7.71 & 1.17 \\
\hline BPS CS 22964-0074 & 194929.40 & -394238.8 & SPM & -2.45 & HK & -346 & 10 & BSL & 14.46 & 0.39 \\
\hline BPS CS 22873-0072 & 195205.48 & -613738.4 & U & -2.50 & HK & -83 & 10 & BSL & 14.64 & 0.41 \\
\hline BPS CS 22964-0092 & 195210.88 & -412415.1 & SPM & -0.70 & HK & -10 & 10 & BSL & 14.73 & 0.64 \\
\hline $\mathrm{V}^{*} \mathrm{DN}$ Pav & 195210.95 & -634023.0 & GSC & -1.54 & $\mathrm{~L}$ & -69 & 12 & L & 12.42 & $\cdots$ \\
\hline BPS CS 22964-0052 & 195219.62 & -380708.9 & SPM & -1.15 & HK & -127 & 10 & BSL & 15.01 & 0.56 \\
\hline BPS CS 22964-0064 & 195232.04 & -391024.3 & SPM & -0.70 & HK & 63 & 10 & BSL & 14.50 & 0.75 \\
\hline V* V2202 Sgr & 195243.92 & -385752.2 & SPM & -2.25 & BSL & -110 & 10 & BSL & 14.76 & 0.58 \\
\hline BPS CS 22873-0055 & 195349.80 & $\begin{array}{lll}-59 & 40 & 00.3\end{array}$ & U & -2.88 & B99 & 207 & 10 & HK & 12.65 & 0.93 \\
\hline BPS CS 22964-0138 & 195452.28 & -380826.8 & SPM & -1.68 & HK & -35 & 10 & BSL & 14.30 & 0.42 \\
\hline HD 188031 & 195458.65 & -423839.6 & HIP & -2.04 & BSL & -139 & 10 & BSL & 10.14 & 0.44 \\
\hline BPS CS 22964-0111 & 195519.84 & -404419.5 & SPM & -1.62 & HK & 55 & 10 & BSL & 14.69 & 0.45 \\
\hline BPS CS 22964-0149 & 195529.75 & -373406.7 & SPM & -0.88 & HK & 3 & 10 & BSL & 14.75 & 0.77 \\
\hline $\mathrm{V}^{*}$ GK Pav & 195655.51 & -592922.9 & $\mathrm{U}$ & -1.91 & BSL & 158 & 10 & BSL & 14.03 & 0.42 \\
\hline BPS CS 22964-0115 & 195728.56 & $\begin{array}{lll}-40 & 00 & 03.1\end{array}$ & SPM & -2.32 & HK & -216 & 10 & BSL & 14.94 & 0.43 \\
\hline $\mathrm{V}^{*} \mathrm{BP} \mathrm{Pav}$ & 195800.18 & -654409.7 & HIP & -1.48 & F98 & 348 & 14 & F98 & 12.54 & $\ldots$ \\
\hline BD-18 5550 & 195849.73 & -181210.3 & HIP & -2.89 & B99 & -126 & 1 & $\mathrm{CY}$ & 9.28 & 0.71 \\
\hline BPS CS 22873-0106 & 195945.42 & $\begin{array}{lll}-58 & 47 & 02.5\end{array}$ & U & -1.52 & HK & 124 & 10 & BSL & 14.19 & 0.63 \\
\hline BPS CS 22964-0172 & 200027.82 & -400147.2 & SPM & -0.13 & HK & 2 & 10 & BSL & 14.49 & 0.81 \\
\hline$V^{*}$ V1711 Sgr & 200107.99 & -303038.9 & HIP & -1.10 & BSL & -76 & 1 & BSL & 10.60 & 0.70 \\
\hline BPS CS 22964-0183 & $2001 \quad 12.76$ & -404909.5 & SPM & -2.60 & HK & 97 & 10 & BSL & 14.48 & 0.43 \\
\hline BPS CS 22964-0157 & 200141.63 & -375814.3 & SPM & -1.66 & HK & 34 & 10 & BSL & 14.49 & 0.52 \\
\hline BPS CS 22964-0181 & 200201.50 & -404224.4 & SPM & -0.82 & HK & 119 & 10 & BSL & 13.60 & 0.92 \\
\hline BPS CS 22964-0176 & 200207.12 & -402125.7 & SPM & -2.61 & HK & -54 & 10 & BSL & 15.33 & 0.44 \\
\hline BPS CS 22964-0158 & 200239.15 & -375948.6 & $\mathrm{SPM}$ & -1.90 & HK & -217 & 10 & BSL & 14.74 & 0.48 \\
\hline BPS CS 22964-0161 & 200325.56 & -383834.9 & SPM & -2.30 & HK & 40 & 10 & BSL & 14.41 & 0.49 \\
\hline HD 190287 & 200538.29 & -345510.5 & HIP & -1.59 & B99 & 135 & 3 & $\mathrm{CY}$ & 8.53 & 0.75 \\
\hline BPS CS 22964-0214 & 200549.98 & -392742.4 & SPM & -2.47 & HK & 43 & 10 & BSL & 13.66 & 0.40 \\
\hline BPS CS 22873-0139 & 200555.07 & $\begin{array}{llll}-59 & 17 & 10.9\end{array}$ & $\mathrm{U}$ & -3.03 & HK & 243 & 10 & BSL & 13.84 & 0.37 \\
\hline BPS CS 22873-0128 & 200703.79 & -583457.7 & U & -2.88 & B99 & 206 & 10 & HK & 13.03 & 0.69 \\
\hline BPS CS 22964-0232 & 200758.58 & -375416.5 & SPM & -0.44 & HK & -40 & 10 & BSL & 14.44 & 0.78 \\
\hline $\mathrm{V}^{*} \mathrm{HY}$ Tel & 200813.42 & $\begin{array}{lll}-55 & 14 & 02.2\end{array}$ & STN & -0.17 & $\mathrm{~L}$ & 48 & 14 & L & 12.61 & $\cdots$ \\
\hline
\end{tabular}


TABLE 1A. (continued)

\begin{tabular}{|c|c|c|c|c|c|c|c|c|c|c|}
\hline Star & RA (2000.0) & DEC & $\begin{array}{c}\text { Source }^{\mathrm{a}} \\
(4)\end{array}$ & $\begin{array}{c}{[\mathrm{Fe} / \mathrm{H}]} \\
(\mathrm{dex}) \\
(5)\end{array}$ & $\begin{array}{c}\text { Source }^{b} \\
(6)\end{array}$ & $\begin{array}{c}\mathrm{RV} \\
(\mathrm{km} / \mathrm{s}) \\
(7)\end{array}$ & $\begin{array}{c}\sigma_{\mathrm{RV}} \\
(\mathrm{km} / \mathrm{s}) \\
(8)\end{array}$ & $\begin{array}{c}\text { Source }{ }^{\mathrm{c}} \\
(9)\end{array}$ & $\begin{array}{c}V \\
(\mathrm{mag}) \\
(10)\end{array}$ & $\begin{array}{c}B-V \\
(\mathrm{mag}) \\
(11)\end{array}$ \\
\hline BPS CS 22964-0231 & 200834.76 & -380157.3 & GSC & -1.09 & HK & -13 & 10 & BSL & 14.60 & 0.46 \\
\hline BPS CS 22873-0137 & 200836.69 & -590132.4 & U & -1.21 & HK & -58 & 10 & BSL & 13.46 & 0.39 \\
\hline$V^{*}$ V1644 Sgr & 200914.89 & -293321.8 & SPM & -2.51 & L & -37 & 14 & L & 12.20 & $\cdots$ \\
\hline $\mathrm{V}^{*} \mathrm{TW}$ Cap & 201428.42 & -135007.8 & HIP & -1.20 & BSL & -32 & 1 & BSL & 10.70 & 0.30 \\
\hline BPS CS 22943-0001 & 201602.44 & -473728.3 & $\mathrm{U}$ & -1.96 & HK & -185 & 10 & BSL & $(14.00)$ & $(0.50)$ \\
\hline BPS CS 22943-0069 & 201627.03 & -444918.5 & $\mathrm{U}$ & -1.97 & HK & -2 & 10 & BSL & $(14.60)$ & $(0.40)$ \\
\hline HD 192718 & 201637.90 & -072636.7 & HIP & -0.87 & BSL & -111 & 10 & BSL & 8.39 & 0.58 \\
\hline BPS CS 22943-0053 & $2017 \quad 11.31$ & -425727.3 & $\mathrm{U}$ & -1.90 & HK & 162 & 10 & BSL & $(14.15)$ & $(0.45)$ \\
\hline BPS CS 22943-0078 & $2017 \quad 15.83$ & -451318.7 & $\mathrm{U}$ & -1.67 & HK & -17 & 10 & BSL & $(14.23)$ & $(0.57)$ \\
\hline BPS CS 22885-0040 & 201807.96 & -380557.0 & SPM & -3.03 & BSL & 58 & 10 & BSL & 15.24 & 0.40 \\
\hline BPS CS 22943-0067 & $2018 \quad 10.55$ & -443138.8 & U & -0.06 & HK & -58 & 10 & BSL & $(14.47)$ & $(0.63)$ \\
\hline BPS CS 22943-0059 & 201900.59 & -431721.6 & U & -2.60 & HK & 260 & 10 & BSL & $(14.49)$ & $(0.41)$ \\
\hline $\mathrm{V}^{*} \mathrm{HV} \mathrm{Pav}$ & 201905.08 & -564932.6 & STN & -1.64 & $\mathrm{~L}$ & -14 & 36 & L & 13.07 & $\ldots$ \\
\hline BPS CS 22885-0054 & 201907.85 & $-39 \quad 12 \quad 29.5$ & SPM & -1.97 & HK & 91 & 10 & BSL & 14.78 & 0.44 \\
\hline BPS CS 22943-0087 & 201918.47 & -462727.5 & U & -1.98 & $\mathrm{HK}$ & 452 & 10 & BSL & $(14.24)$ & $(0.56)$ \\
\hline BPS CS 22873-0166 & 201922.20 & -613014.6 & GSC & -2.90 & B99 & -20 & 10 & HK & 11.82 & 0.99 \\
\hline BPS CS 22885-0069 & 201924.05 & -412244.6 & SPM & -2.24 & HK & 7 & 10 & BSL & 13.72 & 0.69 \\
\hline BPS CS 22885-0067 & 201941.17 & -405116.4 & GSC & -1.94 & HK & 31 & 10 & BSL & 13.99 & 0.60 \\
\hline BPS CS 22950-0050 & 201953.63 & -132435.8 & U & -2.13 & HK & -103 & 10 & BSL & 13.91 & 0.69 \\
\hline HD 193242 & 201954.31 & -195445.7 & HIP & -1.76 & BSL & -128 & 10 & BSL & 9.15 & 0.76 \\
\hline BPS CS 22885-0105 & 202030.51 & -384702.5 & SPM & -2.19 & HK & -222 & 10 & BSL & 14.36 & 0.68 \\
\hline V* V1645 Sgr & 202044.46 & $\begin{array}{lll}-41 & 07 & 05.4\end{array}$ & SPM & -1.74 & $\mathrm{~L}$ & -330 & 14 & L & 11.96 & $\ldots$ \\
\hline BPS CS 22885-0096 & 202051.18 & -395330.1 & SPM & -3.60 & B99 & -248 & 10 & HK & 13.33 & 0.69 \\
\hline BPS CS 22885-0094 & 202102.86 & -403056.2 & SPM & -1.19 & HK & 34 & 10 & BSL & 13.62 & 0.61 \\
\hline V* BX Del & 202120.50 & +1827 13.0 & U & -0.63 & BSL & 30 & 30 & BSL & 11.60 & 0.00 \\
\hline BPS CS 22950-0046 & 202128.38 & -131633.4 & $\mathrm{U}$ & -3.40 & B99 & 111 & 10 & HK & 14.22 & 0.91 \\
\hline BPS CS 22950-0063 & 202133.92 & -150204.6 & U & -2.55 & HK & -141 & 10 & BSL & 14.22 & 0.72 \\
\hline BPS CS 22943-0107 & 202238.86 & -460857.4 & $\mathrm{U}$ & -1.36 & HK & -90 & 10 & BSL & $(14.84)$ & $(0.46)$ \\
\hline BPS CS 22955-0038 & 202248.46 & $\begin{array}{lll}-23 & 02 & 25.2\end{array}$ & $\mathrm{U}$ & -1.51 & HK & -134 & 10 & BSL & $(14.25)$ & $(0.55)$ \\
\hline BPS CS 22943-0132 & 202250.80 & -431338.7 & $\mathrm{U}$ & -2.40 & HK & 3 & 10 & BSL & 13.36 & $(0.48)$ \\
\hline BPS CS 22943-0109 & $2022 \quad 57.35$ & -454050.5 & $\mathrm{U}$ & -1.94 & HK & 119 & 10 & BSL & $(14.28)$ & $(0.82)$ \\
\hline V* V1646 Sgr & 202308.64 & -311740.6 & GSC & -1.41 & L & 142 & 14 & L & 12.06 & $\ldots$ \\
\hline BPS CS 22943-0110 & 202317.46 & -453234.7 & U & -1.26 & HK & -60 & 10 & BSL & $(14.44)$ & $(0.46)$ \\
\hline BPS CS 22943-0134 & 202428.06 & $-42 \quad 4912.8$ & U & -2.20 & HK & -135 & 10 & BSL & {$[15.60]$} & {$[0.45]$} \\
\hline BPS CS 22885-0106 & 202442.05 & -383626.6 & SPM & -2.12 & HK & -135 & 10 & BSL & 14.97 & 0.44 \\
\hline BPS CS 22943-0092 & 202444.30 & -471550.7 & U & -1.57 & HK & 91 & 10 & BSL & $(13.68)$ & $(0.52)$ \\
\hline BPS CS 22955-0032 & 202452.93 & -244957.6 & $\mathrm{U}$ & -2.37 & HK & -64 & 10 & BSL & 14.58 & $(0.63)$ \\
\hline BPS CS 22950-0078 & 202457.89 & -162955.3 & $\mathrm{U}$ & -2.17 & HK & 14 & 10 & BSL & 14.62 & 0.41 \\
\hline BPS CS 22950-0102 & 202459.78 & -145708.4 & U & -0.20 & HK & 33 & 10 & BSL & $(13.92)$ & $(0.58)$ \\
\hline $\mathrm{V}^{*}$ CK Del & 202502.05 & +111237.3 & GSC & -1.26 & L & -90 & 23 & L & 12.29 & $\ldots$ \\
\hline
\end{tabular}


TABLE 1A. (continued)

\begin{tabular}{|c|c|c|c|c|c|c|c|c|c|c|}
\hline $\begin{array}{l}\text { Star } \\
(1)\end{array}$ & $\begin{array}{c}\text { RA (2000.0) } \\
(2)\end{array}$ & DEC & $\begin{array}{c}\text { Source }^{\mathrm{a}} \\
(4)\end{array}$ & $\begin{array}{c}{[\mathrm{Fe} / \mathrm{H}]} \\
(\mathrm{dex}) \\
(5)\end{array}$ & $\begin{array}{c}\text { Source }^{b} \\
(6)\end{array}$ & $\begin{array}{c}\mathrm{RV} \\
(\mathrm{km} / \mathrm{s}) \\
(7)\end{array}$ & $\begin{array}{c}\sigma_{\mathrm{RV}} \\
(\mathrm{km} / \mathrm{s}) \\
(8)\end{array}$ & $\begin{array}{c}\text { Source }^{\mathrm{c}} \\
(9)\end{array}$ & $\begin{array}{c}V \\
(\mathrm{mag}) \\
(10)\end{array}$ & $\begin{array}{c}B-V \\
(\mathrm{mag}) \\
(11)\end{array}$ \\
\hline BPS CS 22943-0097 & $2026 \quad 15.29$ & -463514.5 & STN & -2.20 & HK & 70 & 10 & BSL & $(13.47)$ & $(0.43)$ \\
\hline BPS CS 22943-0137 & 202629.01 & -430042.1 & $\mathrm{U}$ & -3.22 & B99 & -20 & 10 & HK & (14.47) & $(0.53)$ \\
\hline V* V2279 Sgr & 202703.35 & -404014.3 & STN & -1.16 & $\mathrm{~L}$ & 55 & 14 & $\mathrm{~L}$ & 12.63 & $\ldots$ \\
\hline BPS CS 22943-0095 & 202729.98 & -465056.6 & STN & -2.17 & HK & -166 & 10 & BSL & 11.76 & $(0.44)$ \\
\hline BPS CS 22885-0143 & $2027 \quad 42.14$ & -392339.4 & SPM & -2.51 & HK & -69 & 10 & BSL & 13.93 & 0.75 \\
\hline BPS CS 22950-0096 & 202809.44 & -154220.5 & STN & -1.62 & HK & -72 & 10 & BSL & 13.90 & 0.46 \\
\hline BPS CS 22943-0164 & 202826.20 & -460131.4 & $\mathrm{U}$ & -1.97 & HK & 216 & 10 & BSL & $(14.46)$ & $(0.44)$ \\
\hline BPS CS 22955-0047 & 202827.24 & -230355.8 & $\mathrm{U}$ & -1.55 & HK & 36 & 10 & BSL & 14.30 & 0.40 \\
\hline BPS CS 22885-0203 & 203019.25 & -374216.7 & SPM & -2.17 & HK & -32 & 10 & BSL & 14.23 & 0.41 \\
\hline BPS CS 22955-0117 & 203019.99 & -242831.8 & SPM & -2.02 & HK & 186 & 10 & BSL & 14.40 & 0.44 \\
\hline $\mathrm{V}^{*} \mathrm{SV}$ Mic & 203020.04 & $-4358 \quad 14.3$ & GSC & -0.96 & BSL & -171 & 30 & BSL & 14.40 & $\ldots$ \\
\hline BPS CS 22950-0147 & $2031 \quad 11.17$ & -142824.9 & $\mathrm{U}$ & -1.97 & HK & -163 & 10 & BSL & 14.60 & 0.46 \\
\hline BPS CS 22955-0101 & 203133.30 & -263004.2 & SPM & -0.94 & HK & -169 & 10 & BSL & 13.78 & 0.53 \\
\hline BPS CS 22955-0121 & 203137.46 & -234738.1 & U & -1.62 & HK & -16 & 10 & BSL & $(14.56)$ & $(0.44)$ \\
\hline CD-23 16310 & 203203.19 & -233252.0 & HIP & -2.44 & BSL & -84 & 10 & BSL & 10.38 & 0.84 \\
\hline BPS CS 22955-0107 & 203210.62 & -254352.1 & GSC & -1.34 & HK & 72 & 10 & BSL & 14.31 & 0.45 \\
\hline BPS CS 22885-0175 & 203228.40 & -391018.9 & SPM & -0.79 & HK & -24 & 10 & BSL & 13.76 & 0.85 \\
\hline V* V341 Aql & 203231.54 & +003507.3 & HIP & -1.22 & F98 & -79 & 2 & F98 & 10.85 & $\ldots$ \\
\hline BPS CS 22950-0153 & 203234.50 & -154758.2 & STN & -1.84 & $\mathrm{HK}$ & -161 & 10 & BSL & 13.74 & 0.56 \\
\hline HD 195636 & 203248.98 & -092150.9 & HIP & -2.80 & B99 & -258 & 1 & $\mathrm{CY}$ & 9.54 & 0.64 \\
\hline BPS CS 22943-0192 & 203259.72 & -445921.2 & $\mathrm{U}$ & -1.56 & HK & 199 & 10 & BSL & $(14.41)$ & $(0.39)$ \\
\hline BPS CS 22950-0173 & 203531.48 & -155327.3 & $\mathrm{U}$ & -2.70 & HK & 60 & 10 & BSL & 14.04 & 0.41 \\
\hline BPS CS 22955-0153 & 203534.04 & -264054.4 & SPM & -1.54 & HK & -47 & 10 & BSL & 14.49 & 0.42 \\
\hline BPS CS 22943-0201 & 203628.79 & -434929.7 & $\mathrm{U}$ & -2.54 & HK & 37 & 10 & BSL & {$[15.20]$} & [0.41] \\
\hline BPS CS 22879-0012 & $2037 \quad 14.02$ & $\begin{array}{lll}-41 & 02 & 33.4\end{array}$ & SPM & -2.70 & HK & -40 & 10 & BSL & 14.72 & 0.39 \\
\hline BD-17 6036 & 203715.95 & -163357.7 & HIP & -2.70 & ATT & 19 & 1 & $\mathrm{CY}$ & 10.52 & 0.84 \\
\hline BPS CS 22955-0134 & 203723.44 & -222700.8 & SPM & -1.71 & HK & -99 & 10 & BSL & 13.58 & 0.46 \\
\hline BPS CS $22880-0013$ & 203728.05 & -201431.4 & $\mathrm{U}$ & -2.50 & HK & -179 & 10 & BSL & 13.68 & 0.39 \\
\hline $\mathrm{V}^{*} \mathrm{AA}$ Aql & $2038 \quad 15.06$ & -025324.3 & GSC & -0.58 & $\mathrm{~L}$ & -32 & 4 & L & 11.74 & $\ldots$ \\
\hline BPS CS 22879-0029 & 203851.79 & -383646.3 & SPM & -2.16 & HK & -161 & 10 & BSL & 14.45 & 0.44 \\
\hline BPS CS 22879-0034 & 203851.98 & -381346.0 & SPM & -1.10 & BSL & 48 & 10 & BSL & 14.25 & 0.28 \\
\hline BPS CS 22940-0050 & 203934.49 & -614033.8 & $\mathrm{U}$ & -1.92 & HK & 217 & 10 & BSL & 14.88 & 0.50 \\
\hline $\mathrm{V}^{*} \mathrm{FH} \mathrm{Vul}$ & 204019.89 & +221324.7 & STN & -0.85 & L & 46 & 30 & L & 12.88 & $\ldots$ \\
\hline BPS CS 22955-0169 & 204042.71 & $\begin{array}{lll}-25 & 07 & 10.3\end{array}$ & SPM & -0.50 & $\mathrm{HK}$ & 26 & 10 & BSL & 14.58 & 0.61 \\
\hline HD 196892 & 204049.35 & $-18 \quad 4729.5$ & HIP & -1.11 & B99 & -34 & 10 & HK & 8.24 & 0.49 \\
\hline BPS CS 22940-0078 & $2041 \quad 15.19$ & $\begin{array}{lll}-59 & 46 & 00.8\end{array}$ & GSC & -1.28 & $\mathrm{HK}$ & 8 & 10 & BSL & 13.62 & 0.47 \\
\hline BPS CS 22940-0077 & 204133.67 & -595035.1 & $\mathrm{U}$ & -2.24 & HK & 41 & 10 & BSL & 14.13 & 0.53 \\
\hline BPS CS 22955-0165 & 204143.18 & $\begin{array}{lll}-25 & 18 & 10.4\end{array}$ & $\mathrm{U}$ & -1.66 & HK & -190 & 10 & BSL & 14.89 & 0.43 \\
\hline $\mathrm{V}^{*} \mathrm{VX}$ Ind & 204151.39 & $-4706 \quad 42.8$ & STN & -0.91 & $\mathrm{~L}$ & 123 & 14 & L & 12.54 & $\ldots$ \\
\hline BPS CS 22955-0174 & 204205.05 & -234912.6 & SPM & -2.22 & HK & 8 & 10 & BSL & 14.38 & 0.50 \\
\hline
\end{tabular}


TABLE 1A. (continued)

\begin{tabular}{|c|c|c|c|c|c|c|c|c|c|c|}
\hline $\begin{array}{l}\text { Star } \\
(1)\end{array}$ & $\begin{array}{c}\text { RA (2000.0) } \\
(2)\end{array}$ & DEC & $\begin{array}{c}\text { Source }^{\mathrm{a}} \\
(4)\end{array}$ & $\begin{array}{c}{[\mathrm{Fe} / \mathrm{H}]} \\
(\mathrm{dex}) \\
(5)\end{array}$ & $\begin{array}{c}\text { Source }^{b} \\
(6)\end{array}$ & $\begin{array}{c}\mathrm{RV} \\
(\mathrm{km} / \mathrm{s}) \\
(7)\end{array}$ & $\begin{array}{c}\sigma_{\mathrm{RV}} \\
(\mathrm{km} / \mathrm{s}) \\
(8)\end{array}$ & $\begin{array}{c}\text { Source }^{\mathrm{c}} \\
(9)\end{array}$ & $\begin{array}{c}V \\
(\mathrm{mag}) \\
(10)\end{array}$ & $\begin{array}{c}B-V \\
(\mathrm{mag}) \\
(11)\end{array}$ \\
\hline BPS CS 22879-0051 & 204236.47 & -391828.7 & SPM & -2.50 & HK & -25 & 10 & BSL & 13.89 & 0.39 \\
\hline BPS CS $22940-0070$ & $2042 \quad 39.21$ & $\begin{array}{lll}-61 & 40 & 39.1\end{array}$ & $\mathrm{U}$ & -2.30 & HK & 75 & 10 & BSL & 14.87 & 0.48 \\
\hline BD-20 6008 & 204248.78 & -200039.3 & HIP & -2.63 & BSL & 21 & 10 & BSL & 9.84 & 0.86 \\
\hline BPS CS $22880-0037$ & 204300.53 & $-18 \quad 1404.8$ & $\mathrm{U}$ & -1.48 & HK & -31 & 10 & BSL & 14.34 & 0.46 \\
\hline BPS CS 22940-0071 & 204302.31 & -605800.5 & $\mathrm{U}$ & -1.45 & HK & -59 & 10 & BSL & 14.26 & 0.45 \\
\hline BPS CS 22940-0082 & 204331.56 & -584154.7 & $\mathrm{U}$ & -1.94 & HK & 128 & 10 & BSL & 14.28 & 0.63 \\
\hline BPS CS 22879-0066 & 204342.55 & -412446.1 & SPM & -2.06 & HK & 279 & 10 & BSL & 13.63 & 0.40 \\
\hline BPS CS 22880-0058 & 204356.11 & -213632.3 & NPM & -2.57 & HK & -41 & 10 & BSL & 14.54 & 0.37 \\
\hline CD-30 18140 & 204406.33 & $\begin{array}{lll}-30 & 00 & 04.8\end{array}$ & HIP & -2.07 & B99 & 18 & 10 & HK & 9.94 & 0.43 \\
\hline BD-15 5781 & 204534.54 & -143115.0 & HIP & -2.47 & $\mathrm{ATT}$ & -76 & 1 & $\mathrm{CY}$ & 10.72 & 0.99 \\
\hline BPS CS 22880-0086 & 204547.53 & -183332.2 & $\mathrm{U}$ & -2.61 & HK & -90 & 10 & BSL & 14.41 & 0.64 \\
\hline BPS CS 22880-0074 & 204603.19 & -205913.3 & U & -2.36 & HK & 97 & 10 & BSL & 13.27 & 0.57 \\
\hline BPS CS 22879-0103 & 204710.14 & -372652.4 & SPM & -1.96 & HK & -45 & 10 & BSL & 14.30 & 0.49 \\
\hline V* DX Del & 204728.35 & +122750.6 & HIP & -0.39 & F98 & -52 & 3 & F98 & 9.94 & $\ldots$ \\
\hline BPS CS 22879-0097 & 204846.56 & -383049.2 & SPM & -2.30 & HK & 26 & 10 & BSL & 14.22 & 0.46 \\
\hline BPS CS 22880-0067 & 204907.94 & -213454.6 & U & -2.75 & HK & -188 & 10 & BSL & 15.08 & 0.51 \\
\hline BPS CS 22879-0098 & 204918.38 & -382459.8 & SPM & -2.38 & HK & -49 & 10 & BSL & 13.30 & 0.76 \\
\hline BPS CS 22879-0094 & 205008.35 & -384255.5 & SPM & -2.44 & HK & 37 & 10 & BSL & 15.20 & 0.42 \\
\hline BPS CS $22880-0110$ & 205021.73 & -185652.1 & $\mathrm{U}$ & -2.32 & HK & 60 & 10 & BSL & 15.01 & 0.43 \\
\hline $\mathrm{V}^{*} \mathrm{FK}$ Vul & 205231.03 & +222611.8 & STN & -0.95 & $\mathrm{~L}$ & -76 & 30 & L & 12.87 & $\cdots$ \\
\hline V* BV Del & 205311.64 & +160930.8 & $\mathrm{U}$ & -0.69 & $\mathrm{~L}$ & -45 & 33 & $\mathrm{~L}$ & 12.89 & $\ldots$ \\
\hline BPS CS $22880-0127$ & 205314.94 & -220405.1 & SPM & -1.58 & HK & -102 & 10 & BSL & 13.55 & 0.62 \\
\hline CD-48 13714 & 205402.88 & $-48 \quad 1251.8$ & HIP & -0.81 & BSL & 34 & 10 & BSL & 10.69 & 0.50 \\
\hline BPS CS 22940-0121 & 205510.90 & -580054.9 & $\mathrm{U}$ & -2.42 & HK & -3 & 10 & BSL & 14.16 & 0.55 \\
\hline BPS CS 22940-0099 & 205521.25 & -605432.0 & $\mathrm{U}$ & -0.28 & $\mathrm{HK}$ & -111 & 10 & BSL & 14.33 & 0.57 \\
\hline BD-14 5890 & 205609.15 & -133116.9 & HIP & -2.07 & B99 & 118 & 1 & $\mathrm{CY}$ & 10.21 & 0.89 \\
\hline $\mathrm{V}^{*} \mathrm{UY}$ Cyg & 205628.31 & +302540.4 & HIP & -0.80 & F98 & 3 & 10 & F98 & 11.11 & $\ldots$ \\
\hline CD-37 14010 & 205727.36 & -363252.7 & HIP & -2.55 & BSL & -200 & 10 & BSL & 9.74 & 0.99 \\
\hline $\mathrm{V}^{*} \mathrm{BT}$ Aqr & 205748.42 & -054106.8 & STN & -0.29 & L & -52 & 11 & L & 12.34 & $\ldots$ \\
\hline $\mathrm{V}^{*}$ DH Cyg & 205827.97 & +473850.3 & $\mathrm{U}$ & -0.63 & BSL & -49 & 10 & BSL & $\ldots$ & $\cdots$ \\
\hline $\mathrm{V}^{*}$ XZ Mic & 205854.79 & -385629.1 & SPM & -1.22 & $\mathrm{~L}$ & -19 & 14 & $\mathrm{~L}$ & 13.02 & $\ldots$ \\
\hline BPS CS 22879-0141 & 205959.54 & -393932.9 & SPM & -1.36 & HK & 62 & 10 & BSL & 15.11 & 0.62 \\
\hline BPS CS 22879-0144 & 210006.09 & -390353.3 & SPM & -2.01 & HK & -197 & 10 & BSL & 15.13 & 0.40 \\
\hline HD 199854 & 210013.65 & -150652.0 & HIP & -1.94 & BSL & 13 & 10 & BSL & 8.95 & 0.39 \\
\hline BPS CS 22897-0012 & 210039.43 & -643602.3 & $\mathrm{U}$ & -1.28 & HK & 73 & 10 & BSL & $(14.87)$ & $(0.43)$ \\
\hline $\mathrm{V}^{*} \mathrm{AA} \mathrm{Mic}$ & 210041.51 & -391051.3 & GSC & -1.17 & BSL & -85 & 30 & BSL & $\ldots$ & $\ldots$ \\
\hline V* RV Cap & 210128.86 & -151345.2 & HIP & -1.61 & F98 & -99 & 4 & F98 & 11.04 & $\ldots$ \\
\hline BPS CS 22897-0019 & 210225.07 & -634910.3 & $\mathrm{U}$ & -1.34 & $\mathrm{HK}$ & -14 & 10 & BSL & $(14.81)$ & $(0.39)$ \\
\hline BPS CS 29501-0014 & 210306.14 & $\begin{array}{lll}-35 & 16 & 37.7\end{array}$ & $\mathrm{U}$ & -1.62 & $\mathrm{HK}$ & -43 & 10 & BSL & (12.30) & $(0.50)$ \\
\hline BPS CS 22897-0008 & 210311.83 & -650509.0 & $\mathrm{U}$ & -3.34 & B99 & 259 & 10 & HK & 13.33 & 0.69 \\
\hline
\end{tabular}


TABLE 1A. (continued)

\begin{tabular}{|c|c|c|c|c|c|c|c|c|c|c|}
\hline $\begin{array}{l}\text { Star } \\
\text { (1) }\end{array}$ & RA (2000.0) & DEC & $\begin{array}{c}\text { Source }^{\mathrm{a}} \\
(4)\end{array}$ & $\begin{array}{c}{[\mathrm{Fe} / \mathrm{H}]} \\
(\mathrm{dex}) \\
(5)\end{array}$ & $\begin{array}{c}\text { Source }^{b} \\
(6)\end{array}$ & $\begin{array}{c}\mathrm{RV} \\
(\mathrm{km} / \mathrm{s}) \\
(7)\end{array}$ & $\begin{array}{c}\sigma_{\mathrm{RV}} \\
(\mathrm{km} / \mathrm{s}) \\
(8)\end{array}$ & $\begin{array}{c}\text { Source }^{\mathrm{c}} \\
(9)\end{array}$ & $\begin{array}{c}V \\
(\mathrm{mag}) \\
(10)\end{array}$ & $\begin{array}{c}B-V \\
(\mathrm{mag}) \\
(11)\end{array}$ \\
\hline BPS CS 22897-0001 & 210331.48 & -662158.1 & $\mathrm{U}$ & -2.08 & HK & -71 & 10 & BSL & 14.04 & 0.46 \\
\hline BPS CS $22898-0040$ & 210352.06 & -220054.7 & SPM & -2.06 & HK & 153 & 10 & BSL & 14.84 & 0.42 \\
\hline BPS CS 22937-0006 & 210401.38 & $-41 \quad 1235.1$ & SPM & -2.06 & HK & -89 & 10 & BSL & 14.84 & 0.40 \\
\hline BPS CS 30492-0034 & 210430.99 & -380255.0 & SPM & -2.01 & HK & 54 & 10 & BSL & 13.95 & 0.83 \\
\hline BPS CS 30492-0016 & 210449.73 & $-4014 \quad 47.4$ & SPM & -2.30 & HK & 46 & 10 & BSL & 13.89 & 0.45 \\
\hline BPS CS 29501-0004 & 210458.06 & -365718.4 & SPM & -1.83 & BSL & -68 & 10 & BSL & 14.00 & 0.55 \\
\hline BPS CS 29501-0012 & 210522.45 & -353645.3 & SPM & -1.80 & HK & -231 & 10 & BSL & 14.30 & 0.73 \\
\hline BPS CS 30492-0008 & 210543.81 & -411954.2 & SPM & -0.17 & HK & -13 & 10 & BSL & 13.94 & 0.78 \\
\hline BPS CS 22898-0027 & 210545.10 & -183657.6 & STN & -2.36 & B99 & -54 & 10 & HK & 12.76 & 0.50 \\
\hline BPS CS 30492-0001 & 210555.22 & $\begin{array}{lll}-42 & 10 & 09.7\end{array}$ & $\mathrm{SPM}$ & -2.10 & HK & -132 & 10 & BSL & 13.50 & 0.44 \\
\hline BPS CS 22898-0047 & 210559.65 & -210657.0 & $\mathrm{U}$ & -2.54 & HK & -50 & 10 & BSL & 14.24 & 0.56 \\
\hline CD-62 1346 & 210602.94 & -613343.7 & HIP & -1.56 & RL & 127 & 10 & BSL & 9.84 & 0.71 \\
\hline BPS CS 22937-0008 & 210608.41 & -405358.0 & SPM & -0.44 & HK & 50 & 10 & BSL & 13.72 & 0.73 \\
\hline HD 200654 & 210634.58 & -495747.9 & HIP & -2.93 & B99 & -48 & 5 & $\mathrm{CY}$ & 9.09 & 0.58 \\
\hline BPS CS 22897-0017 & $2107 \quad 17.52$ & -633859.1 & $\mathrm{U}$ & -1.97 & HK & 7 & 10 & BSL & 14.33 & 0.42 \\
\hline BPS CS 22937-0004 & 210740.31 & -412751.0 & SPM & -1.97 & HK & 47 & 10 & BSL & 14.71 & 0.41 \\
\hline BPS CS 22937-0039 & 210744.50 & -410317.5 & SPM & -0.87 & HK & 8 & 10 & BSL & 14.51 & 0.54 \\
\hline BPS CS 29501-0032 & 210752.48 & $-35 \quad 1247.3$ & SPM & -2.40 & HK & -150 & 10 & BSL & 14.53 & 0.47 \\
\hline BPS CS 30492-0050 & 210825.62 & -392733.2 & SPM & -1.48 & HK & 72 & 10 & BSL & 14.25 & 0.54 \\
\hline BPS CS 22937-0034 & 210837.60 & -400512.6 & SPM & -1.92 & HK & 83 & 10 & BSL & 14.43 & 0.60 \\
\hline BPS CS 22937-0019 & 210838.94 & -383701.1 & SPM & -1.50 & HK & -53 & 10 & BSL & 13.60 & 0.68 \\
\hline BPS CS 30492-0047 & 210921.62 & -385853.6 & SPM & -0.97 & HK & -71 & 10 & BSL & 14.75 & 0.57 \\
\hline BPS CS 29501-0028 & 210937.04 & -335058.3 & SPM & -1.88 & HK & 19 & 10 & BSL & 13.72 & 0.50 \\
\hline $\mathrm{V}^{*} \mathrm{CP}$ Aqr & $21 \quad 1012.84$ & -014316.5 & $\mathrm{STN}$ & -0.90 & $\mathrm{~L}$ & 29 & 21 & L & 11.71 & $\ldots$ \\
\hline BPS CS 22898-0043 & $21 \quad 1036.77$ & -214451.7 & SPM & -2.69 & HK & 113 & 10 & BSL & 14.06 & 0.43 \\
\hline BPS CS 22937-0023 & 211037.44 & -382422.7 & SPM & -1.38 & HK & -249 & 10 & BSL & 14.74 & 0.60 \\
\hline BPS CS 22897-0042 & 211048.72 & -661852.0 & $\mathrm{U}$ & -1.02 & HK & 55 & 10 & BSL & 14.33 & 0.34 \\
\hline $\mathrm{V}^{*} \mathrm{~V}$ Ind & $21 \quad 1129.96$ & -450427.7 & HIP & -1.50 & F98 & 202 & 2 & F98 & 9.96 & $\cdots$ \\
\hline $\mathrm{V}^{*} \mathrm{AN}$ Mic & 211134.06 & -420237.4 & GSC & -1.25 & BSL & -65 & 30 & BSL & $\ldots$ & $\ldots$ \\
\hline BPS CS 22937-0042 & 211201.27 & -412620.4 & SPM & -2.11 & HK & -123 & 10 & BSL & 13.49 & 0.62 \\
\hline BPS CS 29501-0043 & 211316.80 & -363425.3 & SPM & -1.36 & HK & 20 & 10 & BSL & 14.83 & 0.70 \\
\hline BPS CS 30492-0095 & 211405.10 & -383459.9 & SPM & -1.02 & HK & -44 & 10 & BSL & 13.94 & 0.61 \\
\hline BPS CS 22898-0062 & $21 \quad 14 \quad 10.31$ & -184242.9 & $\mathrm{U}$ & -1.87 & HK & -16 & 10 & BSL & 13.80 & 0.63 \\
\hline BPS CS 22897-0028 & $21 \quad 14 \quad 11.42$ & -624742.8 & U & -1.96 & HK & 38 & 10 & BSL & 14.16 & 0.40 \\
\hline BPS CS $30492-0080$ & 211421.39 & $\begin{array}{lll}-41 & 03 & 48.4\end{array}$ & SPM & -1.20 & HK & -115 & 10 & BSL & 14.78 & 0.66 \\
\hline BPS CS 22937-0072 & 211440.62 & -372451.7 & SPM & -2.19 & HK & -152 & 10 & BSL & 14.01 & 0.51 \\
\hline $\mathrm{V}^{*} \mathrm{SW}$ Aqr & $21 \quad 1517.87$ & +00 0435.4 & HIP & -1.63 & F98 & -50 & 7 & F98 & 11.18 & $\cdots$ \\
\hline BPS CS 22937-0073 & 211529.60 & -372450.9 & SPM & -2.04 & HK & -167 & 10 & BSL & 14.22 & 0.43 \\
\hline $\mathrm{V}^{*} \mathrm{RR} \mathrm{Mic}$ & 211549.32 & -431139.9 & HIP & -1.40 & BSL & 55 & 1 & BSL & 11.40 & $\ldots$ \\
\hline BPS CS 29501-0056 & 211558.21 & -354442.9 & SPM & -1.64 & HK & 13 & 10 & BSL & 14.35 & 0.71 \\
\hline
\end{tabular}


TABLE 1A. (continued)

\begin{tabular}{|c|c|c|c|c|c|c|c|c|c|c|}
\hline $\begin{array}{l}\text { Star } \\
(1)\end{array}$ & RA (2000.0) & DEC & $\begin{array}{c}\text { Source }^{\mathrm{a}} \\
(4)\end{array}$ & $\begin{array}{c}{[\mathrm{Fe} / \mathrm{H}]} \\
(\mathrm{dex}) \\
(5)\end{array}$ & $\begin{array}{c}\text { Source }^{b} \\
(6)\end{array}$ & $\begin{array}{c}\mathrm{RV} \\
(\mathrm{km} / \mathrm{s}) \\
(7)\end{array}$ & $\begin{array}{c}\sigma_{\mathrm{RV}} \\
(\mathrm{km} / \mathrm{s}) \\
(8)\end{array}$ & $\begin{array}{c}\text { Source }^{\mathrm{c}} \\
(9)\end{array}$ & $\begin{array}{c}V \\
(\mathrm{mag}) \\
(10)\end{array}$ & $\begin{array}{c}B-V \\
(\mathrm{mag}) \\
(11)\end{array}$ \\
\hline BPS CS 29501-0055 & 211608.36 & -362928.0 & SPM & -2.19 & HK & -24 & 10 & BSL & 13.83 & 0.64 \\
\hline BPS CS 22937-0054 & 211610.75 & -403515.5 & SPM & -1.78 & HK & 54 & 10 & BSL & 14.80 & 0.42 \\
\hline $\mathrm{V}^{*} \mathrm{Z}$ Mic & 211622.70 & -301703.0 & HIP & -1.10 & F98 & -61 & 2 & F98 & 11.65 & $\ldots$ \\
\hline $\mathrm{BD}+424040$ & 211644.50 & +424106.3 & $\mathrm{ACT}$ & -0.99 & BSL & -263 & 10 & BSL & 8.60 & 0.70 \\
\hline BPS CS 22937-0060 & 211713.11 & -391005.6 & SPM & -0.87 & HK & 51 & 10 & BSL & 14.36 & 0.35 \\
\hline BPS CS 22937-0057 & $21 \quad 17 \quad 18.77$ & -401156.0 & SPM & -1.38 & HK & 23 & 10 & BSL & 14.56 & 0.58 \\
\hline BPS CS 29501-0082 & $21 \quad 1725.41$ & -345341.3 & SPM & -1.84 & HK & 212 & 10 & BSL & 14.93 & 0.64 \\
\hline BPS CS 30492-0114 & 211737.57 & -390606.1 & SPM & -1.78 & HK & -104 & 10 & BSL & 13.40 & 0.50 \\
\hline [MFF90] 95942134 & 211814.14 & +041802.7 & STN & $-1.32:$ & $\mathrm{MFF}$ & -165 & 10 & BSL & 12.01 & $\cdots$ \\
\hline $\mathrm{V}^{*}$ AS Mic & 211814.75 & -421317.3 & GSC & -1.63 & BSL & 4 & 30 & BSL & 14.40 & $\cdots$ \\
\hline [MFF90] 9594299 & 211904.45 & +035301.7 & STN & $-0.65:$ & MFF & -1 & 10 & BSL & 11.69 & $\ldots$ \\
\hline BPS CS 29501-0095 & 211905.64 & -372001.1 & $\mathrm{SPM}$ & -1.94 & BSL & -205 & 10 & BSL & 14.75 & 0.51 \\
\hline [M93] 19171 & 211926.91 & -465053.2 & $\mathrm{U}$ & -2.37 & BSL & 48 & 10 & BSL & 14.51 & 0.97 \\
\hline $\mathrm{V}^{*}$ YZ Cap & 211932.42 & -150701.0 & HIP & -1.06 & F98 & -105 & 3 & F98 & 11.30 & $\ldots$ \\
\hline BPS CS 29501-0075 & 211942.70 & -333401.2 & SPM & -2.20 & HK & 129 & 10 & BSL & 13.71 & 0.64 \\
\hline [M93] 18103 & 211951.43 & -452055.3 & $\mathrm{U}$ & -1.49 & BSL & -90 & 10 & BSL & 13.71 & 0.89 \\
\hline [M93] 18082 & 212004.13 & -454347.4 & $\mathrm{U}$ & -1.41 & BSL & -37 & 10 & BSL & 14.23 & 0.74 \\
\hline [M93] 28118 & 212006.13 & -443515.6 & $\mathrm{U}$ & -1.86 & BSL & 250 & 10 & BSL & 12.62 & 1.43 \\
\hline BPS CS 29506-0019 & 212013.98 & -181246.4 & $\mathrm{U}$ & -2.26 & HK & -207 & 10 & BSL & 14.36 & $(0.67)$ \\
\hline BPS CS 29506-0007 & 212028.66 & -204622.9 & $\mathrm{U}$ & -2.70 & HK & 46 & 10 & BSL & 14.18 & 0.38 \\
\hline [MFF90] 9594249 & 212032.40 & +031659.2 & STN & $-0.79:$ & MFF & -134 & 10 & BSL & 11.16 & $\cdots$ \\
\hline [M93] 18050 & 212033.77 & -462529.5 & U & -1.23 & BSL & -26 & 10 & BSL & 13.61 & 0.86 \\
\hline [MFF90] 959422 & 212034.30 & +024755.5 & $\mathrm{U}$ & $-1.53:$ & $\mathrm{MFF}$ & -140 & 10 & BSL & 12.46 & $\cdots$ \\
\hline [MFF90] 9594272 & 212038.80 & +032857.7 & $\mathrm{U}$ & $-1.72:$ & $\mathrm{MFF}$ & -13 & 10 & BSL & 12.42 & $\cdots$ \\
\hline [MFF90] 9594226 & 212043.73 & +025733.4 & $\mathrm{STN}$ & $-0.62:$ & MFF & -17 & 10 & BSL & 11.59 & $\cdots$ \\
\hline [M93] 27036 & 212052.52 & $\begin{array}{lll}-43 & 10 & 02.4\end{array}$ & $\mathrm{STN}$ & -0.63 & BSL & -38 & 10 & BSL & 11.44 & 1.52 \\
\hline [M93] 18045 & 212109.86 & -445044.9 & $\mathrm{U}$ & -0.95 & BSL & 29 & 10 & BSL & 13.61 & 0.96 \\
\hline [M93] 27009 & 212110.46 & -435207.3 & U & -1.42 & BSL & -95 & 10 & BSL & 14.24 & 0.77 \\
\hline $\mathrm{V}^{*} \mathrm{DM}$ Cyg & 212111.58 & +32 1129.1 & $\mathrm{STN}$ & -0.14 & L & 12 & 23 & L & 11.49 & $\cdots$ \\
\hline [M93] 18002 & 212114.29 & $-4616 \quad 45.5$ & $\mathrm{U}$ & -1.34 & BSL & 19 & 10 & BSL & 14.61 & 0.79 \\
\hline [M93] 18006 & 212126.05 & -454919.4 & GSC & -1.40 & BSL & 90 & 10 & BSL & 11.88 & 0.99 \\
\hline [M93] 17062 & 212136.88 & -464222.7 & $\mathrm{U}$ & -2.16 & BSL & -205 & 10 & BSL & 14.11 & 1.14 \\
\hline BPS CS 30492-0116 & 212138.41 & -395612.8 & SPM & -2.05 & HK & -49 & 10 & BSL & 13.48 & 0.76 \\
\hline [M93] 17089 & 212140.16 & -453810.1 & $\mathrm{STN}$ & -2.35 & BSL & -164 & 10 & BSL & 13.09 & 0.72 \\
\hline BPS CS 30492-0110 & 212145.83 & -380857.8 & $\mathrm{SPM}$ & -2.71 & HK & 44 & 10 & BSL & 14.63 & 0.75 \\
\hline [M93] 26113 & 212148.98 & -424120.8 & SPM & -1.21 & BSL & -37 & 10 & BSL & 13.22 & 0.87 \\
\hline [M93] 17072 & 212154.63 & -454008.8 & U & -2.24 & BSL & -79 & 10 & BSL & 13.09 & 2.10 \\
\hline BPS CS 30492-0113 & 212155.82 & -390832.1 & $\mathrm{SPM}$ & -1.76 & HK & 219 & 10 & BSL & 14.11 & 0.43 \\
\hline [M93] 26049 & 212206.72 & -425504.6 & $\mathrm{U}$ & -2.12 & BSL & 123 & 10 & BSL & 13.43 & 1.06 \\
\hline BPS CS 29501-0099 & 212224.20 & -363314.0 & SPM & -0.19 & HK & 10 & 10 & BSL & 13.99 & 0.72 \\
\hline
\end{tabular}


TABLE 1A. (continued)

\begin{tabular}{|c|c|c|c|c|c|c|c|c|c|c|}
\hline Star & $\begin{array}{c}\text { RA (2000.0) } \\
\text { (2) }\end{array}$ & DEC & $\begin{array}{c}\text { Source }^{\mathrm{a}} \\
(4)\end{array}$ & $\begin{array}{c}{[\mathrm{Fe} / \mathrm{H}]} \\
(\mathrm{dex}) \\
(5)\end{array}$ & $\begin{array}{c}\text { Source }^{b} \\
(6)\end{array}$ & $\begin{array}{c}\mathrm{RV} \\
(\mathrm{km} / \mathrm{s}) \\
(7)\end{array}$ & $\begin{array}{c}\sigma_{\mathrm{RV}} \\
(\mathrm{km} / \mathrm{s}) \\
(8)\end{array}$ & $\begin{array}{c}\text { Source }^{\mathrm{c}} \\
(9)\end{array}$ & $\begin{array}{c}V \\
(\mathrm{mag}) \\
(10)\end{array}$ & $\begin{array}{c}B-V \\
(\mathrm{mag}) \\
(11)\end{array}$ \\
\hline [M93] 17020 & 212224.56 & -461938.7 & GSC & -0.63 & BSL & -57 & 10 & BSL & 11.52 & 1.50 \\
\hline [MFF90] 95931108 & 212229.01 & +041415.4 & U & -0.65 : & MFF & -11 & 10 & BSL & 12.83 & $\ldots$ \\
\hline [M93] 26023 & 212230.16 & -432252.8 & $\mathrm{U}$ & -1.14 & BSL & -117 & 10 & BSL & 13.38 & 0.87 \\
\hline [M93] 17005 & 212240.90 & -460619.0 & $\mathrm{U}$ & -1.85 & BSL & 92 & 10 & BSL & 13.78 & 0.74 \\
\hline [M93] 16090 & 212245.89 & -464103.2 & STN & -2.63 & BSL & -70 & 10 & BSL & 12.28 & 0.84 \\
\hline BPS CS 29506-0030 & 212251.41 & -190556.2 & $\mathrm{U}$ & -0.64 & HK & 37 & 10 & BSL & $(14.80)$ & $(0.40)$ \\
\hline [M93] 25104 & 212255.35 & -443148.0 & $\mathrm{U}$ & -1.55 & BSL & -90 & 10 & BSL & 14.16 & $\ldots$ \\
\hline [M93] 16070 & 212323.83 & -444720.3 & $\mathrm{U}$ & -0.95 & BSL & -16 & 10 & BSL & 13.80 & 0.98 \\
\hline [M93] 25072 & 212324.59 & -435256.4 & STN & -2.24 & BSL & -66 & 10 & BSL & 13.64 & 0.77 \\
\hline [MFF90] 9591136 & 212329.05 & +070817.5 & STN & $-0.71:$ & MFF & -5 & 10 & BSL & 11.84 & $\cdots$ \\
\hline [MFF90] 9591194 & 212343.30 & +062207.2 & STN & -0.80 & B99 & -66 & 10 & HK & 11.24 & 0.85 \\
\hline [MFF90] 9593187 & 212357.33 & +042352.8 & $\mathrm{U}$ & -1.22 : & $\mathrm{MFF}$ & 5 & 10 & BSL & 12.80 & $\cdots$ \\
\hline [MFF90] 9591158 & 212358.89 & +065150.1 & STN & -0.87 & B99 & 3 & 10 & HK & 11.92 & 0.88 \\
\hline BPS CS 29506-0036 & 212440.79 & -201305.8 & $\mathrm{U}$ & -1.66 & HK & -60 & 10 & BSL & (13.98) & $(0.52)$ \\
\hline [MFF90] 95911142 & 212440.92 & +054610.5 & $\mathrm{U}$ & $-0.79:$ & $\mathrm{MFF}$ & -62 & 10 & BSL & 12.11 & $\ldots$ \\
\hline [M93] 14080 & 212447.25 & -461710.5 & STN & -1.39 & BSL & -70 & 10 & BSL & 12.17 & 0.81 \\
\hline [M93] 28066 & 212449.58 & -440743.1 & $\mathrm{U}$ & -1.14 & BSL & 16 & 10 & BSL & 13.42 & 0.99 \\
\hline [MFF90] 95931167 & 212456.55 & +034247.2 & STN & -1.76 : & MFF & -25 & 10 & BSL & 11.08 & $\cdots$ \\
\hline [MFF90] 9591151 & 212508.96 & +065630.9 & STN & -0.61 & B99 & 4 & 10 & HK & 11.47 & 0.98 \\
\hline [M93] 28065 & 212512.21 & -430824.1 & U & -1.54 & BSL & 44 & 10 & BSL & 13.19 & 1.04 \\
\hline BPS CS 22937-0090 & 212516.74 & -413643.4 & SPM & -2.08 & HK & 26 & 10 & BSL & 15.25 & 0.49 \\
\hline [M93] 14053 & 212517.36 & -455336.7 & $\mathrm{U}$ & -1.37 & BSL & -36 & 10 & BSL & 15.29 & 0.81 \\
\hline [M93] 23058 & 212531.67 & -444431.6 & STN & -1.91 & BSL & 161 & 10 & BSL & 12.13 & 1.35 \\
\hline BPS CS 29506-0057 & 212532.52 & -212343.7 & U & -0.83 & HK & -28 & 10 & BSL & $(14.26)$ & $(0.44)$ \\
\hline [MFF90] 95911163 & 212542.83 & +052949.6 & $\mathrm{U}$ & $-1.06:$ & $\mathrm{MFF}$ & -211 & 10 & BSL & 12.31 & 1.37 \\
\hline [MFF90] 959112 & 212550.48 & +073152.2 & $\mathrm{U}$ & $-0.88:$ & $\mathrm{MFF}$ & 79 & 10 & BSL & 12.45 & $\cdots$ \\
\hline [M93] 24010 & 212551.41 & -431846.7 & U & -1.75 & BSL & -99 & 10 & BSL & 12.94 & 0.67 \\
\hline [M93] 13101 & 212553.33 & -460611.3 & STN & -1.07 & BSL & -80 & 10 & BSL & 13.51 & 0.74 \\
\hline [MFF90] 95931190 & 212555.43 & +032735.0 & STN & -0.69 : & $\mathrm{MFF}$ & -1 & 10 & BSL & 12.41 & $\cdots$ \\
\hline [MFF90] 9591139 & 212630.15 & +070551.3 & STN & -0.66 : & MFF & -9 & 10 & BSL & 10.82 & $\cdots$ \\
\hline [MFF90] 959113 & 212645.53 & +072926.7 & STN & -0.62 & B99 & -46 & 10 & HK & 11.52 & 0.95 \\
\hline BPS CS 30492-0133 & 212645.71 & -405152.0 & $\mathrm{SPM}$ & -2.10 & HK & 15 & 10 & BSL & 13.72 & 0.81 \\
\hline [M93] 13016 & 212648.92 & -465913.0 & $\mathrm{U}$ & -1.58 & BSL & 12 & 10 & BSL & 13.49 & 1.13 \\
\hline $\mathrm{V}^{*}$ AO Peg & 212704.89 & +183801.9 & STN & -0.92 & L & 115 & 35 & L & 12.83 & $\cdots$ \\
\hline [M93] 12085 & 212709.87 & -464342.4 & STN & -1.52 & BSL & -73 & 10 & BSL & 13.43 & $\cdots$ \\
\hline [M93] 12046 & 212741.63 & -463104.4 & $\mathrm{U}$ & -2.02 & BSL & -3 & 10 & BSL & 13.69 & 0.66 \\
\hline [MFF90] 95932190 & 212750.94 & +030714.9 & $\mathrm{U}$ & -0.66 : & $\mathrm{MFF}$ & -19 & 10 & BSL & 13.18 & $\cdots$ \\
\hline [M93] 19047 & 212751.59 & -470342.9 & $\mathrm{U}$ & -1.20 & BSL & 63 & 10 & BSL & 13.21 & 0.81 \\
\hline [MFF90] 9591244 & 212755.74 & +064630.0 & $\mathrm{U}$ & $-1.54:$ & MFF & -51 & 10 & BSL & 12.68 & $\cdots$ \\
\hline [MFF90] 9593212 & 212800.65 & +050637.8 & $\mathrm{U}$ & $-0.61:$ & $\mathrm{MFF}$ & -42 & 10 & BSL & 12.75 & $\cdots$ \\
\hline
\end{tabular}


TABLE 1A. (continued)

\begin{tabular}{|c|c|c|c|c|c|c|c|c|c|c|}
\hline Star & $\begin{array}{c}\text { RA (2000.0) } \\
(2)\end{array}$ & $\begin{array}{l}\text { DEC } \\
(3)\end{array}$ & $\begin{array}{c}\text { Source }^{\mathrm{a}} \\
(4)\end{array}$ & $\begin{array}{c}{[\mathrm{Fe} / \mathrm{H}]} \\
(\mathrm{dex}) \\
(5)\end{array}$ & $\begin{array}{c}\text { Source }^{\mathrm{b}} \\
(6)\end{array}$ & $\begin{array}{c}\mathrm{RV} \\
(\mathrm{km} / \mathrm{s}) \\
(7)\end{array}$ & $\begin{array}{c}\sigma_{\mathrm{RV}} \\
(\mathrm{km} / \mathrm{s}) \\
(8)\end{array}$ & $\begin{array}{c}\text { Source }^{\mathrm{c}} \\
(9)\end{array}$ & $\begin{array}{c}V \\
(\mathrm{mag}) \\
(10)\end{array}$ & $\begin{array}{c}B-V \\
(\mathrm{mag}) \\
(11)\end{array}$ \\
\hline BD-03 5215 & 212801.31 & -030740.7 & HIP & -1.57 & B99 & -294 & 1 & $\mathrm{CY}$ & 10.17 & 0.72 \\
\hline $\mathrm{V}^{*} \mathrm{SS}$ Gru & 212806.32 & -370935.3 & SPM & -2.35 & $\mathrm{~L}$ & -63 & 14 & L & 12.77 & $\ldots$ \\
\hline [MFF90] 9593287 & 212832.07 & +041404.5 & STN & -1.20 & $\mathrm{MFF}$ & -315 & 10 & BSL & 11.38 & 1.33 \\
\hline [M93] 12029 & 212840.52 & -452708.3 & STN & -2.56 & BSL & -85 & 10 & BSL & 12.92 & 0.66 \\
\hline HD 235518 & 212844.17 & +504933.2 & HIP & -2.57 & BSL & -23 & 10 & BSL & 8.27 & 1.23 \\
\hline [MFF90] 9593298 & 212846.32 & +040912.4 & $\mathrm{STN}$ & $-1.26:$ & $\mathrm{MFF}$ & -35 & 10 & BSL & 12.17 & $\cdots$ \\
\hline $\mathrm{BD}+463330$ & 212848.98 & +470654.5 & STN & -1.93 & BSL & -9 & 10 & BSL & 9.30 & 0.90 \\
\hline [M93] 22013 & 212915.93 & -423621.9 & SPM & -2.96 & BSL & -124 & 10 & BSL & 12.94 & 1.02 \\
\hline BPS CS 29506-0089 & 212919.26 & -220200.3 & $\mathrm{U}$ & -1.58 & HK & -295 & 10 & BSL & $(13.76)$ & $(0.44)$ \\
\hline $\mathrm{BD}+473427$ & 212924.26 & +475523.8 & $\mathrm{ACT}$ & -0.64 & BSL & 6 & 10 & BSL & 9.60 & 0.90 \\
\hline HD 204543 & 212928.21 & -033055.0 & HIP & -1.76 & B99 & -98 & 1 & $\mathrm{CY}$ & 8.28 & 0.89 \\
\hline [M93] 11079 & 212928.92 & -460920.0 & $\mathrm{U}$ & -1.42 & BSL & -32 & 10 & BSL & 13.78 & 0.74 \\
\hline [M93] 21083 & 212940.76 & -443450.3 & $\mathrm{STN}$ & -0.91 & BSL & 60 & 10 & BSL & 12.72 & 1.06 \\
\hline [M93] 19019 & 212942.58 & -465159.1 & $\mathrm{U}$ & -1.33 & BSL & -122 & 10 & BSL & 14.21 & 0.78 \\
\hline [M93] 11059 & 212958.38 & $\begin{array}{lll}-45 & 04 & 03.4\end{array}$ & $\mathrm{STN}$ & -1.83 & BSL & -34 & 10 & BSL & 12.06 & 0.66 \\
\hline [M93] 19013 & 213027.43 & -445551.6 & $\mathrm{U}$ & -2.31 & BSL & -254 & 10 & BSL & 14.27 & 0.73 \\
\hline BPS CS 29506-0090 & 213028.88 & $-22 \quad 1041.2$ & SPM & -3.00 & HK & -17 & 10 & BSL & 14.33 & 0.40 \\
\hline [M93] 21043 & 213030.59 & -433004.1 & U & -1.24 & BSL & 7 & 10 & BSL & 13.48 & 0.95 \\
\hline [M93] 11024 & 213031.38 & -455816.4 & $\mathrm{U}$ & -0.81 & BSL & -4 & 10 & BSL & 14.35 & 1.18 \\
\hline [MFF90] 9593259 & 213034.41 & +043617.5 & $\mathrm{U}$ & $-0.89:$ & $\mathrm{MFF}$ & -72 & 10 & BSL & 12.69 & $\cdots$ \\
\hline BPS CS 29506-0069 & 213054.10 & -174644.4 & $\mathrm{U}$ & -2.04 & HK & -95 & 10 & BSL & $(14.78)$ & $(0.42)$ \\
\hline [M93] 21004 & 213054.47 & -442337.1 & $\mathrm{U}$ & -0.87 & BSL & 142 & 10 & BSL & 12.65 & 0.88 \\
\hline BPS CS 29506-0068 & 213114.12 & -174028.0 & $\mathrm{U}$ & -1.26 & $\mathrm{HK}$ & -12 & 10 & BSL & $(14.12)$ & $(0.68)$ \\
\hline [M93] 44096 & 213115.70 & -434304.8 & $\mathrm{U}$ & -3.62 & BSL & -31 & 10 & BSL & 14.71 & 0.98 \\
\hline [MFF90] 95932108 & 213120.92 & +040560.0 & $\mathrm{U}$ & $-0.91:$ & $\mathrm{MFF}$ & -13 & 10 & BSL & 13.27 & $\cdots$ \\
\hline [M93] 44078 & 213125.78 & -441626.4 & $\mathrm{U}$ & -1.75 & BSL & 232 & 10 & BSL & 14.67 & 0.71 \\
\hline [M93] 44088 & 213127.77 & $-42 \quad 47 \quad 13.7$ & SPM & -2.34 & BSL & -447 & 10 & BSL & 13.54 & 0.80 \\
\hline [M93] 46144 & 213139.47 & -444450.7 & $\mathrm{U}$ & -1.95 & BSL & 149 & 10 & BSL & 13.89 & 0.72 \\
\hline [M93] 34041 & 213145.55 & -463108.2 & $\mathrm{U}$ & -1.27 & BSL & -38 & 10 & BSL & 13.62 & 1.07 \\
\hline [M93] 44052 & 213154.40 & -423541.0 & SPM & -3.44 & BSL & -25 & 10 & BSL & 14.43 & 1.09 \\
\hline [M93] 46143 & 213155.16 & -42 2709.8 & SPM & -1.26 & BSL & 37 & 10 & BSL & 14.12 & 0.93 \\
\hline BPS CS 22897-0102 & 213157.25 & -66 2129.4 & $\mathrm{U}$ & -2.12 & $\mathrm{HK}$ & -72 & 10 & BSL & 14.76 & 0.51 \\
\hline [M93] 44032 & 213208.07 & -424141.1 & GSC & -0.80 & BSL & 42 & 10 & BSL & 14.40 & $\ldots$ \\
\hline [M93] 44005 & 213220.49 & -435139.1 & $\mathrm{STN}$ & -1.57 & BSL & 36 & 10 & BSL & 12.48 & 0.99 \\
\hline BPS CS 22897-0099 & 213221.81 & -654442.4 & $\mathrm{U}$ & -2.41 & HK & 39 & 10 & BSL & 14.41 & 0.69 \\
\hline BPS CS 22948-0012 & 213222.21 & -380725.5 & SPM & -0.69 & HK & -1 & 10 & BSL & 12.90 & 0.80 \\
\hline BPS CS 29495-0005 & 213249.17 & -273408.5 & SPM & -2.20 & HK & -145 & 10 & BSL & 14.32 & 0.41 \\
\hline BPS CS 29506-0109 & 213257.39 & $-1852 \quad 36.2$ & U & -1.96 & HK & 60 & 10 & BSL & $(14.31)$ & $(0.49)$ \\
\hline BPS CS 22948-0006 & 213317.70 & -393942.7 & SPM & -2.09 & HK & 73 & 10 & BSL & 15.07 & 0.49 \\
\hline [M93] 43059 & 213335.15 & -442810.1 & $\mathrm{U}$ & -1.83 & BSL & 7 & 10 & BSL & 13.41 & 0.75 \\
\hline
\end{tabular}


TABLE 1A. (continued)

\begin{tabular}{|c|c|c|c|c|c|c|c|c|c|c|}
\hline Star & RA (2000.0) & DEC & $\begin{array}{c}\text { Source }^{\mathrm{a}} \\
(4)\end{array}$ & $\begin{array}{c}{[\mathrm{Fe} / \mathrm{H}]} \\
(\mathrm{dex}) \\
(5)\end{array}$ & $\begin{array}{c}\text { Source }^{b} \\
(6)\end{array}$ & $\begin{array}{c}\mathrm{RV} \\
(\mathrm{km} / \mathrm{s}) \\
(7)\end{array}$ & $\begin{array}{c}\sigma_{\mathrm{RV}} \\
(\mathrm{km} / \mathrm{s}) \\
(8)\end{array}$ & $\begin{array}{c}\text { Source }^{c} \\
(9)\end{array}$ & $\begin{array}{c}V \\
(\mathrm{mag}) \\
(10)\end{array}$ & $\begin{array}{c}B-V \\
(\mathrm{mag}) \\
(11)\end{array}$ \\
\hline [M93] 33058 & 213340.06 & -455031.5 & $\mathrm{U}$ & -1.05 & BSL & 9 & 10 & BSL & 12.31 & 1.42 \\
\hline [M93] 33015 & 213401.62 & -444827.6 & $\mathrm{STN}$ & -1.62 & BSL & 51 & 10 & BSL & 12.20 & 1.05 \\
\hline $\mathrm{V}^{*} \mathrm{Z}$ Gru & 213432.16 & -490830.5 & $\mathrm{v}$ & -1.55 & BSL & 23 & 30 & BSL & 12.30 & $\ldots$ \\
\hline [M93] 42163 & 213439.69 & -442524.6 & $\mathrm{U}$ & -1.32 & BSL & 75 & 10 & BSL & 13.53 & 0.89 \\
\hline [M93] 32141 & 213442.78 & -445836.8 & STN & -1.72 & BSL & -70 & 10 & BSL & 12.59 & 0.93 \\
\hline [M93] 42146 & 213444.86 & -432202.3 & $\mathrm{U}$ & -2.04 & BSL & 259 & 10 & BSL & 13.89 & 0.83 \\
\hline BPS CS 29495-0002 & 213446.98 & $-2757 \quad 17.6$ & SPM & -2.19 & HK & -161 & 10 & BSL & 13.32 & 0.50 \\
\hline [M93] 37006 & 213525.68 & -470135.1 & GSC & -2.18 & BSL & 57 & 10 & BSL & 13.97 & 0.69 \\
\hline $\mathrm{BD}+433979$ & 213528.15 & +435814.0 & $\mathrm{ACT}$ & -0.74 & BSL & -47 & 10 & BSL & 9.20 & 0.90 \\
\hline BPS CS 22948-0024 & 213531.67 & -391209.7 & SPM & -2.03 & HK & -84 & 10 & BSL & 14.65 & 0.59 \\
\hline [M93] 46112 & 213531.75 & -423157.3 & SPM & -1.19 & BSL & 6 & 10 & BSL & 14.32 & 0.79 \\
\hline BPS CS 29506-0106 & 213532.01 & -195816.0 & $\mathrm{U}$ & -1.51 & HK & -17 & 10 & BSL & $(14.99)$ & $(0.51)$ \\
\hline $\mathrm{V}^{*} \mathrm{SX}$ Aqr & 213608.47 & +031350.4 & HIP & -1.87 & F98 & -165 & 3 & F98 & 11.78 & $\ldots$ \\
\hline $\mathrm{V}^{*} \mathrm{RY}$ Oct & 213609.38 & -771813.3 & HIP & -1.83 & F98 & 37 & 10 & F98 & 12.06 & $\cdots$ \\
\hline [M93] 32022 & 213632.42 & -452550.7 & $\mathrm{U}$ & -1.78 & BSL & -34 & 10 & BSL & 13.69 & 0.71 \\
\hline BPS CS 29495-0041 & 213633.29 & $-28 \quad 1848.3$ & SPM & -2.93 & HK & 77 & 10 & BSL & 13.30 & 0.90 \\
\hline [M93] 32010 & 213649.89 & -461156.9 & $\mathrm{v}$ & -1.60 & BSL & -9 & 10 & BSL & 13.49 & 0.72 \\
\hline HD 205547 & 213706.37 & -404052.7 & HIP & -1.85 & BSL & 47 & 10 & BSL & 8.60 & 1.14 \\
\hline BPS CS 22948-0019 & 213707.85 & $\begin{array}{llll}-37 & 51 & 04.2\end{array}$ & SPM & -1.96 & HK & 4 & 10 & BSL & 15.43 & 0.55 \\
\hline [M93] 36131 & 213721.12 & -450320.6 & $\mathrm{U}$ & -1.61 & BSL & 87 & 10 & BSL & 13.76 & 0.93 \\
\hline [M93] 41114 & 213721.14 & -434603.7 & STN & -2.07 & BSL & 47 & 10 & BSL & 13.38 & 0.80 \\
\hline [M93] 45105 & 213721.14 & $\begin{array}{lll}-43 & 46 & 03.7\end{array}$ & $\mathrm{STN}$ & -2.02 & BSL & 33 & 10 & BSL & 13.36 & 0.80 \\
\hline [M93] 31181 & 213722.33 & $\begin{array}{lll}-45 & 01 & 04.3\end{array}$ & $\mathrm{U}$ & -1.88 & BSL & -23 & 10 & BSL & 14.15 & 0.76 \\
\hline BPS CS 22948-0041 & 213728.48 & -413456.2 & $\mathrm{v}$ & -2.35 & HK & 12 & 10 & BSL & 14.49 & 0.66 \\
\hline BPS CS 22897-0125 & 213742.73 & -640335.7 & GSC & -1.84 & HK & 7 & 10 & BSL & 13.72 & 0.42 \\
\hline [M93] 31167 & 213743.70 & -451827.3 & $\mathrm{U}$ & -2.20 & BSL & -129 & 10 & BSL & 14.24 & 0.78 \\
\hline [M93] 45080 & 213756.70 & $\begin{array}{lll}-43 & 07 & 18.4\end{array}$ & $\mathrm{U}$ & -1.16 & BSL & 28 & 10 & BSL & 13.38 & 0.83 \\
\hline $\mathrm{V}^{*} \mathrm{RR}$ Gru & 213803.52 & -444112.3 & STN & -0.45 & L & 37 & 14 & L & 12.54 & $\cdots$ \\
\hline [M93] 31121 & 213856.65 & -461610.4 & $\mathrm{U}$ & -1.80 & BSL & 66 & 10 & BSL & 14.04 & 0.65 \\
\hline BPS CS 22897-0126 & 213906.28 & -640529.3 & $\mathrm{U}$ & -2.02 & HK & 16 & 10 & BSL & 14.87 & 0.37 \\
\hline $\mathrm{BD}+404598$ & 213906.42 & +404927.0 & $\mathrm{ACT}$ & -0.72 & BSL & -166 & 10 & BSL & 9.00 & 0.70 \\
\hline BPS CS 29495-0031 & 213911.52 & -243103.9 & SPM & -2.12 & HK & -225 & 10 & BSL & 13.25 & 0.58 \\
\hline [M93] 36067 & 213914.58 & -450637.9 & U & -1.36 & BSL & 40 & 10 & BSL & 13.88 & 0.76 \\
\hline [M93] 31083 & 213940.98 & -463346.5 & $\mathrm{U}$ & -1.76 & BSL & -19 & 10 & BSL & 13.99 & 0.75 \\
\hline BPS CS 22948-0057 & 214015.80 & -385639.3 & SPM & -2.07 & HK & -17 & 10 & BSL & 13.98 & 0.59 \\
\hline BPS CS 22951-0018 & 214016.98 & -450737.6 & $\mathrm{U}$ & -1.64 & HK & 136 & 10 & BSL & $(14.29)$ & $(0.61)$ \\
\hline BPS CS 22951-0025 & 214033.45 & -430331.4 & $\mathrm{U}$ & -1.78 & HK & 114 & 10 & BSL & $(14.40)$ & $(0.50)$ \\
\hline BPS CS 22951-0027 & 214041.49 & -425307.9 & GSC & -2.03 & HK & -208 & 10 & BSL & 14.85 & 0.56 \\
\hline BPS CS 22951-0014 & 214056.89 & -454344.5 & $\mathrm{U}$ & -1.00 & HK & -22 & 10 & BSL & $(13.98)$ & $(0.52)$ \\
\hline BPS CS 22944-0014 & $2141 \quad 12.21$ & -143332.0 & $\mathrm{U}$ & -2.17 & HK & -370 & 10 & BSL & 14.18 & 0.43 \\
\hline
\end{tabular}


TABLE 1A. (continued)

\begin{tabular}{|c|c|c|c|c|c|c|c|c|c|c|}
\hline $\begin{array}{l}\text { Star } \\
(1)\end{array}$ & $\begin{array}{c}\text { RA (2000.0) } \\
(2)\end{array}$ & $\begin{array}{c}\text { DEC } \\
(3)\end{array}$ & $\begin{array}{c}\text { Source }^{\mathrm{a}} \\
(4)\end{array}$ & $\begin{array}{c}{[\mathrm{Fe} / \mathrm{H}]} \\
(\mathrm{dex}) \\
(5)\end{array}$ & $\begin{array}{c}\text { Source }^{b} \\
(6)\end{array}$ & $\begin{array}{c}\mathrm{RV} \\
(\mathrm{km} / \mathrm{s}) \\
(7)\end{array}$ & $\begin{array}{c}\sigma_{\mathrm{RV}} \\
(\mathrm{km} / \mathrm{s}) \\
(8)\end{array}$ & $\begin{array}{c}\text { Source }^{c} \\
(9)\end{array}$ & $\begin{array}{c}V \\
(\mathrm{mag}) \\
(10)\end{array}$ & $\begin{array}{c}B-V \\
(\mathrm{mag}) \\
(11)\end{array}$ \\
\hline$V^{*}$ CG Peg & $2141 \quad 16.79$ & +244624.5 & HIP & -0.50 & F98 & -6 & 2 & F98 & 11.18 & $\cdots$ \\
\hline BPS CS 22944-0011 & 214126.41 & -151246.5 & STN & -2.06 & HK & -97 & 10 & BSL & 12.00 & 0.41 \\
\hline [M93] 36018 & 214130.53 & -461312.6 & U & -1.12 & BSL & 33 & 10 & BSL & 14.39 & 0.73 \\
\hline BPS CS 22944-0002 & 214157.09 & -164402.6 & $\mathrm{U}$ & -1.56 & HK & -388 & 10 & BSL & 14.63 & 0.40 \\
\hline [M93] 34118 & 214205.68 & -444636.8 & STN & -1.92 & BSL & -189 & 10 & BSL & 12.33 & 0.83 \\
\hline [M93] 34119 & $2142 \quad 22.39$ & -462346.3 & U & -0.75 & BSL & -80 & 10 & BSL & 12.92 & 0.97 \\
\hline HD 235596 & 214232.91 & +514434.9 & $\mathrm{ACT}$ & -0.72 & BSL & -22 & 10 & BSL & 9.40 & 1.00 \\
\hline BPS CS 22956-0017 & 214239.06 & -643337.2 & U & -2.93 & BSL & -11 & 10 & BSL & 14.29 & 0.16 \\
\hline BPS CS 22956-0021 & 214324.72 & -641445.6 & $\mathrm{U}$ & -2.32 & HK & 36 & 10 & BSL & 14.70 & 0.38 \\
\hline HD 206739 & 214423.92 & -114622.6 & HIP & -1.56 & B99 & -58 & 1 & $\mathrm{CY}$ & 8.56 & 1.00 \\
\hline HD 206642 & 214429.41 & -383307.7 & HIP & -0.75 & $\mathrm{C} 97$ & -58 & 10 & BSL & 6.28 & 1.13 \\
\hline BPS CS 22956-0013 & 214429.49 & $\begin{array}{lll}-65 & 09 & 03.2\end{array}$ & $\mathrm{v}$ & -0.81 & BSL & -40 & 10 & BSL & {$[13.10]$} & $\ldots$ \\
\hline $\mathrm{V}^{*} \mathrm{UW}$ Oct & 214438.39 & -803344.2 & GSC & -0.49 & L & 66 & 25 & L & 12.86 & $\ldots$ \\
\hline BPS CS 22948-0066 & 214451.17 & -372754.9 & SPM & -3.10 & B99 & -169 & 10 & HK & 13.47 & 0.63 \\
\hline BPS CS 22944-0039 & $2145 \quad 12.29$ & -144124.3 & U & -2.12 & HK & -253 & 10 & BSL & 14.30 & 0.55 \\
\hline BD-09 5831 & 214530.52 & -082435.1 & HIP & -1.83 & B99 & 15 & 1 & $\mathrm{CY}$ & 10.39 & 0.98 \\
\hline BPS CS 22951-0005 & 214546.60 & -460458.2 & $\mathrm{U}$ & -2.17 & HK & 235 & 10 & BSL & {$[14.90]$} & [0.39] \\
\hline BPS CS 22948-0045 & 214548.46 & $\begin{array}{lll}-42 & 05 & 01.5\end{array}$ & SPM & -0.69 & HK & -79 & 10 & BSL & 14.16 & 0.78 \\
\hline BPS CS 29493-0037 & 214550.08 & -315337.1 & SPM & -2.13 & HK & -115 & 10 & BSL & 14.49 & 0.49 \\
\hline BPS CS 22948-0075 & 214618.06 & -382546.4 & SPM & -2.06 & HK & -46 & 10 & BSL & 15.05 & 0.39 \\
\hline BPS CS 22951-0042 & 214621.00 & -450720.7 & STN & -1.57 & BSL & 248 & 10 & BSL & {$[13.70]$} & $\cdots$ \\
\hline BPS CS 22951-0033 & 214635.77 & -430628.4 & $\mathrm{U}$ & -0.84 & HK & 11 & 10 & BSL & $(14.29)$ & $(0.51)$ \\
\hline BPS CS 22948-0070 & 214637.02 & -373357.5 & SPM & -0.85 & HK & 2 & 10 & BSL & 14.14 & 0.59 \\
\hline BPS CS 22948-0067 & 214654.01 & -371134.1 & SPM & -1.45 & HK & -37 & 10 & BSL & 14.08 & 0.40 \\
\hline $\mathrm{V}^{*} \mathrm{RS}$ Oct & 214715.45 & -873906.4 & STN & -1.60 & $\mathrm{~L}$ & 215 & 14 & L & 12.80 & $\ldots$ \\
\hline BPS CS 22948-0079 & 214729.20 & -392519.0 & SPM & -1.69 & HK & -55 & 10 & BSL & 13.72 & 0.35 \\
\hline BPS CS 29493-0023 & 214730.16 & -275506.6 & SPM & -2.50 & HK & 37 & 10 & BSL & 14.59 & 0.38 \\
\hline BPS CS 29495-0074 & 214732.60 & -273138.0 & SPM & -0.20 & HK & 50 & 10 & BSL & 13.97 & 0.71 \\
\hline BPS CS 29495-0071 & 214737.68 & -260936.9 & SPM & -1.86 & HK & -43 & 10 & BSL & 13.26 & 0.62 \\
\hline BPS CS 22944-0035 & 214739.72 & -135538.3 & U & -1.90 & HK & -188 & 10 & BSL & 14.02 & 0.52 \\
\hline BPS CS 22944-0032 & 214743.25 & -134020.7 & $\mathrm{U}$ & -2.46 & HK & 30 & 10 & BSL & 13.28 & 0.59 \\
\hline HD 206998 & 214743.98 & -553915.0 & HIP & -1.12 & BSL & -90 & 10 & BSL & 8.69 & 0.55 \\
\hline BPS CS 29493-0044 & 214757.61 & -315056.6 & SPM & -2.02 & HK & -29 & 10 & BSL & 14.67 & 0.43 \\
\hline BPS CS 22956-0004 & 214832.23 & -655619.5 & $\mathrm{U}$ & -2.17 & HK & -23 & 10 & BSL & (13.59) & $(0.51)$ \\
\hline BPS CS 22951-0037 & 214840.62 & -442529.3 & $\mathrm{U}$ & -2.16 & HK & 59 & 10 & BSL & (14.66) & $(0.44)$ \\
\hline BPS CS 29493-0043 & 214917.37 & -315918.4 & SPM & -0.95 & HK & -76 & 10 & BSL & 14.52 & 0.55 \\
\hline BPS CS 29493-0050 & 214926.68 & $-2945 \quad 24.5$ & SPM & -2.79 & HK & -95 & 10 & BSL & 14.39 & 0.38 \\
\hline BPS CS 22948-0077 & 214949.80 & -383754.2 & SPM & -1.45 & HK & 131 & 10 & BSL & 14.92 & 0.72 \\
\hline BPS CS 29493-0049 & 215017.51 & -295437.8 & SPM & -1.28 & HK & -61 & 10 & BSL & 14.19 & 0.52 \\
\hline BPS CS 22944-0061 & 215018.26 & -134524.0 & U & -2.54 & HK & 153 & 10 & BSL & 14.35 & 0.43 \\
\hline
\end{tabular}


TABLE 1A. (continued)

\begin{tabular}{|c|c|c|c|c|c|c|c|c|c|c|}
\hline $\begin{array}{l}\text { Star } \\
(1)\end{array}$ & $\begin{array}{c}\text { RA (2000.0) } \\
(2)\end{array}$ & DEC & $\begin{array}{c}\text { Source }^{\mathrm{a}} \\
(4)\end{array}$ & $\begin{array}{c}{[\mathrm{Fe} / \mathrm{H}]} \\
(\mathrm{dex}) \\
(5)\end{array}$ & $\begin{array}{c}\text { Source }^{b} \\
(6)\end{array}$ & $\begin{array}{c}\mathrm{RV} \\
(\mathrm{km} / \mathrm{s}) \\
(7)\end{array}$ & $\begin{array}{c}\sigma_{\mathrm{RV}} \\
(\mathrm{km} / \mathrm{s}) \\
(8)\end{array}$ & $\begin{array}{c}\text { Source }^{\mathrm{c}} \\
(9)\end{array}$ & $\begin{array}{c}V \\
(\mathrm{mag}) \\
(10)\end{array}$ & $\begin{array}{c}B-V \\
(\mathrm{mag}) \\
(11)\end{array}$ \\
\hline BPS CS 22956-0015 & 215020.49 & -644904.3 & $\mathrm{U}$ & -2.44 & HK & -22 & 10 & BSL & 14.19 & 0.39 \\
\hline BPS CS 29495-0080 & 215022.11 & -271053.3 & SPM & -1.75 & HK & -46 & 10 & BSL & 14.10 & 0.57 \\
\hline BPS CS 22951-0059 & 215025.98 & -444849.2 & $\mathrm{U}$ & -2.40 & HK & 80 & 10 & BSL & $(14.44)$ & $(0.56)$ \\
\hline BPS CS 22948-0093 & 215031.53 & $\begin{array}{lll}-41 & 07 & 49.2\end{array}$ & SPM & -3.72 & HK & 395 & 10 & BSL & 15.18 & 0.36 \\
\hline $\mathrm{BD}+434063$ & 215040.42 & +435042.7 & $\mathrm{ACT}$ & -1.05 & BSL & 18 & 10 & BSL & 9.80 & 0.70 \\
\hline BPS CS 22951-0052 & 215053.72 & -465523.2 & U & -1.19 & BSL & 42 & 10 & BSL & 13.90 & $\ldots$ \\
\hline BPS CS 22948-0091 & 215105.01 & -413756.0 & SPM & -2.01 & HK & 80 & 10 & BSL & 14.12 & 0.61 \\
\hline BPS CS 22951-0069 & $2151 \quad 13.47$ & $-42 \quad 4756.1$ & U & -2.06 & HK & 26 & 10 & BSL & $(14.08)$ & $(0.52)$ \\
\hline BPS CS 29493-0052 & 215128.44 & -292418.1 & SPM & -1.77 & HK & -121 & 10 & BSL & 13.80 & 0.44 \\
\hline BPS CS 22948-0104 & 215145.77 & -375230.8 & SPM & -2.42 & HK & 7 & 10 & BSL & 13.96 & 0.59 \\
\hline V* RT Gru & 215158.48 & -455907.0 & STN & -1.49 & $\mathrm{~L}$ & -67 & 10 & $\mathrm{~L}$ & 12.79 & $\cdots$ \\
\hline $\mathrm{V}^{*}$ AV Peg & 215202.79 & +223429.4 & HIP & -0.08 & F98 & -58 & 1 & F98 & 10.50 & $\cdots$ \\
\hline BPS CS 22951-0065 & 215208.42 & -430410.8 & $\mathrm{U}$ & -1.11 & HK & -15 & 10 & BSL & $(14.71)$ & $(0.49)$ \\
\hline BPS CS 29493-0058 & 215219.74 & -273959.5 & SPM & -1.96 & HK & 142 & 10 & BSL & 14.13 & 0.77 \\
\hline BPS CS 29493-0062 & 215231.02 & -280937.3 & SPM & -2.24 & HK & 15 & 10 & BSL & 13.17 & 0.46 \\
\hline BPS CS 29493-0084 & 215318.77 & -322112.6 & U & -1.96 & HK & 133 & 10 & BSL & $(14.71)$ & $(0.39)$ \\
\hline BPS CS 29493-0059 & 215325.61 & -275718.6 & SPM & -2.34 & HK & -151 & 10 & BSL & 13.54 & 0.85 \\
\hline $\mathrm{V}^{*} \mathrm{SS}$ Oct & 215335.36 & $-8246 \quad 43.5$ & HIP & -1.60 & F98 & 312 & 14 & F98 & 11.91 & $\cdots$ \\
\hline HD 208069 & 215425.92 & -301523.5 & HIP & -1.83 & ATT & -167 & 10 & BSL & 9.22 & 0.69 \\
\hline BPS CS 29493-0064 & 215427.01 & -283608.9 & $\mathrm{SPM}$ & -1.23 & HK & 48 & 10 & BSL & 13.81 & 0.58 \\
\hline BPS CS 22951-0055 & 215444.84 & -463126.6 & $\mathrm{U}$ & -1.84 & HK & 362 & 10 & BSL & $(14.78)$ & $(0.42)$ \\
\hline BPS CS 29493-0066 & 215512.24 & -290049.9 & SPM & -0.74 & HK & -31 & 10 & BSL & 13.64 & 0.94 \\
\hline BPS CS 29493-0061 & 215519.48 & $-28 \quad 1500.9$ & SPM & -1.96 & HK & -10 & 10 & BSL & 13.21 & 0.54 \\
\hline BPS CS 22881-0017 & 215543.03 & -393145.7 & SPM & -2.16 & HK & 31 & 10 & BSL & 14.22 & 0.57 \\
\hline BPS CS 29493-0060 & 215601.41 & $\begin{array}{lll}-28 & 00 & 03.1\end{array}$ & SPM & -1.75 & HK & -132 & 10 & BSL & 14.81 & 0.62 \\
\hline $\mathrm{BD}+473617$ & 215702.84 & +482249.9 & $\mathrm{ACT}$ & -1.76 & BSL & -22 & 10 & BSL & 10.30 & 0.60 \\
\hline BPS CS 22965-0016 & 215707.91 & -043407.7 & $\mathrm{U}$ & -2.45 & HK & -90 & 10 & BSL & 14.05 & 0.75 \\
\hline HD 208360 & 215708.26 & -494151.9 & HIP & -1.96 & BSL & 78 & 10 & BSL & 7.63 & 0.64 \\
\hline BPS CS 29493-0095 & 215720.43 & -295908.4 & SPM & -2.19 & HK & -186 & 10 & BSL & 14.73 & 0.48 \\
\hline BPS CS 29493-0094 & 215727.37 & $\begin{array}{lll}-30 & 08 & 02.4\end{array}$ & SPM & -2.44 & HK & -205 & 10 & BSL & 14.12 & 0.38 \\
\hline BPS CS 22951-0077 & 215753.21 & -430808.1 & $\mathrm{U}$ & -2.17 & HK & 122 & 10 & BSL & 13.61 & 0.50 \\
\hline BPS CS 22956-0050 & 215805.83 & -651327.3 & $\mathrm{U}$ & -2.82 & HK & 2 & 10 & BSL & 14.27 & 0.68 \\
\hline BPS CS 22956-0052 & 215819.51 & -660827.4 & $\mathrm{U}$ & -1.62 & HK & 153 & 10 & BSL & $(14.90)$ & $(0.40)$ \\
\hline BPS CS 22881-0010 & 215825.31 & -405327.1 & SPM & -2.04 & HK & -8 & 10 & BSL & 14.80 & 0.40 \\
\hline BPS CS 22881-0006 & 215853.48 & -413625.4 & SPM & -1.48 & HK & -164 & 10 & BSL & 14.98 & 0.41 \\
\hline BPS CS 29493-0090 & 215905.78 & $-31 \quad 1612.1$ & SPM & -3.09 & HK & 262 & 10 & BSL & 14.07 & 0.79 \\
\hline BPS CS 22965-0035 & 215929.52 & -062745.5 & NPM & -1.88 & HK & -142 & 10 & BSL & 14.64 & $(0.35)$ \\
\hline BPS CS 22881-0008 & 215931.67 & -412412.1 & SPM & -2.47 & HK & 188 & 10 & BSL & 14.29 & 0.83 \\
\hline BPS CS 22965-0029 & 215932.19 & -033637.5 & $\mathrm{U}$ & -2.26 & HK & -32 & 10 & BSL & 13.76 & $(0.62)$ \\
\hline $\mathrm{BD}+463527$ & 220000.81 & +465826.4 & $\mathrm{ACT}$ & -0.60 & BSL & -92 & 10 & BSL & 9.30 & 0.90 \\
\hline
\end{tabular}


TABLE 1A. (continued)

\begin{tabular}{|c|c|c|c|c|c|c|c|c|c|c|}
\hline $\begin{array}{l}\text { Star } \\
(1)\end{array}$ & $\begin{array}{c}\text { RA (2000.0) } \\
(2)\end{array}$ & DEC & $\begin{array}{c}\text { Source }^{\mathrm{a}} \\
(4)\end{array}$ & $\begin{array}{c}{[\mathrm{Fe} / \mathrm{H}]} \\
(\mathrm{dex}) \\
(5)\end{array}$ & $\begin{array}{c}\text { Source }^{b} \\
(6)\end{array}$ & $\begin{array}{c}\mathrm{RV} \\
(\mathrm{km} / \mathrm{s}) \\
(7)\end{array}$ & $\begin{array}{c}\sigma_{\mathrm{RV}} \\
(\mathrm{km} / \mathrm{s}) \\
(8)\end{array}$ & $\begin{array}{c}\text { Source }^{c} \\
(9)\end{array}$ & $\begin{array}{c}V \\
(\mathrm{mag}) \\
(10)\end{array}$ & $\begin{array}{c}B-V \\
(\mathrm{mag}) \\
(11)\end{array}$ \\
\hline BPS CS 29493-0092 & 220002.01 & -302022.5 & SPM & -1.94 & HK & -57 & 10 & BSL & 14.02 & 0.82 \\
\hline BPS CS 22951-0117 & 220017.85 & -435125.9 & STN & -2.05 & BSL & -149 & 10 & BSL & 13.86 & 0.41 \\
\hline BPS CS 22892-0014 & 220121.72 & $-14 \quad 1651.4$ & U & -1.76 & HK & 2 & 10 & BSL & 14.32 & 0.44 \\
\hline $\mathrm{V}^{*} \mathrm{TZ}$ Aqr & 220155.53 & -053602.8 & STN & -1.24 & L & -35 & 12 & L & 12.01 & $\ldots$ \\
\hline BPS CS 22881-0028 & 220253.84 & -405404.0 & SPM & -1.96 & HK & -105 & 10 & BSL & 14.31 & 0.39 \\
\hline $\mathrm{V}^{*} \mathrm{BV}$ Aqr & 220254.00 & -213132.0 & HIP & -1.42 & F98 & -239 & 3 & F98 & 10.90 & $\ldots$ \\
\hline BPS CS 22881-0032 & 220300.59 & -411426.2 & SPM & -2.91 & HK & -35 & 10 & BSL & 15.23 & 0.36 \\
\hline BPS CS 22965-0052 & 220303.91 & -031156.4 & U & -1.92 & HK & -242 & 10 & BSL & $(13.94)$ & $(0.66)$ \\
\hline BPS CS 22951-0108 & 220318.02 & $\begin{array}{lll}-46 & 02 & 42.1\end{array}$ & $\mathrm{U}$ & -2.19 & HK & 20 & 10 & BSL & 14.30 & 0.46 \\
\hline BPS CS 22956-0081 & 220411.87 & -625051.5 & $\mathrm{U}$ & -2.50 & HK & 210 & 10 & BSL & 15.18 & 0.38 \\
\hline $\mathrm{BD}+463563$ & 220415.27 & +472416.6 & $\mathrm{ACT}$ & -1.75 & BSL & 0 & 10 & BSL & 10.10 & 0.70 \\
\hline V* SX Gru & 220421.42 & -452505.4 & GSC & -1.63 & BSL & 28 & 30 & BSL & 14.20 & $\cdots$ \\
\hline BPS CS 22951-0115 & 220443.33 & -441036.6 & $\mathrm{U}$ & -1.74 & BSL & 50 & 10 & BSL & 14.03 & 0.40 \\
\hline BPS CS 22892-0005 & 220454.00 & -155010.4 & $\mathrm{U}$ & -1.76 & HK & -110 & 10 & BSL & 14.10 & 0.43 \\
\hline BPS CS 22965-0043 & 220530.62 & -062151.8 & $\mathrm{U}$ & -1.36 & HK & -5 & 10 & BSL & 13.88 & $(0.51)$ \\
\hline BPS CS 22965-0054 & 220630.20 & -023234.0 & $\mathrm{U}$ & -3.15 & HK & -298 & 10 & BSL & 14.65 & 1.34 \\
\hline BPS CS 22892-0024 & 220643.99 & -150903.5 & $\mathrm{U}$ & -2.19 & HK & -83 & 10 & BSL & 14.74 & 0.49 \\
\hline $\mathrm{BD}+473694$ & $2206 \quad 48.54$ & +475209.2 & $\mathrm{ACT}$ & -0.77 & BSL & -113 & 10 & BSL & 9.40 & 0.90 \\
\hline BPS CS 22965-0068 & 220712.92 & -042914.9 & $\mathrm{U}$ & -1.75 & HK & -178 & 10 & BSL & $(13.71)$ & $(0.59)$ \\
\hline BPS CS 22956-0062 & 220726.32 & -660856.7 & $\mathrm{U}$ & -2.08 & HK & 189 & 10 & BSL & 14.84 & 0.47 \\
\hline BPS CS 22892-0025 & 220742.89 & -151459.0 & $\mathrm{U}$ & -2.32 & HK & 168 & 10 & BSL & 14.03 & 0.39 \\
\hline BPS CS 22881-0036 & 220758.41 & -404415.6 & SPM & -2.42 & HK & 50 & 10 & BSL & 13.96 & 0.48 \\
\hline BPS CS 22960-0010 & 220825.57 & -445355.9 & $\mathrm{U}$ & -2.77 & HK & 41 & 10 & BSL & 14.21 & 0.57 \\
\hline BPS CS 22881-0040 & 220841.12 & -394512.1 & SPM & -2.27 & HK & 133 & 10 & BSL & 13.33 & 0.69 \\
\hline BPS CS 22892-0041 & 220901.75 & -124751.3 & U & -1.96 & HK & 39 & 10 & BSL & 14.33 & 0.40 \\
\hline BPS CS 22881-0039 & 220935.42 & -402551.2 & SPM & -3.24 & HK & 74 & 10 & BSL & 15.14 & 0.39 \\
\hline HD 210295 & 220941.47 & -133618.7 & HIP & -1.36 & B99 & -19 & 10 & HK & 9.55 & 0.89 \\
\hline BPS CS 22965-0076 & 221006.49 & -062328.0 & $\mathrm{U}$ & -1.14 & HK & -1 & 10 & BSL & $(14.59)$ & $(0.41)$ \\
\hline BPS CS 22965-0087 & 221124.41 & -025810.0 & $\mathrm{U}$ & -1.90 & HK & -45 & 10 & BSL & {$[15.10]$} & {$[0.45]$} \\
\hline BPS CS 22960-0029 & 221135.54 & -442522.1 & $\mathrm{U}$ & -2.50 & HK & 47 & 10 & BSL & 13.47 & $(0.74)$ \\
\hline BPS CS 22965-0075 & 221158.14 & -065234.1 & $\mathrm{U}$ & -1.96 & HK & -234 & 10 & BSL & 14.44 & $(0.48)$ \\
\hline BPS CS 22892-0046 & 221224.00 & -145325.4 & $\mathrm{U}$ & -1.54 & HK & -98 & 10 & BSL & 14.55 & 0.41 \\
\hline BPS CS 29512-0007 & 221247.01 & -093451.5 & $\mathrm{U}$ & -0.44 & HK & 68 & 10 & BSL & (13.77) & $(0.63)$ \\
\hline BPS CS 22956-0085 & 221250.96 & -624538.7 & $\mathrm{U}$ & -2.02 & HK & 100 & 10 & BSL & (14.44) & $(0.46)$ \\
\hline $\mathrm{V}^{*} \mathrm{VV}$ Peg & 221303.91 & +182703.9 & STN & -1.88 & $\mathrm{~L}$ & 13 & 8 & L & 11.79 & $\ldots$ \\
\hline BPS CS 22886-0008 & 221324.41 & -093419.1 & U & -2.16 & HK & -54 & 10 & BSL & 14.44 & 0.47 \\
\hline BPS CS 22886-0012 & 221325.87 & -084342.8 & $\mathrm{U}$ & -2.39 & HK & -81 & 10 & BSL & 14.52 & 0.47 \\
\hline BPS CS 29512-0030 & 221352.03 & -104700.9 & NPM & -2.50 & HK & -73 & 10 & BSL & 14.64 & $(0.43)$ \\
\hline BPS CS 22881-0060 & 221414.73 & $\begin{array}{lll}-40 & 02 & 10.4\end{array}$ & SPM & -2.12 & HK & -97 & 10 & BSL & 14.27 & 0.40 \\
\hline HD 211075 & 221420.04 & +1801 13.4 & HIP & -0.61 & BSL & 6 & 10 & BSL & 8.19 & 1.22 \\
\hline
\end{tabular}


TABLE 1A. (continued)

\begin{tabular}{|c|c|c|c|c|c|c|c|c|c|c|}
\hline $\begin{array}{l}\text { Star } \\
(1)\end{array}$ & $\begin{array}{c}\text { RA (2000.0) } \\
(2)\end{array}$ & DEC & $\begin{array}{c}\text { Source }^{\mathrm{a}} \\
(4)\end{array}$ & $\begin{array}{c}{[\mathrm{Fe} / \mathrm{H}]} \\
(\mathrm{dex}) \\
(5)\end{array}$ & $\begin{array}{c}\text { Source }^{b} \\
(6)\end{array}$ & $\begin{array}{c}\mathrm{RV} \\
(\mathrm{km} / \mathrm{s}) \\
(7)\end{array}$ & $\begin{array}{c}\sigma_{\mathrm{RV}} \\
(\mathrm{km} / \mathrm{s}) \\
(8)\end{array}$ & $\begin{array}{c}\text { Source }^{c} \\
(9)\end{array}$ & $\begin{array}{c}V \\
(\mathrm{mag}) \\
(10)\end{array}$ & $\begin{array}{c}B-V \\
(\operatorname{mag}) \\
(11)\end{array}$ \\
\hline BPS CS 29512-0031 & 221425.04 & -105139.2 & $\mathrm{U}$ & -1.35 & HK & 23 & 10 & BSL & $(14.15)$ & $(0.65)$ \\
\hline $\mathrm{V}^{*} \mathrm{YZ}$ Aqr & 221430.60 & -105546.5 & GSC & -1.55 & $\mathrm{~L}$ & -150 & 14 & L & 12.65 & $\ldots$ \\
\hline BPS CS 29512-0032 & 221430.60 & -105546.1 & STN & -1.76 & HK & -138 & 10 & BSL & $(12.17)$ & $(0.43)$ \\
\hline BPS CS 22960-0027 & 221433.78 & -435206.9 & $\mathrm{U}$ & -1.29 & HK & -81 & 10 & BSL & $(14.36)$ & $(0.44)$ \\
\hline HD 235766 & 221447.17 & +504715.4 & $\mathrm{ACT}$ & -2.35 & BSL & -314 & 10 & BSL & 9.10 & 1.10 \\
\hline BPS CS 22960-0036 & 221450.90 & -464808.0 & U & -1.17 & HK & 25 & 10 & BSL & $(14.38)$ & $(0.42)$ \\
\hline BPS CS 22956-0105 & $22 \quad 1519.14$ & -654833.3 & $\mathrm{U}$ & -0.67 & HK & -60 & 10 & BSL & $(14.27)$ & $(0.53)$ \\
\hline BPS CS 29512-0024 & 221521.21 & -09 1959.9 & $\mathrm{U}$ & -1.39 & HK & -17 & 10 & BSL & $(13.35)$ & $(0.55)$ \\
\hline V* DH Peg & 221525.63 & +06 4921.4 & HIP & -1.24 & F98 & -70 & 1 & F98 & 9.55 & $\ldots$ \\
\hline BPS CS 22886-0029 & $22 \quad 16 \quad 11.22$ & -100636.6 & $\mathrm{U}$ & -0.65 & HK & -19 & 10 & BSL & $(13.92)$ & $(0.58)$ \\
\hline BPS CS 29512-0037 & 221618.84 & -125449.8 & $\mathrm{U}$ & -1.26 & HK & 7 & 10 & BSL & $(12.20)$ & $(0.80)$ \\
\hline BPS CS 22886-0022 & 221623.66 & -084223.0 & $\mathrm{U}$ & -1.56 & HK & -191 & 10 & BSL & 13.97 & 0.40 \\
\hline BPS CS 22956-0102 & 221636.55 & -651944.9 & $\mathrm{U}$ & -2.55 & HK & -1 & 10 & BSL & 15.10 & 0.44 \\
\hline BPS CS 22881-0070 & $22 \quad 1637.05$ & -404406.1 & SPM & -2.70 & HK & -120 & 10 & BSL & 14.39 & 0.37 \\
\hline $\mathrm{V}^{*}$ TY Gru & 221639.42 & -395617.9 & SPM & -2.16 & BSL & -5 & 10 & BSL & 14.04 & 0.60 \\
\hline BPS CS 22886-0018 & 221645.13 & -072143.9 & $\mathrm{U}$ & -1.99 & HK & -221 & 10 & BSL & 14.59 & 0.38 \\
\hline BPS CS 22956-0106 & 221650.34 & -660504.6 & $\mathrm{U}$ & -2.79 & HK & 201 & 10 & BSL & 14.47 & 0.38 \\
\hline BPS CS 29512-0026 & 221652.03 & -095433.1 & $\mathrm{U}$ & -1.96 & HK & -226 & 10 & BSL & $(14.68)$ & $(0.42)$ \\
\hline BPS CS 22960-0048 & $22 \quad 1701.54$ & -451216.6 & $\mathrm{U}$ & -3.22 & HK & -85 & 10 & BSL & 14.99 & 0.65 \\
\hline BPS CS 22892-0052 & 221701.63 & -163926.7 & $\mathrm{U}$ & -3.01 & B99 & 10 & 10 & HK & 13.18 & 0.78 \\
\hline BPS CS 22886-0028 & 221809.40 & -100150.1 & U & -2.17 & HK & -192 & 10 & BSL & 15.49 & 0.40 \\
\hline BPS CS 22886-0026 & 221842.80 & -094241.6 & NPM & -1.78 & HK & -70 & 10 & BSL & 14.89 & 0.43 \\
\hline BPS CS 22960-0040 & 221852.05 & $\begin{array}{lll}-46 & 03 & 11.2\end{array}$ & $\mathrm{U}$ & -1.76 & HK & 109 & 10 & BSL & $(15.38)$ & $(0.42)$ \\
\hline BPS CS 22960-0037 & 221853.53 & -463934.9 & $\mathrm{U}$ & -1.98 & HK & 95 & 10 & BSL & $(15.36)$ & $(0.44)$ \\
\hline BPS CS 22892-0056 & 221912.14 & -160708.8 & $\mathrm{U}$ & -1.84 & HK & 55 & 10 & BSL & 14.11 & 0.46 \\
\hline BPS CS 22956-0110 & 221943.51 & -664121.9 & $\mathrm{U}$ & -2.79 & HK & -97 & 10 & BSL & 14.79 & 0.38 \\
\hline BPS CS 29512-0043 & 222002.61 & -114307.1 & $\mathrm{U}$ & -2.26 & HK & -1 & 10 & BSL & 13.42 & $(0.51)$ \\
\hline HD 211744 & $22 \quad 2009.93$ & -442152.6 & HIP & -1.03 & B99 & 1 & 10 & HK & 9.12 & 0.75 \\
\hline BPS CS 22886-0044 & 222011.49 & -094137.2 & $\mathrm{U}$ & -1.71 & $\mathrm{HK}$ & -143 & 10 & BSL & 14.23 & 0.51 \\
\hline $\mathrm{V}^{*} \mathrm{UW}$ Gru & 222013.13 & -543328.8 & STN & -1.68 & $\mathrm{~L}$ & 81 & 36 & $\mathrm{~L}$ & 13.20 & $\cdots$ \\
\hline BPS CS 22886-0042 & 222025.82 & -102319.8 & $\mathrm{U}$ & -2.67 & HK & -218 & 10 & BSL & 13.26 & 0.77 \\
\hline BPS CS 22960-0041 & 222040.83 & -455350.3 & $\mathrm{U}$ & -1.67 & HK & 29 & 10 & BSL & $(15.40)$ & $(0.40)$ \\
\hline BPS CS 29512-0048 & 222048.31 & -122520.0 & $\mathrm{U}$ & -1.15 & HK & -25 & 10 & BSL & $(13.61)$ & $(0.49)$ \\
\hline BPS CS 22956-0126 & 222052.38 & -633743.1 & $\mathrm{U}$ & -1.66 & HK & 166 & 10 & BSL & $(15.03)$ & $(0.37)$ \\
\hline BPS CS 22875-0004 & $22 \quad 2103.44$ & -403123.6 & SPM & -1.56 & HK & -123 & 10 & BSL & 13.84 & 0.37 \\
\hline BPS CS 22886-0055 & 222108.00 & -080049.5 & U & -2.06 & HK & -100 & 10 & BSL & $(14.57)$ & $(0.43)$ \\
\hline $\mathrm{V}^{*} \mathrm{CQ} \mathrm{Lac}$ & 222110.47 & +3943 14.2 & GSC & -2.04 & $\mathrm{~L}$ & 20 & 30 & L & 12.43 & $\ldots$ \\
\hline BPS CS 29512-0047 & 222118.99 & -124123.0 & $\mathrm{U}$ & -0.84 & HK & -1 & 10 & BSL & $(13.97)$ & $(0.63)$ \\
\hline BPS CS 29512-0052 & 222121.05 & -100558.7 & $\mathrm{U}$ & -0.36 & HK & -4 & 10 & BSL & $(13.82)$ & $(0.58)$ \\
\hline BPS CS 22956-0116 & 222124.29 & -653358.9 & $\mathrm{U}$ & -2.24 & HK & 49 & 10 & BSL & (13.33) & $(0.47)$ \\
\hline
\end{tabular}


TABLE 1A. (continued)

\begin{tabular}{|c|c|c|c|c|c|c|c|c|c|c|}
\hline $\begin{array}{l}\text { Star } \\
(1)\end{array}$ & $\begin{array}{c}\text { RA (2000.0) } \\
(2)\end{array}$ & $\begin{array}{c}\text { DEC } \\
(3)\end{array}$ & $\begin{array}{c}\text { Source }^{\mathrm{a}} \\
(4)\end{array}$ & $\begin{array}{c}{[\mathrm{Fe} / \mathrm{H}]} \\
(\mathrm{dex}) \\
(5)\end{array}$ & $\begin{array}{c}\text { Source }^{b} \\
(6)\end{array}$ & $\begin{array}{c}\mathrm{RV} \\
(\mathrm{km} / \mathrm{s}) \\
(7)\end{array}$ & $\begin{array}{c}\sigma_{\mathrm{RV}} \\
(\mathrm{km} / \mathrm{s}) \\
(8)\end{array}$ & $\begin{array}{c}\text { Source }^{c} \\
(9)\end{array}$ & $\begin{array}{c}V \\
(\mathrm{mag}) \\
(10)\end{array}$ & $\begin{array}{c}B-V \\
(\mathrm{mag}) \\
(11)\end{array}$ \\
\hline BPS CS 22960-0060 & $22 \quad 2204.22$ & $\begin{array}{lll}-42 & 08 & 02.4\end{array}$ & SPM & -2.30 & HK & -64 & 10 & BSL & 15.57 & 0.32 \\
\hline BPS CS 29512-0069 & 222231.83 & -123357.3 & $\mathrm{U}$ & -1.07 & HK & -55 & 10 & BSL & $(14.71)$ & $(0.69)$ \\
\hline BPS CS 22886-0043 & $22 \quad 2233.78$ & -101408.7 & $\mathrm{U}$ & -1.97 & HK & -61 & 10 & BSL & 14.72 & 0.46 \\
\hline BPS CS 22875-0012 & 222342.57 & $\begin{array}{lll}-39 & 12 & 10.1\end{array}$ & SPM & -2.12 & HK & -37 & 10 & BSL & 14.49 & 0.40 \\
\hline BPS CS 22960-0062 & 222343.23 & -421203.5 & SPM & -1.76 & HK & 37 & 10 & BSL & 13.57 & 0.51 \\
\hline BPS CS 22960-0064 & 222400.13 & -423516.0 & SPM & -2.98 & HK & -74 & 10 & BSL & 13.82 & 0.58 \\
\hline BPS CS 22960-0068 & 222401.51 & -431823.7 & U & -2.44 & HK & -34 & 10 & BSL & {$[15.60]$} & {$[0.39]$} \\
\hline HD 212516 & 222434.43 & +160900.5 & HIP & -0.64 & BSL & 44 & 10 & BSL & 8.72 & 1.62 \\
\hline BPS CS 22956-0117 & 222442.93 & -645827.0 & $\mathrm{U}$ & -1.99 & HK & 33 & 10 & BSL & $(14.08)$ & $(0.52)$ \\
\hline BPS CS 29512-0066 & 222449.86 & -110038.8 & $\mathrm{U}$ & -1.96 & HK & -226 & 10 & BSL & (13.80) & $(0.50)$ \\
\hline BPS CS 22886-0064 & 222509.95 & -091158.2 & $\mathrm{U}$ & -1.80 & HK & -320 & 10 & BSL & 14.42 & 0.62 \\
\hline BPS CS 22960-0080 & $22 \quad 2511.73$ & -451520.7 & $\mathrm{U}$ & -1.88 & HK & 14 & 10 & BSL & $(15.53)$ & $(0.37)$ \\
\hline BPS CS 29512-0072 & 222547.86 & -123317.3 & $\mathrm{U}$ & -0.13 & HK & 20 & 10 & BSL & $(13.95)$ & $(0.65)$ \\
\hline BPS CS 22960-0078 & 222605.60 & -443449.0 & $\mathrm{U}$ & -1.76 & HK & -14 & 10 & BSL & $(14.16)$ & $(0.44)$ \\
\hline BPS CS 22956-0114 & 222608.19 & -653306.7 & $\mathrm{U}$ & -2.62 & BSL & -47 & 10 & BSL & 14.04 & 0.60 \\
\hline V* UY Gru & 222620.23 & -405928.9 & SPM & -1.78 & BSL & 119 & 10 & BSL & 14.47 & 0.45 \\
\hline BPS CS 22960-0089 & 222641.42 & -464402.7 & $\mathrm{U}$ & -1.87 & HK & 95 & 10 & BSL & $(15.01)$ & $(0.39)$ \\
\hline BPS CS 22886-0080 & 222656.63 & -074719.1 & $\mathrm{U}$ & -1.57 & HK & -114 & 10 & BSL & $(14.34)$ & $(0.56)$ \\
\hline BPS CS 29512-0083 & 222704.17 & -085058.3 & $\mathrm{U}$ & -0.13 & HK & -3 & 10 & BSL & $(12.62)$ & $(0.58)$ \\
\hline BPS CS 22956-0121 & 222720.02 & -642814.4 & $\mathrm{U}$ & -2.60 & HK & 114 & 10 & BSL & 14.86 & 0.39 \\
\hline $\mathrm{V}^{*}$ AE Peg & 222721.50 & +164816.7 & STN & -1.84 & $\mathrm{~L}$ & -409 & 23 & L & 12.79 & $\cdots$ \\
\hline BPS CS 29512-0076 & 222743.06 & -112124.2 & NPM & -1.97 & HK & -8 & 10 & BSL & 13.96 & $(0.48)$ \\
\hline BPS CS 22960-0088 & 222743.15 & -463526.6 & $\mathrm{U}$ & -0.99 & HK & 29 & 10 & BSL & {$[15.10]$} & {$[0.62]$} \\
\hline $\mathrm{V}^{*} \mathrm{BN}$ Aqr & $22 \quad 2748.78$ & -072902.2 & NPM & -1.33 & $\mathrm{~L}$ & -182 & 30 & L & 12.52 & $\ldots$ \\
\hline BPS CS 22875-0028 & 222826.43 & -393953.2 & SPM & -1.38 & HK & 70 & 10 & BSL & 13.53 & 0.58 \\
\hline BPS CS 29512-0073 & 222836.81 & -122441.7 & U & -2.33 & HK & -75 & 10 & BSL & $(13.92)$ & $(0.58)$ \\
\hline BPS CS 22875-0029 & 222925.08 & -385747.4 & SPM & -2.54 & HK & 70 & 10 & BSL & 13.68 & 0.40 \\
\hline BPS CS 22960-0105 & 222949.97 & -432528.1 & U & -0.70 & HK & -13 & 10 & BSL & $(12.98)$ & $(0.62)$ \\
\hline BPS CS 22875-0026 & 223114.06 & -394109.8 & SPM & -2.04 & HK & -114 & 10 & BSL & 14.07 & 0.54 \\
\hline HD 213487 & 223203.30 & -213556.6 & HIP & -1.25 & BSL & 3 & 10 & BSL & 9.87 & 0.84 \\
\hline HD 213467 & $2232 \quad 07.70$ & -311023.6 & HIP & -1.45 & ATT & -73 & 10 & BSL & 8.52 & 0.69 \\
\hline HD 213657 & 223347.10 & -420311.6 & HIP & -1.98 & B99 & 48 & 10 & HK & 9.65 & 0.43 \\
\hline BPS CS 22887-0005 & 223430.69 & -102633.8 & $\mathrm{U}$ & -2.02 & HK & -130 & 10 & BSL & 14.70 & $(0.49)$ \\
\hline BPS CS 22887-0007 & 223512.59 & -094024.9 & $\mathrm{U}$ & -2.45 & HK & -80 & 10 & BSL & $(14.62)$ & $(0.58)$ \\
\hline $\mathrm{V}^{*}$ AA Aqr & 223603.82 & -100055.2 & GSC & -2.09 & $\mathrm{~L}$ & -20 & 14 & L & 12.37 & $\ldots$ \\
\hline BPS CS 22887-0006 & 223616.69 & -09 5243.3 & $\mathrm{v}$ & -1.84 & HK & -305 & 10 & BSL & 16.00 & 0.49 \\
\hline HD 214161 & 223708.04 & -403038.4 & HIP & -2.16 & ATT & -371 & 10 & BSL & 9.10 & 0.77 \\
\hline HD 214362 & 223758.56 & -223839.7 & HIP & -1.77 & B99 & -92 & 1 & $\mathrm{CY}$ & 9.10 & 0.50 \\
\hline BPS CS 22887-0018 & 223808.78 & -095516.4 & $\mathrm{U}$ & -1.34 & BSL & -80 & 10 & BSL & {$[13.40]$} & $\cdots$ \\
\hline BPS CS 22875-0038 & 223826.78 & -391238.9 & SPM & -1.28 & HK & -86 & 10 & BSL & 13.94 & 0.58 \\
\hline
\end{tabular}


TABLE 1A. (continued)

\begin{tabular}{|c|c|c|c|c|c|c|c|c|c|c|}
\hline $\begin{array}{l}\text { Star } \\
(1)\end{array}$ & $\begin{array}{c}\text { RA (2000.0) } \\
(2)\end{array}$ & DEC & $\begin{array}{c}\text { Source }^{\mathrm{a}} \\
(4)\end{array}$ & $\begin{array}{c}{[\mathrm{Fe} / \mathrm{H}]} \\
(\mathrm{dex}) \\
(5)\end{array}$ & $\begin{array}{c}\text { Source }^{b} \\
(6)\end{array}$ & $\begin{array}{c}\mathrm{RV} \\
(\mathrm{km} / \mathrm{s}) \\
(7)\end{array}$ & $\begin{array}{c}\sigma_{\mathrm{RV}} \\
(\mathrm{km} / \mathrm{s}) \\
(8)\end{array}$ & $\begin{array}{c}\text { Source }^{\mathrm{c}} \\
(9)\end{array}$ & $\begin{array}{c}V \\
(\mathrm{mag}) \\
(10)\end{array}$ & $\begin{array}{c}B-V \\
(\mathrm{mag}) \\
(11)\end{array}$ \\
\hline $\mathrm{V}^{*} \mathrm{RZ}$ Cep & 223913.05 & +645128.9 & HIP & -1.77 & F98 & 9 & 3 & F98 & 9.47 & $\cdots$ \\
\hline HD 214925 & 224159.42 & -204703.3 & HIP & -2.14 & BSL & -328 & 10 & BSL & 9.30 & 1.49 \\
\hline V* RW Gru & $2242 \quad 07.00$ & -440912.4 & STN & -2.00 & L & 0 & 10 & L & 12.39 & $\ldots$ \\
\hline$V^{*}$ DX Cep & $2244 \quad 19.10$ & +835702.6 & STN & -1.83 & $\mathrm{~L}$ & -6 & 30 & $\mathrm{~L}$ & 12.67 & $\ldots$ \\
\hline BPS CS 22887-0048 & 224644.63 & -110842.6 & STN & -1.72 & $\mathrm{HK}$ & -84 & 10 & BSL & $(12.70)$ & $(0.40)$ \\
\hline HD 215601 & 224648.05 & -315218.1 & HIP & -1.56 & ATT & -35 & 10 & BSL & 8.46 & 0.78 \\
\hline BPS CS 22887-0047 & 224735.23 & $-1047 \quad 16.3$ & $\mathrm{U}$ & -1.66 & HK & -24 & 10 & BSL & $(13.77)$ & $(0.53)$ \\
\hline HD 215801 & 224829.40 & -460347.9 & HIP & -2.45 & BSL & -86 & 10 & BSL & 10.04 & 0.45 \\
\hline HD 216143 & 225031.13 & -065448.6 & HIP & -2.16 & B99 & -116 & 1 & $\mathrm{CY}$ & 7.80 & 0.95 \\
\hline BPS CS 22938-0027 & 225245.61 & -674243.3 & $\mathrm{U}$ & -0.44 & HK & -15 & 10 & BSL & (13.30) & $(0.60)$ \\
\hline $\mathrm{V}^{*} \mathrm{BH} \mathrm{Peg}$ & 225301.05 & +154717.2 & HIP & -1.22 & F98 & -275 & 3 & F98 & 10.46 & $\ldots$ \\
\hline BPS CS 22887-0054 & 225324.05 & -105920.5 & $\mathrm{U}$ & -1.13 & BSL & 27 & 10 & BSL & {$[14.10]$} & $\ldots$ \\
\hline BPS CS 22893-0005 & 225350.73 & -095914.9 & $\mathrm{U}$ & -2.25 & HK & -84 & 10 & BSL & 14.22 & 0.53 \\
\hline $\mathrm{V}^{*} \mathrm{BO}$ Aqr & 225408.16 & -122138.5 & GSC & -1.80 & $\mathrm{~L}$ & -24 & 13 & L & 12.11 & $\ldots$ \\
\hline BPS CS 22893-0006 & 225440.10 & -100325.6 & $\mathrm{U}$ & -1.11 & HK & -58 & 10 & BSL & 14.28 & 0.53 \\
\hline BPS CS 22893-0010 & $2258 \quad 11.74$ & -115644.6 & $\mathrm{U}$ & -2.46 & HK & -26 & 10 & BSL & 14.74 & 0.59 \\
\hline BPS CS 30493-0016 & 230051.17 & -343941.0 & SPM & -1.26 & HK & 7 & 10 & BSL & 13.88 & 0.89 \\
\hline BPS CS 22893-0011 & 230052.32 & -103524.2 & $\mathrm{U}$ & -2.04 & HK & -116 & 10 & BSL & 14.54 & 0.43 \\
\hline BPS CS 30493-0014 & 230101.55 & -345036.0 & SPM & -2.20 & HK & 24 & 10 & BSL & 14.82 & 0.42 \\
\hline HD 217515 & 230127.84 & -181818.1 & HIP & -0.82 & BSL & -5 & 10 & BSL & 9.40 & 0.36 \\
\hline BPS CS 22888-0010 & 230200.02 & $-3341 \quad 18.0$ & SPM & -1.97 & HK & -116 & 10 & BSL & 14.45 & 0.40 \\
\hline BPS CS 22893-0030 & 230205.68 & -075846.8 & $\mathrm{U}$ & -2.45 & HK & 6 & 10 & BSL & 14.21 & 0.46 \\
\hline BPS CS 30493-0001 & 230252.83 & -370120.0 & SPM & -1.76 & HK & -142 & 10 & BSL & 13.06 & 0.54 \\
\hline CD-63 1588 & 230316.05 & -624900.7 & HIP & -1.36 & BSL & 108 & 10 & BSL & 9.38 & 0.84 \\
\hline BPS CS 22938-0078 & 230337.93 & $\begin{array}{lll}-63 & 01 & 49.2\end{array}$ & $\mathrm{U}$ & -1.26 & HK & 176 & 10 & BSL & $(14.44)$ & $(0.46)$ \\
\hline BPS CS 22893-0015 & 230346.12 & $-1147 \quad 11.2$ & $\mathrm{U}$ & -2.69 & HK & 205 & 10 & BSL & 14.80 & 0.44 \\
\hline HD 217808 & 230352.00 & -443542.0 & HIP & -2.18 & ATT & 155 & 10 & BSL & 9.30 & 0.74 \\
\hline BPS CS 22888-0014 & 230415.50 & -332944.5 & SPM & -2.40 & HK & -109 & 10 & BSL & 14.44 & 0.40 \\
\hline BPS CS 29521-0051 & 230423.01 & +074511.0 & U & -1.92 & HK & -236 & 10 & BSL & 14.00 & 0.44 \\
\hline BPS CS 22888-0002 & 230437.23 & -363957.4 & SPM & -2.20 & HK & 41 & 10 & BSL & 14.70 & 0.42 \\
\hline BPS CS 22893-0032 & 230503.20 & -083011.4 & $\mathrm{U}$ & -1.18 & HK & -158 & 10 & BSL & 14.98 & 0.47 \\
\hline BPS CS 22888-0001 & 230543.58 & -364532.3 & SPM & -1.98 & HK & 6 & 10 & BSL & 14.90 & 0.45 \\
\hline BPS CS 22938-0056 & 230556.34 & -670756.0 & $\mathrm{U}$ & -1.38 & HK & 19 & 10 & BSL & $(13.56)$ & $(0.44)$ \\
\hline HD 218367 & $23 \quad 07 \quad 17.52$ & +151618.8 & $\mathrm{ACT}$ & -0.74 & BSL & 11 & 10 & BSL & 10.40 & 0.20 \\
\hline BPS CS 30493-0029 & 230828.11 & -333927.3 & SPM & -0.64 & HK & -34 & 10 & BSL & 14.36 & 0.76 \\
\hline BPS CS 30493-0031 & 230938.30 & -342805.9 & SPM & -0.17 & HK & 43 & 10 & BSL & 14.17 & 0.80 \\
\hline $\mathrm{V}^{*} \mathrm{YY}$ Tuc & 231100.58 & -582007.3 & STN & -1.82 & $\mathrm{~L}$ & 56 & 9 & $\mathrm{~L}$ & 11.98 & $\ldots$ \\
\hline HD 218810 & $23 \quad 11 \quad 11.65$ & -300005.2 & HIP & -0.65 & BSL & 8 & 10 & BSL & 8.40 & 0.39 \\
\hline BPS CS 22888-0039 & 231122.20 & -333517.0 & SPM & -1.78 & HK & 11 & 10 & BSL & 14.56 & 0.39 \\
\hline HD 218857 & 231124.63 & $\begin{array}{lll}-16 & 15 & 03.2\end{array}$ & HIP & -1.94 & B99 & -170 & 1 & $\mathrm{CY}$ & 8.94 & 0.72 \\
\hline
\end{tabular}


TABLE 1A. (continued)

\begin{tabular}{|c|c|c|c|c|c|c|c|c|c|c|}
\hline $\begin{array}{l}\text { Star } \\
(1)\end{array}$ & $\begin{array}{c}\text { RA (2000.0) } \\
(2)\end{array}$ & DEC & $\begin{array}{c}\text { Source }^{\mathrm{a}} \\
(4)\end{array}$ & $\begin{array}{c}{[\mathrm{Fe} / \mathrm{H}]} \\
(\mathrm{dex}) \\
(5)\end{array}$ & $\begin{array}{c}\text { Source }^{b} \\
(6)\end{array}$ & $\begin{array}{c}\mathrm{RV} \\
(\mathrm{km} / \mathrm{s}) \\
(7)\end{array}$ & $\begin{array}{c}\sigma_{\mathrm{RV}} \\
(\mathrm{km} / \mathrm{s}) \\
(8)\end{array}$ & $\begin{array}{c}\text { Source }^{\mathrm{c}} \\
(9)\end{array}$ & $\begin{array}{c}V \\
(\mathrm{mag}) \\
(10)\end{array}$ & $\begin{array}{c}B-V \\
(\mathrm{mag}) \\
(11)\end{array}$ \\
\hline BPS CS 22888-0031 & 231132.43 & -352642.6 & SPM & -3.08 & HK & -126 & 10 & BSL & 14.90 & 0.41 \\
\hline $\mathrm{V}^{*}$ AR Oct & $23 \quad 12 \quad 14.63$ & -743446.2 & GSC & -0.51 & $\mathrm{~L}$ & -18 & 40 & L & 13.12 & $\ldots$ \\
\hline BPS CS 30493-0049 & $2312 \quad 21.27$ & -362203.7 & SPM & -0.61 & HK & 9 & 10 & BSL & 13.86 & 0.67 \\
\hline BPS CS 22888-0044 & 231230.01 & $\begin{array}{lll}-33 & 10 & 26.0\end{array}$ & SPM & -2.24 & HK & 112 & 10 & BSL & 14.57 & 0.56 \\
\hline BPS CS 30493-0050 & $23 \quad 13 \quad 17.15$ & $-3501 \quad 16.4$ & SPM & -1.21 & HK & -53 & 10 & BSL & 14.66 & 0.55 \\
\hline BPS CS 30493-0058 & $2313 \quad 18.44$ & -333038.7 & SPM & -2.30 & HK & -71 & 10 & BSL & 15.00 & 0.50 \\
\hline BPS CS 29513-0006 & 231329.03 & -403634.3 & $\mathrm{U}$ & -1.02 & HK & -2 & 10 & BSL & $(14.77)$ & $(0.53)$ \\
\hline BPS CS 29513-0002 & 231338.63 & -421405.0 & SPM & -1.03 & HK & 31 & 10 & BSL & 13.58 & 1.15 \\
\hline $\mathrm{V}^{*}$ AP Gru & 231412.46 & -503911.7 & $\mathrm{v}$ & -1.92 & L & 101 & 11 & L & 12.98 & $\ldots$ \\
\hline BPS CS 22888-0029 & 231420.76 & -354522.6 & SPM & -1.48 & HK & 152 & 10 & BSL & 14.72 & 0.43 \\
\hline BPS CS 29513-0014 & 231424.90 & -375306.9 & SPM & -2.24 & HK & 40 & 10 & BSL & 13.80 & 0.71 \\
\hline BPS CS 29513-0015 & 231433.07 & -373604.9 & SPM & -2.02 & HK & 50 & 10 & BSL & 14.25 & 0.37 \\
\hline BPS CS 30493-0060 & 231436.46 & -331442.2 & SPM & -0.44 & HK & -12 & 10 & BSL & 14.65 & 0.76 \\
\hline BPS CS 29513-0009 & 231442.22 & -393448.7 & SPM & -1.14 & HK & 87 & 10 & BSL & 14.59 & 0.46 \\
\hline HD 219221 & 231447.31 & -485945.9 & HIP & -0.67 & BSL & 28 & 10 & BSL & 8.52 & 0.40 \\
\hline BPS CS 29513-0003 & 231531.71 & -415619.6 & SPM & -2.15 & HK & -103 & 10 & BSL & 13.52 & 0.49 \\
\hline BPS CS 22888-0026 & 231600.48 & -370542.8 & SPM & -1.78 & HK & 88 & 10 & BSL & 14.52 & 0.73 \\
\hline BPS CS 30493-0065 & 231623.69 & -340937.5 & SPM & -1.20 & HK & -48 & 10 & BSL & 13.84 & 0.63 \\
\hline BPS CS 30493-0071 & 231630.78 & -353435.8 & SPM & -2.20 & HK & -3 & 10 & BSL & 13.21 & 0.42 \\
\hline BPS CS 22949-0008 & 231658.72 & -032053.0 & GSC & -2.30 & HK & -149 & 10 & BSL & 14.15 & 0.46 \\
\hline BPS CS 29513-0010 & $23 \quad 1702.16$ & $-3852 \quad 10.0$ & SPM & -1.67 & HK & 29 & 10 & BSL & 13.89 & 0.31 \\
\hline BPS CS 30493-0072 & 231705.16 & -362156.9 & SPM & -1.77 & HK & 20 & 10 & BSL & 14.67 & 0.60 \\
\hline BPS CS 22938-0065 & $23 \quad 17 \quad 12.34$ & -653711.0 & $\mathrm{U}$ & -1.96 & HK & 255 & 10 & BSL & $(14.54)$ & $(0.46)$ \\
\hline BPS CS 22949-0018 & 231757.55 & -062736.8 & GSC & -1.54 & HK & -122 & 10 & BSL & 13.90 & 0.49 \\
\hline $\mathrm{V}^{*} \mathrm{AC}$ And & $23 \quad 18 \quad 02.35$ & +484658.4 & HIP & -1.16 & BSL & -50 & 1 & BSL & $\ldots$ & $\ldots$ \\
\hline BPS CS 29513-0016 & 231838.57 & -373025.0 & SPM & -1.97 & HK & 48 & 10 & BSL & 13.97 & 0.49 \\
\hline BPS CS 30493-0064 & 231908.32 & -340915.1 & SPM & -1.15 & HK & -58 & 10 & BSL & 16.46 & 0.52 \\
\hline $\mathrm{V}^{*} \mathrm{DN}$ Aqr & $23 \quad 1917.17$ & -241258.7 & HIP & -1.66 & F98 & -220 & 6 & F98 & 11.20 & $\cdots$ \\
\hline BPS CS 22949-0019 & 231917.70 & -064147.2 & GSC & -1.97 & HK & -136 & 10 & BSL & 15.23 & 0.44 \\
\hline BPS CS 22949-0014 & 231931.14 & -032643.4 & GSC & -1.48 & HK & -205 & 10 & BSL & 14.55 & 0.46 \\
\hline V* DZ Peg & 232007.06 & +160406.8 & STN & -1.52 & $\mathrm{~L}$ & -294 & 11 & $\mathrm{~L}$ & 12.00 & $\ldots$ \\
\hline BPS CS 22888-0047 & 232019.85 & -334546.8 & SPM & -2.25 & HK & -161 & 10 & BSL & 14.56 & 0.44 \\
\hline BPS CS 22945-0017 & 232055.55 & -631536.6 & $\mathrm{U}$ & -2.72 & HK & 91 & 10 & BSL & 14.43 & 0.39 \\
\hline BPS CS 22888-0057 & $2321 \quad 14.76$ & $-3544 \quad 43.5$ & SPM & -2.06 & HK & -63 & 10 & BSL & 15.02 & 0.39 \\
\hline BPS CS 22949-0030 & 232122.82 & -034555.0 & GSC & -2.44 & HK & -79 & 10 & BSL & 13.85 & 0.42 \\
\hline HD 220127 & 232143.40 & -492912.6 & HIP & -1.79 & ATT & 63 & 10 & BSL & 10.14 & 0.69 \\
\hline BPS CS 22949-0029 & 232204.33 & -035057.9 & GSC & -2.10 & HK & 5 & 10 & BSL & 14.63 & 0.47 \\
\hline BPS CS 29513-0032 & $23 \quad 22 \quad 19.07$ & -394424.3 & SPM & -2.02 & HK & -216 & 10 & BSL & 14.44 & 0.53 \\
\hline $\mathrm{V}^{*} \mathrm{UZ} \mathrm{Scl}$ & 232247.03 & $\begin{array}{lll}-30 & 07 & 09.7\end{array}$ & SPM & -1.14 & $\mathrm{~L}$ & -4 & 10 & L & 12.34 & $\ldots$ \\
\hline BPS CS 22949-0026 & 232302.35 & -051251.4 & $\mathrm{U}$ & -1.98 & HK & 208 & 10 & BSL & 15.23 & 0.45 \\
\hline
\end{tabular}


TABLE 1A. (continued)

\begin{tabular}{|c|c|c|c|c|c|c|c|c|c|c|}
\hline Star & $\begin{array}{c}\text { RA (2000.0) } \\
(2)\end{array}$ & DEC & $\begin{array}{c}\text { Source }^{\mathrm{a}} \\
(4)\end{array}$ & $\begin{array}{c}{[\mathrm{Fe} / \mathrm{H}]} \\
(\mathrm{dex}) \\
(5)\end{array}$ & $\begin{array}{c}\text { Source }^{b} \\
(6)\end{array}$ & $\begin{array}{c}\mathrm{RV} \\
(\mathrm{km} / \mathrm{s}) \\
(7)\end{array}$ & $\begin{array}{c}\sigma_{\mathrm{RV}} \\
(\mathrm{km} / \mathrm{s}) \\
(8)\end{array}$ & $\begin{array}{c}\text { Source }{ }^{\mathrm{c}} \\
(9)\end{array}$ & $\begin{array}{c}V \\
(\mathrm{mag}) \\
(10)\end{array}$ & $\begin{array}{c}B-V \\
(\mathrm{mag}) \\
(11)\end{array}$ \\
\hline BPS CS 29513-0035 & 232320.18 & $\begin{array}{lll}-39 & 06 & 12.6\end{array}$ & SPM & -1.90 & HK & 96 & 10 & BSL & 15.05 & 0.59 \\
\hline BPS CS 29513-0033 & 232424.72 & -392437.2 & SPM & -1.63 & HK & 51 & 10 & BSL & 14.72 & 0.85 \\
\hline BPS CS 22888-0049 & 232457.21 & -342323.4 & SPM & -1.84 & HK & 21 & 10 & BSL & 14.63 & 0.42 \\
\hline BPS CS 29513-0031 & 232511.35 & -395928.2 & SPM & -2.79 & HK & 295 & 10 & BSL & 15.09 & 0.35 \\
\hline BPS CS 22949-0043 & 232526.59 & -044935.2 & GSC & -2.30 & HK & -77 & 10 & BSL & 14.97 & 0.44 \\
\hline BPS CS 29513-0034 & 232536.16 & $\begin{array}{lll}-39 & 08 & 04.4\end{array}$ & SPM & -1.76 & HK & 212 & 10 & BSL & 15.02 & 0.58 \\
\hline HD 220662 & 232541.62 & -235620.5 & HIP & -1.59 & C97 & -78 & 1 & $\mathrm{CY}$ & 10.11 & 1.03 \\
\hline BPS CS 22949-0050 & 232604.22 & $\begin{array}{lll}-06 & 02 & 46.3\end{array}$ & GSC & -1.38 & HK & 37 & 10 & BSL & 15.04 & 0.47 \\
\hline BPS CS 22949-0048 & 232607.38 & -055006.3 & GSC & -3.17 & B99 & -129 & 10 & HK & 13.66 & 0.78 \\
\hline BPS CS 22949-0040 & 232629.30 & -035231.0 & GSC & -1.66 & HK & -56 & 10 & BSL & 14.64 & 0.46 \\
\hline BPS CS 22949-0037 & 232632.65 & -023936.4 & GSC & -3.99 & B99 & -115 & 10 & HK & 14.36 & 0.73 \\
\hline HD 220838 & 232716.48 & -265858.1 & HIP & -1.74 & B99 & -23 & 1 & $\mathrm{CY}$ & 9.38 & 1.16 \\
\hline BPS CS 22949-0046 & 232744.32 & -051425.3 & GSC & -0.62 & BSL & -36 & 10 & BSL & 14.95 & 0.31 \\
\hline BPS CS 22949-0052 & 232745.45 & -064659.7 & GSC & -2.02 & HK & -1 & 10 & BSL & 13.85 & 0.39 \\
\hline BPS CS 22941-0011 & 232754.94 & -331454.7 & SPM & -2.16 & HK & 17 & 10 & BSL & 15.35 & 0.36 \\
\hline $\mathrm{V}^{*} \mathrm{RV}$ Phe & 232831.41 & -472712.9 & HIP & -1.69 & F98 & 99 & 2 & F98 & 11.94 & $\cdots$ \\
\hline BPS CS 22941-0007 & 232833.67 & -343053.5 & GSC & -1.70 & HK & -88 & 10 & BSL & 14.43 & 0.38 \\
\hline BPS CS 29513-0048 & 232911.05 & -405533.2 & SPM & -1.49 & HK & 98 & 10 & BSL & 14.80 & 0.69 \\
\hline HD 221170 & 232928.82 & +302558.3 & HIP & -2.12 & B99 & -122 & 1 & CY & 7.67 & 1.03 \\
\hline $\mathrm{V}^{*} \mathrm{BK}$ Tuc & 232933.26 & -723239.4 & STN & -1.82 & $\mathrm{~L}$ & 121 & 14 & L & 12.91 & $\cdots$ \\
\hline BPS CS 29513-0040 & 233008.96 & $-38 \quad 18 \quad 19.7$ & SPM & -1.54 & HK & -24 & 10 & BSL & 14.56 & 0.78 \\
\hline BPS CS 22952-0004 & 233027.49 & -042641.1 & $\mathrm{U}$ & -2.06 & HK & -183 & 10 & BSL & 13.49 & 0.42 \\
\hline BPS CS 22949-0055 & 233027.54 & -042643.9 & GSC & -1.96 & HK & -174 & 10 & BSL & 13.50 & 0.42 \\
\hline BPS CS 22945-0028 & 233113.57 & -662958.0 & $\mathrm{U}$ & -2.59 & HK & 378 & 10 & BSL & 14.64 & 0.68 \\
\hline BPS CS 22894-0010 & 233145.05 & +00 5518.8 & $\mathrm{U}$ & -0.65 & HK & -40 & 10 & BSL & 14.90 & 0.57 \\
\hline $\mathrm{V}^{*} \mathrm{DM}$ And & 233200.74 & +351148.0 & STN & -2.32 & L & -265 & 30 & L & 12.80 & $\cdots$ \\
\hline BPS CS 22941-0014 & 233202.08 & -331320.4 & SPM & -1.84 & HK & 23 & 10 & BSL & 14.59 & 0.62 \\
\hline BPS CS 22894-0004 & 233239.45 & -005609.8 & $\mathrm{U}$ & -2.06 & HK & 0 & 10 & BSL & 14.17 & 0.41 \\
\hline BPS CS 29496-0003 & 233241.16 & -312127.5 & SPM & -2.20 & HK & -31 & 10 & BSL & 13.97 & 0.63 \\
\hline BPS CS 22941-0017 & 233250.19 & -335925.5 & SPM & -2.40 & HK & 114 & 10 & BSL & 15.00 & 0.56 \\
\hline BPS CS 22941-0020 & 233250.60 & -344139.9 & SPM & -2.17 & HK & 49 & 10 & BSL & 14.69 & 0.39 \\
\hline BPS CS 22952-0002 & 233303.82 & -051245.2 & $\mathrm{U}$ & -1.98 & HK & 144 & 10 & BSL & 14.38 & 0.57 \\
\hline BPS CS 22894-0009 & 233328.86 & +005733.0 & $\mathrm{U}$ & -0.98 & HK & -3 & 10 & BSL & 14.14 & 0.52 \\
\hline BPS CS 22894-0005 & 233347.53 & -003208.9 & $\mathrm{U}$ & -1.48 & HK & -155 & 10 & BSL & 14.51 & 0.47 \\
\hline BPS CS 22941-0027 & 233458.09 & -365205.7 & SPM & -2.57 & HK & -96 & 10 & BSL & 14.05 & 0.35 \\
\hline BPS CS 22941-0021 & 233500.98 & -345247.5 & SPM & -1.48 & HK & -83 & 10 & BSL & 13.89 & 0.42 \\
\hline $\mathrm{V}^{*} \mathrm{BK}$ And & 233506.03 & +410610.4 & $\mathrm{STN}$ & -0.08 & L & 98 & 53 & L & 12.99 & $\cdots$ \\
\hline BPS CS 29513-0053 & 233515.89 & $\begin{array}{lll}-41 & 0309.8\end{array}$ & SPM & -1.21 & HK & -43 & 10 & BSL & 14.74 & 0.52 \\
\hline BPS CS 22952-0012 & 233518.17 & -040104.9 & $\mathrm{U}$ & -1.01 & HK & -2 & 10 & BSL & $(14.61)$ & $(0.39)$ \\
\hline BPS CS 22894-0017 & 233623.40 & +00 0415.3 & $\mathrm{U}$ & -1.66 & HK & -67 & 10 & BSL & 14.13 & 0.48 \\
\hline
\end{tabular}


TABLE 1A. (continued)

\begin{tabular}{|c|c|c|c|c|c|c|c|c|c|c|}
\hline $\begin{array}{l}\text { Star } \\
(1)\end{array}$ & $\begin{array}{c}\text { RA (2000.0) } \\
(2)\end{array}$ & DEC & $\begin{array}{c}\text { Source }^{\mathrm{a}} \\
(4)\end{array}$ & $\begin{array}{c}{[\mathrm{Fe} / \mathrm{H}]} \\
(\mathrm{dex}) \\
(5)\end{array}$ & $\begin{array}{c}\text { Source }^{b} \\
(6)\end{array}$ & $\begin{array}{c}\mathrm{RV} \\
(\mathrm{km} / \mathrm{s}) \\
(7)\end{array}$ & $\begin{array}{c}\sigma_{\mathrm{RV}} \\
(\mathrm{km} / \mathrm{s}) \\
(8)\end{array}$ & $\begin{array}{c}\text { Source }^{\mathrm{c}} \\
(9)\end{array}$ & $\begin{array}{c}V \\
(\mathrm{mag}) \\
(10)\end{array}$ & $\begin{array}{c}B-V \\
(\mathrm{mag}) \\
(11)\end{array}$ \\
\hline BPS CS 22945-0024 & 233642.42 & -653543.8 & $\mathrm{U}$ & -2.86 & HK & 143 & 10 & BSL & 14.36 & 0.72 \\
\hline BPS CS 22952-0011 & 233718.58 & -020828.0 & STN & -2.70 & HK & -325 & 10 & BSL & 13.75 & 0.40 \\
\hline BPS CS 22952-0015 & 233728.69 & -054756.1 & $\mathrm{U}$ & -3.26 & B99 & -16 & 10 & HK & 13.28 & 0.75 \\
\hline BPS CS 29496-0021 & 233735.12 & -312606.1 & SPM & -2.27 & $\mathrm{HK}$ & -29 & 10 & BSL & 13.43 & 0.57 \\
\hline BPS CS 29496-0022 & 233757.46 & -312936.6 & SPM & -2.25 & HK & 37 & 10 & BSL & 14.59 & 0.35 \\
\hline $\mathrm{V}^{*} \mathrm{BR}$ Aqr & 233832.88 & -09 1907.4 & HIP & -0.74 & F98 & 30 & 1 & F98 & 11.42 & $\ldots$ \\
\hline BPS CS 29496-0014 & 233832.89 & -281803.4 & SPM & -1.87 & HK & 4 & 10 & BSL & 13.44 & 0.52 \\
\hline BPS CS 29499-0017 & 233840.16 & -231934.1 & SPM & -2.22 & HK & 34 & 10 & BSL & 13.95 & 0.52 \\
\hline BPS CS 22952-0018 & 233859.01 & -042658.6 & $\mathrm{U}$ & -1.66 & HK & -67 & 10 & BSL & 13.79 & 0.45 \\
\hline BPS CS 22894-0019 & 233918.98 & +000345.0 & $\mathrm{U}$ & -2.90 & $\mathrm{BCM}$ & 27 & 10 & BSL & 13.92 & 0.43 \\
\hline BPS CS 29499-0010 & 233939.60 & -250655.9 & SPM & -1.45 & HK & 4 & 10 & BSL & 15.22 & 0.37 \\
\hline BPS CS 29499-0003 & 233942.97 & -265855.2 & SPM & -1.88 & HK & -68 & 10 & BSL & 14.29 & 0.37 \\
\hline BPS CS 29496-0013 & 233957.38 & -281936.1 & SPM & -2.50 & HK & 32 & 10 & BSL & 14.72 & 0.23 \\
\hline BPS CS 22941-0043 & 234020.18 & -325237.6 & SPM & -2.04 & HK & 128 & 10 & BSL & 15.02 & 0.40 \\
\hline HD 222434 & 234041.51 & -344146.2 & HIP & -1.90 & C97 & 13 & 1 & $\mathrm{CY}$ & 8.82 & 1.08 \\
\hline BPS CS 22945-0029 & $2341 \quad 12.43$ & -665917.6 & $\mathrm{U}$ & -2.54 & HK & 365 & 10 & BSL & 15.26 & 0.42 \\
\hline BPS CS 29499-0027 & 234143.46 & -225019.6 & SPM & -1.78 & HK & 64 & 10 & BSL & 14.57 & 0.48 \\
\hline BPS CS 22952-0021 & 234145.33 & -033631.9 & $\mathrm{U}$ & -1.87 & HK & -168 & 10 & BSL & 14.59 & 0.42 \\
\hline BPS CS 22952-0020 & 234206.78 & -041007.1 & $\mathrm{U}$ & -1.96 & HK & -55 & 10 & BSL & 13.89 & 0.42 \\
\hline $\mathrm{V}^{*} \mathrm{VZ}$ Peg & 234216.29 & +245458.6 & HIP & -1.80 & F98 & -264 & 1 & F98 & 11.90 & $\cdots$ \\
\hline $\mathrm{V}^{*}$ AT And & 234230.84 & +430052.1 & HIP & -1.18 & F98 & -228 & 1 & F98 & 10.71 & $\ldots$ \\
\hline HD 222766 & 234334.56 & -075522.6 & HIP & -1.40 & BSL & -92 & 10 & BSL & 10.11 & 0.67 \\
\hline BPS CS 22894-0038 & 234347.78 & +020142.4 & $\mathrm{U}$ & -1.58 & HK & -8 & 10 & BSL & 14.60 & 0.53 \\
\hline BPS CS 29499-0034 & 234351.02 & -263404.7 & SPM & -1.67 & HK & 15 & 10 & BSL & 14.67 & 0.39 \\
\hline BPS CS 22941-0046 & 234413.56 & -344525.1 & SPM & -1.62 & HK & -35 & 10 & BSL & 14.53 & 0.47 \\
\hline BPS CS 22894-0046 & 234431.05 & -002610.4 & U & -1.76 & HK & 8 & 10 & BSL & 14.83 & 0.47 \\
\hline $\mathrm{V}^{*} \mathrm{KK}$ Peg & 234437.15 & +301008.4 & $\mathrm{U}$ & -0.87 & BSL & -149 & 30 & BSL & $\ldots$ & $\ldots$ \\
\hline BPS CS 22894-0047 & 234442.51 & -012850.4 & $\mathrm{U}$ & -1.62 & HK & -51 & 10 & BSL & 13.97 & 0.56 \\
\hline BPS CS 22945-0043 & 234508.27 & -635153.0 & $\mathrm{U}$ & -1.54 & HK & 138 & 10 & BSL & $(14.21)$ & $(0.39)$ \\
\hline HD 222925 & 234517.42 & -615442.0 & HIP & -1.19 & $\mathrm{ATT}$ & -45 & 10 & BSL & 9.02 & 0.57 \\
\hline BPS CS 22894-0041 & 234541.46 & +011250.5 & $\mathrm{U}$ & -1.76 & HK & -81 & 10 & BSL & 13.51 & 0.46 \\
\hline BPS CS 29517-0005 & 234549.70 & -151950.5 & $\mathrm{U}$ & -2.37 & HK & -134 & 10 & BSL & $(14.10)$ & $(0.70)$ \\
\hline BPS CS 29517-0010 & $2346 \quad 11.41$ & -140951.2 & NPM & -1.29 & HK & -12 & 10 & BSL & $(14.85)$ & $(0.45)$ \\
\hline $\mathrm{V}^{*}$ KL Peg & 234657.08 & +295101.6 & $\mathrm{U}$ & -1.03 & BSL & -317 & 30 & BSL & $\ldots$ & $\ldots$ \\
\hline BPS CS 22945-0044 & $2347 \quad 12.90$ & -633552.1 & $\mathrm{U}$ & -1.36 & HK & 83 & 10 & BSL & $(14.61)$ & $(0.59)$ \\
\hline BPS CS 22945-0031 & 234731.89 & -673035.8 & $\mathrm{U}$ & -2.44 & HK & 197 & 10 & BSL & 14.77 & 0.38 \\
\hline HD 223224 & 234752.51 & $-5516 \quad 52.6$ & HIP & -1.16 & $\mathrm{ATT}$ & 52 & 10 & BSL & 9.57 & 0.76 \\
\hline BPS CS 22894-0054 & 234828.63 & +002357.9 & $\mathrm{U}$ & -1.01 & HK & 6 & 10 & BSL & 14.18 & 0.39 \\
\hline BPS CS 22876-0003 & 234856.03 & -362910.8 & SPM & -1.98 & HK & 77 & 10 & BSL & 13.84 & 0.45 \\
\hline BPS CS 29517-0004 & 234927.09 & -155738.8 & $\mathrm{U}$ & -1.26 & HK & -33 & 10 & BSL & $(14.03)$ & $(0.37)$ \\
\hline
\end{tabular}


TABlE 1A. (continued)

\begin{tabular}{|c|c|c|c|c|c|c|c|c|c|c|c|}
\hline $\begin{array}{l}\text { Star } \\
\text { (1) }\end{array}$ & $\begin{array}{c}\text { RA (2000.0) } \\
(2)\end{array}$ & DEC & $\begin{array}{c}\text { Source } \\
\text { (4) }\end{array}$ & $\begin{array}{c}{[\mathrm{Fe} / \mathrm{H}]} \\
(\mathrm{dex}) \\
(5)\end{array}$ & $\begin{array}{c}\text { Source }^{b} \\
(6)\end{array}$ & $\begin{array}{c}\mathrm{RV} \\
(\mathrm{km} / \mathrm{s}) \\
(7)\end{array}$ & $\begin{array}{c}\sigma_{\mathrm{RV}} \\
(\mathrm{km} / \mathrm{s}) \\
(8)\end{array}$ & $\begin{array}{c}\text { Source }^{c} \\
(9)\end{array}$ & $\begin{array}{c}V \\
(\mathrm{mag}) \\
(10)\end{array}$ & $\begin{array}{c}B-V \\
(\mathrm{mag}) \\
(11)\end{array}$ & $\begin{array}{r}\text { Sour } \\
(12)\end{array}$ \\
\hline BPS CS 22894-0049 & 234952.80 & -015745.9 & $\mathrm{U}$ & -2.20 & HK & -23 & 10 & BSL & 14.46 & 0.45 & B \\
\hline BPS CS 22894-0050 & 235006.86 & -014201.0 & $\mathrm{U}$ & -0.69 & HK & -122 & 10 & BSL & $(14.62)$ & $(0.68)$ & \\
\hline BPS CS 29517-0012 & 235040.52 & -132814.1 & $\mathrm{U}$ & -1.06 & HK & -70 & 10 & BSL & $(14.28)$ & $(0.42)$ & \\
\hline BPS CS 29499-0058 & 235215.90 & -255818.3 & SPM & -2.44 & HK & 143 & 10 & BSL & 13.76 & 0.39 & B \\
\hline BPS CS 29517-0014 & 235221.23 & -145002.8 & $\mathrm{U}$ & -1.38 & HK & 18 & 10 & BSL & $(14.25)$ & $(0.45)$ & \\
\hline BPS CS 22945-0058 & 235237.73 & -660501.3 & $\mathrm{U}$ & -2.32 & HK & -36 & 10 & BSL & 14.96 & 0.36 & B \\
\hline BPS CS 29499-0053 & 235245.55 & -234345.3 & SPM & -1.71 & HK & 0 & 10 & BSL & 14.87 & 0.55 & $\mathrm{SF}$ \\
\hline BPS CS 22945-0056 & 235319.81 & -652939.5 & $\mathrm{U}$ & -2.54 & HK & 222 & 10 & BSL & 14.09 & 0.40 & B \\
\hline BPS CS 22876-0016 & 235336.38 & $-32 \quad 2722.2$ & SPM & -1.17 & HK & 86 & 10 & BSL & 14.09 & 0.31 & $\mathrm{SF}$ \\
\hline BPS CS 29517-0018 & 235337.84 & -172309.0 & $\mathrm{U}$ & -2.10 & HK & 151 & 10 & BSL & 14.50 & 0.44 & $\mathrm{~B}$ \\
\hline BPS CS 29499-0060 & 235340.25 & -265844.2 & SPM & -2.63 & B99 & -60 & 10 & HK & 13.03 & 0.37 & B \\
\hline BPS CS 29517-0025 & 235428.82 & -135956.3 & $\mathrm{U}$ & -2.30 & HK & -186 & 10 & BSL & $(14.15)$ & $(0.55)$ & \\
\hline BPS CS 29517-0023 & 235503.00 & -145143.2 & $\mathrm{U}$ & -2.06 & HK & -86 & 10 & BSL & (14.39) & $(0.51)$ & \\
\hline$V^{*}$ KM Peg & 235545.11 & +290952.0 & $\mathrm{U}$ & -1.65 & BSL & -164 & 30 & BSL & $\ldots$ & $\ldots$ & \\
\hline BPS CS 22957-0013 & 235549.05 & -052252.1 & $\mathrm{U}$ & -2.69 & HK & -217 & 10 & BSL & 14.08 & 0.73 & B \\
\hline BPS CS 22945-0073 & 235609.77 & $\begin{array}{lll}-62 & 40 & 04.1\end{array}$ & $\mathrm{U}$ & -2.30 & HK & 106 & 10 & BSL & 14.70 & 0.39 & $\mathrm{~B}$ \\
\hline BPS CS 29499-0065 & 235623.61 & -251855.5 & SPM & -2.70 & HK & -11 & 10 & BSL & 15.24 & 0.38 & B \\
\hline $\mathrm{V}^{*}$ KN Peg & 235646.57 & +314022.6 & $\mathrm{U}$ & -1.65 & BSL & -74 & 30 & BSL & $\ldots$ & $\ldots$ & \\
\hline BPS CS 22945-0068 & 235716.29 & $-64 \quad 1219.2$ & $\mathrm{U}$ & -1.88 & HK & 15 & 10 & BSL & 15.19 & 0.36 & B \\
\hline BPS CS 22957-0016 & 235716.84 & -055427.8 & $\mathrm{U}$ & -1.38 & HK & -193 & 10 & BSL & 14.34 & 0.45 & $\mathrm{~B}$ \\
\hline BPS CS 29499-0068 & $2357 \quad 17.19$ & -245757.9 & SPM & -2.26 & HK & -28 & 10 & BSL & 14.20 & 0.52 & $\mathrm{SF}$ \\
\hline BPS CS 22957-0019 & 235731.31 & -065704.1 & $\mathrm{U}$ & -2.06 & HK & -31 & 10 & BSL & 13.71 & 0.41 & B \\
\hline BPS CS 22957-0015 & 235750.65 & $-0557 \quad 17.0$ & NPM & -2.10 & HK & -59 & 10 & BSL & 14.85 & 0.44 & B \\
\hline BPS CS 22957-0024 & 235834.53 & -043322.0 & $\mathrm{U}$ & -2.57 & HK & -37 & 10 & BSL & 14.30 & 0.38 & B \\
\hline BPS CS 22957-0028 & 235906.04 & -033810.2 & $\mathrm{U}$ & -2.02 & HK & -54 & 10 & BSL & 14.82 & 0.40 & B. \\
\hline BPS CS 22876-0027 & 235934.88 & -353934.7 & SPM & -2.07 & HK & -100 & 10 & BSL & 14.36 & 0.39 & B \\
\hline
\end{tabular}

aPositions: U: USNO-A 2.0; HIP: Hipparcos; SPM: SPM 2.0; STN: STARNET; ACT: ACT; NPM: NPM1; SBD: SIMBAD; GSC: GSC 1 uncertain

b Abundances: HK: HK survey; B99: Beers et al. 1999; C97: Cayrel et al. 1997; McW: McWilliam et al. 1995; BCM: Bonifacio, Centurion, \& Lambert 1995; BSL: values adopted in the original BSL catalog; MFF: Morrison, Flynn, Freeman 1990; W99: Wilhelm et al. 1999; L: Layder F98: Fernley et al. 1998

'Radial Velocities: HK: HK survey; BSL: values adopted in the original BSL catalog; L: Layden 1994, Layden et al. 1996; F98: Fernley et al. in Chiba \& Yoshii 1998; ATT: Anthony-Twarog \& Twarog 1994

dPhotometry: BPS: HK survey; SBD: SIMBAD; HIP: Hipparcos; GSC: GSC 1.2; SC1: Schuster et al. 1996; SC2: Schuster et al. 2000; SA 2000; BSL: values adopted in the original BSL catalog; TP: This paper; L: intensity averaged $<V>$ from Layden 1994, Layden et al. 199 $<V>$ from Fernley et al. 1998 
Table 1B. Distance Estimates and Measured Proper Motions

\begin{tabular}{|c|c|c|c|c|c|c|c|c|c|c|}
\hline $\begin{array}{l}\text { Star } \\
(1)\end{array}$ & $\begin{array}{c}D_{p h o} \\
(\mathrm{kpc}) \\
(2)\end{array}$ & $\begin{array}{c}D_{H I P} \\
(\mathrm{kpc}) \\
(3)\end{array}$ & $\begin{array}{c}\sigma_{\pi_{H I P}} / \pi_{H I P} \\
(4)\end{array}$ & $\begin{array}{c}D_{B S L} \\
(\mathrm{kpc}) \\
(5)\end{array}$ & $\begin{array}{c}D_{\text {adopt }} \\
(\mathrm{kpc}) \\
\quad(6)\end{array}$ & $\begin{array}{c}\mu_{\alpha^{*}} \\
(\mathrm{mas} / \mathrm{yr}) \\
(7)\end{array}$ & $\begin{array}{c}\mu_{\delta} \\
\text { (mas/yr) } \\
(8)\end{array}$ & $\begin{array}{c}\sigma_{\mu_{\alpha^{*}}} \\
(\mathrm{mas} / \mathrm{yr}) \\
(9)\end{array}$ & $\begin{array}{c}\sigma_{\mu_{\delta}} \\
(\mathrm{mas} / \mathrm{yr}) \\
(10)\end{array}$ & Sour \\
\hline BPS CS 22957-0026 & 0.601 & $\ldots$ & $\ldots$ & 0.700 & 0.601 & 108. & -53 & 4.6 & 4.6 & STN \\
\hline $\mathrm{V}^{*}$ GV Peg & 3.586 & $\ldots$ & $\ldots$ & $\ldots$ & 3.586 & 6.9 & -30.7 & 5 & 5 & NPM \\
\hline BPS CS 29517-0037 & 1.325 & $\ldots$ & $\ldots$ & 1.300 & 1.325 & $\ldots$ & $\ldots$ & $\ldots$ & $\ldots$ & $\ldots$ \\
\hline BPS CS 22957-0025 & 1.337 & $\ldots$ & $\cdots$ & 2.100 & 1.337 & $\ldots$ & $\ldots$ & $\cdots$ & $\ldots$ & $\ldots$ \\
\hline BPS CS 22957-0022 & 2.407 & $\cdots$ & $\cdots$ & 2.200 & 2.407 & 5. & -29 & 4.3 & 4.3 & STN \\
\hline BPS CS 29517-0029 & 0.874 & $\cdots$ & $\cdots$ & 0.900 & 0.874 & 6.6 & -38.5 & 5 & 5 & NPM \\
\hline BPS CS 29517-0034 & 0.855 & $\ldots$ & $\ldots$ & 0.900 & 0.855 & $\ldots$ & $\ldots$ & $\ldots$ & $\ldots$ & $\ldots$ \\
\hline $\mathrm{V}^{*} \mathrm{KO}$ Peg & $\ldots$ & $\cdots$ & $\cdots$ & 13.182 & 13.182 & $\cdots$ & $\cdots$ & $\cdots$ & $\cdots$ & $\cdots$ \\
\hline BPS CS 22945-0063 & 6.447 & $\ldots$ & $\ldots$ & 1.700 & 6.447 & $\ldots$ & $\ldots$ & $\ldots$ & $\ldots$ & $\ldots$ \\
\hline $\mathrm{V}^{*} \mathrm{RU} \mathrm{Scl}$ & 0.801 & 1.587 & 2.65 & 0.855 & 0.801 & 53.7 & -18.4 & 1.2 & 0.9 & HIP,SPM, \\
\hline BPS CS 22957-0031 & 1.107 & $\cdots$ & $\cdots$ & 1.300 & 1.107 & $\cdots$ & $\cdots$ & $\ldots$ & $\cdots$ & $\cdots$ \\
\hline BPS CS 22957-0036 & 1.099 & $\ldots$ & $\ldots$ & 1.200 & 1.099 & $\ldots$ & $\ldots$ & $\ldots$ & $\ldots$ & $\ldots$ \\
\hline BPS CS 29517-0040 & 1.038 & $\ldots$ & $\ldots$ & 1.200 & 1.038 & $\ldots$ & $\ldots$ & $\ldots$ & $\ldots$ & $\ldots$ \\
\hline $\mathrm{V}^{*} \mathrm{UU}$ Cet & 1.884 & 0.154 & 0.64 & 1.888 & 1.884 & 30.3 & -1.3 & 3.0 & 1.7 & HIP,STN,N \\
\hline $\mathrm{V}^{*} \mathrm{AO}$ Tuc & $\ldots$ & $\ldots$ & $\ldots$ & 1.230 & 1.230 & 15.6 & -49.5 & 2.6 & 3.0 & $\mathrm{ACT}, \mathrm{STN}$ \\
\hline [FF93] 513440 & 2.467 & $\ldots$ & $\cdots$ & 1.122 & 2.467 & 6.3 & -3.6 & 1.7 & 1.7 & SPM,STN \\
\hline BPS CS 22876-0040 & 1.682 & $\ldots$ & $\cdots$ & 1.700 & 1.682 & -7.0 & -18.8 & 2.6 & 2.6 & SPM \\
\hline BPS CS 29503-0010 & 0.697 & $\ldots$ & $\cdots$ & 0.900 & 0.697 & -9.1 & -2.2 & 2.8 & 2.4 & SPM \\
\hline BPS CS 29517-0042 & 1.066 & $\cdots$ & $\cdots$ & 1.300 & 1.066 & $\ldots$ & $\ldots$ & $\cdots$ & $\ldots$ & $\ldots$ \\
\hline BPS CS 29517-0047 & 2.354 & $\cdots$ & $\cdots$ & 5.700 & 2.354 & $\cdots$ & $\cdots$ & $\cdots$ & $\cdots$ & $\cdots$ \\
\hline HD 20 & 0.169 & 0.714 & 0.94 & $\cdots$ & 0.169 & 132.7 & -40.7 & 0.9 & 0.7 & HIP,SPM, \\
\hline BPS CS 29517-0048 & 1.105 & $\cdots$ & $\cdots$ & 1.200 & 1.105 & $\ldots$ & $\ldots$ & $\cdots$ & $\cdots$ & $\ldots$ \\
\hline HD 97 & 0.342 & $<0$ & $\ldots$ & 0.500 & 0.342 & -23.3 & -178.5 & 1.2 & 0.7 & HIP,ACT, \\
\hline BPS CS 29503-0013 & 1.046 & $\ldots$ & $\cdots$ & 1.100 & 1.046 & 5.3 & -16.1 & 5.8 & 6.2 & SPM \\
\hline $\mathrm{V}^{*} \mathrm{IQ}$ Peg & $\ldots$ & $\cdots$ & $\cdots$ & 12.941 & 12.941 & $\ldots$ & $\ldots$ & $\cdots$ & $\cdots$ & $\ldots$ \\
\hline [FF93] 513390 & 1.135 & $\ldots$ & $\ldots$ & 1.556 & 1.135 & -10.2 & -4.7 & 1.6 & 1.6 & $\mathrm{SPM}, \mathrm{ACT}$ \\
\hline $\mathrm{V}^{*} \mathrm{NN}$ And & $\ldots$ & $\ldots$ & $\ldots$ & 17.060 & 17.060 & $\ldots$ & $\ldots$ & $\ldots$ & $\ldots$ & $\ldots$ \\
\hline BPS CS 29503-0022 & $\ldots$ & $\cdots$ & $\cdots$ & 1.500 & 1.500 & 3.3 & -19.5 & 7.2 & 6.6 & SPM \\
\hline BPS CS 22876-0032 & 0.553 & $\ldots$ & $\ldots$ & 0.509 & 0.553 & 81.2 & -126.5 & 2.2 & 4.9 & SPM,STN \\
\hline BPS CS 29503-0017 & 0.997 & $\cdots$ & $\cdots$ & 1.200 & 0.997 & -9.7 & -66.1 & 6.9 & 7.1 & $\mathrm{SPM}$ \\
\hline BPS CS 29503-0026 & 0.608 & $\cdots$ & $\cdots$ & 0.900 & 0.608 & 44.1 & -4.8 & 3.0 & 2.7 & SPM \\
\hline BPS CS 29503-0015 & 1.146 & $\cdots$ & $\cdots$ & 1.200 & 1.146 & -0.6 & -39.8 & 3.2 & 2.6 & SPM \\
\hline $\mathrm{V}^{*} \mathrm{NQ}$ And & $\ldots$ & $\cdots$ & $\cdots$ & 14.996 & 14.996 & $\cdots$ & $\ldots$ & $\cdots$ & $\cdots$ & $\cdots$ \\
\hline $\mathrm{V}^{*} \mathrm{RY}$ Psc & 2.050 & $\ldots$ & $\ldots$ & 2.137 & 2.050 & 39.9 & -7.7 & 5 & 5 & NPM \\
\hline HD 868 & 0.827 & $\ldots$ & $\ldots$ & 0.478 & 0.827 & 21.0 & 13.7 & 0.9 & 1.0 & $\mathrm{SPM}, \mathrm{ACT}$ \\
\hline HD 891 & 0.924 & 0.260 & 0.38 & 0.835 & 0.924 & 13.6 & -20.4 & 0.8 & 0.7 & HIP,SPM, \\
\hline HD 941 & 0.280 & 0.154 & 0.15 & 0.141 & 0.280 & 127.6 & -75.2 & 0.9 & 0.5 & HIP,SPM, \\
\hline BPS CS 29503-0031 & 0.682 & $\ldots$ & $\ldots$ & 0.900 & 0.682 & 16.7 & -17.7 & 1.8 & 1.5 & SPM \\
\hline HD 991 & 0.558 & 1.449 & 1.57 & 0.476 & 0.558 & 48.1 & -44.6 & 0.9 & 0.6 & HIP,SPM, \\
\hline BPS CS 30339-0002 & 0.715 & $\cdots$ & $\cdots$ & 1.100 & 0.715 & -1.7 & -78.6 & 2.7 & 1.8 & SPM,STN \\
\hline
\end{tabular}


TABLE 1B. (continued)

\begin{tabular}{|c|c|c|c|c|c|c|c|c|c|c|}
\hline $\begin{array}{l}\text { Star } \\
(1)\end{array}$ & $\begin{array}{c}D_{p h o} \\
(\mathrm{kpc}) \\
(2)\end{array}$ & $\begin{array}{c}D_{H I P} \\
(\mathrm{kpc}) \\
(3)\end{array}$ & $\begin{array}{c}\sigma_{\pi_{H I P}} / \pi_{H I P} \\
(4)\end{array}$ & $\begin{array}{c}D_{B S L} \\
(\mathrm{kpc}) \\
(5)\end{array}$ & $\begin{array}{l}D_{\text {adopt }} \\
(\mathrm{kpc}) \\
(6)\end{array}$ & $\begin{array}{c}\mu_{\alpha^{*}} \\
(\mathrm{mas} / \mathrm{yr}) \\
(7)\end{array}$ & $\begin{array}{c}\mu_{\delta} \\
(\mathrm{mas} / \mathrm{yr}) \\
(8)\end{array}$ & $\begin{array}{c}\sigma_{\mu_{\alpha *}} \\
(\mathrm{mas} / \mathrm{yr}) \\
(9)\end{array}$ & $\begin{array}{c}\sigma_{\mu_{\delta}} \\
(\mathrm{mas} / \mathrm{yr}) \\
(10)\end{array}$ & \\
\hline BPS CS 29503-0042 & 0.687 & $\cdots$ & $\cdots$ & 0.900 & 0.687 & 9.7 & -23.3 & 4.1 & 3.5 & SPM \\
\hline BPS CS 30339-0019 & 1.082 & $\ldots$ & $\ldots$ & 0.900 & 1.082 & 31.6 & -59.2 & 3.3 & 2.5 & SPM \\
\hline BPS CS 30339-0007 & 0.733 & $\ldots$ & $\ldots$ & 1.100 & 0.733 & 11.8 & -77.3 & 2.9 & 3.2 & SPM \\
\hline CD-23 72 & 0.186 & 1.163 & 1.84 & 0.600 & 0.186 & 15.2 & -23.7 & 1.0 & 0.8 & HIP,SPM, \\
\hline BPS CS 30339-0010 & 1.044 & $\cdots$ & $\cdots$ & 1.100 & 1.044 & -13.7 & -21.9 & 3.4 & 6.4 & SPM \\
\hline BPS CS 29503-0043 & 2.019 & $\cdots$ & $\cdots$ & 1.100 & 2.019 & 86.4 & -43.3 & 2.8 & 2.7 & SPM \\
\hline BPS CS 30339-0015 & 1.832 & $\cdots$ & $\cdots$ & 2.200 & 1.832 & 44.8 & -30.6 & 4.9 & 2.0 & SPM \\
\hline BPS CS 30339-0009 & 0.709 & $\cdots$ & $\cdots$ & 0.900 & 0.709 & 66.5 & -37.2 & 3.4 & 3.3 & SPM \\
\hline BPS CS 30339-0005 & 0.674 & $\ldots$ & $\ldots$ & 0.900 & 0.674 & -1.6 & -33.8 & 4.7 & 5.9 & SPM \\
\hline [FF93] 513135 & 1.797 & $\cdots$ & $\ldots$ & 1.500 & 1.797 & 15.7 & -0.2 & 1.1 & 1.4 & SPM,ACT \\
\hline CD-35 87 & 1.509 & $\cdots$ & $\cdots$ & 2.070 & 1.509 & 9.5 & 1.3 & 1.1 & 1.3 & SPM,ACT \\
\hline BPS CS 29503-0037 & 0.983 & $\cdots$ & $\cdots$ & 0.800 & 0.983 & 4.4 & -15.4 & 3.8 & 3.5 & SPM,STN \\
\hline BPS CS 22882-0005 & 2.638 & $\cdots$ & $\cdots$ & 2.400 & 2.638 & 12.1 & -3.0 & 2.1 & 2.0 & SPM \\
\hline BPS CS 22882-0001 & 7.465 & $\cdots$ & $\cdots$ & 6.900 & 7.465 & 8.5 & -4.9 & 2.2 & 2.6 & SPM \\
\hline BPS CS 30339-0040 & $\cdots$ & $\cdots$ & $\cdots$ & 1.200 & 1.200 & 1.8 & -7.4 & 3.2 & 2.9 & SPM \\
\hline HD 1638 & 0.980 & 2.381 & 3.24 & 0.666 & 0.980 & 18.5 & -16.5 & 0.8 & 0.9 & HIP,SPM, \\
\hline BPS CS 30339-0037 & 0.803 & $\ldots$ & $\ldots$ & 0.900 & 0.803 & 82.0 & -5.9 & 4.3 & 2.5 & SPM \\
\hline BPS CS 30339-0028 & 1.410 & $\ldots$ & $\cdots$ & 1.100 & 1.410 & 71.6 & -13.2 & 6.5 & 6.5 & SPM \\
\hline NGC 104 LEE 3516 & 8.884 & $\cdots$ & $\cdots$ & 4.169 & 8.884 & $\cdots$ & $\ldots$ & $\cdots$ & $\cdots$ & $\cdots$ \\
\hline BPS CS 22882-0006 & 1.103 & $\cdots$ & $\cdots$ & 1.100 & 1.103 & 18.1 & -56.1 & 2.2 & 2.2 & SPM \\
\hline BPS CS 29503-0049 & 0.745 & $\cdots$ & $\cdots$ & 1.200 & 0.745 & 49.1 & -11.5 & 2.2 & 4.2 & $\mathrm{SPM}$ \\
\hline BPS CS 30339-0052 & 9.457 & $\ldots$ & $\ldots$ & 1.900 & 9.457 & -1.8 & -1.1 & 2.6 & 2.6 & SPM \\
\hline BPS CS 30339-0041 & 1.983 & $\cdots$ & $\cdots$ & 3.800 & 1.983 & -3.8 & -2.1 & 3.9 & 4.0 & SPM \\
\hline BPS CS 29503-0054 & 1.608 & $\cdots$ & $\cdots$ & 1.000 & 1.608 & 13.9 & -16.2 & 6.3 & 5.8 & SPM \\
\hline BPS CS 22882-0009 & 1.996 & $\cdots$ & $\cdots$ & 2.000 & 1.996 & 0.1 & -31.4 & 2.9 & 2.9 & SPM \\
\hline BPS CS 30339-0053 & 1.733 & $\cdots$ & $\cdots$ & 1.300 & 1.733 & 46.2 & -22.5 & 2.5 & 2.4 & SPM \\
\hline BPS CS 22882-0036 & 1.315 & $\cdots$ & $\cdots$ & 1.200 & 1.315 & 26.7 & -1.2 & 2.1 & 2.1 & SPM \\
\hline $\mathrm{V}^{*} \mathrm{SW}$ And & 0.552 & $<0$ & $\cdots$ & 0.562 & 0.552 & -5.7 & -19.7 & 1.1 & 0.8 & $\mathrm{HIP}, \mathrm{ACT}$, \\
\hline BPS CS 30339-0046 & 9.889 & $\cdots$ & $\cdots$ & 1.700 & 9.889 & -2.2 & 0.6 & 2.8 & 2.9 & SPM \\
\hline BPS CS 30339-0061 & 0.479 & $\cdots$ & $\cdots$ & 0.700 & 0.479 & 28.6 & -29.8 & 2.0 & 2.6 & SPM \\
\hline BPS CS 30339-0062 & $\cdots$ & $\cdots$ & $\cdots$ & 1.200 & 1.200 & -1.0 & -3.8 & 3.4 & 2.7 & SPM \\
\hline BPS CS 30339-0057 & $\ldots$ & $\cdots$ & $\cdots$ & 1.800 & 1.800 & -1.1 & -37.9 & 2.1 & 2.3 & SPM \\
\hline [FF93] 514765 & 1.559 & $\cdots$ & $\cdots$ & 0.642 & 1.559 & 14.4 & -16.5 & 1.6 & 0.9 & $\mathrm{SPM}, \mathrm{ACT}$ \\
\hline BPS CS 30339-0069 & 1.534 & $\ldots$ & $\ldots$ & 1.400 & 1.534 & 23.6 & -20.2 & 2.9 & 3.0 & SPM \\
\hline BPS CS 22882-0030 & 1.594 & $\ldots$ & $\ldots$ & 1.600 & 1.594 & 20.3 & -7.9 & 2.0 & 1.6 & SPM \\
\hline BPS CS 22882-0012 & 2.007 & $\cdots$ & $\cdots$ & 2.000 & 2.007 & 36.6 & -18.0 & 1.9 & 1.8 & SPM \\
\hline BPS CS 30339-0064 & 0.668 & $\ldots$ & $\cdots$ & 0.700 & 0.668 & -1.7 & -63.2 & 2.9 & 2.9 & SPM \\
\hline HD 2665 & 0.179 & 0.474 & 0.43 & 0.330 & 0.179 & 40.3 & -64.4 & 0.8 & 0.5 & HIP,ACT, \\
\hline HD 2743 & 1.184 & $\ldots$ & $\ldots$ & 0.552 & 1.184 & 25.6 & -9.2 & 1.2 & 1.1 & $\mathrm{SPM}, \mathrm{ACT}$ \\
\hline BPS CS 30339-0055 & 0.819 & $\cdots$ & $\cdots$ & 1.000 & 0.819 & 41.2 & -6.0 & 1.4 & 1.8 & $\mathrm{SPM}$ \\
\hline
\end{tabular}


TABle 1B. (continued)

\begin{tabular}{|c|c|c|c|c|c|c|c|c|c|c|}
\hline $\begin{array}{l}\text { Star } \\
(1)\end{array}$ & $\begin{array}{c}D_{p h o} \\
(\mathrm{kpc}) \\
(2)\end{array}$ & $\begin{array}{c}D_{H I P} \\
(\mathrm{kpc}) \\
(3)\end{array}$ & $\begin{array}{c}\sigma_{\pi_{H I P}} / \pi_{H I P} \\
(4)\end{array}$ & $\begin{array}{c}D_{B S L} \\
(\mathrm{kpc}) \\
(5)\end{array}$ & $\begin{array}{c}D_{\text {adopt }} \\
(\mathrm{kpc}) \\
(6)\end{array}$ & $\begin{array}{c}\mu_{\alpha^{*}} \\
(\mathrm{mas} / \mathrm{yr}) \\
(7)\end{array}$ & $\begin{array}{c}\mu_{\delta} \\
(\mathrm{mas} / \mathrm{yr}) \\
(8)\end{array}$ & $\begin{array}{c}\sigma_{\mu_{\alpha *}} \\
(\mathrm{mas} / \mathrm{yr}) \\
(9)\end{array}$ & $\begin{array}{c}\sigma_{\mu_{\delta}} \\
(\mathrm{mas} / \mathrm{yr}) \\
(10)\end{array}$ & \\
\hline BPS CS 22882-0016 & 0.746 & $\ldots$ & $\ldots$ & 1.200 & 0.746 & 10.8 & -18.0 & 1.8 & 2.4 & SPM \\
\hline HD 2796 & 0.383 & $<0$ & $\ldots$ & 0.422 & 0.383 & -2.0 & -52.1 & 1.1 & 0.6 & $\mathrm{HIP}, t$ \\
\hline HD 2857 & 0.716 & 0.559 & 0.93 & 0.468 & 0.716 & -11.8 & -66.1 & 0.7 & 0.8 & HIP,$t$ \\
\hline HD 3010 & 0.823 & $\ldots$ & $\ldots$ & 0.721 & 0.823 & 33.7 & 0.5 & 0.9 & 1.4 & SPM, \\
\hline HD 3008 & 1.893 & 0.347 & 0.51 & 1.780 & 1.893 & 15.4 & -8.3 & 1.2 & 0.7 & HIP,, \\
\hline $\mathrm{V}^{*} \mathrm{RX}$ Cet & 1.392 & 0.370 & 0.97 & 1.349 & 1.392 & -25.2 & -61.4 & 1.8 & 1.5 & HIP,$t$ \\
\hline BPS CS 22882-0029 & 1.483 & $\ldots$ & $\ldots$ & 1.400 & 1.483 & 12.9 & -9.2 & 2.1 & 2.5 & SPM \\
\hline BPS CS 30339-0080 & 1.509 & $\ldots$ & $\ldots$ & 1.500 & 1.509 & 21.8 & -10.8 & 3.5 & 2.0 & SPM \\
\hline HD 3179 & 0.181 & 0.338 & 0.53 & $\ldots$ & 0.181 & 39.6 & -37.2 & 1.2 & 0.8 & HIP, $\mathrm{S}$ \\
\hline BPS CS 30339-0073 & 3.041 & $\ldots$ & $\ldots$ & 2.900 & 3.041 & $\ldots$ & $\ldots$ & $\cdots$ & $\cdots$ & $\ldots$ \\
\hline [FF93] 514560 & 1.011 & $\ldots$ & $\ldots$ & 0.487 & 1.011 & 19.7 & -19.4 & 1.0 & 1.4 & SPM, \\
\hline HD 3232 & 0.627 & 0.355 & 0.43 & 0.192 & 0.627 & 15.2 & -75.5 & 1.1 & 0.7 & HIP, \\
\hline HD 3276 & 1.425 & $\ldots$ & $\ldots$ & 0.500 & 1.425 & -9.4 & -10.7 & 1.3 & 1.2 & SPM, \\
\hline BPS CS 29527-0031 & 4.326 & $\ldots$ & $\ldots$ & 4.093 & 4.326 & $\ldots$ & $\ldots$ & $\ldots$ & $\ldots$ & $\ldots$ \\
\hline BPS CS 22882-0024 & 1.234 & $\ldots$ & $\ldots$ & 1.200 & 1.234 & 51.6 & -42.1 & 2.4 & 2.7 & SPM \\
\hline BPS CS 30339-0076 & $\ldots$ & $\ldots$ & $\ldots$ & 0.800 & 0.800 & 3.1 & -10.4 & 2.2 & 2.6 & SPM, \\
\hline BPS CS 22882-0026 & 1.151 & $\ldots$ & $\ldots$ & 1.200 & 1.151 & 1.8 & -11.4 & 4.2 & 1.9 & SPM \\
\hline BPS CS 22882-0021 & 0.946 & $\ldots$ & $\ldots$ & 0.900 & 0.946 & 20.1 & -28.1 & 5.3 & 4.4 & SPM \\
\hline [FF93] 514450 & 2.212 & $\ldots$ & $\ldots$ & 0.401 & 2.212 & 11.9 & -5.3 & 1.7 & 1.7 & SPM, \\
\hline HD 3567 & 0.085 & 0.104 & 0.14 & 0.062 & 0.085 & 21.7 & -546.7 & 0.7 & 0.8 & HIP, \\
\hline HD 3582 & 1.456 & $\ldots$ & $\ldots$ & 0.461 & 1.456 & 5.7 & 3.4 & 1.9 & 1.4 & SPM, \\
\hline HD 3633 & 1.250 & $\ldots$ & $\ldots$ & 0.474 & 1.250 & 3.7 & -30.3 & 0.9 & 1.0 & SPM, \\
\hline BPS CS 29497-0033 & 4.644 & $\ldots$ & $\ldots$ & $\ldots$ & 4.644 & 1.6 & -9.4 & 4.0 & 2.7 & SPM \\
\hline BPS CS 22170-0021 & 1.095 & $\ldots$ & $\ldots$ & 1.100 & 1.095 & $\ldots$ & $\ldots$ & $\ldots$ & $\ldots$ & $\ldots$ \\
\hline$-29201 \mathrm{~W} 1$ & $\ldots$ & $\ldots$ & $\ldots$ & 4.018 & 4.018 & 19.9 & 1.5 & 1.6 & 1.7 & SPM, \\
\hline HD 4024 & 0.961 & $\ldots$ & $\ldots$ & 0.990 & 0.961 & 7.3 & -30.4 & 2.1 & 1.5 & SPM, \\
\hline [FF93] 514210 & 2.149 & $\ldots$ & $\ldots$ & 0.673 & 2.149 & -4.2 & -13.5 & 1.6 & 0.9 & SPM, \\
\hline HD 4261 & 0.310 & 0.209 & 0.21 & 0.657 & 0.310 & 33.6 & -7.4 & 0.9 & 0.7 & $\mathrm{HIP}, \mathrm{S}$ \\
\hline HD 4306 & 0.618 & 0.208 & 0.29 & 0.620 & 0.618 & 60.9 & 20.8 & 0.8 & 0.9 & HIP,$A$ \\
\hline BPS CS 22942-0003 & 1.531 & $\ldots$ & $\cdots$ & 1.600 & 1.531 & -32.8 & -47.7 & 2.9 & 2.9 & SPM \\
\hline [FF93] 514133 & 1.362 & $\ldots$ & $\ldots$ & 0.542 & 1.362 & 11.8 & 5.4 & 2.1 & 1.9 & $\mathrm{SPM}$, \\
\hline BPS CS 22942-0002 & 2.699 & $\ldots$ & $\ldots$ & 2.000 & 2.699 & 18.8 & -7.5 & 6.6 & 6.8 & SPM \\
\hline CD-38 245 & 3.754 & $<0$ & $\ldots$ & 3.344 & 3.754 & 15.2 & -8.5 & 1.5 & 1.6 & HIP,, \\
\hline [FF93] 514104 & 1.132 & $\ldots$ & $\ldots$ & 2.099 & 1.132 & 17.5 & -11.6 & 1.8 & 2.0 & SPM, \\
\hline CD-29 222 & 2.163 & $\ldots$ & $\ldots$ & 1.746 & 2.163 & 3.1 & -14.5 & 0.9 & 0.9 & SPM, \\
\hline$-33302 \mathrm{~S} 1$ & 0.668 & $\ldots$ & $\ldots$ & 3.020 & 0.668 & 101.0 & -15.5 & 3.8 & 2.8 & SPM, \\
\hline BD-11 145 & 1.968 & 0.641 & 1.53 & 2.080 & 1.968 & -0.8 & -26.5 & 1.4 & 0.9 & HIP, \\
\hline [FF93] 514033 & 1.799 & $\ldots$ & $\ldots$ & 1.879 & 1.799 & 4.6 & 6.0 & 1.2 & 1.3 & SPM, \\
\hline BPS CS 22170-0040 & $4.212 / 0.719$ & $\cdots$ & $\cdots$ & 0.900 & 4.212 & $\ldots$ & $\ldots$ & $\cdots$ & $\cdots$ & $\ldots$ \\
\hline $\mathrm{V}^{*} \mathrm{ZZ}$ And & 2.896 & $\cdots$ & $\cdots$ & $\ldots$ & 2.896 & 25.0 & -18.0 & 5 & 5 & NPM \\
\hline
\end{tabular}


TABle 1B. (continued)

\begin{tabular}{|c|c|c|c|c|c|c|c|c|c|c|}
\hline $\begin{array}{l}\text { Star } \\
(1)\end{array}$ & $\begin{array}{c}D_{p h o} \\
(\mathrm{kpc}) \\
(2)\end{array}$ & $\begin{array}{c}D_{H I P} \\
(\mathrm{kpc}) \\
(3)\end{array}$ & $\begin{array}{c}\sigma_{\pi_{H I P}} / \pi_{H I P} \\
(4)\end{array}$ & $\begin{array}{c}D_{B S L} \\
(\mathrm{kpc}) \\
(5)\end{array}$ & $\begin{array}{c}D_{\text {adopt }} \\
(\mathrm{kpc}) \\
(6)\end{array}$ & $\begin{array}{c}\mu_{\alpha^{*}} \\
(\mathrm{mas} / \mathrm{yr}) \\
(7)\end{array}$ & $\begin{array}{c}\mu_{\delta} \\
(\mathrm{mas} / \mathrm{yr}) \\
(8)\end{array}$ & $\begin{array}{c}\sigma_{\mu_{\alpha *}} \\
(\mathrm{mas} / \mathrm{yr}) \\
(9)\end{array}$ & $\begin{array}{c}\sigma_{\mu_{\delta}} \\
(\mathrm{mas} / \mathrm{yr}) \\
(10)\end{array}$ & \\
\hline $\mathrm{V}^{*} \mathrm{AE}$ Tuc & 1.826 & $\ldots$ & $\ldots$ & $\ldots$ & 1.826 & $\ldots$ & $\ldots$ & $\ldots$ & $\ldots$ & $\ldots$ \\
\hline HD 5098 & 0.171 & 0.153 & 0.14 & 0.685 & 0.171 & 37.4 & 33.4 & 0.7 & 0.6 & HIP, \\
\hline BPS CS 22942-0010 & 1.043 & $\ldots$ & $\ldots$ & 1.000 & 1.043 & 22.4 & 0.8 & 4.9 & 4.7 & SPM \\
\hline $\mathrm{E}-29.0 .069$ & 1.711 & $\ldots$ & $\ldots$ & 1.706 & 1.711 & -4.8 & -6.6 & 2.4 & 1.8 & SPM, \\
\hline E-29.3.052 & $\ldots$ & $\ldots$ & $\ldots$ & 2.696 & 2.696 & 6.0 & 2.2 & 7.7 & 10.6 & SPM \\
\hline BPS CS 22942-0011 & 2.241 & $\ldots$ & $\ldots$ & 2.100 & 2.241 & 4.2 & -11.5 & 1.7 & 4.3 & SPM \\
\hline$-28285 \mathrm{~S} 1$ & 1.694 & $\ldots$ & $\ldots$ & 4.325 & 1.694 & $\ldots$ & $\ldots$ & $\ldots$ & $\ldots$ & $\ldots$ \\
\hline E-30.3.078 & $\ldots$ & $\ldots$ & $\ldots$ & 2.108 & 2.108 & 15.8 & -7.4 & 2.1 & 2.2 & SPM \\
\hline $\mathrm{V}^{*} \mathrm{AG}$ Tuc & 2.947 & $\ldots$ & $\ldots$ & 2.655 & 2.947 & $\ldots$ & $\ldots$ & $\ldots$ & $\ldots$ & $\ldots$ \\
\hline BPS CS 22166-0007 & 1.909 & $\cdots$ & $\ldots$ & 1.600 & 1.909 & $\ldots$ & $\ldots$ & $\ldots$ & $\ldots$ & $\cdots$ \\
\hline E-30.5.084 & 3.541 & $\ldots$ & $\ldots$ & 3.215 & 3.541 & -3.6 & -17.2 & 1.5 & 2.2 & SPM, \\
\hline-24 396E1 & 0.752 & $\ldots$ & $\ldots$ & 2.138 & 0.752 & $\ldots$ & $\ldots$ & $\ldots$ & $\ldots$ & $\ldots$ \\
\hline HD 5426 & 0.664 & 5.882 & 9.41 & 0.760 & 0.664 & 24.1 & -52.3 & 1.1 & 0.7 & HIP, \\
\hline HD 5422 & 0.665 & 0.292 & 0.34 & 0.394 & 0.665 & -6.1 & -6.7 & 1.1 & 0.7 & HIP,$A$ \\
\hline BPS CS 22942-0021 & $5.278 / 1.347$ & $\ldots$ & $\ldots$ & 5.600 & 5.278 & $\ldots$ & $\ldots$ & $\ldots$ & $\ldots$ & $\ldots$ \\
\hline E-30.1.080 & 3.046 & $\ldots$ & $\ldots$ & 2.654 & 3.046 & 15.1 & -8.4 & 1.7 & 2.1 & SPM, \\
\hline CD-28 292 & 2.903 & $\ldots$ & $\ldots$ & 2.158 & 2.903 & 3.2 & -7.5 & 1.1 & 1.4 & SPM, \\
\hline E-29.1.079 & 5.399 & $\ldots$ & $\ldots$ & 4.757 & 5.399 & 2.4 & -17.1 & 1.5 & 1.6 & SPM \\
\hline BD-20 170 & 0.398 & 0.952 & 1.66 & 1.280 & 0.398 & 24.4 & -5.2 & 1.2 & 0.8 & HIP,$A$ \\
\hline CD-30 288 & 0.359 & $\ldots$ & $\ldots$ & 0.827 & 0.359 & 17.7 & -0.1 & 1.6 & 1.1 & SPM, \\
\hline $\mathrm{V}^{*} \mathrm{~W}$ Tuc & 1.431 & 0.205 & 0.37 & 1.426 & 1.431 & 4.3 & -19.8 & 1.5 & 1.7 & HIP,$A$ \\
\hline Bok II 274 & 1.909 & $\ldots$ & $\ldots$ & 0.762 & 1.909 & 4. & -1 & 4.9 & 4.9 & STN \\
\hline Bok III 233 & 1.909 & $\ldots$ & $\ldots$ & 4.846 & 1.909 & $\ldots$ & $\ldots$ & $\ldots$ & $\ldots$ & $\ldots$ \\
\hline Bok II 284 & 1.466 & $\ldots$ & $\ldots$ & 2.258 & 1.466 & 9.4 & -13.9 & 1.3 & 1.6 & SPM \\
\hline BPS CS 22166-0016 & 1.705 & $\ldots$ & $\ldots$ & 1.700 & 1.705 & 36. & -23 & 5.3 & 5.3 & STN \\
\hline CD-30 298 & 1.011 & 0.943 & 2.10 & 1.300 & 1.011 & 66.8 & 23.5 & 0.8 & 0.8 & HIP,S \\
\hline E-29.3.099 & 2.489 & $\ldots$ & $\ldots$ & 2.606 & 2.489 & -8.0 & -17.3 & 1.5 & 1.5 & SPM \\
\hline Bok III 281 & 0.370 & $\ldots$ & $\ldots$ & 1.601 & 0.370 & 44.7 & -1.1 & 1.6 & 1.4 & SPM, \\
\hline BPS CS 22942-0024 & 1.126 & $\ldots$ & $\ldots$ & 1.200 & 1.126 & -15.8 & -44.9 & 5.9 & 5.9 & SPM \\
\hline Bok III 167 & 2.271 & $\cdots$ & $\cdots$ & 0.621 & 2.271 & $\ldots$ & $\ldots$ & $\cdots$ & $\cdots$ & $\cdots$ \\
\hline CD-31 394 & 2.534 & $\ldots$ & $\ldots$ & 1.219 & 2.534 & 4.6 & -5.2 & 1.3 & 1.4 & SPM, \\
\hline $\mathrm{V}^{*} \mathrm{WY} \mathrm{Scl}$ & 3.012 & $\ldots$ & $\ldots$ & $\ldots$ & 3.012 & 11.5 & -13.9 & 2.9 & 3.8 & SPM \\
\hline Bok II 256 & 1.878 & $\ldots$ & $\ldots$ & 1.305 & 1.878 & $\ldots$ & $\ldots$ & $\ldots$ & $\ldots$ & $\ldots$ \\
\hline Bok III 179 & 3.006 & $\ldots$ & $\ldots$ & 2.944 & 3.006 & -0.1 & -18.7 & 1.4 & 1.3 & SPM, \\
\hline $\mathrm{V}^{*} \mathrm{RU}$ Cet & 1.629 & 0.331 & 1.05 & 1.542 & 1.629 & 22.0 & -25.0 & 2.4 & 1.7 & HIP, \\
\hline$-28308 \mathrm{~S} 1$ & 1.655 & $\ldots$ & $\ldots$ & 5.321 & 1.655 & -7.8 & -28.9 & 3.9 & 3.7 & SPM \\
\hline$-290055 \mathrm{D}$ & 10.492 & $\ldots$ & $\ldots$ & 9.200 & 10.492 & 1.7 & -6.6 & 5.5 & 4.9 & SPM \\
\hline E-29.0.138 & 0.261 & 0.329 & 0.48 & 0.641 & 0.261 & 29.4 & -47.6 & 1.2 & 1.0 & HIP, $\mathrm{S}$ \\
\hline HD 6054 & 1.398 & $\ldots$ & $\ldots$ & 1.393 & 1.398 & 4.6 & -5.9 & 1.1 & 1.0 & SPM, \\
\hline$-290055 \mathrm{E}$ & 5.251 & $\ldots$ & $\ldots$ & 3.800 & 5.251 & 11.8 & 1.1 & 4.8 & 4.7 & SPM \\
\hline
\end{tabular}


TABLE 1B. (continued)

\begin{tabular}{|c|c|c|c|c|c|c|c|c|c|c|}
\hline $\begin{array}{l}\text { Star } \\
(1)\end{array}$ & $\begin{array}{c}D_{p h o} \\
(\mathrm{kpc}) \\
(2)\end{array}$ & $\begin{array}{c}D_{H I P} \\
(\mathrm{kpc}) \\
(3)\end{array}$ & $\begin{array}{c}\sigma_{\pi_{H I P}} / \pi_{H I P} \\
(4)\end{array}$ & $\begin{array}{c}D_{B S L} \\
(\mathrm{kpc}) \\
(5)\end{array}$ & $\begin{array}{c}D_{\text {adopt }} \\
\text { (kpc) } \\
(6)\end{array}$ & $\begin{array}{c}\mu_{\alpha^{*}} \\
(\mathrm{mas} / \mathrm{yr}) \\
(7)\end{array}$ & $\begin{array}{c}\mu_{\delta} \\
(\mathrm{mas} / \mathrm{yr}) \\
(8)\end{array}$ & $\begin{array}{c}\sigma_{\mu_{\alpha^{*}}} \\
(\mathrm{mas} / \mathrm{yr}) \\
(9)\end{array}$ & $\begin{array}{c}\sigma_{\mu_{\delta}} \\
(\mathrm{mas} / \mathrm{yr}) \\
(10)\end{array}$ & Sou \\
\hline$-300055 \mathrm{~A}$ & 18.957 & $\ldots$ & $\ldots$ & 21.700 & 18.957 & -3.4 & -2.3 & 2.4 & 2.4 & SPM \\
\hline BPS CS 22166-0022 & 0.981 & $\ldots$ & $\ldots$ & 1.500 & 0.981 & $\ldots$ & $\ldots$ & $\ldots$ & $\ldots$ & $\ldots$ \\
\hline BPS CS 22942-0029 & 1.488 & $\ldots$ & $\ldots$ & 1.500 & 1.488 & 30.8 & -7.6 & 1.6 & 2.5 & SPM \\
\hline$-290055 \mathrm{C}$ & 12.937 & $\ldots$ & $\ldots$ & 11.500 & 12.937 & 1.1 & -4.2 & 5.3 & 5.1 & SPM \\
\hline CD-30 314 & $\ldots$ & $\ldots$ & $\ldots$ & 2.857 & 2.857 & 16. & -20 & 6.4 & 6.4 & STN \\
\hline Bok III 82 & 2.166 & $\ldots$ & $\ldots$ & 1.273 & 2.166 & $\ldots$ & $\ldots$ & $\ldots$ & $\ldots$ & $\ldots$ \\
\hline BPS CS 22953-0003 & 4.510 & $\ldots$ & $\ldots$ & 3.900 & 4.510 & $\ldots$ & $\ldots$ & $\ldots$ & $\ldots$ & $\ldots$ \\
\hline HD 6446 & 0.619 & 0.510 & 0.29 & 0.580 & 0.619 & 39.3 & -49.0 & 0.5 & 0.6 & HIP,ACT, \\
\hline$-310100 \mathrm{C}$ & 6.633 & $\ldots$ & $\ldots$ & 7.400 & 6.633 & 3.0 & -2.2 & 2.0 & 2.0 & SPM \\
\hline$-320100 \mathrm{~A}$ & 31.020 & $\ldots$ & $\ldots$ & 21.700 & 31.020 & -2.1 & -0.2 & 6.0 & 4.0 & SPM \\
\hline$-290100 \mathrm{C}$ & 12.857 & $\ldots$ & $\ldots$ & 10.000 & 12.857 & -2.3 & -2.8 & 2.0 & 2.0 & SPM \\
\hline [FF93] 515282 & 1.668 & $\ldots$ & $\ldots$ & 3.251 & 1.668 & 4.2 & -33.2 & 2.4 & 1.7 & SPM,STN \\
\hline CD-29 308 & 0.190 & $\ldots$ & $\ldots$ & 1.768 & 0.190 & -23.5 & -18.7 & 1.1 & 1.4 & SPM,ACT \\
\hline BPS CS 22942-0033 & 1.071 & $\ldots$ & $\ldots$ & 1.100 & 1.071 & 16.0 & -1.2 & 4.7 & 4.8 & SPM \\
\hline HD 6255 & 1.237 & 1.282 & 1.79 & 0.616 & 1.237 & 18.1 & -8.2 & 0.7 & 0.9 & HIP,SPM, \\
\hline HD 6268 & 0.611 & 0.515 & 0.54 & 0.700 & 0.611 & -28.8 & -34.9 & 0.7 & 0.9 & HIP,SPM, \\
\hline$-280100 \mathrm{~A}$ & 11.129 & $\ldots$ & $\ldots$ & 8.400 & 11.129 & 4.9 & -0.3 & 2.5 & 2.5 & SPM \\
\hline$-300100 \mathrm{~F}$ & 16.591 & $\ldots$ & $\ldots$ & 14.600 & 16.591 & -4.8 & -1.3 & 2.0 & 2.0 & SPM \\
\hline HD 6229 & 0.135 & 0.408 & 0.50 & 0.430 & 0.135 & 13.9 & -21.6 & 0.6 & 0.5 & HIP,ACT, \\
\hline BPS CS 22166-0024 & 0.908 & $\ldots$ & $\cdots$ & 0.900 & 0.908 & $\ldots$ & $\ldots$ & $\cdots$ & $\cdots$ & .. \\
\hline BPS CS 22942-0035 & 4.305 & $\ldots$ & $\ldots$ & 3.900 & 4.305 & 4.3 & -15.3 & 2.0 & 2.0 & SPM \\
\hline [FF93] 507215 & 2.243 & $\ldots$ & $\ldots$ & 1.324 & 2.243 & -3.6 & -12.9 & 0.9 & 1.0 & SPM,ACT \\
\hline $\mathrm{V}^{*} \mathrm{DR}$ And & 2.122 & $\ldots$ & $\ldots$ & $\ldots$ & 2.122 & 31.0 & -11.9 & 4.4 & 4.4 & STN,NPM \\
\hline$-300100 \mathrm{~A}$ & 18.622 & $\ldots$ & $\ldots$ & 20.600 & 18.622 & -3.0 & -1.6 & 2.4 & 2.4 & SPM \\
\hline HD 6461 & 0.103 & 0.529 & 0.52 & 0.223 & 0.103 & 64.0 & 49.6 & 0.7 & 0.5 & $\mathrm{HIP}, \mathrm{ACT}$, \\
\hline CD-28 337 & 1.461 & $\ldots$ & $\ldots$ & 1.811 & 1.461 & $\ldots$ & $\ldots$ & $\ldots$ & $\ldots$ & $\ldots$ \\
\hline CD-29 324 & 1.080 & $\ldots$ & $\ldots$ & 1.329 & 1.080 & $\ldots$ & $\ldots$ & $\ldots$ & $\ldots$ & $\ldots$ \\
\hline Bok III 45 & 4.175 & $\ldots$ & $\ldots$ & 1.723 & 4.175 & 4. & -13 & 5.8 & 5.8 & STN \\
\hline CD-31 434 & 0.755 & $\ldots$ & $\ldots$ & 2.051 & 0.755 & 3.2 & -24.3 & 1.0 & 1.2 & $\mathrm{SPM}, \mathrm{ACT}$ \\
\hline$-310100 \mathrm{~A}$ & 8.183 & $\cdots$ & $\cdots$ & 7.700 & 8.183 & -1.5 & -2.2 & 2.8 & 2.8 & SPM \\
\hline CD-30 339 & 3.148 & $\ldots$ & $\ldots$ & 1.303 & 3.148 & $\ldots$ & $\ldots$ & $\ldots$ & $\ldots$ & $\ldots$ \\
\hline BPS CS 29514-0004 & $\ldots$ & $\ldots$ & $\ldots$ & 2.100 & 2.100 & 4.6 & -21.9 & 2.1 & 2.1 & SPM \\
\hline Bok III 43 & 0.864 & $\ldots$ & $\ldots$ & 0.848 & 0.864 & $\ldots$ & $\ldots$ & $\ldots$ & $\ldots$ & $\ldots$ \\
\hline [FF93] 507267 & 1.816 & $\ldots$ & $\ldots$ & 1.265 & 1.816 & 7.2 & -9.3 & 1.4 & 1.4 & SPM,STN \\
\hline BPS CS 29514-0007 & 1.018 & $\ldots$ & $\ldots$ & 1.100 & 1.018 & 91.1 & -67.4 & 5.8 & 5.4 & SPM \\
\hline$-300100 \mathrm{~B}$ & 10.786 & $\ldots$ & $\ldots$ & 15.100 & 10.786 & -3.8 & -2.3 & 2.1 & 2.1 & SPM \\
\hline$-28336 \mathrm{~N} 1$ & 0.963 & $\ldots$ & $\ldots$ & 3.236 & 0.963 & 19.4 & -18.7 & 2.4 & 1.7 & SPM,STN \\
\hline$-290100 \mathrm{E}$ & 1.942 & $\ldots$ & $\ldots$ & 1.600 & 1.942 & 29.9 & -3.0 & 2.3 & 1.8 & SPM \\
\hline $\mathrm{V}^{*} \mathrm{AE} \mathrm{Scl}$ & 2.382 & $\cdots$ & $\ldots$ & $\ldots$ & 2.382 & $\ldots$ & $\ldots$ & $\cdots$ & $\ldots$ & $\ldots$ \\
\hline BPS CS 22953-0005 & 1.957 & $\cdots$ & $\cdots$ & 1.800 & 1.957 & $\cdots$ & $\cdots$ & $\cdots$ & $\cdots$ & $\cdots$ \\
\hline
\end{tabular}


TABLE 1B. (continued)

\begin{tabular}{|c|c|c|c|c|c|c|c|c|c|c|}
\hline $\begin{array}{l}\text { Star } \\
(1)\end{array}$ & $\begin{array}{c}D_{\text {pho }} \\
(\mathrm{kpc}) \\
(2)\end{array}$ & $\begin{array}{c}D_{H I P} \\
(\mathrm{kpc}) \\
(3)\end{array}$ & $\begin{array}{c}\sigma_{\pi_{H I P}} / \pi_{H I P} \\
(4)\end{array}$ & $\begin{array}{c}D_{B S L} \\
(\mathrm{kpc}) \\
(5)\end{array}$ & $\begin{array}{c}D_{\text {adopt }} \\
\text { (kpc) } \\
(6)\end{array}$ & $\begin{array}{c}\mu_{\alpha^{*}} \\
(\mathrm{mas} / \mathrm{yr}) \\
(7)\end{array}$ & $\begin{array}{c}\mu_{\delta} \\
(\mathrm{mas} / \mathrm{yr}) \\
(8)\end{array}$ & $\begin{array}{c}\sigma_{\mu_{\alpha *}} \\
(\mathrm{mas} / \mathrm{yr}) \\
(9)\end{array}$ & $\begin{array}{c}\sigma_{\mu_{\delta}} \\
(\mathrm{mas} / \mathrm{yr}) \\
(10)\end{array}$ & Sou \\
\hline BPS CS 29514-0001 & 0.961 & $\ldots$ & $\ldots$ & 1.200 & 0.961 & -3.4 & -19.0 & 3.9 & 4.0 & SPM \\
\hline CD-33 410 & 1.228 & $\ldots$ & $\ldots$ & 2.070 & 1.228 & -9.0 & -22.1 & 2.0 & 0.9 & SPM,ACT \\
\hline BPS CS 22953-0011 & 1.582 & $\ldots$ & $\ldots$ & 1.300 & 1.582 & $\ldots$ & $\ldots$ & $\ldots$ & $\ldots$ & $\ldots$ \\
\hline$-290105 \mathrm{H}$ & 3.260 & $\ldots$ & $\ldots$ & 4.300 & 3.260 & -2.8 & -2.1 & 2.3 & 1.7 & SPM \\
\hline [FF93] 515144 & 1.198 & $\ldots$ & $\ldots$ & 0.879 & 1.198 & 15.6 & -26.6 & 1.2 & 1.4 & SPM,ACT \\
\hline [FF93] 507329 & 1.454 & $\ldots$ & $\ldots$ & 1.387 & 1.454 & -1.0 & -27.6 & 1.4 & 1.3 & $\mathrm{SPM}, \mathrm{ACT}$ \\
\hline BPS CS 22183-0031 & 2.968 & $\ldots$ & $\ldots$ & 1.900 & 2.968 & $\ldots$ & $\ldots$ & $\ldots$ & $\ldots$ & $\ldots$ \\
\hline$-290105 \mathrm{G}$ & 4.253 & $\ldots$ & $\ldots$ & 2.900 & 4.253 & -0.3 & -7.7 & 2.4 & 2.4 & SPM \\
\hline-290105 B & 2.243 & $\ldots$ & $\ldots$ & 3.200 & 2.243 & 7.2 & -23.4 & 2.5 & 2.1 & SPM \\
\hline BPS CS 29514-0018 & 0.661 & $\ldots$ & $\ldots$ & 0.800 & 0.661 & 15.4 & -6.6 & 1.9 & 2.0 & SPM \\
\hline [FF93] 507335 & 1.403 & $\ldots$ & $\ldots$ & 1.854 & 1.403 & 26.8 & 14.6 & 1.9 & 1.3 & $\mathrm{SPM}, \mathrm{ACT}$ \\
\hline-23 418W1 & 0.965 & $\ldots$ & $\ldots$ & 2.858 & 0.965 & $\ldots$ & $\ldots$ & $\ldots$ & $\ldots$ & $\ldots$ \\
\hline HD 6755 & 0.108 & 0.129 & 0.12 & 0.160 & 0.129 & 627.9 & 76.6 & 0.7 & 0.5 & $\mathrm{HIP}, \mathrm{ACT}$ \\
\hline BPS CS 29514-0017 & 2.189 & $\ldots$ & $\ldots$ & 1.800 & 2.189 & 52.3 & -23.9 & 1.9 & 2.0 & SPM \\
\hline HD 7041 & 0.250 & 0.152 & 0.16 & 0.396 & 0.250 & 133.8 & 13.4 & 0.8 & 1.1 & $\mathrm{HIP}, \mathrm{ACT}$, \\
\hline BPS CS 22953-0016 & 2.363 & $\ldots$ & $\ldots$ & 2.900 & 2.363 & $\ldots$ & $\ldots$ & $\cdots$ & $\ldots$ & $\ldots$ \\
\hline HD 6833 & 0.229 & 0.230 & 0.18 & 0.310 & 0.229 & 20.8 & 35.2 & 0.6 & 0.5 & HIP,ACT, \\
\hline [FF93] 515107 & 0.831 & $\ldots$ & $\ldots$ & 0.666 & 0.831 & 21.9 & 17.2 & 1.6 & 1.3 & $\mathrm{SPM}, \mathrm{ACT}$ \\
\hline CD-33 426 & 1.196 & $\cdots$ & $\ldots$ & 1.862 & 1.196 & 0.2 & -5.8 & 0.8 & 1.4 & $\mathrm{SPM}, \mathrm{ACT}$ \\
\hline $\mathrm{V}^{*} \mathrm{TZ}$ Phe & 2.367 & $\ldots$ & $\ldots$ & $\ldots$ & 2.367 & 14.6 & -5.4 & 2.0 & 1.9 & SPM,STN \\
\hline CD-27 379 & 0.814 & $\ldots$ & $\cdots$ & 1.175 & 0.814 & -6.4 & -17.2 & 1.5 & 1.2 & $\mathrm{SPM}, \mathrm{ACT}$ \\
\hline E-30.0.203 & 1.581 & $\ldots$ & $\ldots$ & 3.613 & 1.581 & 12.8 & 5.4 & 1.6 & 1.6 & SPM,STN \\
\hline$-290105 \mathrm{E}$ & 4.813 & $\ldots$ & $\ldots$ & 2.900 & 4.813 & 13.1 & -4.4 & 2.8 & 2.0 & SPM \\
\hline BPS CS 29514-0012 & 0.783 & $\ldots$ & $\ldots$ & 1.000 & 0.783 & 7.0 & -3.0 & 1.8 & 4.4 & SPM \\
\hline HD 7150 & 1.266 & $\ldots$ & $\ldots$ & 0.255 & 1.266 & 18.2 & -36.0 & 0.9 & 1.0 & $\mathrm{SPM}, \mathrm{ACT}$ \\
\hline BPS CS 29514-0014 & $\ldots$ & $\ldots$ & $\ldots$ & 1.300 & 1.300 & 36.8 & -49.2 & 3.1 & 2.4 & SPM \\
\hline$-290105 \mathrm{~N}$ & 10.231 & $\ldots$ & $\ldots$ & 6.500 & 10.231 & -3.4 & -2.0 & 2.2 & 2.9 & SPM \\
\hline BPS CS 22946-0003 & 1.263 & $\ldots$ & $\ldots$ & 2.100 & 1.263 & $\ldots$ & $\ldots$ & $\ldots$ & $\ldots$ & $\ldots$ \\
\hline$-290110 \mathrm{~A}$ & 4.488 & $\ldots$ & $\ldots$ & 5.200 & 4.488 & -4.3 & -16.4 & 2.2 & 2.3 & SPM \\
\hline BPS CS 22946-0002 & 1.537 & $\cdots$ & $\cdots$ & 1.300 & 1.537 & $\cdots$ & $\ldots$ & $\cdots$ & $\cdots$ & $\ldots$ \\
\hline BPS CS 22953-0023 & 1.090 & $\ldots$ & $\ldots$ & 1.200 & 1.090 & $\ldots$ & $\ldots$ & $\ldots$ & $\ldots$ & $\ldots$ \\
\hline [FF93] 507458 & 2.597 & $\ldots$ & $\ldots$ & 2.109 & 2.597 & -0.9 & -1.8 & 1.7 & 1.5 & SPM,STN \\
\hline [FF93] 507463 & 1.589 & $\ldots$ & $\ldots$ & 1.592 & 1.589 & 7.7 & 1.1 & 1.4 & 1.5 & $\mathrm{SPM}, \mathrm{ACT}$ \\
\hline$-310110 \mathrm{C}$ & 5.225 & $\ldots$ & $\ldots$ & 3.500 & 5.225 & 16.7 & -8.2 & 2.0 & 2.2 & SPM \\
\hline BPS CS 22174-0012 & 0.901 & $\ldots$ & $\ldots$ & 0.800 & 0.901 & $\ldots$ & $\ldots$ & $\ldots$ & $\ldots$ & $\ldots$ \\
\hline$-300110 \mathrm{C}$ & 16.814 & $\ldots$ & $\ldots$ & 14.700 & 16.814 & -1.4 & -3.0 & 3.3 & 1.8 & SPM \\
\hline Bok IV 43 & 0.379 & $\ldots$ & $\ldots$ & 3.519 & 0.379 & $\ldots$ & $\ldots$ & $\ldots$ & $\ldots$ & $\ldots$ \\
\hline $\mathrm{V}^{*} \mathrm{RU}$ Psc & 0.802 & 0.455 & 0.74 & 0.655 & 0.802 & 97.8 & -40.2 & 0.8 & 0.6 & HIP,ACT, \\
\hline$-290110 \mathrm{C}$ & 10.484 & $\ldots$ & $\ldots$ & 10.100 & 10.484 & 0.6 & -3.4 & 2.8 & 2.3 & SPM \\
\hline HD 7595 & 1.252 & $\cdots$ & $\cdots$ & 0.917 & 1.252 & 3.7 & -21.6 & 1.5 & 1.1 & $\mathrm{SPM}, \mathrm{ACT}$ \\
\hline
\end{tabular}


TABLE 1B. (continued)

\begin{tabular}{|c|c|c|c|c|c|c|c|c|c|c|}
\hline $\begin{array}{l}\text { Star } \\
(1)\end{array}$ & $\begin{array}{c}D_{p h o} \\
(\mathrm{kpc}) \\
(2)\end{array}$ & $\begin{array}{c}D_{H I P} \\
(\mathrm{kpc}) \\
(3)\end{array}$ & $\begin{array}{c}\sigma_{\pi_{H I P}} / \pi_{H I P} \\
(4)\end{array}$ & $\begin{array}{c}D_{B S L} \\
(\mathrm{kpc}) \\
(5)\end{array}$ & $\begin{array}{c}D_{\text {adopt }} \\
(\mathrm{kpc}) \\
\quad(6)\end{array}$ & $\begin{array}{c}\mu_{\alpha^{*}} \\
(\mathrm{mas} / \mathrm{yr}) \\
(7)\end{array}$ & $\begin{array}{c}\mu_{\delta} \\
(\mathrm{mas} / \mathrm{yr}) \\
(8)\end{array}$ & $\begin{array}{c}\sigma_{\mu_{\alpha^{*}}} \\
(\mathrm{mas} / \mathrm{yr}) \\
(9)\end{array}$ & $\begin{array}{c}\sigma_{\mu_{\delta}} \\
(\mathrm{mas} / \mathrm{yr}) \\
(10)\end{array}$ & Sou \\
\hline$-310110 \mathrm{~B}$ & 3.197 & $\ldots$ & $\ldots$ & 2.000 & 3.197 & 3.5 & -5.0 & 2.6 & 2.8 & SPM \\
\hline [FF93] 516565 & 2.550 & $\ldots$ & $\ldots$ & 0.508 & 2.550 & 4.3 & -15.8 & 1.4 & 1.5 & SPM,ACT \\
\hline$-310110 \mathrm{~A}$ & 3.941 & $\cdots$ & $\cdots$ & 3.500 & 3.941 & 4.5 & -3.7 & 2.8 & 2.3 & SPM \\
\hline CD-27 412 & 0.514 & $\cdots$ & $\cdots$ & $\ldots$ & 0.514 & 6.0 & -8.4 & 0.8 & 1.0 & SPM,ACT \\
\hline$-290110 \mathrm{D}$ & 15.678 & $\cdots$ & $\cdots$ & 12.800 & 15.678 & 5.5 & -5.2 & 3.0 & 2.9 & SPM \\
\hline $\mathrm{V}^{*} \mathrm{XX}$ And & 1.028 & 0.667 & 1.43 & 0.959 & 1.028 & 54.5 & -34.5 & 1.1 & 1.2 & $\mathrm{HIP}, \mathrm{ACT}$ \\
\hline$-300115 \mathrm{~B}$ & 12.971 & $\ldots$ & $\cdots$ & 13.300 & 12.971 & 1.9 & -3.4 & 4.5 & 5.3 & SPM \\
\hline$-300115 \mathrm{C}$ & 6.015 & $\cdots$ & $\cdots$ & 3.000 & 6.015 & 5.5 & -11.8 & 4.9 & 5.2 & SPM \\
\hline$-270115 \mathrm{~A}$ & 17.137 & $\cdots$ & $\cdots$ & 16.900 & 17.137 & 1.0 & -0.2 & 3.3 & 4.4 & SPM \\
\hline BPS CS 29514-0031 & 0.804 & $\cdots$ & $\cdots$ & 0.700 & 0.804 & 27.7 & -10.2 & 6.0 & 5.7 & SPM \\
\hline $\mathrm{V}^{*} \mathrm{VW} \mathrm{Scl}$ & 1.117 & 0.602 & 1.28 & $\cdots$ & 1.117 & 25.3 & -39.1 & 0.8 & 0.7 & $\mathrm{HIP}, \mathrm{SPM}$ \\
\hline $\mathrm{V}^{*} \mathrm{AM}$ Tuc & 1.591 & 4.545 & 8.77 & 1.514 & 1.591 & 23.0 & -29.2 & 1.7 & 1.8 & HIP,STN \\
\hline BPS CS 29514-0034 & 2.470 & $\ldots$ & $\cdots$ & 1.700 & 2.470 & 5.8 & -12.8 & 5.0 & 5.7 & SPM \\
\hline Bok IV 16 & 1.736 & $\cdots$ & $\cdots$ & 1.287 & 1.736 & 19. & -21 & 6.0 & 6.0 & $\mathrm{STN}$ \\
\hline HD 7983 & 0.052 & 0.067 & 0.08 & 0.051 & 0.067 & -229.8 & -458.6 & 1.1 & 0.9 & $\mathrm{HIP}, \mathrm{ACT}$, \\
\hline BPS CS 22174-0020 & 1.644 & $\ldots$ & $\ldots$ & 1.800 & 1.644 & $\ldots$ & $\ldots$ & $\cdots$ & $\cdots$ & $\ldots$ \\
\hline $\mathrm{V}^{*} \mathrm{SS}$ Psc & $\ldots$ & $\cdots$ & $\cdots$ & 1.122 & 1.122 & -0.6 & -3.2 & 1.0 & 0.8 & ACT,STN \\
\hline-310115 A & 24.879 & $\cdots$ & $\cdots$ & 18.300 & 24.879 & 3.4 & -4.4 & 4.7 & 4.8 & SPM \\
\hline HD 8186 & 0.429 & $\cdots$ & $\cdots$ & 0.520 & 0.429 & 12.8 & -24.6 & 1.1 & 0.9 & ACT,STN \\
\hline BPS CS 22946-0017 & 1.381 & $\cdots$ & $\cdots$ & 1.500 & 1.381 & $\cdots$ & $\cdots$ & $\cdots$ & $\cdots$ & $\cdots$ \\
\hline BPS CS 29514-0037 & 0.852 & $\cdots$ & $\cdots$ & 1.000 & 0.852 & 30.3 & -40.7 & 2.1 & 2.6 & SPM \\
\hline$-300115 \mathrm{~A}$ & 20.278 & $\ldots$ & $\ldots$ & 15.100 & 20.278 & 0.6 & -6.4 & 3.1 & 3.1 & SPM \\
\hline-290115 A & 13.923 & $\cdots$ & $\cdots$ & 12.600 & 13.923 & 1.2 & -0.9 & 2.7 & 2.6 & SPM \\
\hline BPS CS 22946-0012 & 1.137 & $\cdots$ & $\cdots$ & 1.300 & 1.137 & $\cdots$ & $\cdots$ & $\cdots$ & $\cdots$ & $\ldots$ \\
\hline BPS CS 22946-0014 & 1.023 & $\cdots$ & $\cdots$ & 1.000 & 1.023 & $\cdots$ & $\cdots$ & $\cdots$ & $\cdots$ & $\cdots$ \\
\hline BPS CS 22953-0037 & 0.900 & $\cdots$ & $\cdots$ & 1.000 & 0.900 & $\cdots$ & $\ldots$ & $\cdots$ & $\cdots$ & $\cdots$ \\
\hline BPS CS 29504-0012 & 1.789 & $\cdots$ & $\cdots$ & 1.500 & 1.789 & 8.1 & -15.8 & 2.9 & 2.9 & SPM \\
\hline HD 8724 & 0.650 & 0.329 & 0.31 & 0.650 & 0.650 & 51.9 & -76.2 & 0.8 & 0.5 & $\mathrm{HIP}, \mathrm{ACT}$, \\
\hline [FF93] 516243 & 1.980 & $\cdots$ & $\cdots$ & 1.057 & 1.980 & 11.4 & -3.3 & 0.9 & 1.1 & $\mathrm{SPM}, \mathrm{ACT}$ \\
\hline $\mathrm{V}^{*} \mathrm{XY}$ And & 3.559 & $\cdots$ & $\cdots$ & $\cdots$ & 3.559 & 13.4 & -2.8 & 5 & 5 & NPM \\
\hline BPS CS 29504-0002 & 0.905 & $\cdots$ & $\cdots$ & 1.000 & 0.905 & -15.2 & -51.6 & 3.2 & 1.8 & SPM \\
\hline HD 9051 & 0.453 & 1.724 & 2.22 & 0.450 & 0.453 & 52.9 & -17.1 & 0.9 & 0.7 & HIP,SPM, \\
\hline BPS CS 29504-0009 & 1.284 & $\ldots$ & $\cdots$ & 0.900 & 1.284 & 2.4 & -4.4 & 3.5 & 5.1 & SPM \\
\hline HD 9360 & 1.003 & $\cdots$ & $\cdots$ & 1.432 & 1.003 & -4.5 & -3.8 & 1.9 & 1.0 & $\mathrm{SPM}, \mathrm{ACT}$ \\
\hline BPS CS 29504-0006 & 1.327 & $\cdots$ & $\cdots$ & 1.400 & 1.327 & -2.0 & 9.9 & 2.3 & 2.4 & SPM \\
\hline $\mathrm{V}^{*} \mathrm{RR}$ Cet & 0.650 & $<0$ & $\cdots$ & 0.608 & 0.650 & 10.5 & -44.0 & 0.9 & 0.8 & $\mathrm{HIP}, \mathrm{ACT}$, \\
\hline BPS CS 22180-0013 & $\cdots$ & $\cdots$ & $\cdots$ & 0.700 & 0.700 & 8. & 8. & 3.4 & 3.4 & STN \\
\hline BPS CS 22953-0031 & 0.821 & $\cdots$ & $\cdots$ & 1.200 & 0.821 & $\cdots$ & $\cdots$ & $\cdots$ & $\cdots$ & $\cdots$ \\
\hline BPS CS 22180-0014 & 0.799 & $\cdots$ & $\cdots$ & 0.800 & 0.799 & $\cdots$ & $\cdots$ & $\cdots$ & $\cdots$ & $\cdots$ \\
\hline BPS CS 22180-0005 & 0.845 & $\cdots$ & $\cdots$ & 1.400 & 0.845 & $\cdots$ & $\cdots$ & $\cdots$ & $\cdots$ & $\cdots$ \\
\hline
\end{tabular}


TABLE 1B. (continued)

\begin{tabular}{|c|c|c|c|c|c|c|c|c|c|c|}
\hline $\begin{array}{l}\text { Star } \\
(1)\end{array}$ & $\begin{array}{l}D_{p h o} \\
(\mathrm{kpc}) \\
(2)\end{array}$ & $\begin{array}{c}D_{H I P} \\
(\mathrm{kpc}) \\
(3)\end{array}$ & $\begin{array}{c}\sigma_{\pi_{H I P}} / \pi_{H I P} \\
(4)\end{array}$ & $\begin{array}{c}D_{B S L} \\
(\mathrm{kpc}) \\
(5)\end{array}$ & $\begin{array}{l}D_{\text {adopt }} \\
\text { (kpc) } \\
(6)\end{array}$ & $\begin{array}{c}\mu_{\alpha^{*}} \\
(\mathrm{mas} / \mathrm{yr}) \\
(7)\end{array}$ & $\begin{array}{c}\mu_{\delta} \\
(\mathrm{mas} / \mathrm{yr}) \\
(8)\end{array}$ & $\begin{array}{c}\sigma_{\mu_{\alpha^{*}}} \\
(\mathrm{mas} / \mathrm{yr}) \\
(9)\end{array}$ & $\begin{array}{c}\sigma_{\mu_{\delta}} \\
(\mathrm{mas} / \mathrm{yr}) \\
(10)\end{array}$ & Sou \\
\hline BPS CS 22180-0010 & 1.572 & $\ldots$ & $\cdots$ & 1.200 & 1.572 & $\cdots$ & $\cdots$ & $\cdots$ & $\cdots$ & $\ldots$ \\
\hline HD 9731 & 0.307 & 0.304 & 0.26 & 1.986 & 0.307 & 24.8 & 12.7 & 0.9 & 0.5 & HIP,SPM, \\
\hline $\mathrm{V}^{*} \mathrm{VX} \mathrm{Scl}$ & 2.046 & $<0$ & $\ldots$ & $\ldots$ & 2.046 & 36.0 & -25.4 & 1.7 & 1.4 & HIP,SPM, \\
\hline BPS CS 22180-0034 & 1.080 & $\ldots$ & $\ldots$ & 1.200 & 1.080 & $\ldots$ & $\ldots$ & $\ldots$ & $\ldots$ & $\ldots$ \\
\hline BD-18 271 & 2.399 & $<0$ & $\ldots$ & 1.135 & 2.399 & 6.6 & -25.3 & 1.1 & 1.1 & HIP,ACT \\
\hline HD 10141 & 0.818 & $\ldots$ & $\ldots$ & 2.333 & 0.818 & 23.9 & -24.4 & 1.7 & 1.2 & SPM,ACT \\
\hline BPS CS 29504-0032 & 0.456 & $\ldots$ & $\ldots$ & 0.600 & 0.456 & -1.8 & -29.6 & 2.7 & 2.4 & SPM \\
\hline HD 10413 & 0.952 & $\ldots$ & $\ldots$ & 1.862 & 0.952 & 16.2 & 7.6 & 2.3 & 1.7 & $\mathrm{SPM}, \mathrm{ACT}$ \\
\hline HD 10607 & 0.054 & 0.071 & 0.05 & 0.058 & 0.071 & 320.78 & -452.10 & 0.72 & 1.11 & HIP \\
\hline BPS CS 22958-0008 & $\ldots$ & $\ldots$ & $\ldots$ & 1.100 & 1.100 & $\ldots$ & $\ldots$ & $\ldots$ & $\ldots$ & $\ldots$ \\
\hline BPS CS 22180-0030 & 2.822 & $\ldots$ & $\cdots$ & 2.600 & 2.822 & $\ldots$ & $\ldots$ & $\ldots$ & $\ldots$ & $\ldots$ \\
\hline $\mathrm{V}^{*} \mathrm{SV} \mathrm{Scl}$ & 1.453 & 0.180 & 0.37 & 1.406 & 1.453 & -2.6 & -35.5 & 1.2 & 1.0 & HIP,SPM, \\
\hline BPS CS 22958-0014 & 1.598 & $\ldots$ & $\ldots$ & 1.400 & 1.598 & $\ldots$ & $\ldots$ & $\ldots$ & $\ldots$ & $\ldots$ \\
\hline BPS CS 29504-0044 & 0.703 & $\ldots$ & $\ldots$ & 0.900 & 0.703 & -21.0 & -35.7 & 3.4 & 3.6 & SPM \\
\hline BPS CS 22958-0004 & 0.874 & $\ldots$ & $\ldots$ & 1.100 & 0.874 & $\ldots$ & $\ldots$ & $\ldots$ & $\ldots$ & $\ldots$ \\
\hline BPS CS 22958-0002 & 2.472 & $\ldots$ & $\ldots$ & 2.800 & 2.472 & -2 & -60 & 5.9 & 5.9 & STN \\
\hline BPS CS 22958-0026 & 0.553 & $\ldots$ & $\ldots$ & 0.700 & 0.553 & $\ldots$ & $\ldots$ & $\ldots$ & $\ldots$ & $\ldots$ \\
\hline HD 11569 & 0.117 & 0.106 & 0.08 & 0.132 & 0.106 & 268.9 & -31.8 & 0.6 & 0.6 & HIP,ACT \\
\hline BD-10 388 & 0.130 & 0.311 & 0.54 & 0.097 & 0.130 & 254.9 & 96.0 & 1.5 & 1.5 & HIP,ACT \\
\hline BPS CS 22958-0025 & 1.570 & $\cdots$ & $\cdots$ & 1.200 & 1.570 & $\cdots$ & $\cdots$ & $\cdots$ & $\cdots$ & $\ldots$ \\
\hline BPS CS 22958-0019 & $\ldots$ & $\ldots$ & $\ldots$ & 1.300 & 1.300 & $\ldots$ & $\ldots$ & $\ldots$ & $\ldots$ & $\ldots$ \\
\hline HD 11582 & 0.222 & 0.312 & 0.43 & 0.278 & 0.222 & 22.8 & -210.6 & 0.8 & 0.7 & HIP,SPM, \\
\hline $\mathrm{V}^{*}$ CI And & 1.822 & $<0$ & $\ldots$ & $\ldots$ & 1.822 & 0.6 & -1.8 & 2.4 & 1.9 & HIP,STN,I \\
\hline BPS CS 22958-0068 & $\ldots$ & $\ldots$ & $\ldots$ & 1.300 & 1.300 & $\ldots$ & $\ldots$ & $\ldots$ & $\ldots$ & $\ldots$ \\
\hline $\mathrm{V}^{*} \mathrm{U}$ Tri & 2.184 & $\ldots$ & $\ldots$ & 2.570 & 2.184 & 10.0 & -13.2 & 4.4 & 4.4 & STN,NPM \\
\hline BPS CS 22958-0036 & 0.986 & $\cdots$ & $\ldots$ & 0.800 & 0.986 & $\ldots$ & $\ldots$ & $\ldots$ & $\ldots$ & $\ldots$ \\
\hline BPS CS 22958-0037 & $\ldots$ & $\ldots$ & $\ldots$ & 2.100 & 2.100 & $\ldots$ & $\ldots$ & $\ldots$ & $\ldots$ & $\ldots$ \\
\hline BPS CS 22958-0061 & 1.091 & $\ldots$ & $\ldots$ & 1.300 & 1.091 & $\ldots$ & $\ldots$ & $\ldots$ & $\ldots$ & $\ldots$ \\
\hline BPS CS 22958-0038 & 0.712 & $\ldots$ & $\ldots$ & 0.900 & 0.712 & 57. & 17. & 9.1 & 9.1 & STN \\
\hline BPS CS 22958-0042 & 1.254 & $\cdots$ & $\cdots$ & 1.400 & 1.254 & $\cdots$ & $\cdots$ & $\cdots$ & $\cdots$ & $\cdots$ \\
\hline BPS CS 22958-0041 & 1.692 & $\ldots$ & $\ldots$ & 1.700 & 1.692 & $\ldots$ & $\ldots$ & $\ldots$ & $\ldots$ & $\ldots$ \\
\hline BPS CS 22958-0065 & 1.190 & $\ldots$ & $\ldots$ & 1.300 & 1.190 & $\ldots$ & $\ldots$ & $\ldots$ & $\ldots$ & $\ldots$ \\
\hline BPS CS 22958-0073 & 1.355 & $\ldots$ & $\ldots$ & 1.200 & 1.355 & $\ldots$ & $\ldots$ & $\ldots$ & $\ldots$ & $\ldots$ \\
\hline $\mathrm{V}^{*}$ SS For & 0.769 & 0.281 & 0.40 & $\ldots$ & 0.769 & 29.4 & -70.5 & 1.1 & 1.1 & HIP,SPM, \\
\hline BPS CS 22171-0037 & 1.690 & $\ldots$ & $\ldots$ & 1.700 & 1.690 & $\ldots$ & $\ldots$ & $\ldots$ & $\ldots$ & $\ldots$ \\
\hline BPS CS 22958-0079 & 1.527 & $\ldots$ & $\ldots$ & 1.300 & 1.527 & $\cdots$ & $\ldots$ & $\cdots$ & $\cdots$ & $\cdots$ \\
\hline BPS CS 22171-0031 & 1.010 & $\ldots$ & $\ldots$ & 1.000 & 1.010 & $\ldots$ & $\ldots$ & $\ldots$ & $\ldots$ & $\ldots$ \\
\hline BPS CS 22171-0034 & 0.702 & $\ldots$ & $\ldots$ & 0.700 & 0.702 & 49. & -53 & 4.7 & 4.7 & STN \\
\hline HD 13359 & 0.334 & 1.250 & 1.80 & 0.470 & 0.334 & -2.3 & -13.7 & 0.9 & 0.7 & HIP,ACT, \\
\hline BPS CS 22958-0049 & 1.044 & $\cdots$ & $\ldots$ & 1.200 & 1.044 & $\ldots$ & $\ldots$ & $\ldots$ & $\ldots$ & $\ldots$ \\
\hline
\end{tabular}


TABLE 1B. (continued)

\begin{tabular}{|c|c|c|c|c|c|c|c|c|c|c|}
\hline $\begin{array}{l}\text { Star } \\
(1)\end{array}$ & $\begin{array}{c}D_{\text {pho }} \\
(\mathrm{kpc}) \\
(2)\end{array}$ & $\begin{array}{c}D_{H I P} \\
(\mathrm{kpc}) \\
(3)\end{array}$ & $\begin{array}{c}\sigma_{\pi_{H I P}} / \pi_{H I P} \\
(4)\end{array}$ & $\begin{array}{c}D_{B S L} \\
(\mathrm{kpc}) \\
(5)\end{array}$ & $\begin{array}{c}D_{\text {adopt }} \\
\text { (kpc) } \\
(6)\end{array}$ & $\begin{array}{c}\mu_{\alpha^{*}} \\
(\mathrm{mas} / \mathrm{yr}) \\
(7)\end{array}$ & $\begin{array}{c}\mu_{\delta} \\
(\mathrm{mas} / \mathrm{yr}) \\
(8)\end{array}$ & $\begin{array}{c}\sigma_{\mu_{\alpha *}} \\
(\mathrm{mas} / \mathrm{yr}) \\
(9)\end{array}$ & $\begin{array}{c}\sigma_{\mu_{\delta}} \\
(\mathrm{mas} / \mathrm{yr}) \\
(10)\end{array}$ & Sou \\
\hline BPS CS 22958-0074 & 1.308 & $\ldots$ & $\ldots$ & 1.300 & 1.308 & $\ldots$ & $\ldots$ & $\cdots$ & $\ldots$ & $\ldots$ \\
\hline BPS CS 22958-0053 & $\ldots$ & $\ldots$ & $\ldots$ & 1.600 & 1.600 & $\ldots$ & $\ldots$ & $\ldots$ & $\ldots$ & $\ldots$ \\
\hline BPS CS 22958-0052 & 1.094 & $\ldots$ & $\ldots$ & 1.400 & 1.094 & $\ldots$ & $\ldots$ & $\ldots$ & $\ldots$ & $\ldots$ \\
\hline HD 13889 & 0.092 & 0.082 & 0.11 & 0.076 & 0.082 & 45.6 & -171.1 & 0.9 & 0.7 & $\mathrm{HIP}, \mathrm{ACT}$ \\
\hline BPS CS 22958-0082 & 1.205 & $\ldots$ & $\ldots$ & 1.200 & 1.205 & $\ldots$ & $\ldots$ & $\ldots$ & $\ldots$ & $\ldots$ \\
\hline $\mathrm{V}^{*} \mathrm{RV}$ Cet & 1.128 & 0.429 & 0.87 & 1.014 & 1.128 & 27.0 & -19.1 & 1.4 & 0.8 & $\mathrm{HIP}, \mathrm{ACT}$, \\
\hline HD 13979 & 0.453 & 0.518 & 0.65 & 0.910 & 0.453 & 30.3 & -47.6 & 0.7 & 0.8 & $\mathrm{HIP}, \mathrm{ACT}$ \\
\hline BPS CS 22958-0083 & 5.457 & $\ldots$ & $\ldots$ & 5.000 & 5.457 & $\ldots$ & $\ldots$ & $\ldots$ & $\ldots$ & $\ldots$ \\
\hline $\mathrm{V}^{*} \mathrm{RW}$ Ari & $\ldots$ & $\ldots$ & $\ldots$ & 2.061 & 2.061 & -6.6 & -17.2 & 2.9 & 2.9 & STN,NPM \\
\hline $\mathrm{V}^{*} \mathrm{TW} \mathrm{Hyi}$ & 2.957 & $\ldots$ & $\ldots$ & 2.924 & 2.957 & $\ldots$ & $\ldots$ & $\cdots$ & $\cdots$ & $\ldots$ \\
\hline BD-22 395 & 1.249 & 0.235 & 0.45 & 1.580 & 1.249 & 34.5 & -31.7 & 1.0 & 0.9 & $\mathrm{HIP}, \mathrm{ACT}$, \\
\hline HD 14829 & 0.512 & 0.227 & 0.45 & 0.813 & 0.512 & 30.2 & -45.0 & 1.2 & 0.8 & $\mathrm{HIP}, \mathrm{ACT}$, \\
\hline HD 15061 & 1.267 & $\ldots$ & $\ldots$ & 0.720 & 1.267 & 16.7 & -17.0 & 0.8 & 0.8 & ACT,STN \\
\hline HD 15395 & 0.086 & 0.085 & 0.08 & 0.063 & 0.085 & 49.8 & -115.9 & 0.8 & 0.9 & HIP,ACT, \\
\hline $\mathrm{BD}+44493$ & 0.487 & 0.200 & 0.25 & 0.190 & 0.487 & 119.1 & -34.4 & 1.0 & 0.8 & HIP,ACT \\
\hline V* RZ Cet & 1.687 & $<0$ & $\ldots$ & 1.652 & 1.687 & 22.9 & 1.9 & 2.0 & 2.1 & HIP,STN \\
\hline BD-17 484 & 0.174 & 0.185 & 0.31 & 0.156 & 0.174 & 400.2 & -61.5 & 1.2 & 0.8 & $\mathrm{HIP}, \mathrm{ACT}$ \\
\hline BPS CS 22184-0001 & 1.267 & $\ldots$ & $\ldots$ & 1.400 & 1.267 & $\ldots$ & $\ldots$ & $\ldots$ & $\ldots$ & $\ldots$ \\
\hline BPS CS 22189-0004 & 0.746 & $\ldots$ & $\ldots$ & 0.600 & 0.746 & $\ldots$ & $\ldots$ & $\cdots$ & $\ldots$ & $\ldots$ \\
\hline HD 16031 & 0.111 & 0.115 & 0.21 & 0.080 & 0.111 & 62.6 & -183.8 & 0.8 & 0.9 & $\mathrm{HIP}, \mathrm{ACT}$, \\
\hline $\mathrm{V}^{*}$ CS Eri & 0.464 & 0.308 & 0.30 & $\ldots$ & 0.464 & 96.8 & 6.8 & 0.6 & 0.7 & HIP,ACT \\
\hline BPS CS 22189-0007 & 1.265 & $\ldots$ & $\ldots$ & 1.000 & 1.265 & 6. & -31 & 3.9 & 3.9 & STN \\
\hline BPS CS 22954-0004 & 1.067 & $\ldots$ & $\ldots$ & 1.000 & 1.067 & $\ldots$ & $\ldots$ & $\ldots$ & $\ldots$ & $\ldots$ \\
\hline CD-50 776 & 0.286 & 0.513 & 0.61 & 1.119 & 0.286 & 81.4 & 1.0 & 1.0 & 0.7 & HIP,ACT, \\
\hline BPS CS 22954-0015 & 0.562 & $\ldots$ & $\ldots$ & 0.700 & 0.562 & 27. & -56 & 4.7 & 4.7 & STN \\
\hline HD 17072 & 0.131 & 0.132 & 0.07 & 0.076 & 0.132 & 64.1 & -12.1 & 0.5 & 0.5 & $\mathrm{HIP}, \mathrm{ACT}$ \\
\hline BPS CS 22189-0009 & 6.678 & $\ldots$ & $\ldots$ & 6.700 & 6.678 & $\ldots$ & $\ldots$ & $\ldots$ & $\ldots$ & $\ldots$ \\
\hline HD 17233 & $\ldots$ & 0.176 & 0.16 & 0.127 & 0.127 & 65.11 & 92.32 & 0.83 & 0.91 & HIP \\
\hline BPS CS 22189-0018 & 2.020 & $\ldots$ & $\ldots$ & 1.900 & 2.020 & $\ldots$ & $\ldots$ & $\ldots$ & $\ldots$ & $\ldots$ \\
\hline BPS CS 22189-0027 & 1.007 & $\cdots$ & $\cdots$ & 1.000 & 1.007 & $\cdots$ & $\cdots$ & $\cdots$ & $\cdots$ & $\cdots$ \\
\hline BPS CS 22189-0029 & 5.361 & $\ldots$ & $\ldots$ & 5.100 & 5.361 & $\ldots$ & $\ldots$ & $\ldots$ & $\ldots$ & $\ldots$ \\
\hline BD-10 548 & 0.368 & 0.513 & 1.02 & 0.984 & 0.368 & 81.2 & -11.7 & 0.8 & 0.9 & $\mathrm{HIP}, \mathrm{ACT}$ \\
\hline CD-36 1052 & 0.792 & 14.286 & 21.00 & 0.704 & 0.792 & 1.9 & 10.4 & 0.9 & 0.8 & HIP,SPM, \\
\hline BPS CS 22184-0040 & 1.188 & $\ldots$ & $\ldots$ & 1.200 & 1.188 & 28. & -3 . & 5.3 & 5.3 & STN \\
\hline BPS CS 22189-0036 & 1.116 & $\ldots$ & $\ldots$ & 1.200 & 1.116 & $\ldots$ & $\ldots$ & $\ldots$ & $\ldots$ & $\ldots$ \\
\hline $\mathrm{V}^{*} \mathrm{BK}$ Eri & 2.451 & $\ldots$ & $\ldots$ & $\ldots$ & 2.451 & 32.8 & -4.5 & 2.6 & 2.6 & STN,NPM \\
\hline BPS CS 22954-0011 & $\ldots$ & $\ldots$ & $\ldots$ & 1.500 & 1.500 & $\ldots$ & $\ldots$ & $\ldots$ & $\ldots$ & $\ldots$ \\
\hline$-610250 \mathrm{G}$ & 21.698 & $\ldots$ & $\ldots$ & 20.000 & 21.698 & $\ldots$ & $\ldots$ & $\cdots$ & $\ldots$ & $\ldots$ \\
\hline $\mathrm{V}^{*} \mathrm{BY}$ Eri & $\ldots$ & $\cdots$ & $\ldots$ & 2.535 & 2.535 & 37. & 0. & 4.2 & 4.2 & STN \\
\hline$-600250 \mathrm{M}$ & 23.545 & $\cdots$ & $\cdots$ & 21.100 & 23.545 & $\ldots$ & $\cdots$ & $\cdots$ & $\cdots$ & $\cdots$ \\
\hline
\end{tabular}


TABLE 1B. (continued)

\begin{tabular}{|c|c|c|c|c|c|c|c|c|c|c|}
\hline $\begin{array}{l}\text { Star } \\
(1)\end{array}$ & $\begin{array}{c}D_{p h o} \\
(\mathrm{kpc}) \\
(2)\end{array}$ & $\begin{array}{c}D_{H I P} \\
(\mathrm{kpc}) \\
(3)\end{array}$ & $\begin{array}{c}\sigma_{\pi_{H I P}} / \pi_{H I P} \\
(4)\end{array}$ & $\begin{array}{c}D_{B S L} \\
(\mathrm{kpc}) \\
(5)\end{array}$ & $\begin{array}{l}D_{\text {adopt }} \\
(\mathrm{kpc}) \\
(6)\end{array}$ & $\begin{array}{c}\mu_{\alpha^{*}} \\
(\mathrm{mas} / \mathrm{yr}) \\
(7)\end{array}$ & $\begin{array}{c}\mu_{\delta} \\
\text { (mas/yr) } \\
(8)\end{array}$ & $\begin{array}{c}\sigma_{\mu_{\alpha^{*}}} \\
(\mathrm{mas} / \mathrm{yr}) \\
(9)\end{array}$ & $\begin{array}{c}\sigma_{\mu_{\delta}} \\
(\mathrm{mas} / \mathrm{yr}) \\
(10)\end{array}$ & Sou \\
\hline BPS CS 22963-0004 & 2.946 & $\cdots$ & $\cdots$ & 2.300 & 2.946 & $\ldots$ & $\ldots$ & $\ldots$ & $\ldots$ & $\ldots$ \\
\hline CD-30 1121 & 0.629 & 0.483 & 0.84 & 1.050 & 0.629 & 33.1 & -25.4 & 0.9 & 0.8 & HIP,SPM, \\
\hline$-580250 \mathrm{G}$ & 16.350 & $\ldots$ & $\ldots$ & 16.300 & 16.350 & $\ldots$ & $\ldots$ & $\cdots$ & $\ldots$ & $\ldots$ \\
\hline BPS CS 22968-0009 & 1.421 & $\cdots$ & $\cdots$ & 1.200 & 1.421 & $\cdots$ & $\cdots$ & $\cdots$ & $\cdots$ & $\cdots$ \\
\hline BPS CS 22963-0009 & 0.680 & $\cdots$ & $\cdots$ & 0.900 & 0.680 & $\cdots$ & $\cdots$ & $\cdots$ & $\cdots$ & $\cdots$ \\
\hline BPS CS 22968-0001 & 1.501 & $\cdots$ & $\cdots$ & 1.500 & 1.501 & $\cdots$ & $\cdots$ & $\cdots$ & $\cdots$ & $\cdots$ \\
\hline BPS CS 22968-0007 & 1.595 & $\ldots$ & $\cdots$ & 1.300 & 1.595 & $\ldots$ & $\cdots$ & $\ldots$ & $\cdots$ & $\ldots$ \\
\hline HD 18710 & 0.666 & 0.211 & 0.59 & 0.670 & 0.666 & -5.7 & 17.8 & 0.9 & 2.0 & $\mathrm{HIP}, \mathrm{ACT}$ \\
\hline $\mathrm{V}^{*} \mathrm{SW}$ For & 2.305 & $\ldots$ & $\cdots$ & 2.158 & 2.305 & $\cdots$ & $\cdots$ & $\cdots$ & $\cdots$ & $\ldots$ \\
\hline$-600250 \mathrm{~A}$ & 9.579 & $\cdots$ & $\cdots$ & 8.900 & 9.579 & $\cdots$ & $\cdots$ & $\cdots$ & $\cdots$ & $\cdots$ \\
\hline BPS CS 22963-0005 & 0.691 & $\cdots$ & $\cdots$ & 1.200 & 0.691 & $\cdots$ & $\cdots$ & $\cdots$ & $\cdots$ & $\ldots$ \\
\hline$-610300 \mathrm{C}$ & 13.589 & $\cdots$ & $\cdots$ & 10.900 & 13.589 & $\cdots$ & $\cdots$ & $\cdots$ & $\cdots$ & $\cdots$ \\
\hline BPS CS 22963-0023 & 1.487 & $\cdots$ & $\cdots$ & 0.900 & 1.487 & $\cdots$ & $\cdots$ & $\cdots$ & $\cdots$ & $\cdots$ \\
\hline BPS CS 22968-0014 & 5.876 & $\cdots$ & $\cdots$ & 4.500 & 5.876 & $\cdots$ & $\ldots$ & $\cdots$ & $\cdots$ & $\cdots$ \\
\hline $\mathrm{V}^{*} \mathrm{X}$ Ari & 0.547 & 1.124 & 1.74 & 0.459 & 0.547 & 65.8 & -88.7 & 1.6 & 1.5 & $\mathrm{HIP}, \mathrm{ACT}$, \\
\hline$-580300 \mathrm{D}$ & 17.897 & $\ldots$ & $\ldots$ & 18.200 & 17.897 & $\ldots$ & $\ldots$ & $\cdots$ & $\ldots$ & $\ldots$ \\
\hline $\mathrm{V}^{*} \mathrm{TU}$ Per & 1.310 & $\cdots$ & $\ldots$ & 1.247 & 1.310 & $\ldots$ & $\ldots$ & $\cdots$ & $\ldots$ & $\ldots$ \\
\hline HD 20038 & 0.245 & 0.337 & 0.28 & 0.469 & 0.245 & -22.8 & -9.0 & 0.7 & 0.9 & HIP,ACT, \\
\hline HD 19697 & 0.953 & $\cdots$ & $\cdots$ & 0.828 & 0.953 & 7.0 & -36.1 & 0.9 & 1.9 & $\mathrm{ACT}, \mathrm{STN}$ \\
\hline $\mathrm{V}^{*} \mathrm{RX}$ For & 1.751 & $\cdots$ & $\cdots$ & 1.811 & 1.751 & $\cdots$ & $\cdots$ & $\cdots$ & $\cdots$ & $\cdots$ \\
\hline $\mathrm{V}^{*} \mathrm{SV}$ Eri & 0.672 & 0.820 & 1.45 & 0.673 & 0.672 & 17.5 & -50.1 & 0.9 & 1.6 & HIP,ACT, \\
\hline BPS CS 22968-0043 & 1.171 & $\ldots$ & $\ldots$ & 1.800 & 1.171 & $\ldots$ & $\ldots$ & $\ldots$ & $\ldots$ & $\ldots$ \\
\hline $\mathrm{V}^{*} \mathrm{UY}$ Eri & $\ldots$ & $<0$ & $\cdots$ & 2.000 & 2.000 & 24.8 & -9.8 & 2.3 & 2.0 & HIP,STN, \\
\hline HD 20305 & 0.687 & 1.515 & 2.14 & 0.420 & 0.687 & 6.8 & -0.6 & 0.8 & 0.8 & $\mathrm{HIP}, \mathrm{ACT}$, \\
\hline BPS CS 22968-0029 & 1.160 & $\ldots$ & $\cdots$ & 1.300 & 1.160 & $\cdots$ & $\cdots$ & $\cdots$ & $\cdots$ & $\cdots$ \\
\hline BPS CS 22185-0007 & 4.393 & $\cdots$ & $\cdots$ & 2.800 & 4.393 & $\cdots$ & $\cdots$ & $\cdots$ & $\cdots$ & $\cdots$ \\
\hline BPS CS 22968-0030 & 1.850 & $\cdots$ & $\cdots$ & 1.200 & 1.850 & $\cdots$ & $\cdots$ & $\cdots$ & $\cdots$ & $\cdots$ \\
\hline BPS CS 22968-0039 & 1.671 & $\cdots$ & $\cdots$ & 2.100 & 1.671 & $\cdots$ & $\cdots$ & $\cdots$ & $\cdots$ & $\cdots$ \\
\hline BPS CS 22185-0006 & 0.946 & $\cdots$ & $\cdots$ & 1.000 & 0.946 & $\cdots$ & $\cdots$ & $\cdots$ & $\cdots$ & $\cdots$ \\
\hline V* UU Hor & 2.375 & $\cdots$ & $\cdots$ & $\cdots$ & 2.375 & 15. & -6 & 4.1 & 4.1 & STN \\
\hline$-580310 \mathrm{~A}$ & 7.340 & $\cdots$ & $\cdots$ & 6.700 & 7.340 & $\cdots$ & $\cdots$ & $\cdots$ & $\cdots$ & $\cdots$ \\
\hline BPS CS 22968-0026 & 1.047 & $\cdots$ & $\cdots$ & 1.200 & 1.047 & $\cdots$ & $\cdots$ & $\cdots$ & $\cdots$ & $\cdots$ \\
\hline$-610320 \mathrm{C}$ & 8.177 & $\cdots$ & $\cdots$ & 7.600 & 8.177 & $\cdots$ & $\cdots$ & $\cdots$ & $\cdots$ & $\cdots$ \\
\hline BPS CS 22185-0002 & 0.822 & $\cdots$ & $\cdots$ & 0.700 & 0.822 & $\cdots$ & $\cdots$ & $\cdots$ & $\cdots$ & $\ldots$ \\
\hline HD 21022 & 1.115 & 1.724 & 2.07 & 1.081 & 1.115 & 31.1 & -32.9 & 0.7 & 0.9 & HIP,SPM, \\
\hline $\mathrm{V}^{*} \mathrm{RX}$ Men & 4.771 & $\cdots$ & $\cdots$ & $\cdots$ & 4.771 & $\cdots$ & $\cdots$ & $\cdots$ & $\cdots$ & $\cdots$ \\
\hline BPS CS 22172-0019 & 1.098 & $\cdots$ & $\cdots$ & 1.100 & 1.098 & $\cdots$ & $\cdots$ & $\cdots$ & $\cdots$ & $\cdots$ \\
\hline BPS CS 22968-0033 & 1.325 & $\cdots$ & $\cdots$ & 1.600 & 1.325 & $\cdots$ & $\cdots$ & $\cdots$ & $\cdots$ & $\cdots$ \\
\hline $\mathrm{V}^{*} \mathrm{~V}$ Ret & 4.474 & $\cdots$ & $\cdots$ & $\cdots$ & 4.474 & $\cdots$ & $\cdots$ & $\cdots$ & $\cdots$ & $\cdots$ \\
\hline BPS CS 22968-0032 & $\cdots$ & $\cdots$ & $\cdots$ & 1.500 & 1.500 & $\cdots$ & $\cdots$ & $\cdots$ & $\cdots$ & $\cdots$ \\
\hline
\end{tabular}


TABLE 1B. (continued)

\begin{tabular}{|c|c|c|c|c|c|c|c|c|c|c|}
\hline $\begin{array}{l}\text { Star } \\
(1)\end{array}$ & $\begin{array}{c}D_{p h o} \\
(\mathrm{kpc}) \\
(2)\end{array}$ & $\begin{array}{c}D_{H I P} \\
(\mathrm{kpc}) \\
(3)\end{array}$ & $\begin{array}{c}\sigma_{\pi_{H I P}} / \pi_{H I P} \\
(4)\end{array}$ & $\begin{array}{c}D_{B S L} \\
(\mathrm{kpc}) \\
(5)\end{array}$ & $\begin{array}{c}D_{\text {adopt }} \\
(\mathrm{kpc}) \\
\quad(6)\end{array}$ & $\begin{array}{c}\mu_{\alpha^{*}} \\
(\mathrm{mas} / \mathrm{yr}) \\
(7)\end{array}$ & $\begin{array}{c}\mu_{\delta} \\
\text { (mas/yr) } \\
(8)\end{array}$ & $\begin{array}{c}\sigma_{\mu_{\alpha^{*}}} \\
(\mathrm{mas} / \mathrm{yr}) \\
(9)\end{array}$ & $\begin{array}{c}\sigma_{\mu_{\delta}} \\
(\mathrm{mas} / \mathrm{yr}) \\
(10)\end{array}$ & \\
\hline $\mathrm{V}^{*} \mathrm{X}$ Ret & 1.547 & $\ldots$ & $\ldots$ & 1.897 & 1.547 & $\ldots$ & $\ldots$ & $\ldots$ & $\ldots$ & $\ldots$ \\
\hline BPS CS 22185-0018 & 0.595 & $\ldots$ & $\ldots$ & 1.000 & 0.595 & $\ldots$ & $\ldots$ & $\ldots$ & $\ldots$ & $\ldots$ \\
\hline$-600320 \mathrm{~L}$ & 14.211 & $\ldots$ & $\ldots$ & 15.600 & 14.211 & $\ldots$ & $\ldots$ & $\ldots$ & $\cdots$ & $\ldots$ \\
\hline HD 21581 & 0.223 & 0.234 & 0.28 & 0.472 & 0.223 & 67.1 & -78.6 & 0.9 & 0.8 & HIP, \\
\hline $\mathrm{V}^{*} \mathrm{SX}$ For & 1.277 & $<0$ & $\cdots$ & 1.330 & 1.277 & 36.5 & -9.3 & 0.9 & 1.2 & HIP,, \\
\hline BPS CS 22172-0029 & 2.266 & $\ldots$ & $\cdots$ & 2.800 & 2.266 & $\ldots$ & $\cdots$ & $\cdots$ & $\cdots$ & $\cdots$ \\
\hline BPS CS 22172-0025 & 0.568 & $\cdots$ & $\cdots$ & 1.000 & 0.568 & 14. & 28. & 4.8 & 4.8 & STN \\
\hline $\mathrm{V}^{*} \mathrm{SS} \mathrm{Tau}$ & 1.701 & $\cdots$ & $\cdots$ & 1.820 & 1.701 & 7.7 & 2.8 & 5 & 5 & NPM \\
\hline $\mathrm{V}^{*} \mathrm{TV}$ For & 4.762 & $\ldots$ & $\ldots$ & $\ldots$ & 4.762 & $\ldots$ & $\ldots$ & $\ldots$ & $\ldots$ & $\ldots$ \\
\hline CD-24 1782 & 0.587 & 0.226 & 0.40 & 0.518 & 0.587 & 78.4 & -124.3 & 1.0 & 0.8 & $\mathrm{HIP}, \mathrm{A}$ \\
\hline HD 22879 & 0.020 & 0.024 & 0.02 & 0.018 & 0.024 & 690.8 & -214.2 & 0.8 & 0.9 & HIP, \\
\hline HD 23332 & 0.347 & 0.613 & 0.50 & 0.626 & 0.347 & 3.1 & 22.2 & 0.7 & 0.8 & HIP,$A$ \\
\hline $\mathrm{V}^{*} \mathrm{TW}$ For & 5.768 & $\ldots$ & $\cdots$ & $\cdots$ & 5.768 & $\cdots$ & $\cdots$ & $\cdots$ & $\cdots$ & $\cdots$ \\
\hline BPS CS 29529-0004 & 0.850 & $\cdots$ & $\cdots$ & 1.000 & 0.850 & $\ldots$ & $\ldots$ & $\ldots$ & $\ldots$ & $\ldots$ \\
\hline BPS CS 29529-0012 & 1.568 & $\cdots$ & $\cdots$ & 0.400 & 1.568 & $\cdots$ & $\ldots$ & $\ldots$ & $\ldots$ & $\ldots$ \\
\hline HD 23592 & 0.650 & 0.599 & 0.60 & 0.665 & 0.650 & 14.0 & 24.9 & 0.9 & 1.0 & HIP, \\
\hline BPS CS 29529-0028 & $\ldots$ & $\ldots$ & $\cdots$ & 1.400 & 1.400 & $\ldots$ & $\cdots$ & $\cdots$ & $\ldots$ & $\ldots$ \\
\hline BPS CS 29529-0025 & $\cdots$ & $\cdots$ & $\cdots$ & $\ldots$ & $\ldots$ & $\cdots$ & $\cdots$ & $\cdots$ & $\cdots$ & $\cdots$ \\
\hline BPS CS 29529-0024 & $\cdots$ & $\cdots$ & $\cdots$ & $\cdots$ & $\cdots$ & $\cdots$ & $\cdots$ & $\cdots$ & $\cdots$ & $\cdots$ \\
\hline BPS CS 29529-0026 & 0.846 & $\cdots$ & $\cdots$ & 1.000 & 0.846 & $\cdots$ & $\cdots$ & $\cdots$ & $\cdots$ & $\cdots$ \\
\hline HD 23798 & 0.995 & 1.408 & 1.34 & 1.040 & 0.995 & 18.5 & -1.7 & 0.6 & 0.7 & HIP, $\mathrm{s}$ \\
\hline BPS CS 29529-0005 & 1.303 & $\ldots$ & $\ldots$ & 5.600 & 1.303 & $\ldots$ & $\ldots$ & $\ldots$ & $\ldots$ & $\ldots$ \\
\hline BPS CS 29529-0002 & 0.397 & $\cdots$ & $\cdots$ & 0.700 & 0.397 & $\cdots$ & $\cdots$ & $\cdots$ & $\cdots$ & $\cdots$ \\
\hline BPS CS 29529-0021 & $\ldots$ & $\cdots$ & $\cdots$ & 1.400 & 1.400 & $\cdots$ & $\cdots$ & $\cdots$ & $\cdots$ & $\cdots$ \\
\hline $8578 \mathrm{~N} 1$ & $\ldots$ & $\cdots$ & $\cdots$ & 1.622 & 1.622 & $\cdots$ & $\cdots$ & $\cdots$ & $\cdots$ & $\cdots$ \\
\hline BPS CS 29529-0042 & 0.853 & $\cdots$ & $\cdots$ & 1.100 & 0.853 & $\cdots$ & $\cdots$ & $\cdots$ & $\cdots$ & $\cdots$ \\
\hline BPS CS 22190-0007 & 5.506 & $\cdots$ & $\cdots$ & 5.300 & 5.506 & $\cdots$ & $\cdots$ & $\cdots$ & $\cdots$ & $\cdots$ \\
\hline BPS CS 29529-0039 & 0.763 & $\cdots$ & $\cdots$ & 1.300 & 0.763 & $\cdots$ & $\cdots$ & $\cdots$ & $\cdots$ & $\cdots$ \\
\hline BPS CS 29529-0034 & 1.456 & $\cdots$ & $\cdots$ & 1.000 & 1.456 & $\cdots$ & $\cdots$ & $\cdots$ & $\cdots$ & $\cdots$ \\
\hline BPS CS 29529-0040 & 3.223 & $\cdots$ & $\cdots$ & 1.600 & 3.223 & $\cdots$ & $\cdots$ & $\cdots$ & $\cdots$ & $\cdots$ \\
\hline BPS CS 29529-0045 & 0.682 & $\cdots$ & $\cdots$ & 1.000 & 0.682 & $\cdots$ & $\cdots$ & $\cdots$ & $\cdots$ & $\cdots$ \\
\hline BPS CS 30494-0003 & 1.318 & $\cdots$ & $\cdots$ & 2.500 & 1.318 & 18. & -6 & 6.0 & 6.0 & STN \\
\hline BPS CS 22173-0002 & 0.746 & $\ldots$ & $\ldots$ & 0.800 & 0.746 & $\ldots$ & $\ldots$ & $\cdots$ & $\cdots$ & $\ldots$ \\
\hline BPS CS 29529-0054 & 3.096 & $\cdots$ & $\cdots$ & 3.600 & 3.096 & $\cdots$ & $\cdots$ & $\cdots$ & $\cdots$ & $\cdots$ \\
\hline BPS CS 29529-0055 & $6.293 / 1.659$ & $\cdots$ & $\cdots$ & $\cdots$ & 6.293 & $\cdots$ & $\cdots$ & $\cdots$ & $\cdots$ & $\cdots$ \\
\hline HD 26169 & 0.365 & 0.353 & 0.28 & 0.489 & 0.365 & 137.4 & 75.9 & 0.6 & 0.8 & $\mathrm{HIP}, A$ \\
\hline BPS CS 22173-0007 & 1.388 & $\ldots$ & $\cdots$ & 1.400 & 1.388 & $\ldots$ & $\cdots$ & $\cdots$ & $\cdots$ & $\cdots$ \\
\hline BPS CS 22169-0008 & 1.475 & $\cdots$ & $\cdots$ & 1.800 & 1.475 & $\cdots$ & $\cdots$ & $\cdots$ & $\cdots$ & $\cdots$ \\
\hline BPS CS 22173-0025 & 0.636 & $\cdots$ & $\cdots$ & 0.600 & 0.636 & 39. & 41. & 5.4 & 5.4 & STN \\
\hline HD 25532 & 0.083 & 0.228 & 0.28 & 0.400 & 0.083 & 91.8 & -116.9 & 0.7 & 0.9 & HIP,$A$ \\
\hline
\end{tabular}


TABLE 1B. (continued)

\begin{tabular}{|c|c|c|c|c|c|c|c|c|c|c|}
\hline $\begin{array}{l}\text { Star } \\
(1)\end{array}$ & $\begin{array}{c}D_{p h o} \\
(\mathrm{kpc}) \\
(2)\end{array}$ & $\begin{array}{c}D_{H I P} \\
(\mathrm{kpc}) \\
(3)\end{array}$ & $\begin{array}{c}\sigma_{\pi_{H I P}} / \pi_{H I P} \\
(4)\end{array}$ & $\begin{array}{c}D_{B S L} \\
(\mathrm{kpc}) \\
(5)\end{array}$ & $\begin{array}{c}D_{\text {adopt }} \\
(\mathrm{kpc}) \\
(6)\end{array}$ & $\begin{array}{c}\mu_{\alpha^{*}} \\
(\mathrm{mas} / \mathrm{yr}) \\
(7)\end{array}$ & $\begin{array}{c}\mu_{\delta} \\
(\mathrm{mas} / \mathrm{yr}) \\
(8)\end{array}$ & $\begin{array}{c}\sigma_{\mu_{\alpha *}} \\
(\mathrm{mas} / \mathrm{yr}) \\
(9)\end{array}$ & $\begin{array}{c}\sigma_{\mu_{\delta}} \\
(\mathrm{mas} / \mathrm{yr}) \\
(10)\end{array}$ & Sou \\
\hline BPS CS 30494-0016 & 1.306 & $\cdots$ & $\cdots$ & 1.500 & 1.306 & $\cdots$ & $\cdots$ & $\cdots$ & $\cdots$ & $\cdots$ \\
\hline BPS CS 22173-0014 & 0.922 & $\ldots$ & $\ldots$ & 0.900 & 0.922 & $\ldots$ & $\ldots$ & $\ldots$ & $\ldots$ & $\ldots$ \\
\hline BPS CS 22173-0015 & 0.654 & $\ldots$ & $\ldots$ & 0.700 & 0.654 & $\ldots$ & $\ldots$ & $\cdots$ & $\ldots$ & $\ldots$ \\
\hline BPS CS 22173-0021 & 0.916 & $\cdots$ & $\cdots$ & 1.000 & 0.916 & $\cdots$ & $\cdots$ & $\cdots$ & $\cdots$ & $\cdots$ \\
\hline BPS CS 22169-0012 & 0.859 & $\cdots$ & $\cdots$ & 1.200 & 0.859 & $\cdots$ & $\cdots$ & $\cdots$ & $\cdots$ & $\cdots$ \\
\hline BPS CS 22169-0014 & 0.984 & $\cdots$ & $\cdots$ & 1.200 & 0.984 & $\cdots$ & $\cdots$ & $\cdots$ & $\cdots$ & $\cdots$ \\
\hline BPS CS 29529-0075 & $\ldots$ & $\cdots$ & $\cdots$ & 1.800 & 1.800 & $\cdots$ & $\cdots$ & $\cdots$ & $\cdots$ & $\cdots$ \\
\hline BPS CS 22169-0017 & 0.503 & $\cdots$ & $\cdots$ & 1.100 & 0.503 & $\cdots$ & $\cdots$ & $\cdots$ & $\cdots$ & $\cdots$ \\
\hline BPS CS 22177-0009 & 1.114 & $\cdots$ & $\cdots$ & 1.300 & 1.114 & $\cdots$ & $\cdots$ & $\cdots$ & $\cdots$ & $\cdots$ \\
\hline BPS CS 29529-0093 & $\cdots$ & $\cdots$ & $\cdots$ & 1.200 & 1.200 & $\cdots$ & $\cdots$ & $\cdots$ & $\cdots$ & $\cdots$ \\
\hline BPS CS 30494-0038 & 0.465 & $\cdots$ & $\cdots$ & 0.700 & 0.465 & $\cdots$ & $\ldots$ & $\ldots$ & $\ldots$ & $\ldots$ \\
\hline HD 26297 & 0.570 & 0.781 & 0.79 & 0.520 & 0.570 & 44.7 & -4.9 & 0.7 & 0.6 & HIP,ACT, \\
\hline BPS CS 29529-0092 & 1.307 & $\cdots$ & $\cdots$ & 1.400 & 1.307 & $\cdots$ & $\cdots$ & $\cdots$ & $\cdots$ & $\cdots$ \\
\hline BPS CS 22177-0010 & 1.162 & $\cdots$ & $\cdots$ & 1.300 & 1.162 & $\cdots$ & $\cdots$ & $\cdots$ & $\cdots$ & $\cdots$ \\
\hline BPS CS 30494-0049 & $\ldots$ & $\cdots$ & $\cdots$ & 1.200 & 1.200 & $\ldots$ & $\ldots$ & $\ldots$ & $\ldots$ & $\ldots$ \\
\hline $\mathrm{V}^{*} \mathrm{XY}$ Eri & 3.020 & $\cdots$ & $\cdots$ & $\ldots$ & 3.020 & 11.3 & 6.8 & 5 & 5 & NPM \\
\hline BPS CS 30494-0045 & 0.780 & $\cdots$ & $\cdots$ & 1.100 & 0.780 & $\cdots$ & $\ldots$ & $\cdots$ & $\cdots$ & $\ldots$ \\
\hline BPS CS 22169-0035 & 6.993 & $\cdots$ & $\cdots$ & 5.900 & 6.993 & 1. & -4 & 3.4 & 3.4 & STN \\
\hline BPS CS 22186-0005 & 3.361 & $\cdots$ & $\cdots$ & 3.000 & 3.361 & 19.4 & 0.5 & 2.7 & 1.9 & SPM \\
\hline $\mathrm{BD}+06648$ & 1.387 & 0.388 & 0.48 & 1.116 & 1.387 & 29.1 & -39.5 & 0.8 & 1.1 & HIP,ACT, \\
\hline BPS CS 22186-0002 & 0.653 & $\cdots$ & $\cdots$ & 0.800 & 0.653 & 51.7 & -1.9 & 2.5 & 1.7 & SPM \\
\hline BPS CS 22169-0019 & 0.889 & $\ldots$ & $\ldots$ & 0.900 & 0.889 & $\ldots$ & $\ldots$ & $\ldots$ & $\ldots$ & $\ldots$ \\
\hline BPS CS 22186-0017 & 0.802 & $\cdots$ & $\cdots$ & 0.900 & 0.802 & 91.4 & 34.6 & 2.6 & 2.6 & SPM \\
\hline BPS CS 22182-0022 & 0.610 & $\ldots$ & $\cdots$ & 0.600 & 0.610 & 43.5 & 4.8 & 3.6 & 3.0 & SPM \\
\hline $\mathrm{V}^{*}$ AR Per & 0.612 & 0.543 & 0.89 & 0.594 & 0.612 & -5.6 & -9.2 & 1.3 & 1.1 & $\mathrm{HIP}, \mathrm{ACT}$, \\
\hline HD 27145 & 0.119 & 0.172 & 0.18 & 0.451 & 0.119 & 65.7 & 43.4 & 0.8 & 0.7 & HIP,ACT, \\
\hline BPS CS 22182-0021 & 0.954 & $\cdots$ & $\cdots$ & 1.300 & 0.954 & 4.8 & 6.0 & 2.3 & 2.4 & SPM \\
\hline BPS CS 22186-0023 & 2.763 & $\cdots$ & $\cdots$ & 2.300 & 2.763 & 7.7 & -1.8 & 2.3 & 2.6 & SPM \\
\hline BPS CS 22182-0024 & 0.577 & $\cdots$ & $\cdots$ & 0.600 & 0.577 & 25.5 & 16.0 & 2.2 & 2.7 & SPM \\
\hline BPS CS 22186-0022 & 0.890 & $\cdots$ & $\cdots$ & 0.900 & 0.890 & 66.1 & 18.0 & 5.2 & 3.9 & $\mathrm{SPM}$ \\
\hline BPS CS 22182-0047 & 0.576 & $\cdots$ & $\cdots$ & 0.900 & 0.576 & 72.5 & -63.9 & 3.1 & 3.1 & SPM,STN \\
\hline HD 27686 & 0.555 & $\cdots$ & $\cdots$ & 0.280 & 0.555 & -4.7 & -9.0 & 0.9 & 0.9 & $\mathrm{ACT}, \mathrm{STN}$ \\
\hline HD 27928 & 0.679 & 0.424 & 0.49 & 0.680 & 0.679 & 27.2 & -66.5 & 0.8 & 0.7 & HIP,SPM, \\
\hline BPS CS 22182-0033 & 1.276 & $\ldots$ & $\cdots$ & 1.500 & 1.276 & 32.6 & -5.2 & 2.3 & 2.3 & SPM \\
\hline BPS CS 22182-0025 & 1.281 & $\cdots$ & $\cdots$ & 1.300 & 1.281 & 42.7 & -27.1 & 3.8 & 2.2 & SPM \\
\hline HD 28085 & 0.269 & $\cdots$ & $\cdots$ & 0.240 & 0.269 & -12.7 & -6.1 & 0.9 & 2.3 & $\mathrm{ACT}, \mathrm{STN}$ \\
\hline BPS CS 22186-0050 & 0.793 & $\cdots$ & $\cdots$ & 0.800 & 0.793 & 21.4 & 43.3 & 3.0 & 2.2 & SPM \\
\hline BPS CS 22191-0017 & 0.929 & $\cdots$ & $\cdots$ & 0.900 & 0.929 & $\cdots$ & $\cdots$ & $\cdots$ & $\cdots$ & $\cdots$ \\
\hline CD-57 949 & 0.379 & 0.690 & 0.57 & 0.655 & 0.379 & 31.6 & 11.3 & 1.0 & 0.9 & HIP,ACT, \\
\hline $\mathrm{V}^{*}$ HP Eri & $\cdots$ & 1.515 & 1.56 & 1.627 & 1.627 & -13.6 & -54.2 & 0.8 & 0.9 & $\mathrm{HIP}, \mathrm{ACT}$, \\
\hline
\end{tabular}


TABLE 1B. (continued)

\begin{tabular}{|c|c|c|c|c|c|c|c|c|c|c|}
\hline $\begin{array}{l}\text { Star } \\
(1)\end{array}$ & $\begin{array}{c}D_{p h o} \\
(\mathrm{kpc}) \\
(2)\end{array}$ & $\begin{array}{c}D_{H I P} \\
(\mathrm{kpc}) \\
(3)\end{array}$ & $\begin{array}{c}\sigma_{\pi_{H I P}} / \pi_{H I P} \\
(4)\end{array}$ & $\begin{array}{c}D_{B S L} \\
(\mathrm{kpc}) \\
(5)\end{array}$ & $\begin{array}{c}D_{\text {adopt }} \\
(\mathrm{kpc}) \\
(6)\end{array}$ & $\begin{array}{c}\mu_{\alpha^{*}} \\
(\mathrm{mas} / \mathrm{yr}) \\
(7)\end{array}$ & $\begin{array}{c}\mu_{\delta} \\
\text { (mas/yr) } \\
(8)\end{array}$ & $\begin{array}{c}\sigma_{\mu_{\alpha^{*}}} \\
(\mathrm{mas} / \mathrm{yr}) \\
(9)\end{array}$ & $\begin{array}{c}\sigma_{\mu_{\delta}} \\
(\mathrm{mas} / \mathrm{yr}) \\
(10)\end{array}$ & Sou \\
\hline HD 30229 & 0.357 & 1.075 & 0.96 & 0.426 & 0.357 & 28.4 & -41.4 & 0.9 & 1.0 & HIP,ACT \\
\hline BPS CS 22191-0024 & 3.560 & $\ldots$ & $\ldots$ & 5.400 & 3.560 & $\ldots$ & $\ldots$ & $\ldots$ & $\ldots$ & $\ldots$ \\
\hline $\mathrm{V}^{*} \mathrm{BC}$ Eri & $\ldots$ & 0.429 & 0.88 & 1.164 & 1.164 & 18.1 & -5.1 & 0.8 & 1.1 & HIP,ACT, \\
\hline BPS CS 22191-0029 & 5.363 & $\cdots$ & $\cdots$ & 4.900 & 5.363 & $\ldots$ & $\ldots$ & $\cdots$ & $\cdots$ & $\ldots$ \\
\hline $\mathrm{V}^{*} \mathrm{RX}$ Eri & 0.595 & 0.926 & 1.19 & 0.597 & 0.595 & -15.9 & -13.5 & 1.0 & 0.7 & HIP,ACT, \\
\hline BD-22 928 & 0.689 & $\ldots$ & $\ldots$ & 1.628 & 0.689 & 7.6 & 21.3 & 1.1 & 3.4 & $\mathrm{ACT}, \mathrm{STN}$ \\
\hline $\mathrm{V}^{*} \mathrm{U} \mathrm{Pic}$ & 1.311 & 0.662 & 1.09 & 1.202 & 1.311 & -2.6 & 0.8 & 1.5 & 1.9 & HIP,STN \\
\hline CPD-62 394 & 1.427 & $<0$ & $\cdots$ & 1.460 & 1.427 & 41.1 & 8.9 & 0.9 & 1.1 & HIP,ACT \\
\hline HD 31128 & 0.088 & 0.064 & 0.08 & 0.062 & 0.064 & 164.3 & -28.6 & 0.7 & 0.9 & HIP,SPM, \\
\hline $\mathrm{V}^{*} \mathrm{U} \mathrm{Cae}$ & 1.942 & $\ldots$ & $\cdots$ & $\ldots$ & 1.942 & 16.3 & 5.2 & 1.7 & 3.4 & SPM,STN \\
\hline$V^{*}$ BB Eri & 1.407 & 0.376 & 1.14 & 1.466 & 1.407 & 31.9 & 0.3 & 2.1 & 2.1 & HIP,STN,I \\
\hline $\mathrm{V}^{*} \mathrm{U}$ Lep & 0.975 & 0.316 & 0.60 & 1.047 & 0.975 & 41.3 & -59.6 & 0.9 & 1.2 & HIP,ACT \\
\hline HD 32546 & 0.526 & 4.762 & 4.90 & 0.750 & 0.526 & 18.4 & 30.3 & 1.0 & 0.7 & HIP,ACT \\
\hline $\mathrm{V}^{*} \mathrm{~V}$ Cae & 2.934 & $\ldots$ & $\cdots$ & $\cdots$ & 2.934 & 4.7 & -28.0 & 2.3 & 1.8 & SPM,STN \\
\hline HD 268957 & 0.556 & 0.246 & 0.26 & 0.605 & 0.556 & 30.0 & 129.3 & 1.1 & 1.2 & HIP,ACT, \\
\hline V* SW Dor & 4.121 & $\ldots$ & $\ldots$ & $\ldots$ & 4.121 & $\ldots$ & $\ldots$ & $\ldots$ & $\ldots$ & $\ldots$ \\
\hline $\mathrm{V}^{*} \mathrm{SX}$ Dor & 3.892 & $\cdots$ & $\cdots$ & $\cdots$ & 3.892 & $\cdots$ & $\ldots$ & $\cdots$ & $\cdots$ & $\ldots$ \\
\hline $\mathrm{V}^{*}$ V964 Ori & 2.642 & $\ldots$ & $\ldots$ & $\ldots$ & 2.642 & 7.0 & -12.1 & 5 & 5 & NPM \\
\hline HD 33073 & 0.190 & 1.515 & 2.08 & 0.243 & 0.190 & 25.1 & -11.8 & 0.9 & 0.8 & HIP,SPM, \\
\hline $\mathrm{V}^{*} \mathrm{TX}$ Men & 3.999 & $\ldots$ & $\cdots$ & $\ldots$ & 3.999 & $\cdots$ & $\ldots$ & $\cdots$ & $\cdots$ & $\ldots$ \\
\hline $\mathrm{V}^{*} \mathrm{~T}$ Men & 2.872 & $\ldots$ & $\ldots$ & $\ldots$ & 2.872 & $\ldots$ & $\ldots$ & $\cdots$ & $\ldots$ & $\ldots$ \\
\hline CPD-57 752 & 0.411 & 0.238 & 0.24 & 0.437 & 0.411 & -28.2 & -2.5 & 1.1 & 0.8 & HIP,ACT, \\
\hline HD 34048 & 0.665 & 2.083 & 2.10 & 0.818 & 0.665 & 48.7 & -41.5 & 1.1 & 1.3 & HIP,ACT \\
\hline HD 33771 & 0.803 & 0.610 & 0.66 & 1.450 & 0.803 & 79.8 & -30.1 & 0.6 & 0.7 & HIP,SPM, \\
\hline HD 33605 & $\ldots$ & 0.144 & 0.21 & 0.755 & 0.755 & 0.4 & 5.2 & 0.8 & 0.6 & HIP,ACT, \\
\hline HD 34328 & 0.071 & 0.069 & 0.07 & 0.072 & 0.069 & 935.4 & 515.4 & 1.0 & 0.9 & HIP,ACT \\
\hline $\mathrm{V}^{*} \mathrm{RY} \mathrm{Col}$ & 1.042 & 0.833 & 1.11 & 1.071 & 1.042 & 34.1 & 23.1 & 0.8 & 0.9 & HIP,SPM, \\
\hline HD 241894 & 0.692 & $\ldots$ & $\ldots$ & 0.904 & 0.692 & 3.1 & 4.8 & 0.9 & 1.3 & $\mathrm{ACT}, \mathrm{STN}$ \\
\hline HD 242106 & $\ldots$ & 0.164 & 0.25 & 0.355 & 0.355 & 7.5 & -34.2 & 1.1 & 0.8 & HIP,ACT, \\
\hline $\mathrm{V}^{*} \mathrm{RT}$ Col & 2.517 & $\cdots$ & $\cdots$ & $\cdots$ & 2.517 & -5.7 & -5.7 & 3.3 & 3.3 & SPM \\
\hline HD 273975 & 0.162 & 0.156 & 0.22 & 0.123 & 0.162 & 184.3 & -239.8 & 0.8 & 1.4 & HIP,ACT \\
\hline HD 34414 & 0.337 & 0.209 & 0.29 & 0.281 & 0.337 & 2.3 & -16.2 & 0.8 & 0.6 & HIP,ACT, \\
\hline HD 35179 & 0.372 & 0.935 & 1.33 & 0.690 & 0.372 & -8.4 & -21.3 & 0.9 & 0.7 & HIP,ACT, \\
\hline HD 35238 & 0.223 & 0.228 & 0.19 & 0.199 & 0.223 & -30.8 & -8.6 & 0.7 & 0.5 & HIP,ACT, \\
\hline $\mathrm{V}^{*} \mathrm{RT}$ Dor & 2.205 & $\ldots$ & $\cdots$ & $\ldots$ & 2.205 & $\ldots$ & $\ldots$ & $\cdots$ & $\cdots$ & $\ldots$ \\
\hline HD 36054 & 0.315 & 0.189 & 0.25 & 0.407 & 0.315 & 1.8 & -4.7 & 0.7 & 0.5 & HIP,ACT, \\
\hline HD 36702 & 1.061 & 1.471 & 1.15 & 1.356 & 1.061 & 3.8 & -18.3 & 0.6 & 0.7 & HIP,ACT, \\
\hline HD 274939 & 0.387 & 0.347 & 0.37 & 0.540 & 0.387 & 11.4 & -122.5 & 1.1 & 1.1 & HIP,ACT, \\
\hline HD 244759 & 0.558 & $\ldots$ & $\cdots$ & 0.433 & 0.558 & 5.9 & -12.7 & 1.4 & 2.0 & ACT,STN \\
\hline HD 36931 & 0.671 & $\cdots$ & $\cdots$ & 0.302 & 0.671 & -2.7 & -2.1 & 1.3 & 3.4 & ACT,STN \\
\hline
\end{tabular}


TABle 1B. (continued)

\begin{tabular}{|c|c|c|c|c|c|c|c|c|c|c|}
\hline $\begin{array}{l}\text { Star } \\
(1)\end{array}$ & $\begin{array}{c}D_{p h o} \\
(\mathrm{kpc}) \\
(2)\end{array}$ & $\begin{array}{c}D_{H I P} \\
(\mathrm{kpc}) \\
(3)\end{array}$ & $\begin{array}{c}\sigma_{\pi_{H I P}} / \pi_{H I P} \\
(4)\end{array}$ & $\begin{array}{c}D_{B S L} \\
(\mathrm{kpc}) \\
(5)\end{array}$ & $\begin{array}{c}D_{\text {adopt }} \\
(\mathrm{kpc}) \\
\quad(6)\end{array}$ & $\begin{array}{c}\mu_{\alpha^{*}} \\
(\mathrm{mas} / \mathrm{yr}) \\
(7)\end{array}$ & $\begin{array}{c}\mu_{\delta} \\
(\mathrm{mas} / \mathrm{yr}) \\
(8)\end{array}$ & $\begin{array}{c}\sigma_{\mu_{\alpha^{*}}} \\
(\mathrm{mas} / \mathrm{yr}) \\
(9)\end{array}$ & $\begin{array}{c}\sigma_{\mu_{\delta}} \\
(\mathrm{mas} / \mathrm{yr}) \\
(10)\end{array}$ & $\begin{array}{l}\text { Source } \\
\text { (11) }\end{array}$ \\
\hline $\mathrm{V}^{*} \mathrm{HH}$ Aur & $\cdots$ & 0.237 & 0.30 & 0.372 & 0.372 & -8.3 & -12.5 & 1.0 & 0.6 & HIP,ACT,STN \\
\hline HD 246114 & 0.453 & $\ldots$ & $\ldots$ & 0.501 & 0.453 & 2.5 & -1.7 & 1.9 & 0.8 & ACT,STN \\
\hline HD 37828 & 0.310 & 0.261 & 0.21 & 0.393 & 0.310 & 37.5 & -48.1 & 0.6 & 0.5 & HIP,ACT,STN \\
\hline HD 246316 & $\ldots$ & $\ldots$ & $\cdots$ & 0.402 & 0.402 & 7.4 & -24.8 & 1.3 & 1.2 & $\mathrm{ACT}, \mathrm{STN}$ \\
\hline HD 246370 & 0.417 & $\ldots$ & $\ldots$ & 0.382 & 0.417 & 0.8 & 2.6 & 1.2 & 1.4 & ACT,STN \\
\hline HD 38893 & 0.136 & 0.361 & 0.35 & 0.319 & 0.136 & 0.0 & 20.0 & 0.6 & 0.6 & HIP,ACT \\
\hline HD 38510 & 0.041 & 0.063 & 0.06 & 0.046 & 0.063 & -82.2 & 289.4 & 0.6 & 0.8 & HIP,SPM,ACT \\
\hline HD 38504 & 0.223 & 0.298 & 0.36 & 0.282 & 0.223 & -19.7 & -17.1 & 0.7 & 0.6 & HIP,ACT,STN \\
\hline HD 247593 & 0.539 & $\ldots$ & $\cdots$ & 0.594 & 0.539 & -4.7 & 9.7 & 0.8 & 1.3 & $\mathrm{ACT}, \mathrm{STN}$ \\
\hline $\mathrm{V}^{*} \mathrm{UU}$ Men & 4.368 & $\cdots$ & $\cdots$ & $\cdots$ & 4.368 & $\cdots$ & $\cdots$ & $\cdots$ & $\cdots$ & $\cdots$ \\
\hline HD 38708 & $\cdots$ & 7.692 & 9.69 & 2.500 & 2.500 & -1.9 & -2.5 & 1.2 & 0.8 & HIP,ACT,STN \\
\hline HD 248271 & 0.806 & $\ldots$ & $\cdots$ & 0.878 & 0.806 & 3.6 & 2.2 & 0.8 & 2.4 & $\mathrm{ACT}, \mathrm{STN}$ \\
\hline HD 248412 & 1.108 & $\ldots$ & $\cdots$ & 1.113 & 1.108 & -4.3 & -7.1 & 2.1 & 1.3 & ACT,STN \\
\hline HD 40361 & 0.459 & 0.429 & 0.38 & 0.852 & 0.459 & -18.1 & 22.5 & 0.7 & 0.6 & HIP,ACT,STN \\
\hline $\mathrm{BD}+231114$ & 1.019 & $\ldots$ & $\cdots$ & 0.643 & 1.019 & -17 & -20 & 3.5 & 3.5 & STN \\
\hline HD 249499 & 1.648 & $\cdots$ & $\ldots$ & 0.264 & 1.648 & 2.6 & -1.8 & 1.0 & 1.5 & $\mathrm{ACT}, \mathrm{STN}$ \\
\hline HD 41010 & 0.495 & $\cdots$ & $\cdots$ & 0.445 & 0.495 & -7.3 & -24.1 & 2.1 & 0.9 & $\mathrm{ACT}, \mathrm{STN}$ \\
\hline HD 250915 & 0.664 & $\cdots$ & $\ldots$ & 0.513 & 0.664 & -15 & -4 & 3.8 & 3.8 & STN \\
\hline HD 41115 & 0.264 & $\cdots$ & $\cdots$ & 0.278 & 0.264 & -2.3 & -13.7 & 1.2 & 1.9 & $\mathrm{ACT}, \mathrm{STN}$ \\
\hline HD 41667 & 0.533 & 0.478 & 0.45 & 0.640 & 0.533 & 11.0 & 69.5 & 0.6 & 0.7 & HIP,SPM,ACT \\
\hline HD 251432 & 0.815 & $\cdots$ & $\cdots$ & 1.060 & 0.815 & 1.0 & -1.5 & 1.0 & 1.5 & $\mathrm{ACT}, \mathrm{STN}$ \\
\hline HD 251549 & 1.233 & $<0$ & $\ldots$ & 0.912 & 1.233 & -5.6 & 1.9 & 2.4 & 1.9 & HIP,STN \\
\hline $\mathrm{V}^{*} \mathrm{VW}$ Dor & 1.494 & $\cdots$ & $\cdots$ & $\ldots$ & 1.494 & $\cdots$ & $\cdots$ & $\cdots$ & $\cdots$ & $\ldots$ \\
\hline HD 252041 & $\ldots$ & $\cdots$ & $\cdots$ & 0.667 & 0.667 & -0.4 & -5.5 & 1.5 & 1.2 & $\mathrm{ACT}, \mathrm{STN}$ \\
\hline HD 41996 & 0.098 & $\cdots$ & $\cdots$ & 0.242 & 0.098 & $\ldots$ & $\cdots$ & $\cdots$ & $\cdots$ & $\cdots$ \\
\hline HD 41994 & 0.399 & 0.361 & 0.45 & 0.267 & 0.399 & -0.5 & -4.5 & 0.9 & 0.6 & HIP,ACT,STN \\
\hline HD 252370 & 0.685 & $\cdots$ & $\cdots$ & 0.537 & 0.685 & -1.5 & -6.8 & 1.7 & 0.9 & ACT,STN \\
\hline HD 252477 & $\ldots$ & $\ldots$ & $\cdots$ & 0.406 & 0.406 & -3.5 & -7.5 & 0.9 & 0.9 & $\mathrm{ACT}, \mathrm{STN}$ \\
\hline HD 44165 & 0.165 & 0.197 & 0.11 & 0.205 & 0.197 & -11.1 & 19.6 & 0.5 & 0.6 & HIP,ACT,STN \\
\hline V* RX Col & 2.448 & $<0$ & $\cdots$ & $\cdots$ & 2.448 & 7.5 & 6.4 & 1.7 & 2.2 & HIP,SPM,STN \\
\hline HD 43039 & 0.047 & 0.052 & 0.04 & 0.047 & 0.052 & -71.6 & -261.7 & 0.7 & 0.6 & HIP,ACT \\
\hline HD 253911 & $\ldots$ & $\cdots$ & $\cdots$ & 0.345 & 0.345 & 8.0 & -21.6 & 0.9 & 2.4 & $\mathrm{ACT}, \mathrm{STN}$ \\
\hline HD 44007 & 0.176 & 0.193 & 0.20 & 0.379 & 0.176 & 82.0 & -40.8 & 1.0 & 0.9 & HIP,ACT,STN \\
\hline HD 255236 & 0.525 & 0.410 & 0.62 & 0.488 & 0.525 & -2.8 & -0.5 & 0.8 & 1.2 & HIP,ACT,STN \\
\hline BD-19 1422 & 0.397 & 3.448 & 4.17 & 0.577 & 0.397 & 13.9 & -25.2 & 0.8 & 0.7 & HIP,ACT,STN \\
\hline HD 45282 & 0.101 & 0.136 & 0.13 & 0.106 & 0.101 & 6.4 & -90.7 & 0.6 & 0.7 & HIP,ACT,STN \\
\hline HD 45610 & 0.147 & 0.167 & 0.11 & 0.178 & 0.167 & 82.6 & -88.6 & 0.6 & 0.6 & HIP,ACT,STN \\
\hline $\mathrm{V}^{*} \mathrm{RZ}$ Cam & 2.249 & $\cdots$ & $\cdots$ & $\cdots$ & 2.249 & 14.6 & -7.3 & 2.9 & 2.9 & STN,NPM \\
\hline$V^{*}$ V338 Pup & 0.536 & 0.637 & 0.56 & 0.520 & 0.536 & 71.6 & -44.9 & 0.6 & 0.6 & HIP,ACT,STN \\
\hline V* ST Pup & $\ldots$ & $<0$ & $\cdots$ & 2.600 & 2.600 & -1.1 & 3.3 & 1.0 & 0.8 & HIP,ACT,STN \\
\hline
\end{tabular}


TABle 1B. (continued)

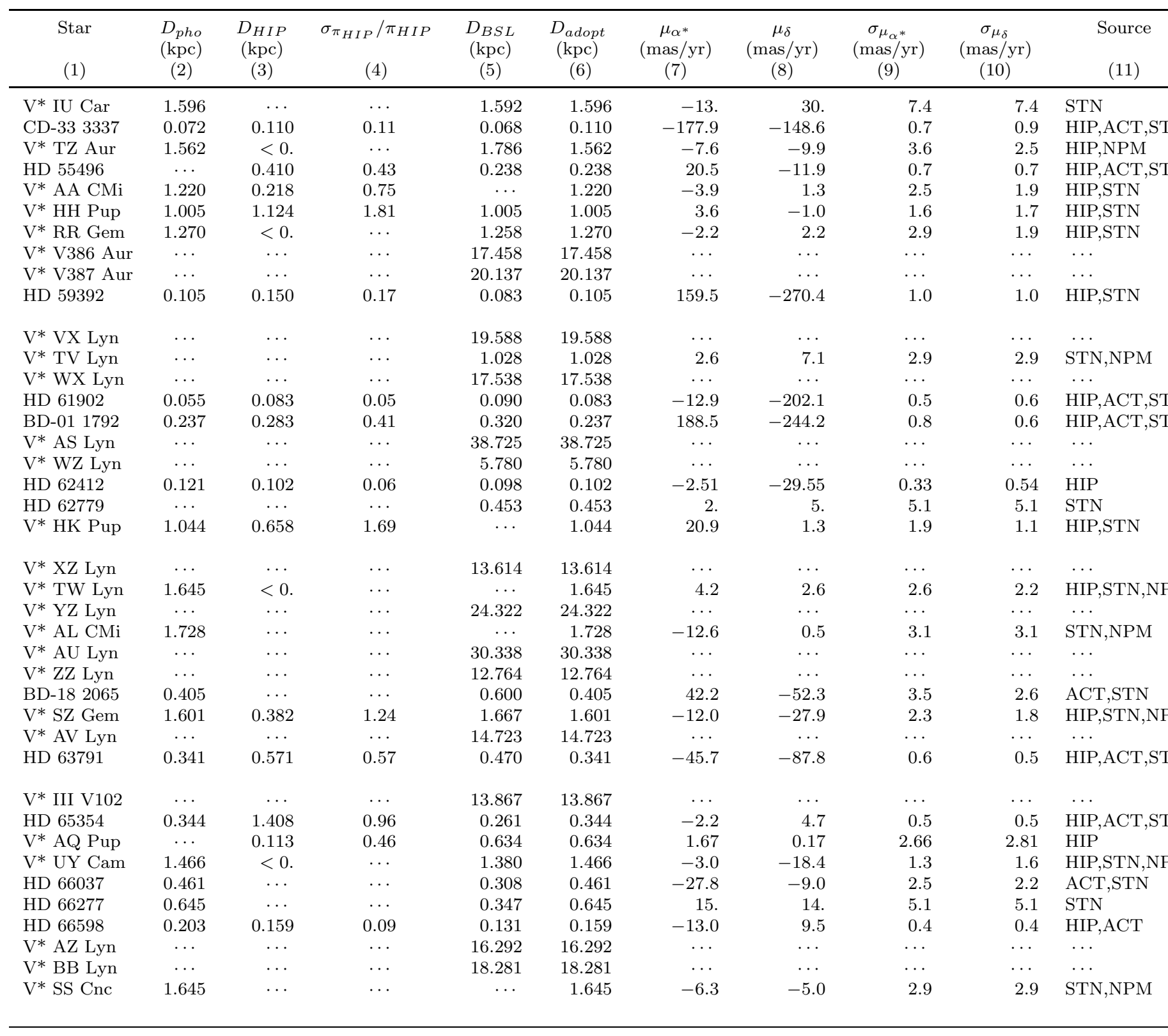


TABle 1B. (continued)

\begin{tabular}{|c|c|c|c|c|c|c|c|c|c|c|}
\hline $\begin{array}{l}\text { Star } \\
(1)\end{array}$ & $\begin{array}{c}D_{p h o} \\
(\mathrm{kpc}) \\
(2)\end{array}$ & $\begin{array}{c}D_{H I P} \\
(\mathrm{kpc}) \\
(3)\end{array}$ & $\begin{array}{c}\sigma_{\pi_{H I P}} / \pi_{H I P} \\
(4)\end{array}$ & $\begin{array}{c}D_{B S L} \\
(\mathrm{kpc}) \\
(5)\end{array}$ & $\begin{array}{c}D_{\text {adopt }} \\
\text { (kpc) } \\
(6)\end{array}$ & $\begin{array}{c}\mu_{\alpha^{*}} \\
(\mathrm{mas} / \mathrm{yr}) \\
(7)\end{array}$ & $\begin{array}{c}\mu_{\delta} \\
(\mathrm{mas} / \mathrm{yr}) \\
(8)\end{array}$ & $\begin{array}{c}\sigma_{\mu_{\alpha^{*}}} \\
(\mathrm{mas} / \mathrm{yr}) \\
(9)\end{array}$ & $\begin{array}{c}\sigma_{\mu_{\delta}} \\
(\mathrm{mas} / \mathrm{yr}) \\
(10)\end{array}$ & $\begin{array}{c}\text { Source } \\
\text { (11) }\end{array}$ \\
\hline HD 67380 & 0.569 & 0.240 & 0.31 & 0.433 & 0.569 & -8.2 & 21.2 & 0.8 & 0.9 & HIP,ACT,STN \\
\hline$V^{*}$ XX Pup & 1.216 & 0.633 & 1.53 & 1.380 & 1.216 & -21.8 & -3.6 & 1.8 & 1.9 & HIP,STN \\
\hline $\mathrm{V}^{*}$ SZ Lyn & $\ldots$ & 0.448 & 0.61 & 0.581 & 0.581 & -8.6 & -26.9 & 0.9 & 0.9 & HIP,ACT,STN,I \\
\hline $\mathrm{V}^{*} \mathrm{BC}$ Lyn & $\ldots$ & $\ldots$ & $\ldots$ & 20.796 & 20.796 & $\ldots$ & $\ldots$ & $\ldots$ & $\ldots$ & ... \\
\hline HD 68298 & 0.442 & $\ldots$ & $\ldots$ & 0.310 & 0.442 & -5.7 & 10.2 & 2.0 & 2.4 & ACT,STN \\
\hline$V^{*}$ DD Hya & 1.942 & $<0$ & $\ldots$ & $\ldots$ & 1.942 & -0.5 & -6.7 & 2.8 & 2.4 & HIP,STN,NPM \\
\hline HD 68960 & 0.093 & $\ldots$ & $\ldots$ & 0.573 & 0.093 & -3.5 & 2.5 & 1.9 & 1.0 & ACT,STN \\
\hline HD 69083 & 0.076 & 0.667 & 0.73 & 0.306 & 0.076 & -9.47 & 8.18 & 0.83 & 0.97 & HIP \\
\hline CD-44 4311 & $\ldots$ & 0.388 & 0.43 & 1.150 & 1.150 & -12.8 & 1.8 & 0.7 & 0.7 & HIP,ACT,STN \\
\hline HD 70765 & 0.461 & $\ldots$ & $\ldots$ & 0.481 & 0.461 & 7.6 & -7.1 & 1.5 & 1.0 & ACT,STN \\
\hline HD 70714 & 0.456 & $\ldots$ & $\ldots$ & 0.270 & 0.456 & -4.5 & 7.7 & 3.5 & 1.7 & ACT,STN \\
\hline$V^{*}$ BB Pup & 1.639 & $\ldots$ & $\ldots$ & 1.584 & 1.639 & -15.8 & 10.5 & 5 & 5 & NPM \\
\hline HD 71160 & 0.605 & 0.505 & 0.43 & 0.387 & 0.605 & -18.6 & -2.1 & 0.5 & 0.5 & HIP,ACT,STN \\
\hline $\mathrm{V}^{*} \mathrm{AS} \mathrm{Cnc}$ & 2.338 & $\ldots$ & $\ldots$ & $\ldots$ & 2.338 & 23.2 & -2.9 & 2.9 & 2.9 & STN,NPM \\
\hline HD 71584 & 0.641 & 0.588 & 0.41 & 0.368 & 0.641 & -2.1 & 5.7 & 0.5 & 0.6 & HIP,ACT,STN \\
\hline HD 71624 & 0.583 & $\ldots$ & $\ldots$ & 0.396 & 0.583 & -2.7 & 10.9 & 1.6 & 1.2 & ACT,STN \\
\hline HD 71670 & 0.575 & $\ldots$ & $\ldots$ & 0.370 & 0.575 & 6.4 & -10.6 & 2.8 & 1.1 & $\mathrm{ACT}, \mathrm{STN}$ \\
\hline HD 72348 & 0.085 & 0.166 & 0.11 & 0.244 & 0.166 & -47.7 & 16.0 & 0.6 & 0.6 & HIP,STN \\
\hline BD-20 2583 & 0.750 & 0.322 & 0.57 & 0.977 & 0.750 & 26.9 & -13.8 & 1.0 & 0.9 & HIP,ACT,STN, \\
\hline $\mathrm{V}^{*} \mathrm{TT}$ Cnc & 1.311 & 0.513 & 1.54 & 1.288 & 1.311 & -45.0 & -33.0 & 2.2 & 1.9 & HIP,STN,NPM \\
\hline HD 73075 & 0.147 & 0.154 & 0.11 & 0.219 & 0.154 & -8.0 & -21.7 & 0.6 & 0.5 & HIP,ACT,STN \\
\hline HD 73676 & 0.362 & 0.420 & 0.38 & 0.479 & 0.362 & -17.3 & -8.8 & 0.6 & 0.6 & HIP,ACT,STN \\
\hline HD 73700 & 0.352 & 0.633 & 0.51 & 0.457 & 0.352 & -5.8 & 8.8 & 0.8 & 0.6 & HIP,ACT,STN \\
\hline HD 73808 & 0.289 & $\ldots$ & $\ldots$ & 0.336 & 0.289 & -13.6 & 36.4 & 2.4 & 0.9 & $\mathrm{ACT}, \mathrm{STN}$ \\
\hline V* GL Hya & 2.842 & $\ldots$ & $\ldots$ & $\ldots$ & 2.842 & -13.4 & -9.0 & 5 & 5 & NPM \\
\hline HD 74000 & 0.107 & 0.138 & 0.18 & 0.078 & 0.107 & 351.8 & -484.8 & 1.2 & 1.0 & HIP,ACT \\
\hline HD 74617 & 0.489 & 1.471 & 1.04 & 0.322 & 0.489 & -7.3 & -0.2 & 0.5 & 0.6 & HIP,ACT,STN \\
\hline HD 74721 & 0.402 & 2.941 & 4.29 & 0.393 & 0.402 & -41.5 & -112.7 & 1.1 & 0.9 & HIP,ACT,STN \\
\hline BD-12 2669 & 0.260 & 0.174 & 0.26 & 0.142 & 0.260 & -331.6 & -161.6 & 1.4 & 1.0 & $\mathrm{HIP}, \mathrm{ACT}$ \\
\hline HD 74462 & 0.538 & 0.645 & 0.75 & 0.710 & 0.538 & 54.4 & -92.9 & 0.5 & 0.6 & HIP,ACT,STN, \\
\hline $\mathrm{V}^{*} \mathrm{SV} \mathrm{Vol}$ & 1.930 & $\ldots$ & $\ldots$ & $\ldots$ & 1.930 & 12. & -13 & 3.7 & 3.7 & STN \\
\hline HD 75745 & 0.169 & $\ldots$ & $\ldots$ & 0.106 & 0.169 & -52.2 & 20.9 & 4.2 & 2.8 & $\mathrm{ACT}, \mathrm{STN}$ \\
\hline HD 75837 & 0.258 & $\ldots$ & $\ldots$ & 0.210 & 0.258 & -17.2 & 22.3 & 3.0 & 1.0 & $\mathrm{ACT}, \mathrm{STN}$ \\
\hline $\mathrm{V}^{*} \mathrm{GO} \mathrm{Hya}$ & 1.953 & $\ldots$ & $\ldots$ & $\ldots$ & 1.953 & -2.3 & -9.8 & 5 & 5 & NPM \\
\hline$V^{*}$ DG Hya & 1.966 & $\ldots$ & $\ldots$ & $\ldots$ & 1.966 & -10.8 & -25.2 & 5 & 5 & NPM \\
\hline $\mathrm{V}^{*} \mathrm{AN}$ Cnc & 3.108 & $\ldots$ & $\ldots$ & $\ldots$ & 3.108 & -5.1 & -25.3 & 5 & 5 & NPM \\
\hline HD 77119 & 0.165 & $\ldots$ & $\ldots$ & 0.203 & 0.165 & -19.0 & 27.0 & 3.1 & 0.9 & $\mathrm{ACT}, \mathrm{STN}$ \\
\hline HD 77142 & 0.220 & 0.283 & 0.29 & 0.649 & 0.220 & -29.7 & 0.0 & 1.1 & 0.8 & HIP,ACT,STN \\
\hline $\mathrm{V}^{*} \mathrm{DH}$ Hya & 1.931 & $\ldots$ & $\ldots$ & 1.977 & 1.931 & -23.7 & -6.7 & 5 & 5 & NPM \\
\hline HD 77489 & 0.169 & 0.240 & 0.18 & 0.277 & 0.169 & -52.6 & 25.3 & 0.6 & 0.6 & HIP,ACT,STN \\
\hline
\end{tabular}


TABle 1B. (continued)

\begin{tabular}{|c|c|c|c|c|c|c|c|c|c|c|}
\hline $\begin{array}{l}\text { Star } \\
\text { (1) }\end{array}$ & $\begin{array}{c}D_{p h o} \\
(\mathrm{kpc}) \\
(2)\end{array}$ & $\begin{array}{c}D_{H I P} \\
(\mathrm{kpc}) \\
(3)\end{array}$ & $\begin{array}{c}\sigma_{\pi_{H I P}} / \pi_{H I P} \\
(4)\end{array}$ & $\begin{array}{c}D_{B S L} \\
(\mathrm{kpc}) \\
(5)\end{array}$ & $\begin{array}{c}D_{\text {adopt }} \\
\text { (kpc) } \\
(6)\end{array}$ & $\begin{array}{c}\mu_{\alpha^{*}} \\
(\mathrm{mas} / \mathrm{yr}) \\
(7)\end{array}$ & $\begin{array}{c}\mu_{\delta} \\
(\mathrm{mas} / \mathrm{yr}) \\
(8)\end{array}$ & $\begin{array}{c}\sigma_{\mu_{\alpha^{*}}} \\
(\mathrm{mas} / \mathrm{yr}) \\
(9)\end{array}$ & $\begin{array}{c}\sigma_{\mu_{\delta}} \\
(\mathrm{mas} / \mathrm{yr}) \\
(10)\end{array}$ & $\begin{array}{l}\text { Source } \\
\text { (11) }\end{array}$ \\
\hline HD 77567 & $\ldots$ & 0.350 & 0.33 & 0.461 & 0.461 & -30.1 & 45.5 & 1.0 & 0.8 & HIP,STN \\
\hline V* TT Lyn & 0.703 & $<0$ & $\ldots$ & $\ldots$ & 0.703 & -83.5 & -42.3 & 1.2 & 0.8 & HIP,ACT,STN, \\
\hline HD 77927 & 0.473 & $\ldots$ & $\ldots$ & 0.310 & 0.473 & -21.2 & 31.5 & 1.8 & 0.9 & $\mathrm{ACT}, \mathrm{STN}$ \\
\hline HD 77941 & $\ldots$ & $\ldots$ & $\ldots$ & 0.565 & 0.565 & -3.1 & -1.1 & 1.7 & 1.1 & ACT,STN \\
\hline HD 78747 & 0.038 & 0.040 & 0.03 & 0.032 & 0.040 & -64.3 & -132.1 & 0.7 & 0.6 & HIP,ACT,STN \\
\hline HD 78746 & 0.019 & 0.031 & 0.03 & 0.550 & 0.031 & -29.2 & 121.8 & 1.0 & 0.8 & HIP,ACT,STN \\
\hline HD 78737 & 0.096 & 0.154 & 0.17 & 0.084 & 0.096 & 69.2 & -13.1 & 0.7 & 0.6 & HIP,ACT,STN \\
\hline V* XX Hya & 1.595 & $\ldots$ & $\ldots$ & 1.117 & 1.595 & 18.6 & -29.2 & 5 & 5 & NPM \\
\hline HD 79091 & 0.121 & 0.093 & 0.05 & 0.131 & 0.093 & -91.7 & 54.6 & 0.6 & 0.5 & HIP,ACT \\
\hline HD 79134 & 0.238 & 0.317 & 0.26 & 0.290 & 0.238 & 17.1 & -14.6 & 0.8 & 0.8 & HIP,ACT,STN \\
\hline HD 79350 & $\ldots$ & 0.168 & 0.12 & 0.259 & 0.259 & -36.6 & 49.8 & 0.6 & 0.6 & HIP,ACT \\
\hline HD 79349 & 0.792 & 0.351 & 0.28 & 0.547 & 0.792 & -29.9 & 18.5 & 0.6 & 0.6 & HIP,ACT,STN \\
\hline $\mathrm{V}^{*} \mathrm{SZ}$ Hya & 1.313 & $\ldots$ & $\ldots$ & 1.318 & 1.313 & 6.8 & -55.3 & 2.9 & 2.9 & STN,NPM \\
\hline $\mathrm{V}^{*} \mathrm{AQ} \mathrm{Cnc}$ & 1.864 & $\ldots$ & $\ldots$ & $\ldots$ & 1.864 & -19.1 & -35.9 & 3.0 & 3.0 & STN,NPM \\
\hline $\mathrm{V}^{*} \mathrm{RW}$ Cnc & 1.770 & $<0$ & $\ldots$ & 1.629 & 1.770 & 1.3 & -35.1 & 2.3 & 1.6 & HIP,STN,NPM \\
\hline HD 80936 & 0.223 & $\ldots$ & $\ldots$ & 0.184 & 0.223 & -38.8 & 17.5 & 2.5 & 2.8 & $\mathrm{ACT}, \mathrm{STN}$ \\
\hline HD 81223 & 0.320 & 0.295 & 0.20 & 0.637 & 0.320 & -101.7 & 85.3 & 0.8 & 0.6 & HIP,ACT,STN \\
\hline HD 81173 & 0.187 & $\ldots$ & $\ldots$ & 0.714 & 0.187 & -44.7 & 11.7 & 3.0 & 0.9 & $\mathrm{ACT}, \mathrm{STN}$ \\
\hline V* IV Нya & 3.316 & $\ldots$ & $\ldots$ & $\ldots$ & 3.316 & $\ldots$ & $\ldots$ & $\ldots$ & $\cdots$ & $\ldots$ \\
\hline HD 81290 & 0.357 & 0.265 & 0.26 & 0.257 & 0.357 & -10.9 & -12.3 & 1.0 & 0.6 & HIP,ACT,STN \\
\hline HD 81590 & 0.632 & $\ldots$ & $\ldots$ & 0.762 & 0.632 & $\ldots$ & $\ldots$ & $\ldots$ & $\ldots$ & $\ldots$ \\
\hline HD 81713 & 0.292 & 0.380 & 0.46 & 0.452 & 0.292 & -7.6 & 23.5 & 0.9 & 0.8 & HIP,ACT,STN \\
\hline HD 82004 & 0.366 & $\ldots$ & $\ldots$ & 0.356 & 0.366 & -23.2 & 6.0 & 1.2 & 1.8 & $\mathrm{ACT}, \mathrm{STN}$ \\
\hline HD 82225 & 0.613 & $\ldots$ & $\ldots$ & 0.466 & 0.613 & $\ldots$ & $\ldots$ & $\cdots$ & $\cdots$ & $\ldots$ \\
\hline CD-36 5714 & 0.217 & $<0$ & $\ldots$ & 0.427 & 0.217 & -12.0 & -15.9 & 0.9 & 1.2 & HIP,STN \\
\hline HD 82403 & 0.250 & $\ldots$ & $\ldots$ & 0.368 & 0.250 & -38.0 & 2.6 & 3.9 & 3.4 & $\mathrm{ACT}, \mathrm{STN}$ \\
\hline HD 82456 & 0.160 & $\ldots$ & $\ldots$ & 0.504 & 0.160 & -63.3 & 33.1 & 2.7 & 1.4 & $\mathrm{ACT}, \mathrm{STN}$ \\
\hline $\mathrm{V}^{*} \mathrm{WW}$ Leo & 2.191 & $\ldots$ & $\ldots$ & $\ldots$ & 2.191 & -0.3 & -26.3 & 5 & 5 & NPM \\
\hline HD 82590 & 0.570 & 0.418 & 0.56 & 0.567 & 0.570 & 34.1 & -125.2 & 1.4 & 0.8 & HIP,ACT,STN \\
\hline HD 83155 & 0.172 & $\ldots$ & $\cdots$ & 0.331 & 0.172 & -44.0 & 35.2 & 4.4 & 3.6 & $\mathrm{ACT}, \mathrm{STN}$ \\
\hline HD 83212 & 0.569 & 0.510 & 0.50 & 0.710 & 0.569 & -16.3 & -20.6 & 0.7 & 0.6 & HIP,ACT,STN, \\
\hline $\mathrm{V}^{*} \mathrm{UU}$ Hya & 2.082 & $\ldots$ & $\ldots$ & 1.355 & 2.082 & -15.5 & -4.7 & 3.0 & 3.0 & STN,NPM \\
\hline HD 233666 & 0.166 & 0.820 & 0.98 & 0.620 & 0.166 & 12.3 & -29.1 & 0.9 & 0.7 & $\mathrm{HIP}, \mathrm{ACT}, \mathrm{STN}$, \\
\hline HD 84903 & 0.466 & $<0$ & $\ldots$ & 0.760 & 0.466 & -19.6 & -4.4 & 0.5 & 0.7 & HIP, ACT,STN \\
\hline HD 85454 & $\ldots$ & $\ldots$ & $\ldots$ & 0.594 & 0.594 & -6.0 & 1.6 & 3.8 & 2.4 & $\mathrm{ACT}, \mathrm{STN}$ \\
\hline HD 237846 & 1.560 & 50.000 & 80.50 & 1.000 & 1.560 & 1.5 & -9.1 & 1.0 & 0.9 & HIP,ACT,STN, \\
\hline $\mathrm{V}^{*} \mathrm{~T}$ Sex & 0.713 & 0.420 & 0.72 & 0.695 & 0.713 & -23.8 & -23.3 & 1.5 & 0.7 & HIP, ACT,STN, \\
\hline $\mathrm{V}^{*} \mathrm{SU}$ Leo & 3.684 & $\ldots$ & $\ldots$ & $\ldots$ & 3.684 & 6.0 & -8.6 & 5 & 5 & NPM \\
\hline HD 85773 & 1.664 & 0.246 & 0.32 & 1.620 & 1.664 & -16.4 & -22.3 & 0.9 & 0.8 & HIP,ACT,STN, \\
\hline HD 86268 & 0.356 & 0.935 & 0.70 & 0.325 & 0.356 & -44.6 & 31.2 & 0.6 & 0.6 & HIP,ACT,STN \\
\hline
\end{tabular}


TABle 1B. (continued)

\begin{tabular}{|c|c|c|c|c|c|c|c|c|c|c|}
\hline $\begin{array}{l}\text { Star } \\
(1)\end{array}$ & $\begin{array}{c}D_{p h o} \\
(\mathrm{kpc}) \\
(2)\end{array}$ & $\begin{array}{c}D_{H I P} \\
(\mathrm{kpc}) \\
(3)\end{array}$ & $\begin{array}{c}\sigma_{\pi_{H I P}} / \pi_{H I P} \\
(4)\end{array}$ & $\begin{array}{c}D_{B S L} \\
(\mathrm{kpc}) \\
(5)\end{array}$ & $\begin{array}{l}D_{\text {adopt }} \\
(\mathrm{kpc}) \\
(6)\end{array}$ & $\begin{array}{c}\mu_{\alpha^{*}} \\
(\mathrm{mas} / \mathrm{yr}) \\
(7)\end{array}$ & $\begin{array}{c}\mu_{\delta} \\
(\mathrm{mas} / \mathrm{yr}) \\
(8)\end{array}$ & $\begin{array}{c}\sigma_{\mu_{\alpha^{*}}} \\
(\mathrm{mas} / \mathrm{yr}) \\
(9)\end{array}$ & $\begin{array}{c}\sigma_{\mu_{\delta}} \\
(\mathrm{mas} / \mathrm{yr}) \\
(10)\end{array}$ & $\begin{array}{l}\text { Source } \\
(11)\end{array}$ \\
\hline CD-24 8642 & 0.309 & 0.513 & 0.69 & 0.539 & 0.309 & 3.6 & -4.9 & 1.1 & 0.7 & HIP,ACT,STN \\
\hline HD 87030 & 0.082 & 0.157 & 0.11 & 0.145 & 0.157 & -54.7 & 23.2 & 0.6 & 0.6 & HIP,ACT \\
\hline HD 87103 & 0.624 & 0.366 & 0.31 & 0.394 & 0.624 & -25.5 & 17.4 & 0.8 & 0.7 & HIP,ACT,STN \\
\hline HD 87254 & 0.421 & 0.226 & 0.16 & 0.278 & 0.421 & 48.8 & -12.0 & 0.7 & 0.5 & HIP,ACT,STN \\
\hline HD 86986 & 0.298 & 0.265 & 0.25 & 0.281 & 0.298 & 144.9 & -208.2 & 0.7 & 0.5 & HIP,ACT,STN \\
\hline $\mathrm{V}^{*} \mathrm{X} \mathrm{LMi}$ & 2.201 & $\ldots$ & $\ldots$ & 2.208 & 2.201 & 9.3 & -20.0 & 3.2 & 3.2 & STN,NPM \\
\hline V* DI Leo & 4.063 & $\cdots$ & $\cdots$ & $\ldots$ & 4.063 & $\ldots$ & $\ldots$ & $\cdots$ & $\cdots$ & $\ldots$ \\
\hline $\mathrm{V}^{*} \mathrm{RR}$ Leo & 1.016 & $<0$ & $\cdots$ & 1.057 & 1.016 & -16.9 & -10.1 & 1.6 & 0.9 & HIP,ACT,STN, \\
\hline HD 88226 & 0.302 & 0.303 & 0.21 & 0.355 & 0.302 & 11.6 & -35.4 & 0.6 & 0.6 & HIP,ACT,STN \\
\hline V* WZ Hya & 1.021 & 0.345 & 0.85 & 1.081 & 1.021 & -4.8 & -15.6 & 2.0 & 1.5 & HIP,STN,NPM \\
\hline HD 88609 & 1.003 & 1.587 & 1.81 & 0.953 & 1.003 & 2.3 & -31.5 & 0.9 & 0.7 & HIP,ACT,STN, \\
\hline $\mathrm{V}^{*}$ WY Ant & 1.037 & $<0$ & $\cdots$ & 1.056 & 1.037 & 35.6 & -48.2 & 1.2 & 1.4 & HIP,ACT \\
\hline V* RV Leo & 4.240 & $\cdots$ & $\cdots$ & $\ldots$ & 4.240 & $\cdots$ & $\ldots$ & $\cdots$ & $\cdots$ & $\ldots$ \\
\hline V* V LMi & 1.555 & $\cdots$ & $\cdots$ & 1.730 & 1.555 & 22.8 & -36.7 & 3.2 & 3.2 & STN,NPM \\
\hline $\mathrm{V}^{*} \mathrm{RV}$ Cha & 3.265 & $\ldots$ & $\cdots$ & $\ldots$ & 3.265 & $\cdots$ & $\ldots$ & $\ldots$ & $\cdots$ & $\ldots$ \\
\hline HD 90862 & 0.946 & 0.676 & 0.86 & 0.400 & 0.946 & 2.2 & 0.8 & 0.8 & 0.6 & HIP,ACT,STN \\
\hline $\mathrm{BD}+302034$ & 2.484 & 100.000 & 174.00 & 1.820 & 2.484 & -22.7 & -10.8 & 1.6 & 1.0 & HIP,ACT,STN, \\
\hline HD 92205 & 0.128 & $\ldots$ & $\ldots$ & 0.485 & 0.128 & -26 & -2 & 5.0 & 5.0 & STN \\
\hline $\mathrm{BD}+092384$ & 0.459 & $\cdots$ & $\cdots$ & 0.455 & 0.459 & -12.0 & -119.3 & 2.9 & 4.9 & ACT,STN \\
\hline CD-30 8626 & 0.445 & 0.478 & 0.66 & 0.624 & 0.445 & -13.0 & -42.6 & 0.8 & 0.9 & HIP,SPM,ACT \\
\hline $\mathrm{V}^{*}$ FS Vel & 1.560 & $\cdots$ & $\cdots$ & 1.778 & 1.560 & 42. & -9 & 4.0 & 4.0 & STN \\
\hline V* RV Sex & 2.013 & $\ldots$ & $\ldots$ & $\ldots$ & 2.013 & -9.8 & -8.5 & 5 & 5 & NPM \\
\hline HD 93529 & 0.371 & 0.270 & 0.33 & 0.725 & 0.371 & -19.3 & -47.2 & 0.7 & 0.9 & HIP,ACT,STN \\
\hline $\mathrm{V}^{*} \mathrm{AF}$ Vel & 1.061 & 0.446 & 0.95 & 1.282 & 1.061 & 41.0 & -14.3 & 1.4 & 1.5 & HIP,STN \\
\hline HD 94510 & 0.021 & 0.030 & 0.02 & 0.043 & 0.030 & 79.7 & 38.3 & 0.5 & 0.5 & HIP,ACT \\
\hline $\mathrm{V}^{*}$ SW Leo & 2.943 & $\cdots$ & $\cdots$ & $\ldots$ & 2.943 & -7.4 & -6.8 & 5 & 5 & NPM \\
\hline HD 95096 & 0.627 & 0.395 & 0.34 & 0.407 & 0.627 & 8.9 & -5.3 & 0.8 & 0.8 & HIP,ACT \\
\hline HD 95338 & 0.020 & 0.037 & 0.03 & 0.409 & 0.037 & -416.0 & -119.8 & 0.9 & 0.7 & HIP,ACT \\
\hline $\mathrm{V}^{*} \mathrm{SZ}$ Leo & 2.224 & $\cdots$ & $\cdots$ & 1.675 & 2.224 & -16.0 & -25.5 & 5 & 5 & NPM \\
\hline-181055 A & 18.968 & $\cdots$ & $\cdots$ & 18.000 & 18.968 & $\cdots$ & $\cdots$ & $\cdots$ & $\cdots$ & $\cdots$ \\
\hline-191055 A & 3.992 & $\cdots$ & $\cdots$ & 4.200 & 3.992 & $\cdots$ & $\cdots$ & $\cdots$ & $\cdots$ & $\cdots$ \\
\hline$-171100 \mathrm{H}$ & 7.480 & $\cdots$ & $\cdots$ & 8.400 & 7.480 & $\cdots$ & $\cdots$ & $\cdots$ & $\cdots$ & $\cdots$ \\
\hline$-181100 \mathrm{~K}$ & 14.330 & $\cdots$ & $\cdots$ & 13.000 & 14.330 & $\cdots$ & $\cdots$ & $\cdots$ & $\cdots$ & $\cdots$ \\
\hline$-161100 \mathrm{~A}$ & 17.643 & $\cdots$ & $\cdots$ & 15.900 & 17.643 & $\cdots$ & $\cdots$ & $\cdots$ & $\cdots$ & $\cdots$ \\
\hline$-171100 \mathrm{E}$ & 20.273 & $\cdots$ & $\cdots$ & 22.500 & 20.273 & $\cdots$ & $\cdots$ & $\cdots$ & $\cdots$ & $\cdots$ \\
\hline$-161100 \mathrm{C}$ & 7.969 & $\cdots$ & $\cdots$ & 9.500 & 7.969 & $\cdots$ & $\cdots$ & $\cdots$ & $\cdots$ & $\cdots$ \\
\hline-171100 D & 18.354 & $\cdots$ & $\cdots$ & 17.900 & 18.354 & $\cdots$ & $\cdots$ & $\cdots$ & $\cdots$ & $\cdots$ \\
\hline$-171100 \mathrm{C}$ & 12.131 & $\cdots$ & $\cdots$ & 11.900 & 12.131 & $\ldots$ & $\ldots$ & $\ldots$ & $\ldots$ & $\ldots$ \\
\hline$-171100 \mathrm{~B}$ & 6.538 & $\cdots$ & $\cdots$ & 6.100 & 6.538 & $\cdots$ & $\cdots$ & $\cdots$ & $\cdots$ & $\cdots$ \\
\hline-171100 I & 25.896 & $\cdots$ & $\cdots$ & 24.700 & 25.896 & $\cdots$ & $\cdots$ & $\cdots$ & $\cdots$ & $\cdots$ \\
\hline
\end{tabular}


TABle 1B. (continued)

\begin{tabular}{|c|c|c|c|c|c|c|c|c|c|c|}
\hline $\begin{array}{l}\text { Star } \\
\text { (1) }\end{array}$ & $\begin{array}{c}D_{p h o} \\
(\mathrm{kpc}) \\
(2)\end{array}$ & $\begin{array}{c}D_{H I P} \\
(\mathrm{kpc}) \\
(3)\end{array}$ & $\begin{array}{c}\sigma_{\pi_{H I P}} / \pi_{H I P} \\
(4)\end{array}$ & $\begin{array}{c}D_{B S L} \\
(\mathrm{kpc}) \\
(5)\end{array}$ & $\begin{array}{c}D_{\text {adopt }} \\
(\mathrm{kpc}) \\
(6)\end{array}$ & $\begin{array}{c}\mu_{\alpha^{*}} \\
(\mathrm{mas} / \mathrm{yr}) \\
(7)\end{array}$ & $\begin{array}{c}\mu_{\delta} \\
\text { (mas/yr) } \\
(8)\end{array}$ & $\begin{array}{c}\sigma_{\mu_{\alpha^{*}}} \\
(\mathrm{mas} / \mathrm{yr}) \\
(9)\end{array}$ & $\begin{array}{c}\sigma_{\mu_{\delta}} \\
(\mathrm{mas} / \mathrm{yr}) \\
(10)\end{array}$ & $\begin{array}{l}\text { Source } \\
\text { (11) }\end{array}$ \\
\hline$-171100 \mathrm{~A}$ & 16.379 & $\cdots$ & $\cdots$ & 16.300 & 16.379 & $\cdots$ & $\cdots$ & $\cdots$ & $\cdots$ & $\cdots$ \\
\hline$-181100 \mathrm{~B}$ & 5.424 & $\ldots$ & $\ldots$ & 5.600 & 5.424 & $\ldots$ & $\ldots$ & $\ldots$ & $\ldots$ & $\ldots$ \\
\hline$-181100 \mathrm{~A}$ & 14.477 & $\ldots$ & $\ldots$ & 14.500 & 14.477 & $\ldots$ & $\ldots$ & $\ldots$ & $\ldots$ & $\ldots$ \\
\hline$-181105 \mathrm{H}$ & 5.710 & $\ldots$ & $\cdots$ & 5.200 & 5.710 & $\cdots$ & $\ldots$ & $\ldots$ & $\cdots$ & $\cdots$ \\
\hline$-161105 \mathrm{E}$ & 5.056 & $\cdots$ & $\cdots$ & 5.700 & 5.056 & $\cdots$ & $\cdots$ & $\cdots$ & $\cdots$ & $\cdots$ \\
\hline$-191105 \mathrm{C}$ & 16.528 & $\ldots$ & $\ldots$ & 17.300 & 16.528 & $\cdots$ & $\cdots$ & $\ldots$ & $\ldots$ & $\cdots$ \\
\hline-191105 B & 11.311 & $\ldots$ & $\ldots$ & 11.000 & 11.311 & $\ldots$ & $\ldots$ & $\cdots$ & $\cdots$ & $\cdots$ \\
\hline$-191105 \mathrm{~F}$ & 21.564 & $\ldots$ & $\ldots$ & 22.000 & 21.564 & $\cdots$ & $\ldots$ & $\cdots$ & $\ldots$ & $\ldots$ \\
\hline$-161105 \mathrm{~A}$ & 6.535 & $\cdots$ & $\ldots$ & 7.400 & 6.535 & $\ldots$ & $\ldots$ & $\ldots$ & $\ldots$ & $\ldots$ \\
\hline$-171105 \mathrm{H}$ & 3.363 & $\cdots$ & $\cdots$ & 3.200 & 3.363 & $\cdots$ & $\cdots$ & $\cdots$ & $\cdots$ & $\cdots$ \\
\hline$-191105 \mathrm{E}$ & 11.088 & $\ldots$ & $\ldots$ & 9.600 & 11.088 & $\cdots$ & $\ldots$ & $\ldots$ & $\ldots$ & $\ldots$ \\
\hline HD 97320 & 0.050 & 0.056 & 0.04 & 0.043 & 0.056 & 159.2 & -201.3 & 0.7 & 0.7 & HIP,ACT \\
\hline-171105 A & 22.513 & $\ldots$ & $\cdots$ & 24.300 & 22.513 & $\ldots$ & $\ldots$ & $\ldots$ & $\cdots$ & $\ldots$ \\
\hline $\mathrm{V}^{*} \mathrm{TV}$ Leo & 1.977 & $\ldots$ & $\cdots$ & 1.811 & 1.977 & 9.9 & 0.5 & 3.6 & 3.6 & STN,NPM \\
\hline $\mathrm{V}^{*}$ BT Leo & 2.895 & $\ldots$ & $\ldots$ & $\ldots$ & 2.895 & -9.0 & -3.4 & 5 & 5 & NPM \\
\hline -191105 D & 20.863 & $\ldots$ & $\ldots$ & 20.400 & 20.863 & -5.5 & -2.1 & 5 & 5 & NPM \\
\hline$-181105 \mathrm{~K}$ & 11.397 & $\ldots$ & $\ldots$ & 11.900 & 11.397 & $\ldots$ & $\ldots$ & $\ldots$ & $\ldots$ & $\ldots$ \\
\hline$-181110 \mathrm{D}$ & 6.959 & $\ldots$ & $\ldots$ & 6.300 & 6.959 & $\ldots$ & $\ldots$ & $\cdots$ & $\ldots$ & $\ldots$ \\
\hline$-171110 \mathrm{~F}$ & 2.893 & $\cdots$ & $\cdots$ & 3.200 & 2.893 & $\cdots$ & $\cdots$ & $\cdots$ & $\cdots$ & $\cdots$ \\
\hline$-191110 \mathrm{E}$ & 13.023 & $\cdots$ & $\cdots$ & 13.600 & 13.023 & $\ldots$ & $\ldots$ & $\cdots$ & $\ldots$ & $\ldots$ \\
\hline$-161110 \mathrm{E}$ & 6.607 & $\cdots$ & $\cdots$ & 6.800 & 6.607 & $\cdots$ & $\ldots$ & $\cdots$ & $\cdots$ & $\cdots$ \\
\hline$-191110 \mathrm{D}$ & 2.652 & $\ldots$ & $\ldots$ & 3.500 & 2.652 & $\ldots$ & $\ldots$ & $\ldots$ & $\ldots$ & $\ldots$ \\
\hline$-191110 \mathrm{C}$ & 13.121 & $\ldots$ & $\ldots$ & 13.200 & 13.121 & $\ldots$ & $\ldots$ & $\ldots$ & $\ldots$ & $\ldots$ \\
\hline$-161110 \mathrm{C}$ & 4.611 & $\ldots$ & $\ldots$ & 5.000 & 4.611 & $\cdots$ & $\ldots$ & $\ldots$ & $\cdots$ & $\cdots$ \\
\hline-171110 B & 19.581 & $\ldots$ & $\cdots$ & 19.100 & 19.581 & $\cdots$ & $\ldots$ & $\ldots$ & $\cdots$ & $\ldots$ \\
\hline-161110 B & 12.271 & $\cdots$ & $\cdots$ & 11.500 & 12.271 & $\cdots$ & $\cdots$ & $\cdots$ & $\ldots$ & $\ldots$ \\
\hline-161110 A & 13.832 & $\cdots$ & $\cdots$ & 13.800 & 13.832 & $\cdots$ & $\cdots$ & $\cdots$ & $\cdots$ & $\cdots$ \\
\hline-191110 A & 21.701 & $\cdots$ & $\cdots$ & 20.300 & 21.701 & $\cdots$ & $\cdots$ & $\cdots$ & $\cdots$ & $\cdots$ \\
\hline-191115 D & 16.859 & $\cdots$ & $\cdots$ & 15.600 & 16.859 & $\cdots$ & $\cdots$ & $\cdots$ & $\cdots$ & $\cdots$ \\
\hline$-171115 \mathrm{E}$ & 9.644 & $\cdots$ & $\cdots$ & 10.600 & 9.644 & $\cdots$ & $\cdots$ & $\cdots$ & $\cdots$ & $\cdots$ \\
\hline$-171115 \mathrm{~F}$ & 4.492 & $\cdots$ & $\ldots$ & 4.500 & 4.492 & $\ldots$ & $\ldots$ & $\cdots$ & $\cdots$ & $\cdots$ \\
\hline$-161115 \mathrm{C}$ & 19.418 & $\cdots$ & $\ldots$ & 18.100 & 19.418 & $\cdots$ & $\cdots$ & $\cdots$ & $\cdots$ & $\cdots$ \\
\hline$-191115 \mathrm{E}$ & 23.585 & $\cdots$ & $\ldots$ & 22.400 & 23.585 & $\cdots$ & $\cdots$ & $\cdots$ & $\ldots$ & $\cdots$ \\
\hline-161115 B & 9.702 & $\cdots$ & $\cdots$ & 9.700 & 9.702 & $\cdots$ & $\cdots$ & $\cdots$ & $\cdots$ & $\cdots$ \\
\hline-161115 A & 9.050 & $\cdots$ & $\cdots$ & 8.000 & 9.050 & $\cdots$ & $\cdots$ & $\cdots$ & $\cdots$ & $\cdots$ \\
\hline-181115 A & 20.706 & $\cdots$ & $\cdots$ & 20.700 & 20.706 & $\cdots$ & $\cdots$ & $\cdots$ & $\cdots$ & $\cdots$ \\
\hline-191115 A & 6.085 & $\cdots$ & $\cdots$ & 5.900 & 6.085 & $\ldots$ & $\ldots$ & $\ldots$ & $\ldots$ & $\ldots$ \\
\hline$-171115 \mathrm{~A}$ & 20.463 & $\cdots$ & $\cdots$ & 21.400 & 20.463 & $\cdots$ & $\cdots$ & $\cdots$ & $\cdots$ & $\cdots$ \\
\hline-191120 A & 8.570 & $\cdots$ & $\cdots$ & 9.300 & 8.570 & $\cdots$ & $\ldots$ & $\cdots$ & $\cdots$ & $\cdots$ \\
\hline $\mathrm{V}^{*}$ AN Leo & 2.082 & $\cdots$ & $\cdots$ & $\ldots$ & 2.082 & 2.4 & -29.1 & 3.3 & 3.3 & STN,NPM \\
\hline
\end{tabular}


TABLE 1B. (continued)

\begin{tabular}{|c|c|c|c|c|c|c|c|c|c|c|}
\hline $\begin{array}{l}\text { Star } \\
(1)\end{array}$ & $\begin{array}{c}D_{p h o} \\
(\mathrm{kpc}) \\
(2)\end{array}$ & $\begin{array}{c}D_{H I P} \\
(\mathrm{kpc}) \\
(3)\end{array}$ & $\begin{array}{c}\sigma_{\pi_{H I P}} / \pi_{H I P} \\
(4)\end{array}$ & $\begin{array}{c}D_{B S L} \\
(\mathrm{kpc}) \\
(5)\end{array}$ & $\begin{array}{c}D_{\text {adopt }} \\
(\mathrm{kpc}) \\
\quad(6)\end{array}$ & $\begin{array}{c}\mu_{\alpha^{*}} \\
(\mathrm{mas} / \mathrm{yr}) \\
(7)\end{array}$ & $\begin{array}{c}\mu_{\delta} \\
\text { (mas/yr) } \\
(8)\end{array}$ & $\begin{array}{c}\sigma_{\mu_{\alpha^{*}}} \\
(\mathrm{mas} / \mathrm{yr}) \\
(9)\end{array}$ & $\begin{array}{c}\sigma_{\mu_{\delta}} \\
(\mathrm{mas} / \mathrm{yr}) \\
(10)\end{array}$ & Sou \\
\hline $\mathrm{V}^{*} \mathrm{RX}$ Leo & 1.764 & $\ldots$ & $\ldots$ & 1.754 & 1.764 & 8.2 & -28.5 & 3.5 & 3.3 & STN,NPM \\
\hline HD 99383 & 0.067 & 0.091 & 0.13 & 0.069 & 0.067 & -124.6 & 173.7 & 1.2 & 0.9 & HIP,ACT, \\
\hline $\mathrm{V}^{*}$ AE Leo & 2.414 & $\ldots$ & $\ldots$ & $\ldots$ & 2.414 & 23.8 & -12.5 & 5 & 5 & NPM \\
\hline HD 99513 & 0.327 & $\ldots$ & $\cdots$ & 0.283 & 0.327 & -28.4 & -7.1 & 1.9 & 2.2 & $\mathrm{ACT}, \mathrm{STN}$ \\
\hline $\mathrm{V}^{*} \mathrm{~W}$ Crt & 1.312 & $<0$ & $\cdots$ & 1.355 & 1.312 & -21.4 & -9.4 & 2.1 & 1.6 & HIP,STN,I \\
\hline $\mathrm{BD}+042466$ & $\ldots$ & $<0$ & $\ldots$ & 2.032 & 2.032 & -21.1 & -60.6 & 1.2 & 1.6 & HIP,ACT, \\
\hline HD 99833 & 0.098 & 0.613 & 0.57 & 0.105 & 0.098 & -29.5 & -112.2 & 0.8 & 0.7 & HIP,ACT \\
\hline HD 99978 & 0.309 & 0.251 & 0.26 & 0.560 & 0.309 & 10.1 & -26.3 & 1.0 & 1.0 & HIP,STN \\
\hline $\mathrm{V}^{*} \mathrm{TU} \mathrm{UMa}$ & 0.681 & $<0$ & $\ldots$ & 0.676 & 0.681 & -70.4 & -52.6 & 1.0 & 1.0 & $\mathrm{HIP}, \mathrm{ACT}$ \\
\hline $\mathrm{V}^{*}$ AX Leo & 2.103 & 0.127 & 0.68 & $\cdots$ & 2.103 & -21.0 & -25.6 & 3.2 & 2.9 & HIP,STN,P \\
\hline$V^{*}$ SS Leo & 1.231 & 0.893 & 2.01 & 1.282 & 1.231 & -23.7 & -26.0 & 1.3 & 1.2 & HIP,ACT \\
\hline HD 100906 & 0.183 & 1.852 & 2.52 & 0.423 & 0.183 & -14.0 & 8.1 & 0.9 & 0.8 & HIP,ACT \\
\hline HD 101063 & 0.200 & 0.943 & 1.05 & 0.417 & 0.200 & -314.5 & -15.0 & 0.9 & 0.6 & HIP,SPM \\
\hline $\mathrm{V}^{*} \mathrm{SU}$ Dra & 0.703 & 0.901 & 1.04 & 0.670 & 0.703 & -46.8 & -76.9 & 0.7 & 0.6 & HIP,ACT, \\
\hline $\mathrm{V}^{*}$ BX Leo & 1.506 & 0.358 & 1.16 & $\ldots$ & 1.506 & -27.4 & -13.6 & 2.3 & 2.0 & HIP,STN, $\mathrm{N}$ \\
\hline $\mathrm{V}^{*}$ ST Leo & 1.376 & $<0$ & $\ldots$ & 1.500 & 1.376 & -8.8 & -36.2 & 2.2 & 1.7 & HIP,STN,I \\
\hline $\mathrm{V}^{*}$ AA Leo & 2.043 & $\ldots$ & $\ldots$ & $\ldots$ & 2.043 & -2.6 & -33.5 & 5 & 5 & NPM \\
\hline HD 102200 & 0.080 & 0.080 & 0.10 & 0.066 & 0.080 & 60.5 & -110.1 & 0.7 & 0.8 & HIP,ACT \\
\hline $\mathrm{V}^{*} \mathrm{BI}$ Cen & 1.131 & $\ldots$ & $\cdots$ & 1.419 & 1.131 & $\cdots$ & $\ldots$ & $\cdots$ & $\cdots$ & $\cdots$ \\
\hline $\mathrm{V}^{*} \mathrm{KS}$ Cen & 2.834 & $\cdots$ & $\cdots$ & $\ldots$ & 2.834 & $\cdots$ & $\cdots$ & $\cdots$ & $\cdots$ & $\cdots$ \\
\hline $\mathrm{V}^{*} \mathrm{X}$ Crt & 1.533 & $<0$ & $\cdots$ & 1.479 & 1.533 & -1.4 & -39.8 & 1.7 & 1.4 & HIP,STN, \\
\hline HD 102780 & 0.804 & 0.699 & 0.84 & 0.429 & 0.804 & 3.8 & 2.8 & 0.9 & 0.6 & $\mathrm{HIP}, \mathrm{ACT}$ \\
\hline HD 102866 & 0.450 & $\cdots$ & $\cdots$ & 0.724 & 0.450 & $\ldots$ & $\cdots$ & $\cdots$ & $\cdots$ & $\ldots$ \\
\hline CD-70 889 & 0.562 & 0.595 & 0.59 & 1.086 & 0.562 & -115.2 & 13.1 & 1.0 & 0.8 & HIP,ACT, \\
\hline $\mathrm{BD}+222411$ & 2.496 & $<0$ & $\ldots$ & 2.430 & 2.496 & -8.0 & -14.6 & 1.3 & 0.8 & HIP,ACT \\
\hline $\mathrm{V}^{*} \mathrm{TY}$ Vir & $\ldots$ & 1.389 & 1.51 & 1.180 & 1.180 & -35.0 & -48.0 & 0.8 & 0.5 & HIP,ACT, \\
\hline HD 103295 & 0.220 & 3.226 & 4.16 & 0.484 & 0.220 & -53.6 & 14.6 & 0.9 & 0.7 & HIP,SPM, \\
\hline BD-01 2582 & 0.626 & 0.336 & 0.45 & 0.540 & 0.626 & -21.6 & -118.1 & 1.0 & 0.6 & HIP,ACT, \\
\hline [OM87] 115221.21-0 & $\cdots$ & $\cdots$ & $\cdots$ & 1.900 & 1.900 & 17. & -31 & 5.2 & 5.2 & STN \\
\hline HD 103545 & 0.858 & 1.667 & 2.05 & $\cdots$ & 0.858 & -49.6 & -38.3 & 1.3 & 0.9 & HIP,ACT, \\
\hline CD-35 7576 & 0.697 & $<0$ & $\cdots$ & 0.714 & 0.697 & -53.2 & -8.2 & 1.0 & 0.7 & HIP,SPM, \\
\hline HD 104053 & 1.075 & $\ldots$ & $\cdots$ & 0.708 & 1.075 & -27.3 & -21.2 & 3.3 & 2.6 & $\mathrm{ACT}, \mathrm{STN}$ \\
\hline $\mathrm{V}^{*} \mathrm{SW}$ Cru & 1.361 & $\ldots$ & $\ldots$ & 1.445 & 1.361 & $\ldots$ & $\ldots$ & $\cdots$ & $\cdots$ & $\ldots$ \\
\hline HD 233891 & 0.355 & 2.439 & 2.73 & 0.630 & 0.355 & -23.3 & 8.7 & 0.7 & 0.7 & HIP,ACT, \\
\hline $\mathrm{V}^{*}$ GK Com & $\ldots$ & 0.490 & 0.43 & 0.736 & 0.736 & -94.7 & -27.7 & 0.9 & 0.5 & HIP,STN \\
\hline BD+09 2574 & 1.076 & $<0$ & $\cdots$ & 1.650 & 1.076 & -10.5 & -46.3 & 1.5 & 0.9 & HIP,STN,I \\
\hline HD 104785 & 0.520 & 0.758 & 0.73 & 0.244 & 0.520 & -26.3 & -93.7 & 1.0 & 0.4 & HIP,ACT, \\
\hline HD 104893 & 1.816 & 6.667 & 8.33 & 1.829 & 1.816 & -33.2 & -8.0 & 1.0 & 0.7 & HIP,SPM, \\
\hline V* IK Hya & 0.713 & 0.426 & 0.60 & $\cdots$ & 0.713 & -61.6 & -50.7 & 1.1 & 0.8 & HIP,SPM, \\
\hline CD-37 7677 & 0.631 & $<0$ & $\cdots$ & 0.769 & 0.631 & -6.5 & -29.0 & 0.7 & 0.9 & HIP,SPM, \\
\hline
\end{tabular}


TABLE 1B. (continued)

\begin{tabular}{|c|c|c|c|c|c|c|c|c|c|c|}
\hline $\begin{array}{l}\text { Star } \\
(1)\end{array}$ & $\begin{array}{l}D_{p h o} \\
(\mathrm{kpc}) \\
(2)\end{array}$ & $\begin{array}{c}D_{H I P} \\
(\mathrm{kpc}) \\
(3)\end{array}$ & $\begin{array}{c}\sigma_{\pi_{H I P}} / \pi_{H I P} \\
(4)\end{array}$ & $\begin{array}{c}D_{B S L} \\
(\mathrm{kpc}) \\
(5)\end{array}$ & $\begin{array}{c}D_{\text {adopt }} \\
\text { (kpc) } \\
(6)\end{array}$ & $\begin{array}{c}\mu_{\alpha^{*}} \\
(\mathrm{mas} / \mathrm{yr}) \\
(7)\end{array}$ & $\begin{array}{c}\mu_{\delta} \\
(\mathrm{mas} / \mathrm{yr}) \\
(8)\end{array}$ & $\begin{array}{c}\sigma_{\mu_{\alpha^{*}}} \\
(\mathrm{mas} / \mathrm{yr}) \\
(9)\end{array}$ & $\begin{array}{c}\sigma_{\mu_{\delta}} \\
(\mathrm{mas} / \mathrm{yr}) \\
(10)\end{array}$ & Sou \\
\hline $\mathrm{V}^{*} \mathrm{UU}$ Vir & 0.901 & $<0$ & $\ldots$ & 1.005 & 0.901 & -43.5 & -2.3 & 1.2 & 0.8 & HIP,ACT, \\
\hline HD 105546 & 0.112 & 0.510 & 0.46 & 0.410 & 0.112 & -30.5 & -70.4 & 0.5 & 0.6 & HIP,ACT, \\
\hline CD-28 9325 & 2.537 & $\ldots$ & $\ldots$ & 2.800 & 2.537 & 13.6 & -19.2 & 1.6 & 1.7 & SPM,ACT \\
\hline $\mathrm{V}^{*} \mathrm{~V}$ Com & 3.262 & $\ldots$ & $\ldots$ & 3.499 & 3.262 & -10.8 & 0.4 & 3.6 & 3.6 & STN,NPM \\
\hline HD 105740 & 0.322 & 0.247 & 0.30 & 0.817 & 0.322 & -26.7 & -92.6 & 1.2 & 0.5 & HIP,ACT, \\
\hline $\mathrm{V}^{*} \mathrm{AB} \mathrm{UMa}$ & 1.019 & 1.099 & 2.09 & $\ldots$ & 1.019 & -15.6 & -14.3 & 1.0 & 1.1 & HIP,ACT, \\
\hline HD 105976 & 0.715 & $\ldots$ & $\ldots$ & 0.500 & 0.715 & $\ldots$ & $\ldots$ & $\ldots$ & $\ldots$ & $\ldots$ \\
\hline HD 106373 & 0.145 & 0.450 & 0.49 & $\ldots$ & 0.145 & -105.3 & 22.0 & 0.7 & 0.6 & HIP,SPM, \\
\hline HD 106383 & 0.156 & 0.208 & 0.21 & 0.332 & 0.156 & -80.1 & 10.7 & 0.9 & 0.7 & $\mathrm{HIP}, \mathrm{ACT}$, \\
\hline CD-28 9374 & 0.313 & 0.149 & 0.29 & 0.598 & 0.313 & -25.1 & -120.0 & 1.1 & 0.8 & HIP,SPM, \\
\hline HD 106411 & 0.067 & 0.106 & 0.13 & 0.065 & 0.067 & -5.5 & -207.2 & 0.8 & 0.7 & $\mathrm{HIP}, \mathrm{ACT}$, \\
\hline HD 106670 & 0.188 & 0.377 & 0.46 & 0.425 & 0.188 & -69.1 & -9.6 & 0.9 & 0.7 & $\mathrm{HIP}, \mathrm{ACT}$, \\
\hline V* LN Cen & 4.554 & $\ldots$ & $\ldots$ & $\ldots$ & 4.554 & $\ldots$ & $\ldots$ & $\ldots$ & $\ldots$ & $\ldots$ \\
\hline $\mathrm{V}^{*} \mathrm{IW}$ Com & $\ldots$ & 0.847 & 0.81 & 1.072 & 1.072 & 7.1 & -8.7 & 0.8 & 0.5 & $\mathrm{HIP}, \mathrm{ACT}$, \\
\hline $\mathrm{V}^{*} \mathrm{SW}$ Dra & 0.897 & 4.762 & 5.48 & 0.972 & 0.897 & -22.0 & -8.5 & 0.7 & 0.9 & $\mathrm{HIP}, \mathrm{ACT}$, \\
\hline HD 107363 & 0.676 & 3.333 & 5.20 & 0.290 & 0.676 & 8.8 & -53.2 & 1.7 & 1.1 & HIP,STN \\
\hline $\mathrm{V}^{*} \mathrm{UV}$ Vir & 1.656 & $\ldots$ & $\ldots$ & 1.853 & 1.656 & -21.0 & -23.7 & 3.0 & 3.0 & STN,NPM \\
\hline HD 107752 & 1.335 & 2.174 & 3.46 & 1.620 & 1.335 & -49.4 & -37.0 & 1.3 & 0.8 & $\mathrm{HIP}, \mathrm{ACT}$, \\
\hline [MFF90] PHI 2/2 8 & $\ldots$ & $\ldots$ & $\ldots$ & 2.900 & 2.900 & $\ldots$ & $\ldots$ & $\ldots$ & $\cdots$ & $\ldots$ \\
\hline $\mathrm{BD}+312360$ & 0.901 & $\cdots$ & $\cdots$ & 1.238 & 0.901 & $\cdots$ & $\cdots$ & $\cdots$ & $\cdots$ & $\cdots$ \\
\hline [MFF90] PHI 2/2 7 & $\cdots$ & $\ldots$ & $\ldots$ & 6.100 & 6.100 & $\ldots$ & $\ldots$ & $\ldots$ & $\ldots$ & $\ldots$ \\
\hline [MFF90] PHI $2 / 24$ & $\ldots$ & $\ldots$ & $\ldots$ & 4.200 & 4.200 & $\ldots$ & $\ldots$ & $\ldots$ & $\ldots$ & $\ldots$ \\
\hline $\mathrm{BD}+292287$ & 0.683 & $\ldots$ & $\ldots$ & 0.636 & 0.683 & -3.0 & -15.2 & 1.3 & 0.9 & $\mathrm{ACT}, \mathrm{STN}$ \\
\hline MA 300141 & $\ldots$ & $\ldots$ & $\ldots$ & 7.685 & 7.685 & $\ldots$ & $\ldots$ & $\ldots$ & $\ldots$ & $\ldots$ \\
\hline [MFF90] PHI 2/1 66 & $\ldots$ & $\ldots$ & $\ldots$ & 2.900 & 2.900 & $\ldots$ & $\ldots$ & $\ldots$ & $\ldots$ & $\ldots$ \\
\hline MA 300142 & $\ldots$ & $\ldots$ & $\ldots$ & 3.145 & 3.145 & -21 & 2. & 5.2 & 5.2 & STN \\
\hline HD 108317 & 0.159 & 0.221 & 0.23 & 0.220 & 0.159 & -167.5 & -25.0 & 1.1 & 0.7 & $\mathrm{HIP}, \mathrm{ACT}$, \\
\hline MA 300157 & $\ldots$ & $\ldots$ & $\ldots$ & 3.678 & 3.678 & 12. & -22 & 4.6 & 4.6 & STN \\
\hline HD 108405 & 0.068 & 0.077 & 0.10 & 0.057 & 0.077 & -320.5 & -216.0 & 0.6 & 1.2 & $\mathrm{HIP}, \mathrm{ACT}$ \\
\hline MA 300162 & $\cdots$ & $\cdots$ & $\cdots$ & 6.481 & 6.481 & $\ldots$ & $\ldots$ & $\cdots$ & $\cdots$ & $\cdots$ \\
\hline [MFF90] PHI 2/2 97 & 2.419 & $\ldots$ & $\ldots$ & 2.900 & 2.419 & -13.8 & -8.1 & 2.7 & 3.4 & SPM \\
\hline [MFF90] PHI 2/1 52 & $\ldots$ & $\ldots$ & $\ldots$ & 5.800 & 5.800 & $\ldots$ & $\ldots$ & $\ldots$ & $\ldots$ & $\ldots$ \\
\hline MA 300168 & $\ldots$ & $\ldots$ & $\ldots$ & 8.159 & 8.159 & $\ldots$ & $\ldots$ & $\ldots$ & $\ldots$ & $\ldots$ \\
\hline [MFF90] PHI 2/1 49 & $\ldots$ & $\ldots$ & $\ldots$ & 1.900 & 1.900 & $\ldots$ & $\ldots$ & $\ldots$ & $\ldots$ & $\ldots$ \\
\hline HD 108577 & 0.686 & 0.426 & 0.64 & 1.230 & 0.686 & -76.6 & -45.3 & 1.2 & 0.7 & $\mathrm{HIP}, \mathrm{ACT}$, \\
\hline $\mathrm{BD}+422309$ & 1.028 & 2.128 & 3.74 & 1.023 & 1.028 & -20.6 & -32.6 & 0.9 & 0.9 & $\mathrm{HIP}, \mathrm{ACT}$, \\
\hline $\mathrm{V}^{*} \mathrm{IX}$ Com & $\ldots$ & 2.273 & 2.48 & 1.241 & 1.241 & -19.5 & -12.8 & 1.2 & 0.6 & $\mathrm{HIP}, \mathrm{ACT}$, \\
\hline $\mathrm{BD}+042621$ & 1.593 & 1.562 & 3.08 & 1.920 & 1.593 & -21.8 & -36.4 & 1.5 & 0.8 & $\mathrm{HIP}, \mathrm{ACT}$, \\
\hline $\mathrm{V}^{*} \mathrm{RR}$ CVn & 2.313 & $\ldots$ & $\ldots$ & 2.594 & 2.313 & -15.8 & -31.7 & 5 & 5 & NPM \\
\hline $\mathrm{BD}+302282$ & 0.905 & $\ldots$ & $\ldots$ & 1.744 & 0.905 & 3. & 13. & 4.6 & 4.6 & STN \\
\hline
\end{tabular}


TABLE 1B. (continued)

\begin{tabular}{|c|c|c|c|c|c|c|c|c|c|c|}
\hline $\begin{array}{l}\text { Star } \\
(1)\end{array}$ & $\begin{array}{l}D_{p h o} \\
(\mathrm{kpc}) \\
(2)\end{array}$ & $\begin{array}{c}D_{H I P} \\
(\mathrm{kpc}) \\
(3)\end{array}$ & $\begin{array}{c}\sigma_{\pi_{H I P}} / \pi_{H I P} \\
(4)\end{array}$ & $\begin{array}{c}D_{B S L} \\
(\mathrm{kpc}) \\
(5)\end{array}$ & $\begin{array}{c}D_{\text {adopt }} \\
\text { (kpc) } \\
(6)\end{array}$ & $\begin{array}{c}\mu_{\alpha^{*}} \\
(\mathrm{mas} / \mathrm{yr}) \\
(7)\end{array}$ & $\begin{array}{c}\mu_{\delta} \\
(\mathrm{mas} / \mathrm{yr}) \\
(8)\end{array}$ & $\begin{array}{c}\sigma_{\mu_{\alpha^{*}}} \\
(\mathrm{mas} / \mathrm{yr}) \\
(9)\end{array}$ & $\begin{array}{c}\sigma_{\mu_{\delta}} \\
(\mathrm{mas} / \mathrm{yr}) \\
(10)\end{array}$ & Sou \\
\hline [MFF90] PHI 2/1 43 & $\cdots$ & $\ldots$ & $\cdots$ & 5.000 & 5.000 & $\cdots$ & $\ldots$ & $\cdots$ & $\cdots$ & $\cdots$ \\
\hline $\mathrm{V}^{*} \mathrm{UZ} \mathrm{CVn}$ & 2.043 & 0.062 & 0.44 & $\ldots$ & 2.043 & -7.5 & -25.7 & 2.6 & 2.8 & $\mathrm{HIP}, \mathrm{STN}$ \\
\hline V* SV Hya & 0.864 & 0.180 & 0.37 & 0.895 & 0.864 & -33.6 & 12.3 & 1.1 & 0.8 & HIP,SPM, \\
\hline HD 108976 & 0.087 & 0.079 & 0.08 & 0.766 & 0.079 & -13.9 & -8.0 & 0.9 & 0.8 & $\mathrm{HIP}, \mathrm{ACT}$, \\
\hline MA 30210 & 0.521 & $\ldots$ & $\ldots$ & 4.471 & 0.521 & -24 & -15 & 4.0 & 4.0 & STN \\
\hline [MFF90] PHI 2/2 49 & $\ldots$ & $\ldots$ & $\ldots$ & 2.600 & 2.600 & $\ldots$ & $\ldots$ & $\ldots$ & $\ldots$ & $\ldots$ \\
\hline [MFF90] PHI $2 / 246$ & 8.530 & $\ldots$ & $\ldots$ & 8.500 & 8.530 & -7.2 & -3.0 & 2.4 & 2.1 & SPM \\
\hline $\mathrm{V}^{*} \mathrm{~S}$ Com & 1.646 & $<0$ & $\ldots$ & 1.659 & 1.646 & -17.1 & -15.4 & 1.0 & 1.3 & $\mathrm{HIP}, \mathrm{ACT}$, \\
\hline MA 30225 & 2.162 & $\ldots$ & $\ldots$ & 9.602 & 2.162 & $\ldots$ & $\ldots$ & $\ldots$ & $\ldots$ & $\ldots$ \\
\hline [MFF90] PHI 2/2 40 & 2.432 & $\ldots$ & $\cdots$ & 2.300 & 2.432 & -14.6 & 2.5 & 2.1 & 2.2 & SPM,STN \\
\hline CD-33 8486 & 1.115 & $<0$ & $\ldots$ & 1.300 & 1.115 & -32.9 & -7.4 & 1.4 & 0.9 & HIP,SPM, \\
\hline [OM87] 123129.29-0 & 4.865 & $\ldots$ & $\ldots$ & 4.400 & 4.865 & -19.8 & -13.3 & 1.5 & 1.2 & SPM,STN \\
\hline $\mathrm{BD}+302294$ & 1.810 & $\ldots$ & $\ldots$ & 1.934 & 1.810 & -12.7 & -19.3 & 3.0 & 1.8 & $\mathrm{ACT}, \mathrm{STN}$ \\
\hline $\mathrm{BD}+302297$ & $\ldots$ & $\ldots$ & $\ldots$ & 2.506 & 2.506 & -5 . & -2 & 3.9 & 3.9 & STN \\
\hline [MFF90] PHI 2/2 23 & $\ldots$ & $\ldots$ & ... & 10.000 & 10.000 & $\ldots$ & $\ldots$ & $\ldots$ & $\ldots$ & $\ldots$ \\
\hline $\mathrm{V}^{*}$ EM Mus & 1.815 & $\ldots$ & $\ldots$ & $\ldots$ & 1.815 & $\ldots$ & $\ldots$ & $\ldots$ & $\ldots$ & $\ldots$ \\
\hline $\mathrm{V}^{*} \mathrm{SV} \mathrm{CVn}$ & 2.646 & $\ldots$ & $\ldots$ & 1.950 & 2.646 & 1.2 & -25.5 & 5 & 5 & NPM \\
\hline $\mathrm{BD}+262368$ & 1.259 & $\ldots$ & $\ldots$ & 1.633 & 1.259 & -1.9 & -23.0 & 1.8 & 1.5 & $\mathrm{ACT}, \mathrm{STN}$ \\
\hline $\mathrm{V}^{*} \mathrm{BQ}$ Vir & 2.253 & $\ldots$ & $\ldots$ & $\ldots$ & 2.253 & -2.7 & -13.9 & 5 & 5 & NPM \\
\hline MA 300285 & $\ldots$ & $\cdots$ & $\cdots$ & 2.624 & 2.624 & $\cdots$ & $\ldots$ & $\cdots$ & $\cdots$ & $\cdots$ \\
\hline HD 109823 & 0.833 & 1.020 & 0.88 & $\ldots$ & 0.833 & -13.8 & -19.3 & 0.8 & 0.5 & $\mathrm{HIP}, \mathrm{ACT}$, \\
\hline MA 300289 & $\ldots$ & $\ldots$ & $\ldots$ & 2.944 & 2.944 & $\ldots$ & $\ldots$ & $\ldots$ & $\ldots$ & $\ldots$ \\
\hline MA 300290 & $\ldots$ & $\ldots$ & $\ldots$ & 3.155 & 3.155 & $\ldots$ & $\ldots$ & $\ldots$ & $\ldots$ & $\ldots$ \\
\hline $\mathrm{V}^{*} \mathrm{FU}$ Vir & 2.351 & $\ldots$ & $\ldots$ & $\ldots$ & 2.351 & 13.3 & -7.7 & 5 & 5 & NPM \\
\hline MA 300293 & $\ldots$ & $\ldots$ & $\ldots$ & 1.981 & 1.981 & $\ldots$ & $\ldots$ & $\ldots$ & $\ldots$ & $\ldots$ \\
\hline [MFF90] PHI 4/2 34 & $\ldots$ & $\ldots$ & $\ldots$ & 2.700 & 2.700 & $\ldots$ & $\ldots$ & $\cdots$ & $\cdots$ & $\ldots$ \\
\hline HD 109995 & 0.252 & 0.203 & 0.18 & 0.236 & 0.252 & -114.7 & -144.2 & 0.8 & 0.7 & $\mathrm{HIP}, \mathrm{ACT}$, \\
\hline [MFF90] PHI 4/2 15 & $\ldots$ & $\ldots$ & $\ldots$ & 2.600 & 2.600 & $\ldots$ & $\ldots$ & $\ldots$ & $\ldots$ & $\ldots$ \\
\hline [MFF90] PHI 4/3 58 & $\ldots$ & $\ldots$ & $\ldots$ & 3.900 & 3.900 & $\ldots$ & $\ldots$ & $\ldots$ & $\ldots$ & $\ldots$ \\
\hline $\mathrm{V}^{*} \mathrm{U}$ Com & 1.659 & 0.289 & 0.79 & 1.659 & 1.659 & -50.2 & -13.1 & 1.3 & 1.4 & $\mathrm{HIP}, \mathrm{ACT}$, \\
\hline HD 110184 & 1.226 & 1.000 & 0.99 & 1.075 & 1.226 & -14.4 & -9.6 & 1.0 & 0.5 & $\mathrm{HIP}, \mathrm{ACT}$, \\
\hline $\mathrm{V}^{*} \mathrm{SW} \mathrm{CVn}$ & 2.709 & $\ldots$ & $\ldots$ & 2.716 & 2.709 & -9.6 & -19.8 & 5 & 5 & NPM \\
\hline $\mathrm{V}^{*} \mathrm{KR}$ Vir & $\ldots$ & 0.575 & 0.78 & $\ldots$ & $\ldots$ & 6.9 & -32.0 & 0.9 & 0.7 & $\mathrm{HIP}, \mathrm{ACT}$, \\
\hline$[\mathrm{M} 60 \mathrm{~b}]+31351$ & 1.371 & $\ldots$ & $\ldots$ & 1.151 & 1.371 & -28.7 & -5.8 & 2.2 & 2.3 & $\mathrm{ACT}, \mathrm{STN}$ \\
\hline [MFF90] PHI 4/3 20 & $\ldots$ & $\ldots$ & $\ldots$ & 1.300 & 1.300 & -11 & 4. & 6.0 & 6.0 & STN \\
\hline [MFF90] PHI 4/3 15 & $\ldots$ & $\ldots$ & $\ldots$ & 2.300 & 2.300 & $\ldots$ & $\ldots$ & $\ldots$ & $\cdots$ & $\ldots$ \\
\hline [MFF90] PHI 4/3 13 & $\ldots$ & $\ldots$ & $\ldots$ & 1.900 & 1.900 & -18 & 16. & 6.6 & 6.6 & STN \\
\hline $\mathrm{BD}+282144$ & 1.367 & $\ldots$ & $\cdots$ & 1.373 & 1.367 & -17.4 & -8.5 & 2.9 & 2.9 & STN,NPM \\
\hline [MFF90] PHI 4/3 4 & 0.804 & $\cdots$ & $\cdots$ & 1.300 & 0.804 & $\ldots$ & $\ldots$ & $\cdots$ & $\cdots$ & $\ldots$ \\
\hline [MFF90] PHI 4/3 1 & $\ldots$ & $\cdots$ & $\cdots$ & 7.100 & 7.100 & -17 & 2. & 6.6 & 6.6 & STN \\
\hline
\end{tabular}


TABle 1B. (continued)

\begin{tabular}{|c|c|c|c|c|c|c|c|c|c|c|}
\hline $\begin{array}{l}\text { Star } \\
\text { (1) }\end{array}$ & $\begin{array}{c}D_{p h o} \\
(\mathrm{kpc}) \\
(2)\end{array}$ & $\begin{array}{c}D_{H I P} \\
(\mathrm{kpc}) \\
(3)\end{array}$ & $\begin{array}{c}\sigma_{\pi_{H I P}} / \pi_{H I P} \\
(4)\end{array}$ & $\begin{array}{c}D_{B S L} \\
(\mathrm{kpc}) \\
(5)\end{array}$ & $\begin{array}{c}D_{\text {adopt }} \\
\text { (kpc) } \\
(6)\end{array}$ & $\begin{array}{c}\mu_{\alpha^{*}} \\
(\mathrm{mas} / \mathrm{yr}) \\
(7)\end{array}$ & $\begin{array}{c}\mu_{\delta} \\
(\mathrm{mas} / \mathrm{yr}) \\
(8)\end{array}$ & $\begin{array}{c}\sigma_{\mu_{\alpha^{*}}} \\
(\mathrm{mas} / \mathrm{yr}) \\
(9)\end{array}$ & $\begin{array}{c}\sigma_{\mu_{\delta}} \\
(\mathrm{mas} / \mathrm{yr}) \\
(10)\end{array}$ & Sc \\
\hline HD 110621 & 0.156 & 0.142 & 0.21 & 0.106 & 0.156 & -221.7 & -17.3 & 0.8 & 0.8 & $\mathrm{HIP}, \mathrm{AC}]$ \\
\hline [OM87] 124102.16-0 & 0.818 & $\ldots$ & $\ldots$ & 1.100 & 0.818 & -17.4 & 9.5 & 1.5 & 2.3 & SPM,ST \\
\hline [MFF90] PHI 4/1 178 & $\ldots$ & $\ldots$ & $\ldots$ & 1.900 & 1.900 & $\ldots$ & $\ldots$ & $\ldots$ & $\ldots$ & $\ldots$ \\
\hline [MFF90] PHI 4/1 170 & $\ldots$ & $\ldots$ & $\ldots$ & 4.300 & 4.300 & -43. & 5. & 5.2 & 5.2 & STN \\
\hline $\mathrm{BD}+262386$ & 1.325 & $\ldots$ & $\ldots$ & 1.013 & 1.325 & $\ldots$ & $\ldots$ & $\ldots$ & $\cdots$ & $\ldots$ \\
\hline [MFF90] PHI 4/1 167 & $\ldots$ & $\ldots$ & $\ldots$ & 9.100 & 9.100 & $\ldots$ & $\ldots$ & $\ldots$ & $\ldots$ & $\ldots$ \\
\hline UP 25091 & 1.649 & $\ldots$ & $\ldots$ & 1.022 & 1.649 & -7 & -3 & 3.8 & 3.8 & STN \\
\hline MA 34370 & 4.651 & $\ldots$ & $\ldots$ & 1.790 & 4.651 & -7 & -2 . & 8.6 & 8.6 & STN \\
\hline$[\mathrm{M} 60 \mathrm{~b}]+34382$ & 1.612 & $\ldots$ & $\ldots$ & 1.011 & 1.612 & 8. & -1 . & 8.9 & 8.9 & STN \\
\hline [MFF90] PHI 4/2 121 & $\ldots$ & $\ldots$ & $\cdots$ & 2.200 & 2.200 & $\ldots$ & $\cdots$ & $\cdots$ & $\cdots$ & $\cdots$ \\
\hline [MFF90] PHI 4/1 99 & 10.738 & $\ldots$ & $\ldots$ & 12.000 & 10.738 & -1.5 & -6.4 & 2.5 & 4.4 & SPM \\
\hline CD-27 8864 & 1.770 & $\ldots$ & $\ldots$ & 2.100 & 1.770 & -32.3 & -16.3 & 1.6 & 0.9 & SPM,AC \\
\hline $\mathrm{BD}+332273$ & 1.458 & $\ldots$ & $\ldots$ & 2.386 & 1.458 & -23 & -14 & 7.9 & 7.9 & STN \\
\hline [MFF90] PHI 4/2 105 & $\ldots$ & $\ldots$ & $\ldots$ & 1.400 & 1.400 & $\ldots$ & $\ldots$ & $\ldots$ & $\ldots$ & $\ldots$ \\
\hline UP 29143 & $\ldots$ & $\ldots$ & $\ldots$ & 1.202 & 1.202 & $\ldots$ & $\ldots$ & $\ldots$ & $\ldots$ & $\ldots$ \\
\hline [MFF90] PHI 4/1 65 & $\ldots$ & $\ldots$ & $\ldots$ & 1.400 & 1.400 & $\ldots$ & $\ldots$ & $\ldots$ & $\ldots$ & $\ldots$ \\
\hline [MFF90] PHI 4/1 62 & $\ldots$ & $\ldots$ & $\ldots$ & 3.300 & 3.300 & $\ldots$ & $\ldots$ & $\ldots$ & $\ldots$ & $\ldots$ \\
\hline $\mathrm{V}^{*} \mathrm{Z} \mathrm{CVn}$ & 1.913 & $\ldots$ & $\ldots$ & 1.819 & 1.913 & -7.9 & -39.7 & 5 & 5 & NPM \\
\hline HD 111464 & 0.315 & 0.236 & 0.17 & 0.219 & 0.315 & -20.9 & 19.8 & 0.5 & 0.5 & $\mathrm{HIP}, \mathrm{AC}$ \\
\hline [MFF90] PHI 4/2 73 & $\ldots$ & $\ldots$ & $\cdots$ & 3.100 & 3.100 & $\ldots$ & $\cdots$ & $\cdots$ & $\cdots$ & $\ldots$ \\
\hline [MFF90] PHI 4/2 68 & $\ldots$ & $\ldots$ & $\cdots$ & 6.600 & 6.600 & 4. & 3. & 5.1 & 5.1 & STN \\
\hline HD 111721 & 0.162 & 0.304 & 0.34 & 0.181 & 0.162 & -273.8 & -321.8 & 0.9 & 0.7 & $\mathrm{HIP}, \mathrm{AC}]$ \\
\hline [MFF90] PHI 4/1 21 & $\ldots$ & $\ldots$ & $\ldots$ & 3.800 & 3.800 & $\ldots$ & $\ldots$ & $\ldots$ & $\ldots$ & $\ldots$ \\
\hline $\mathrm{BD}+272181$ & 1.566 & $\ldots$ & $\ldots$ & 1.819 & 1.566 & 46.9 & 51.0 & 3.5 & 3.5 & STN,NP \\
\hline [MFF90] PHI 4/1 7 & $\ldots$ & $\ldots$ & $\ldots$ & 4.000 & 4.000 & -6 . & 7. & 5.0 & 5.0 & STN \\
\hline $\mathrm{V}^{*}$ AS Vir & 1.731 & $\ldots$ & $\ldots$ & 1.923 & 1.731 & 11.4 & -36.9 & 5 & 5 & NPM \\
\hline HD 111980 & 0.042 & 0.080 & 0.11 & 0.039 & 0.080 & 300.6 & -795.3 & 0.9 & 0.7 & HIP,AC] \\
\hline HD 111971 & 0.051 & 0.065 & 0.10 & 0.035 & 0.065 & -48.6 & 79.3 & 1.1 & 0.8 & HIP,AC] \\
\hline HD 112126 & 1.302 & 0.280 & 0.31 & 0.880 & 1.302 & -27.9 & -24.5 & 1.0 & 0.9 & $\mathrm{HIP}, \mathrm{AC}$ \\
\hline $\mathrm{BD}+282162$ & 2.221 & $\cdots$ & $\cdots$ & 0.982 & 2.221 & 0.1 & -22.2 & 3.5 & 3.5 & STN,NP \\
\hline $\mathrm{V}^{*}$ AT Vir & 1.432 & 0.535 & 1.22 & 1.438 & 1.432 & -60.5 & -22.3 & 1.4 & 1.2 & $\mathrm{HIP}, \mathrm{AC}]$ \\
\hline $\mathrm{BD}+272191$ & 0.232 & $\ldots$ & $\ldots$ & 0.398 & 0.232 & -68.2 & -2.1 & 2.3 & 0.9 & $\mathrm{ACT}, \mathrm{ST}$ \\
\hline $\mathrm{BD}+272196$ & 2.137 & $\ldots$ & $\ldots$ & 1.153 & 2.137 & $\ldots$ & $\ldots$ & $\ldots$ & $\ldots$ & $\ldots$ \\
\hline $\mathrm{BD}+102495$ & 0.518 & 0.424 & 0.63 & 0.980 & 0.518 & -44.6 & 31.7 & 1.2 & 1.0 & HIP,STN \\
\hline Weis 60529 & 3.429 & $\ldots$ & $\ldots$ & 1.689 & 3.429 & -22 & -8 & 4.8 & 4.8 & STN \\
\hline Weis 62013 & 8.313 & $\ldots$ & $\ldots$ & 0.467 & 8.313 & $\ldots$ & $\ldots$ & $\ldots$ & $\ldots$ & $\ldots$ \\
\hline Weis 60443 & 2.182 & $\ldots$ & $\ldots$ & 5.174 & 2.182 & $\ldots$ & $\ldots$ & $\ldots$ & $\ldots$ & $\ldots$ \\
\hline Weis 62339 & 0.899 & $\ldots$ & $\ldots$ & 2.146 & 0.899 & -33 . & 2 . & 4.9 & 4.9 & STN \\
\hline Weis 62021 & 14.068 & $\ldots$ & $\ldots$ & 1.736 & 14.068 & $\ldots$ & $\ldots$ & $\ldots$ & $\cdots$ & $\ldots$ \\
\hline HD 113002 & 0.140 & 0.290 & 0.32 & 0.360 & 0.140 & -154.5 & -56.9 & 1.0 & 0.7 & HIP,STN \\
\hline
\end{tabular}


TABLE 1B. (continued)

\begin{tabular}{|c|c|c|c|c|c|c|c|c|c|c|}
\hline $\begin{array}{l}\text { Star } \\
(1)\end{array}$ & $\begin{array}{c}D_{p h o} \\
(\mathrm{kpc}) \\
(2)\end{array}$ & $\begin{array}{c}D_{H I P} \\
(\mathrm{kpc}) \\
(3)\end{array}$ & $\begin{array}{c}\sigma_{\pi_{H I P}} / \pi_{H I P} \\
(4)\end{array}$ & $\begin{array}{c}D_{B S L} \\
(\mathrm{kpc}) \\
(5)\end{array}$ & $\begin{array}{c}D_{\text {adopt }} \\
(\mathrm{kpc}) \\
\quad(6)\end{array}$ & $\begin{array}{c}\mu_{\alpha^{*}} \\
(\mathrm{mas} / \mathrm{yr}) \\
(7)\end{array}$ & $\begin{array}{c}\mu_{\delta} \\
\text { (mas } / \mathrm{yr}) \\
(8)\end{array}$ & $\begin{array}{c}\sigma_{\mu_{\alpha^{*}}} \\
(\mathrm{mas} / \mathrm{yr}) \\
(9)\end{array}$ & $\begin{array}{c}\sigma_{\mu_{\delta}} \\
(\mathrm{mas} / \mathrm{yr}) \\
(10)\end{array}$ & $\begin{array}{c}\text { Source } \\
\text { (11) }\end{array}$ \\
\hline Weis 33496 & 0.700 & $\ldots$ & $\ldots$ & 1.310 & 0.700 & $\ldots$ & $\ldots$ & $\ldots$ & $\ldots$ & $\ldots$ \\
\hline $\mathrm{BD}+292354$ & 0.216 & $\ldots$ & $\ldots$ & 0.508 & 0.216 & -78.5 & -7.1 & 1.9 & 1.0 & ACT,STN,N \\
\hline Weis 64018 & 15.888 & $\ldots$ & $\ldots$ & 3.858 & 15.888 & $\ldots$ & $\ldots$ & $\ldots$ & $\ldots$ & $\ldots$ \\
\hline HD 112911 & 0.528 & 0.256 & 0.21 & 0.242 & 0.528 & 11.9 & -0.9 & 0.6 & 0.6 & HIP,ACT \\
\hline Weis 63200 & 2.708 & $\ldots$ & $\ldots$ & 3.529 & 2.708 & $\ldots$ & $\ldots$ & $\ldots$ & $\cdots$ & $\ldots$ \\
\hline HD 113083 & 0.036 & 0.054 & 0.06 & 0.036 & 0.054 & -476.4 & -202.5 & 1.1 & 0.6 & HIP,ACT \\
\hline Weis 63150 & 4.928 & $\ldots$ & $\ldots$ & 3.545 & 4.928 & $\ldots$ & $\ldots$ & $\cdots$ & $\cdots$ & $\ldots$ \\
\hline $\mathrm{BD}+292356$ & 0.640 & $\ldots$ & $\ldots$ & 1.046 & 0.640 & -9 & -23 & 4.0 & 4.0 & STN \\
\hline CD-32 9096 & 3.201 & 0.476 & 0.83 & 3.800 & 3.201 & 10.3 & -14.0 & 1.0 & 0.7 & HIP,SPM,AC \\
\hline Weis 63059 & 3.423 & $\ldots$ & $\cdots$ & 2.664 & 3.423 & -1 & -3 & 4.4 & 4.4 & STN \\
\hline Weis 31775 & 1.513 & $\cdots$ & $\cdots$ & 0.760 & 1.513 & $\ldots$ & $\ldots$ & $\cdots$ & $\ldots$ & $\cdots$ \\
\hline Weis 35550 & 0.377 & $\cdots$ & $\cdots$ & 1.310 & 0.377 & -35 & 4. & 3.9 & 3.9 & STN \\
\hline CD-33 8772 & 1.634 & $\cdots$ & $\cdots$ & 2.200 & 1.634 & -34.8 & 2.3 & 0.9 & 0.9 & $\mathrm{SPM}, \mathrm{ACT}, \mathrm{S}$ \\
\hline Weis 62864 & 1.084 & $\cdots$ & $\cdots$ & 2.531 & 1.084 & $\ldots$ & $\cdots$ & $\cdots$ & $\cdots$ & $\cdots$ \\
\hline Weis 62467 & 1.313 & $\cdots$ & $\cdots$ & 2.195 & 1.313 & -7 & 4. & 4.8 & 4.8 & STN \\
\hline Weis 61345 & 9.242 & $\ldots$ & $\ldots$ & 6.448 & 9.242 & $\ldots$ & $\ldots$ & $\ldots$ & $\cdots$ & $\ldots$ \\
\hline Weis 62990 & 1.400 & $\cdots$ & $\cdots$ & 3.247 & 1.400 & $\ldots$ & $\cdots$ & $\cdots$ & $\cdots$ & $\ldots$ \\
\hline Weis 62981 & 1.713 & $\cdots$ & $\cdots$ & 2.591 & 1.713 & -6 & -86 & 4.2 & 4.2 & STN \\
\hline $\mathrm{BD}+282176$ & 1.225 & $\cdots$ & $\cdots$ & 0.866 & 1.225 & -15.1 & 9.2 & 1.1 & 1.1 & $\mathrm{ACT}, \mathrm{STN}, \mathrm{Nl}$ \\
\hline Weis 62561 & 1.325 & $\cdots$ & $\cdots$ & 1.287 & 1.325 & -31 & 1. & 4.8 & 4.8 & STN \\
\hline Weis 61803 & 1.582 & $\cdots$ & $\cdots$ & 3.626 & 1.582 & $\cdots$ & $\cdots$ & $\cdots$ & $\cdots$ & $\cdots$ \\
\hline Weis 61514 & 7.597 & $\ldots$ & $\ldots$ & 1.784 & 7.597 & $\ldots$ & $\ldots$ & $\ldots$ & $\ldots$ & $\ldots$ \\
\hline Weis 61766 & 3.157 & $\cdots$ & $\cdots$ & 4.182 & 3.157 & $\cdots$ & $\ldots$ & $\cdots$ & $\cdots$ & $\cdots$ \\
\hline $\mathrm{BD}+122547$ & 1.864 & 0.258 & 0.42 & 1.780 & 1.864 & 4.7 & -54.3 & 0.9 & 1.1 & HIP,ACT,ST \\
\hline Weis 61574 & 5.826 & $\cdots$ & $\cdots$ & 2.362 & 5.826 & $\cdots$ & $\ldots$ & $\cdots$ & $\cdots$ & $\cdots$ \\
\hline Weis 61730 & 2.682 & $\cdots$ & $\cdots$ & 1.539 & 2.682 & $\ldots$ & $\ldots$ & $\ldots$ & $\cdots$ & $\ldots$ \\
\hline $\mathrm{V}^{*} \mathrm{RY}$ Com & 2.194 & $\cdots$ & $\cdots$ & 2.421 & 2.194 & -6.1 & -17.8 & 5 & 5 & NPM \\
\hline $\mathrm{BD}+302364$ & 2.312 & $\cdots$ & $\cdots$ & 0.885 & 2.312 & $\cdots$ & $\ldots$ & $\cdots$ & $\cdots$ & $\cdots$ \\
\hline Basel SA 57-1416 & 0.835 & $\cdots$ & $\cdots$ & 0.900 & 0.835 & $\cdots$ & $\cdots$ & $\cdots$ & $\cdots$ & $\cdots$ \\
\hline Basel SA 57-1372 & 1.707 & $\cdots$ & $\cdots$ & 1.200 & 1.707 & $\cdots$ & $\cdots$ & $\cdots$ & $\cdots$ & $\cdots$ \\
\hline Basel SA 57-1284 & 0.670 & $\cdots$ & $\cdots$ & 0.500 & 0.670 & $\cdots$ & $\cdots$ & $\cdots$ & $\cdots$ & $\cdots$ \\
\hline Basel SA 57-1378 & 0.515 & $\cdots$ & $\cdots$ & 0.600 & 0.515 & $\cdots$ & $\cdots$ & $\cdots$ & $\cdots$ & $\cdots$ \\
\hline Basel SA 57-1373 & 2.034 & $\cdots$ & $\cdots$ & 3.500 & 2.034 & $\cdots$ & $\cdots$ & $\cdots$ & $\cdots$ & $\cdots$ \\
\hline Basel SA 57-1374 & 0.977 & $\cdots$ & $\cdots$ & 1.500 & 0.977 & $\cdots$ & $\cdots$ & $\cdots$ & $\cdots$ & $\cdots$ \\
\hline Basel SA 57-1302 & $\cdots$ & $\cdots$ & $\cdots$ & 1.200 & 1.200 & $\cdots$ & $\cdots$ & $\cdots$ & $\cdots$ & $\cdots$ \\
\hline Basel SA 57-1383 & $\cdots$ & $\cdots$ & $\cdots$ & 2.800 & 2.800 & $\cdots$ & $\cdots$ & $\cdots$ & $\cdots$ & $\cdots$ \\
\hline Basel SA 57-1375 & 3.708 & $\cdots$ & $\cdots$ & 7.000 & 3.708 & $\cdots$ & $\cdots$ & $\cdots$ & $\cdots$ & $\cdots$ \\
\hline Basel SA 57-1299 & 7.516 & $\cdots$ & $\cdots$ & 6.900 & 7.516 & $\cdots$ & $\cdots$ & $\cdots$ & $\cdots$ & $\cdots$ \\
\hline Basel SA 57-1148 & 1.138 & $\cdots$ & $\cdots$ & 2.800 & 1.138 & $\cdots$ & $\cdots$ & $\cdots$ & $\cdots$ & $\cdots$ \\
\hline Basel SA 57-1110 & 0.489 & $\cdots$ & $\cdots$ & 0.400 & 0.489 & $\cdots$ & $\cdots$ & $\cdots$ & $\cdots$ & $\cdots$ \\
\hline
\end{tabular}


TABle 1B. (continued)

\begin{tabular}{|c|c|c|c|c|c|c|c|c|c|}
\hline $\begin{array}{l}\text { Star } \\
\text { (1) }\end{array}$ & $\begin{array}{c}D_{p h o} \\
(\mathrm{kpc}) \\
(2)\end{array}$ & $\begin{array}{c}D_{H I P} \\
(\mathrm{kpc}) \\
(3)\end{array}$ & $\begin{array}{c}\sigma_{\pi_{H I P}} / \pi_{H I P} \\
(4)\end{array}$ & $\begin{array}{c}D_{B S L} \\
(\mathrm{kpc}) \\
(5)\end{array}$ & $\begin{array}{c}D_{\text {adopt }} \\
(\mathrm{kpc}) \\
\quad(6)\end{array}$ & $\begin{array}{c}\mu_{\alpha^{*}} \\
(\mathrm{mas} / \mathrm{yr}) \\
(7)\end{array}$ & $\begin{array}{c}\mu_{\delta} \\
(\mathrm{mas} / \mathrm{yr}) \\
(8)\end{array}$ & $\begin{array}{c}\sigma_{\mu_{\alpha *}} \\
(\mathrm{mas} / \mathrm{yr}) \\
(9)\end{array}$ & $\begin{array}{c}\sigma_{\mu_{\delta}} \\
(\mathrm{mas} / \mathrm{yr}) \\
(10)\end{array}$ \\
\hline $\mathrm{BD}+312450$ & 1.696 & $\cdots$ & $\cdots$ & 0.623 & 1.696 & $\cdots$ & $\cdots$ & $\ldots$ & $\cdots$ \\
\hline Basel SA 57-1144 & 0.668 & $\ldots$ & $\ldots$ & 0.100 & 0.668 & $\ldots$ & $\ldots$ & $\ldots$ & $\ldots$ \\
\hline Basel SA 57-1147 & 0.990 & $\ldots$ & $\ldots$ & 5.400 & 0.990 & $\ldots$ & $\ldots$ & $\ldots$ & $\ldots$ \\
\hline Basel SA 57-1146 & 2.716 & $\cdots$ & $\cdots$ & 0.200 & 2.716 & $\cdots$ & $\cdots$ & $\cdots$ & $\cdots$ \\
\hline Basel SA 57-1142 & 1.542 & $\cdots$ & $\cdots$ & 1.600 & 1.542 & $\cdots$ & $\cdots$ & $\cdots$ & $\cdots$ \\
\hline Weis 51212 & 3.307 & $\cdots$ & $\cdots$ & 2.134 & 3.307 & $\cdots$ & $\cdots$ & $\cdots$ & $\cdots$ \\
\hline Basel SA 57-1141 & 1.278 & $\cdots$ & $\cdots$ & 2.500 & 1.278 & $\cdots$ & $\cdots$ & $\cdots$ & $\ldots$ \\
\hline Basel SA 57-1152 & 1.553 & $\cdots$ & $\cdots$ & 3.000 & 1.553 & $\cdots$ & $\cdots$ & $\cdots$ & $\cdots$ \\
\hline Basel SA 57-1140 & 1.103 & $\cdots$ & $\cdots$ & 4.000 & 1.103 & $\cdots$ & $\cdots$ & $\cdots$ & $\cdots$ \\
\hline Basel SA 57-0996 & 0.773 & $\cdots$ & $\cdots$ & 0.700 & 0.773 & $\cdots$ & $\cdots$ & $\cdots$ & $\cdots$ \\
\hline Basel SA 57-1060 & 0.551 & $\ldots$ & $\ldots$ & 1.300 & 0.551 & $\ldots$ & $\ldots$ & $\ldots$ & $\cdots$ \\
\hline Basel SA 57-0993 & 0.580 & $\cdots$ & $\cdots$ & 0.600 & 0.580 & $\cdots$ & $\cdots$ & $\cdots$ & $\cdots$ \\
\hline Basel SA 57-1003 & 0.618 & $\cdots$ & $\cdots$ & 0.700 & 0.618 & $\cdots$ & $\cdots$ & $\cdots$ & $\cdots$ \\
\hline Basel SA 57-0987 & 0.598 & $\cdots$ & $\cdots$ & 1.100 & 0.598 & $\cdots$ & $\cdots$ & $\cdots$ & $\cdots$ \\
\hline Basel SA 57-1132 & 1.260 & $\ldots$ & $\ldots$ & 1.700 & 1.260 & $\ldots$ & $\ldots$ & $\ldots$ & $\ldots$ \\
\hline Basel SA 57-0982 & 24.836 & $\ldots$ & $\ldots$ & 24.300 & 24.836 & $\ldots$ & $\ldots$ & $\ldots$ & $\ldots$ \\
\hline Basel SA 57-0983 & 0.995 & $\cdots$ & $\cdots$ & 3.300 & 0.995 & $\cdots$ & $\cdots$ & $\cdots$ & $\cdots$ \\
\hline Basel SA 57-1156 & 1.015 & $\cdots$ & $\cdots$ & 0.700 & 1.015 & $\cdots$ & $\cdots$ & $\cdots$ & $\cdots$ \\
\hline Basel SA 57-1157 & 1.428 & $\cdots$ & $\cdots$ & 1.800 & 1.428 & $\cdots$ & $\cdots$ & $\cdots$ & $\cdots$ \\
\hline Basel SA 57-1131 & 1.362 & $\cdots$ & $\cdots$ & 5.800 & 1.362 & $\cdots$ & $\cdots$ & $\cdots$ & $\cdots$ \\
\hline Basel SA 57-0978 & 0.673 & $\cdots$ & $\cdots$ & 0.900 & 0.673 & $\cdots$ & $\cdots$ & $\cdots$ & $\cdots$ \\
\hline Basel SA 57-1012 & 0.706 & $\ldots$ & $\ldots$ & 0.700 & 0.706 & $\ldots$ & $\ldots$ & $\ldots$ & $\ldots$ \\
\hline Basel SA 57-0977 & 0.455 & $\cdots$ & $\cdots$ & 0.400 & 0.455 & $\cdots$ & $\cdots$ & $\cdots$ & $\cdots$ \\
\hline Basel SA 57-1117 & 0.668 & $\cdots$ & $\cdots$ & 0.500 & 0.668 & $\cdots$ & $\cdots$ & $\cdots$ & $\cdots$ \\
\hline Basel SA 57-0966 & 11.374 & $\cdots$ & $\cdots$ & 11.400 & 11.374 & $\ldots$ & $\ldots$ & $\ldots$ & $\cdots$ \\
\hline Basel SA 57-1016 & 0.582 & $\cdots$ & $\cdots$ & 0.500 & 0.582 & -9.1 & -3.8 & 5 & 5 \\
\hline $\mathrm{V}^{*} \mathrm{Z}$ Com & 4.091 & $\cdots$ & $\cdots$ & 4.365 & 4.091 & -7.7 & -18.5 & 5 & 5 \\
\hline Basel SA 57-0692 & $\ldots$ & $\cdots$ & $\cdots$ & 2.800 & 2.800 & $\ldots$ & $\ldots$ & $\cdots$ & $\cdots$ \\
\hline Basel SA 57-0715 & $\cdots$ & $\cdots$ & $\cdots$ & 2.800 & 2.800 & $\cdots$ & $\ldots$ & $\cdots$ & $\cdots$ \\
\hline Basel SA 57-0789 & 1.070 & $\cdots$ & $\cdots$ & 0.600 & 1.070 & 28.6 & -94.9 & 5 & 5 \\
\hline Basel SA 57-0792 & 1.185 & $\ldots$ & $\ldots$ & 3.000 & 1.185 & $\ldots$ & $\ldots$ & $\ldots$ & $\ldots$ \\
\hline Basel SA 57-0827 & 0.758 & $\cdots$ & $\cdots$ & 2.800 & 0.758 & $\cdots$ & $\cdots$ & $\cdots$ & $\cdots$ \\
\hline Basel SA 57-0786 & 3.234 & $\cdots$ & $\cdots$ & 4.700 & 3.234 & $\ldots$ & $\ldots$ & $\cdots$ & $\cdots$ \\
\hline Basel SA 57-0758 & 1.553 & $\cdots$ & $\cdots$ & 1.100 & 1.553 & -7.6 & -9.6 & 5 & 5 \\
\hline Basel SA 57-0760 & 0.783 & $\cdots$ & $\cdots$ & 0.900 & 0.783 & 0.2 & -13.5 & 5 & 5 \\
\hline Basel SA 57-0712 & 8.120 & $\cdots$ & $\cdots$ & 8.100 & 8.120 & $\cdots$ & $\cdots$ & $\cdots$ & $\cdots$ \\
\hline Basel SA 57-0709 & 1.041 & $\cdots$ & $\cdots$ & 1.400 & 1.041 & $\cdots$ & $\cdots$ & $\cdots$ & $\cdots$ \\
\hline Basel SA 57-0699 & 0.827 & $\cdots$ & $\cdots$ & 0.900 & 0.827 & $\cdots$ & $\cdots$ & $\cdots$ & $\cdots$ \\
\hline Basel SA 57-0825 & 3.584 & $\cdots$ & $\cdots$ & 1.000 & 3.584 & $\cdots$ & $\cdots$ & $\cdots$ & $\cdots$ \\
\hline Basel SA 57-0718 & 8.097 & $\cdots$ & $\cdots$ & 11.900 & 8.097 & $\cdots$ & $\cdots$ & $\cdots$ & $\cdots$ \\
\hline
\end{tabular}


TABLE 1B. (continued)

\begin{tabular}{|c|c|c|c|c|c|c|c|c|c|c|}
\hline $\begin{array}{l}\text { Star } \\
(1)\end{array}$ & $\begin{array}{c}D_{p h o} \\
(\mathrm{kpc}) \\
(2)\end{array}$ & $\begin{array}{c}D_{H I P} \\
(\mathrm{kpc}) \\
(3)\end{array}$ & $\begin{array}{c}\sigma_{\pi_{H I P}} / \pi_{H I P} \\
(4)\end{array}$ & $\begin{array}{c}D_{B S L} \\
(\mathrm{kpc}) \\
(5)\end{array}$ & $\begin{array}{c}D_{\text {adopt }} \\
(\mathrm{kpc}) \\
\quad(6)\end{array}$ & $\begin{array}{c}\mu_{\alpha^{*}} \\
(\mathrm{mas} / \mathrm{yr}) \\
(7)\end{array}$ & $\begin{array}{c}\mu_{\delta} \\
\text { (mas/yr) } \\
(8)\end{array}$ & $\begin{array}{c}\sigma_{\mu_{\alpha *}} \\
(\mathrm{mas} / \mathrm{yr}) \\
(9)\end{array}$ & $\begin{array}{c}\sigma_{\mu_{\delta}} \\
(\mathrm{mas} / \mathrm{yr}) \\
(10)\end{array}$ & Sou \\
\hline Basel SA 57-0650 & 0.936 & $\cdots$ & $\cdots$ & 1.000 & 0.936 & $\cdots$ & $\cdots$ & $\cdots$ & $\cdots$ & $\cdots$ \\
\hline Basel SA 57-0719 & 1.057 & $\ldots$ & $\ldots$ & 6.400 & 1.057 & $\ldots$ & $\ldots$ & $\ldots$ & $\ldots$ & $\ldots$ \\
\hline Basel SA 57-0708 & 1.842 & $\ldots$ & $\ldots$ & 1.500 & 1.842 & $\ldots$ & $\ldots$ & $\ldots$ & $\ldots$ & $\ldots$ \\
\hline Basel SA 57-0799 & 31.265 & $\ldots$ & $\cdots$ & 28.400 & 31.265 & $\cdots$ & $\cdots$ & $\cdots$ & $\cdots$ & $\ldots$ \\
\hline Basel SA 57-0720 & 2.457 & $\cdots$ & $\cdots$ & 3.900 & 2.457 & $\cdots$ & $\ldots$ & $\cdots$ & $\cdots$ & $\cdots$ \\
\hline Basel SA 57-0739 & 1.598 & $\cdots$ & $\cdots$ & 0.100 & 1.598 & 7.1 & -26.9 & 5 & 5 & NPM \\
\hline Basel SA 57-0802 & 1.323 & $\cdots$ & $\cdots$ & 1.600 & 1.323 & $\ldots$ & $\ldots$ & $\cdots$ & $\cdots$ & $\ldots$ \\
\hline Weis 50158 & 0.509 & $\cdots$ & $\cdots$ & 2.575 & 0.509 & -37 & -14 & 4.3 & 4.3 & STN \\
\hline Basel SA 57-0803 & 21.769 & $\cdots$ & $\cdots$ & 19.500 & 21.769 & $\cdots$ & $\cdots$ & $\cdots$ & $\cdots$ & $\ldots$ \\
\hline Basel SA 57-0677 & 1.782 & $\cdots$ & $\cdots$ & 6.400 & 1.782 & $\cdots$ & $\cdots$ & $\cdots$ & $\cdots$ & $\cdots$ \\
\hline Basel SA 57-0659 & 10.826 & $\cdots$ & $\cdots$ & 10.200 & 10.826 & $\cdots$ & $\cdots$ & $\ldots$ & $\ldots$ & $\ldots$ \\
\hline Basel SA 57-0608 & 1.864 & $\cdots$ & $\cdots$ & 4.900 & 1.864 & $\cdots$ & $\cdots$ & $\cdots$ & $\cdots$ & $\cdots$ \\
\hline Basel SA 57-0808 & 0.449 & $\cdots$ & $\cdots$ & 1.200 & 0.449 & $\ldots$ & $\cdots$ & $\cdots$ & $\cdots$ & $\cdots$ \\
\hline Basel SA 57-0664 & 0.440 & $\cdots$ & $\cdots$ & 0.500 & 0.440 & $\cdots$ & $\cdots$ & $\cdots$ & $\cdots$ & $\cdots$ \\
\hline Basel SA 57-0671 & 0.424 & $\cdots$ & $\cdots$ & 0.400 & 0.424 & $\cdots$ & $\cdots$ & $\cdots$ & $\ldots$ & $\cdots$ \\
\hline Basel SA 57-0488 & 0.718 & $\ldots$ & $\ldots$ & 0.700 & 0.718 & $\ldots$ & $\ldots$ & $\ldots$ & $\ldots$ & $\ldots$ \\
\hline Basel SA 57-0564 & 1.439 & $\ldots$ & $\ldots$ & 5.000 & 1.439 & $\ldots$ & $\ldots$ & $\ldots$ & $\ldots$ & $\ldots$ \\
\hline Basel SA 57-0559 & 8.646 & $\cdots$ & $\cdots$ & 9.600 & 8.646 & $\cdots$ & $\cdots$ & $\cdots$ & $\cdots$ & $\cdots$ \\
\hline Basel SA 57-0467 & 0.642 & $\cdots$ & $\cdots$ & 0.700 & 0.642 & $\cdots$ & $\cdots$ & $\cdots$ & $\cdots$ & $\cdots$ \\
\hline Basel SA 57-0548 & 0.632 & $\cdots$ & $\cdots$ & 0.700 & 0.632 & $\cdots$ & $\cdots$ & $\ldots$ & $\cdots$ & $\cdots$ \\
\hline Basel SA 57-0546 & 0.800 & $\ldots$ & $\ldots$ & 0.900 & 0.800 & $\ldots$ & $\ldots$ & $\ldots$ & $\ldots$ & $\ldots$ \\
\hline Weis 50001 & 3.237 & $\ldots$ & $\ldots$ & 0.930 & 3.237 & -8 & -13 & 4.3 & 4.3 & STN \\
\hline CD-33 8865 & 3.402 & 0.176 & 0.45 & 3.400 & 3.402 & -11.7 & -4.2 & 1.4 & 1.3 & $\mathrm{HIP,SPM}$ \\
\hline Basel SA 57-0455 & 4.002 & $\ldots$ & $\cdots$ & 6.400 & 4.002 & $\ldots$ & $\cdots$ & $\cdots$ & $\cdots$ & $\ldots$ \\
\hline Basel SA 57-0505 & 2.707 & $\ldots$ & $\ldots$ & 7.600 & 2.707 & $\ldots$ & $\ldots$ & $\cdots$ & $\ldots$ & $\ldots$ \\
\hline Basel SA 57-0735 & $\ldots$ & $\cdots$ & $\cdots$ & 0.300 & 0.300 & $\ldots$ & $\ldots$ & $\cdots$ & $\ldots$ & $\cdots$ \\
\hline BPS CS 22877-0013 & 2.133 & $\cdots$ & $\cdots$ & 2.100 & 2.133 & $\cdots$ & $\cdots$ & $\cdots$ & $\cdots$ & $\cdots$ \\
\hline CD-31 10167 & 0.302 & $\cdots$ & $\cdots$ & 2.200 & 0.302 & $\cdots$ & $\cdots$ & $\cdots$ & $\cdots$ & $\cdots$ \\
\hline BPS CS 22877-0016 & 0.519 & $\cdots$ & $\cdots$ & 1.100 & 0.519 & $\cdots$ & $\cdots$ & $\cdots$ & $\cdots$ & $\cdots$ \\
\hline BPS CS 22877-0015 & 0.653 & $\cdots$ & $\cdots$ & 0.700 & 0.653 & $\cdots$ & $\cdots$ & $\cdots$ & $\cdots$ & $\cdots$ \\
\hline BPS CS 22877-0001 & 1.676 & $\cdots$ & $\cdots$ & 1.600 & 1.676 & -21 & -19 & 4.0 & 4.0 & STN \\
\hline BPS CS 22877-0011 & 3.036 & $\cdots$ & $\ldots$ & 2.600 & 3.036 & $\ldots$ & $\ldots$ & $\cdots$ & $\cdots$ & $\ldots$ \\
\hline HD 115338 & 0.730 & $\ldots$ & $\ldots$ & 0.295 & 0.730 & -70.1 & -87.8 & 4.0 & 4.1 & $\mathrm{ACT}, \mathrm{STN}$ \\
\hline HD 115444 & 0.703 & 0.282 & 0.32 & 1.030 & 0.703 & 4.9 & -60.8 & 0.9 & 0.8 & HIP,ACT, \\
\hline $\mathrm{V}^{*} \mathrm{YZ}$ Vir & $\ldots$ & $\cdots$ & $\cdots$ & 10.800 & 10.800 & $\ldots$ & $\ldots$ & $\cdots$ & $\cdots$ & $\ldots$ \\
\hline $\mathrm{V}^{*} \mathrm{ST}$ Com & 1.386 & $<0$ & $\cdots$ & 1.380 & 1.386 & -21.9 & -35.7 & 1.1 & 1.0 & $\mathrm{HIP}, \mathrm{ACT}$ \\
\hline BPS CS 22877-0029 & $\ldots$ & $\ldots$ & $\cdots$ & 1.200 & 1.200 & $\ldots$ & $\ldots$ & $\ldots$ & $\ldots$ & $\ldots$ \\
\hline HD 115772 & 0.216 & 0.474 & 0.77 & 0.609 & 0.216 & -1.2 & -24.0 & 0.8 & 0.7 & HIP,SPM, \\
\hline $\mathrm{V}^{*} \mathrm{AV}$ Vir & 1.646 & 0.331 & 1.00 & 1.614 & 1.646 & 8.5 & -37.3 & 2.2 & 1.7 & HIP,STN,I \\
\hline HD 116064 & 0.084 & 0.064 & 0.09 & 0.058 & 0.064 & -754.2 & 103.6 & 1.5 & 1.2 & HIP,ACT \\
\hline
\end{tabular}


TABLE 1B. (continued)

\begin{tabular}{|c|c|c|c|c|c|c|c|c|c|c|}
\hline $\begin{array}{l}\text { Star } \\
(1)\end{array}$ & $\begin{array}{c}D_{p h o} \\
(\mathrm{kpc}) \\
(2)\end{array}$ & $\begin{array}{c}D_{H I P} \\
(\mathrm{kpc}) \\
(3)\end{array}$ & $\begin{array}{c}\sigma_{\pi_{H I P}} / \pi_{H I P} \\
(4)\end{array}$ & $\begin{array}{c}D_{B S L} \\
(\mathrm{kpc}) \\
(5)\end{array}$ & $\begin{array}{c}D_{\text {adopt }} \\
(\mathrm{kpc}) \\
(6)\end{array}$ & $\begin{array}{c}\mu_{\alpha^{*}} \\
(\mathrm{mas} / \mathrm{yr}) \\
(7)\end{array}$ & $\begin{array}{c}\mu_{\delta} \\
\text { (mas/yr) } \\
(8)\end{array}$ & $\begin{array}{c}\sigma_{\mu_{\alpha^{*}}} \\
(\mathrm{mas} / \mathrm{yr}) \\
(9)\end{array}$ & $\begin{array}{c}\sigma_{\mu_{\delta}} \\
(\mathrm{mas} / \mathrm{yr}) \\
(10)\end{array}$ & Sou \\
\hline $\mathrm{V}^{*} \mathrm{BC}$ Vir & 2.012 & $\cdots$ & $\cdots$ & 2.080 & 2.012 & 13.3 & -17.5 & 3.1 & 3.1 & STN,NPM \\
\hline BPS CS 22877-0051 & 1.137 & $\ldots$ & $\ldots$ & 1.400 & 1.137 & $\ldots$ & $\ldots$ & $\ldots$ & $\ldots$ & $\ldots$ \\
\hline $\mathrm{V}^{*}$ AM Vir & 1.370 & $<0$. & $\cdots$ & 1.374 & 1.370 & 2.5 & -45.2 & 2.1 & 1.4 & HIP,STN,I \\
\hline BPS CS 22877-0024 & 1.453 & $\ldots$ & $\cdots$ & 0.800 & 1.453 & $\cdots$ & $\ldots$ & $\ldots$ & $\ldots$ & $\ldots$ \\
\hline $\mathrm{V}^{*} \mathrm{AU}$ Vir & 1.525 & $<0$ & $\cdots$ & 1.479 & 1.525 & -13.0 & -24.4 & 1.0 & 0.9 & $\mathrm{HIP}, \mathrm{ACT}$ \\
\hline $\mathrm{V}^{*} \mathrm{~W}$ Vir & $\ldots$ & $<0$ & $\cdots$ & 2.500 & 2.500 & -4.5 & 0.8 & 0.9 & 0.7 & $\mathrm{HIP}, \mathrm{ACT}$ \\
\hline $\mathrm{V}^{*} \mathrm{SX} \mathrm{UMa}$ & 1.142 & 1.429 & 1.99 & 1.117 & 1.142 & -67.9 & 2.7 & 1.1 & 1.2 & HIP,ACT, \\
\hline V* FX Нya & 2.283 & $\ldots$ & $\cdots$ & $\ldots$ & 2.283 & 2.6 & -7.0 & 4.1 & 4.1 & $\mathrm{SPM}$ \\
\hline $\mathrm{V}^{*} \mathrm{WW}$ Vir & 2.803 & $\cdots$ & $\cdots$ & $\ldots$ & 2.803 & $\ldots$ & $\ldots$ & $\cdots$ & $\cdots$ & $\cdots$ \\
\hline [OM87] 132605.43-0 & 0.334 & $\cdots$ & $\cdots$ & 0.890 & 0.334 & -25.0 & -3.2 & 2.3 & 2.8 & SPM,STN \\
\hline BPS CS 22877-0060 & 1.113 & $\ldots$ & $\ldots$ & 1.200 & 1.113 & $\ldots$ & $\ldots$ & $\ldots$ & $\cdots$ & $\cdots$ \\
\hline HD 117220 & 0.220 & 0.314 & 0.42 & 0.415 & 0.220 & -58.8 & -21.8 & 0.9 & 0.6 & HIP,SPM, \\
\hline $\mathrm{BD}+032782$ & 1.818 & 0.256 & 0.36 & $\ldots$ & 1.818 & -31.0 & -7.2 & 0.9 & 0.8 & $\mathrm{HIP}, \mathrm{ACT}$ \\
\hline $\mathrm{V}^{*} \mathrm{RV} \mathrm{UMa}$ & 1.028 & 1.515 & 2.17 & 1.072 & 1.028 & -27.8 & -42.6 & 1.2 & 1.3 & $\mathrm{HIP}, \mathrm{ACT}$ \\
\hline HD 117880 & 0.452 & 0.208 & 0.23 & 0.468 & 0.452 & -85.5 & -139.9 & 0.9 & 0.6 & $\mathrm{HIP}, \mathrm{ACT}$ \\
\hline BPS CS 22889-0006 & 1.283 & $\ldots$ & $\ldots$ & 1.200 & 1.283 & $\ldots$ & $\ldots$ & $\ldots$ & $\ldots$ & $\ldots$ \\
\hline HD 118055 & 1.185 & 1.515 & 1.88 & 1.300 & 1.185 & -24.6 & -14.8 & 0.8 & 0.7 & HIP,ACT \\
\hline $\mathrm{V}^{*} \mathrm{WY}$ Vir & 4.015 & $\cdots$ & $\cdots$ & $\ldots$ & 4.015 & -17.4 & -11.7 & 5 & 5 & NPM \\
\hline $\mathrm{BD}+182757$ & 0.853 & 3.571 & 4.89 & 1.580 & 0.853 & -18.3 & -28.9 & 0.9 & 0.9 & HIP,ACT, \\
\hline$V^{*}$ V671 Cen & 2.120 & $\cdots$ & $\cdots$ & $\cdots$ & 2.120 & -11.3 & -26.6 & 1.6 & 2.0 & SPM,STN \\
\hline BPS CS 22889-0029 & 0.785 & $\cdots$ & $\ldots$ & 1.200 & 0.785 & $\cdots$ & $\cdots$ & $\cdots$ & $\cdots$ & $\ldots$ \\
\hline BPS CS 22889-0038 & 1.109 & $\ldots$ & $\ldots$ & 1.300 & 1.109 & $\ldots$ & $\ldots$ & $\cdots$ & $\ldots$ & $\ldots$ \\
\hline HD 119516 & 0.406 & 20.000 & 27.80 & $\cdots$ & 0.406 & -50.9 & -15.3 & 1.0 & 0.9 & $\mathrm{HIP}, \mathrm{ACT}$, \\
\hline BPS CS 22889-0045 & 0.637 & $\ldots$ & $\ldots$ & 0.900 & 0.637 & $\ldots$ & $\ldots$ & $\cdots$ & $\cdots$ & $\ldots$ \\
\hline $\mathrm{V}^{*} \mathrm{RZ} \mathrm{CVn}$ & 1.599 & $<0$ & $\cdots$ & 1.258 & 1.599 & -53.8 & -0.6 & 2.1 & 1.5 & HIP,STN,I \\
\hline BPS CS 22889-0050 & 1.041 & $\ldots$ & $\ldots$ & 1.300 & 1.041 & $\ldots$ & $\ldots$ & $\cdots$ & $\cdots$ & $\ldots$ \\
\hline CD-33 9314 & 0.784 & 0.350 & 0.98 & 1.000 & 0.784 & 18.4 & -58.3 & 1.4 & 0.8 & HIP,SPM, \\
\hline $\mathrm{V}^{*} \mathrm{RV}$ Oct & 0.978 & 2.381 & 3.31 & $\cdots$ & 0.978 & -33.6 & 16.9 & 0.9 & 1.2 & HIP,ACT, \\
\hline HD 120183 & 0.285 & 0.203 & 0.23 & 0.244 & 0.285 & -37.5 & -42.8 & 1.1 & 0.8 & $\mathrm{HIP}, \mathrm{ACT}$, \\
\hline BPS CS 22889-0064 & 0.966 & $\cdots$ & $\cdots$ & 1.100 & 0.966 & $\cdots$ & $\cdots$ & $\cdots$ & $\cdots$ & $\cdots$ \\
\hline BPS CS 22889-0047 & 1.066 & $\cdots$ & $\cdots$ & 1.400 & 1.066 & $\cdots$ & $\cdots$ & $\cdots$ & $\cdots$ & $\cdots$ \\
\hline $\mathrm{V}^{*} \mathrm{SS}$ CVn & 1.741 & $<0$ & $\cdots$ & 1.690 & 1.741 & 6.8 & -43.6 & 1.4 & 1.5 & HIP,STN,I \\
\hline $\mathrm{V}^{*} \mathrm{RX} \mathrm{CVn}$ & 2.417 & $\cdots$ & $\cdots$ & 2.333 & 2.417 & 5.3 & -0.5 & 3.6 & 3.6 & STN,NPM \\
\hline $\mathrm{V}^{*} \mathrm{BB}$ Vir & 1.218 & $\ldots$ & $\cdots$ & 1.300 & 1.218 & -47.2 & -4.2 & 2.6 & 2.3 & $\mathrm{ACT}, \mathrm{STN}$ \\
\hline HD 120559 & 0.023 & 0.025 & 0.02 & 0.049 & 0.025 & -360.10 & -412.65 & 0.77 & 0.67 & HIP \\
\hline HD 121135 & 0.431 & 0.578 & 0.75 & 0.810 & 0.431 & -33.8 & -18.6 & 0.9 & 0.7 & HIP,ACT, \\
\hline $\mathrm{V}^{*} \mathrm{FY}$ Нya & 2.377 & $\ldots$ & $\cdots$ & 2.312 & 2.377 & $\ldots$ & $\ldots$ & $\cdots$ & $\cdots$ & $\ldots$ \\
\hline HD 121261 & 1.283 & 0.917 & 1.35 & 1.122 & 1.283 & -32.7 & -11.0 & 0.9 & 0.7 & HIP,SPM, \\
\hline $\mathrm{V}^{*}$ V499 Cen & 1.140 & $<0$ & $\cdots$ & 1.132 & 1.140 & 8.7 & -25.6 & 0.8 & 1.1 & HIP,SPM, \\
\hline $\mathrm{V}^{*} \mathrm{ST}$ CVn & 1.346 & $<0$ & $\cdots$ & 1.472 & 1.346 & -15.4 & -14.1 & 2.0 & 1.6 & HIP,STN,I \\
\hline
\end{tabular}


TABLE 1B. (continued)

\begin{tabular}{|c|c|c|c|c|c|c|c|c|c|c|}
\hline $\begin{array}{l}\text { Star } \\
(1)\end{array}$ & $\begin{array}{c}D_{\text {pho }} \\
(\mathrm{kpc}) \\
(2)\end{array}$ & $\begin{array}{c}D_{H I P} \\
(\mathrm{kpc}) \\
(3)\end{array}$ & $\begin{array}{c}\sigma_{\pi_{H I P}} / \pi_{H I P} \\
(4)\end{array}$ & $\begin{array}{c}D_{B S L} \\
(\mathrm{kpc}) \\
(5)\end{array}$ & $\begin{array}{c}D_{\text {adopt }} \\
\text { (kpc) } \\
(6)\end{array}$ & $\begin{array}{c}\mu_{\alpha^{*}} \\
(\mathrm{mas} / \mathrm{yr}) \\
(7)\end{array}$ & $\begin{array}{c}\mu_{\delta} \\
(\mathrm{mas} / \mathrm{yr}) \\
(8)\end{array}$ & $\begin{array}{c}\sigma_{\mu_{\alpha^{*}}} \\
(\mathrm{mas} / \mathrm{yr}) \\
(9)\end{array}$ & $\begin{array}{c}\sigma_{\mu_{\delta}} \\
(\mathrm{mas} / \mathrm{yr}) \\
(10)\end{array}$ & Sou \\
\hline V* UY Boo & 1.250 & 0.485 & 0.93 & 1.218 & 1.250 & 1.1 & -47.8 & 0.8 & 0.7 & HIP,ACT, \\
\hline $\mathrm{V}^{*} \mathrm{RU} \mathrm{CVn}$ & 1.818 & $\ldots$ & $\ldots$ & 1.976 & 1.818 & -25.0 & -1.3 & 4.4 & 4.4 & STN,NPM \\
\hline HD 122196 & 0.060 & 0.102 & 0.14 & 0.055 & 0.060 & -451.6 & -81.7 & 0.7 & 0.7 & HIP,SPM, \\
\hline HD 122563 & 0.280 & 0.266 & 0.19 & 0.283 & 0.280 & -188.9 & -70.1 & 0.6 & 0.4 & HIP,ACT \\
\hline$V^{*}$ V674 Cen & 1.453 & $\ldots$ & $\ldots$ & $\ldots$ & 1.453 & -52.7 & -21.3 & 1.8 & 1.4 & SPM,STN \\
\hline HD 122956 & 0.288 & 0.303 & 0.27 & 0.253 & 0.288 & -92.9 & -45.2 & 0.7 & 0.6 & HIP,ACT, \\
\hline $\mathrm{V}^{*} \mathrm{AD}$ Vir & 2.889 & $\ldots$ & $\ldots$ & $\ldots$ & 2.889 & -19.7 & -6.0 & 5 & 5 & NPM \\
\hline $\mathrm{V}^{*} \mathrm{~W} \mathrm{CVn}$ & 0.956 & 0.870 & 1.47 & 0.954 & 0.956 & -19.1 & -15.2 & 0.9 & 1.2 & HIP,ACT \\
\hline $\mathrm{V}^{*} \mathrm{AL}$ Vir & $\ldots$ & 2.632 & 4.16 & 1.700 & 1.700 & -2.7 & -1.8 & 0.9 & 0.7 & $\mathrm{HIP}, \mathrm{ACT}$ \\
\hline $\mathrm{BD}+092860$ & 0.533 & 0.201 & 0.45 & 1.400 & 0.533 & 10.1 & -44.0 & 1.8 & 1.4 & HIP,ACT \\
\hline HD 124358 & 0.904 & 0.862 & 1.31 & 0.873 & 0.904 & -95.4 & -28.1 & 0.8 & 0.8 & HIP,ACT, \\
\hline BPS CS 22883-0004 & 1.034 & $\ldots$ & $\ldots$ & 1.100 & 1.034 & $\ldots$ & $\ldots$ & $\ldots$ & $\ldots$ & $\ldots$ \\
\hline $\mathrm{V}^{*}$ GS Hya & 2.520 & $\ldots$ & $\ldots$ & $\ldots$ & 2.520 & $\ldots$ & $\ldots$ & $\ldots$ & $\ldots$ & $\ldots$ \\
\hline HD 124884 & 1.192 & $\ldots$ & $\ldots$ & 0.365 & 1.192 & -7.3 & -12.8 & 0.9 & 0.9 & $\mathrm{ACT}, \mathrm{STN}$ \\
\hline $\mathrm{V}^{*}$ CM Boo & 1.900 & $\ldots$ & $\ldots$ & $\ldots$ & 1.900 & $\ldots$ & $\ldots$ & $\ldots$ & $\ldots$ & $\ldots$ \\
\hline $\mathrm{BD}+092870$ & 1.739 & 0.446 & 0.71 & 1.480 & 1.739 & -56.6 & 1.7 & 1.3 & 0.7 & HIP,ACT \\
\hline $\mathrm{V}^{*} \mathrm{TV}$ Boo & 1.304 & $<0$ & $\ldots$ & 1.230 & 1.304 & -3.3 & -35.5 & 0.7 & 1.1 & $\mathrm{HIP}, \mathrm{ACT}$ \\
\hline $\mathrm{V}^{*} \mathrm{XX}$ Vir & $\ldots$ & $\ldots$ & $\ldots$ & 3.000 & 3.000 & -13 & -13 & 3.6 & 3.6 & STN \\
\hline BPS CS 22883-0021 & $\ldots$ & $\ldots$ & $\ldots$ & 1.500 & 1.500 & $\ldots$ & $\ldots$ & $\ldots$ & $\ldots$ & $\ldots$ \\
\hline BD+01 2916 & 2.093 & 0.680 & 1.04 & 1.979 & 2.093 & -22.6 & -13.4 & 0.8 & 0.9 & $\mathrm{HIP}, \mathrm{ACT}$, \\
\hline BPS CS 22883-0030 & 1.199 & $\ldots$ & $\ldots$ & 1.200 & 1.199 & $\ldots$ & $\ldots$ & $\ldots$ & $\ldots$ & $\ldots$ \\
\hline BD+08 2856 & 1.701 & $<0$ & $\ldots$ & 2.300 & 1.701 & 9.4 & -21.1 & 1.3 & 1.1 & HIP,ACT, \\
\hline BPS CS 22883-0037 & 2.550 & $\ldots$ & $\ldots$ & 7.300 & 2.550 & $\ldots$ & $\ldots$ & $\ldots$ & $\ldots$ & $\ldots$ \\
\hline $\mathrm{V}^{*} \mathrm{Y}$ Oct & 1.740 & $\ldots$ & $\ldots$ & $\ldots$ & 1.740 & $\ldots$ & $\ldots$ & $\ldots$ & $\ldots$ & $\ldots$ \\
\hline HD 126238 & 0.186 & 0.262 & 0.25 & 0.208 & 0.186 & 26.1 & -104.6 & 0.7 & 0.5 & HIP,ACT, \\
\hline HD 126587 & 0.523 & 0.714 & 1.03 & 0.623 & 0.523 & -15.5 & -52.5 & 1.0 & 1.0 & HIP,ACT, \\
\hline $\mathrm{V}^{*} \mathrm{AE}$ Vir & 3.181 & $\ldots$ & $\ldots$ & $\ldots$ & 3.181 & -0.2 & -17.7 & 5 & 5 & NPM \\
\hline $\mathrm{V}^{*} \mathrm{RY}$ Lib & 2.818 & $\ldots$ & $\ldots$ & $\ldots$ & 2.818 & -16.8 & -4.5 & 5 & 5 & NPM \\
\hline $\mathrm{V}^{*} \mathrm{SW}$ Boo & 2.120 & $\ldots$ & $\ldots$ & 2.270 & 2.120 & -45.8 & 1.3 & 5 & 5 & NPM \\
\hline $\mathrm{V}^{*} \mathrm{ST}$ Vir & 1.329 & $<0$ & $\cdots$ & 1.528 & 1.329 & -4.6 & -19.0 & 2.2 & 2.0 & HIP,STN,I \\
\hline $\mathrm{V}^{*} \mathrm{AF}$ Vir & 1.661 & $<0$ & $\ldots$ & $\ldots$ & 1.661 & -56.1 & -0.8 & 2.4 & 2.1 & HIP,STN,I \\
\hline $\mathrm{BD}+182890$ & 0.660 & 0.658 & 0.95 & 0.930 & 0.660 & -13.8 & -98.4 & 0.7 & 0.7 & $\mathrm{HIP}, \mathrm{ACT}$, \\
\hline $\mathrm{V}^{*} \mathrm{KS}$ Hya & 3.412 & $\ldots$ & $\ldots$ & 3.300 & 3.412 & -40 . & 10. & 7.1 & 7.1 & STN \\
\hline BPS CS 22874-0018 & 0.816 & $\ldots$ & $\ldots$ & 0.700 & 0.816 & $\ldots$ & $\ldots$ & $\ldots$ & $\ldots$ & $\ldots$ \\
\hline $\mathrm{V}^{*} \mathrm{RS}$ Boo & 0.787 & 1.299 & 2.04 & 0.875 & 0.787 & 5.5 & 2.3 & 1.0 & 0.8 & $\mathrm{HIP}, \mathrm{ACT}$ \\
\hline $\mathrm{V}^{*} \mathrm{SV}$ Boo & 3.179 & $\ldots$ & $\ldots$ & 3.006 & 3.179 & -1.7 & -22.8 & 5 & 5 & NPM \\
\hline BPS CS 22871-0012 & 7.077 & $\ldots$ & $\ldots$ & 1.100 & 7.077 & $\ldots$ & $\ldots$ & $\ldots$ & $\ldots$ & $\ldots$ \\
\hline HD 128188 & 0.736 & 0.730 & 1.29 & 0.110 & 0.736 & -9.7 & -9.7 & 1.0 & 0.9 & HIP,ACT, \\
\hline BPS CS 22874-0038 & 3.464 & $\ldots$ & $\ldots$ & 2.400 & 3.464 & $\ldots$ & $\ldots$ & $\ldots$ & $\ldots$ & $\ldots$ \\
\hline BPS CS 22874-0049 & $\ldots$ & $\cdots$ & $\cdots$ & 1.400 & 1.400 & $\cdots$ & $\cdots$ & $\cdots$ & $\cdots$ & $\cdots$ \\
\hline
\end{tabular}


TABLE 1B. (continued)

\begin{tabular}{|c|c|c|c|c|c|c|c|c|c|c|}
\hline $\begin{array}{l}\text { Star } \\
(1)\end{array}$ & $\begin{array}{c}D_{p h o} \\
(\mathrm{kpc}) \\
(2)\end{array}$ & $\begin{array}{c}D_{H I P} \\
(\mathrm{kpc}) \\
(3)\end{array}$ & $\begin{array}{c}\sigma_{\pi_{H I P}} / \pi_{H I P} \\
(4)\end{array}$ & $\begin{array}{c}D_{B S L} \\
(\mathrm{kpc}) \\
(5)\end{array}$ & $\begin{array}{c}D_{\text {adopt }} \\
\text { (kpc) } \\
(6)\end{array}$ & $\begin{array}{c}\mu_{\alpha^{*}} \\
(\mathrm{mas} / \mathrm{yr}) \\
(7)\end{array}$ & $\begin{array}{c}\mu_{\delta} \\
(\mathrm{mas} / \mathrm{yr}) \\
(8)\end{array}$ & $\begin{array}{c}\sigma_{\mu_{\alpha^{*}}} \\
(\mathrm{mas} / \mathrm{yr}) \\
(9)\end{array}$ & $\begin{array}{c}\sigma_{\mu_{\delta}} \\
(\mathrm{mas} / \mathrm{yr}) \\
(10)\end{array}$ & Sou \\
\hline HD 128279 & 0.144 & 0.168 & 0.22 & 0.194 & 0.144 & 67.2 & -342.6 & 1.1 & 0.9 & HIP,SPM, \\
\hline $\mathrm{V}^{*} \mathrm{DO}$ Vir & 4.299 & $\ldots$ & $\ldots$ & $\ldots$ & 4.299 & -27.2 & 6.8 & 5 & 5 & NPM \\
\hline BPS CS 22871-0035 & 1.300 & $\ldots$ & $\ldots$ & 1.600 & 1.300 & $\ldots$ & $\ldots$ & $\ldots$ & $\ldots$ & $\ldots$ \\
\hline BPS CS 22871-0070 & 1.090 & $\ldots$ & $\ldots$ & 1.200 & 1.090 & $\ldots$ & $\ldots$ & $\ldots$ & $\ldots$ & $\ldots$ \\
\hline BPS CS 22871-0060 & 1.063 & $\ldots$ & $\ldots$ & 4.600 & 1.063 & -9 & 6. & 7.9 & 7.9 & STN \\
\hline $\mathrm{V}^{*} \mathrm{SZ} \mathrm{Boo}$ & 2.580 & $\ldots$ & $\ldots$ & 2.594 & 2.580 & -7.7 & -8.5 & 5 & 5 & NPM \\
\hline BPS CS 22871-0095 & 1.305 & $\ldots$ & $\ldots$ & 1.300 & 1.305 & $\ldots$ & $\ldots$ & $\ldots$ & $\ldots$ & $\ldots$ \\
\hline $\mathrm{V}^{*} \mathrm{TW}$ Boo & 1.349 & $<0$ & $\ldots$ & 1.336 & 1.349 & -2.0 & -54.4 & 1.1 & 0.7 & $\mathrm{HIP}, \mathrm{ACT}$ \\
\hline BPS CS 22874-0091 & 0.962 & $\ldots$ & $\ldots$ & 1.000 & 0.962 & $\ldots$ & $\ldots$ & $\ldots$ & $\ldots$ & $\ldots$ \\
\hline $\mathrm{V}^{*} \mathrm{RU}$ Boo & 4.012 & $\cdots$ & $\cdots$ & 4.018 & 4.012 & -13.5 & -3.2 & 5 & 5 & NPM \\
\hline BPS CS 22874-0094 & 0.786 & $\ldots$ & $\ldots$ & 0.400 & 0.786 & $\ldots$ & $\ldots$ & $\ldots$ & $\ldots$ & $\ldots$ \\
\hline $\mathrm{V}^{*} \mathrm{AE}$ Boo & 0.981 & 0.725 & 1.35 & $\ldots$ & 0.981 & -25.6 & -36.7 & 1.0 & 0.8 & HIP, ACT, \\
\hline $\mathrm{V}^{*} \mathrm{TY}$ Aps & 1.387 & 2.083 & 5.15 & 1.086 & 1.387 & -37.3 & 9.2 & 1.6 & 1.9 & HIP,STN \\
\hline BPS CS 22874-0123 & 1.149 & $\ldots$ & $\ldots$ & 1.200 & 1.149 & $\ldots$ & $\ldots$ & $\ldots$ & $\ldots$ & $\ldots$ \\
\hline BPS CS 22871-0105 & $\ldots$ & $\ldots$ & $\ldots$ & 1.200 & 1.200 & $\ldots$ & $\ldots$ & $\ldots$ & $\ldots$ & $\ldots$ \\
\hline BPS CS 22874-0119 & 0.607 & $\ldots$ & $\ldots$ & 1.300 & 0.607 & $\ldots$ & $\ldots$ & $\ldots$ & $\ldots$ & $\ldots$ \\
\hline $\mathrm{V}^{*} \mathrm{BT}$ Dra & 1.646 & 0.532 & 0.85 & $\ldots$ & 1.646 & 2.8 & -32.8 & 1.6 & 1.7 & HIP,STN,I \\
\hline $\mathrm{V}^{*} \mathrm{XZ}$ Aps & 1.768 & 1.408 & 4.27 & $\ldots$ & 1.768 & -20.3 & 14.4 & 2.0 & 2.4 & HIP,STN \\
\hline BPS CS 22871-0102 & 0.799 & $\ldots$ & $\ldots$ & 0.800 & 0.799 & $\ldots$ & $\ldots$ & $\ldots$ & $\ldots$ & $\ldots$ \\
\hline $\mathrm{V}^{*} \mathrm{UY}$ Aps & 2.913 & $\cdots$ & $\cdots$ & $\ldots$ & 2.913 & $\cdots$ & $\cdots$ & $\cdots$ & $\ldots$ & $\ldots$ \\
\hline HD 132475 & 0.047 & 0.092 & 0.11 & 0.033 & 0.092 & -559.3 & -499.4 & 0.8 & 0.8 & HIP,ACT \\
\hline BD-11 3853 & 0.122 & 0.301 & 0.61 & 0.155 & 0.122 & -150.3 & -37.3 & 1.5 & 1.1 & HIP,ACT, \\
\hline HD 133461 & 0.724 & 0.980 & 1.34 & 0.334 & 0.724 & 3.5 & -29.8 & 0.8 & 0.7 & HIP,ACT, \\
\hline BD-08 3901 & 0.509 & $<0$ & $\ldots$ & 0.609 & 0.509 & -28.9 & -3.8 & 1.5 & 1.3 & HIP,ACT, \\
\hline $\mathrm{BD}+302611$ & 1.294 & 0.290 & 0.38 & 1.480 & 1.294 & 16.0 & -8.9 & 0.7 & 0.8 & HIP,ACT, \\
\hline $\mathrm{V}^{*}$ XX Lib & 1.671 & $\ldots$ & $\ldots$ & $\ldots$ & 1.671 & $\ldots$ & $\ldots$ & $\ldots$ & $\ldots$ & $\ldots$ \\
\hline $\mathrm{BD}+182976$ & 2.170 & 0.433 & 0.74 & 1.730 & 2.170 & -29.9 & -35.0 & 1.7 & 1.4 & HIP,ACT, \\
\hline HD 135148 & 2.026 & $<0$ & $\ldots$ & 1.400 & 2.026 & -27.8 & 3.2 & 1.0 & 1.0 & HIP,STN \\
\hline BPS CS 22890-0011 & 1.366 & $\ldots$ & $\ldots$ & 1.300 & 1.366 & $\ldots$ & $\ldots$ & $\ldots$ & $\cdots$ & $\ldots$ \\
\hline $\mathrm{V}^{*}$ AP Ser & 1.210 & $<0$ & $\cdots$ & 1.009 & 1.210 & -40.5 & -43.6 & 2.0 & 1.7 & HIP,STN,I \\
\hline$V^{*}$ BH Ser & 2.730 & $\ldots$ & $\ldots$ & $\ldots$ & 2.730 & -7.8 & -15.1 & 5 & 5 & NPM \\
\hline $\mathrm{V}^{*} \mathrm{DF}$ Ser & 2.283 & $\ldots$ & $\ldots$ & $\ldots$ & 2.283 & 4.6 & -5.5 & 5 & 5 & NPM \\
\hline BPS CS 22890-0024 & 1.532 & $\ldots$ & $\ldots$ & 1.300 & 1.532 & $\ldots$ & $\ldots$ & $\ldots$ & $\ldots$ & $\ldots$ \\
\hline HD 135449 & 0.501 & 0.183 & 0.25 & 0.568 & 0.501 & -131.9 & -41.3 & 0.9 & 0.9 & HIP,SPM, \\
\hline $\mathrm{V}^{*} \mathrm{BF}$ Ser & $\ldots$ & $<0$ & $\ldots$ & 2.600 & 2.600 & -15.2 & -11.3 & 2.1 & 2.0 & HIP,STN,I \\
\hline $\mathrm{V}^{*} \mathrm{UU}$ Boo & 2.164 & $\ldots$ & $\ldots$ & 2.177 & 2.164 & 8.6 & -33.8 & 5 & 5 & NPM \\
\hline $\mathrm{BD}+172844$ & 0.864 & 0.546 & 0.62 & 0.251 & 0.864 & -30.3 & -3.5 & 0.9 & 0.7 & HIP,ACT, \\
\hline BPS CS 22890-0064 & 1.419 & $\ldots$ & $\ldots$ & 1.400 & 1.419 & $\ldots$ & $\ldots$ & $\ldots$ & $\ldots$ & $\ldots$ \\
\hline $\mathrm{V}^{*} \mathrm{TV}$ Lib & 1.428 & $\cdots$ & $\cdots$ & 1.698 & 1.428 & 0.5 & 10.4 & 5 & 5 & NPM \\
\hline BPS CS 22890-0029 & 0.868 & $\cdots$ & $\cdots$ & 1.000 & 0.868 & $\cdots$ & $\ldots$ & $\ldots$ & $\cdots$ & $\ldots$ \\
\hline
\end{tabular}


TABLE 1B. (continued)

\begin{tabular}{|c|c|c|c|c|c|c|c|c|c|c|}
\hline $\begin{array}{l}\text { Star } \\
(1)\end{array}$ & $\begin{array}{c}D_{p h o} \\
(\mathrm{kpc}) \\
(2)\end{array}$ & $\begin{array}{c}D_{H I P} \\
(\mathrm{kpc}) \\
(3)\end{array}$ & $\begin{array}{c}\sigma_{\pi_{H I P}} / \pi_{H I P} \\
(4)\end{array}$ & $\begin{array}{c}D_{B S L} \\
(\mathrm{kpc}) \\
(5)\end{array}$ & $\begin{array}{c}D_{\text {adopt }} \\
(\mathrm{kpc}) \\
(6)\end{array}$ & $\begin{array}{c}\mu_{\alpha^{*}} \\
(\mathrm{mas} / \mathrm{yr}) \\
(7)\end{array}$ & $\begin{array}{c}\mu_{\delta} \\
\text { (mas/yr) } \\
(8)\end{array}$ & $\begin{array}{c}\sigma_{\mu_{\alpha^{*}}} \\
(\mathrm{mas} / \mathrm{yr}) \\
(9)\end{array}$ & $\begin{array}{c}\sigma_{\mu_{\delta}} \\
(\mathrm{mas} / \mathrm{yr}) \\
(10)\end{array}$ & Sou \\
\hline $\mathrm{V}^{*} \mathrm{AB}$ Aps & 2.740 & $\cdots$ & $\cdots$ & $\cdots$ & 2.740 & $\cdots$ & $\cdots$ & $\cdots$ & $\cdots$ & $\cdots$ \\
\hline BPS CS 22890-0063 & 0.904 & $\ldots$ & $\cdots$ & 0.900 & 0.904 & $\ldots$ & $\cdots$ & $\cdots$ & $\ldots$ & $\ldots$ \\
\hline HD 136316 & 0.388 & 0.926 & 0.88 & 0.540 & 0.388 & -28.6 & -64.5 & 0.7 & 0.8 & HIP,ACT \\
\hline $\mathrm{V}^{*} \mathrm{TV} \mathrm{CrB}$ & 1.864 & $<0$ & $\cdots$ & $\ldots$ & 1.864 & -3.9 & -7.1 & 2.5 & 2.5 & HIP,STN,I \\
\hline $\mathrm{V}^{*}$ FW Lup & 0.380 & 0.382 & 0.50 & $\ldots$ & 0.380 & -4.4 & -16.1 & 0.9 & 1.0 & HIP,ACT, \\
\hline $\mathrm{BD}+013070$ & 0.536 & 0.417 & 0.90 & 0.480 & 0.536 & -36.9 & 64.3 & 0.9 & 0.8 & HIP,ACT, \\
\hline BPS CS 22890-0095 & 0.994 & $\ldots$ & $\ldots$ & 1.400 & 0.994 & $\ldots$ & $\ldots$ & $\cdots$ & $\cdots$ & $\ldots$ \\
\hline $\mathrm{V}^{*}$ CS Ser & 2.180 & $\ldots$ & $\cdots$ & $\ldots$ & 2.180 & 25.5 & -29.1 & 3.8 & 3.8 & STN,NPM \\
\hline $\mathrm{V}^{*} \mathrm{ST}$ Boo & 1.216 & 2.128 & 3.72 & $\ldots$ & 1.216 & -15.1 & -11.9 & 1.2 & 1.1 & HIP,ACT \\
\hline$V^{*}$ VY Ser & 0.789 & $<0$ & $\cdots$ & 0.798 & 0.789 & -104.2 & -9.5 & 0.9 & 0.8 & HIP,ACT \\
\hline BPS CS 22884-0005 & 1.374 & $\cdots$ & $\ldots$ & 2.900 & 1.374 & $\cdots$ & $\cdots$ & $\cdots$ & $\cdots$ & $\ldots$ \\
\hline BPS CS 30312-0006 & 1.294 & $\cdots$ & $\cdots$ & 1.100 & 1.294 & $\cdots$ & $\cdots$ & $\cdots$ & $\cdots$ & $\cdots$ \\
\hline BPS CS 30312-0002 & $\ldots$ & $\cdots$ & $\cdots$ & 1.400 & 1.400 & $\ldots$ & $\cdots$ & $\cdots$ & $\cdots$ & $\ldots$ \\
\hline $\mathrm{V}^{*}$ AR Ser & 1.730 & $\cdots$ & $\cdots$ & 1.086 & 1.730 & -39.0 & 3.7 & 3.7 & 3.7 & STN,NPM \\
\hline CD-33 10593 & 0.098 & 0.163 & 0.24 & 0.093 & 0.098 & -48.0 & 58.9 & 1.1 & 1.0 & HIP,SPM, \\
\hline BPS CS 30312-0059 & 3.680 & $\ldots$ & $\ldots$ & 3.500 & 3.680 & $\ldots$ & $\ldots$ & $\ldots$ & $\ldots$ & $\ldots$ \\
\hline $\mathrm{V}^{*} \mathrm{CG} \mathrm{Lib}$ & 1.266 & $<0$ & $\cdots$ & 1.549 & 1.266 & -63.3 & -18.3 & 1.6 & 1.9 & HIP,SPM \\
\hline BPS CS 30312-0067 & $\ldots$ & $\cdots$ & $\cdots$ & 1.700 & 1.700 & $\ldots$ & $\ldots$ & $\cdots$ & $\cdots$ & $\ldots$ \\
\hline BPS CS 22884-0033 & 0.910 & $\cdots$ & $\cdots$ & 1.200 & 0.910 & $\cdots$ & $\cdots$ & $\cdots$ & $\cdots$ & $\cdots$ \\
\hline BPS CS 30312-0044 & 3.381 & $\cdots$ & $\cdots$ & 5.300 & 3.381 & $\cdots$ & $\cdots$ & $\cdots$ & $\cdots$ & $\cdots$ \\
\hline BPS CS 30312-0062 & 2.346 & $\cdots$ & $\ldots$ & 0.900 & 2.346 & -7 & -8 & 4.0 & 4.0 & STN \\
\hline BPS CS 22884-0065 & 0.628 & $\ldots$ & $\ldots$ & 0.700 & 0.628 & $\ldots$ & $\ldots$ & $\ldots$ & $\ldots$ & $\ldots$ \\
\hline HD 140283 & 0.024 & 0.057 & 0.06 & 0.022 & 0.057 & -1115.6 & -302.4 & 0.9 & 0.8 & HIP,ACT \\
\hline BPS CS 30312-0100 & 2.294 & $\cdots$ & $\cdots$ & 2.800 & 2.294 & $\ldots$ & $\ldots$ & $\cdots$ & $\cdots$ & $\ldots$ \\
\hline $\mathrm{V}^{*}$ AR Aps & 4.007 & $\cdots$ & $\cdots$ & $\ldots$ & 4.007 & $\cdots$ & $\cdots$ & $\cdots$ & $\cdots$ & $\ldots$ \\
\hline BPS CS 22884-0097 & 3.648 & $\ldots$ & $\ldots$ & 1.800 & 3.648 & $\ldots$ & $\ldots$ & $\ldots$ & $\cdots$ & $\ldots$ \\
\hline HD 141531 & 1.320 & 0.316 & 0.56 & 1.140 & 1.320 & -10.8 & -54.7 & 0.9 & 1.7 & HIP,ACT, \\
\hline BPS CS 22884-0108 & 1.003 & $\cdots$ & $\cdots$ & 1.000 & 1.003 & $\ldots$ & $\ldots$ & $\cdots$ & $\cdots$ & $\ldots$ \\
\hline $\mathrm{V}^{*}$ V765 Sco & 2.805 & $\cdots$ & $\cdots$ & $\cdots$ & 2.805 & $\cdots$ & $\cdots$ & $\cdots$ & $\cdots$ & $\cdots$ \\
\hline V* VY Lib & 1.366 & $<0$ & $\cdots$ & 1.521 & 1.366 & 1.2 & -53.4 & 2.3 & 2.0 & HIP,STN,I \\
\hline $\mathrm{BD}+053098$ & 1.103 & 0.403 & 0.93 & 1.530 & 1.103 & -44.3 & -51.8 & 2.2 & 2.3 & HIP,ACT, \\
\hline $\mathrm{V}^{*}$ AN Ser & 0.954 & $<0$ & $\cdots$ & 1.107 & 0.954 & -2.0 & -7.4 & 2.4 & 1.9 & HIP,STN,I \\
\hline$V^{*}$ AT Ser & 1.512 & 2.041 & 6.10 & 1.577 & 1.512 & -3.0 & -6.0 & 2.5 & 2.5 & HIP,STN,I \\
\hline HD 141864 & 0.305 & 0.735 & 0.76 & 0.510 & 0.305 & 0.3 & -4.3 & 0.6 & 0.9 & HIP,ACT \\
\hline $\mathrm{V}^{*} \mathrm{VX}$ Aps & 1.777 & $\cdots$ & $\cdots$ & $\cdots$ & 1.777 & $\ldots$ & $\ldots$ & $\cdots$ & $\cdots$ & $\ldots$ \\
\hline HD 142948 & 0.164 & 0.180 & 0.18 & 0.408 & 0.164 & -23.8 & -35.9 & 1.0 & 1.0 & HIP,ACT, \\
\hline $\mathrm{V}^{*}$ AR Her & 1.302 & 0.806 & 1.35 & 1.380 & 1.302 & -61.4 & 16.8 & 1.5 & 1.4 & HIP,ACT, \\
\hline $\mathrm{V}^{*}$ AV Ser & 1.192 & $\ldots$ & $\cdots$ & 1.459 & 1.192 & 8.0 & 6.5 & 5 & 5 & NPM \\
\hline $\mathrm{V}^{*}$ AW Ser & 2.662 & $\ldots$ & $\cdots$ & $\cdots$ & 2.662 & -3.3 & -13.9 & 3.3 & 3.3 & STN,NPM \\
\hline HD 145293 & 0.550 & 0.195 & 0.34 & 0.561 & 0.550 & -44.7 & -57.6 & 0.9 & 1.3 & $\mathrm{HIP}, \mathrm{ACT}$, \\
\hline
\end{tabular}


TABle 1B. (continued)

\begin{tabular}{|c|c|c|c|c|c|c|c|c|c|c|}
\hline $\begin{array}{l}\text { Star } \\
(1)\end{array}$ & $\begin{array}{c}D_{p h o} \\
(\mathrm{kpc}) \\
(2)\end{array}$ & $\begin{array}{c}D_{H I P} \\
(\mathrm{kpc}) \\
(3)\end{array}$ & $\begin{array}{c}\sigma_{\pi_{H I P}} / \pi_{H I P} \\
(4)\end{array}$ & $\begin{array}{c}D_{B S L} \\
(\mathrm{kpc}) \\
(5)\end{array}$ & $\begin{array}{c}D_{\text {adopt }} \\
(\mathrm{kpc}) \\
(6)\end{array}$ & $\begin{array}{c}\mu_{\alpha^{*}} \\
(\mathrm{mas} / \mathrm{yr}) \\
(7)\end{array}$ & $\begin{array}{c}\mu_{\delta} \\
\text { (mas/yr) } \\
(8)\end{array}$ & $\begin{array}{c}\sigma_{\mu_{\alpha^{*}}} \\
(\mathrm{mas} / \mathrm{yr}) \\
(9)\end{array}$ & $\begin{array}{c}\sigma_{\mu_{\delta}} \\
(\mathrm{mas} / \mathrm{yr}) \\
(10)\end{array}$ & \\
\hline BPS CS 22872-0027 & 4.131 & $\cdots$ & $\cdots$ & 1.200 & 4.131 & $\ldots$ & $\ldots$ & $\ldots$ & $\ldots$ & $\cdots$ \\
\hline $\mathrm{V}^{*} \mathrm{RV} \mathrm{CrB}$ & 1.403 & $<0$ & $\ldots$ & 1.259 & 1.403 & -21.9 & -26.5 & 0.8 & 1.5 & HIP, \\
\hline $\mathrm{V}^{*} \mathrm{BS}$ Aps & 2.033 & $\ldots$ & $\cdots$ & $\ldots$ & 2.033 & -6 & -4 & 4.2 & 4.2 & STN \\
\hline $\mathrm{V}^{*} \mathrm{~V} 1023 \mathrm{Oph}$ & 2.198 & $\cdots$ & $\cdots$ & $\ldots$ & 2.198 & -8 & 1. & 4.1 & 4.1 & STN \\
\hline BPS CS 22872-0036 & 1.357 & $\cdots$ & $\ldots$ & 1.300 & 1.357 & $\cdots$ & $\ldots$ & $\cdots$ & $\cdots$ & $\ldots$ \\
\hline BPS CS 22872-0054 & 0.568 & $\ldots$ & $\cdots$ & 0.800 & 0.568 & $\ldots$ & $\cdots$ & $\cdots$ & $\cdots$ & $\cdots$ \\
\hline $\mathrm{V}^{*} \mathrm{~V} 445 \mathrm{Oph}$ & 0.827 & 12.500 & 30.88 & 0.798 & 0.827 & -10.7 & 5.9 & 2.7 & 2.1 & HIP, \\
\hline $\mathrm{V}^{*} \mathrm{~V} 413 \mathrm{Oph}$ & 1.426 & $\ldots$ & $\ldots$ & $\ldots$ & 1.426 & -11.0 & -16.2 & 5 & 5 & NPM \\
\hline BPS CS 22872-0079 & 1.234 & $\cdots$ & $\cdots$ & 1.100 & 1.234 & $\ldots$ & $\ldots$ & $\cdots$ & $\cdots$ & $\cdots$ \\
\hline BPS CS 22872-0102 & 2.185 & $\cdots$ & $\cdots$ & 1.000 & 2.185 & $\cdots$ & $\cdots$ & $\cdots$ & $\cdots$ & $\cdots$ \\
\hline BD+11 2998 & 0.120 & 0.308 & 0.38 & 0.510 & 0.120 & -109.4 & -55.3 & 0.8 & 0.8 & $\mathrm{HIP}, \mathrm{L}$ \\
\hline V* VX Her & 0.990 & 2.128 & 3.87 & 0.982 & 0.990 & -52.1 & 19.9 & 1.0 & 0.8 & HIP, \\
\hline V* V716 Oph & $\ldots$ & $\ldots$ & $\cdots$ & 1.977 & 1.977 & -28 & -13 & 4.0 & 4.0 & STN \\
\hline BPS CS 22878-0017 & $6.246 / 1.209$ & $\cdots$ & $\cdots$ & 1.200 & 6.246 & $\cdots$ & $\cdots$ & $\cdots$ & $\cdots$ & $\cdots$ \\
\hline BPS CS 22878-0013 & 0.587 & $\cdots$ & $\cdots$ & 0.700 & 0.587 & -27 . & -34 . & 7.1 & 7.1 & STN \\
\hline $\mathrm{V}^{*} \mathrm{UV}$ Oct & 0.554 & 0.676 & 0.64 & 0.499 & 0.554 & -58.6 & -120.3 & 0.8 & 0.9 & HIP, \\
\hline $\mathrm{BD}+093223$ & 0.255 & 0.532 & 0.75 & 0.780 & 0.255 & -33.6 & -106.2 & 1.2 & 1.2 & $\mathrm{HIP}, \mathrm{t}$ \\
\hline BPS CS 22878-0003 & 0.951 & $\cdots$ & $\cdots$ & 1.100 & 0.951 & $\ldots$ & $\ldots$ & $\cdots$ & $\cdots$ & $\cdots$ \\
\hline BPS CS 22878-0002 & 1.003 & $\cdots$ & $\cdots$ & 1.200 & 1.003 & $\cdots$ & $\cdots$ & $\cdots$ & $\cdots$ & $\cdots$ \\
\hline $\mathrm{V}^{*} \mathrm{RW}$ Dra & 1.657 & 0.556 & 1.13 & 1.563 & 1.657 & -8.1 & -5.2 & 2.6 & 2.5 & HIP, \\
\hline BPS CS 22878-0027 & 1.107 & $\cdots$ & $\cdots$ & 1.300 & 1.107 & $\cdots$ & $\cdots$ & $\cdots$ & $\cdots$ & $\cdots$ \\
\hline $\mathrm{V}^{*}$ GY Her & 2.558 & $\ldots$ & $\ldots$ & $\ldots$ & 2.558 & 6.7 & 9.7 & 4.4 & 4.4 & STN, \\
\hline BPS CS 22878-0073 & 1.181 & $\cdots$ & $\cdots$ & 1.300 & 1.181 & $\ldots$ & $\cdots$ & $\cdots$ & $\cdots$ & $\cdots$ \\
\hline $\mathrm{V}^{*}$ AF Her & 2.906 & $\cdots$ & $\cdots$ & 2.655 & 2.906 & -16.2 & -7.3 & 5 & 5 & NPM \\
\hline BPS CS 22878-0076 & 1.845 & $\cdots$ & $\cdots$ & 6.300 & 1.845 & $\ldots$ & $\cdots$ & $\cdots$ & $\cdots$ & $\cdots$ \\
\hline $\mathrm{V}^{*}$ AG Her & 2.747 & $\cdots$ & $\cdots$ & 2.766 & 2.747 & -16.4 & -16.2 & 3.2 & 3.2 & STN, \\
\hline $\mathrm{V}^{*}$ CK Aps & 2.458 & $\cdots$ & $\cdots$ & $\cdots$ & 2.458 & $\ldots$ & $\ldots$ & $\cdots$ & $\cdots$ & $\cdots$ \\
\hline BPS CS 22878-0101 & 7.478 & $\cdots$ & $\cdots$ & 6.300 & 7.478 & $\cdots$ & $\cdots$ & $\cdots$ & $\cdots$ & $\cdots$ \\
\hline BPS CS 22878-0121 & 1.413 & $\cdots$ & $\cdots$ & 4.400 & 1.413 & $\cdots$ & $\cdots$ & $\cdots$ & $\cdots$ & $\cdots$ \\
\hline BPS CS 22878-0116 & $5.974 / 1.089$ & $\cdots$ & $\cdots$ & 1.100 & 5.974 & $\cdots$ & $\cdots$ & $\cdots$ & $\cdots$ & $\cdots$ \\
\hline $\mathrm{V}^{*} \mathrm{CW}$ Her & 2.458 & $\cdots$ & $\cdots$ & $\cdots$ & 2.458 & -14.6 & 12.1 & 4.6 & 4.6 & STN, \\
\hline $\mathrm{V}^{*} \mathrm{SW}$ Her & 4.756 & $\ldots$ & $\cdots$ & 4.365 & 4.756 & -11.1 & 0.3 & 5 & 5 & NPM \\
\hline $\mathrm{V}^{*} \mathrm{RW} \operatorname{Tr} \mathrm{A}$ & 1.120 & 0.746 & 2.46 & 1.318 & 1.120 & -6.8 & -4.5 & 1.0 & 1.8 & $\mathrm{HIP}$, \\
\hline $\mathrm{V}^{*} \mathrm{DD}$ Aps & 2.331 & $\ldots$ & $\cdots$ & $\ldots$ & 2.331 & $\cdots$ & $\ldots$ & $\cdots$ & $\cdots$ & $\cdots$ \\
\hline $\mathrm{V}^{*} \mathrm{DI}$ Aps & 2.779 & $\cdots$ & $\cdots$ & $\cdots$ & 2.779 & $\cdots$ & $\cdots$ & $\cdots$ & $\cdots$ & $\cdots$ \\
\hline $\mathrm{V}^{*}$ VZ Her & 1.375 & 0.543 & 0.96 & 1.479 & 1.375 & -19.2 & -16.3 & 1.0 & 1.4 & $\mathrm{HIP}$, \\
\hline $\mathrm{V}^{*} \mathrm{~V} 452 \mathrm{Oph}$ & 1.662 & $\ldots$ & $\cdots$ & $\cdots$ & 1.662 & -3.6 & -1.0 & 5 & 5 & NPM \\
\hline $\mathrm{V}^{*} \mathrm{DL}$ Her & 1.886 & $\cdots$ & $\cdots$ & $\cdots$ & 1.886 & 11.3 & -1.3 & 5 & 5 & NPM \\
\hline V* V394 Her & 2.606 & $\cdots$ & $\cdots$ & $\cdots$ & 2.606 & -6.7 & 6.7 & 5 & 5 & NPM \\
\hline $\mathrm{BD}+173248$ & 0.287 & 0.272 & 0.41 & 0.740 & 0.287 & -46.7 & -24.2 & 0.8 & 0.7 & $\mathrm{HIP}$, \\
\hline
\end{tabular}


TABle 1B. (continued)

\begin{tabular}{|c|c|c|c|c|c|c|c|c|c|c|}
\hline $\begin{array}{l}\text { Star } \\
(1)\end{array}$ & $\begin{array}{c}D_{p h o} \\
(\mathrm{kpc}) \\
(2)\end{array}$ & $\begin{array}{c}D_{H I P} \\
(\mathrm{kpc}) \\
(3)\end{array}$ & $\begin{array}{c}\sigma_{\pi_{H I P}} / \pi_{H I P} \\
(4)\end{array}$ & $\begin{array}{c}D_{B S L} \\
(\mathrm{kpc}) \\
(5)\end{array}$ & $\begin{array}{l}D_{\text {adopt }} \\
(\mathrm{kpc}) \\
(6)\end{array}$ & $\begin{array}{c}\mu_{\alpha^{*}} \\
(\mathrm{mas} / \mathrm{yr}) \\
(7)\end{array}$ & $\begin{array}{c}\mu_{\delta} \\
(\mathrm{mas} / \mathrm{yr}) \\
(8)\end{array}$ & $\begin{array}{c}\sigma_{\mu_{\alpha^{*}}} \\
(\mathrm{mas} / \mathrm{yr}) \\
(9)\end{array}$ & $\begin{array}{c}\sigma_{\mu_{\delta}} \\
(\mathrm{mas} / \mathrm{yr}) \\
(10)\end{array}$ & $\begin{array}{l}\text { Source } \\
\text { (11) }\end{array}$ \\
\hline $\mathrm{V}^{*}$ ST Oph & 1.475 & $\cdots$ & $\cdots$ & 1.514 & 1.475 & -3 & -1 & 3.7 & 3.7 & STN \\
\hline$V^{*}$ V784 Oph & 1.952 & $\ldots$ & $\ldots$ & $\ldots$ & 1.952 & $\ldots$ & $\ldots$ & $\ldots$ & $\ldots$ & $\ldots$ \\
\hline $\mathrm{V}^{*} \mathrm{EL}$ Aps & 2.570 & $\ldots$ & $\ldots$ & $\ldots$ & 2.570 & 4. & -12 & 4.7 & 4.7 & STN \\
\hline$V^{*}$ V494 Sco & 0.659 & $\cdots$ & $\cdots$ & 1.225 & 0.659 & -1 & 0. & 4.8 & 4.8 & STN \\
\hline V* CE Her & $\ldots$ & $\cdots$ & $\cdots$ & 2.800 & 2.800 & $\cdots$ & $\cdots$ & $\cdots$ & $\cdots$ & $\ldots$ \\
\hline $\mathrm{V}^{*} \mathrm{~V} 816 \mathrm{Oph}$ & 1.889 & $\ldots$ & $\ldots$ & $\ldots$ & 1.889 & 4. & -16 & 3.7 & 3.7 & STN \\
\hline HD 160617 & 0.065 & 0.115 & 0.14 & 0.057 & 0.065 & -61.5 & -395.6 & 1.4 & 1.1 & HIP,ACT \\
\hline $\mathrm{V}^{*} \mathrm{~V} 554 \mathrm{Oph}$ & $\ldots$ & $\ldots$ & $\ldots$ & 4.100 & 4.100 & $\ldots$ & $\ldots$ & $\cdots$ & $\cdots$ & $\ldots$ \\
\hline $\mathrm{V}^{*} \mathrm{LU}$ Aps & 2.945 & $\cdots$ & $\cdots$ & $\ldots$ & 2.945 & -16 & -18 & 3.9 & 3.9 & STN \\
\hline $\mathrm{V}^{*}$ ER Aps & 3.112 & $\cdots$ & $\cdots$ & $\cdots$ & 3.112 & $\cdots$ & $\cdots$ & $\cdots$ & $\cdots$ & $\cdots$ \\
\hline PSD 157-558 & $\cdots$ & $\cdots$ & $\cdots$ & 0.100 & 0.100 & $\cdots$ & $\ldots$ & $\cdots$ & $\cdots$ & $\cdots$ \\
\hline V* TX Pav & 2.234 & $\cdots$ & $\ldots$ & $\ldots$ & 2.234 & $\ldots$ & $\ldots$ & $\cdots$ & $\cdots$ & $\cdots$ \\
\hline HD 161770 & 0.093 & 0.094 & 0.14 & 0.429 & 0.093 & -143.1 & -209.3 & 1.7 & 1.0 & HIP,ACT,STN \\
\hline V* TY Pav & 2.386 & $\ldots$ & $\cdots$ & 2.060 & 2.386 & $\ldots$ & $\ldots$ & $\cdots$ & $\cdots$ & $\cdots$ \\
\hline V* CQ Sco & $\cdots$ & $\ldots$ & $\ldots$ & 8.200 & 8.200 & $\cdots$ & $\cdots$ & $\cdots$ & $\cdots$ & $\cdots$ \\
\hline V* V709 Sco & $\ldots$ & $\ldots$ & $\ldots$ & 6.800 & 6.800 & $\cdots$ & $\ldots$ & $\ldots$ & $\ldots$ & $\ldots$ \\
\hline $\mathrm{V}^{*}$ EX Aps & 1.797 & $\ldots$ & $\ldots$ & $\ldots$ & 1.797 & $\cdots$ & $\ldots$ & $\cdots$ & $\cdots$ & $\ldots$ \\
\hline CD-68 1881 & 1.191 & $\cdots$ & $\cdots$ & 1.270 & 1.191 & 21. & -19 & 3.7 & 3.7 & STN \\
\hline $\mathrm{V}^{*} \mathrm{TW}$ Her & 1.190 & 1.667 & 2.73 & 1.355 & 1.190 & 1.9 & -4.1 & 1.2 & 0.8 & HIP,ACT,STN, \\
\hline V* WY Pav & 1.700 & 0.909 & 3.56 & $\cdots$ & 1.700 & -31.4 & -31.6 & 2.8 & 2.2 & HIP,STN \\
\hline V* MR Ara & $\cdots$ & $\cdots$ & $\cdots$ & 4.700 & 4.700 & -5 & -22 & 3.8 & 3.8 & $\mathrm{STN}$ \\
\hline$V^{*}$ V690 Sco & $\ldots$ & $\ldots$ & $\ldots$ & 0.925 & 0.925 & -3 & 4. & 3.9 & 3.9 & STN \\
\hline $\mathrm{V}^{*}$ V446 Sco & $\ldots$ & $\ldots$ & $\ldots$ & 9.600 & 9.600 & $\ldots$ & $\ldots$ & $\cdots$ & $\cdots$ & $\ldots$ \\
\hline $\mathrm{V}^{*} \mathrm{~S}$ Ara & 0.854 & 1.961 & 4.22 & 0.955 & 0.854 & -26.9 & -10.1 & 1.8 & 1.0 & HIP,ACT,STN \\
\hline $\mathrm{V}^{*} \mathrm{KQ} \mathrm{CrA}$ & $\ldots$ & $\ldots$ & $\cdots$ & 9.300 & 9.300 & -5 & -17 & 4.4 & 4.4 & STN \\
\hline $\mathrm{V}^{*} \mathrm{~V} 478 \mathrm{Oph}$ & $\ldots$ & $\ldots$ & $\cdots$ & 5.500 & 5.500 & $\cdots$ & $\ldots$ & $\cdots$ & $\cdots$ & $\ldots$ \\
\hline $\mathrm{V}^{*}$ MS Ara & 1.668 & 0.100 & 0.40 & $\cdots$ & 1.668 & -2.88 & -37.39 & 4.82 & 2.93 & HIP \\
\hline $\mathrm{V}^{*}$ V1438 Sgr & $\cdots$ & $\cdots$ & $\cdots$ & 2.729 & 2.729 & $\ldots$ & $\ldots$ & $\cdots$ & $\cdots$ & $\cdots$ \\
\hline HD 165195 & 0.585 & 0.455 & 0.47 & 0.277 & 0.585 & -27.5 & -82.0 & 0.6 & 0.6 & HIP,ACT,STN \\
\hline $\mathrm{V}^{*} \mathrm{WW} \mathrm{CrA}$ & 1.721 & $\cdots$ & $\cdots$ & $\cdots$ & 1.721 & $\cdots$ & $\cdots$ & $\cdots$ & $\cdots$ & $\cdots$ \\
\hline $\mathrm{V}^{*}$ IN Ara & 1.769 & $\cdots$ & $\cdots$ & $\cdots$ & 1.769 & -20 & -3 & 6.0 & 6.0 & STN \\
\hline HD 166161 & 0.130 & 0.308 & 0.37 & 0.192 & 0.130 & -98.0 & -191.1 & 1.1 & 0.7 & HIP,ACT,STN \\
\hline V* V675 Sgr & 0.793 & 0.233 & 0.41 & 0.794 & 0.793 & 28.4 & -22.4 & 1.6 & 1.1 & HIP,ACT,STN \\
\hline $\mathrm{V}^{*}$ BC Dra & 1.525 & 0.595 & 0.93 & $\cdots$ & 1.525 & -19.2 & 31.8 & 1.2 & 1.0 & HIP,ACT,STN \\
\hline $\mathrm{V}^{*} \mathrm{~V} 455 \mathrm{Oph}$ & 1.763 & 0.265 & 1.11 & $\cdots$ & 1.763 & -31.0 & -26.6 & 2.7 & 2.6 & HIP,STN \\
\hline HD 166913 & 0.057 & 0.062 & 0.06 & 0.051 & 0.062 & -259.1 & -125.7 & 1.0 & 0.8 & HIP,ACT,STN \\
\hline V* BI Tel & 2.254 & $\ldots$ & $\cdots$ & $\ldots$ & 2.254 & $\ldots$ & $\ldots$ & $\cdots$ & $\cdots$ & $\ldots$ \\
\hline$V^{*}$ BD Dra & 2.462 & $\cdots$ & $\cdots$ & $\cdots$ & 2.462 & -28.8 & 0.7 & 5 & 5 & NPM \\
\hline$V^{*}$ V1290 Sgr & $\cdots$ & $\ldots$ & $\cdots$ & 9.700 & 9.700 & 5. & -7 & 6.2 & 6.2 & STN \\
\hline $\mathrm{V}^{*}$ IO Lyr & 1.570 & 1.538 & 3.63 & $\cdots$ & 1.570 & -14.5 & 29.5 & 2.1 & 2.2 & HIP,STN,NPM \\
\hline
\end{tabular}


TABLE 1B. (continued)

\begin{tabular}{|c|c|c|c|c|c|c|c|c|c|c|}
\hline $\begin{array}{l}\text { Star } \\
(1)\end{array}$ & $\begin{array}{c}D_{p h o} \\
(\mathrm{kpc}) \\
(2)\end{array}$ & $\begin{array}{c}D_{H I P} \\
(\mathrm{kpc}) \\
(3)\end{array}$ & $\begin{array}{c}\sigma_{\pi_{H I P}} / \pi_{H I P} \\
(4)\end{array}$ & $\begin{array}{c}D_{B S L} \\
(\mathrm{kpc}) \\
(5)\end{array}$ & $\begin{array}{c}D_{\text {adopt }} \\
(\mathrm{kpc}) \\
(6)\end{array}$ & $\begin{array}{c}\mu_{\alpha^{*}} \\
(\mathrm{mas} / \mathrm{yr}) \\
(7)\end{array}$ & $\begin{array}{c}\mu_{\delta} \\
\text { (mas/yr) } \\
(8)\end{array}$ & $\begin{array}{c}\sigma_{\mu_{\alpha^{*}}} \\
(\mathrm{mas} / \mathrm{yr}) \\
(9)\end{array}$ & $\begin{array}{c}\sigma_{\mu_{\delta}} \\
(\mathrm{mas} / \mathrm{yr}) \\
(10)\end{array}$ & Sou \\
\hline$V^{*}$ V1189 Sgr & $\cdots$ & $\cdots$ & $\cdots$ & 6.000 & 6.000 & $\cdots$ & $\cdots$ & $\cdots$ & $\cdots$ & $\cdots$ \\
\hline $\mathrm{V}^{*} \mathrm{~V} 592 \mathrm{CrA}$ & 1.939 & $\cdots$ & $\cdots$ & $\ldots$ & 1.939 & $\ldots$ & $\ldots$ & $\cdots$ & $\cdots$ & $\ldots$ \\
\hline $\mathrm{V}^{*} \mathrm{AE}$ Dra & 2.466 & $\cdots$ & $\cdots$ & $\ldots$ & 2.466 & -8.3 & -2.4 & 5 & 5 & NPM \\
\hline V* V1303 Sgr & $\cdots$ & $\cdots$ & $\cdots$ & 7.000 & 7.000 & $\ldots$ & $\ldots$ & $\ldots$ & $\ldots$ & $\cdots$ \\
\hline HD 170737 & 0.129 & 0.121 & 0.11 & 0.260 & 0.121 & 91.0 & -47.3 & 0.6 & 0.8 & $\mathrm{HIP}, \mathrm{ACT}$, \\
\hline $\mathrm{V}^{*} \mathrm{AC}$ Her & $\ldots$ & 1.429 & 1.56 & 0.910 & 0.910 & -3.6 & 0.9 & 0.8 & 0.8 & HIP,STN \\
\hline $\mathrm{V}^{*} \mathrm{KX}$ Lyr & 0.985 & $\ldots$ & $\ldots$ & 1.005 & 0.985 & $\ldots$ & $\cdots$ & $\cdots$ & $\cdots$ & $\ldots$ \\
\hline $\mathrm{V}^{*}$ CC Lyr & $\ldots$ & 0.769 & 2.26 & 9.100 & 9.100 & 1.1 & -8.9 & 1.8 & 2.2 & HIP,STN,I \\
\hline $\mathrm{V}^{*} \mathrm{BH} \mathrm{Pav}$ & 2.111 & $\ldots$ & $\ldots$ & $\ldots$ & 2.111 & $\ldots$ & $\ldots$ & $\ldots$ & $\ldots$ & $\ldots$ \\
\hline HD 171496 & 0.396 & 0.180 & 0.24 & 0.240 & 0.396 & -11.7 & 1.7 & 1.5 & 0.8 & $\mathrm{HIP}, \mathrm{ACT}$, \\
\hline $\mathrm{V}^{*} \mathrm{Y}$ Lyr & 3.054 & $\cdots$ & $\cdots$ & 2.754 & 3.054 & -0.8 & -6.6 & 5 & 5 & NPM \\
\hline $\mathrm{V}^{*} \mathrm{LX}$ Lyr & 2.912 & $\cdots$ & $\cdots$ & $\ldots$ & 2.912 & -15 & -23 & 4.2 & 4.2 & STN \\
\hline BPS CS 22959-0035 & $\ldots$ & $\cdots$ & $\cdots$ & 1.100 & 1.100 & $\ldots$ & $\ldots$ & $\cdots$ & $\cdots$ & $\ldots$ \\
\hline BPS CS 22959-0007 & 1.110 & $\cdots$ & $\cdots$ & 1.100 & 1.110 & $\cdots$ & $\cdots$ & $\cdots$ & $\cdots$ & $\ldots$ \\
\hline $\mathrm{V}^{*} \mathrm{CN}$ Lyr & 1.035 & $<0$ & $\ldots$ & $\ldots$ & 1.035 & 0.3 & -7.3 & 1.4 & 1.7 & HIP,STN,I \\
\hline V* V1640 Sgr & 1.970 & $\ldots$ & $\ldots$ & $\cdots$ & 1.970 & -1 & 0. & 4.2 & 4.2 & STN \\
\hline V* RZ Lyr & 1.445 & $\cdots$ & $\cdots$ & 1.465 & 1.445 & 10.2 & 19.9 & 5 & 5 & NPM \\
\hline BPS CS 22959-0072 & 0.838 & $\ldots$ & $\cdots$ & 0.900 & 0.838 & $\ldots$ & $\cdots$ & $\cdots$ & $\cdots$ & $\ldots$ \\
\hline HD 175305 & 0.107 & 0.162 & 0.09 & 0.150 & 0.162 & 319.3 & 80.8 & 0.6 & 0.5 & $\mathrm{HIP}, \mathrm{ACT}$ \\
\hline $\mathrm{V}^{*} \mathrm{EZ} \mathrm{Lyr}$ & 1.379 & 50.000 & 318.50 & 1.028 & 1.379 & -1.59 & 13.10 & 5.92 & 8.23 & HIP \\
\hline $\mathrm{V}^{*} \mathrm{~V} 413 \mathrm{CrA}$ & 0.866 & 1.515 & 3.11 & 1.047 & 0.866 & -31.1 & -24.9 & 1.0 & 1.3 & HIP,ACT, \\
\hline V* AP Her & $\ldots$ & $<0$ & $\ldots$ & 2.200 & 2.200 & -0.9 & -8.9 & 1.1 & 1.4 & HIP,ACT, \\
\hline BPS CS 22959-0090 & 1.264 & $\cdots$ & $\cdots$ & 1.000 & 1.264 & $\cdots$ & $\ldots$ & $\cdots$ & $\cdots$ & $\cdots$ \\
\hline $\mathrm{V}^{*}$ CX Lyr & 2.056 & $\cdots$ & $\cdots$ & $\ldots$ & 2.056 & -4.9 & -13.0 & 5 & 5 & NPM \\
\hline BPS CS 22959-0074 & 4.370 & $\ldots$ & $\cdots$ & 4.300 & 4.370 & $\cdots$ & $\ldots$ & $\cdots$ & $\cdots$ & $\ldots$ \\
\hline HD 174578 & 1.098 & 1.064 & 1.60 & 1.490 & 1.098 & -5.5 & 24.9 & 1.0 & 0.8 & $\mathrm{HIP}, \mathrm{ACT}$, \\
\hline BPS CS 22959-0092 & 0.536 & $\cdots$ & $\cdots$ & 0.800 & 0.536 & $\cdots$ & $\cdots$ & $\cdots$ & $\cdots$ & $\ldots$ \\
\hline BPS CS 22959-0095 & 0.750 & $\cdots$ & $\cdots$ & 0.600 & 0.750 & $\cdots$ & $\cdots$ & $\cdots$ & $\cdots$ & $\cdots$ \\
\hline BPS CS 22959-0097 & 1.615 & $\cdots$ & $\cdots$ & 7.700 & 1.615 & $\cdots$ & $\cdots$ & $\cdots$ & $\cdots$ & $\cdots$ \\
\hline BPS CS 22959-0139 & 1.092 & $\cdots$ & $\cdots$ & 1.200 & 1.092 & $\cdots$ & $\cdots$ & $\cdots$ & $\cdots$ & $\cdots$ \\
\hline HD 175606 & 0.143 & 0.306 & 0.47 & 0.095 & 0.143 & 17.4 & -161.3 & 1.6 & 1.2 & HIP,ACT, \\
\hline HD 176203 & 0.177 & 0.304 & 0.41 & 0.324 & 0.177 & 12.5 & -129.6 & 0.9 & 0.9 & HIP,ACT, \\
\hline $\mathrm{V}^{*} \mathrm{MT}$ Tel & 0.465 & 0.990 & 1.25 & $\cdots$ & 0.465 & -137.2 & -114.7 & 0.8 & 0.7 & HIP,ACT, \\
\hline $\mathrm{V}^{*} \mathrm{NR}$ Lyr & 2.099 & $\ldots$ & $\cdots$ & $\ldots$ & 2.099 & -3 & 26. & 8.3 & 8.3 & STN \\
\hline BPS CS 22947-0049 & 0.863 & $\cdots$ & $\cdots$ & 2.100 & 0.863 & $\cdots$ & $\cdots$ & $\cdots$ & $\cdots$ & $\cdots$ \\
\hline $\mathrm{V}^{*} \mathrm{XZ}$ Dra & 0.722 & 0.500 & 0.49 & 0.809 & 0.722 & 11.0 & 4.8 & 0.8 & 1.1 & HIP,ACT, \\
\hline BPS CS 22959-0192 & 0.477 & $\cdots$ & $\cdots$ & 0.700 & 0.477 & $\cdots$ & $\cdots$ & $\cdots$ & $\cdots$ & $\ldots$ \\
\hline $\mathrm{V}^{*} \mathrm{FN}$ Lyr & 2.389 & $\cdots$ & $\cdots$ & $\cdots$ & 2.389 & $\ldots$ & $\ldots$ & $\cdots$ & $\cdots$ & $\cdots$ \\
\hline HD 178443 & 0.394 & $<0$ & $\cdots$ & 0.435 & 0.394 & -10.9 & -47.9 & 0.9 & 2.3 & HIP,ACT, \\
\hline BPS CS 22947-0101 & 0.574 & $\cdots$ & $\cdots$ & 0.600 & 0.574 & $\cdots$ & $\cdots$ & $\cdots$ & $\cdots$ & $\cdots$ \\
\hline
\end{tabular}


TABLE 1B. (continued)

\begin{tabular}{|c|c|c|c|c|c|c|c|c|c|c|}
\hline $\begin{array}{l}\text { Star } \\
(1)\end{array}$ & $\begin{array}{c}D_{p h o} \\
(\mathrm{kpc}) \\
(2)\end{array}$ & $\begin{array}{c}D_{H I P} \\
(\mathrm{kpc}) \\
(3)\end{array}$ & $\begin{array}{c}\sigma_{\pi_{H I P}} / \pi_{H I P} \\
(4)\end{array}$ & $\begin{array}{c}D_{B S L} \\
(\mathrm{kpc}) \\
(5)\end{array}$ & $\begin{array}{l}D_{\text {adopt }} \\
\text { (kpc) } \\
(6)\end{array}$ & $\begin{array}{c}\mu_{\alpha^{*}} \\
(\operatorname{mas} / \mathrm{yr}) \\
(7)\end{array}$ & $\begin{array}{c}\mu_{\delta} \\
(\mathrm{mas} / \mathrm{yr}) \\
(8)\end{array}$ & $\begin{array}{c}\sigma_{\mu_{\alpha^{*}}} \\
(\mathrm{mas} / \mathrm{yr}) \\
(9)\end{array}$ & $\begin{array}{c}\sigma_{\mu_{\delta}} \\
(\mathrm{mas} / \mathrm{yr}) \\
(10)\end{array}$ & So \\
\hline BPS CS 22959-0225 & 0.871 & $\cdots$ & $\cdots$ & 0.800 & 0.871 & $\ldots$ & $\ldots$ & $\ldots$ & $\ldots$ & $\ldots$ \\
\hline BPS CS 22959-0231 & 1.506 & $\ldots$ & $\ldots$ & 1.200 & 1.506 & $\ldots$ & $\ldots$ & $\ldots$ & $\ldots$ & $\ldots$ \\
\hline BPS CS 22947-0322 & 1.724 & $\ldots$ & $\ldots$ & 6.500 & 1.724 & $\ldots$ & $\ldots$ & $\ldots$ & $\ldots$ & $\ldots$ \\
\hline BPS CS 22891-0089 & 1.394 & $\ldots$ & $\ldots$ & 1.300 & 1.394 & $\ldots$ & $\ldots$ & $\ldots$ & $\ldots$ & $\ldots$ \\
\hline BPS CS 22959-0219 & 0.624 & $\ldots$ & $\ldots$ & 0.900 & 0.624 & $\ldots$ & $\ldots$ & $\ldots$ & $\ldots$ & $\ldots$ \\
\hline $\mathrm{V}^{*}$ BK Dra & 1.303 & 0.763 & 1.04 & $\ldots$ & 1.303 & -15.6 & 28.8 & 0.9 & 1.1 & HIP,ACT \\
\hline BPS CS 22947-0187 & 2.612 & $\ldots$ & $\ldots$ & 1.500 & 2.612 & -1 & -21 & 4.2 & 4.2 & STN \\
\hline BPS CS 22896-0015 & 2.538 & $\ldots$ & $\ldots$ & 1.900 & 2.538 & $\ldots$ & $\ldots$ & $\cdots$ & $\ldots$ & $\ldots$ \\
\hline HD 181007 & 0.396 & 0.373 & 0.56 & 0.613 & 0.396 & -41.9 & -170.2 & 0.9 & 0.9 & HIP,ACT \\
\hline$V^{*}$ V1130 Sgr & 2.225 & $\ldots$ & $\ldots$ & $\ldots$ & 2.225 & 6. & -9 & 5.7 & 5.7 & STN \\
\hline BPS CS 22891-0115 & 7.661 & $\ldots$ & $\ldots$ & 7.600 & 7.661 & $\ldots$ & $\ldots$ & $\ldots$ & $\ldots$ & $\ldots$ \\
\hline BPS CS 22896-0028 & 1.009 & $\ldots$ & $\ldots$ & 1.000 & 1.009 & $\ldots$ & $\ldots$ & $\ldots$ & $\ldots$ & $\ldots$ \\
\hline HD 181743 & 0.092 & 0.088 & 0.16 & 0.089 & 0.092 & -67.9 & -815.2 & 1.0 & 1.4 & HIP,ACT \\
\hline BPS CS 22896-0072 & $\ldots$ & $\ldots$ & $\ldots$ & 1.600 & 1.600 & $\ldots$ & $\ldots$ & $\cdots$ & $\ldots$ & $\ldots$ \\
\hline $\mathrm{V}^{*} \mathrm{RR}$ Lyr & 0.255 & 0.228 & 0.13 & 0.258 & 0.255 & -109.1 & -195.6 & 0.4 & 0.5 & HIP,ACT \\
\hline BPS CS 22896-0044 & 0.821 & $\ldots$ & $\ldots$ & 1.000 & 0.821 & $\ldots$ & $\ldots$ & $\cdots$ & $\ldots$ & $\ldots$ \\
\hline BPS CS 22891-0184 & 4.386 & $\ldots$ & $\ldots$ & 4.100 & 4.386 & $\ldots$ & $\ldots$ & $\ldots$ & $\ldots$ & $\ldots$ \\
\hline BPS CS 22891-0155 & 1.118 & $\ldots$ & $\ldots$ & 1.200 & 1.118 & $\ldots$ & $\ldots$ & $\ldots$ & $\ldots$ & $\ldots$ \\
\hline $\mathrm{V}^{*} \mathrm{BN}$ Vul & 0.736 & 0.170 & 0.36 & 0.669 & 0.736 & -43.2 & -33.7 & 1.2 & 1.2 & HIP,ACT \\
\hline BPS CS 22896-0055 & 3.840 & $\ldots$ & $\cdots$ & 3.600 & 3.840 & $\ldots$ & $\ldots$ & $\cdots$ & $\cdots$ & $\ldots$ \\
\hline BPS CS 22891-0194 & 1.097 & $\ldots$ & $\ldots$ & 1.100 & 1.097 & $\ldots$ & $\ldots$ & $\cdots$ & $\cdots$ & $\ldots$ \\
\hline V* V440 Sgr & 0.784 & $<0$ & $\ldots$ & 0.755 & 0.784 & -3.9 & -54.7 & 1.1 & 0.9 & HIP,SPM \\
\hline $\mathrm{V}^{*} \mathrm{XZ}$ Cyg & 0.573 & 0.439 & 0.38 & 0.646 & 0.573 & 83.6 & -24.7 & 1.0 & 0.8 & HIP,ACT \\
\hline$V^{*}$ V894 Cyg & 2.329 & $\ldots$ & $\ldots$ & $\ldots$ & 2.329 & $\ldots$ & $\ldots$ & $\ldots$ & $\ldots$ & $\ldots$ \\
\hline $\mathrm{V}^{*} \mathrm{HH}$ Tel & 1.892 & $\ldots$ & $\ldots$ & $\ldots$ & 1.892 & -18 & -15 & 4.3 & 4.3 & STN \\
\hline V* WY Dra & 2.413 & $\ldots$ & $\ldots$ & $\ldots$ & 2.413 & -11.1 & 8.8 & 5 & 5 & NPM \\
\hline BPS CS 22896-0089 & 5.287 & $\ldots$ & $\ldots$ & 5.100 & 5.287 & $\ldots$ & $\ldots$ & $\cdots$ & $\ldots$ & $\ldots$ \\
\hline HD 184266 & 0.214 & 0.305 & 0.29 & 0.257 & 0.214 & 130.4 & -197.3 & 0.8 & 0.5 & HIP,ACT \\
\hline BPS CS 22896-0115 & 1.101 & $\ldots$ & $\ldots$ & 1.100 & 1.101 & $\ldots$ & $\ldots$ & $\cdots$ & $\ldots$ & $\ldots$ \\
\hline BPS CS 22891-0221 & 2.229 & $\cdots$ & $\cdots$ & 1.800 & 2.229 & $\cdots$ & $\cdots$ & $\cdots$ & $\cdots$ & $\cdots$ \\
\hline BPS CS 22891-0200 & 9.941 & $\ldots$ & $\ldots$ & 7.800 & 9.941 & $\ldots$ & $\ldots$ & $\ldots$ & $\ldots$ & $\ldots$ \\
\hline $\mathrm{V}^{*} \mathrm{XZ}$ Oct & 3.110 & $\ldots$ & $\ldots$ & $\ldots$ & 3.110 & $\ldots$ & $\ldots$ & $\ldots$ & $\ldots$ & $\ldots$ \\
\hline BPS CS 22896-0110 & 1.265 & $\ldots$ & $\ldots$ & 3.700 & 1.265 & $\ldots$ & $\ldots$ & $\ldots$ & $\ldots$ & $\ldots$ \\
\hline HD 184711 & 0.876 & 0.317 & 0.37 & 1.126 & 0.876 & 3.0 & -50.9 & 0.8 & 0.8 & HIP,SPM \\
\hline BPS CS 22891-0206 & 1.209 & $\ldots$ & $\ldots$ & 1.400 & 1.209 & $\ldots$ & $\ldots$ & $\ldots$ & $\ldots$ & $\ldots$ \\
\hline $\mathrm{V}^{*} \mathrm{BN}$ Pav & 2.204 & 1.471 & 7.35 & $\ldots$ & 2.204 & 0.80 & -4.50 & 4.49 & 4.22 & HIP \\
\hline HD 232078 & 0.833 & 0.621 & 0.69 & 0.600 & 0.833 & 12.3 & -32.1 & 0.6 & 0.6 & HIP,ACT \\
\hline BPS CS 22896-0152 & 0.776 & $\ldots$ & $\ldots$ & 4.000 & 0.776 & $\ldots$ & $\ldots$ & $\cdots$ & $\ldots$ & $\ldots$ \\
\hline BPS CS 22896-0136 & 1.308 & $\ldots$ & $\ldots$ & 1.400 & 1.308 & $\ldots$ & $\ldots$ & $\ldots$ & $\ldots$ & $\ldots$ \\
\hline BPS CS 22891-0209 & 3.928 & $\cdots$ & $\cdots$ & 3.200 & 3.928 & $\cdots$ & $\ldots$ & $\cdots$ & $\ldots$ & $\ldots$ \\
\hline
\end{tabular}


TABLE 1B. (continued)

\begin{tabular}{|c|c|c|c|c|c|c|c|c|c|c|}
\hline $\begin{array}{l}\text { Star } \\
(1)\end{array}$ & $\begin{array}{c}D_{p h o} \\
(\mathrm{kpc}) \\
(2)\end{array}$ & $\begin{array}{c}D_{H I P} \\
(\mathrm{kpc}) \\
(3)\end{array}$ & $\begin{array}{c}\sigma_{\pi_{H I P}} / \pi_{H I P} \\
(4)\end{array}$ & $\begin{array}{c}D_{B S L} \\
(\mathrm{kpc}) \\
(5)\end{array}$ & $\begin{array}{l}D_{\text {adopt }} \\
\text { (kpc) } \\
(6)\end{array}$ & $\begin{array}{c}\mu_{\alpha^{*}} \\
(\mathrm{mas} / \mathrm{yr}) \\
(7)\end{array}$ & $\begin{array}{c}\mu_{\delta} \\
(\mathrm{mas} / \mathrm{yr}) \\
(8)\end{array}$ & $\begin{array}{c}\sigma_{\mu_{\alpha^{*}}} \\
(\mathrm{mas} / \mathrm{yr}) \\
(9)\end{array}$ & $\begin{array}{c}\sigma_{\mu_{\delta}} \\
(\mathrm{mas} / \mathrm{yr}) \\
(10)\end{array}$ & Sou \\
\hline BPS CS 22896-0150 & 1.148 & $\ldots$ & $\ldots$ & 0.400 & 1.148 & $\ldots$ & $\ldots$ & $\ldots$ & $\ldots$ & $\ldots$ \\
\hline BPS CS 22896-0154 & 3.060 & $\ldots$ & $\ldots$ & 1.800 & 3.060 & $\ldots$ & $\ldots$ & $\ldots$ & $\ldots$ & $\ldots$ \\
\hline HD 186478 & 1.017 & 0.746 & 0.93 & 0.742 & 1.017 & -20.9 & -84.4 & 1.0 & 0.7 & HIP,ACT, \\
\hline BPS CS 22873-0015 & 0.849 & $\ldots$ & $\ldots$ & 4.800 & 0.849 & $\ldots$ & $\ldots$ & $\ldots$ & $\ldots$ & $\ldots$ \\
\hline $\mathrm{V}^{*} \mathrm{~V} 796 \mathrm{Sgr}$ & 1.903 & $\ldots$ & $\ldots$ & $\ldots$ & 1.903 & 15.1 & -36.8 & 1.7 & 1.7 & SPM,STN \\
\hline BPS CS 22964-0024 & 0.864 & $\ldots$ & $\ldots$ & 0.800 & 0.864 & 10.2 & -30.6 & 2.9 & 2.8 & SPM \\
\hline BPS CS 22964-0019 & 6.029 & $\ldots$ & $\ldots$ & 4.400 & 6.029 & -5.7 & -2.3 & 2.3 & 2.5 & SPM \\
\hline $\mathrm{V}^{*}$ V2194 Sgr & 1.639 & $\ldots$ & $\ldots$ & 1.100 & 1.639 & -15.0 & -17.3 & 4.6 & 5.1 & SPM \\
\hline HD 187111 & 0.583 & 0.503 & 0.55 & 0.680 & 0.583 & 37.0 & -58.4 & 1.0 & 0.5 & $\mathrm{HIP}, \mathrm{ACT}$, \\
\hline BPS CS 22964-0074 & 1.240 & $\ldots$ & $\ldots$ & 1.100 & 1.240 & 29.7 & -66.4 & 3.7 & 4.0 & SPM \\
\hline BPS CS 22873-0072 & 1.305 & $\ldots$ & $\ldots$ & 1.500 & 1.305 & $\ldots$ & $\ldots$ & $\ldots$ & $\ldots$ & $\ldots$ \\
\hline BPS CS 22964-0092 & $\ldots$ & $\ldots$ & $\ldots$ & 1.500 & 1.500 & 11.9 & -5.4 & 2.1 & 2.8 & SPM \\
\hline $\mathrm{V}^{*} \mathrm{DN} \mathrm{Pav}$ & 2.340 & $\ldots$ & $\ldots$ & 2.323 & 2.340 & $\ldots$ & $\ldots$ & $\ldots$ & $\ldots$ & $\ldots$ \\
\hline BPS CS 22964-0052 & 1.151 & $\ldots$ & $\ldots$ & 1.100 & 1.151 & 6.1 & -2.6 & 2.1 & 2.1 & SPM \\
\hline BPS CS 22964-0064 & 0.685 & $\ldots$ & $\ldots$ & 1.000 & 0.685 & -3.1 & -3.4 & 2.5 & 4.1 & SPM \\
\hline V* V2202 Sgr & 6.575 & $\ldots$ & $\ldots$ & 6.500 & 6.575 & 2.4 & -3.3 & 2.0 & 2.0 & SPM \\
\hline BPS CS 22873-0055 & 6.514 & $\ldots$ & $\ldots$ & 5.200 & 6.514 & $\ldots$ & $\ldots$ & $\ldots$ & $\ldots$ & $\ldots$ \\
\hline BPS CS 22964-0138 & 0.952 & $\ldots$ & $\ldots$ & 1.000 & 0.952 & 7.8 & -24.3 & 3.5 & 2.7 & SPM \\
\hline HD 188031 & 0.163 & 0.209 & 0.44 & 0.122 & 0.163 & 3.0 & -435.9 & 2.3 & 1.9 & HIP,SPM \\
\hline BPS CS 22964-0111 & 1.211 & $\ldots$ & $\ldots$ & 1.200 & 1.211 & 3.1 & -6.4 & 2.1 & 3.4 & SPM \\
\hline BPS CS 22964-0149 & 1.364 & $\ldots$ & $\ldots$ & 1.900 & 1.364 & -5.9 & -7.4 & 5.3 & 4.0 & SPM \\
\hline $\mathrm{V}^{*}$ GK Pav & 4.719 & $\ldots$ & $\ldots$ & 4.600 & 4.719 & $\ldots$ & $\ldots$ & $\ldots$ & $\ldots$ & $\ldots$ \\
\hline BPS CS 22964-0115 & 1.370 & $\ldots$ & $\ldots$ & 1.500 & 1.370 & -32.3 & -55.0 & 3.1 & 3.1 & SPM \\
\hline $\mathrm{V}^{*} \mathrm{BP}$ Pav & 2.243 & $<0$ & $\ldots$ & $\ldots$ & 2.243 & -25.48 & -30.76 & 3.19 & 3.23 & HIP \\
\hline BD-18 5550 & 0.441 & 0.526 & 0.76 & 1.391 & 0.441 & 11.4 & -91.4 & 1.4 & 0.8 & $\mathrm{HIP}, \mathrm{ACT}$, \\
\hline BPS CS 22873-0106 & 1.430 & $\ldots$ & $\ldots$ & 5.900 & 1.430 & $\ldots$ & $\ldots$ & $\ldots$ & $\ldots$ & $\ldots$ \\
\hline BPS CS 22964-0172 & $\ldots$ & $\ldots$ & $\ldots$ & 1.100 & 1.100 & -0.6 & -11.7 & 2.7 & 2.7 & SPM \\
\hline $\mathrm{V}^{*}$ V1711 Sgr & $\ldots$ & 0.427 & 1.02 & 5.200 & 5.200 & -6.7 & -2.5 & 1.2 & 0.8 & HIP,SPM, \\
\hline BPS CS 22964-0183 & 1.141 & $\ldots$ & $\ldots$ & 1.300 & 1.141 & -1.1 & -45.6 & 2.9 & 2.9 & SPM \\
\hline BPS CS 22964-0157 & 1.408 & $\cdots$ & $\cdots$ & 1.200 & 1.408 & -22.0 & -66.4 & 2.7 & 2.8 & SPM \\
\hline BPS CS 22964-0181 & 3.193 & $\ldots$ & $\ldots$ & 7.400 & 3.193 & -12.0 & -5.2 & 3.0 & 3.0 & SPM \\
\hline BPS CS 22964-0176 & 1.666 & $\ldots$ & $\ldots$ & 1.900 & 1.666 & -16.1 & -27.5 & 3.5 & 3.5 & SPM \\
\hline BPS CS 22964-0158 & 1.079 & $\ldots$ & $\ldots$ & 1.300 & 1.079 & 3.6 & -14.4 & 3.0 & 3.1 & SPM \\
\hline BPS CS 22964-0161 & 0.962 & $\ldots$ & $\ldots$ & 1.200 & 0.962 & -25.2 & -44.5 & 2.7 & 2.7 & SPM \\
\hline HD 190287 & 0.178 & 0.140 & 0.21 & 0.226 & 0.178 & -35.9 & -166.8 & 1.4 & 0.8 & $\mathrm{HIP}, \mathrm{ACT}$, \\
\hline BPS CS 22964-0214 & 0.818 & $\ldots$ & $\ldots$ & 0.900 & 0.818 & 12.0 & -96.1 & 6.6 & 6.0 & SPM \\
\hline BPS CS 22873-0139 & 0.970 & $\ldots$ & $\ldots$ & 1.000 & 0.970 & $\ldots$ & $\ldots$ & $\ldots$ & $\ldots$ & $\ldots$ \\
\hline BPS CS 22873-0128 & 3.557 & $\ldots$ & $\ldots$ & 2.500 & 3.557 & $\ldots$ & $\ldots$ & $\ldots$ & $\ldots$ & $\ldots$ \\
\hline BPS CS 22964-0232 & 0.660 & $\cdots$ & $\cdots$ & 1.000 & 0.660 & 9.5 & -2.0 & 2.9 & 2.9 & SPM \\
\hline $\mathrm{V}^{*} \mathrm{HY}$ Tel & 2.056 & $\cdots$ & $\cdots$ & $\ldots$ & 2.056 & 3. & -9 & 6.1 & 6.1 & $\mathrm{STN}$ \\
\hline
\end{tabular}


TABLE 1B. (continued)

\begin{tabular}{|c|c|c|c|c|c|c|c|c|c|c|}
\hline $\begin{array}{l}\text { Star } \\
(1)\end{array}$ & $\begin{array}{c}D_{p h o} \\
(\mathrm{kpc}) \\
(2)\end{array}$ & $\begin{array}{c}D_{H I P} \\
(\mathrm{kpc}) \\
(3)\end{array}$ & $\begin{array}{c}\sigma_{\pi_{H I P}} / \pi_{H I P} \\
(4)\end{array}$ & $\begin{array}{c}D_{B S L} \\
(\mathrm{kpc}) \\
(5)\end{array}$ & $\begin{array}{c}D_{\text {adopt }} \\
(\mathrm{kpc}) \\
(6)\end{array}$ & $\begin{array}{c}\mu_{\alpha^{*}} \\
(\mathrm{mas} / \mathrm{yr}) \\
(7)\end{array}$ & $\begin{array}{c}\mu_{\delta} \\
(\mathrm{mas} / \mathrm{yr}) \\
(8)\end{array}$ & $\begin{array}{c}\sigma_{\mu_{\alpha *}} \\
(\mathrm{mas} / \mathrm{yr}) \\
(9)\end{array}$ & $\begin{array}{c}\sigma_{\mu_{\delta}} \\
(\mathrm{mas} / \mathrm{yr}) \\
(10)\end{array}$ & \\
\hline BPS CS 22964-0231 & $5.306 / 0.956$ & $\cdots$ & $\cdots$ & 5.900 & 5.306 & $\cdots$ & $\cdots$ & $\cdots$ & $\cdots$ & $\cdots$ \\
\hline BPS CS 22873-0137 & $3.434 / 0.773$ & $\ldots$ & $\ldots$ & 0.600 & 3.434 & $\ldots$ & $\ldots$ & $\ldots$ & $\ldots$ & $\ldots$ \\
\hline V* V1644 Sgr & 1.952 & $\ldots$ & $\ldots$ & $\ldots$ & 1.952 & -7.1 & -33.4 & 3.7 & 2.5 & SPM \\
\hline $\mathrm{V}^{*} \mathrm{TW}$ Cap & $\ldots$ & $<0$ & $\cdots$ & 4.800 & 4.800 & -3.3 & -12.2 & 1.6 & 0.9 & HIP, \\
\hline BPS CS 22943-0001 & 0.852 & $\cdots$ & $\cdots$ & 1.200 & 0.852 & $\cdots$ & $\ldots$ & $\cdots$ & $\cdots$ & $\cdots$ \\
\hline BPS CS 22943-0069 & 1.367 & $\ldots$ & $\ldots$ & 1.400 & 1.367 & $\ldots$ & $\ldots$ & $\cdots$ & $\ldots$ & $\ldots$ \\
\hline HD 192718 & 0.040 & 0.058 & 0.07 & 0.037 & 0.058 & 311.4 & -129.5 & 0.7 & 0.8 & HIP, \\
\hline BPS CS 22943-0053 & 0.995 & $\ldots$ & $\ldots$ & 1.400 & 0.995 & $\ldots$ & $\ldots$ & $\cdots$ & $\cdots$ & $\cdots$ \\
\hline BPS CS 22943-0078 & 0.811 & $\cdots$ & $\cdots$ & 1.100 & 0.811 & $\ldots$ & $\ldots$ & $\cdots$ & $\cdots$ & $\cdots$ \\
\hline BPS CS 22885-0040 & 1.706 & $\cdots$ & $\cdots$ & 1.900 & 1.706 & -25.7 & -12.1 & 3.1 & 2.6 & SPM \\
\hline BPS CS 22943-0067 & 0.866 & $\ldots$ & $\ldots$ & 1.000 & 0.866 & $\ldots$ & $\ldots$ & $\ldots$ & $\ldots$ & $\ldots$ \\
\hline BPS CS 22943-0059 & 1.345 & $\cdots$ & $\cdots$ & 1.400 & 1.345 & $\cdots$ & $\cdots$ & $\cdots$ & $\cdots$ & $\cdots$ \\
\hline $\mathrm{V}^{*} \mathrm{HV}$ Pav & 2.976 & $\cdots$ & $\cdots$ & $\cdots$ & 2.976 & -7 & -26 & 4.7 & 4.7 & STN \\
\hline BPS CS 22885-0054 & 1.243 & $\cdots$ & $\cdots$ & 1.400 & 1.243 & -18.8 & -24.3 & 2.1 & 2.8 & SPM \\
\hline BPS CS 22943-0087 & $\ldots$ & $\ldots$ & $\ldots$ & 1.500 & 1.500 & $\ldots$ & $\ldots$ & $\ldots$ & $\ldots$ & $\ldots$ \\
\hline BPS CS 22873-0166 & 5.263 & $\ldots$ & $\ldots$ & 4.100 & 5.263 & $\ldots$ & $\ldots$ & $\ldots$ & $\ldots$ & $\ldots$ \\
\hline BPS CS 22885-0069 & 3.772 & $\ldots$ & $\ldots$ & 5.300 & 3.772 & -2.6 & -9.5 & 2.5 & 1.9 & SPM \\
\hline BPS CS 22885-0067 & 1.601 & $\cdots$ & $\cdots$ & 1.400 & 1.601 & $\cdots$ & $\cdots$ & $\cdots$ & $\cdots$ & $\cdots$ \\
\hline BPS CS 22950-0050 & 3.501 & $\cdots$ & $\cdots$ & 2.500 & 3.501 & $\ldots$ & $\ldots$ & $\cdots$ & $\cdots$ & $\cdots$ \\
\hline HD 193242 & 0.314 & 0.459 & 0.62 & 0.529 & 0.314 & -21.0 & -97.7 & 1.4 & 1.1 & HIP, \\
\hline BPS CS 22885-0105 & 4.525 & $\cdots$ & $\cdots$ & 4.000 & 4.525 & 1.1 & -11.7 & 2.3 & 2.3 & SPM \\
\hline V* V1645 Sgr & 1.771 & $\ldots$ & $\ldots$ & $\ldots$ & 1.771 & 29.4 & -42.3 & 1.5 & 1.3 & SPM \\
\hline BPS CS 22885-0096 & 3.971 & $\cdots$ & $\cdots$ & 3.118 & 3.971 & -0.7 & -9.1 & 3.1 & 2.4 & SPM \\
\hline BPS CS 22885-0094 & 0.551 & $\cdots$ & $\cdots$ & 0.700 & 0.551 & -17.2 & -28.3 & 2.8 & 3.6 & SPM \\
\hline V* BX Del & $\ldots$ & $\cdots$ & $\cdots$ & 1.355 & 1.355 & $\ldots$ & $\ldots$ & $\cdots$ & $\cdots$ & $\cdots$ \\
\hline BPS CS 22950-0046 & 13.221 & $\cdots$ & $\cdots$ & 8.900 & 13.221 & $\cdots$ & $\cdots$ & $\ldots$ & $\ldots$ & $\ldots$ \\
\hline BPS CS 22950-0063 & 6.885 & $\cdots$ & $\cdots$ & 6.200 & 6.885 & $\cdots$ & $\cdots$ & $\cdots$ & $\cdots$ & $\cdots$ \\
\hline BPS CS 22943-0107 & 1.267 & $\cdots$ & $\cdots$ & 0.800 & 1.267 & $\cdots$ & $\cdots$ & $\cdots$ & $\cdots$ & $\cdots$ \\
\hline BPS CS 22955-0038 & 0.836 & $\cdots$ & $\cdots$ & 0.900 & 0.836 & $\cdots$ & $\cdots$ & $\cdots$ & $\cdots$ & $\cdots$ \\
\hline BPS CS 22943-0132 & $\cdots$ & $\cdots$ & $\cdots$ & 1.200 & 1.200 & $\cdots$ & $\cdots$ & $\cdots$ & $\cdots$ & $\cdots$ \\
\hline BPS CS 22943-0109 & 6.273 & $\ldots$ & $\ldots$ & 9.900 & 6.273 & $\ldots$ & $\ldots$ & $\ldots$ & $\ldots$ & $\ldots$ \\
\hline $\mathrm{V}^{*} \mathrm{~V} 1646 \mathrm{Sgr}$ & 1.762 & $\cdots$ & $\ldots$ & $\ldots$ & 1.762 & $\cdots$ & $\cdots$ & $\ldots$ & $\ldots$ & $\ldots$ \\
\hline BPS CS 22943-0110 & 1.045 & $\cdots$ & $\cdots$ & 1.100 & 1.045 & $\cdots$ & $\cdots$ & $\cdots$ & $\cdots$ & $\cdots$ \\
\hline BPS CS 22943-0134 & 2.021 & $\ldots$ & $\ldots$ & 1.500 & 2.021 & $\ldots$ & $\ldots$ & $\ldots$ & $\ldots$ & $\ldots$ \\
\hline BPS CS 22885-0106 & 1.390 & $\ldots$ & $\ldots$ & 1.600 & 1.390 & -2.1 & -38.2 & 2.1 & 2.1 & SPM \\
\hline BPS CS 22943-0092 & 0.674 & $\cdots$ & $\cdots$ & 0.800 & 0.674 & $\cdots$ & $\ldots$ & $\ldots$ & $\cdots$ & $\ldots$ \\
\hline BPS CS 22955-0032 & 0.988 & $\cdots$ & $\cdots$ & 0.900 & 0.988 & $\cdots$ & $\cdots$ & $\cdots$ & $\cdots$ & $\cdots$ \\
\hline BPS CS 22950-0078 & 1.239 & $\cdots$ & $\cdots$ & 1.200 & 1.239 & $\cdots$ & $\cdots$ & $\cdots$ & $\cdots$ & $\cdots$ \\
\hline BPS CS 22950-0102 & 0.747 & $\cdots$ & $\cdots$ & 0.800 & 0.747 & $\cdots$ & $\cdots$ & $\ldots$ & $\ldots$ & $\cdots$ \\
\hline $\mathrm{V}^{*}$ CK Del & 1.773 & $\cdots$ & $\cdots$ & $\ldots$ & 1.773 & $\ldots$ & $\cdots$ & $\cdots$ & $\cdots$ & $\cdots$ \\
\hline
\end{tabular}


TABle 1B. (continued)

\begin{tabular}{|c|c|c|c|c|c|c|c|c|c|c|}
\hline $\begin{array}{l}\text { Star } \\
(1)\end{array}$ & $\begin{array}{c}D_{p h o} \\
(\mathrm{kpc}) \\
(2)\end{array}$ & $\begin{array}{c}D_{H I P} \\
(\mathrm{kpc}) \\
(3)\end{array}$ & $\begin{array}{c}\sigma_{\pi_{H I P}} / \pi_{H I P} \\
(4)\end{array}$ & $\begin{array}{c}D_{B S L} \\
(\mathrm{kpc}) \\
(5)\end{array}$ & $\begin{array}{c}D_{\text {adopt }} \\
\text { (kpc) } \\
(6)\end{array}$ & $\begin{array}{c}\mu_{\alpha^{*}} \\
(\mathrm{mas} / \mathrm{yr}) \\
(7)\end{array}$ & $\begin{array}{c}\mu_{\delta} \\
(\mathrm{mas} / \mathrm{yr}) \\
(8)\end{array}$ & $\begin{array}{c}\sigma_{\mu_{\alpha^{*}}} \\
(\mathrm{mas} / \mathrm{yr}) \\
(9)\end{array}$ & $\begin{array}{c}\sigma_{\mu_{\delta}} \\
(\mathrm{mas} / \mathrm{yr}) \\
(10)\end{array}$ & \\
\hline BPS CS 22943-0097 & 0.782 & $\ldots$ & $\ldots$ & 0.700 & 0.782 & 8. & -18 & 5.2 & 5.2 & STN \\
\hline BPS CS 22943-0137 & $\ldots$ & $\ldots$ & $\ldots$ & 2.000 & 2.000 & $\ldots$ & $\ldots$ & $\ldots$ & $\ldots$ & $\ldots$ \\
\hline $\mathrm{V}^{*} \mathrm{~V} 2279 \mathrm{Sgr}$ & 2.329 & $\ldots$ & $\ldots$ & $\ldots$ & 2.329 & -3 & -4 & 5.9 & 5.9 & STN \\
\hline BPS CS 22943-0095 & 0.347 & $\ldots$ & $\ldots$ & 0.400 & 0.347 & 42. & -63 & 3.7 & 3.7 & STN \\
\hline BPS CS 22885-0143 & 7.050 & $\ldots$ & $\ldots$ & 6.500 & 7.050 & -3.2 & -6.8 & 3.5 & 4.6 & SPM \\
\hline BPS CS 22950-0096 & 0.749 & $\ldots$ & $\ldots$ & 0.800 & 0.749 & -4 & -24 & 5.0 & 5.0 & STN \\
\hline BPS CS 22943-0164 & 1.182 & $\ldots$ & $\ldots$ & 1.200 & 1.182 & $\ldots$ & $\ldots$ & $\ldots$ & $\ldots$ & $\ldots$ \\
\hline BPS CS 22955-0047 & 4.936 & $\ldots$ & $\ldots$ & 5.000 & 4.936 & $\ldots$ & $\ldots$ & $\ldots$ & $\ldots$ & $\ldots$ \\
\hline BPS CS 22885-0203 & 1.058 & $\ldots$ & $\ldots$ & 1.100 & 1.058 & 18.0 & -55.3 & 1.9 & 2.1 & SPM \\
\hline BPS CS 22955-0117 & 1.041 & $\cdots$ & $\cdots$ & 1.100 & 1.041 & -21.0 & -45.9 & 3.0 & 3.0 & SPM \\
\hline $\mathrm{V}^{*} \mathrm{SV}$ Mic & $\ldots$ & $\ldots$ & $\ldots$ & 1.000 & 1.000 & $\ldots$ & $\ldots$ & $\ldots$ & $\ldots$ & $\cdots$ \\
\hline BPS CS 22950-0147 & 1.100 & $\ldots$ & $\ldots$ & 1.100 & 1.100 & $\ldots$ & $\ldots$ & $\ldots$ & $\ldots$ & $\ldots$ \\
\hline BPS CS 22955-0101 & 0.668 & $\ldots$ & $\ldots$ & 0.900 & 0.668 & -13.2 & -51.8 & 2.6 & 2.5 & SPM \\
\hline BPS CS 22955-0121 & 1.209 & $\ldots$ & $\ldots$ & 0.800 & 1.209 & $\ldots$ & $\ldots$ & $\ldots$ & $\ldots$ & $\ldots$ \\
\hline CD-23 16310 & 1.461 & $<0$ & $\ldots$ & 2.240 & 1.461 & 14.9 & -20.2 & 1.1 & 0.8 & $\mathrm{HIP}, \mathrm{S}$ \\
\hline BPS CS 22955-0107 & 0.929 & $\ldots$ & $\ldots$ & 0.900 & 0.929 & $\ldots$ & $\ldots$ & $\ldots$ & $\ldots$ & $\ldots$ \\
\hline BPS CS 22885-0175 & 1.716 & $\ldots$ & $\ldots$ & 3.700 & 1.716 & 5.5 & -3.7 & 2.5 & 2.5 & SPM \\
\hline $\mathrm{V}^{*} \mathrm{~V} 341 \mathrm{Aql}$ & 0.967 & 0.129 & 0.75 & 1.052 & 0.967 & 24.5 & -27.8 & 2.4 & 1.3 & HIP,$A$ \\
\hline BPS CS 22950-0153 & 1.203 & $\ldots$ & $\ldots$ & 0.800 & 1.203 & 27. & -21 & 5.2 & 5.2 & STN \\
\hline HD 195636 & 0.373 & 1.351 & 2.39 & 0.800 & 0.373 & -64.9 & -91.4 & 1.4 & 1.2 & HIP,$A$ \\
\hline BPS CS 22943-0192 & 1.324 & $\ldots$ & $\ldots$ & 0.900 & 1.324 & $\ldots$ & $\ldots$ & $\ldots$ & $\ldots$ & $\ldots$ \\
\hline BPS CS 22950-0173 & 1.005 & $\ldots$ & $\ldots$ & 0.900 & 1.005 & $\ldots$ & $\ldots$ & $\ldots$ & $\ldots$ & $\ldots$ \\
\hline BPS CS 22955-0153 & 1.084 & $\ldots$ & $\ldots$ & 1.000 & 1.084 & 6.9 & -47.0 & 2.1 & 2.2 & SPM \\
\hline BPS CS 22943-0201 & 1.870 & $\ldots$ & $\ldots$ & 1.600 & 1.870 & $\ldots$ & $\ldots$ & $\ldots$ & $\ldots$ & $\ldots$ \\
\hline BPS CS 22879-0012 & 1.515 & $\ldots$ & $\ldots$ & 1.500 & 1.515 & 22.3 & -16.0 & 1.6 & 3.8 & SPM \\
\hline BD-17 6036 & 1.676 & 0.606 & 1.62 & 1.680 & 1.676 & -30.9 & -39.8 & 1.9 & 1.7 & HIP,$A$ \\
\hline BPS CS 22955-0134 & 0.733 & $\ldots$ & $\ldots$ & 0.800 & 0.733 & 6.5 & -11.3 & 4.3 & 4.3 & SPM, \\
\hline BPS CS 22880-0013 & 0.906 & $\ldots$ & $\ldots$ & 0.700 & 0.906 & $\ldots$ & $\ldots$ & $\ldots$ & $\ldots$ & $\ldots$ \\
\hline $\mathrm{V}^{*} \mathrm{AA}$ Aql & 1.380 & $\ldots$ & $\ldots$ & 1.607 & 1.380 & -18.0 & -15.4 & 5 & 5 & NPM \\
\hline BPS CS 22879-0029 & 1.114 & $\cdots$ & $\cdots$ & 1.300 & 1.114 & 21.6 & -44.7 & 1.6 & 2.3 & SPM \\
\hline BPS CS 22879-0034 & 1.724 & $\ldots$ & $\ldots$ & 5.200 & 1.724 & 5.6 & -6.5 & 2.6 & 2.1 & SPM \\
\hline BPS CS 22940-0050 & 1.136 & $\ldots$ & $\ldots$ & 1.400 & 1.136 & $\ldots$ & $\ldots$ & $\ldots$ & $\ldots$ & $\ldots$ \\
\hline $\mathrm{V}^{*} \mathrm{FH} \mathrm{Vul}$ & 2.380 & $\ldots$ & $\ldots$ & $\ldots$ & 2.380 & -3 . & -9. & 5.8 & 5.8 & STN \\
\hline BPS CS 22955-0169 & $\ldots$ & $\ldots$ & $\ldots$ & 1.200 & 1.200 & -0.4 & 11.1 & 2.0 & 2.0 & SPM \\
\hline HD 196892 & 0.056 & 0.063 & 0.08 & 0.048 & 0.063 & 44.0 & -428.0 & 1.1 & 0.9 & HIP,$A$ \\
\hline BPS CS 22940-0078 & $3.573 / 0.627$ & $\ldots$ & $\ldots$ & 0.700 & 3.573 & $\ldots$ & $\ldots$ & $\ldots$ & $\ldots$ & $\ldots$ \\
\hline BPS CS 22940-0077 & 1.613 & $\ldots$ & $\ldots$ & 4.700 & 1.613 & $\ldots$ & $\ldots$ & $\ldots$ & $\ldots$ & $\ldots$ \\
\hline BPS CS 22955-0165 & 1.255 & $\ldots$ & $\ldots$ & 1.300 & 1.255 & $\ldots$ & $\ldots$ & $\ldots$ & $\cdots$ & $\ldots$ \\
\hline $\mathrm{V}^{*} \mathrm{VX}$ Ind & 2.155 & $\cdots$ & $\cdots$ & $\ldots$ & 2.155 & -14 & -2 . & 4.6 & 4.6 & STN \\
\hline BPS CS 22955-0174 & 5.781 & $\cdots$ & $\cdots$ & 5.200 & 5.781 & -3.1 & -7.6 & 2.0 & 2.0 & SPM \\
\hline
\end{tabular}


TABle 1B. (continued)

\begin{tabular}{|c|c|c|c|c|c|c|c|c|c|c|}
\hline $\begin{array}{l}\text { Star } \\
(1)\end{array}$ & $\begin{array}{c}D_{p h o} \\
(\mathrm{kpc}) \\
(2)\end{array}$ & $\begin{array}{c}D_{H I P} \\
(\mathrm{kpc}) \\
(3)\end{array}$ & $\begin{array}{c}\sigma_{\pi_{H I P}} / \pi_{H I P} \\
(4)\end{array}$ & $\begin{array}{c}D_{B S L} \\
(\mathrm{kpc}) \\
(5)\end{array}$ & $\begin{array}{c}D_{\text {adopt }} \\
(\mathrm{kpc}) \\
(6)\end{array}$ & $\begin{array}{c}\mu_{\alpha^{*}} \\
(\mathrm{mas} / \mathrm{yr}) \\
(7)\end{array}$ & $\begin{array}{c}\mu_{\delta} \\
\text { (mas/yr) } \\
(8)\end{array}$ & $\begin{array}{c}\sigma_{\mu_{\alpha^{*}}} \\
(\mathrm{mas} / \mathrm{yr}) \\
(9)\end{array}$ & $\begin{array}{c}\sigma_{\mu_{\delta}} \\
(\mathrm{mas} / \mathrm{yr}) \\
(10)\end{array}$ & \\
\hline BPS CS 22879-0051 & 0.993 & $\ldots$ & $\cdots$ & 1.000 & 0.993 & 3.7 & -67.6 & 2.9 & 3.9 & SPM \\
\hline BPS CS 22940-0070 & 7.238 & $\ldots$ & $\ldots$ & 6.500 & 7.238 & $\ldots$ & $\ldots$ & $\ldots$ & $\ldots$ & $\ldots$ \\
\hline BD-20 6008 & 1.370 & $<0$ & $\cdots$ & 1.620 & 1.370 & -14.9 & -7.1 & 1.2 & 0.9 & HIP, \\
\hline BPS CS 22880-0037 & $5.257 / 0.933$ & $\cdots$ & $\cdots$ & 0.800 & 5.257 & $\ldots$ & $\cdots$ & $\cdots$ & $\cdots$ & $\cdots$ \\
\hline BPS CS 22940-0071 & $5.047 / 0.913$ & $\cdots$ & $\cdots$ & 4.900 & 5.047 & $\cdots$ & $\cdots$ & $\cdots$ & $\cdots$ & $\cdots$ \\
\hline BPS CS 22940-0082 & 2.354 & $\cdots$ & $\cdots$ & 5.600 & 2.354 & $\ldots$ & $\ldots$ & $\cdots$ & $\ldots$ & $\cdots$ \\
\hline BPS CS 22879-0066 & 0.814 & $\cdots$ & $\cdots$ & 0.800 & 0.814 & -24.9 & -60.2 & 3.1 & 2.2 & SPM \\
\hline BPS CS 22880-0058 & 1.360 & $\cdots$ & $\cdots$ & 1.000 & 1.360 & -24.0 & -12.1 & 5 & 5 & NPM \\
\hline CD-30 18140 & 0.152 & 0.137 & 0.21 & 0.114 & 0.152 & -61.6 & -322.4 & 1.4 & 0.7 & HIP,$\varsigma$ \\
\hline BD-15 5781 & 2.913 & $<0$ & $\cdots$ & 1.950 & 2.913 & 6.1 & -21.2 & 1.0 & 1.6 & $\mathrm{HIP}$, \\
\hline BPS CS 22880-0086 & 4.835 & $\cdots$ & $\ldots$ & 2.100 & 4.835 & $\cdots$ & $\ldots$ & $\cdots$ & $\ldots$ & $\cdots$ \\
\hline BPS CS 22880-0074 & 1.543 & $\cdots$ & $\cdots$ & 0.900 & 1.543 & $\cdots$ & $\cdots$ & $\cdots$ & $\cdots$ & $\cdots$ \\
\hline BPS CS 22879-0103 & 5.458 & $\ldots$ & $\cdots$ & 5.300 & 5.458 & -3.0 & -9.2 & 2.3 & 2.6 & SPM \\
\hline $\mathrm{V}^{*} \mathrm{DX}$ Del & 0.570 & 0.645 & 0.99 & 0.649 & 0.570 & 14.1 & 8.2 & 1.4 & 0.9 & HIP,, \\
\hline BPS CS 22879-0097 & 5.424 & $\ldots$ & $\cdots$ & 5.100 & 5.424 & -1.2 & -18.3 & 2.4 & 2.4 & SPM \\
\hline BPS CS 22880-0067 & 2.674 & $\cdots$ & $\ldots$ & 1.700 & 2.674 & $\ldots$ & $\ldots$ & $\cdots$ & $\ldots$ & $\ldots$ \\
\hline BPS CS 22879-0098 & 5.000 & $\cdots$ & $\cdots$ & 5.100 & 5.000 & -7.2 & -14.1 & 6.0 & 6.2 & SPM \\
\hline BPS CS 22879-0094 & 1.689 & $\cdots$ & $\cdots$ & 1.900 & 1.689 & 3.5 & -32.0 & 2.3 & 2.3 & SPM \\
\hline BPS CS 22880-0110 & 1.455 & $\cdots$ & $\cdots$ & 1.300 & 1.455 & $\cdots$ & $\cdots$ & $\cdots$ & $\cdots$ & $\cdots$ \\
\hline $\mathrm{V}^{*} \mathrm{FK} \mathrm{Vul}$ & 2.326 & $\cdots$ & $\cdots$ & $\cdots$ & 2.326 & -3.7 & -20.6 & 2.8 & 2.8 & STN, \\
\hline $\mathrm{V}^{*} \mathrm{BV}$ Del & 2.431 & $\cdots$ & $\cdots$ & $\ldots$ & 2.431 & $\cdots$ & $\ldots$ & $\cdots$ & $\cdots$ & $\cdots$ \\
\hline BPS CS 22880-0127 & 0.546 & $\ldots$ & $\ldots$ & 0.700 & 0.546 & -1.7 & -21.6 & 3.3 & 2.6 & SPM \\
\hline CD-48 13714 & 0.160 & 0.156 & 0.33 & 0.181 & 0.160 & 46.1 & -156.6 & 1.8 & 0.8 & HIP, \\
\hline BPS CS 22940-0121 & 2.147 & $\ldots$ & $\cdots$ & 4.700 & 2.147 & $\cdots$ & $\ldots$ & $\cdots$ & $\cdots$ & $\cdots$ \\
\hline BPS CS 22940-0099 & 0.808 & $\cdots$ & $\cdots$ & 0.900 & 0.808 & $\ldots$ & $\ldots$ & $\cdots$ & $\cdots$ & $\cdots$ \\
\hline BD-14 5890 & 1.428 & 0.676 & 1.26 & 1.120 & 1.428 & -16.0 & -82.5 & 0.9 & 1.3 & HIP, \\
\hline V* UY Cyg & 0.922 & 2.381 & 4.90 & 1.122 & 0.922 & -3.8 & -11.4 & 1.1 & 0.8 & HIP, \\
\hline CD-37 14010 & 1.679 & 0.405 & 0.70 & 2.060 & 1.679 & 24.4 & -40.9 & 0.8 & 0.8 & HIP, \\
\hline $\mathrm{V}^{*} \mathrm{BT}$ Aqr & 1.850 & $\cdots$ & $\cdots$ & $\cdots$ & 1.850 & 1.7 & -6.8 & 2.9 & 2.9 & STN, \\
\hline $\mathrm{V}^{*} \mathrm{DH}$ Cyg & $\cdots$ & $\cdots$ & $\cdots$ & 1.300 & 1.300 & $\cdots$ & $\cdots$ & $\cdots$ & $\cdots$ & $\cdots$ \\
\hline $\mathrm{V}^{*}$ XZ Mic & 2.822 & $\cdots$ & $\cdots$ & $\cdots$ & 2.822 & -8.7 & -18.9 & 1.9 & 2.2 & SPM \\
\hline BPS CS 22879-0141 & 1.093 & $\cdots$ & $\cdots$ & 1.200 & 1.093 & -1.3 & -36.9 & 3.6 & 5.0 & SPM \\
\hline BPS CS 22879-0144 & 1.617 & $\ldots$ & $\cdots$ & 1.600 & 1.617 & 13.6 & -46.4 & 4.9 & 4.6 & SPM \\
\hline HD 199854 & 0.466 & 0.763 & 1.11 & 0.432 & 0.466 & -10.2 & 0.6 & 1.3 & 1.0 & HIP, \\
\hline BPS CS 22897-0012 & 1.428 & $\cdots$ & $\cdots$ & 1.200 & 1.428 & $\cdots$ & $\cdots$ & $\cdots$ & $\cdots$ & $\cdots$ \\
\hline $\mathrm{V}^{*} \mathrm{AA} \mathrm{Mic}$ & $\cdots$ & $\cdots$ & $\cdots$ & 1.200 & 1.200 & $\cdots$ & $\ldots$ & $\cdots$ & $\cdots$ & $\cdots$ \\
\hline $\mathrm{V}^{*} \mathrm{RV}$ Cap & 1.154 & $<0$ & $\cdots$ & 1.081 & 1.154 & 21.1 & -107.3 & 0.9 & 1.4 & HIP, \\
\hline BPS CS 22897-0019 & 1.696 & $\cdots$ & $\cdots$ & 1.000 & 1.696 & $\cdots$ & $\cdots$ & $\cdots$ & $\cdots$ & $\cdots$ \\
\hline BPS CS 29501-0014 & 0.376 & $\cdots$ & $\cdots$ & 0.800 & 0.376 & $\cdots$ & $\cdots$ & $\cdots$ & $\cdots$ & $\cdots$ \\
\hline BPS CS 22897-0008 & 4.108 & $\cdots$ & $\cdots$ & 4.900 & 4.108 & $\cdots$ & $\cdots$ & $\cdots$ & $\cdots$ & $\cdots$ \\
\hline
\end{tabular}


TABle 1B. (continued)

\begin{tabular}{|c|c|c|c|c|c|c|c|c|c|c|}
\hline $\begin{array}{l}\text { Star } \\
(1)\end{array}$ & $\begin{array}{c}D_{p h o} \\
(\mathrm{kpc}) \\
(2)\end{array}$ & $\begin{array}{c}D_{H I P} \\
(\mathrm{kpc}) \\
(3)\end{array}$ & $\begin{array}{c}\sigma_{\pi_{H I P}} / \pi_{H I P} \\
(4)\end{array}$ & $\begin{array}{c}D_{B S L} \\
(\mathrm{kpc}) \\
(5)\end{array}$ & $\begin{array}{l}D_{\text {adopt }} \\
(\mathrm{kpc}) \\
(6)\end{array}$ & $\begin{array}{c}\mu_{\alpha^{*}} \\
(\mathrm{mas} / \mathrm{yr}) \\
(7)\end{array}$ & $\begin{array}{c}\mu_{\delta} \\
\text { (mas/yr) } \\
(8)\end{array}$ & $\begin{array}{c}\sigma_{\mu_{\alpha *}} \\
(\mathrm{mas} / \mathrm{yr}) \\
(9)\end{array}$ & $\begin{array}{c}\sigma_{\mu_{\delta}} \\
(\mathrm{mas} / \mathrm{yr}) \\
(10)\end{array}$ & $\mathrm{S}$ \\
\hline BPS CS 22897-0001 & 0.894 & $\cdots$ & $\cdots$ & 1.000 & 0.894 & $\cdots$ & $\cdots$ & $\cdots$ & $\cdots$ & \\
\hline BPS CS 22898-0040 & 1.322 & $\ldots$ & $\ldots$ & 1.400 & 1.322 & -14.8 & -46.8 & 2.1 & 2.1 & SPM \\
\hline BPS CS 22937-0006 & 1.432 & $\ldots$ & $\ldots$ & 1.400 & 1.432 & 0.3 & -34.5 & 4.0 & 1.9 & SPM \\
\hline BPS CS 30492-0034 & 5.705 & $\cdots$ & $\cdots$ & 1.900 & 5.705 & 2.1 & -18.2 & 3.6 & 3.8 & SPM \\
\hline BPS CS 30492-0016 & 0.875 & $\cdots$ & $\cdots$ & 1.400 & 0.875 & 32.5 & -88.6 & 3.0 & 4.8 & SPM \\
\hline BPS CS 29501-0004 & 0.774 & $\cdots$ & $\cdots$ & 1.100 & 0.774 & 11.5 & -10.6 & 3.1 & 3.1 & SPM \\
\hline BPS CS 29501-0012 & 0.693 & $\cdots$ & $\cdots$ & 1.000 & 0.693 & 50.8 & -53.3 & 4.7 & 5.7 & SPM \\
\hline BPS CS 30492-0008 & 0.561 & $\cdots$ & $\cdots$ & 0.700 & 0.561 & 4.9 & -8.5 & 2.5 & 3.1 & SPM \\
\hline BPS CS 22898-0027 & 0.469 & $\cdots$ & $\cdots$ & 0.800 & 0.469 & -10 & -73 & 6.5 & 6.5 & STN \\
\hline BPS CS 30492-0001 & 0.767 & $\cdots$ & $\cdots$ & 0.900 & 0.767 & 15.2 & -48.3 & 3.5 & 2.5 & SPM \\
\hline BPS CS 22898-0047 & 2.628 & $\ldots$ & $\cdots$ & 1.800 & 2.628 & $\cdots$ & $\ldots$ & $\cdots$ & $\cdots$ & $\cdots$ \\
\hline CD-62 1346 & 0.299 & 0.196 & 0.34 & 0.440 & 0.299 & -14.5 & -102.2 & 0.7 & 1.1 & $\mathrm{HIP}, \mathrm{A}$ \\
\hline BPS CS 22937-0008 & 0.510 & $\ldots$ & $\cdots$ & 0.700 & 0.510 & -13.6 & -2.8 & 5.2 & 3.9 & SPM \\
\hline HD 200654 & 0.260 & 0.312 & 0.39 & 0.380 & 0.260 & 193.1 & -274.2 & 0.9 & 0.8 & HIP, \\
\hline BPS CS 22897-0017 & 1.081 & $\ldots$ & $\cdots$ & 1.200 & 1.081 & $\ldots$ & $\ldots$ & $\cdots$ & $\cdots$ & $\cdots$ \\
\hline BPS CS 22937-0004 & 1.316 & $\ldots$ & $\ldots$ & 1.300 & 1.316 & 27.1 & -9.4 & 2.2 & 4.2 & SPM \\
\hline BPS CS 22937-0039 & 0.915 & $\cdots$ & $\cdots$ & 1.000 & 0.915 & 5.4 & -14.4 & 2.2 & 2.1 & SPM \\
\hline BPS CS 29501-0032 & 1.211 & $\cdots$ & $\cdots$ & 1.200 & 1.211 & 14.4 & -25.1 & 2.3 & 2.3 & SPM \\
\hline BPS CS 30492-0050 & 0.840 & $\cdots$ & $\cdots$ & 1.100 & 0.840 & -29.1 & -45.5 & 2.1 & 2.1 & SPM \\
\hline BPS CS 22937-0034 & 0.884 & $\cdots$ & $\cdots$ & 1.100 & 0.884 & 10.3 & -31.5 & 2.3 & 2.2 & SPM \\
\hline BPS CS 22937-0019 & $\cdots$ & $\cdots$ & $\cdots$ & 1.000 & 1.000 & 8.1 & -4.2 & 2.5 & 2.4 & SPM \\
\hline BPS CS 30492-0047 & 0.974 & $\cdots$ & $\cdots$ & 0.900 & 0.974 & 8.6 & -3.0 & 2.1 & 2.1 & SPM \\
\hline BPS CS 29501-0028 & 0.755 & $\cdots$ & $\cdots$ & 0.900 & 0.755 & 9.3 & -17.7 & 4.1 & 3.1 & SPM \\
\hline $\mathrm{V}^{*} \mathrm{CP}$ Aqr & 1.453 & $\cdots$ & $\cdots$ & $\cdots$ & 1.453 & -8.9 & -16.4 & 2.9 & 2.9 & STN, \\
\hline BPS CS 22898-0043 & 5.239 & $\cdots$ & $\cdots$ & 4.700 & 5.239 & -0.2 & -11.1 & 4.3 & 4.6 & SPM \\
\hline BPS CS 22937-0023 & 0.950 & $\cdots$ & $\cdots$ & 1.100 & 0.950 & 18.6 & -36.2 & 2.3 & 2.3 & SPM \\
\hline BPS CS 22897-0042 & $5.111 / 1.595$ & $\ldots$ & $\cdots$ & 5.600 & 5.111 & $\ldots$ & $\ldots$ & $\cdots$ & $\cdots$ & $\ldots$ \\
\hline $\mathrm{V}^{*} \mathrm{~V}$ Ind & 0.702 & 0.667 & 1.06 & 0.727 & 0.702 & -71.9 & -83.5 & 1.2 & 0.9 & HIP,$A$ \\
\hline $\mathrm{V}^{*}$ AN Mic & $\cdots$ & $\cdots$ & $\cdots$ & 0.800 & 0.800 & $\cdots$ & $\cdots$ & $\cdots$ & $\cdots$ & $\cdots$ \\
\hline BPS CS 22937-0042 & 1.912 & $\cdots$ & $\cdots$ & 4.400 & 1.912 & 8.1 & -12.5 & 4.6 & 5.6 & SPM \\
\hline BPS CS 29501-0043 & 0.866 & $\cdots$ & $\cdots$ & 1.100 & 0.866 & -1.8 & -24.8 & 2.5 & 2.0 & SPM \\
\hline BPS CS 30492-0095 & 0.633 & $\cdots$ & $\cdots$ & 0.800 & 0.633 & 22.5 & -40.7 & 5.5 & 4.6 & SPM \\
\hline BPS CS 22898-0062 & 1.752 & $\cdots$ & $\cdots$ & 1.800 & 1.752 & $\cdots$ & $\cdots$ & $\cdots$ & $\cdots$ & $\cdots$ \\
\hline BPS CS 22897-0028 & 1.045 & $\cdots$ & $\cdots$ & 1.100 & 1.045 & $\cdots$ & $\ldots$ & $\cdots$ & $\cdots$ & $\cdots$ \\
\hline BPS CS 30492-0080 & $\cdots$ & $\cdots$ & $\cdots$ & 1.100 & 1.100 & 7.9 & -13.2 & 2.3 & 2.3 & SPM \\
\hline BPS CS 22937-0072 & 1.514 & $\cdots$ & $\cdots$ & 4.700 & 1.514 & -1.7 & -1.7 & 2.5 & 2.1 & SPM \\
\hline $\mathrm{V}^{*} \mathrm{SW}$ Aqr & 1.189 & 0.870 & 2.14 & 1.265 & 1.189 & -42.0 & -59.8 & 2.0 & 1.7 & HIP, $\mathrm{S}$ \\
\hline BPS CS 22937-0073 & 1.005 & $\cdots$ & $\cdots$ & 1.100 & 1.005 & -0.9 & -30.0 & 3.4 & 2.4 & SPM \\
\hline $\mathrm{V}^{*} \mathrm{RR}$ Mic & $\cdots$ & $<0$ & $\cdots$ & 8.900 & 8.900 & -4.7 & -8.3 & 2.1 & 1.8 & HIP, $\mathrm{S}$ \\
\hline BPS CS 29501-0056 & 1.768 & $\cdots$ & $\cdots$ & 1.900 & 1.768 & 24.9 & -10.2 & 2.2 & 2.1 & SPM \\
\hline
\end{tabular}


TABLE 1B. (continued)

\begin{tabular}{|c|c|c|c|c|c|c|c|c|c|c|}
\hline $\begin{array}{l}\text { Star } \\
(1)\end{array}$ & $\begin{array}{c}D_{p h o} \\
(\mathrm{kpc}) \\
(2)\end{array}$ & $\begin{array}{c}D_{H I P} \\
(\mathrm{kpc}) \\
(3)\end{array}$ & $\begin{array}{c}\sigma_{\pi_{H I P}} / \pi_{H I P} \\
(4)\end{array}$ & $\begin{array}{c}D_{B S L} \\
(\mathrm{kpc}) \\
(5)\end{array}$ & $\begin{array}{c}D_{\text {adopt }} \\
(\mathrm{kpc}) \\
(6)\end{array}$ & $\begin{array}{c}\mu_{\alpha^{*}} \\
(\mathrm{mas} / \mathrm{yr}) \\
(7)\end{array}$ & $\begin{array}{c}\mu_{\delta} \\
\text { (mas/yr) } \\
(8)\end{array}$ & $\begin{array}{c}\sigma_{\mu_{\alpha *}} \\
(\mathrm{mas} / \mathrm{yr}) \\
(9)\end{array}$ & $\begin{array}{c}\sigma_{\mu_{\delta}} \\
(\mathrm{mas} / \mathrm{yr}) \\
(10)\end{array}$ & Sou \\
\hline BPS CS 29501-0055 & 0.668 & $\ldots$ & $\ldots$ & 0.900 & 0.668 & -27.3 & -26.6 & 2.8 & 2.7 & SPM \\
\hline BPS CS 22937-0054 & 1.301 & $\ldots$ & $\ldots$ & 1.400 & 1.301 & 30.0 & -32.9 & 2.1 & 2.1 & SPM \\
\hline $\mathrm{V}^{*} \mathrm{Z}$ Mic & 1.368 & $<0$ & $\ldots$ & 1.486 & 1.368 & 16.1 & -7.8 & 1.9 & 1.3 & HIP,SPM, \\
\hline $\mathrm{BD}+424040$ & 0.090 & $\cdots$ & $\cdots$ & 1.000 & 0.090 & 162.3 & 97.3 & 1.5 & 1.3 & $\mathrm{ACT}, \mathrm{STN}$ \\
\hline BPS CS 22937-0060 & 1.809 & $\cdots$ & $\cdots$ & 1.000 & 1.809 & -1.0 & -9.6 & 8.4 & 6.6 & $\mathrm{SPM}$ \\
\hline BPS CS 22937-0057 & 0.900 & $\cdots$ & $\ldots$ & 0.900 & 0.900 & -25.9 & -31.2 & 2.2 & 2.2 & SPM \\
\hline BPS CS 29501-0082 & 1.038 & $\cdots$ & $\cdots$ & 1.200 & 1.038 & -14.5 & -58.9 & 4.1 & 4.3 & SPM \\
\hline BPS CS 30492-0114 & 0.637 & $\cdots$ & $\cdots$ & 0.800 & 0.637 & -5.5 & -33.7 & 6.3 & 6.3 & SPM \\
\hline [MFF90] 95942134 & $\ldots$ & $\cdots$ & $\cdots$ & 2.900 & 2.900 & 5. & -23 & 4.8 & 4.8 & STN \\
\hline $\mathrm{V}^{*} \mathrm{AS}$ Mic & $\cdots$ & $\cdots$ & $\cdots$ & 1.100 & 1.100 & $\cdots$ & $\cdots$ & $\cdots$ & $\cdots$ & $\cdots$ \\
\hline [MFF90] 9594299 & $\cdots$ & $\ldots$ & $\cdots$ & 1.500 & 1.500 & -12 & -12 & 3.9 & 3.9 & STN \\
\hline BPS CS 29501-0095 & 1.794 & $\cdots$ & $\cdots$ & 1.300 & 1.794 & 8.7 & -46.0 & 1.7 & 1.9 & SPM \\
\hline [M93] 19171 & 15.485 & $\cdots$ & $\cdots$ & 16.300 & 15.485 & $\ldots$ & $\ldots$ & $\cdots$ & $\cdots$ & $\cdots$ \\
\hline $\mathrm{V}^{*}$ YZ Cap & 1.208 & 0.337 & 0.85 & 1.355 & 1.208 & -14.4 & -20.0 & 1.6 & 0.8 & $\mathrm{HIP}, \mathrm{ACT}$, \\
\hline BPS CS 29501-0075 & 0.628 & $\cdots$ & $\cdots$ & 0.900 & 0.628 & -6.8 & -56.5 & 4.1 & 4.1 & $\mathrm{SPM}$ \\
\hline [M93] 18103 & 4.486 & $\ldots$ & $\ldots$ & 5.273 & 4.486 & $\ldots$ & $\ldots$ & $\cdots$ & $\cdots$ & $\ldots$ \\
\hline [M93] 18082 & 2.258 & $\cdots$ & $\ldots$ & 2.913 & 2.258 & $\cdots$ & $\ldots$ & $\cdots$ & $\ldots$ & $\cdots$ \\
\hline [M93] 28118 & 8.569 & $\ldots$ & $\cdots$ & 10.210 & 8.569 & $\cdots$ & $\ldots$ & $\cdots$ & $\cdots$ & $\cdots$ \\
\hline BPS CS 29506-0019 & $\cdots$ & $\cdots$ & $\cdots$ & 1.500 & 1.500 & $\cdots$ & $\cdots$ & $\cdots$ & $\cdots$ & $\cdots$ \\
\hline BPS CS 29506-0007 & 1.128 & $\cdots$ & $\cdots$ & 1.400 & 1.128 & $\cdots$ & $\cdots$ & $\cdots$ & $\cdots$ & $\cdots$ \\
\hline [MFF90] 9594249 & $\cdots$ & $\cdots$ & $\cdots$ & 0.730 & 0.730 & 6. & 9. & 5.5 & 5.5 & STN \\
\hline [M93] 18050 & 3.043 & $\cdots$ & $\cdots$ & 3.516 & 3.043 & $\cdots$ & $\cdots$ & $\cdots$ & $\cdots$ & $\cdots$ \\
\hline [MFF90] 959422 & $\ldots$ & $\ldots$ & $\ldots$ & 2.800 & 2.800 & $\cdots$ & $\cdots$ & $\ldots$ & $\cdots$ & $\ldots$ \\
\hline [MFF90] 9594272 & $\cdots$ & $\cdots$ & $\cdots$ & 4.500 & 4.500 & $\cdots$ & $\cdots$ & $\cdots$ & $\cdots$ & $\cdots$ \\
\hline [MFF90] 9594226 & $\ldots$ & $\cdots$ & $\cdots$ & 1.100 & 1.100 & -4 & -2 & 4.7 & 4.7 & STN \\
\hline [M93] 27036 & 3.457 & $\cdots$ & $\cdots$ & 3.072 & 3.457 & 2. & -8 & 4.5 & 4.5 & STN \\
\hline [M93] 18045 & 3.715 & $\cdots$ & $\cdots$ & 4.252 & 3.715 & $\cdots$ & $\cdots$ & $\cdots$ & $\cdots$ & $\cdots$ \\
\hline [M93] 27009 & 2.633 & $\cdots$ & $\cdots$ & 3.622 & 2.633 & $\cdots$ & $\cdots$ & $\cdots$ & $\cdots$ & $\cdots$ \\
\hline $\mathrm{V}^{*} \mathrm{DM}$ Cyg & 0.994 & $\cdots$ & $\cdots$ & $\cdots$ & 0.994 & 11.9 & 1.3 & 3.0 & 3.0 & STN,NPM \\
\hline [M93] 18002 & 3.578 & $\cdots$ & $\cdots$ & 4.056 & 3.578 & $\cdots$ & $\cdots$ & $\cdots$ & $\cdots$ & $\cdots$ \\
\hline [M93] 18006 & 2.675 & $\cdots$ & $\cdots$ & 2.940 & 2.675 & $\cdots$ & $\cdots$ & $\cdots$ & $\cdots$ & $\cdots$ \\
\hline [M93] 17062 & 15.549 & $\cdots$ & $\cdots$ & 17.482 & 15.549 & $\cdots$ & $\cdots$ & $\cdots$ & $\cdots$ & $\cdots$ \\
\hline BPS CS 30492-0116 & 3.298 & $\cdots$ & $\cdots$ & 3.200 & 3.298 & 13.3 & -11.5 & 2.6 & 2.5 & SPM \\
\hline [M93] 17089 & 3.115 & $\cdots$ & $\cdots$ & 4.007 & 3.115 & 2. & -14 & 4.1 & 4.1 & STN \\
\hline BPS CS 30492-0110 & 9.116 & $\cdots$ & $\cdots$ & 9.400 & 9.116 & 8.1 & -3.7 & 2.3 & 2.3 & SPM \\
\hline [M93] 26113 & 2.594 & $\cdots$ & $\cdots$ & 2.468 & 2.594 & 0.8 & -7.5 & 6.7 & 7.8 & SPM \\
\hline [M93] 17072 & $\cdots$ & $\cdots$ & $\cdots$ & 11.673 & 11.673 & $\cdots$ & $\cdots$ & $\cdots$ & $\cdots$ & $\cdots$ \\
\hline BPS CS 30492-0113 & 1.008 & $\cdots$ & $\cdots$ & 0.900 & 1.008 & -31.5 & -65.7 & 2.3 & 2.2 & SPM \\
\hline [M93] 26049 & 10.992 & $\cdots$ & $\cdots$ & 10.912 & 10.992 & $\cdots$ & $\cdots$ & $\cdots$ & $\cdots$ & $\cdots$ \\
\hline BPS CS 29501-0099 & 0.593 & $\cdots$ & $\cdots$ & 0.600 & 0.593 & -15.9 & 6.2 & 6.1 & 6.2 & SPM \\
\hline
\end{tabular}


TABLE 1B. (continued)

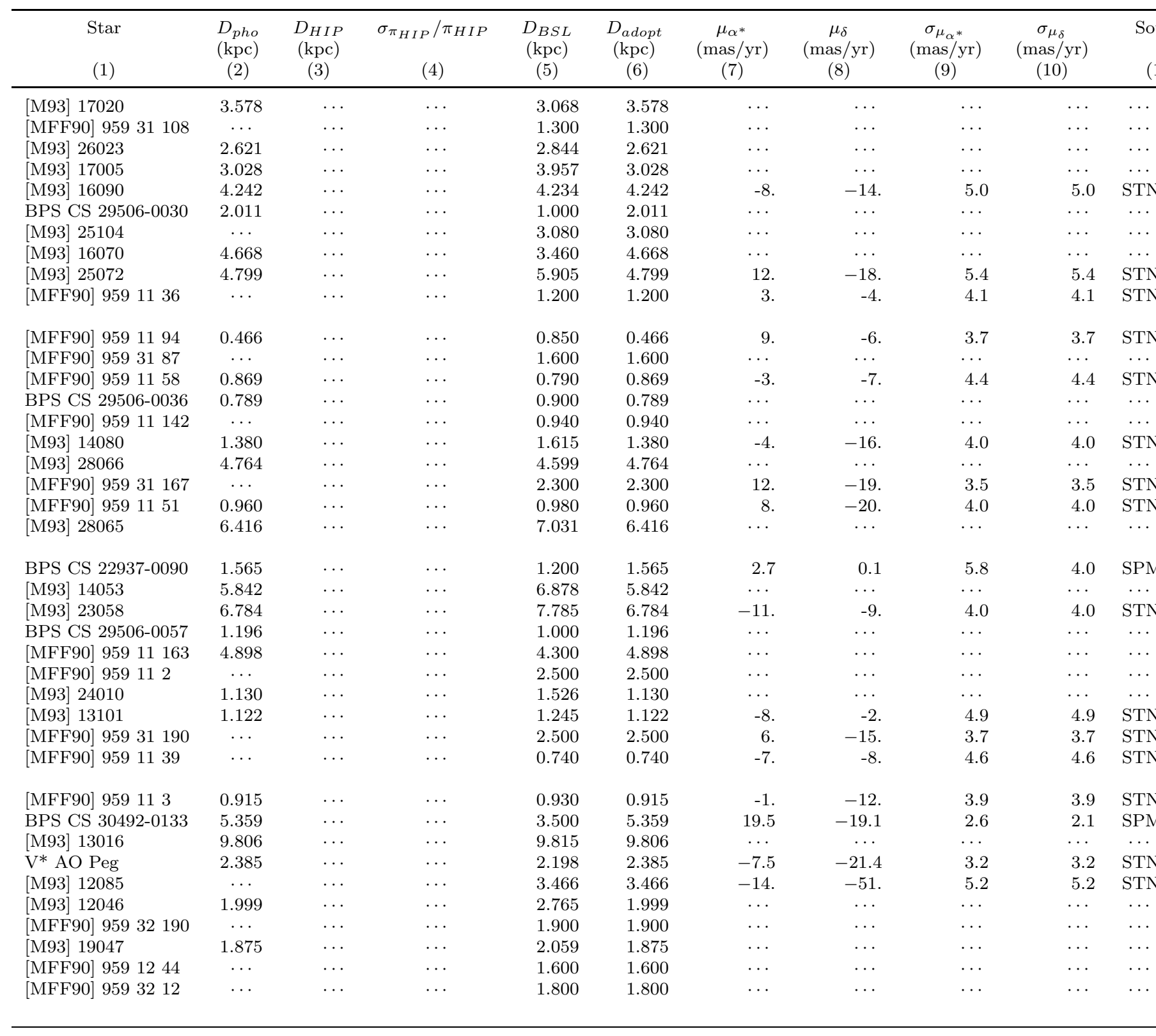


TABle 1B. (continued)

\begin{tabular}{|c|c|c|c|c|c|c|c|c|c|c|}
\hline $\begin{array}{l}\text { Star } \\
(1)\end{array}$ & $\begin{array}{c}D_{p h o} \\
(\mathrm{kpc}) \\
(2)\end{array}$ & $\begin{array}{c}D_{H I P} \\
(\mathrm{kpc}) \\
(3)\end{array}$ & $\begin{array}{c}\sigma_{\pi_{H I P}} / \pi_{H I P} \\
(4)\end{array}$ & $\begin{array}{c}D_{B S L} \\
(\mathrm{kpc}) \\
(5)\end{array}$ & $\begin{array}{l}D_{\text {adopt }} \\
(\mathrm{kpc}) \\
(6)\end{array}$ & $\begin{array}{c}\mu_{\alpha^{*}} \\
(\mathrm{mas} / \mathrm{yr}) \\
(7)\end{array}$ & $\begin{array}{c}\mu_{\delta} \\
(\operatorname{mas} / \mathrm{yr}) \\
(8)\end{array}$ & $\begin{array}{c}\sigma_{\mu_{\alpha *}} \\
(\mathrm{mas} / \mathrm{yr}) \\
(9)\end{array}$ & $\begin{array}{c}\sigma_{\mu_{\delta}} \\
(\mathrm{mas} / \mathrm{yr}) \\
(10)\end{array}$ & So \\
\hline BD-03 5215 & 0.327 & 0.474 & 0.96 & 0.920 & 0.327 & 19.7 & -23.5 & 1.4 & 1.3 & $\mathrm{HIP}, \mathrm{ACT}$ \\
\hline V* SS Gru & 2.748 & $\ldots$ & $\ldots$ & $\ldots$ & 2.748 & -13.2 & -17.8 & 1.6 & 1.5 & SPM,STI \\
\hline [MFF90] 9593287 & 3.350 & $\cdots$ & $\cdots$ & 3.000 & 3.350 & 7. & 9. & 3.7 & 3.7 & STN \\
\hline [M93] 12029 & 2.532 & $\cdots$ & $\cdots$ & 2.738 & 2.532 & -13 & -12 & 4.1 & 4.1 & STN \\
\hline HD 235518 & 1.179 & 4.762 & 3.48 & 0.532 & 1.179 & -0.3 & -2.7 & 0.6 & 0.6 & $\mathrm{HIP}, \mathrm{ACT}$ \\
\hline [MFF90] 9593298 & $\ldots$ & $\ldots$ & $\ldots$ & 1.300 & 1.300 & 15. & -7 & 3.7 & 3.7 & STN \\
\hline $\mathrm{BD}+463330$ & 0.563 & $\cdots$ & $\cdots$ & 0.239 & 0.563 & 1. & -1 & 3.1 & 3.1 & STN \\
\hline [M93] 22013 & 9.055 & $\cdots$ & $\cdots$ & 8.217 & 9.055 & 1.7 & -4.1 & 2.8 & 3.2 & SPM \\
\hline BPS CS 29506-0089 & 0.827 & $\cdots$ & $\cdots$ & 0.900 & 0.827 & $\cdots$ & $\ldots$ & $\cdots$ & $\cdots$ & $\ldots$ \\
\hline $\mathrm{BD}+473427$ & 0.266 & $\cdots$ & $\cdots$ & 0.501 & 0.266 & 14.8 & -5.8 & 2.2 & 0.9 & $\mathrm{ACT}, \mathrm{STI}$ \\
\hline HD 204543 & 0.456 & $<0$ & $\ldots$ & 0.490 & 0.456 & -3.5 & -48.3 & 0.9 & 0.9 & HIP,ACT \\
\hline [M93] 11079 & 1.931 & $\cdots$ & $\cdots$ & 2.370 & 1.931 & $\cdots$ & $\cdots$ & $\cdots$ & $\cdots$ & $\cdots$ \\
\hline [M93] 21083 & 3.804 & $\cdots$ & $\cdots$ & 3.172 & 3.804 & 5. & -15 & 4.3 & 4.3 & STN \\
\hline [M93] 19019 & 2.763 & $\cdots$ & $\cdots$ & 3.162 & 2.763 & $\cdots$ & $\cdots$ & $\cdots$ & $\cdots$ & $\cdots$ \\
\hline [M93] 11059 & 0.831 & $\ldots$ & $\ldots$ & 1.028 & 0.831 & -11 & -20 & 4.6 & 4.6 & STN \\
\hline [M93] 19013 & 6.030 & $\ldots$ & $\ldots$ & 7.060 & 6.030 & $\ldots$ & $\ldots$ & $\cdots$ & $\cdots$ & $\cdots$ \\
\hline BPS CS 29506-0090 & 1.143 & $\cdots$ & $\cdots$ & 1.400 & 1.143 & -9.5 & -41.1 & 3.6 & 3.3 & SPM \\
\hline [M93] 21043 & 4.472 & $\cdots$ & $\cdots$ & 4.611 & 4.472 & $\ldots$ & $\ldots$ & $\cdots$ & $\cdots$ & $\ldots$ \\
\hline [M93] 11024 & 10.052 & $\cdots$ & $\cdots$ & 4.878 & 10.052 & $\cdots$ & $\cdots$ & $\cdots$ & $\cdots$ & $\cdots$ \\
\hline [MFF90] 9593259 & $\cdots$ & $\cdots$ & $\cdots$ & 3.200 & 3.200 & $\cdots$ & $\cdots$ & $\cdots$ & $\cdots$ & $\cdots$ \\
\hline BPS CS 29506-0069 & 1.435 & $\cdots$ & $\cdots$ & 1.500 & 1.435 & $\ldots$ & $\ldots$ & $\ldots$ & $\ldots$ & $\ldots$ \\
\hline [M93] 21004 & 1.621 & $\ldots$ & $\ldots$ & 1.503 & 1.621 & $\ldots$ & $\ldots$ & $\ldots$ & $\ldots$ & $\ldots$ \\
\hline BPS CS 29506-0068 & 1.267 & $\cdots$ & $\cdots$ & 2.200 & 1.267 & $\cdots$ & $\cdots$ & $\cdots$ & $\cdots$ & $\cdots$ \\
\hline [M93] 44096 & 19.083 & $\cdots$ & $\cdots$ & 18.569 & 19.083 & $\cdots$ & $\cdots$ & $\cdots$ & $\cdots$ & $\cdots$ \\
\hline [MFF90] 95932108 & $\ldots$ & $\cdots$ & $\cdots$ & 4.600 & 4.600 & $\cdots$ & $\cdots$ & $\cdots$ & $\cdots$ & $\cdots$ \\
\hline [M93] 44078 & 4.316 & $\cdots$ & $\cdots$ & 4.344 & 4.316 & $\cdots$ & $\ldots$ & $\cdots$ & $\cdots$ & $\cdots$ \\
\hline [M93] 44088 & 5.782 & $\cdots$ & $\cdots$ & 6.568 & 5.782 & 0.0 & -11.9 & 3.6 & 2.7 & SPM \\
\hline [M93] 46144 & 3.299 & $\cdots$ & $\cdots$ & 4.048 & 3.299 & $\cdots$ & $\ldots$ & $\cdots$ & $\cdots$ & $\ldots$ \\
\hline [M93] 34041 & 7.342 & $\cdots$ & $\cdots$ & 6.902 & 7.342 & $\cdots$ & $\cdots$ & $\cdots$ & $\cdots$ & $\cdots$ \\
\hline [M93] 44052 & 19.852 & $\cdots$ & $\cdots$ & 18.298 & 19.852 & -1.2 & -9.4 & 3.5 & 7.9 & SPM \\
\hline [M93] 46143 & 5.836 & $\cdots$ & $\cdots$ & 5.731 & 5.836 & 0.3 & -13.8 & 4.5 & 9.7 & SPM \\
\hline BPS CS 22897-0102 & 2.100 & $\cdots$ & $\cdots$ & 2.000 & 2.100 & $\cdots$ & $\cdots$ & $\cdots$ & $\cdots$ & $\cdots$ \\
\hline [M93] 44032 & $\ldots$ & $\cdots$ & $\cdots$ & 3.720 & 3.720 & $\cdots$ & $\cdots$ & $\cdots$ & $\cdots$ & $\cdots$ \\
\hline [M93] 44005 & 4.121 & $\cdots$ & $\cdots$ & 4.555 & 4.121 & -2 & -14 & 5.2 & 5.2 & STN \\
\hline BPS CS 22897-0099 & 6.263 & $\cdots$ & $\cdots$ & 5.600 & 6.263 & $\cdots$ & $\cdots$ & $\cdots$ & $\cdots$ & $\cdots$ \\
\hline BPS CS 22948-0012 & 0.610 & $\cdots$ & $\cdots$ & 1.300 & 0.610 & -8.9 & -25.0 & 3.1 & 2.0 & SPM \\
\hline BPS CS 29495-0005 & 1.124 & $\cdots$ & $\cdots$ & 1.500 & 1.124 & 18.2 & -22.4 & 2.7 & 2.7 & SPM \\
\hline BPS CS 29506-0109 & 1.001 & $\cdots$ & $\cdots$ & 1.200 & 1.001 & $\cdots$ & $\cdots$ & $\cdots$ & $\cdots$ & $\ldots$ \\
\hline BPS CS 22948-0006 & 8.048 & $\cdots$ & $\cdots$ & 7.800 & 8.048 & 1.5 & -8.7 & 2.3 & 2.3 & SPM \\
\hline [M93] 43059 & 2.723 & $\cdots$ & $\cdots$ & 3.268 & 2.723 & $\cdots$ & $\ldots$ & $\cdots$ & $\cdots$ & $\ldots$ \\
\hline
\end{tabular}


TABLE 1B. (continued)

\begin{tabular}{|c|c|c|c|c|c|c|c|c|c|c|}
\hline $\begin{array}{l}\text { Star } \\
(1)\end{array}$ & $\begin{array}{c}D_{\text {pho }} \\
(\mathrm{kpc}) \\
(2)\end{array}$ & $\begin{array}{c}D_{H I P} \\
(\mathrm{kpc}) \\
(3)\end{array}$ & $\begin{array}{c}\sigma_{\pi_{H I P}} / \pi_{H I P} \\
(4)\end{array}$ & $\begin{array}{c}D_{B S L} \\
(\mathrm{kpc}) \\
(5)\end{array}$ & $\begin{array}{c}D_{\text {adopt }} \\
(\mathrm{kpc}) \\
(6)\end{array}$ & $\begin{array}{c}\mu_{\alpha^{*}} \\
(\mathrm{mas} / \mathrm{yr}) \\
(7)\end{array}$ & $\begin{array}{c}\mu_{\delta} \\
\text { (mas/yr) } \\
(8)\end{array}$ & $\begin{array}{c}\sigma_{\mu_{\alpha^{*}}} \\
(\mathrm{mas} / \mathrm{yr}) \\
(9)\end{array}$ & $\begin{array}{c}\sigma_{\mu_{\delta}} \\
(\mathrm{mas} / \mathrm{yr}) \\
(10)\end{array}$ & $\begin{array}{r}\text { Sou } \\
(1\end{array}$ \\
\hline [M93] 33058 & 5.750 & $\ldots$ & $\ldots$ & 5.176 & 5.750 & $\ldots$ & $\ldots$ & $\ldots$ & $\ldots$ & $\cdots$ \\
\hline [M93] 33015 & 4.819 & $\ldots$ & $\ldots$ & 4.864 & 4.819 & -15 & -14 & 4.6 & 4.6 & STN \\
\hline V* Z Gru & $\ldots$ & $\ldots$ & $\ldots$ & 1.986 & 1.986 & $\ldots$ & $\ldots$ & $\ldots$ & $\ldots$ & $\ldots$ \\
\hline [M93] 42163 & 3.768 & $\cdots$ & $\cdots$ & 4.012 & 3.768 & $\cdots$ & $\cdots$ & $\cdots$ & $\cdots$ & $\cdots$ \\
\hline [M93] 32141 & 4.509 & $\cdots$ & $\cdots$ & 4.534 & 4.509 & 1. & -3 & 4.6 & 4.6 & STN \\
\hline [M93] 42146 & 6.242 & $\cdots$ & $\cdots$ & 7.087 & 6.242 & $\ldots$ & $\ldots$ & $\cdots$ & $\cdots$ & $\cdots$ \\
\hline BPS CS 29495-0002 & 0.643 & $\cdots$ & $\cdots$ & 0.900 & 0.643 & 67.4 & -44.5 & 2.3 & 2.1 & SPM \\
\hline [M93] 37006 & 3.457 & $\cdots$ & $\cdots$ & 4.555 & 3.457 & $\cdots$ & $\ldots$ & $\cdots$ & $\cdots$ & $\cdots$ \\
\hline $\mathrm{BD}+433979$ & 0.178 & $\cdots$ & $\cdots$ & 0.298 & 0.178 & 15.9 & 29.3 & 2.4 & 0.8 & $\mathrm{ACT}, \mathrm{STN}$ \\
\hline BPS CS 22948-0024 & 2.348 & $\cdots$ & $\cdots$ & 2.900 & 2.348 & 18.9 & -6.4 & 2.3 & 2.3 & SPM \\
\hline [M93] 46112 & 2.862 & $\cdots$ & $\cdots$ & 2.672 & 2.862 & 5.9 & -14.4 & 6.4 & 6.5 & SPM \\
\hline BPS CS 29506-0106 & 1.250 & $\cdots$ & $\cdots$ & 1.300 & 1.250 & $\cdots$ & $\ldots$ & $\cdots$ & $\cdots$ & $\cdots$ \\
\hline $\mathrm{V}^{*} \mathrm{SX}$ Aqr & 1.676 & $<0$ & $\cdots$ & 1.629 & 1.676 & -41.4 & -46.9 & 2.5 & 1.8 & $\mathrm{HIP}, \mathrm{STN}$, \\
\hline $\mathrm{V}^{*} \mathrm{RY}$ Oct & 1.735 & 0.173 & 0.66 & $\ldots$ & 1.735 & -5.3 & -25.6 & 2.9 & 2.7 & $\mathrm{HIP}, \mathrm{STN}$ \\
\hline [M93] 32022 & 2.260 & $\ldots$ & $\ldots$ & 2.993 & 2.260 & $\ldots$ & $\ldots$ & $\ldots$ & $\ldots$ & $\ldots$ \\
\hline BPS CS 29495-0041 & 7.975 & $\cdots$ & $\cdots$ & 4.200 & 7.975 & -0.5 & -12.5 & 3.2 & 2.0 & SPM \\
\hline [M93] 32010 & 1.865 & $\cdots$ & $\ldots$ & 2.358 & 1.865 & $\ldots$ & $\ldots$ & $\ldots$ & $\ldots$ & $\ldots$ \\
\hline HD 205547 & 1.102 & 0.469 & 0.67 & 1.270 & 1.102 & -33.1 & -40.6 & 0.7 & 0.6 & HIP,SPM, \\
\hline BPS CS 22948-0019 & 1.515 & $\cdots$ & $\cdots$ & 1.600 & 1.515 & -2.9 & -23.2 & 2.3 & 2.3 & SPM \\
\hline [M93] 36131 & 6.340 & $\cdots$ & $\cdots$ & 7.364 & 6.340 & $\cdots$ & $\ldots$ & $\cdots$ & $\cdots$ & $\cdots$ \\
\hline [M93] 41114 & 5.161 & $\cdots$ & $\cdots$ & 5.204 & 5.161 & -8 & -14 & 5.0 & 5.0 & STN \\
\hline [M93] 45105 & 4.914 & $\ldots$ & $\ldots$ & 4.928 & 4.914 & -8 & -14 & 5.0 & 5.0 & STN \\
\hline [M93] 31181 & 4.339 & $\cdots$ & $\cdots$ & 5.083 & 4.339 & $\cdots$ & $\cdots$ & $\cdots$ & $\cdots$ & $\cdots$ \\
\hline BPS CS 22948-0041 & 5.219 & $\cdots$ & $\cdots$ & 4.800 & 5.219 & $\cdots$ & $\cdots$ & $\cdots$ & $\cdots$ & $\cdots$ \\
\hline BPS CS 22897-0125 & 0.796 & $\cdots$ & $\cdots$ & 0.900 & 0.796 & $\cdots$ & $\cdots$ & $\cdots$ & $\cdots$ & $\cdots$ \\
\hline [M93] 31167 & 7.799 & $\cdots$ & $\cdots$ & 7.873 & 7.799 & $\cdots$ & $\cdots$ & $\cdots$ & $\cdots$ & $\cdots$ \\
\hline [M93] 45080 & 2.310 & $\cdots$ & $\cdots$ & 2.350 & 2.310 & $\cdots$ & $\cdots$ & $\cdots$ & $\cdots$ & $\cdots$ \\
\hline $\mathrm{V}^{*} \mathrm{RR}$ Gru & 2.133 & $\cdots$ & $\cdots$ & $\cdots$ & 2.133 & -13 & -7 & 4.6 & 4.6 & STN \\
\hline [M93] 31121 & 1.805 & $\cdots$ & $\cdots$ & 2.591 & 1.805 & $\cdots$ & $\cdots$ & $\cdots$ & $\cdots$ & $\cdots$ \\
\hline BPS CS 22897-0126 & 1.524 & $\cdots$ & $\cdots$ & 1.500 & 1.524 & $\cdots$ & $\cdots$ & $\cdots$ & $\cdots$ & $\cdots$ \\
\hline $\mathrm{BD}+404598$ & 0.096 & $\cdots$ & $\cdots$ & 0.286 & 0.096 & -17.0 & 30.8 & 2.3 & 0.8 & $\mathrm{ACT}, \mathrm{STN}$ \\
\hline BPS CS 29495-0031 & $\cdots$ & $\cdots$ & $\cdots$ & 1.100 & 1.100 & 17.0 & -50.8 & 3.1 & 4.0 & SPM \\
\hline [M93] 36067 & 2.287 & $\cdots$ & $\cdots$ & 2.469 & 2.287 & $\cdots$ & $\cdots$ & $\cdots$ & $\cdots$ & $\cdots$ \\
\hline [M93] 31083 & 3.484 & $\cdots$ & $\cdots$ & 4.263 & 3.484 & $\cdots$ & $\cdots$ & $\cdots$ & $\cdots$ & $\cdots$ \\
\hline BPS CS 22948-0057 & 1.849 & $\cdots$ & $\cdots$ & 2.100 & 1.849 & -9.1 & -1.3 & 6.0 & 3.9 & SPM \\
\hline BPS CS 22951-0018 & $\cdots$ & $\cdots$ & $\cdots$ & 1.400 & 1.400 & $\cdots$ & $\cdots$ & $\cdots$ & $\cdots$ & $\cdots$ \\
\hline BPS CS 22951-0025 & 0.993 & $\cdots$ & $\cdots$ & 0.800 & 0.993 & $\cdots$ & $\cdots$ & $\cdots$ & $\cdots$ & $\cdots$ \\
\hline BPS CS 22951-0027 & 2.297 & $\cdots$ & $\cdots$ & 1.700 & 2.297 & $\cdots$ & $\cdots$ & $\cdots$ & $\cdots$ & $\cdots$ \\
\hline BPS CS 22951-0014 & 0.740 & $\cdots$ & $\cdots$ & 1.000 & 0.740 & $\cdots$ & $\cdots$ & $\cdots$ & $\cdots$ & $\cdots$ \\
\hline BPS CS 22944-0014 & 0.983 & $\cdots$ & $\cdots$ & 1.100 & 0.983 & $\cdots$ & $\cdots$ & $\cdots$ & $\cdots$ & $\cdots$ \\
\hline
\end{tabular}


TABLE 1B. (continued)

\begin{tabular}{|c|c|c|c|c|c|c|c|c|c|c|}
\hline $\begin{array}{l}\text { Star } \\
(1)\end{array}$ & $\begin{array}{c}D_{\text {pho }} \\
(\mathrm{kpc}) \\
(2)\end{array}$ & $\begin{array}{c}D_{H I P} \\
(\mathrm{kpc}) \\
(3)\end{array}$ & $\begin{array}{c}\sigma_{\pi_{H I P}} / \pi_{H I P} \\
(4)\end{array}$ & $\begin{array}{c}D_{B S L} \\
(\mathrm{kpc}) \\
(5)\end{array}$ & $\begin{array}{c}D_{\text {adopt }} \\
\text { (kpc) } \\
(6)\end{array}$ & $\begin{array}{c}\mu_{\alpha^{*}} \\
(\mathrm{mas} / \mathrm{yr}) \\
(7)\end{array}$ & $\begin{array}{c}\mu_{\delta} \\
(\mathrm{mas} / \mathrm{yr}) \\
(8)\end{array}$ & $\begin{array}{c}\sigma_{\mu_{\alpha^{*}}} \\
(\mathrm{mas} / \mathrm{yr}) \\
(9)\end{array}$ & $\begin{array}{c}\sigma_{\mu_{\delta}} \\
(\mathrm{mas} / \mathrm{yr}) \\
(10)\end{array}$ & Soul \\
\hline $\mathrm{V}^{*}$ CG Peg & 1.069 & 0.476 & 0.98 & 1.091 & 1.069 & -1.3 & -3.6 & 1.6 & 1.3 & HIP,STN, \\
\hline BPS CS 22944-0011 & 0.399 & $\ldots$ & $\ldots$ & 0.400 & 0.399 & -2 . & -101 & 4.5 & 4.5 & STN \\
\hline [M93] 36018 & 1.772 & $\ldots$ & $\ldots$ & 1.958 & 1.772 & $\ldots$ & $\ldots$ & $\ldots$ & $\ldots$ & $\ldots$ \\
\hline BPS CS 22944-0002 & 6.101 & $\ldots$ & $\ldots$ & 6.100 & 6.101 & $\ldots$ & $\ldots$ & $\ldots$ & $\ldots$ & $\ldots$ \\
\hline [M93] 34118 & 3.212 & $\ldots$ & $\ldots$ & 3.195 & 3.212 & 19. & -30 & 4.2 & 4.2 & STN \\
\hline [M93] 34119 & 2.719 & $\ldots$ & $\ldots$ & 2.146 & 2.719 & $\ldots$ & $\ldots$ & $\ldots$ & $\ldots$ & $\ldots$ \\
\hline HD 235596 & 0.391 & $\ldots$ & $\ldots$ & 1.064 & 0.391 & 6.8 & -0.6 & 2.0 & 1.1 & $\mathrm{ACT}, \mathrm{STN}$ \\
\hline BPS CS 22956-0017 & 1.425 & $\ldots$ & $\ldots$ & 1.100 & 1.425 & $\ldots$ & $\ldots$ & $\ldots$ & $\ldots$ & $\ldots$ \\
\hline BPS CS 22956-0021 & 1.436 & $\ldots$ & $\ldots$ & 1.300 & 1.436 & $\ldots$ & $\ldots$ & $\ldots$ & $\ldots$ & $\ldots$ \\
\hline HD 206739 & 0.629 & 1.449 & 1.80 & 0.510 & 0.629 & 42.1 & -30.4 & 0.8 & 0.7 & $\mathrm{HIP}, \mathrm{ACT}, \mathrm{s}$ \\
\hline HD 206642 & 0.221 & 0.240 & 0.19 & 0.270 & 0.221 & 80.5 & -162.1 & 0.7 & 0.5 & HIP,SPM, \\
\hline BPS CS 22956-0013 & $\ldots$ & $\ldots$ & $\ldots$ & 0.600 & 0.600 & $\ldots$ & $\ldots$ & $\ldots$ & $\ldots$ & $\ldots$ \\
\hline $\mathrm{V}^{*} \mathrm{UW}$ Oct & 2.024 & $\ldots$ & $\ldots$ & $\ldots$ & 2.024 & $\ldots$ & $\ldots$ & $\ldots$ & $\ldots$ & $\ldots$ \\
\hline BPS CS 22948-0066 & 3.141 & $\ldots$ & $\ldots$ & 4.700 & 3.141 & -0.6 & -4.7 & 3.9 & 2.2 & SPM \\
\hline BPS CS 22944-0039 & 1.752 & $\ldots$ & $\ldots$ & 5.300 & 1.752 & $\ldots$ & $\ldots$ & $\ldots$ & $\ldots$ & $\ldots$ \\
\hline BD-09 5831 & 1.682 & $<0$ & $\ldots$ & 1.720 & 1.682 & -2.1 & -16.8 & 1.2 & 0.9 & HIP,ACT,, \\
\hline BPS CS 22951-0005 & 1.615 & $\ldots$ & $\ldots$ & 1.300 & 1.615 & $\ldots$ & $\ldots$ & $\ldots$ & $\ldots$ & $\ldots$ \\
\hline BPS CS 22948-0045 & 1.496 & $\ldots$ & $\ldots$ & 2.400 & 1.496 & 1.0 & -8.6 & 2.6 & 2.6 & SPM \\
\hline BPS CS 29493-0037 & 1.110 & $\ldots$ & $\ldots$ & 1.500 & 1.110 & 9.1 & -13.2 & 1.6 & 2.2 & SPM \\
\hline BPS CS 22948-0075 & 1.692 & $\cdots$ & $\cdots$ & 1.700 & 1.692 & 9.5 & -16.3 & 2.3 & 2.3 & SPM \\
\hline BPS CS 22951-0042 & $\ldots$ & $\ldots$ & $\ldots$ & 0.700 & 0.700 & 9. & -8 & 4.2 & 4.2 & STN \\
\hline BPS CS 22951-0033 & 0.865 & $\ldots$ & $\ldots$ & 1.000 & 0.865 & $\ldots$ & $\ldots$ & $\ldots$ & $\ldots$ & $\ldots$ \\
\hline BPS CS 22948-0070 & 0.712 & $\ldots$ & $\ldots$ & 0.900 & 0.712 & -2.0 & -22.9 & 1.9 & 2.4 & SPM \\
\hline BPS CS 22948-0067 & 1.095 & $\ldots$ & $\ldots$ & 0.800 & 1.095 & 9.7 & -8.5 & 3.5 & 3.8 & SPM \\
\hline $\mathrm{V}^{*} \mathrm{RS}$ Oct & 2.316 & $\ldots$ & $\ldots$ & $\ldots$ & 2.316 & -6 . & -25 & 4.3 & 4.3 & STN \\
\hline BPS CS 22948-0079 & 0.990 & $\ldots$ & $\ldots$ & 0.800 & 0.990 & 16.5 & -27.3 & 6.1 & 2.3 & SPM \\
\hline BPS CS 29493-0023 & 1.409 & $\ldots$ & $\ldots$ & 1.400 & 1.409 & 32.7 & -22.3 & 2.5 & 2.5 & SPM \\
\hline BPS CS 29495-0074 & 0.604 & $\ldots$ & $\ldots$ & 1.000 & 0.604 & 9.0 & -23.6 & 2.6 & 2.6 & SPM \\
\hline BPS CS 29495-0071 & 0.498 & $\ldots$ & $\ldots$ & 0.900 & 0.498 & 2.6 & -16.9 & 3.2 & 2.3 & SPM \\
\hline BPS CS 22944-0035 & 1.356 & $\cdots$ & $\cdots$ & 1.300 & 1.356 & $\cdots$ & $\ldots$ & $\cdots$ & $\cdots$ & $\ldots$ \\
\hline BPS CS 22944-0032 & 2.060 & $\ldots$ & $\ldots$ & 1.400 & 2.060 & $\ldots$ & $\ldots$ & $\ldots$ & $\ldots$ & $\ldots$ \\
\hline HD 206998 & 0.061 & 0.095 & 0.11 & 0.070 & 0.095 & 32.1 & -178.5 & 1.0 & 0.7 & $\mathrm{HIP}, \mathrm{ACT}, \mathrm{S}$ \\
\hline BPS CS 29493-0044 & 1.327 & $\ldots$ & $\ldots$ & 1.500 & 1.327 & -33.5 & -29.1 & 1.7 & 1.9 & SPM \\
\hline BPS CS 22956-0004 & $\ldots$ & $\ldots$ & $\ldots$ & 1.300 & 1.300 & $\ldots$ & $\ldots$ & $\ldots$ & $\ldots$ & $\ldots$ \\
\hline BPS CS 22951-0037 & 1.309 & $\ldots$ & $\ldots$ & 1.200 & 1.309 & $\ldots$ & $\ldots$ & $\ldots$ & $\ldots$ & $\ldots$ \\
\hline BPS CS 29493-0043 & 0.903 & $\ldots$ & $\ldots$ & 1.200 & 0.903 & 27.9 & 3.1 & 2.4 & 1.5 & SPM \\
\hline BPS CS 29493-0050 & 1.255 & $\ldots$ & $\ldots$ & 1.400 & 1.255 & -27.3 & -70.4 & 2.1 & 2.7 & SPM \\
\hline BPS CS 22948-0077 & 0.907 & $\ldots$ & $\ldots$ & 1.200 & 0.907 & -21.5 & -36.3 & 2.6 & 1.6 & SPM \\
\hline BPS CS 29493-0049 & 0.829 & $\cdots$ & $\cdots$ & 1.100 & 0.829 & -10.7 & -53.2 & 2.4 & 3.7 & SPM \\
\hline BPS CS 22944-0061 & 1.206 & $\cdots$ & $\cdots$ & 1.200 & 1.206 & $\ldots$ & $\ldots$ & $\ldots$ & $\ldots$ & $\ldots$ \\
\hline
\end{tabular}


TABLE 1B. (continued)

\begin{tabular}{|c|c|c|c|c|c|c|c|c|c|c|}
\hline $\begin{array}{l}\text { Star } \\
(1)\end{array}$ & $\begin{array}{c}D_{p h o} \\
(\mathrm{kpc}) \\
(2)\end{array}$ & $\begin{array}{c}D_{H I P} \\
(\mathrm{kpc}) \\
(3)\end{array}$ & $\begin{array}{c}\sigma_{\pi_{H I P}} / \pi_{H I P} \\
(4)\end{array}$ & $\begin{array}{c}D_{B S L} \\
(\mathrm{kpc}) \\
(5)\end{array}$ & $\begin{array}{c}D_{\text {adopt }} \\
(\mathrm{kpc}) \\
(6)\end{array}$ & $\begin{array}{c}\mu_{\alpha^{*}} \\
(\mathrm{mas} / \mathrm{yr}) \\
(7)\end{array}$ & $\begin{array}{c}\mu_{\delta} \\
(\mathrm{mas} / \mathrm{yr}) \\
(8)\end{array}$ & $\begin{array}{c}\sigma_{\mu_{\alpha^{*}}} \\
(\mathrm{mas} / \mathrm{yr}) \\
(9)\end{array}$ & $\begin{array}{c}\sigma_{\mu_{\delta}} \\
(\mathrm{mas} / \mathrm{yr}) \\
(10)\end{array}$ & Sou \\
\hline BPS CS 22956-0015 & 1.124 & $\cdots$ & $\cdots$ & 1.000 & 1.124 & $\cdots$ & $\ldots$ & $\cdots$ & $\cdots$ & $\cdots$ \\
\hline BPS CS 29495-0080 & 0.772 & $\ldots$ & $\ldots$ & 1.300 & 0.772 & 38.3 & -138.8 & 2.0 & 2.3 & SPM \\
\hline BPS CS 22951-0059 & 2.364 & $\ldots$ & $\cdots$ & 2.500 & 2.364 & $\ldots$ & $\ldots$ & $\ldots$ & $\ldots$ & $\ldots$ \\
\hline BPS CS 22948-0093 & 1.742 & $\cdots$ & $\cdots$ & 2.000 & 1.742 & -20.1 & -17.7 & 2.8 & 1.7 & SPM \\
\hline $\mathrm{BD}+434063$ & 0.151 & $\cdots$ & $\cdots$ & 1.112 & 0.151 & -2.1 & -8.1 & 0.8 & 1.6 & $\mathrm{ACT}, \mathrm{STN}$ \\
\hline BPS CS 22951-0052 & $\ldots$ & $\cdots$ & $\cdots$ & 0.800 & 0.800 & $\ldots$ & $\ldots$ & $\cdots$ & $\cdots$ & $\ldots$ \\
\hline BPS CS 22948-0091 & 2.164 & $\cdots$ & $\cdots$ & 2.500 & 2.164 & -4.4 & -11.6 & 2.0 & 1.8 & SPM \\
\hline BPS CS 22951-0069 & 0.863 & $\cdots$ & $\cdots$ & 0.900 & 0.863 & $\cdots$ & $\ldots$ & $\cdots$ & $\cdots$ & $\ldots$ \\
\hline BPS CS 29493-0052 & 0.847 & $\ldots$ & $\ldots$ & 0.900 & 0.847 & 35.5 & -62.3 & 3.6 & 4.0 & SPM \\
\hline BPS CS 22948-0104 & 2.759 & $\cdots$ & $\cdots$ & 2.500 & 2.759 & -6.8 & -5.8 & 2.8 & 2.0 & SPM \\
\hline $\mathrm{V}^{*} \mathrm{RT}$ Gru & 2.693 & $\cdots$ & $\cdots$ & 2.630 & 2.693 & 39. & -28 & 3.8 & 3.8 & STN \\
\hline $\mathrm{V}^{*}$ AV Peg & 0.754 & 0.709 & 1.22 & 0.802 & 0.754 & 12.9 & -8.7 & 0.8 & 0.9 & $\mathrm{HIP}, \mathrm{ACT}$ \\
\hline BPS CS 22951-0065 & 1.095 & $\ldots$ & $\cdots$ & 1.000 & 1.095 & $\cdots$ & $\cdots$ & $\cdots$ & $\cdots$ & $\ldots$ \\
\hline BPS CS 29493-0058 & 4.756 & $\cdots$ & $\cdots$ & 2.200 & 4.756 & 10.6 & -2.8 & 1.7 & 1.6 & SPM \\
\hline BPS CS 29493-0062 & 0.611 & $\cdots$ & $\cdots$ & 1.300 & 0.611 & 2.2 & -5.2 & 3.2 & 2.9 & SPM \\
\hline BPS CS 29493-0084 & 1.461 & $\ldots$ & $\cdots$ & 1.400 & 1.461 & $\ldots$ & $\ldots$ & $\cdots$ & $\cdots$ & $\ldots$ \\
\hline BPS CS 29493-0059 & 6.942 & $\cdots$ & $\ldots$ & 4.300 & 6.942 & 2.0 & -12.0 & 3.4 & 4.3 & SPM \\
\hline $\mathrm{V}^{*} \mathrm{SS}$ Oct & 1.651 & 0.383 & 1.07 & $\ldots$ & 1.651 & 3.33 & -34.73 & 2.99 & 2.47 & HIP \\
\hline HD 208069 & 0.285 & 0.444 & 0.58 & 0.376 & 0.285 & 131.5 & -198.9 & 0.9 & 0.6 & HIP,SPM, \\
\hline BPS CS 29493-0064 & 0.629 & $\cdots$ & $\cdots$ & 1.000 & 0.629 & 11.7 & 2.6 & 4.3 & 3.7 & SPM \\
\hline BPS CS 22951-0055 & 1.387 & $\cdots$ & $\cdots$ & 1.200 & 1.387 & $\cdots$ & $\cdots$ & $\cdots$ & $\cdots$ & $\cdots$ \\
\hline BPS CS 29493-0066 & 3.029 & $\cdots$ & $\cdots$ & 10.000 & 3.029 & -0.3 & -1.1 & 2.7 & 3.7 & SPM \\
\hline BPS CS 29493-0061 & 0.553 & $\cdots$ & $\cdots$ & 0.900 & 0.553 & -65.7 & -102.0 & 2.3 & 2.9 & SPM \\
\hline BPS CS 22881-0017 & 1.970 & $\cdots$ & $\cdots$ & 5.300 & 1.970 & 3.6 & -7.6 & 2.3 & 2.3 & SPM \\
\hline BPS CS 29493-0060 & $\cdots$ & $\cdots$ & $\cdots$ & 1.800 & 1.800 & 17.9 & -53.1 & 4.8 & 4.7 & SPM \\
\hline $\mathrm{BD}+473617$ & 0.233 & $\cdots$ & $\cdots$ & 0.365 & 0.233 & -4.0 & -6.7 & 2.8 & 3.0 & $\mathrm{ACT}, \mathrm{STN}$ \\
\hline BPS CS 22965-0016 & 7.191 & $\cdots$ & $\cdots$ & 4.000 & 7.191 & $\cdots$ & $\ldots$ & $\cdots$ & $\cdots$ & $\ldots$ \\
\hline HD 208360 & 0.117 & 0.212 & 0.20 & 0.229 & 0.117 & 36.8 & -254.3 & 0.7 & 0.5 & $\mathrm{HIP}, \mathrm{ACT}$ \\
\hline BPS CS 29493-0095 & 1.269 & $\cdots$ & $\cdots$ & 1.500 & 1.269 & 15.3 & -41.8 & 2.2 & 2.1 & SPM \\
\hline BPS CS 29493-0094 & 1.137 & $\cdots$ & $\cdots$ & 1.400 & 1.137 & 19.8 & -15.2 & 2.3 & 2.3 & SPM \\
\hline BPS CS 22951-0077 & 4.229 & $\cdots$ & $\cdots$ & 3.900 & 4.229 & $\cdots$ & $\cdots$ & $\cdots$ & $\cdots$ & $\cdots$ \\
\hline BPS CS 22956-0050 & 6.000 & $\cdots$ & $\cdots$ & 4.400 & 6.000 & $\cdots$ & $\cdots$ & $\cdots$ & $\cdots$ & $\cdots$ \\
\hline BPS CS 22956-0052 & 1.586 & $\cdots$ & $\cdots$ & 1.100 & 1.586 & $\ldots$ & $\ldots$ & $\cdots$ & $\cdots$ & $\ldots$ \\
\hline BPS CS 22881-0010 & 1.441 & $\cdots$ & $\cdots$ & 1.500 & 1.441 & -7.8 & -70.5 & 1.9 & 1.8 & SPM \\
\hline BPS CS 22881-0006 & 1.446 & $\cdots$ & $\cdots$ & 1.400 & 1.446 & -2.5 & -15.8 & 2.3 & 2.2 & SPM \\
\hline BPS CS 29493-0090 & 9.101 & $\cdots$ & $\cdots$ & 7.500 & 9.101 & 0.1 & -2.6 & 4.6 & 4.7 & SPM \\
\hline BPS CS 22965-0035 & 1.624 & $\cdots$ & $\cdots$ & 0.900 & 1.624 & 59.8 & 1.8 & 5 & 5 & NPM \\
\hline BPS CS 22881-0008 & 11.488 & $\cdots$ & $\cdots$ & 10.100 & 11.488 & 1.0 & -8.4 & 2.2 & 2.2 & SPM \\
\hline BPS CS 22965-0029 & $\cdots$ & $\cdots$ & $\cdots$ & 1.200 & 1.200 & $\cdots$ & $\cdots$ & $\cdots$ & $\cdots$ & $\cdots$ \\
\hline $\mathrm{BD}+463527$ & 0.250 & $\cdots$ & $\cdots$ & 0.277 & 0.250 & 77.2 & -3.2 & 2.2 & 1.2 & $\mathrm{ACT}, \mathrm{STN}$ \\
\hline
\end{tabular}


TABLE 1B. (continued)

\begin{tabular}{|c|c|c|c|c|c|c|c|c|c|c|}
\hline $\begin{array}{l}\text { Star } \\
(1)\end{array}$ & $\begin{array}{c}D_{p h o} \\
(\mathrm{kpc}) \\
(2)\end{array}$ & $\begin{array}{c}D_{H I P} \\
(\mathrm{kpc}) \\
(3)\end{array}$ & $\begin{array}{c}\sigma_{\pi_{H I P}} / \pi_{H I P} \\
(4)\end{array}$ & $\begin{array}{c}D_{B S L} \\
(\mathrm{kpc}) \\
(5)\end{array}$ & $\begin{array}{c}D_{\text {adopt }} \\
(\mathrm{kpc}) \\
\quad(6)\end{array}$ & $\begin{array}{c}\mu_{\alpha^{*}} \\
(\mathrm{mas} / \mathrm{yr}) \\
(7)\end{array}$ & $\begin{array}{c}\mu_{\delta} \\
\text { (mas/yr) } \\
(8)\end{array}$ & $\begin{array}{c}\sigma_{\mu_{\alpha *}} \\
(\mathrm{mas} / \mathrm{yr}) \\
(9)\end{array}$ & $\begin{array}{c}\sigma_{\mu_{\delta}} \\
(\mathrm{mas} / \mathrm{yr}) \\
(10)\end{array}$ & Sou \\
\hline BPS CS 29493-0092 & 5.984 & $\cdots$ & $\cdots$ & 5.400 & 5.984 & -4.4 & 0.5 & 2.5 & 2.5 & SPM \\
\hline BPS CS 22951-0117 & 0.921 & $\ldots$ & $\ldots$ & 0.900 & 0.921 & 10. & -37 & 5.4 & 5.4 & STN \\
\hline BPS CS 22892-0014 & 1.005 & $\ldots$ & $\ldots$ & 1.100 & 1.005 & $\ldots$ & $\ldots$ & $\ldots$ & $\ldots$ & $\ldots$ \\
\hline $\mathrm{V}^{*} \mathrm{TZ}$ Aqr & 1.877 & $\cdots$ & $\cdots$ & 1.870 & 1.877 & 8.5 & -9.0 & 2.8 & 2.8 & STN,NPM \\
\hline BPS CS 22881-0028 & 1.190 & $\cdots$ & $\cdots$ & 1.200 & 1.190 & 41.0 & -20.4 & 2.3 & 2.3 & SPM \\
\hline $\mathrm{V}^{*} \mathrm{BV}$ Aqr & 1.095 & 0.267 & 0.57 & $\ldots$ & 1.095 & -2.1 & -20.6 & 1.5 & 1.2 & $\mathrm{HIP}, \mathrm{ACT}$ \\
\hline BPS CS 22881-0032 & 1.986 & $\ldots$ & $\ldots$ & 2.000 & 1.986 & -0.8 & -3.3 & 2.3 & 2.3 & SPM \\
\hline BPS CS 22965-0052 & 1.159 & $\cdots$ & $\cdots$ & 3.300 & 1.159 & $\ldots$ & $\cdots$ & $\cdots$ & $\cdots$ & $\ldots$ \\
\hline BPS CS 22951-0108 & 1.052 & $\cdots$ & $\cdots$ & 1.200 & 1.052 & $\cdots$ & $\cdots$ & $\cdots$ & $\cdots$ & $\cdots$ \\
\hline BPS CS 22956-0081 & 1.854 & $\cdots$ & $\cdots$ & 1.400 & 1.854 & $\cdots$ & $\cdots$ & $\cdots$ & $\cdots$ & $\cdots$ \\
\hline $\mathrm{BD}+463563$ & 0.290 & $\cdots$ & $\cdots$ & 0.552 & 0.290 & 2.4 & -3.0 & 1.1 & 1.5 & $\mathrm{ACT}, \mathrm{STN}$ \\
\hline $\mathrm{V}^{*} \mathrm{SX}$ Gru & $\cdots$ & $\cdots$ & $\cdots$ & 0.900 & 0.900 & $\cdots$ & $\ldots$ & $\cdots$ & $\cdots$ & $\ldots$ \\
\hline BPS CS 22951-0115 & 0.978 & $\cdots$ & $\cdots$ & 0.900 & 0.978 & $\cdots$ & $\cdots$ & $\cdots$ & $\cdots$ & $\cdots$ \\
\hline BPS CS 22892-0005 & 0.929 & $\cdots$ & $\cdots$ & 1.000 & 0.929 & $\cdots$ & $\cdots$ & $\cdots$ & $\cdots$ & $\cdots$ \\
\hline BPS CS 22965-0043 & 0.743 & $\cdots$ & $\cdots$ & 0.800 & 0.743 & $\cdots$ & $\cdots$ & $\cdots$ & $\cdots$ & $\cdots$ \\
\hline BPS CS 22965-0054 & 22.993 & $\ldots$ & $\ldots$ & 1.700 & 22.993 & $\ldots$ & $\ldots$ & $\cdots$ & $\ldots$ & $\ldots$ \\
\hline BPS CS 22892-0024 & 1.199 & $\ldots$ & $\ldots$ & 1.900 & 1.199 & $\ldots$ & $\ldots$ & $\ldots$ & $\ldots$ & $\ldots$ \\
\hline $\mathrm{BD}+473694$ & 0.291 & $\cdots$ & $\cdots$ & 0.385 & 0.291 & 26.8 & 13.3 & 0.9 & 0.9 & $\mathrm{ACT}, \mathrm{STN}$ \\
\hline BPS CS 22965-0068 & $\cdots$ & $\cdots$ & $\cdots$ & 1.200 & 1.200 & $\cdots$ & $\cdots$ & $\cdots$ & $\cdots$ & $\ldots$ \\
\hline BPS CS 22956-0062 & 1.267 & $\cdots$ & $\cdots$ & 3.300 & 1.267 & $\cdots$ & $\cdots$ & $\cdots$ & $\cdots$ & $\cdots$ \\
\hline BPS CS 22892-0025 & 1.043 & $\ldots$ & $\ldots$ & 1.000 & 1.043 & $\ldots$ & $\ldots$ & $\ldots$ & $\ldots$ & $\ldots$ \\
\hline BPS CS 22881-0036 & 0.903 & $\ldots$ & $\ldots$ & 1.500 & 0.903 & 32.4 & -88.1 & 5.1 & 6.2 & SPM \\
\hline BPS CS 22960-0010 & 2.995 & $\cdots$ & $\cdots$ & 3.100 & 2.995 & $\cdots$ & $\ldots$ & $\cdots$ & $\cdots$ & $\ldots$ \\
\hline BPS CS 22881-0040 & 3.394 & $\cdots$ & $\cdots$ & 4.900 & 3.394 & 2.4 & -9.6 & 3.7 & 2.7 & SPM,STN \\
\hline BPS CS 22892-0041 & 1.107 & $\cdots$ & $\cdots$ & 1.100 & 1.107 & $\cdots$ & $\cdots$ & $\cdots$ & $\cdots$ & $\ldots$ \\
\hline BPS CS 22881-0039 & 9.608 & $\ldots$ & $\ldots$ & 8.100 & 9.608 & -6.7 & -9.7 & 2.0 & 2.0 & SPM \\
\hline HD 210295 & 0.595 & 0.394 & 0.55 & 0.510 & 0.595 & -40.1 & -84.1 & 0.8 & 0.7 & $\mathrm{HIP}, \mathrm{ACT}$ \\
\hline BPS CS 22965-0076 & 1.586 & $\ldots$ & $\cdots$ & 1.000 & 1.586 & $\ldots$ & $\ldots$ & $\cdots$ & $\cdots$ & $\ldots$ \\
\hline BPS CS 22965-0087 & 1.596 & $\cdots$ & $\cdots$ & 1.400 & 1.596 & $\cdots$ & $\cdots$ & $\cdots$ & $\cdots$ & $\cdots$ \\
\hline BPS CS 22960-0029 & 0.578 & $\cdots$ & $\cdots$ & 1.100 & 0.578 & $\cdots$ & $\cdots$ & $\cdots$ & $\cdots$ & $\cdots$ \\
\hline BPS CS 22965-0075 & 1.095 & $\cdots$ & $\cdots$ & 1.200 & 1.095 & $\cdots$ & $\cdots$ & $\cdots$ & $\cdots$ & $\cdots$ \\
\hline BPS CS 22892-0046 & 5.929 & $\cdots$ & $\ldots$ & 1.100 & 5.929 & $\ldots$ & $\ldots$ & $\cdots$ & $\cdots$ & $\ldots$ \\
\hline BPS CS 29512-0007 & 0.580 & $\cdots$ & $\cdots$ & 0.900 & 0.580 & $\cdots$ & $\cdots$ & $\cdots$ & $\cdots$ & $\cdots$ \\
\hline BPS CS 22956-0085 & 1.128 & $\cdots$ & $\cdots$ & 1.200 & 1.128 & $\cdots$ & $\ldots$ & $\cdots$ & $\cdots$ & $\ldots$ \\
\hline $\mathrm{V}^{*}$ VV Peg & 1.703 & $\ldots$ & $\ldots$ & 1.607 & 1.703 & 13.1 & -13.4 & 2.8 & 2.8 & STN,NPM \\
\hline BPS CS 22886-0008 & 1.040 & $\cdots$ & $\cdots$ & 1.300 & 1.040 & $\cdots$ & $\ldots$ & $\cdots$ & $\cdots$ & $\ldots$ \\
\hline BPS CS 22886-0012 & 1.120 & $\cdots$ & $\cdots$ & 1.300 & 1.120 & $\cdots$ & $\ldots$ & $\cdots$ & $\cdots$ & $\ldots$ \\
\hline BPS CS 29512-0030 & 1.400 & $\cdots$ & $\cdots$ & 1.400 & 1.400 & 59.5 & 13.0 & 5 & 5 & NPM \\
\hline BPS CS 22881-0060 & 1.139 & $\ldots$ & $\ldots$ & 1.100 & 1.139 & 27.9 & -64.4 & 1.7 & 2.3 & SPM \\
\hline HD 211075 & 0.557 & 0.369 & 0.41 & 0.476 & 0.557 & 4.5 & -4.6 & 0.7 & 0.8 & $\mathrm{HIP}, \mathrm{ACT}$ \\
\hline
\end{tabular}


TABLE 1B. (continued)

\begin{tabular}{|c|c|c|c|c|c|c|c|c|c|c|}
\hline $\begin{array}{l}\text { Star } \\
(1)\end{array}$ & $\begin{array}{c}D_{p h o} \\
(\mathrm{kpc}) \\
(2)\end{array}$ & $\begin{array}{c}D_{H I P} \\
(\mathrm{kpc}) \\
(3)\end{array}$ & $\begin{array}{c}\sigma_{\pi_{H I P}} / \pi_{H I P} \\
(4)\end{array}$ & $\begin{array}{c}D_{B S L} \\
(\mathrm{kpc}) \\
(5)\end{array}$ & $\begin{array}{c}D_{\text {adopt }} \\
(\mathrm{kpc}) \\
(6)\end{array}$ & $\begin{array}{c}\mu_{\alpha^{*}} \\
(\mathrm{mas} / \mathrm{yr}) \\
(7)\end{array}$ & $\begin{array}{c}\mu_{\delta} \\
\text { (mas/yr) } \\
(8)\end{array}$ & $\begin{array}{c}\sigma_{\mu_{\alpha^{*}}} \\
(\mathrm{mas} / \mathrm{yr}) \\
(9)\end{array}$ & $\begin{array}{c}\sigma_{\mu_{\delta}} \\
(\mathrm{mas} / \mathrm{yr}) \\
(10)\end{array}$ & Soul \\
\hline BPS CS 29512-0031 & $\cdots$ & $\cdots$ & $\cdots$ & 1.600 & 1.600 & $\ldots$ & $\ldots$ & $\ldots$ & $\ldots$ & $\ldots$ \\
\hline $\mathrm{V}^{*} \mathrm{YZ}$ Aqr & 2.436 & $\ldots$ & $\ldots$ & 0.700 & 2.436 & -12.5 & 2.1 & 5 & 5 & NPM \\
\hline BPS CS 29512-0032 & $\ldots$ & $\ldots$ & $\ldots$ & $\ldots$ & $\ldots$ & -5.2 & 6.4 & 3.4 & 3.4 & STN,NPM \\
\hline BPS CS 22960-0027 & 1.043 & $\cdots$ & $\cdots$ & 0.800 & 1.043 & $\ldots$ & $\ldots$ & $\cdots$ & $\cdots$ & $\ldots$ \\
\hline HD 235766 & 0.923 & $\cdots$ & $\cdots$ & 0.619 & 0.923 & -41.9 & -39.0 & 0.9 & 0.9 & $\mathrm{ACT}, \mathrm{STN}$ \\
\hline BPS CS 22960-0036 & 1.148 & $\cdots$ & $\cdots$ & 0.700 & 1.148 & $\ldots$ & $\ldots$ & $\cdots$ & $\ldots$ & $\ldots$ \\
\hline BPS CS 22956-0105 & $\ldots$ & $\cdots$ & $\cdots$ & 1.200 & 1.200 & $\cdots$ & $\cdots$ & $\cdots$ & $\cdots$ & $\cdots$ \\
\hline BPS CS 29512-0024 & 0.544 & $\cdots$ & $\cdots$ & 0.800 & 0.544 & $\cdots$ & $\cdots$ & $\cdots$ & $\cdots$ & $\ldots$ \\
\hline $\mathrm{V}^{*} \mathrm{DH}$ Peg & 0.534 & 6.667 & 9.47 & 0.568 & 0.534 & 21.0 & 1.0 & 1.5 & 0.8 & HIP,ACT,, \\
\hline BPS CS 22886-0029 & 0.658 & $\cdots$ & $\cdots$ & 1.000 & 0.658 & $\cdots$ & $\cdots$ & $\cdots$ & $\cdots$ & $\cdots$ \\
\hline BPS CS 29512-0037 & 1.022 & $\cdots$ & $\cdots$ & 4.100 & 1.022 & $\cdots$ & $\cdots$ & $\cdots$ & $\cdots$ & $\cdots$ \\
\hline BPS CS 22886-0022 & 0.887 & $\cdots$ & $\cdots$ & 0.900 & 0.887 & $\cdots$ & $\cdots$ & $\cdots$ & $\cdots$ & $\cdots$ \\
\hline BPS CS 22956-0102 & 1.614 & $\cdots$ & $\cdots$ & 1.400 & 1.614 & $\cdots$ & $\cdots$ & $\cdots$ & $\cdots$ & $\cdots$ \\
\hline BPS CS 22881-0070 & 1.326 & $\cdots$ & $\cdots$ & 1.400 & 1.326 & 9.1 & -3.0 & 1.7 & 4.9 & SPM \\
\hline $\mathrm{V}^{*} \mathrm{TY}$ Gru & 5.158 & $\cdots$ & $\cdots$ & 5.400 & 5.158 & 9.0 & -0.3 & 4.7 & 1.9 & SPM \\
\hline BPS CS 22886-0018 & 1.266 & $\cdots$ & $\cdots$ & 1.200 & 1.266 & $\cdots$ & $\ldots$ & $\ldots$ & $\ldots$ & $\ldots$ \\
\hline BPS CS 22956-0106 & 1.302 & $\cdots$ & $\cdots$ & 1.100 & 1.302 & $\cdots$ & $\cdots$ & $\cdots$ & $\cdots$ & $\cdots$ \\
\hline BPS CS 29512-0026 & 1.394 & $\cdots$ & $\cdots$ & 1.200 & 1.394 & $\cdots$ & $\cdots$ & $\cdots$ & $\cdots$ & $\cdots$ \\
\hline BPS CS 22960-0048 & 7.221 & $\cdots$ & $\cdots$ & 4.600 & 7.221 & $\cdots$ & $\cdots$ & $\cdots$ & $\cdots$ & $\cdots$ \\
\hline BPS CS 22892-0052 & 5.768 & $\cdots$ & $\cdots$ & 4.700 & 5.768 & $\cdots$ & $\cdots$ & $\cdots$ & $\cdots$ & $\cdots$ \\
\hline BPS CS 22886-0028 & 1.895 & $\cdots$ & $\cdots$ & 2.000 & 1.895 & $\cdots$ & $\cdots$ & $\cdots$ & $\cdots$ & $\ldots$ \\
\hline BPS CS 22886-0026 & 1.405 & $\ldots$ & $\ldots$ & 1.400 & 1.405 & -20.8 & -18.4 & 5 & 5 & NPM \\
\hline BPS CS 22960-0040 & 1.810 & $\cdots$ & $\cdots$ & 1.100 & 1.810 & $\ldots$ & $\ldots$ & $\cdots$ & $\cdots$ & $\cdots$ \\
\hline BPS CS 22960-0037 & 1.766 & $\cdots$ & $\cdots$ & 1.200 & 1.766 & $\cdots$ & $\cdots$ & $\cdots$ & $\cdots$ & $\cdots$ \\
\hline BPS CS 22892-0056 & 0.893 & $\cdots$ & $\cdots$ & 1.000 & 0.893 & $\cdots$ & $\cdots$ & $\cdots$ & $\cdots$ & $\cdots$ \\
\hline BPS CS 22956-0110 & 7.651 & $\cdots$ & $\cdots$ & 1.400 & 7.651 & $\cdots$ & $\cdots$ & $\cdots$ & $\cdots$ & $\cdots$ \\
\hline BPS CS 29512-0043 & 0.671 & $\cdots$ & $\cdots$ & 0.900 & 0.671 & $\cdots$ & $\ldots$ & $\cdots$ & $\cdots$ & $\cdots$ \\
\hline HD 211744 & 0.182 & 0.394 & 0.54 & 0.358 & 0.182 & 50.6 & -35.2 & 1.5 & 0.9 & HIP,ACT,, \\
\hline BPS CS 22886-0044 & 1.335 & $\cdots$ & $\cdots$ & 1.000 & 1.335 & $\cdots$ & $\ldots$ & $\cdots$ & $\cdots$ & $\cdots$ \\
\hline $\mathrm{V}^{*} \mathrm{UW}$ Gru & 3.281 & $\cdots$ & $\cdots$ & $\cdots$ & 3.281 & -20 & -12 & 5.9 & 5.9 & STN \\
\hline BPS CS 22886-0042 & 5.620 & $\cdots$ & $\cdots$ & 4.100 & 5.620 & $\cdots$ & $\cdots$ & $\cdots$ & $\cdots$ & $\cdots$ \\
\hline BPS CS 22960-0041 & 1.887 & $\cdots$ & $\cdots$ & 1.100 & 1.887 & $\cdots$ & $\cdots$ & $\cdots$ & $\cdots$ & $\cdots$ \\
\hline BPS CS 29512-0048 & 0.670 & $\cdots$ & $\cdots$ & 1.000 & 0.670 & $\cdots$ & $\cdots$ & $\cdots$ & $\cdots$ & $\cdots$ \\
\hline BPS CS 22956-0126 & 1.875 & $\cdots$ & $\cdots$ & 1.200 & 1.875 & $\cdots$ & . & $\cdots$ & $\cdots$ & $\cdots$ \\
\hline BPS CS 22875-0004 & 0.987 & $\cdots$ & $\cdots$ & 0.900 & 0.987 & 80.1 & -66.2 & 2.6 & 4.8 & SPM \\
\hline BPS CS 22886-0055 & 1.304 & $\cdots$ & $\cdots$ & 1.400 & 1.304 & $\cdots$ & $\cdots$ & $\cdots$ & $\cdots$ & $\cdots$ \\
\hline $\mathrm{V}^{*}$ CQ Lac & 2.079 & $\cdots$ & $\cdots$ & $\ldots$ & 2.079 & 3.4 & -1.5 & 5 & 5 & NPM \\
\hline BPS CS 29512-0047 & $\cdots$ & $\cdots$ & $\cdots$ & 1.600 & 1.600 & $\cdots$ & $\cdots$ & $\cdots$ & $\cdots$ & $\cdots$ \\
\hline BPS CS 29512-0052 & 0.659 & $\cdots$ & $\cdots$ & 0.900 & 0.659 & $\cdots$ & $\cdots$ & $\cdots$ & $\cdots$ & $\cdots$ \\
\hline BPS CS 22956-0116 & 0.683 & $\cdots$ & $\cdots$ & 0.800 & 0.683 & $\cdots$ & $\cdots$ & $\cdots$ & $\cdots$ & $\cdots$ \\
\hline
\end{tabular}


TABLE 1B. (continued)

\begin{tabular}{|c|c|c|c|c|c|c|c|c|c|c|}
\hline $\begin{array}{l}\text { Star } \\
(1)\end{array}$ & $\begin{array}{c}D_{p h o} \\
(\mathrm{kpc}) \\
(2)\end{array}$ & $\begin{array}{c}D_{H I P} \\
(\mathrm{kpc}) \\
(3)\end{array}$ & $\begin{array}{c}\sigma_{\pi_{H I P}} / \pi_{H I P} \\
(4)\end{array}$ & $\begin{array}{c}D_{B S L} \\
(\mathrm{kpc}) \\
(5)\end{array}$ & $\begin{array}{c}D_{\text {adopt }} \\
\text { (kpc) } \\
(6)\end{array}$ & $\begin{array}{c}\mu_{\alpha^{*}} \\
(\mathrm{mas} / \mathrm{yr}) \\
(7)\end{array}$ & $\begin{array}{c}\mu_{\delta} \\
(\mathrm{mas} / \mathrm{yr}) \\
(8)\end{array}$ & $\begin{array}{c}\sigma_{\mu_{\alpha^{*}}} \\
(\mathrm{mas} / \mathrm{yr}) \\
(9)\end{array}$ & $\begin{array}{c}\sigma_{\mu_{\delta}} \\
(\mathrm{mas} / \mathrm{yr}) \\
(10)\end{array}$ & Sou \\
\hline BPS CS 22960-0060 & 2.452 & $\cdots$ & $\cdots$ & 1.500 & 2.452 & 1.8 & -30.1 & 3.0 & 3.1 & SPM \\
\hline BPS CS 29512-0069 & 1.543 & $\ldots$ & $\ldots$ & 2.200 & 1.543 & $\ldots$ & $\ldots$ & $\ldots$ & $\ldots$ & $\ldots$ \\
\hline BPS CS 22886-0043 & 6.603 & $\ldots$ & $\ldots$ & 6.400 & 6.603 & $\ldots$ & $\ldots$ & $\ldots$ & $\ldots$ & $\ldots$ \\
\hline BPS CS 22875-0012 & 1.290 & $\ldots$ & $\ldots$ & 1.300 & 1.290 & -1.6 & -66.0 & 2.1 & 2.1 & SPM \\
\hline BPS CS 22960-0062 & 0.664 & $\ldots$ & $\ldots$ & 0.900 & 0.664 & 17.4 & -10.8 & 3.0 & 6.4 & SPM \\
\hline BPS CS 22960-0064 & 2.367 & $\ldots$ & $\ldots$ & 3.800 & 2.367 & 7.4 & -11.6 & 4.8 & 4.7 & SPM \\
\hline BPS CS 22960-0068 & 2.304 & $\ldots$ & $\ldots$ & 1.600 & 2.304 & $\ldots$ & $\ldots$ & $\ldots$ & $\ldots$ & $\ldots$ \\
\hline HD 212516 & 1.024 & 0.840 & 1.03 & 0.570 & 1.024 & 3.2 & -6.2 & 0.8 & 0.9 & HIP,ACT, \\
\hline BPS CS 22956-0117 & $\ldots$ & $\ldots$ & $\ldots$ & 1.100 & 1.100 & $\ldots$ & $\ldots$ & $\ldots$ & $\ldots$ & $\ldots$ \\
\hline BPS CS 29512-0066 & 0.777 & $\cdots$ & $\cdots$ & 1.200 & 0.777 & $\ldots$ & $\ldots$ & $\ldots$ & $\ldots$ & $\ldots$ \\
\hline BPS CS 22886-0064 & 1.977 & $\ldots$ & $\ldots$ & 2.100 & 1.977 & $\ldots$ & $\ldots$ & $\ldots$ & $\ldots$ & $\ldots$ \\
\hline BPS CS $22960-0080$ & 2.184 & $\ldots$ & $\ldots$ & 1.200 & 2.184 & $\ldots$ & $\ldots$ & $\ldots$ & $\ldots$ & $\ldots$ \\
\hline BPS CS 29512-0072 & $\ldots$ & $\ldots$ & $\ldots$ & 1.500 & 1.500 & $\ldots$ & $\ldots$ & $\ldots$ & $\ldots$ & $\ldots$ \\
\hline BPS CS 22960-0078 & 0.990 & $\ldots$ & $\ldots$ & 0.900 & 0.990 & $\ldots$ & $\ldots$ & $\ldots$ & $\ldots$ & $\ldots$ \\
\hline BPS CS 22956-0114 & 3.319 & $\ldots$ & $\ldots$ & 2.300 & 3.319 & $\ldots$ & $\ldots$ & $\ldots$ & $\ldots$ & $\ldots$ \\
\hline $\mathrm{V}^{*} \mathrm{UY}$ Gru & 6.081 & $\ldots$ & $\ldots$ & 5.700 & 6.081 & 3.6 & 4.9 & 2.1 & 2.1 & SPM \\
\hline BPS CS 22960-0089 & 1.639 & $\ldots$ & $\ldots$ & 1.200 & 1.639 & $\ldots$ & $\ldots$ & $\ldots$ & $\ldots$ & $\ldots$ \\
\hline BPS CS 22886-0080 & 0.857 & $\ldots$ & $\cdots$ & 1.000 & 0.857 & $\ldots$ & $\ldots$ & $\ldots$ & $\ldots$ & $\ldots$ \\
\hline BPS CS 29512-0083 & 0.415 & $\ldots$ & $\cdots$ & 0.700 & 0.415 & $\cdots$ & $\ldots$ & $\ldots$ & $\ldots$ & $\cdots$ \\
\hline BPS CS 22956-0121 & 1.572 & $\cdots$ & $\cdots$ & 1.600 & 1.572 & $\cdots$ & $\cdots$ & $\cdots$ & $\cdots$ & $\cdots$ \\
\hline $\mathrm{V}^{*} \mathrm{AE}$ Peg & 2.622 & $\ldots$ & $\ldots$ & $\ldots$ & 2.622 & 0. & -40 & 4.6 & 4.6 & STN \\
\hline BPS CS 29512-0076 & 0.870 & $\ldots$ & $\ldots$ & 0.900 & 0.870 & -7.1 & 0.6 & 5 & 5 & NPM \\
\hline BPS CS 22960-0088 & $\ldots$ & $\ldots$ & $\ldots$ & 1.600 & 1.600 & $\ldots$ & $\ldots$ & $\ldots$ & $\ldots$ & $\ldots$ \\
\hline $\mathrm{V}^{*} \mathrm{BN}$ Aqr & 2.250 & $\ldots$ & $\ldots$ & $\ldots$ & 2.250 & 9.7 & -32.6 & 5 & 5 & NPM \\
\hline BPS CS $22875-0028$ & 0.560 & $\ldots$ & $\ldots$ & 0.800 & 0.560 & 34.4 & -23.5 & 4.0 & 2.8 & SPM \\
\hline BPS CS 29512-0073 & 1.562 & $\cdots$ & $\ldots$ & 1.800 & 1.562 & $\ldots$ & $\ldots$ & $\ldots$ & $\ldots$ & $\ldots$ \\
\hline BPS CS 22875-0029 & 4.560 & $\ldots$ & $\ldots$ & 4.100 & 4.560 & 11.8 & -10.6 & 4.7 & 3.4 & SPM,STN \\
\hline BPS CS 22960-0105 & 0.403 & $\ldots$ & $\ldots$ & 0.700 & 0.403 & $\ldots$ & $\ldots$ & $\ldots$ & $\ldots$ & $\ldots$ \\
\hline BPS CS 22875-0026 & 0.832 & $\ldots$ & $\ldots$ & 1.200 & 0.832 & 82.7 & -38.2 & 2.3 & 2.2 & SPM \\
\hline HD 213487 & 0.479 & 0.220 & 0.38 & 0.573 & 0.479 & 4.8 & -4.9 & 1.1 & 0.8 & $\mathrm{HIP}, \mathrm{ACT}$, \\
\hline HD 213467 & 0.141 & 0.209 & 0.24 & 0.186 & 0.141 & 289.8 & -156.5 & 0.8 & 0.7 & HIP,SPM, \\
\hline HD 213657 & 0.101 & 0.176 & 0.27 & 0.101 & 0.101 & -61.1 & -251.0 & 1.0 & 0.7 & HIP,SPM, \\
\hline BPS CS 22887-0005 & 1.213 & $\ldots$ & $\ldots$ & 1.500 & 1.213 & $\ldots$ & $\ldots$ & $\ldots$ & $\ldots$ & $\ldots$ \\
\hline BPS CS 22887-0007 & $\ldots$ & $\ldots$ & $\ldots$ & 2.100 & 2.100 & $\ldots$ & $\ldots$ & $\ldots$ & $\ldots$ & $\ldots$ \\
\hline $\mathrm{V}^{*}$ AA Aqr & 2.212 & $\ldots$ & $\ldots$ & $\ldots$ & 2.212 & 21.1 & -3.0 & 5 & 5 & NPM \\
\hline BPS CS 22887-0006 & 2.168 & $\ldots$ & $\ldots$ & 1.100 & 2.168 & $\ldots$ & $\ldots$ & $\ldots$ & $\ldots$ & $\ldots$ \\
\hline HD 214161 & 0.619 & $<0$ & $\ldots$ & 0.612 & 0.619 & 50.9 & -24.9 & 1.0 & 0.8 & HIP,SPM, \\
\hline HD 214362 & 0.503 & 0.690 & 0.91 & 0.503 & 0.503 & 173.5 & -54.8 & 1.4 & 0.7 & HIP,ACT, \\
\hline BPS CS 22887-0018 & $\ldots$ & $\ldots$ & $\ldots$ & 1.100 & 1.100 & $\ldots$ & $\ldots$ & $\cdots$ & $\cdots$ & $\ldots$ \\
\hline BPS CS 22875-0038 & 0.670 & $\ldots$ & $\cdots$ & 0.800 & 0.670 & -14.9 & -38.1 & 4.5 & 5.0 & SPM \\
\hline
\end{tabular}


TABle 1B. (continued)

\begin{tabular}{|c|c|c|c|c|c|c|c|c|c|c|}
\hline $\begin{array}{l}\text { Star } \\
(1)\end{array}$ & $\begin{array}{c}D_{p h o} \\
(\mathrm{kpc}) \\
(2)\end{array}$ & $\begin{array}{c}D_{H I P} \\
(\mathrm{kpc}) \\
(3)\end{array}$ & $\begin{array}{c}\sigma_{\pi_{H I P}} / \pi_{H I P} \\
(4)\end{array}$ & $\begin{array}{c}D_{B S L} \\
(\mathrm{kpc}) \\
(5)\end{array}$ & $\begin{array}{c}D_{\text {adopt }} \\
(\mathrm{kpc}) \\
(6)\end{array}$ & $\begin{array}{c}\mu_{\alpha^{*}} \\
(\mathrm{mas} / \mathrm{yr}) \\
(7)\end{array}$ & $\begin{array}{c}\mu_{\delta} \\
(\mathrm{mas} / \mathrm{yr}) \\
(8)\end{array}$ & $\begin{array}{c}\sigma_{\mu_{\alpha^{*}}} \\
(\mathrm{mas} / \mathrm{yr}) \\
(9)\end{array}$ & $\begin{array}{c}\sigma_{\mu_{\delta}} \\
(\mathrm{mas} / \mathrm{yr}) \\
(10)\end{array}$ & \\
\hline $\mathrm{V}^{*} \mathrm{RZ}$ Cep & 0.409 & 4.545 & 4.95 & 0.360 & 0.409 & 91.7 & 188.9 & 1.3 & 1.0 & HIP,$A$ \\
\hline HD 214925 & 2.149 & 0.610 & 0.84 & 0.240 & 2.149 & 18.5 & -40.1 & 1.0 & 0.9 & HIP,$A$ \\
\hline $\mathrm{V}^{*} \mathrm{RW}$ Gru & 2.381 & $\ldots$ & $\ldots$ & 2.178 & 2.381 & 4. & -39 . & 5.5 & 5.5 & STN \\
\hline $\mathrm{V}^{*} \mathrm{DX}$ Cep & 2.350 & $\ldots$ & $\ldots$ & $\ldots$ & 2.350 & 17.7 & 8.4 & 2.8 & 2.8 & STN, \\
\hline BPS CS 22887-0048 & 0.593 & $\ldots$ & $\ldots$ & 0.900 & 0.593 & -25 . & -87 & 3.9 & 3.9 & STN \\
\hline HD 215601 & 0.278 & 0.662 & 0.78 & 0.301 & 0.278 & 55.7 & -148.3 & 0.9 & 0.7 & HIP,, \\
\hline BPS CS 22887-0047 & 0.701 & $\ldots$ & $\ldots$ & 1.000 & 0.701 & $\ldots$ & $\ldots$ & $\ldots$ & $\ldots$ & $\ldots$ \\
\hline HD 215801 & 0.159 & 0.207 & 0.30 & 0.102 & 0.159 & 33.1 & -306.2 & 1.4 & 0.7 & $\mathrm{HIP}, t$ \\
\hline HD 216143 & 0.687 & 0.318 & 0.38 & 0.680 & 0.687 & -69.5 & -106.9 & 0.9 & 0.6 & HIP,$A$ \\
\hline BPS CS 22938-0027 & 0.487 & $\ldots$ & $\ldots$ & 0.900 & 0.487 & $\ldots$ & $\ldots$ & $\ldots$ & $\cdots$ & $\ldots$ \\
\hline $\mathrm{V}^{*} \mathrm{BH}$ Peg & 0.816 & $<0$ & $\ldots$ & 0.805 & 0.816 & -25.9 & -63.7 & 0.8 & 0.8 & HIP,$A$ \\
\hline BPS CS 22887-0054 & $\ldots$ & $\ldots$ & $\ldots$ & 0.800 & 0.800 & $\ldots$ & $\ldots$ & $\ldots$ & $\ldots$ & $\ldots$ \\
\hline BPS CS 22893-0005 & 1.760 & $\ldots$ & $\ldots$ & 1.800 & 1.760 & $\ldots$ & $\ldots$ & $\ldots$ & $\ldots$ & $\ldots$ \\
\hline $\mathrm{V}^{*} \mathrm{BO}$ Aqr & 1.967 & $\ldots$ & $\ldots$ & 1.873 & 1.967 & -8.2 & -12.1 & 5 & 5 & NPM \\
\hline BPS CS 22893-0006 & 1.087 & $\ldots$ & $\ldots$ & 1.500 & 1.087 & $\ldots$ & $\ldots$ & $\ldots$ & $\ldots$ & $\ldots$ \\
\hline BPS CS 22893-0010 & 4.057 & $\ldots$ & $\ldots$ & 3.700 & 4.057 & $\ldots$ & $\ldots$ & $\ldots$ & $\ldots$ & $\ldots$ \\
\hline BPS CS 30493-0016 & 4.516 & $\ldots$ & $\ldots$ & 5.700 & 4.516 & 1.9 & -4.7 & 2.7 & 2.7 & SPM \\
\hline BPS CS 22893-0011 & 1.164 & $\ldots$ & $\ldots$ & 1.300 & 1.164 & $\ldots$ & $\ldots$ & $\ldots$ & $\ldots$ & $\ldots$ \\
\hline BPS CS 30493-0014 & 1.422 & $\ldots$ & $\ldots$ & 2.100 & 1.422 & -11.3 & -76.9 & 3.4 & 3.4 & SPM \\
\hline HD 217515 & 0.514 & 0.160 & 0.23 & 0.559 & 0.514 & 12.8 & 14.3 & 0.9 & 1.0 & HIP,$A$ \\
\hline BPS CS 22888-0010 & 1.207 & $\cdots$ & $\ldots$ & 1.200 & 1.207 & 15.6 & -37.6 & 2.3 & 2.3 & SPM \\
\hline BPS CS 22893-0030 & 1.012 & $\ldots$ & $\ldots$ & 1.600 & 1.012 & $\ldots$ & $\ldots$ & $\ldots$ & $\ldots$ & $\ldots$ \\
\hline BPS CS 30493-0001 & 0.501 & $\ldots$ & $\ldots$ & 0.800 & 0.501 & 98.0 & -47.0 & 2.4 & 1.9 & SPM, \\
\hline CD-63 1588 & 0.476 & 1.176 & 1.61 & 0.564 & 0.476 & 69.8 & -67.1 & 1.1 & 1.0 & HIP,$A$ \\
\hline BPS CS 22938-0078 & 1.039 & $\ldots$ & $\ldots$ & 1.200 & 1.039 & $\ldots$ & $\ldots$ & $\ldots$ & $\ldots$ & $\ldots$ \\
\hline BPS CS 22893-0015 & 1.368 & $\ldots$ & $\ldots$ & 1.700 & 1.368 & $\ldots$ & $\ldots$ & $\ldots$ & $\ldots$ & $\ldots$ \\
\hline HD 217808 & 0.603 & 0.299 & 0.41 & 0.720 & 0.603 & 26.1 & -25.4 & 1.0 & 0.7 & $\mathrm{HIP}, \mathrm{S}$ \\
\hline BPS CS 22888-0014 & 1.269 & $\ldots$ & $\ldots$ & 1.400 & 1.269 & 37.7 & -56.3 & 2.3 & 2.3 & SPM \\
\hline BPS CS 29521-0051 & 0.950 & $\ldots$ & $\ldots$ & 0.700 & 0.950 & $\ldots$ & $\ldots$ & $\ldots$ & $\ldots$ & $\ldots$ \\
\hline BPS CS 22888-0002 & 1.348 & $\cdots$ & $\cdots$ & 1.500 & 1.348 & 6.8 & -51.1 & 3.2 & 1.8 & SPM \\
\hline BPS CS 22893-0032 & $6.868 / 1.205$ & $\ldots$ & $\ldots$ & 1.500 & 6.868 & $\ldots$ & $\ldots$ & $\ldots$ & $\ldots$ & $\ldots$ \\
\hline BPS CS 22888-0001 & 1.356 & $\ldots$ & $\ldots$ & 1.500 & 1.356 & -12.7 & -34.4 & 4.6 & 4.6 & SPM \\
\hline BPS CS 22938-0056 & 0.730 & $\ldots$ & $\ldots$ & 1.000 & 0.730 & $\ldots$ & $\ldots$ & $\ldots$ & $\ldots$ & $\ldots$ \\
\hline HD 218367 & $\ldots$ & $\ldots$ & $\ldots$ & 1.542 & 1.542 & 0.1 & 0.0 & 2.1 & 1.2 & $\mathrm{ACT}$ \\
\hline BPS CS 30493-0029 & 0.656 & $\ldots$ & $\ldots$ & 1.100 & 0.656 & 12.0 & 6.3 & 1.7 & 3.4 & SPM \\
\hline BPS CS 30493-0031 & 0.618 & $\ldots$ & $\ldots$ & 0.900 & 0.618 & -13.2 & -15.1 & 1.5 & 1.5 & SPM \\
\hline $\mathrm{V}^{*} \mathrm{YY}$ Tuc & 1.926 & $\ldots$ & $\ldots$ & 1.871 & 1.926 & 5. & -25 . & 4.4 & 4.4 & STN \\
\hline HD 218810 & 0.096 & 0.084 & 0.09 & 0.097 & 0.084 & 73.6 & 17.4 & 1.0 & 0.7 & $\mathrm{HIP}, \mathrm{S}$ \\
\hline BPS CS 22888-0039 & 1.275 & $\ldots$ & $\ldots$ & 1.200 & 1.275 & 77.0 & -20.1 & 1.6 & 2.7 & SPM \\
\hline HD 218857 & 0.307 & 0.285 & 0.40 & 0.493 & 0.307 & -54.6 & -94.4 & 0.9 & 1.1 & HIP,$A$ \\
\hline
\end{tabular}


TABle 1B. (continued)

\begin{tabular}{|c|c|c|c|c|c|c|c|c|c|c|}
\hline $\begin{array}{l}\text { Star } \\
(1)\end{array}$ & $\begin{array}{c}D_{p h o} \\
(\mathrm{kpc}) \\
(2)\end{array}$ & $\begin{array}{c}D_{H I P} \\
(\mathrm{kpc}) \\
(3)\end{array}$ & $\begin{array}{c}\sigma_{\pi_{H I P}} / \pi_{H I P} \\
(4)\end{array}$ & $\begin{array}{c}D_{B S L} \\
(\mathrm{kpc}) \\
(5)\end{array}$ & $\begin{array}{c}D_{\text {adopt }} \\
(\mathrm{kpc}) \\
\quad(6)\end{array}$ & $\begin{array}{c}\mu_{\alpha^{*}} \\
(\mathrm{mas} / \mathrm{yr}) \\
(7)\end{array}$ & $\begin{array}{c}\mu_{\delta} \\
\text { (mas/yr) } \\
(8)\end{array}$ & $\begin{array}{c}\sigma_{\mu_{\alpha^{*}}} \\
(\mathrm{mas} / \mathrm{yr}) \\
(9)\end{array}$ & $\begin{array}{c}\sigma_{\mu_{\delta}} \\
(\mathrm{mas} / \mathrm{yr}) \\
(10)\end{array}$ & \\
\hline BPS CS 22888-0031 & 1.517 & $\ldots$ & $\ldots$ & 1.800 & 1.517 & 57.3 & -30.7 & 1.7 & 4.8 & SPM \\
\hline $\mathrm{V}^{*}$ AR Oct & 2.791 & $\ldots$ & $\ldots$ & $\ldots$ & 2.791 & $\ldots$ & $\ldots$ & $\ldots$ & $\ldots$ & $\ldots$ \\
\hline BPS CS 30493-0049 & $\ldots$ & $\cdots$ & $\cdots$ & 1.200 & 1.200 & 21.3 & -5.2 & 4.9 & 3.0 & SPM \\
\hline BPS CS 22888-0044 & 2.361 & $\cdots$ & $\cdots$ & 2.300 & 2.361 & 21.2 & -2.5 & 1.7 & 1.7 & SPM \\
\hline BPS CS 30493-0050 & 0.972 & $\cdots$ & $\cdots$ & 1.000 & 0.972 & 15.4 & -4.7 & 3.6 & 1.6 & SPM \\
\hline BPS CS 30493-0058 & 1.415 & $\cdots$ & $\cdots$ & 1.300 & 1.415 & 0.1 & -21.0 & 1.7 & 1.6 & SPM \\
\hline BPS CS 29513-0006 & 1.047 & $\cdots$ & $\cdots$ & 1.000 & 1.047 & $\cdots$ & $\ldots$ & $\cdots$ & $\cdots$ & $\cdots$ \\
\hline BPS CS 29513-0002 & $\ldots$ & $\cdots$ & $\cdots$ & 1.200 & 1.200 & 8.8 & 7.7 & 2.5 & 3.4 & SPM \\
\hline $\mathrm{V}^{*}$ AP Gru & 3.106 & $\cdots$ & $\cdots$ & $\ldots$ & 3.106 & $\cdots$ & $\ldots$ & $\cdots$ & $\cdots$ & $\cdots$ \\
\hline BPS CS 22888-0029 & 1.230 & $\cdots$ & $\cdots$ & 1.200 & 1.230 & 14.9 & -33.1 & 4.3 & 3.4 & SPM \\
\hline BPS CS 29513-0014 & 4.184 & $\cdots$ & $\cdots$ & 2.000 & 4.184 & 14.7 & -3.5 & 2.4 & 2.5 & SPM \\
\hline BPS CS 29513-0015 & 1.186 & $\cdots$ & $\cdots$ & 1.000 & 1.186 & -22.8 & -29.0 & 2.5 & 2.2 & SPM \\
\hline BPS CS 30493-0060 & 0.765 & $\cdots$ & $\cdots$ & 0.900 & 0.765 & -3.5 & -11.6 & 1.8 & 3.5 & SPM \\
\hline BPS CS 29513-0009 & 1.092 & $\ldots$ & $\cdots$ & 1.100 & 1.092 & -22.5 & -28.9 & 2.0 & 2.0 & SPM \\
\hline HD 219221 & 0.093 & 0.148 & 0.16 & 0.081 & 0.093 & -35.4 & 2.5 & 0.6 & 0.7 & $\mathrm{HIP}$, \\
\hline BPS CS 29513-0003 & 4.056 & $\ldots$ & $\ldots$ & 2.200 & 4.056 & 5.0 & -14.7 & 2.1 & 1.6 & SPM \\
\hline BPS CS 22888-0026 & 4.151 & $\cdots$ & $\cdots$ & 2.300 & 4.151 & -12.6 & -23.9 & 2.9 & 1.8 & SPM \\
\hline BPS CS 30493-0065 & 0.594 & $\cdots$ & $\cdots$ & 0.900 & 0.594 & 5.5 & -16.4 & 3.3 & 3.4 & SPM \\
\hline BPS CS 30493-0071 & 0.676 & $\cdots$ & $\cdots$ & 1.000 & 0.676 & 19.7 & -8.2 & 2.0 & 2.9 & SPM \\
\hline BPS CS 22949-0008 & 0.962 & $\cdots$ & $\cdots$ & 1.500 & 0.962 & $\cdots$ & $\cdots$ & $\cdots$ & $\cdots$ & $\cdots$ \\
\hline BPS CS 29513-0010 & 1.307 & $\cdots$ & $\cdots$ & 0.900 & 1.307 & 4.3 & -29.5 & 6.0 & 3.7 & SPM \\
\hline BPS CS 30493-0072 & 0.968 & $\ldots$ & $\ldots$ & 0.900 & 0.968 & 2.3 & -4.5 & 4.7 & 2.9 & SPM \\
\hline BPS CS 22938-0065 & 1.169 & $\cdots$ & $\cdots$ & 1.400 & 1.169 & $\cdots$ & $\cdots$ & $\cdots$ & $\cdots$ & $\cdots$ \\
\hline BPS CS 22949-0018 & 0.738 & $\cdots$ & $\cdots$ & 0.900 & 0.738 & $\cdots$ & $\cdots$ & $\cdots$ & $\cdots$ & $\cdots$ \\
\hline $\mathrm{V}^{*} \mathrm{AC}$ And & $\ldots$ & $<0$ & $\cdots$ & 1.111 & 1.111 & -1.5 & -3.1 & 1.1 & 1.0 & $\mathrm{HIP}$, \\
\hline BPS CS 29513-0016 & 0.850 & $\cdots$ & $\cdots$ & 1.000 & 0.850 & 41.8 & -53.0 & 3.5 & 3.1 & SPM \\
\hline BPS CS 30493-0064 & 2.335 & $\cdots$ & $\cdots$ & 1.300 & 2.335 & 9.7 & -6.6 & 4.0 & 3.9 & SPM \\
\hline $\mathrm{V}^{*} \mathrm{DN}$ Aqr & 1.302 & 0.238 & 0.50 & 0.773 & 1.302 & 46.6 & -13.4 & 0.9 & 0.9 & HIP,, \\
\hline BPS CS 22949-0019 & 1.557 & $\cdots$ & $\cdots$ & 1.800 & 1.557 & $\cdots$ & $\cdots$ & $\cdots$ & $\cdots$ & $\cdots$ \\
\hline BPS CS 22949-0014 & $5.848 / 1.036$ & $\cdots$ & $\cdots$ & 1.200 & 5.848 & $\cdots$ & $\cdots$ & $\cdots$ & $\cdots$ & $\cdots$ \\
\hline $\mathrm{V}^{*} \mathrm{DZ}$ Peg & 1.805 & $\cdots$ & $\cdots$ & $\cdots$ & 1.805 & 15.7 & -25.5 & 3.5 & 3.5 & STN, \\
\hline BPS CS 22888-0047 & 1.226 & $\cdots$ & $\cdots$ & 6.300 & 1.226 & 8.7 & -12.6 & 2.0 & 1.9 & SPM \\
\hline BPS CS 22945-0017 & 1.286 & $\cdots$ & $\cdots$ & 1.300 & 1.286 & $\cdots$ & $\ldots$ & $\cdots$ & $\cdots$ & $\cdots$ \\
\hline BPS CS 22888-0057 & 1.629 & $\cdots$ & $\cdots$ & 1.600 & 1.629 & 31.2 & -65.0 & 2.1 & 2.1 & SPM \\
\hline BPS CS 22949-0030 & 0.907 & $\cdots$ & $\cdots$ & 1.000 & 0.907 & $\cdots$ & $\cdots$ & $\cdots$ & $\cdots$ & $\cdots$ \\
\hline HD 220127 & 0.454 & 0.226 & 0.39 & 0.452 & 0.454 & 209.9 & -288.1 & 0.9 & 1.0 & $\mathrm{HIP}$, \\
\hline BPS CS 22949-0029 & 1.129 & $\ldots$ & $\cdots$ & 1.800 & 1.129 & $\ldots$ & $\ldots$ & $\cdots$ & $\cdots$ & $\cdots$ \\
\hline BPS CS 29513-0032 & 0.997 & $\cdots$ & $\cdots$ & 1.000 & 0.997 & 32.5 & 0.6 & 4.9 & 4.0 & SPM \\
\hline $\mathrm{V}^{*} \mathrm{UZ} \mathrm{Scl}$ & 2.107 & $\cdots$ & $\cdots$ & 2.089 & 2.107 & -2.3 & -21.6 & 2.5 & 3.0 & SPM \\
\hline BPS CS 22949-0026 & 1.534 & $\cdots$ & $\cdots$ & 1.800 & 1.534 & $\cdots$ & $\cdots$ & $\cdots$ & $\cdots$ & $\cdots$ \\
\hline
\end{tabular}


TABle 1B. (continued)

\begin{tabular}{|c|c|c|c|c|c|c|c|c|c|c|}
\hline $\begin{array}{l}\text { Star } \\
(1)\end{array}$ & $\begin{array}{c}D_{p h o} \\
(\mathrm{kpc}) \\
(2)\end{array}$ & $\begin{array}{c}D_{H I P} \\
(\mathrm{kpc}) \\
(3)\end{array}$ & $\begin{array}{c}\sigma_{\pi_{H I P}} / \pi_{H I P} \\
(4)\end{array}$ & $\begin{array}{c}D_{B S L} \\
(\mathrm{kpc}) \\
(5)\end{array}$ & $\begin{array}{c}D_{\text {adopt }} \\
(\mathrm{kpc}) \\
(6)\end{array}$ & $\begin{array}{c}\mu_{\alpha^{*}} \\
(\operatorname{mas} / \mathrm{yr}) \\
(7)\end{array}$ & $\begin{array}{c}\mu_{\delta} \\
\text { (mas/yr) } \\
(8)\end{array}$ & $\begin{array}{c}\sigma_{\mu_{\alpha^{*}}} \\
(\mathrm{mas} / \mathrm{yr}) \\
(9)\end{array}$ & $\begin{array}{c}\sigma_{\mu_{\delta}} \\
(\mathrm{mas} / \mathrm{yr}) \\
(10)\end{array}$ & \\
\hline BPS CS 29513-0035 & 1.196 & $\ldots$ & $\cdots$ & 1.200 & 1.196 & -2.8 & -37.3 & 1.7 & 4.2 & SPM \\
\hline BPS CS 29513-0033 & $\ldots$ & $\cdots$ & $\cdots$ & 1.400 & 1.400 & 6.4 & 3.8 & 1.6 & 2.2 & SPM \\
\hline BPS CS 22888-0049 & 1.246 & $\cdots$ & $\cdots$ & 1.300 & 1.246 & 3.8 & -28.7 & 2.1 & 2.0 & SPM \\
\hline BPS CS 29513-0031 & 1.860 & $\cdots$ & $\cdots$ & 1.400 & 1.860 & 5.2 & -3.8 & 1.7 & 1.7 & SPM \\
\hline BPS CS 22949-0043 & 1.446 & $\cdots$ & $\cdots$ & 1.700 & 1.446 & $\ldots$ & $\ldots$ & $\cdots$ & $\cdots$ & $\ldots$ \\
\hline BPS CS 29513-0034 & 1.165 & $\cdots$ & $\cdots$ & 1.100 & 1.165 & -14.6 & -51.6 & 2.5 & 1.7 & SPM \\
\hline HD 220662 & 1.575 & $<0$ & $\cdots$ & 1.350 & 1.575 & 8.5 & -21.1 & 1.4 & 1.0 & HIP, $\mathrm{S}$ \\
\hline BPS CS 22949-0050 & $7.329 / 1.277$ & $\cdots$ & $\cdots$ & 1.500 & 7.329 & $\cdots$ & $\ldots$ & $\cdots$ & $\cdots$ & $\cdots$ \\
\hline BPS CS 22949-0048 & 7.142 & $\cdots$ & $\cdots$ & 6.500 & 7.142 & $\cdots$ & $\cdots$ & $\cdots$ & $\cdots$ & $\cdots$ \\
\hline BPS CS 22949-0040 & 1.096 & $\cdots$ & $\cdots$ & 1.300 & 1.096 & $\cdots$ & $\cdots$ & $\cdots$ & $\cdots$ & $\cdots$ \\
\hline BPS CS 22949-0037 & 7.802 & $\ldots$ & $\cdots$ & 8.200 & 7.802 & $\cdots$ & $\ldots$ & $\ldots$ & $\ldots$ & $\cdots$ \\
\hline HD 220838 & 1.565 & $<0$ & $\cdots$ & 1.250 & 1.565 & 1.6 & -11.7 & 1.4 & 0.9 & HIP, $\mathrm{S}$ \\
\hline BPS CS 22949-0046 & 2.451 & $\cdots$ & $\cdots$ & 1.100 & 2.451 & $\cdots$ & $\ldots$ & $\cdots$ & $\cdots$ & $\cdots$ \\
\hline BPS CS 22949-0052 & 0.926 & $\cdots$ & $\cdots$ & 0.900 & 0.926 & $\cdots$ & $\ldots$ & $\cdots$ & $\cdots$ & $\cdots$ \\
\hline BPS CS 22941-0011 & 2.047 & $\cdots$ & $\cdots$ & 1.700 & 2.047 & 24.5 & -29.7 & 2.6 & 2.5 & SPM \\
\hline $\mathrm{V}^{*} \mathrm{RV}$ Phe & 1.885 & 0.403 & 1.17 & 1.675 & 1.885 & 48.8 & -14.9 & 1.6 & 1.4 & HIP,, \\
\hline BPS CS 22941-0007 & 5.841 & $\ldots$ & $\cdots$ & 1.000 & 5.841 & $\cdots$ & $\ldots$ & $\cdots$ & $\cdots$ & $\ldots$ \\
\hline BPS CS 29513-0048 & 0.890 & $\ldots$ & $\cdots$ & 1.000 & 0.890 & 41.3 & -24.1 & 2.6 & 2.1 & SPM \\
\hline HD 221170 & 0.475 & 0.435 & 0.37 & 0.690 & 0.475 & -16.1 & -54.4 & 0.6 & 0.5 & $\mathrm{HIP}, \mathrm{A}$ \\
\hline $\mathrm{V}^{*} \mathrm{BK}$ Tuc & 2.905 & $\cdots$ & $\cdots$ & $\cdots$ & 2.905 & 16. & -11 & 5.6 & 5.6 & STN \\
\hline BPS CS 29513-0040 & 4.472 & $\cdots$ & $\cdots$ & 3.700 & 4.472 & 8.2 & -3.5 & 2.6 & 2.0 & SPM \\
\hline BPS CS 22952-0004 & 0.726 & $\ldots$ & $\ldots$ & 0.800 & 0.726 & $\ldots$ & $\ldots$ & $\ldots$ & $\ldots$ & $\ldots$ \\
\hline BPS CS 22949-0055 & 0.721 & $\cdots$ & $\cdots$ & 0.800 & 0.721 & $\cdots$ & $\cdots$ & $\cdots$ & $\cdots$ & $\cdots$ \\
\hline BPS CS 22945-0028 & 7.183 & $\cdots$ & $\cdots$ & 4.500 & 7.183 & $\cdots$ & $\cdots$ & $\cdots$ & $\cdots$ & $\cdots$ \\
\hline BPS CS 22894-0010 & 1.047 & $\cdots$ & $\cdots$ & 1.200 & 1.047 & $\cdots$ & $\cdots$ & $\cdots$ & $\cdots$ & $\cdots$ \\
\hline $\mathrm{V}^{*} \mathrm{DM}$ And & 2.649 & $\cdots$ & $\cdots$ & $\ldots$ & 2.649 & 23. & -30 & 9.7 & 11.4 & STN \\
\hline BPS CS 22941-0014 & 2.356 & $\cdots$ & $\cdots$ & 1.800 & 2.356 & 3.7 & -5.2 & 1.7 & 1.5 & SPM \\
\hline BPS CS 22894-0004 & 1.032 & $\cdots$ & $\cdots$ & 1.100 & 1.032 & $\cdots$ & $\ldots$ & $\cdots$ & $\cdots$ & $\cdots$ \\
\hline BPS CS 29496-0003 & 0.732 & $\cdots$ & $\cdots$ & 1.500 & 0.732 & 43.4 & -72.3 & 3.0 & 5.0 & SPM \\
\hline BPS CS 22941-0017 & 3.519 & $\cdots$ & $\cdots$ & 2.400 & 3.519 & -3.4 & -2.7 & 2.7 & 2.6 & SPM \\
\hline BPS CS 22941-0020 & 1.417 & $\cdots$ & $\cdots$ & 1.300 & 1.417 & 38.6 & -28.4 & 2.3 & 1.6 & SPM \\
\hline BPS CS 22952-0002 & 1.772 & $\cdots$ & $\cdots$ & 6.000 & 1.772 & $\cdots$ & $\cdots$ & $\cdots$ & $\cdots$ & $\cdots$ \\
\hline BPS CS 22894-0009 & 0.958 & $\cdots$ & $\cdots$ & 0.900 & 0.958 & $\cdots$ & $\cdots$ & $\cdots$ & $\cdots$ & $\cdots$ \\
\hline BPS CS 22894-0005 & $5.738 / 0.998$ & $\cdots$ & $\cdots$ & 1.200 & 5.738 & $\cdots$ & $\cdots$ & $\cdots$ & $\cdots$ & $\cdots$ \\
\hline BPS CS 22941-0027 & 5.405 & $\cdots$ & $\cdots$ & 4.700 & 5.405 & 8.3 & 3.6 & 2.7 & 2.4 & SPM \\
\hline BPS CS 22941-0021 & 0.860 & $\cdots$ & $\cdots$ & 0.800 & 0.860 & 28.0 & -16.4 & 3.7 & 2.9 & SPM \\
\hline $\mathrm{V}^{*} \mathrm{BK}$ And & 2.113 & $\cdots$ & $\cdots$ & $\cdots$ & 2.113 & 5. & -9 & 3.9 & 3.9 & STN \\
\hline BPS CS 29513-0053 & 1.059 & $\cdots$ & $\cdots$ & 1.000 & 1.059 & 33.2 & -10.4 & 6.7 & 5.5 & SPM \\
\hline BPS CS 22952-0012 & 1.664 & $\cdots$ & $\cdots$ & 1.000 & 1.664 & $\cdots$ & $\cdots$ & $\cdots$ & $\cdots$ & $\cdots$ \\
\hline BPS CS 22894-0017 & 0.846 & $\cdots$ & $\cdots$ & 1.000 & 0.846 & $\cdots$ & $\cdots$ & $\cdots$ & $\cdots$ & $\cdots$ \\
\hline
\end{tabular}


TABle 1B. (continued)

\begin{tabular}{|c|c|c|c|c|c|c|c|c|c|c|}
\hline $\begin{array}{l}\text { Star } \\
(1)\end{array}$ & $\begin{array}{c}D_{p h o} \\
(\mathrm{kpc}) \\
(2)\end{array}$ & $\begin{array}{c}D_{H I P} \\
(\mathrm{kpc}) \\
(3)\end{array}$ & $\begin{array}{c}\sigma_{\pi_{H I P}} / \pi_{H I P} \\
(4)\end{array}$ & $\begin{array}{c}D_{B S L} \\
(\mathrm{kpc}) \\
(5)\end{array}$ & $\begin{array}{c}D_{\text {adopt }} \\
\text { (kpc) } \\
(6)\end{array}$ & $\begin{array}{c}\mu_{\alpha^{*}} \\
(\mathrm{mas} / \mathrm{yr}) \\
(7)\end{array}$ & $\begin{array}{c}\mu_{\delta} \\
(\mathrm{mas} / \mathrm{yr}) \\
(8)\end{array}$ & $\begin{array}{c}\sigma_{\mu_{\alpha *}} \\
(\mathrm{mas} / \mathrm{yr}) \\
(9)\end{array}$ & $\begin{array}{c}\sigma_{\mu_{\delta}} \\
(\mathrm{mas} / \mathrm{yr}) \\
(10)\end{array}$ & \\
\hline BPS CS 22945-0024 & 7.732 & $\cdots$ & $\ldots$ & 10.300 & 7.732 & $\ldots$ & $\ldots$ & $\ldots$ & $\ldots$ & $\ldots$ \\
\hline BPS CS 22952-0011 & 0.906 & $\ldots$ & $\ldots$ & 1.000 & 0.906 & 22. & -7 & 4.5 & 4.5 & STN \\
\hline BPS CS 22952-0015 & 5.322 & $\ldots$ & $\ldots$ & 5.000 & 5.322 & $\ldots$ & $\ldots$ & $\ldots$ & $\ldots$ & $\ldots$ \\
\hline BPS CS 29496-0021 & $\ldots$ & $\ldots$ & $\ldots$ & 1.500 & 1.500 & -4.6 & -71.7 & 3.6 & 4.1 & SPM \\
\hline BPS CS 29496-0022 & 1.524 & $\ldots$ & $\ldots$ & 1.500 & 1.524 & -9.1 & -86.8 & 3.4 & 3.4 & SPM \\
\hline $\mathrm{V}^{*} \mathrm{BR}$ Aqr & 1.304 & $<0$ & $\ldots$ & $\ldots$ & 1.304 & 8.3 & -10.2 & 1.9 & 1.5 & HIP,S \\
\hline BPS CS 29496-0014 & 0.625 & $\ldots$ & $\ldots$ & 1.400 & 0.625 & 30.6 & -76.0 & 3.7 & 5.2 & SPM \\
\hline BPS CS 29499-0017 & $\ldots$ & $\ldots$ & $\ldots$ & 1.700 & 1.700 & -24.9 & -96.4 & 3.5 & 4.0 & SPM \\
\hline BPS CS 22952-0018 & 0.804 & $\ldots$ & $\ldots$ & 0.800 & 0.804 & $\ldots$ & $\ldots$ & $\ldots$ & $\ldots$ & $\ldots$ \\
\hline BPS CS 22894-0019 & 0.924 & $\cdots$ & $\ldots$ & 1.100 & 0.924 & $\ldots$ & $\ldots$ & $\ldots$ & $\ldots$ & $\ldots$ \\
\hline BPS CS 29499-0010 & 2.094 & $\ldots$ & $\ldots$ & 1.300 & 2.094 & 7.1 & -2.3 & 4.4 & 5.7 & SPM \\
\hline BPS CS 29499-0003 & 1.191 & $\ldots$ & $\ldots$ & 1.300 & 1.191 & -19.0 & -42.8 & 3.7 & 3.9 & SPM \\
\hline BPS CS 29496-0013 & 7.285 & $\ldots$ & $\ldots$ & 1.600 & 7.285 & 4.9 & -11.5 & 7.5 & 8.3 & SPM \\
\hline BPS CS 22941-0043 & 1.604 & $\ldots$ & $\ldots$ & 1.400 & 1.604 & 3.4 & -89.3 & 2.8 & 2.8 & SPM \\
\hline HD 222434 & 1.182 & 16.667 & 21.17 & 0.990 & 1.182 & -1.1 & -45.6 & 0.8 & 0.8 & HIP,, \\
\hline BPS CS 22945-0029 & 1.796 & $\ldots$ & $\ldots$ & 2.000 & 1.796 & $\ldots$ & $\ldots$ & $\ldots$ & $\ldots$ & $\ldots$ \\
\hline BPS CS 29499-0027 & 1.115 & $\ldots$ & $\ldots$ & 1.400 & 1.115 & 12.1 & -28.2 & 4.8 & 4.8 & SPM \\
\hline BPS CS 22952-0021 & 1.192 & $\ldots$ & $\ldots$ & 1.300 & 1.192 & $\ldots$ & $\ldots$ & $\ldots$ & $\ldots$ & $\ldots$ \\
\hline BPS CS 22952-0020 & 0.875 & $\ldots$ & $\ldots$ & 0.900 & 0.875 & $\ldots$ & $\ldots$ & $\ldots$ & $\ldots$ & $\ldots$ \\
\hline $\mathrm{V}^{*}$ VZ Peg & 1.797 & $<0$ & $\ldots$ & $\ldots$ & 1.797 & 19.6 & -27.3 & 2.0 & 1.6 & HIP, $\mathrm{S}$ \\
\hline $\mathrm{V}^{*} \mathrm{AT}$ And & 0.880 & $<0$ & $\ldots$ & 0.805 & 0.880 & -9.2 & -50.7 & 1.2 & 0.8 & HIP,$A$ \\
\hline HD 222766 & 0.056 & 0.118 & 0.21 & 0.053 & 0.056 & 611.6 & -164.5 & 0.9 & 0.8 & HIP,$A$ \\
\hline BPS CS 22894-0038 & 1.564 & $\ldots$ & $\ldots$ & 6.100 & 1.564 & $\ldots$ & $\ldots$ & $\ldots$ & $\ldots$ & $\ldots$ \\
\hline BPS CS 29499-0034 & 1.392 & $\ldots$ & $\ldots$ & 1.300 & 1.392 & 15.5 & -17.9 & 2.7 & 2.7 & SPM \\
\hline BPS CS 22941-0046 & 1.066 & $\ldots$ & $\ldots$ & 1.100 & 1.066 & 3.6 & -5.8 & 6.9 & 6.0 & SPM \\
\hline BPS CS 22894-0046 & 6.910 & $\ldots$ & $\ldots$ & 6.800 & 6.910 & $\ldots$ & $\ldots$ & $\ldots$ & $\ldots$ & $\ldots$ \\
\hline $\mathrm{V}^{*} \mathrm{KK}$ Peg & $\ldots$ & $\ldots$ & $\ldots$ & 28.183 & 28.183 & $\ldots$ & $\ldots$ & $\ldots$ & $\ldots$ & $\ldots$ \\
\hline BPS CS 22894-0047 & 1.253 & $\ldots$ & $\ldots$ & 4.600 & 1.253 & $\ldots$ & $\ldots$ & $\ldots$ & $\ldots$ & $\ldots$ \\
\hline BPS CS 22945-0043 & 1.092 & $\ldots$ & $\ldots$ & 1.100 & 1.092 & $\ldots$ & $\ldots$ & $\ldots$ & $\ldots$ & $\ldots$ \\
\hline HD 222925 & 0.389 & 0.273 & 0.25 & 0.561 & 0.389 & 156.0 & -98.5 & 0.9 & 1.0 & HIP,$A$ \\
\hline BPS CS 22894-0041 & 0.701 & $\ldots$ & $\ldots$ & 0.700 & 0.701 & $\ldots$ & $\ldots$ & $\ldots$ & $\ldots$ & $\ldots$ \\
\hline BPS CS 29517-0005 & 4.740 & $\ldots$ & $\ldots$ & 5.500 & 4.740 & $\ldots$ & $\ldots$ & $\ldots$ & $\ldots$ & $\ldots$ \\
\hline BPS CS 29517-0010 & 1.290 & $\ldots$ & $\ldots$ & 1.000 & 1.290 & -3.4 & -14.1 & 5 & 5 & NPM \\
\hline $\mathrm{V}^{*} \mathrm{KL}$ Peg & $\ldots$ & $\ldots$ & $\ldots$ & 18.450 & 18.450 & $\ldots$ & $\ldots$ & $\ldots$ & $\ldots$ & $\ldots$ \\
\hline BPS CS 22945-0044 & 0.906 & $\ldots$ & $\ldots$ & 1.100 & 0.906 & $\ldots$ & $\ldots$ & $\ldots$ & $\ldots$ & $\ldots$ \\
\hline BPS CS 22945-0031 & 1.540 & $\ldots$ & $\ldots$ & 1.300 & 1.540 & $\ldots$ & $\ldots$ & $\ldots$ & $\ldots$ & $\ldots$ \\
\hline HD 223224 & 0.288 & 0.741 & 0.98 & 0.325 & 0.288 & 67.8 & -7.6 & 0.8 & 0.9 & HIP,$A$ \\
\hline BPS CS 22894-0054 & $4.754 / 1.143$ & $\ldots$ & $\ldots$ & 0.900 & 4.754 & $\ldots$ & $\ldots$ & $\cdots$ & $\ldots$ & $\ldots$ \\
\hline BPS CS 22876-0003 & 0.839 & $\cdots$ & $\ldots$ & 0.900 & 0.839 & 5.7 & -32.7 & 2.8 & 3.1 & SPM \\
\hline BPS CS 29517-0004 & 1.309 & $\cdots$ & $\cdots$ & 1.000 & 1.309 & $\ldots$ & $\ldots$ & $\cdots$ & $\cdots$ & $\cdots$ \\
\hline
\end{tabular}


TABle 1B. (continued)

\begin{tabular}{|c|c|c|c|c|c|c|c|c|c|c|}
\hline $\begin{array}{l}\text { Star } \\
(1)\end{array}$ & $\begin{array}{c}D_{p h o} \\
(\mathrm{kpc}) \\
(2)\end{array}$ & $\begin{array}{c}D_{H I P} \\
(\mathrm{kpc}) \\
(3)\end{array}$ & $\begin{array}{c}\sigma_{\pi_{H I P}} / \pi_{H I P} \\
(4)\end{array}$ & $\begin{array}{c}D_{B S L} \\
(\mathrm{kpc}) \\
(5)\end{array}$ & $\begin{array}{c}D_{\text {adopt }} \\
\text { (kpc) } \\
(6)\end{array}$ & $\begin{array}{c}\mu_{\alpha^{*}} \\
(\operatorname{mas} / \mathrm{yr}) \\
(7)\end{array}$ & $\begin{array}{c}\mu_{\delta} \\
(\mathrm{mas} / \mathrm{yr}) \\
(8)\end{array}$ & $\begin{array}{c}\sigma_{\mu_{\alpha^{*}}} \\
(\mathrm{mas} / \mathrm{yr}) \\
(9)\end{array}$ & $\begin{array}{c}\sigma_{\mu_{\delta}} \\
(\mathrm{mas} / \mathrm{yr}) \\
(10)\end{array}$ & So \\
\hline BPS CS 22894-0049 & 1.124 & $\ldots$ & $\ldots$ & 1.300 & 1.124 & $\ldots$ & $\ldots$ & $\ldots$ & $\ldots$ & \\
\hline BPS CS 22894-0050 & $\ldots$ & $\ldots$ & $\ldots$ & 1.700 & 1.700 & $\ldots$ & $\ldots$ & $\ldots$ & $\ldots$ & \\
\hline BPS CS 29517-0012 & 1.178 & $\ldots$ & $\ldots$ & 0.900 & 1.178 & $\ldots$ & $\ldots$ & $\ldots$ & $\ldots$ & \\
\hline BPS CS 29499-0058 & 0.951 & $\ldots$ & $\ldots$ & 1.100 & 0.951 & -5.7 & -57.8 & 2.0 & 3.1 & \\
\hline BPS CS 29517-0014 & 0.991 & $\ldots$ & $\ldots$ & 1.000 & 0.991 & $\ldots$ & $\ldots$ & $\ldots$ & $\ldots$ & \\
\hline BPS CS 22945-0058 & 1.720 & $\ldots$ & $\ldots$ & 1.300 & 1.720 & $\ldots$ & $\ldots$ & $\ldots$ & $\ldots$ & \\
\hline BPS CS 29499-0053 & 1.128 & $\ldots$ & $\ldots$ & 1.300 & 1.128 & 12.4 & -11.0 & 2.2 & 2.4 & \\
\hline BPS CS 22945-0056 & 5.456 & $\cdots$ & $\cdots$ & 5.000 & 5.456 & $\ldots$ & $\ldots$ & $\ldots$ & $\ldots$ & \\
\hline BPS CS 22876-0016 & 1.587 & $\ldots$ & $\cdots$ & 0.700 & 1.587 & 4.3 & -9.2 & 3.5 & 6.4 & \\
\hline BPS CS 29517-0018 & 1.158 & $\cdots$ & $\cdots$ & 1.700 & 1.158 & $\cdots$ & $\cdots$ & $\cdots$ & $\cdots$ & \\
\hline BPS CS 29499-0060 & 0.704 & $\ldots$ & $\ldots$ & 0.900 & 0.704 & 78.7 & 21.9 & 1.9 & 2.3 & \\
\hline BPS CS 29517-0025 & $\ldots$ & $\ldots$ & $\ldots$ & 1.800 & 1.800 & $\ldots$ & $\ldots$ & $\ldots$ & $\ldots$ & \\
\hline BPS CS 29517-0023 & 1.015 & $\ldots$ & $\cdots$ & 1.400 & 1.015 & $\ldots$ & $\ldots$ & $\ldots$ & $\ldots$ & \\
\hline $\mathrm{V}^{*} \mathrm{KM}$ Peg & $\ldots$ & $\ldots$ & $\ldots$ & 27.415 & 27.415 & $\ldots$ & $\ldots$ & $\ldots$ & $\ldots$ & \\
\hline BPS CS 22957-0013 & 7.087 & $\ldots$ & $\ldots$ & 6.100 & 7.087 & $\ldots$ & $\ldots$ & $\ldots$ & $\ldots$ & \\
\hline BPS CS 22945-0073 & 1.440 & $\ldots$ & $\ldots$ & 1.600 & 1.440 & $\ldots$ & $\ldots$ & $\ldots$ & $\ldots$ & \\
\hline BPS CS 29499-0065 & 1.906 & $\ldots$ & $\ldots$ & 1.600 & 1.906 & 24.5 & -36.7 & 6.0 & 7.7 & \\
\hline $\mathrm{V}^{*} \mathrm{KN}$ Peg & $\ldots$ & $\ldots$ & $\ldots$ & 24.099 & 24.099 & $\ldots$ & $\ldots$ & $\ldots$ & $\ldots$ & \\
\hline BPS CS 22945-0068 & 1.843 & $\cdots$ & $\cdots$ & 1.600 & 1.843 & $\cdots$ & $\cdots$ & $\cdots$ & $\cdots$ & \\
\hline BPS CS 22957-0016 & 0.968 & $\cdots$ & $\cdots$ & 1.100 & 0.968 & $\cdots$ & $\cdots$ & $\cdots$ & $\cdots$ & \\
\hline BPS CS 29499-0068 & 0.944 & $\ldots$ & $\ldots$ & 1.200 & 0.944 & -50.4 & -95.4 & 6.0 & 6.3 & \\
\hline BPS CS 22957-0019 & 0.835 & $\cdots$ & $\cdots$ & 0.900 & 0.835 & $\ldots$ & $\ldots$ & $\cdots$ & $\cdots$ & \\
\hline BPS CS 22957-0015 & 1.351 & $\cdots$ & $\cdots$ & 1.600 & 1.351 & 69.8 & -20.8 & 5 & 5 & \\
\hline BPS CS 22957-0024 & 1.225 & $\ldots$ & $\ldots$ & 1.300 & 1.225 & $\ldots$ & $\ldots$ & $\ldots$ & $\ldots$ & \\
\hline BPS CS 22957-0028 & 1.411 & $\cdots$ & $\cdots$ & 1.500 & 1.411 & $\ldots$ & $\ldots$ & $\cdots$ & $\cdots$ & \\
\hline BPS CS 22876-0027 & 1.212 & $\ldots$ & $\ldots$ & 1.200 & 1.212 & 4.8 & -18.4 & 2.7 & 2.7 & \\
\hline
\end{tabular}


TABle 3. Space Motions and Orbital Parameters

\begin{tabular}{|c|c|c|c|c|c|c|c|c|c|c|c|}
\hline $\begin{array}{l}\text { Star } \\
(1)\end{array}$ & $\begin{array}{c}{[\mathrm{Fe} / \mathrm{H}]} \\
(\mathrm{dex}) \\
(2)\end{array}$ & $\begin{array}{c}R \\
(\mathrm{kpc}) \\
(3)\end{array}$ & $\begin{array}{c}Z \\
(\mathrm{kpc}) \\
(4)\end{array}$ & $\begin{array}{c}U \\
(\mathrm{~km} / \mathrm{s}) \\
(5)\end{array}$ & $\begin{array}{c}V \\
(\mathrm{~km} / \mathrm{s}) \\
(6)\end{array}$ & $\begin{array}{c}W \\
(\mathrm{~km} / \mathrm{s}) \\
(7)\end{array}$ & $\begin{array}{c}V_{R} \\
(\mathrm{~km} / \mathrm{s}) \\
(8)\end{array}$ & $\begin{array}{c}V_{\phi} \\
(\mathrm{km} / \mathrm{s}) \\
(9)\end{array}$ & $\begin{array}{c}R_{a p} \\
(\mathrm{kpc}) \\
(10)\end{array}$ & $\begin{array}{c}R_{p r} \\
(\mathrm{kpc}) \\
(11)\end{array}$ & $\begin{array}{c}Z_{\max } \\
(\mathrm{kpc}) \\
(12)\end{array}$ \\
\hline BPS CS 22957-0026 & -1.84 & 8.52 & -0.54 & 186 & -236 & -126 & 185 & -22 & 13.38 & 0.59 & 6.17 \\
\hline $\mathrm{V}^{*}$ GV Peg & -1.99 & 9.86 & -2.05 & -209 & -533 & -241 & -289 & -241 & 76.34 & 7.82 & 56.49 \\
\hline BPS CS 22957-0022 & -2.44 & 8.59 & -2.19 & -120 & -266 & -148 & -125 & -31 & 10.56 & 3.26 & 10.68 \\
\hline BPS CS 29517-0029 & -1.77 & 8.46 & -0.83 & -61 & -143 & -9 & -58 & 79 & 8.83 & 2.02 & 0.92 \\
\hline $\mathrm{V}^{*} \mathrm{RU} \mathrm{Scl}$ & -1.27 & 8.39 & -0.79 & 131 & -137 & -68 & 132 & 81 & 10.60 & 2.02 & 2.00 \\
\hline $\mathrm{V}^{*} \mathrm{UU}$ Cet & -1.28 & 8.37 & -1.82 & 230 & -149 & 66 & 233 & 58 & 15.72 & 1.53 & 7.74 \\
\hline $\mathrm{V}^{*} \mathrm{AO}$ Tuc & -1.29 & 8.05 & -1.03 & -19 & -260 & 139 & -17 & -42 & 8.23 & 1.70 & 5.96 \\
\hline [FF93] 513440 & -0.98 & 7.96 & -2.41 & 17 & -61 & -88 & 16 & 159 & 8.65 & 5.88 & 3.74 \\
\hline BPS CS 22876-0040 & -2.20 & 8.14 & -1.64 & -83 & -92 & 221 & -84 & 127 & 14.11 & 5.92 & 11.28 \\
\hline BPS CS 29503-0010 & -1.88 & 8.41 & -0.69 & -35 & 16 & 36 & -33 & 236 & 10.57 & 8.01 & 1.12 \\
\hline HD 20 & -1.39 & 8.47 & -0.17 & 77 & -70 & 44 & 78 & 149 & 9.43 & 4.26 & 0.83 \\
\hline HD 97 & -1.21 & 8.47 & -0.33 & -188 & -206 & -100 & -188 & 16 & 12.68 & 0.40 & 4.81 \\
\hline BPS CS 29503-0013 & -1.50 & 8.37 & -1.03 & -27 & -67 & -25 & -24 & 154 & 8.51 & 4.80 & 1.21 \\
\hline [FF93] 513390 & -0.72 & 8.27 & -1.11 & -77 & 14 & -26 & -78 & 234 & 11.88 & 7.16 & 1.81 \\
\hline BPS CS 29503-0022 & -2.06 & 8.32 & -1.48 & -56 & -119 & -19 & -54 & 103 & 8.73 & 2.86 & 1.70 \\
\hline BPS CS 22876-0032 & -3.85 & 8.38 & -0.54 & 49 & -376 & 104 & 49 & -156 & 8.89 & 5.60 & 3.14 \\
\hline BPS CS 29503-0017 & -1.98 & 8.39 & -0.98 & -200 & -238 & -23 & -200 & -15 & 12.76 & 0.32 & 2.07 \\
\hline BPS CS 29503-0026 & -1.96 & 8.42 & -0.60 & 100 & -63 & 25 & 102 & 156 & 10.12 & 4.33 & 0.98 \\
\hline BPS CS 29503-0015 & -1.76 & 8.39 & -1.13 & -111 & -183 & 46 & -110 & 39 & 9.76 & 0.94 & 1.62 \\
\hline $\mathrm{V}^{*} \mathrm{RY}$ Psc & -1.39 & 8.72 & -1.83 & 292 & -227 & -84 & 289 & -38 & 23.20 & 0.76 & 5.11 \\
\hline HD 868 & -0.79 & 8.37 & -0.82 & 84 & 21 & -30 & 85 & 241 & 12.82 & 7.10 & 1.35 \\
\hline HD 891 & -0.61 & 8.42 & -0.91 & 4 & -101 & 41 & 6 & 119 & 8.46 & 3.47 & 1.34 \\
\hline HD 941 & -0.86 & 8.47 & -0.28 & 98 & -167 & 62 & 98 & 53 & 9.43 & 1.33 & 1.58 \\
\hline BPS CS 29503-0031 & -2.09 & 8.41 & -0.68 & 6 & -62 & -32 & 7 & 158 & 8.45 & 4.97 & 0.93 \\
\hline HD 991 & -0.88 & 8.39 & -0.55 & 40 & -154 & -31 & 40 & 66 & 8.57 & 1.66 & 0.78 \\
\hline BPS CS 30339-0002 & -1.54 & 8.36 & -0.70 & -129 & -217 & 76 & -129 & 2 & 10.23 & 0.06 & 2.29 \\
\hline BPS CS 29503-0042 & -2.17 & 8.43 & -0.68 & -16 & -72 & 23 & -15 & 148 & 8.50 & 4.48 & 0.80 \\
\hline BPS CS 30339-0019 & -1.77 & 8.32 & -1.07 & -19 & -333 & -40 & -19 & -113 & 8.41 & 3.25 & 1.50 \\
\hline BPS CS 30339-0007 & -1.28 & 8.36 & -0.72 & -96 & -243 & 44 & -96 & -23 & 9.31 & 0.56 & 1.20 \\
\hline CD-23 72 & -1.17 & 8.49 & -0.18 & -9 & -10 & -17 & -9 & 210 & 8.54 & 7.64 & 0.28 \\
\hline BPS CS 30339-0010 & -1.70 & 8.31 & -1.03 & -125 & -52 & -6 & -126 & 167 & 11.21 & 4.49 & 1.42 \\
\hline BPS CS 29503-0043 & -1.33 & 8.31 & -2.00 & 507 & -748 & -104 & 493 & -541 & $\cdots$ & $\cdots$ & $\cdots$ \\
\hline BPS CS 30339-0015 & -2.20 & 8.19 & -1.80 & 185 & -411 & -125 & 186 & -190 & 18.99 & 4.67 & 6.50 \\
\hline BPS CS 30339-0009 & -1.78 & 8.37 & -0.70 & 133 & -203 & 30 & 133 & 18 & 10.08 & 0.41 & 1.30 \\
\hline BPS CS 30339-0005 & -1.50 & 8.37 & -0.66 & -50 & -75 & 91 & -51 & 145 & 9.02 & 4.72 & 2.58 \\
\hline [FF93] 513135 & -0.70 & 8.16 & -1.76 & 94 & -59 & -68 & 91 & 163 & 10.43 & 4.81 & 2.77 \\
\hline CD-35 87 & -0.67 & 8.24 & -1.49 & 67 & -9 & 69 & 65 & 212 & 10.51 & 7.34 & 3.07 \\
\hline BPS CS 29503-0037 & -0.86 & 8.43 & -0.97 & -27 & -60 & -1 & -24 & 161 & 8.59 & 5.05 & 0.99 \\
\hline BPS CS 22882-0005 & -2.31 & 8.18 & -2.62 & 95 & -93 & -78 & 96 & 127 & 10.57 & 3.72 & 3.84 \\
\hline BPS CS 22882-0001 & -2.30 & 7.45 & -7.39 & 164 & -286 & -44 & 164 & -65 & 14.31 & 2.59 & 11.87 \\
\hline
\end{tabular}


TABle 3. (continued)

\begin{tabular}{|c|c|c|c|c|c|c|c|c|c|c|c|}
\hline $\begin{array}{l}\text { Star } \\
(1)\end{array}$ & $\begin{array}{c}{[\mathrm{Fe} / \mathrm{H}]} \\
(\mathrm{dex}) \\
(2)\end{array}$ & $\begin{array}{c}R \\
(\mathrm{kpc}) \\
(3)\end{array}$ & $\begin{array}{c}Z \\
(\mathrm{kpc}) \\
(4)\end{array}$ & $\begin{array}{c}U \\
(\mathrm{~km} / \mathrm{s}) \\
(5)\end{array}$ & $\begin{array}{c}V \\
(\mathrm{~km} / \mathrm{s}) \\
(6)\end{array}$ & $\begin{array}{c}W \\
(\mathrm{~km} / \mathrm{s}) \\
(7)\end{array}$ & $\begin{array}{c}V_{R} \\
(\mathrm{~km} / \mathrm{s}) \\
(8)\end{array}$ & $\begin{array}{c}V_{\phi} \\
(\mathrm{km} / \mathrm{s}) \\
(9)\end{array}$ & $\begin{array}{c}R_{a p} \\
(\mathrm{kpc}) \\
(10)\end{array}$ & $\begin{array}{c}R_{p r} \\
(\mathrm{kpc}) \\
(11)\end{array}$ & $\begin{array}{c}Z_{\max } \\
(\mathrm{kpc}) \\
(12)\end{array}$ \\
\hline BPS CS 30339-0040 & -2.30 & 8.28 & -1.18 & -85 & -59 & -341 & -87 & 160 & 35.26 & 8.23 & 31.67 \\
\hline HD 1638 & -0.88 & 8.37 & -0.97 & 25 & -97 & -25 & 25 & 123 & 8.52 & 3.53 & 1.11 \\
\hline BPS CS 30339-0037 & -2.13 & 8.36 & -0.79 & 294 & -141 & 213 & 294 & 81 & 32.68 & 3.41 & 28.53 \\
\hline BPS CS 30339-0028 & -1.92 & 8.27 & -1.39 & 367 & -297 & -16 & 367 & -74 & 35.63 & 1.27 & 6.32 \\
\hline BPS CS 22882-0006 & -2.30 & 8.37 & -1.10 & -62 & -292 & 79 & -62 & -71 & 8.99 & 2.01 & 2.61 \\
\hline BPS CS 29503-0049 & -2.08 & 8.43 & -0.74 & 129 & -113 & 71 & 129 & 106 & 10.54 & 2.90 & 2.81 \\
\hline BPS CS 30339-0052 & -2.35 & 7.06 & -9.33 & -107 & 7 & -11 & -122 & 220 & 18.58 & 10.22 & 15.57 \\
\hline BPS CS 30339-0041 & -2.59 & 8.15 & -1.94 & -44 & 16 & 46 & -49 & 234 & 11.61 & 7.60 & 2.85 \\
\hline BPS CS 29503-0054 & -0.67 & 8.40 & -1.60 & 21 & -148 & -2 & 23 & 72 & 8.58 & 1.92 & 1.60 \\
\hline BPS CS 22882-0009 & -2.06 & 8.29 & -1.98 & -151 & -248 & 52 & -151 & -27 & 11.21 & 0.64 & 2.69 \\
\hline BPS CS 30339-0053 & -1.48 & 8.24 & -1.71 & 218 & -339 & -90 & 219 & -117 & 16.80 & 2.62 & 4.28 \\
\hline BPS CS 22882-0036 & -1.96 & 8.33 & -1.30 & 129 & -77 & -25 & 129 & 143 & 11.08 & 3.76 & 1.67 \\
\hline $\mathrm{V}^{*} \mathrm{SW}$ And & -0.24 & 8.71 & -0.30 & -48 & -19 & -23 & -38 & 203 & 9.32 & 7.05 & 0.50 \\
\hline BPS CS 30339-0046 & -2.79 & 6.91 & -9.71 & -87 & 86 & -1 & -125 & 293 & 31.63 & 10.93 & 26.16 \\
\hline BPS CS 30339-0061 & -1.57 & 8.44 & -0.47 & 9 & -80 & -28 & 9 & 140 & 8.46 & 4.09 & 0.68 \\
\hline BPS CS 30339-0062 & -2.44 & 8.34 & -1.19 & -33 & -7 & -49 & -34 & 213 & 9.32 & 7.78 & 1.89 \\
\hline BPS CS 30339-0057 & -2.21 & 8.28 & -1.78 & -169 & -260 & 108 & -169 & -41 & 12.70 & 1.03 & 4.74 \\
\hline [FF93] 514765 & -0.62 & 8.26 & -1.53 & 27 & -144 & 38 & 25 & 77 & 8.41 & 2.12 & 1.98 \\
\hline BPS CS 30339-0069 & -3.15 & 8.28 & -1.51 & 64 & -202 & 3 & 64 & 19 & 8.76 & 0.48 & 1.61 \\
\hline BPS CS 22882-0030 & -2.60 & 8.34 & -1.59 & 90 & -117 & -27 & 90 & 103 & 9.53 & 2.75 & 1.80 \\
\hline BPS CS 22882-0012 & -2.50 & 8.35 & -2.00 & 202 & -311 & -30 & 202 & -93 & 14.18 & 2.14 & 3.15 \\
\hline BPS CS 30339-0064 & -1.36 & 8.41 & -0.66 & -127 & -164 & -75 & -127 & 55 & 10.12 & 1.45 & 2.93 \\
\hline HD 2665 & -1.92 & 8.59 & -0.02 & -175 & -334 & -12 & -177 & -110 & 12.56 & 2.51 & 0.21 \\
\hline HD 2743 & -0.62 & 8.39 & -1.18 & 92 & -105 & 30 & 91 & 115 & 9.46 & 3.17 & 1.74 \\
\hline BPS CS 30339-0055 & -0.84 & 8.41 & -0.81 & 113 & -90 & -31 & 113 & 130 & 10.27 & 3.40 & 1.11 \\
\hline BPS CS 22882-0016 & -1.94 & 8.44 & -0.74 & -6 & -63 & 40 & -6 & 157 & 8.48 & 5.01 & 1.13 \\
\hline HD 2796 & -2.31 & 8.51 & -0.38 & -62 & -77 & 50 & -61 & 143 & 9.13 & 4.18 & 1.03 \\
\hline HD 2857 & -1.51 & 8.60 & -0.66 & -174 & -195 & 61 & -173 & 30 & 12.04 & 0.69 & 1.81 \\
\hline HD 3010 & -0.60 & 8.44 & -0.82 & 99 & -52 & -78 & 99 & 168 & 10.83 & 4.98 & 2.36 \\
\hline HD 3008 & -1.90 & 8.68 & -1.81 & 64 & -141 & 54 & 69 & 75 & 9.26 & 2.24 & 3.15 \\
\hline $\mathrm{V}^{*} \mathrm{RX}$ Cet & -1.28 & 8.57 & -1.36 & -363 & -252 & -8 & -363 & -20 & 33.83 & 0.36 & 7.45 \\
\hline BPS CS 22882-0029 & -2.17 & 8.37 & -1.48 & 36 & -90 & 5 & 35 & 130 & 8.64 & 3.86 & 1.53 \\
\hline BPS CS 30339-0080 & -2.02 & 8.34 & -1.50 & 94 & -130 & 82 & 93 & 91 & 9.34 & 2.88 & 4.06 \\
\hline HD 3179 & -0.92 & 8.50 & -0.18 & 4 & -41 & 76 & 4 & 179 & 8.50 & 6.42 & 1.61 \\
\hline [FF93] 514560 & -0.77 & 8.36 & -0.99 & 24 & -116 & 11 & 23 & 104 & 8.46 & 2.85 & 1.05 \\
\hline HD 3232 & -0.76 & 8.52 & -0.62 & -88 & -206 & 6 & -88 & 15 & 9.24 & 0.36 & 0.66 \\
\hline HD 3276 & -0.62 & 8.41 & -1.42 & -99 & -18 & 29 & -99 & 203 & 11.67 & 6.02 & 1.93 \\
\hline BPS CS 22882-0024 & -1.87 & 8.40 & -1.23 & 115 & -356 & -77 & 116 & -136 & 10.95 & 3.79 & 2.67 \\
\hline BPS CS 30339-0076 & -2.18 & 8.40 & -0.79 & -24 & -32 & -29 & -26 & 188 & 8.62 & 6.45 & 1.03 \\
\hline BPS CS 22882-0026 & -2.04 & 8.40 & -1.15 & -26 & -43 & 86 & -27 & 177 & 8.94 & 6.45 & 2.67 \\
\hline
\end{tabular}


TABLE 3. (continued)

\begin{tabular}{|c|c|c|c|c|c|c|c|c|c|c|c|}
\hline $\begin{array}{l}\text { Star } \\
(1)\end{array}$ & $\begin{array}{c}{[\mathrm{Fe} / \mathrm{H}]} \\
(\mathrm{dex}) \\
(2)\end{array}$ & $\begin{array}{c}R \\
(\mathrm{kpc}) \\
(3)\end{array}$ & $\begin{array}{c}Z \\
(\mathrm{kpc}) \\
(4)\end{array}$ & $\begin{array}{c}U \\
(\mathrm{~km} / \mathrm{s}) \\
(5)\end{array}$ & $\begin{array}{c}V \\
(\mathrm{~km} / \mathrm{s}) \\
(6)\end{array}$ & $\begin{array}{c}W \\
(\mathrm{~km} / \mathrm{s}) \\
(7)\end{array}$ & $\begin{array}{c}V_{R} \\
(\mathrm{~km} / \mathrm{s}) \\
(8)\end{array}$ & $\begin{array}{c}V_{\phi} \\
(\mathrm{km} / \mathrm{s}) \\
(9)\end{array}$ & $\begin{array}{c}R_{a p} \\
(\mathrm{kpc}) \\
(10)\end{array}$ & $\begin{array}{c}R_{p r} \\
(\mathrm{kpc}) \\
(11)\end{array}$ & $\begin{array}{c}Z_{\max } \\
(\mathrm{kpc}) \\
(12)\end{array}$ \\
\hline BPS CS 22882-0021 & -2.06 & 8.43 & -0.94 & -1 & -143 & -51 & -2 & 77 & 8.48 & 2.11 & 1.65 \\
\hline [FF93] 514450 & -0.87 & 8.26 & -2.19 & 72 & -98 & 34 & 69 & 124 & 9.06 & 3.84 & 2.88 \\
\hline HD 3567 & -1.29 & 8.51 & -0.08 & -120 & -184 & -14 & -120 & 36 & 9.88 & 0.82 & 0.23 \\
\hline HD 3582 & -0.60 & 8.37 & -1.45 & 40 & 13 & 36 & 38 & 234 & 10.73 & 8.12 & 2.13 \\
\hline HD 3633 & -0.97 & 8.34 & -1.23 & -87 & -156 & -6 & -88 & 63 & 9.19 & 1.57 & 1.40 \\
\hline BPS CS 29497-0033 & -1.90 & 8.40 & -4.64 & -90 & -180 & -72 & -89 & 42 & 9.81 & 2.17 & 8.36 \\
\hline-29 201W1 & -1.20 & 8.33 & -4.01 & 327 & -164 & -49 & 327 & 56 & 30.60 & 1.27 & 16.34 \\
\hline HD 4024 & -0.67 & 8.47 & -0.96 & -54 & -123 & 9 & -54 & 97 & 8.85 & 2.59 & 1.00 \\
\hline [FF93] 514210 & -0.95 & 8.26 & -2.12 & -120 & -83 & 8 & -125 & 132 & 10.82 & 3.62 & 2.69 \\
\hline HD 4261 & -0.70 & 8.48 & -0.31 & 27 & -23 & 9 & 27 & 197 & 8.75 & 6.66 & 0.34 \\
\hline HD 4306 & -2.71 & 8.59 & -0.59 & 164 & -52 & 85 & 167 & 165 & 14.08 & 4.52 & 3.95 \\
\hline BPS CS 22942-0003 & -1.67 & 8.51 & -1.53 & -395 & -153 & -8 & -395 & 71 & 45.14 & 1.24 & 11.71 \\
\hline [FF93] 514133 & -0.65 & 8.38 & -1.35 & 72 & -2 & -19 & 68 & 219 & 10.97 & 7.02 & 1.71 \\
\hline BPS CS 22942-0002 & -2.62 & 8.52 & -2.70 & 142 & -205 & 153 & 142 & 13 & 11.24 & 4.01 & 11.85 \\
\hline CD-38 245 & -3.92 & 8.09 & -3.69 & 134 & -266 & -16 & 137 & -36 & 10.92 & 0.99 & 4.85 \\
\hline [FF93] 514104 & -0.60 & 8.41 & -1.12 & 35 & -93 & -16 & 33 & 127 & 8.64 & 3.68 & 1.18 \\
\hline CD-29 222 & -0.72 & 8.44 & -2.16 & -62 & -131 & 0 & -63 & 89 & 9.11 & 2.50 & 2.30 \\
\hline$-33302 \mathrm{~S} 1$ & -2.20 & 8.46 & -0.67 & 238 & -196 & 68 & 238 & 25 & 15.54 & 0.56 & 4.24 \\
\hline BD-11 145 & -2.02 & 8.79 & -1.89 & -157 & -206 & 27 & -156 & 22 & 11.78 & 0.54 & 2.39 \\
\hline [FF93] 514033 & -0.60 & 8.32 & -1.77 & 50 & 31 & -19 & 42 & 252 & 12.57 & 8.09 & 2.53 \\
\hline $\mathrm{V}^{*} \mathrm{ZZ}$ And & -1.58 & 9.96 & -1.70 & 195 & -303 & -188 & 174 & -120 & 20.84 & 4.72 & 14.22 \\
\hline HD 5098 & -0.39 & 8.51 & -0.17 & 32 & 20 & -28 & 33 & 240 & 10.57 & 8.12 & 0.49 \\
\hline BPS CS 22942-0010 & -1.54 & 8.54 & -1.04 & 86 & -45 & 5 & 87 & 175 & 10.19 & 5.24 & 1.25 \\
\hline E-29.0.069 & -0.71 & 8.48 & -1.71 & -70 & -11 & 17 & -71 & 209 & 10.88 & 6.80 & 2.09 \\
\hline E-29.3.052 & -0.69 & 8.46 & -2.69 & 70 & -8 & -23 & 68 & 213 & 11.51 & 7.25 & 3.40 \\
\hline BPS CS 22942-0011 & -2.40 & 8.56 & -2.24 & -38 & -114 & -17 & -37 & 106 & 8.95 & 3.22 & 2.43 \\
\hline E-30.3.078 & -0.60 & 8.50 & -2.11 & 82 & -136 & 52 & 82 & 85 & 9.35 & 2.61 & 3.57 \\
\hline E-30.5.084 & -0.84 & 8.43 & -3.53 & -217 & -192 & 72 & -217 & 23 & 16.26 & 0.53 & 6.00 \\
\hline HD 5426 & -2.25 & 8.47 & -0.66 & -37 & -169 & 0 & -38 & 51 & 8.62 & 1.25 & 0.67 \\
\hline HD 5422 & -0.61 & 8.56 & -0.66 & -37 & 4 & 14 & -35 & 224 & 9.89 & 7.85 & 0.77 \\
\hline E-30.1.080 & -0.70 & 8.47 & -3.04 & 105 & -214 & -88 & 105 & 8 & 10.77 & 0.22 & 4.69 \\
\hline CD-28 292 & -0.89 & 8.54 & -2.90 & -28 & -99 & -46 & -29 & 121 & 8.98 & 4.18 & 3.58 \\
\hline E-29.1.079 & -1.72 & 8.50 & -5.39 & -200 & -386 & 26 & -196 & -171 & 19.31 & 5.37 & 11.30 \\
\hline BD-20 170 & -1.31 & 8.53 & -0.40 & 24 & -22 & 12 & 24 & 198 & 8.77 & 6.83 & 0.45 \\
\hline CD-30 288 & -0.64 & 8.50 & -0.36 & 16 & -7 & -37 & 15 & 213 & 8.78 & 7.82 & 0.72 \\
\hline $\mathrm{V}^{*} \mathrm{~W}$ Tuc & -1.57 & 8.09 & -1.15 & -67 & -126 & 35 & -75 & 88 & 8.82 & 2.24 & 1.41 \\
\hline Bok II 274 & -0.67 & 8.54 & -1.91 & 14 & -14 & -35 & 13 & 206 & 9.12 & 8.01 & 2.19 \\
\hline Bok II 284 & -0.92 & 8.52 & -1.47 & -8 & -106 & -31 & -8 & 114 & 8.63 & 3.41 & 1.74 \\
\hline BPS CS 22166-0016 & -2.37 & 8.74 & -1.66 & 101 & -335 & 185 & 98 & -119 & 11.56 & 7.12 & 9.62 \\
\hline CD-30 298 & -2.98 & 8.49 & -1.01 & 319 & -74 & -19 & 318 & 148 & 29.73 & 2.76 & 3.31 \\
\hline
\end{tabular}


TABle 3. (continued)

\begin{tabular}{|c|c|c|c|c|c|c|c|c|c|c|c|}
\hline $\begin{array}{l}\text { Star } \\
\text { (1) }\end{array}$ & $\begin{array}{c}{[\mathrm{Fe} / \mathrm{H}]} \\
(\mathrm{dex}) \\
(2)\end{array}$ & $\begin{array}{c}R \\
(\mathrm{kpc}) \\
(3)\end{array}$ & $\begin{array}{c}Z \\
(\mathrm{kpc}) \\
(4)\end{array}$ & $\begin{array}{c}U \\
(\mathrm{~km} / \mathrm{s}) \\
(5)\end{array}$ & $\begin{array}{c}V \\
(\mathrm{~km} / \mathrm{s}) \\
(6)\end{array}$ & $\begin{array}{c}W \\
(\mathrm{~km} / \mathrm{s}) \\
(7)\end{array}$ & $\begin{array}{c}V_{R} \\
(\mathrm{~km} / \mathrm{s}) \\
(8)\end{array}$ & $\begin{array}{c}V_{\phi} \\
(\mathrm{km} / \mathrm{s}) \\
(9)\end{array}$ & $\begin{array}{c}R_{a p} \\
(\mathrm{kpc}) \\
(10)\end{array}$ & $\begin{array}{c}R_{p r} \\
(\mathrm{kpc}) \\
(11)\end{array}$ & $\begin{array}{c}Z_{\max } \\
(\mathrm{kpc}) \\
(12)\end{array}$ \\
\hline E-29.3.099 & -0.63 & 8.51 & -2.49 & -201 & -107 & -37 & -203 & 110 & 14.76 & 3.03 & 6.42 \\
\hline Bok III 281 & -0.65 & 8.49 & -0.37 & 55 & -31 & 45 & 55 & 190 & 9.36 & 6.19 & 0.99 \\
\hline BPS CS 22942-0024 & -2.60 & 8.57 & -1.12 & -215 & -141 & 34 & -214 & 80 & 14.69 & 1.74 & 1.85 \\
\hline CD-31 394 & -0.78 & 8.48 & -2.53 & 2 & -72 & -9 & -1 & 148 & 8.79 & 5.03 & 2.55 \\
\hline $\mathrm{V}^{*} \mathrm{WY} \mathrm{Scl}$ & -1.51 & 8.56 & -3.01 & 18 & -248 & -98 & 19 & -28 & 9.25 & 1.03 & 5.87 \\
\hline Bok III 179 & -2.27 & 8.48 & -3.00 & -159 & -207 & 31 & -159 & 9 & 11.98 & 0.23 & 3.96 \\
\hline $\mathrm{V}^{*} \mathrm{RU}$ Cet & -1.66 & 8.73 & -1.60 & 34 & -234 & -69 & 33 & -15 & 9.04 & 0.44 & 2.96 \\
\hline$-28308 \mathrm{~S} 1$ & -1.80 & 8.53 & -1.65 & -187 & -141 & 22 & -187 & 78 & 13.12 & 1.80 & 2.40 \\
\hline$-290055 \mathrm{D}$ & -1.50 & 8.60 & -10.48 & -123 & -307 & 24 & -117 & -95 & 15.39 & 5.13 & 13.30 \\
\hline E-29.0.138 & -0.69 & 8.50 & -0.26 & -13 & -52 & 111 & -14 & 168 & 8.61 & 6.51 & 3.00 \\
\hline HD 6054 & -0.77 & 8.54 & -1.40 & -5 & -38 & -5 & -6 & 182 & 8.65 & 6.45 & 1.40 \\
\hline$-290055 \mathrm{E}$ & -0.80 & 8.56 & -5.24 & 250 & -131 & -10 & 247 & 97 & 20.57 & 3.26 & 14.66 \\
\hline-300055 A & -1.50 & 8.68 & -18.92 & -378 & 12 & -3 & -406 & 177 & 158.83 & 18.45 & 156.16 \\
\hline BPS CS 22942-0029 & -1.96 & 8.55 & -1.49 & 144 & -156 & -70 & 143 & 65 & 11.39 & 1.62 & 2.73 \\
\hline$-290055 \mathrm{C}$ & -1.60 & 8.69 & -12.92 & -98 & -240 & 2 & -96 & -28 & 15.93 & 3.34 & 15.96 \\
\hline CD-30 314 & -1.22 & 8.53 & -2.85 & 15 & -334 & -29 & 17 & -114 & 9.01 & 3.65 & 3.02 \\
\hline HD 6446 & -1.60 & 8.26 & -0.39 & 5 & -166 & 83 & 3 & 54 & 8.26 & 1.49 & 2.23 \\
\hline$-310100 \mathrm{C}$ & -1.30 & 8.51 & -6.61 & 30 & -99 & -2 & 22 & 122 & 10.60 & 4.97 & 6.68 \\
\hline$-320100 \mathrm{~A}$ & -1.70 & 8.71 & -30.89 & -280 & 167 & 58 & -391 & 275 & $\cdots$ & $\cdots$ & $\cdots$ \\
\hline$-290100 \mathrm{C}$ & -1.30 & 8.75 & -12.84 & -222 & -46 & 74 & -235 & 155 & 35.40 & 9.11 & 32.22 \\
\hline [FF93] 515282 & -0.77 & 8.41 & -1.64 & -134 & -233 & -28 & -133 & -17 & 10.24 & 0.43 & 2.70 \\
\hline CD-29 308 & -0.60 & 8.50 & -0.19 & -36 & 10 & 5 & -36 & 230 & 9.95 & 7.85 & 0.22 \\
\hline BPS CS 22942-0033 & -1.63 & 8.56 & -1.07 & 64 & -39 & -167 & 64 & 181 & 12.55 & 7.12 & 7.04 \\
\hline HD 6255 & -0.70 & 8.55 & -1.24 & 50 & -87 & 47 & 50 & 134 & 8.93 & 4.17 & 1.98 \\
\hline HD 6268 & -2.48 & 8.52 & -0.61 & -134 & -26 & -36 & -134 & 194 & 12.70 & 5.32 & 1.49 \\
\hline$-280100 \mathrm{~A}$ & -1.10 & 8.85 & -11.12 & 199 & -151 & -96 & 195 & 78 & 24.27 & 4.11 & 21.64 \\
\hline$-300100 \mathrm{~F}$ & -1.10 & 8.73 & -16.55 & -378 & 140 & -6 & -423 & 307 & $\cdots$ & $\cdots$ & $\cdots$ \\
\hline HD 6229 & -1.09 & 8.56 & -0.09 & -51 & -60 & 57 & -49 & 160 & 9.03 & 4.99 & 1.07 \\
\hline BPS CS 22942-0035 & -2.44 & 8.81 & -4.29 & -119 & -297 & 96 & -120 & -76 & 12.90 & 2.30 & 6.26 \\
\hline [FF93] 507215 & -0.74 & 8.61 & -2.24 & -122 & -78 & 79 & -122 & 142 & 12.17 & 4.09 & 3.75 \\
\hline $\mathrm{V}^{*} \mathrm{DR}$ And & -1.48 & 9.72 & -1.01 & 178 & -272 & -42 & 168 & -79 & 13.64 & 2.03 & 1.74 \\
\hline$-300100 \mathrm{~A}$ & -1.80 & 8.91 & -18.57 & -308 & 47 & 24 & -344 & 219 & 113.40 & 17.72 & 109.51 \\
\hline HD 6461 & -0.93 & 8.52 & -0.10 & 30 & 14 & 14 & 30 & 234 & 10.02 & 8.07 & 0.22 \\
\hline Bok III 45 & -0.69 & 8.53 & -4.16 & -85 & -246 & 36 & -84 & -30 & 10.35 & 0.88 & 4.54 \\
\hline CD-31 434 & -0.71 & 8.51 & -0.75 & -49 & -64 & 30 & -50 & 155 & 9.02 & 4.70 & 0.96 \\
\hline$-310100 \mathrm{~A}$ & -1.70 & 8.61 & -8.15 & -105 & -28 & -26 & -120 & 183 & 15.43 & 9.35 & 11.82 \\
\hline BPS CS 29514-0004 & -2.24 & 8.62 & -2.10 & -96 & -193 & 18 & -96 & 26 & 9.81 & 0.68 & 2.29 \\
\hline [FF93] 507267 & -0.69 & 8.61 & -1.81 & -2 & -90 & -24 & -2 & 130 & 8.76 & 4.12 & 1.96 \\
\hline BPS CS 29514-0007 & -2.91 & 8.57 & -1.01 & 170 & -505 & -10 & 170 & -285 & 24.02 & 6.74 & 2.62 \\
\hline-300100 B & -1.20 & 8.78 & -10.76 & -236 & 27 & 19 & -257 & 226 & 45.09 & 10.49 & 38.84 \\
\hline
\end{tabular}


TABLE 3. (continued)

\begin{tabular}{|c|c|c|c|c|c|c|c|c|c|c|c|}
\hline $\begin{array}{l}\text { Star } \\
(1)\end{array}$ & $\begin{array}{c}{[\mathrm{Fe} / \mathrm{H}]} \\
(\mathrm{dex}) \\
(2)\end{array}$ & $\begin{array}{c}R \\
(\mathrm{kpc}) \\
(3)\end{array}$ & $\begin{array}{c}Z \\
(\mathrm{kpc}) \\
(4)\end{array}$ & $\begin{array}{c}U \\
(\mathrm{~km} / \mathrm{s}) \\
(5)\end{array}$ & $\begin{array}{c}V \\
(\mathrm{~km} / \mathrm{s}) \\
(6)\end{array}$ & $\begin{array}{c}W \\
(\mathrm{~km} / \mathrm{s}) \\
(7)\end{array}$ & $\begin{array}{c}V_{R} \\
(\mathrm{~km} / \mathrm{s}) \\
(8)\end{array}$ & $\begin{array}{c}V_{\phi} \\
(\mathrm{km} / \mathrm{s}) \\
(9)\end{array}$ & $\begin{array}{c}R_{a p} \\
(\mathrm{kpc}) \\
(10)\end{array}$ & $\begin{array}{c}R_{p r} \\
(\mathrm{kpc}) \\
(11)\end{array}$ & $\begin{array}{c}Z_{\max } \\
(\mathrm{kpc}) \\
(12)\end{array}$ \\
\hline$-28336 \mathrm{~N} 1$ & -1.90 & 8.54 & -0.96 & 18 & -112 & -68 & 18 & 108 & 8.66 & 3.27 & 2.13 \\
\hline BPS CS 29514-0001 & -2.02 & 8.55 & -0.96 & -68 & -53 & -60 & -68 & 167 & 9.52 & 5.50 & 2.14 \\
\hline CD-33 410 & -0.77 & 8.49 & -1.22 & -126 & -63 & 12 & -129 & 154 & 11.37 & 4.14 & 1.56 \\
\hline$-290105 \mathrm{H}$ & -0.70 & 8.61 & -3.25 & -63 & 10 & 5 & -69 & 228 & 12.85 & 8.01 & 4.45 \\
\hline$-290105 \mathrm{G}$ & -0.80 & 8.66 & -4.24 & -100 & -117 & -69 & -104 & 100 & 10.47 & 4.36 & 7.31 \\
\hline$-290105 \mathrm{~B}$ & -0.80 & 8.60 & -2.24 & -87 & -239 & -44 & -86 & -21 & 9.46 & 0.60 & 3.64 \\
\hline BPS CS 29514-0018 & -2.32 & 8.54 & -0.66 & 23 & -35 & -64 & 23 & 185 & 8.82 & 6.60 & 1.59 \\
\hline [FF93] 507335 & -0.63 & 8.63 & -1.40 & 203 & -9 & -92 & 204 & 211 & 21.30 & 5.05 & 4.88 \\
\hline HD 6755 & -1.49 & 8.57 & 0.00 & 114 & -474 & 88 & 111 & -256 & 16.48 & 7.22 & 3.12 \\
\hline $\mathrm{V}^{*} \mathrm{TZ}$ Phe & -1.29 & 8.31 & -2.28 & 82 & -152 & -54 & 77 & 74 & 9.44 & 2.04 & 2.86 \\
\hline CD-27 379 & -0.62 & 8.55 & -0.81 & -63 & -31 & -65 & -64 & 189 & 9.77 & 6.44 & 2.10 \\
\hline E-30.0.203 & -0.65 & 8.56 & -1.58 & 94 & -12 & -19 & 91 & 209 & 11.75 & 6.49 & 2.05 \\
\hline$-290105 \mathrm{E}$ & -0.90 & 8.69 & -4.80 & 176 & -239 & 61 & 176 & -12 & 13.01 & 1.67 & 13.31 \\
\hline BPS CS 29514-0012 & -0.34 & 8.57 & -0.78 & 7 & -12 & -9 & 7 & 208 & 8.65 & 7.77 & 0.80 \\
\hline HD 7150 & -0.97 & 8.58 & -1.26 & -47 & -226 & 48 & -47 & -6 & 8.92 & 0.16 & 1.84 \\
\hline BPS CS 29514-0014 & -2.17 & 8.60 & -1.30 & 0 & -366 & 31 & 0 & -146 & 8.68 & 4.68 & 1.54 \\
\hline$-290105 \mathrm{~N}$ & -0.80 & 9.01 & -10.19 & -200 & 29 & 3 & -220 & 231 & 36.42 & 10.69 & 30.17 \\
\hline$-290110 \mathrm{~A}$ & -1.20 & 8.72 & -4.47 & -285 & -220 & 1 & -285 & -11 & 23.08 & 0.38 & 17.94 \\
\hline [FF93] 507458 & -0.67 & 8.73 & -2.59 & -32 & 0 & 23 & -34 & 220 & 10.82 & 8.32 & 3.06 \\
\hline [FF93] 507463 & -0.60 & 8.66 & -1.58 & 41 & -15 & 28 & 41 & 205 & 9.62 & 7.58 & 1.97 \\
\hline$-310110 \mathrm{C}$ & -0.70 & 8.69 & -5.19 & 210 & -401 & -31 & 222 & -167 & 22.03 & 4.98 & 12.52 \\
\hline$-290110 \mathrm{D}$ & -1.00 & 9.52 & -15.59 & 104 & -553 & -107 & 150 & -315 & 69.43 & 15.02 & 59.47 \\
\hline $\mathrm{V}^{*} \mathrm{XX}$ And & -1.94 & 9.11 & -0.41 & 178 & -207 & -119 & 178 & -1 & 13.63 & 0.04 & 6.37 \\
\hline$-300115 \mathrm{~B}$ & -2.20 & 9.23 & -12.88 & -34 & -234 & -60 & -31 & -19 & 15.68 & 2.15 & 15.63 \\
\hline
\end{tabular}


TABle 3. (continued)

\begin{tabular}{|c|c|c|c|c|c|c|c|c|c|c|c|}
\hline $\begin{array}{l}\text { Star } \\
(1)\end{array}$ & $\begin{array}{c}{[\mathrm{Fe} / \mathrm{H}]} \\
(\mathrm{dex}) \\
(2)\end{array}$ & $\begin{array}{c}R \\
(\mathrm{kpc}) \\
(3)\end{array}$ & $\begin{array}{c}Z \\
(\mathrm{kpc}) \\
(4)\end{array}$ & $\begin{array}{c}U \\
(\mathrm{~km} / \mathrm{s}) \\
(5)\end{array}$ & $\begin{array}{c}V \\
(\mathrm{~km} / \mathrm{s}) \\
(6)\end{array}$ & $\begin{array}{c}W \\
(\mathrm{~km} / \mathrm{s}) \\
(7)\end{array}$ & $\begin{array}{c}V_{R} \\
(\mathrm{~km} / \mathrm{s}) \\
(8)\end{array}$ & $\begin{array}{c}V_{\phi} \\
(\mathrm{km} / \mathrm{s}) \\
(9)\end{array}$ & $\begin{array}{c}R_{a p} \\
(\mathrm{kpc}) \\
(10)\end{array}$ & $\begin{array}{c}R_{p r} \\
(\mathrm{kpc}) \\
(11)\end{array}$ & $\begin{array}{c}Z_{\max } \\
(\mathrm{kpc}) \\
(12)\end{array}$ \\
\hline$-300115 \mathrm{C}$ & -0.80 & 8.86 & -5.98 & -79 & -354 & 1 & -70 & -139 & 11.37 & 5.68 & 6.70 \\
\hline$-270115 \mathrm{~A}$ & -1.30 & 9.92 & -17.05 & 45 & -47 & 34 & 25 & 177 & 19.65 & 14.88 & 17.37 \\
\hline BPS CS 29514-0031 & -0.86 & 8.60 & -0.80 & 46 & -81 & 72 & 46 & 139 & 8.92 & 4.54 & 2.27 \\
\hline V* VW Scl & -0.84 & 8.46 & -1.09 & -26 & -239 & 12 & -25 & -20 & 8.58 & 0.50 & 1.12 \\
\hline $\mathrm{V}^{*}$ AM Tuc & -1.49 & 8.05 & -1.20 & -21 & -290 & 69 & -13 & -72 & 8.17 & 2.02 & 2.34 \\
\hline BPS CS 29514-0034 & -1.75 & 8.76 & -2.45 & -38 & -151 & -33 & -38 & 69 & 9.15 & 2.08 & 3.01 \\
\hline Bok IV 16 & -0.61 & 8.63 & -1.73 & 19 & -223 & -1 & 20 & -3 & 8.81 & 0.07 & 1.73 \\
\hline HD 7983 & -0.68 & 8.52 & -0.06 & -147 & -61 & -40 & -147 & 159 & 12.10 & 4.04 & 0.82 \\
\hline $\mathrm{V}^{*} \mathrm{SS}$ Psc & -0.89 & 9.09 & -0.73 & -14 & 7 & -9 & 1 & 227 & 9.74 & 9.11 & 0.80 \\
\hline$-310115 \mathrm{~A}$ & -1.40 & 10.22 & -24.65 & 0 & -633 & 130 & 127 & -393 & 181.96 & 25.97 & 170.10 \\
\hline HD 8186 & -0.67 & 8.70 & -0.32 & -24 & -54 & 5 & -20 & 167 & 8.79 & 5.29 & 0.33 \\
\hline BPS CS 29514-0037 & -2.16 & 8.58 & -0.85 & -12 & -190 & 51 & -12 & 29 & 8.64 & 0.77 & 1.59 \\
\hline-300115 A & -1.10 & 10.05 & -20.11 & -330 & -514 & 44 & -256 & -361 & 172.85 & 20.39 & 159.00 \\
\hline-290115 A & -1.50 & 9.64 & -13.82 & 3 & -62 & 222 & -19 & 156 & 33.79 & 9.25 & 29.96 \\
\hline BPS CS 29504-0012 & -2.20 & 8.59 & -1.76 & -41 & -115 & 151 & -45 & 104 & 9.80 & 4.59 & 6.71 \\
\hline HD 8724 & -1.64 & 8.83 & -0.46 & -20 & -286 & -57 & -22 & -65 & 8.88 & 1.77 & 1.48 \\
\hline [FF93] 516243 & -0.70 & 8.53 & -1.93 & 57 & -92 & -48 & 51 & 131 & 9.26 & 4.06 & 2.40 \\
\hline $\mathrm{V}^{*} \mathrm{XY}$ And & -0.92 & 10.83 & -1.68 & 124 & -187 & 27 & 128 & 6 & 12.88 & 0.17 & 3.13 \\
\hline BPS CS 29504-0002 & -0.86 & 8.51 & -0.88 & -197 & -114 & 58 & -199 & 101 & 14.15 & 2.29 & 2.05 \\
\hline HD 9051 & -1.50 & 8.57 & -0.45 & 48 & -82 & 93 & 48 & 138 & 8.93 & 4.66 & 2.76 \\
\hline BPS CS 29504-0009 & -1.56 & 8.54 & -1.26 & -11 & -37 & -85 & -16 & 182 & 8.64 & 7.32 & 2.79 \\
\hline HD 9360 & -0.62 & 8.67 & -0.99 & -39 & 11 & 20 & -40 & 231 & 10.75 & 8.04 & 1.25 \\
\hline BPS CS 29504-0006 & -2.93 & 8.54 & -1.29 & 17 & 87 & 83 & 7 & 307 & 20.42 & 8.62 & 4.95 \\
\hline $\mathrm{V}^{*} \mathrm{RR}$ Cet & -1.45 & 8.76 & -0.56 & -78 & -130 & 12 & -76 & 92 & 9.43 & 2.38 & 0.62 \\
\hline BPS CS 22180-0013 & -0.60 & 8.70 & -0.67 & 29 & 19 & 7 & 32 & 238 & 10.77 & 8.35 & 0.82 \\
\hline HD 9731 & -0.65 & 8.55 & -0.30 & 37 & 3 & -23 & 36 & 223 & 9.76 & 7.72 & 0.49 \\
\hline V* VX Scl & -2.25 & 8.62 & -2.00 & 105 & -379 & 144 & 113 & -154 & 12.09 & 6.78 & 8.04 \\
\hline BD-18 271 & -2.17 & 9.09 & -2.32 & -170 & -269 & 173 & -171 & -47 & 16.73 & 1.77 & 12.24 \\
\hline HD 10141 & -0.92 & 8.58 & -0.80 & 11 & -133 & -54 & 10 & 87 & 8.63 & 2.45 & 1.59 \\
\hline BPS CS 29504-0032 & -1.48 & 8.54 & -0.45 & -55 & -29 & 41 & -57 & 190 & 9.55 & 6.13 & 0.89 \\
\hline HD 10413 & -0.66 & 8.64 & -0.93 & 72 & -9 & -5 & 69 & 212 & 10.71 & 6.88 & 1.12 \\
\hline HD 10607 & -1.14 & 8.48 & -0.05 & -14 & -134 & 124 & -14 & 86 & 8.51 & 3.04 & 4.22 \\
\hline $\mathrm{V}^{*} \mathrm{SV} \mathrm{Scl}$ & -1.77 & 8.70 & -1.42 & -180 & -163 & 23 & -181 & 52 & 12.68 & 1.19 & 1.96 \\
\hline BPS CS 29504-0044 & -2.04 & 8.57 & -0.68 & -115 & -92 & -249 & -117 & 126 & 17.39 & 7.29 & 15.20 \\
\hline BPS CS 22958-0002 & -2.37 & 8.18 & -2.10 & -457 & -359 & 411 & -431 & -207 & $\cdots$ & $\cdots$ & $\cdots$ \\
\hline HD 11569 & -1.65 & 8.47 & -0.07 & 88 & -68 & 61 & 87 & 153 & 9.76 & 4.43 & 1.28 \\
\hline BD-10 388 & -2.44 & 8.55 & -0.12 & 153 & -37 & 31 & 154 & 183 & 13.23 & 4.67 & 0.68 \\
\hline HD 11582 & -1.57 & 8.52 & -0.21 & -137 & -170 & 29 & -137 & 49 & 10.42 & 1.13 & 0.51 \\
\hline $\mathrm{V}^{*} \mathrm{CI}$ And & -0.69 & 9.80 & -0.55 & 10 & 19 & -14 & 40 & 236 & 12.13 & 9.10 & 0.72 \\
\hline $\mathrm{V}^{*} \mathrm{U}$ Tri & -0.79 & 10.02 & -1.00 & 52 & -118 & -87 & 65 & 94 & 10.85 & 3.23 & 3.69 \\
\hline
\end{tabular}


TABle 3. (continued)

\begin{tabular}{|c|c|c|c|c|c|c|c|c|c|c|c|}
\hline $\begin{array}{l}\text { Star } \\
(1)\end{array}$ & $\begin{array}{c}{[\mathrm{Fe} / \mathrm{H}]} \\
(\mathrm{dex}) \\
(2)\end{array}$ & $\begin{array}{c}R \\
(\mathrm{kpc}) \\
(3)\end{array}$ & $\begin{array}{c}Z \\
(\mathrm{kpc}) \\
(4)\end{array}$ & $\begin{array}{c}U \\
(\mathrm{~km} / \mathrm{s}) \\
(5)\end{array}$ & $\begin{array}{c}V \\
(\mathrm{~km} / \mathrm{s}) \\
(6)\end{array}$ & $\begin{array}{c}W \\
(\mathrm{~km} / \mathrm{s}) \\
(7)\end{array}$ & $\begin{array}{c}V_{R} \\
(\mathrm{~km} / \mathrm{s}) \\
(8)\end{array}$ & $\begin{array}{c}V_{\phi} \\
(\mathrm{km} / \mathrm{s}) \\
(9)\end{array}$ & $\begin{array}{c}R_{a p} \\
(\mathrm{kpc}) \\
(10)\end{array}$ & $\begin{array}{c}R_{p r} \\
(\mathrm{kpc}) \\
(11)\end{array}$ & $\begin{array}{c}Z_{\max } \\
(\mathrm{kpc}) \\
(12)\end{array}$ \\
\hline BPS CS 22958-0038 & -2.04 & 8.41 & -0.60 & 161 & -114 & -30 & 156 & 113 & 11.56 & 2.67 & 0.94 \\
\hline $\mathrm{V}^{*} \mathrm{SS}$ For & -0.94 & 8.68 & -0.74 & -129 & -230 & 139 & -129 & -12 & 11.30 & 0.40 & 7.25 \\
\hline BPS CS 22171-0034 & -2.12 & 8.80 & -0.63 & -32 & -232 & 86 & -32 & -12 & 8.96 & 0.35 & 2.89 \\
\hline HD 13359 & -1.66 & 8.60 & -0.32 & -16 & -5 & -26 & -17 & 215 & 8.89 & 7.96 & 0.53 \\
\hline HD 13889 & -1.17 & 8.51 & -0.08 & -39 & -66 & -30 & -40 & 153 & 8.77 & 4.55 & 0.42 \\
\hline$V^{*}$ RV Cet & -1.60 & 8.99 & -1.02 & -8 & -161 & 108 & -8 & 59 & 9.08 & 2.11 & 4.65 \\
\hline HD 13979 & -2.61 & 8.62 & -0.43 & -18 & -116 & -28 & -19 & 104 & 8.66 & 2.84 & 0.67 \\
\hline $\mathrm{V}^{*} \mathrm{RW}$ Ari & -1.16 & 9.88 & -1.35 & -143 & -81 & -89 & -132 & 150 & 13.50 & 5.46 & 5.59 \\
\hline BD-22 395 & -2.14 & 8.92 & -1.16 & 42 & -275 & -42 & 43 & -54 & 9.22 & 1.47 & 1.64 \\
\hline HD 14829 & -1.25 & 8.73 & -0.46 & -105 & -119 & 160 & -105 & 101 & 11.28 & 4.10 & 7.45 \\
\hline HD 15061 & -0.91 & 9.38 & -0.80 & 36 & -118 & -34 & 41 & 100 & 9.64 & 2.92 & 1.14 \\
\hline HD 15395 & -1.14 & 8.49 & -0.07 & -33 & -48 & -2 & -34 & 172 & 8.73 & 5.29 & 0.08 \\
\hline $\mathrm{BD}+44493$ & -2.90 & 8.87 & -0.12 & 67 & -296 & 73 & 64 & -78 & 9.33 & 2.18 & 1.92 \\
\hline V* RZ Cet & -1.36 & 9.33 & -1.47 & 117 & -96 & 85 & 118 & 123 & 11.53 & 4.32 & 4.97 \\
\hline BD-17 484 & -1.68 & 8.57 & -0.16 & 267 & -266 & -81 & 268 & -45 & 18.79 & 0.93 & 3.61 \\
\hline HD 16031 & -1.71 & 8.55 & -0.10 & -37 & -83 & -23 & -37 & 137 & 8.74 & 3.88 & 0.33 \\
\hline $\mathrm{V}^{*}$ CS Eri & -1.41 & 8.55 & -0.41 & 123 & -55 & 220 & 119 & 168 & 17.27 & 7.09 & 12.63 \\
\hline BPS CS 22189-0007 & -2.12 & 9.10 & -1.11 & -232 & -131 & 218 & -233 & 87 & 26.38 & 3.40 & 21.02 \\
\hline CD-50 776 & -0.92 & 8.51 & -0.25 & 63 & -69 & 36 & 60 & 152 & 9.09 & 4.45 & 0.67 \\
\hline BPS CS 22954-0015 & -1.67 & 8.83 & -0.46 & -57 & -149 & -2 & -56 & 71 & 9.16 & 1.81 & 0.48 \\
\hline HD 17072 & -1.00 & 8.47 & -0.09 & -4 & -59 & -17 & -6 & 161 & 8.48 & 4.90 & 0.22 \\
\hline HD 17233 & -0.60 & 8.49 & -0.10 & 60 & 24 & 17 & 58 & 244 & 11.62 & 7.71 & 0.31 \\
\hline BD-10 548 & -1.07 & 8.69 & -0.31 & 192 & -111 & -141 & 192 & 109 & 15.68 & 3.15 & 7.64 \\
\hline CD-36 1052 & -2.19 & 8.68 & -0.71 & 92 & -85 & -268 & 87 & 138 & 19.39 & 7.49 & 16.60 \\
\hline BPS CS 22184-0040 & -1.72 & 9.14 & -1.00 & 172 & -112 & -80 & 171 & 109 & 13.94 & 2.85 & 3.18 \\
\hline $\mathrm{V}^{*} \mathrm{BK}$ Eri & -1.64 & 10.02 & -1.92 & 284 & -273 & 41 & 283 & -56 & 24.05 & 1.48 & 10.05 \\
\hline V* BY Eri & -1.16 & 8.77 & -2.17 & 268 & -354 & 85 & 285 & -92 & 23.77 & 2.67 & 14.95 \\
\hline CD-30 1121 & -1.62 & 8.70 & -0.56 & 25 & -139 & -40 & 23 & 81 & 8.78 & 2.18 & 1.00 \\
\hline HD 18710 & -1.05 & 8.55 & -0.56 & 25 & 62 & -2 & 13 & 283 & 14.69 & 8.54 & 0.90 \\
\hline $\mathrm{V}^{*} \mathrm{X}$ Ari & -2.43 & 8.91 & -0.35 & -23 & -275 & -18 & -23 & -55 & 8.97 & 1.40 & 0.47 \\
\hline HD 20038 & -0.87 & 8.49 & -0.19 & -34 & 8 & -23 & -38 & 228 & 9.91 & 7.77 & 0.41 \\
\hline HD 19697 & -0.65 & 9.25 & -0.57 & -33 & -125 & -85 & -31 & 96 & 9.36 & 3.14 & 2.94 \\
\hline V* SV Eri & -1.70 & 8.89 & -0.54 & -84 & -140 & -2 & -85 & 79 & 9.67 & 2.02 & 0.59 \\
\hline V* UY Eri & -1.60 & 9.69 & -1.59 & 163 & -236 & -37 & 163 & -11 & 13.13 & 0.28 & 2.15 \\
\hline HD 20305 & -0.74 & 9.05 & -0.40 & 27 & 1 & -1 & 29 & 221 & 9.92 & 8.25 & 0.44 \\
\hline $\mathrm{V}^{*}$ UU Hor & -1.80 & 8.81 & -1.92 & 41 & -259 & -65 & 46 & -32 & 9.34 & 0.93 & 3.04 \\
\hline HD 21022 & -1.99 & 8.88 & -0.94 & -26 & -251 & 4 & -24 & -32 & 8.98 & 0.82 & 0.94 \\
\hline HD 21581 & -1.63 & 8.66 & -0.15 & 96 & -106 & -97 & 96 & 115 & 10.07 & 3.44 & 2.98 \\
\hline $\mathrm{V}^{*} \mathrm{SX}$ For & -1.66 & 8.91 & -1.05 & 122 & -277 & -55 & 126 & -49 & 10.84 & 1.23 & 1.88 \\
\hline BPS CS 22172-0025 & -1.17 & 8.85 & -0.43 & 70 & 37 & 34 & 66 & 258 & 13.88 & 8.15 & 1.15 \\
\hline
\end{tabular}


TABLE 3. (continued)

\begin{tabular}{|c|c|c|c|c|c|c|c|c|c|c|c|}
\hline $\begin{array}{l}\text { Star } \\
(1)\end{array}$ & $\begin{array}{c}{[\mathrm{Fe} / \mathrm{H}]} \\
(\mathrm{dex}) \\
(2)\end{array}$ & $\begin{array}{c}R \\
(\mathrm{kpc}) \\
(3)\end{array}$ & $\begin{array}{c}Z \\
(\mathrm{kpc}) \\
(4)\end{array}$ & $\begin{array}{c}U \\
(\mathrm{~km} / \mathrm{s}) \\
(5)\end{array}$ & $\begin{array}{c}V \\
(\mathrm{~km} / \mathrm{s}) \\
(6)\end{array}$ & $\begin{array}{c}W \\
(\mathrm{~km} / \mathrm{s}) \\
(7)\end{array}$ & $\begin{array}{c}V_{R} \\
(\mathrm{~km} / \mathrm{s}) \\
(8)\end{array}$ & $\begin{array}{c}V_{\phi} \\
(\mathrm{km} / \mathrm{s}) \\
(9)\end{array}$ & $\begin{array}{c}R_{a p} \\
(\mathrm{kpc}) \\
(10)\end{array}$ & $\begin{array}{c}R_{p r} \\
(\mathrm{kpc}) \\
(11)\end{array}$ & $\begin{array}{c}Z_{\max } \\
(\mathrm{kpc}) \\
(12)\end{array}$ \\
\hline V* SS Tau & -0.28 & 9.83 & -1.06 & 21 & -12 & 62 & 21 & 208 & 10.24 & 9.27 & 2.22 \\
\hline CD-24 1782 & -2.46 & 8.79 & -0.47 & -109 & -401 & -8 & -105 & -184 & 11.22 & 5.37 & 0.63 \\
\hline HD 22879 & -0.78 & 8.52 & -0.02 & 99 & -74 & -36 & 99 & 147 & 10.01 & 3.97 & 0.58 \\
\hline HD 23332 & -0.90 & 8.51 & -0.26 & 25 & 43 & 24 & 18 & 264 & 12.43 & 8.45 & 0.59 \\
\hline HD 23592 & -1.50 & 8.56 & -0.49 & 74 & 48 & 57 & 61 & 271 & 15.00 & 8.14 & 2.00 \\
\hline HD 23798 & -2.20 & 8.91 & -0.78 & 59 & -92 & -10 & 52 & 131 & 9.34 & 3.84 & 0.82 \\
\hline BPS CS 30494-0003 & -2.37 & 9.25 & -0.96 & -20 & -65 & 107 & -29 & 154 & 9.71 & 6.18 & 3.82 \\
\hline HD 26169 & -2.47 & 8.40 & -0.22 & 205 & -119 & 114 & 201 & 107 & 14.76 & 2.78 & 5.65 \\
\hline BPS CS 22173-0025 & -0.97 & 8.86 & -0.46 & 163 & -11 & 66 & 157 & 213 & 16.07 & 5.88 & 2.84 \\
\hline HD 25532 & -1.28 & 8.58 & -0.03 & -105 & -64 & 42 & -104 & 157 & 10.41 & 4.33 & 0.74 \\
\hline HD 26297 & -1.78 & 8.86 & -0.39 & 34 & -80 & 76 & 31 & 141 & 9.01 & 4.71 & 2.03 \\
\hline $\mathrm{V}^{*} \mathrm{XY}$ Eri & -2.08 & 10.55 & -2.01 & 259 & -101 & 0 & 246 & 143 & 23.63 & 3.87 & 5.36 \\
\hline BPS CS 22169-0035 & -3.23 & 13.48 & -4.56 & -81 & -102 & -15 & -100 & 103 & 15.91 & 4.56 & 6.43 \\
\hline BPS CS 22186-0005 & -2.77 & 9.93 & -2.44 & 175 & -293 & 80 & 186 & -38 & 14.76 & 1.55 & 10.54 \\
\hline BD+06 648 & -2.09 & 9.69 & -0.71 & -165 & -296 & 76 & -164 & -78 & 13.63 & 2.11 & 3.03 \\
\hline BPS CS 22186-0002 & -2.22 & 8.74 & -0.47 & 166 & -301 & -138 & 169 & -74 & 13.52 & 2.25 & 7.20 \\
\hline BPS CS 22186-0017 & -2.53 & 8.83 & -0.57 & 335 & -310 & 46 & 339 & -73 & 30.32 & 1.38 & 5.29 \\
\hline BPS CS 22182-0022 & -1.96 & 8.77 & -0.43 & 182 & -236 & -130 & 182 & -9 & 13.60 & 0.25 & 7.05 \\
\hline $\mathrm{V}^{*}$ AR Per & -0.30 & 9.06 & -0.02 & -3 & 7 & -24 & 4 & 227 & 9.53 & 9.03 & 0.33 \\
\hline HD 27145 & -0.70 & 8.61 & -0.05 & 60 & 7 & 23 & 60 & 227 & 10.77 & 7.39 & 0.36 \\
\hline BPS CS 22182-0021 & -1.26 & 8.94 & -0.68 & 31 & 0 & 9 & 18 & 222 & 9.54 & 8.55 & 0.74 \\
\hline BPS CS 22186-0023 & -2.55 & 9.64 & -1.97 & 21 & -94 & 41 & -1 & 128 & 9.82 & 4.45 & 2.48 \\
\hline BPS CS 22182-0024 & -1.56 & 8.76 & -0.41 & 86 & -54 & 3 & 80 & 169 & 10.01 & 5.01 & 0.47 \\
\hline BPS CS 22186-0022 & -1.97 & 8.84 & -0.63 & 241 & -286 & 22 & 245 & -51 & 16.62 & 1.08 & 1.80 \\
\hline BPS CS 22182-0047 & -1.96 & 8.79 & -0.40 & -75 & -226 & 102 & -75 & -9 & 9.49 & 0.27 & 3.75 \\
\hline HD 27686 & -0.79 & 9.01 & -0.22 & -14 & 2 & -17 & -14 & 222 & 9.40 & 8.62 & 0.35 \\
\hline HD 27928 & -2.25 & 8.75 & -0.48 & -165 & -154 & 50 & -168 & 58 & 12.04 & 1.36 & 1.34 \\
\hline BPS CS 22182-0033 & -2.30 & 9.11 & -0.88 & 134 & -261 & -21 & 137 & -31 & 11.18 & 0.74 & 1.09 \\
\hline BPS CS 22182-0025 & -1.96 & 9.09 & -0.89 & 20 & -355 & 33 & 31 & -133 & 9.24 & 4.17 & 1.27 \\
\hline HD 28085 & -0.63 & 8.75 & -0.10 & -36 & 16 & -1 & -36 & 236 & 10.55 & 8.20 & 0.12 \\
\hline BPS CS 22186-0050 & -1.98 & 8.81 & -0.54 & 177 & 3 & 39 & 164 & 233 & 17.65 & 6.08 & 2.00 \\
\hline CD-57 949 & -0.69 & 8.52 & -0.25 & 26 & -33 & 34 & 20 & 188 & 8.64 & 6.38 & 0.58 \\
\hline $\mathrm{V}^{*}$ HP Eri & -1.96 & 9.67 & -0.94 & -296 & -213 & -223 & -296 & -14 & 37.42 & 3.90 & 37.50 \\
\hline HD 30229 & -2.32 & 8.47 & -0.22 & -92 & -262 & -127 & -90 & -45 & 9.46 & 1.52 & 5.33 \\
\hline $\mathrm{V}^{*} \mathrm{BC}$ Eri & -1.16 & 9.33 & -0.65 & 39 & -96 & 38 & 32 & 126 & 9.48 & 3.90 & 1.17 \\
\hline $\mathrm{V}^{*} \mathrm{RX}$ Eri & -1.33 & 8.91 & -0.33 & 1 & -17 & -77 & -5 & 203 & 8.93 & 8.37 & 1.80 \\
\hline BD-22 928 & -0.97 & 8.92 & -0.41 & 56 & 32 & 37 & 46 & 254 & 12.87 & 8.50 & 1.09 \\
\hline $\mathrm{V}^{*} \mathrm{U} \mathrm{Pic}$ & -0.72 & 8.77 & -0.84 & -4 & 7 & -19 & -30 & 226 & 10.02 & 8.30 & 1.05 \\
\hline CPD-62 394 & -2.38 & 8.53 & -0.87 & 101 & -282 & 108 & 109 & -48 & 9.84 & 1.60 & 5.32 \\
\hline HD 31128 & -1.82 & 8.53 & -0.04 & 50 & -85 & -19 & 50 & 135 & 8.88 & 3.77 & 0.24 \\
\hline
\end{tabular}


TABLE 3. (continued)

\begin{tabular}{|c|c|c|c|c|c|c|c|c|c|c|c|c|}
\hline $\begin{array}{l}\text { Star } \\
(1)\end{array}$ & $\begin{array}{c}{[\mathrm{Fe} / \mathrm{H}]} \\
(\mathrm{dex}) \\
(2)\end{array}$ & $\begin{array}{c}R \\
(\mathrm{kpc}) \\
(3)\end{array}$ & $\begin{array}{c}Z \\
(\mathrm{kpc}) \\
(4)\end{array}$ & $\begin{array}{c}U \\
(\mathrm{~km} / \mathrm{s}) \\
(5)\end{array}$ & $\begin{array}{c}V \\
(\mathrm{~km} / \mathrm{s}) \\
(6)\end{array}$ & $\begin{array}{c}W \\
(\mathrm{~km} / \mathrm{s}) \\
(7)\end{array}$ & $\begin{array}{c}V_{R} \\
(\mathrm{~km} / \mathrm{s}) \\
(8)\end{array}$ & $\begin{array}{c}V_{\phi} \\
(\mathrm{km} / \mathrm{s}) \\
(9)\end{array}$ & $\begin{array}{c}R_{a p} \\
(\mathrm{kpc}) \\
(10)\end{array}$ & $\begin{array}{c}R_{p r} \\
(\mathrm{kpc}) \\
(11)\end{array}$ & $\begin{array}{c}Z_{\max } \\
(\mathrm{kpc}) \\
(12)\end{array}$ & (13) \\
\hline $\mathrm{V}^{*} \mathrm{U} \mathrm{Cae}$ & -1.11 & 9.33 & -1.22 & 64 & -57 & 128 & 40 & 170 & 9.93 & 8.18 & 5.05 & 0.1 \\
\hline $\mathrm{V}^{*} \mathrm{BB}$ Eri & -1.32 & 9.43 & -0.79 & 193 & -242 & 33 & 194 & -7 & 13.91 & 0.18 & 2.21 & 0.9 \\
\hline $\mathrm{V}^{*} \mathrm{U}$ Lep & -1.78 & 9.12 & -0.55 & -105 & -333 & 11 & -98 & -118 & 10.44 & 3.23 & 0.63 & 0.5 \\
\hline HD 32546 & -1.30 & 8.45 & -0.31 & 55 & -140 & -64 & 51 & 82 & 8.77 & 2.21 & 1.44 & 0.6 \\
\hline $\mathrm{V}^{*} \mathrm{~V}$ Cae & -2.00 & 9.78 & -1.78 & -367 & -155 & 40 & -372 & -14 & 42.01 & 0.27 & 7.43 & 0.9 \\
\hline HD 268957 & -1.63 & 8.42 & -0.32 & 308 & -185 & -107 & 305 & 51 & 24.40 & 1.02 & 6.86 & 0.9 \\
\hline V* V964 Ori & -1.89 & 10.76 & -1.10 & 76 & -212 & -64 & 75 & 15 & 11.60 & 0.45 & 2.91 & 0.9 \\
\hline HD 33073 & -1.30 & 8.60 & -0.11 & 27 & -56 & -21 & 24 & 164 & 8.72 & 5.09 & 0.29 & 0.2 \\
\hline CPD-57 752 & -0.77 & 8.53 & -0.24 & -21 & 37 & -42 & -31 & 256 & 12.06 & 8.34 & 0.94 & 0.1 \\
\hline HD 34048 & -1.50 & 8.49 & -0.38 & -127 & -295 & -3 & -122 & -83 & 10.08 & 1.98 & 0.47 & $0.6^{\prime}$ \\
\hline HD 33771 & -1.93 & 8.83 & -0.46 & -66 & -221 & 227 & -66 & -5 & 10.97 & 5.68 & 11.67 & 0.3 \\
\hline HD 33605 & -1.51 & 9.25 & -0.09 & 16 & 28 & 16 & 18 & 248 & 11.65 & 9.14 & 0.28 & 0.1 \\
\hline HD 34328 & -1.61 & 8.50 & -0.04 & 200 & -339 & 103 & 201 & -117 & 14.93 & 2.92 & 4.50 & $0.6^{\prime}$ \\
\hline $\mathrm{V}^{*} \mathrm{RY}$ Col & -0.91 & 8.88 & -0.60 & 272 & -402 & -117 & 287 & -157 & 29.26 & 3.41 & 8.55 & 0.7 \\
\hline HD 241894 & -1.22 & 9.19 & -0.06 & 22 & 21 & 22 & 23 & 241 & 11.15 & 8.95 & 0.36 & 0.1 \\
\hline HD 242106 & -0.75 & 8.85 & -0.04 & -42 & -44 & -12 & -42 & 176 & 9.26 & 5.68 & 0.16 & 0.2 \\
\hline $\mathrm{V}^{*} \mathrm{RT}$ Col & -1.43 & 10.01 & -1.33 & 78 & -163 & -210 & 68 & 69 & 13.14 & 6.40 & 12.43 & 0.3 \\
\hline HD 273975 & -1.40 & 8.53 & -0.09 & -109 & -357 & -63 & -107 & -139 & 10.26 & 3.85 & 1.46 & 0.4 \\
\hline HD 34414 & -0.84 & 8.84 & -0.02 & -73 & -16 & 0 & -72 & 204 & 10.53 & 6.50 & 0.03 & 0.2 \\
\hline HD 35179 & -0.67 & 8.77 & -0.16 & -25 & -11 & -25 & -30 & 208 & 9.26 & 7.44 & 0.41 & 0.1 \\
\hline HD 35238 & -0.69 & 8.72 & -0.01 & 26 & 25 & -27 & 26 & 245 & 11.05 & 8.49 & 0.42 & 0.1 \\
\hline HD 36054 & -1.38 & 8.81 & -0.03 & -6 & 4 & 5 & -6 & 224 & 9.06 & 8.70 & 0.07 & 0.0 \\
\hline HD 36702 & -2.06 & 8.94 & -0.55 & -47 & -123 & -56 & -55 & 92 & 9.28 & 2.64 & 1.65 & 0.5 \\
\hline HD 274939 & -1.76 & 8.59 & -0.21 & -184 & -202 & -97 & -184 & 11 & 12.62 & 0.28 & 4.29 & 0.9 \\
\hline HD 244759 & -0.96 & 9.06 & -0.03 & -68 & -24 & 6 & -68 & 196 & 10.42 & 6.39 & 0.08 & 0.2 \\
\hline HD 36931 & -2.09 & 9.17 & -0.01 & -7 & 11 & -4 & -6 & 231 & 9.88 & 9.12 & 0.05 & 0.0 \\
\hline $\mathrm{V}^{*} \mathrm{HH}$ Aur & -0.81 & 8.87 & 0.00 & -69 & 0 & -16 & -69 & 220 & 11.06 & 7.17 & 0.24 & 0.2 \\
\hline HD 246114 & -0.84 & 8.95 & -0.04 & 56 & 0 & 4 & 55 & 220 & 10.61 & 7.51 & 0.07 & $0.1^{\prime}$ \\
\hline HD 37828 & -1.43 & 8.74 & -0.11 & 88 & -163 & -39 & 87 & 58 & 9.50 & 1.44 & 0.68 & 0.7 \\
\hline HD 246316 & -0.85 & 8.90 & -0.03 & -57 & -32 & -3 & -58 & 188 & 9.78 & 6.06 & 0.05 & 0.2 \\
\hline HD 246370 & -1.92 & 8.92 & -0.02 & 0 & 15 & 11 & 0 & 235 & 9.93 & 8.92 & 0.14 & 0.0 \\
\hline HD 38893 & -0.86 & 8.49 & -0.07 & -5 & -95 & -59 & -7 & 125 & 8.50 & 3.65 & 1.09 & 0.4 \\
\hline HD 38510 & -0.92 & 8.53 & -0.03 & 159 & -55 & -63 & 158 & 165 & 13.10 & 4.24 & 1.68 & 0.5 \\
\hline HD 38504 & -0.65 & 8.72 & -0.01 & 19 & 4 & -22 & 18 & 225 & 9.36 & 8.33 & 0.29 & 0.0 \\
\hline HD 247593 & -0.94 & 9.04 & -0.02 & -6 & 39 & 9 & -7 & 259 & 12.39 & 9.02 & 0.15 & 0.11 \\
\hline HD 38708 & -0.61 & 11.00 & 0.03 & -40 & -1 & -28 & -40 & 219 & 12.36 & 9.59 & 0.61 & 0.1 \\
\hline HD 248271 & -0.97 & 9.30 & -0.01 & -18 & 13 & 23 & -19 & 233 & 10.51 & 9.04 & 0.36 & 0.0 \\
\hline HD 248412 & -0.90 & 9.60 & -0.02 & -34 & -7 & -31 & -36 & 213 & 10.46 & 8.23 & 0.54 & 0.1 \\
\hline HD 40361 & -0.91 & 8.63 & -0.22 & 42 & 33 & -24 & 31 & 255 & 11.95 & 8.40 & 0.51 & $0.1^{\prime}$ \\
\hline $\mathrm{BD}+231114$ & -0.66 & 9.52 & -0.01 & -50 & -28 & -110 & -52 & 192 & 11.07 & 7.85 & 3.70 & $0.1^{\prime}$ \\
\hline
\end{tabular}


TABLE 3. (continued)

\begin{tabular}{|c|c|c|c|c|c|c|c|c|c|c|c|c|}
\hline $\begin{array}{l}\text { Star } \\
(1)\end{array}$ & $\begin{array}{c}{[\mathrm{Fe} / \mathrm{H}]} \\
(\mathrm{dex}) \\
(2)\end{array}$ & $\begin{array}{c}R \\
(\mathrm{kpc}) \\
(3)\end{array}$ & $\begin{array}{c}Z \\
(\mathrm{kpc}) \\
(4)\end{array}$ & $\begin{array}{c}U \\
(\mathrm{~km} / \mathrm{s}) \\
(5)\end{array}$ & $\begin{array}{c}V \\
(\mathrm{~km} / \mathrm{s}) \\
(6)\end{array}$ & $\begin{array}{c}W \\
(\mathrm{~km} / \mathrm{s}) \\
(7)\end{array}$ & $\begin{array}{c}V_{R} \\
(\mathrm{~km} / \mathrm{s}) \\
(8)\end{array}$ & $\begin{array}{c}V_{\phi} \\
(\mathrm{km} / \mathrm{s}) \\
(9)\end{array}$ & $\begin{array}{c}R_{a p} \\
(\mathrm{kpc}) \\
(10)\end{array}$ & $\begin{array}{c}R_{p r} \\
(\mathrm{kpc}) \\
(11)\end{array}$ & $\begin{array}{c}Z_{\max } \\
(\mathrm{kpc}) \\
(12)\end{array}$ & (13) \\
\hline HD 249499 & -0.87 & 10.14 & 0.01 & 6 & -12 & 17 & 3 & 209 & 10.15 & 8.92 & 0.27 & 0.06 \\
\hline HD 41010 & -0.77 & 8.99 & 0.03 & -27 & -28 & -37 & -28 & 192 & 9.27 & 6.81 & 0.58 & 0.15 \\
\hline HD 250915 & -0.65 & 9.16 & 0.02 & -55 & 28 & -40 & -57 & 248 & 12.97 & 8.40 & 0.86 & 0.21 \\
\hline HD 41115 & -0.76 & 8.76 & 0.01 & 15 & -4 & -3 & 14 & 216 & 8.98 & 8.08 & 0.04 & 0.05 \\
\hline HD 41667 & -1.18 & 8.76 & -0.21 & 285 & -173 & -39 & 282 & 60 & 20.32 & 1.17 & 1.23 & 0.89 \\
\hline HD 251432 & -1.05 & 9.31 & 0.03 & -16 & 6 & 7 & -18 & 226 & 9.97 & 8.91 & 0.10 & 0.06 \\
\hline HD 251549 & -2.08 & 9.73 & 0.06 & 11 & 36 & -15 & 8 & 256 & 12.98 & 9.71 & 0.29 & 0.14 \\
\hline HD 252041 & -1.76 & 9.16 & 0.05 & -30 & -2 & -4 & -31 & 218 & 9.92 & 8.16 & 0.08 & 0.10 \\
\hline HD 41994 & -1.57 & 8.90 & 0.03 & 2 & 4 & 3 & 1 & 224 & 9.01 & 8.89 & 0.04 & 0.01 \\
\hline HD 252370 & -1.37 & 9.18 & 0.05 & -32 & -4 & -10 & -33 & 216 & 9.94 & 8.03 & 0.14 & 0.11 \\
\hline HD 252477 & -0.89 & 8.90 & 0.03 & 40 & 1 & -2 & 40 & 221 & 10.05 & 7.81 & 0.04 & 0.12 \\
\hline HD 44165 & -0.85 & 8.45 & -0.09 & 28 & 75 & 33 & 22 & 296 & 16.19 & 8.40 & 0.78 & 0.32 \\
\hline $\mathrm{V}^{*} \mathrm{RX}$ Col & -1.70 & 9.69 & -0.96 & 191 & -301 & -36 & 204 & -39 & 15.22 & 0.92 & 1.64 & 0.89 \\
\hline HD 43039 & -0.42 & 8.55 & 0.00 & 10 & -38 & -37 & 10 & 182 & 8.58 & 6.12 & 0.52 & 0.17 \\
\hline HD 253911 & -0.60 & 8.84 & 0.03 & 13 & -27 & 4 & 13 & 193 & 8.90 & 6.78 & 0.06 & 0.14 \\
\hline HD 44007 & -1.58 & 8.63 & -0.04 & 77 & -148 & 14 & 76 & 73 & 9.20 & 1.77 & 0.19 & 0.68 \\
\hline HD 255236 & -0.80 & 9.02 & 0.06 & 5 & 13 & 2 & 4 & 233 & 9.87 & 9.01 & 0.07 & 0.05 \\
\hline BD-19 1422 & -1.64 & 8.77 & -0.10 & -10 & -66 & -3 & -15 & 154 & 8.81 & 4.68 & 0.11 & 0.31 \\
\hline HD 45282 & -1.52 & 8.59 & -0.01 & 239 & -160 & -31 & 239 & 62 & 15.71 & 1.25 & 0.72 & 0.85 \\
\hline HD 45610 & -1.43 & 8.58 & -0.05 & -45 & -105 & 17 & -46 & 114 & 8.84 & 3.06 & 0.21 & 0.49 \\
\hline V* RZ Cam & -1.01 & 10.31 & 0.88 & -199 & -242 & 22 & -200 & -1 & 15.51 & 0.03 & 2.13 & 1.00 \\
\hline$V^{*}$ V338 Pup & -2.16 & 8.65 & -0.20 & -76 & -343 & 30 & -69 & -127 & 9.30 & 3.47 & 0.49 & 0.4 \\
\hline $\mathrm{V}^{*} \mathrm{ST}$ Pup & -0.90 & 9.75 & -0.74 & 47 & -12 & -3 & -3 & 214 & 9.79 & 9.16 & 0.74 & 0.03 \\
\hline V* IU Car & -1.85 & 8.64 & -0.62 & 237 & -275 & -153 & 243 & -14 & 19.10 & 0.38 & 11.16 & 0.96 \\
\hline CD-33 3337 & -1.40 & 8.55 & -0.03 & -23 & -36 & -122 & -25 & 184 & 9.01 & 7.50 & 3.37 & 0.10 \\
\hline $\mathrm{V}^{*} \mathrm{TZ}$ Aur & -0.79 & 9.96 & 0.56 & 63 & -36 & -48 & 65 & 184 & 11.18 & 6.84 & 1.60 & 0.24 \\
\hline HD 55496 & -1.55 & 8.64 & -0.03 & 151 & -262 & -12 & 152 & -39 & 10.94 & 0.87 & 0.16 & 0.85 \\
\hline $\mathrm{V}^{*} \mathrm{AA} \mathrm{CMi}$ & -0.15 & 9.52 & 0.14 & 47 & -6 & -4 & 32 & 217 & 10.31 & 8.39 & 0.16 & 0.10 \\
\hline $\mathrm{V}^{*} \mathrm{HH}$ Pup & -0.50 & 8.75 & -0.26 & -18 & -2 & 18 & -41 & 215 & 9.76 & 7.47 & 0.38 & 0.13 \\
\hline $\mathrm{V}^{*} \mathrm{RR}$ Gem & -0.29 & 9.69 & 0.42 & 56 & 21 & 21 & 52 & 241 & 12.88 & 8.85 & 0.71 & 0.19 \\
\hline HD 59392 & -1.65 & 8.53 & -0.02 & -69 & -291 & -29 & -68 & -72 & 8.99 & 1.78 & 0.41 & 0.67 \\
\hline$V^{*}$ TV Lyn & -0.63 & 9.41 & 0.46 & -29 & 40 & 22 & -25 & 261 & 13.49 & 9.28 & 0.84 & 0.19 \\
\hline HD 61902 & -0.86 & 8.51 & -0.02 & -71 & -60 & -50 & -72 & 159 & 9.44 & 4.69 & 0.90 & 0.3 \\
\hline BD-01 1792 & -0.90 & 8.68 & 0.04 & -206 & -268 & 72 & -206 & -52 & 13.85 & 1.17 & 2.49 & 0.8 \\
\hline HD 62412 & -0.99 & 8.55 & 0.00 & -28 & 23 & -1 & -31 & 242 & 10.60 & 8.21 & 0.01 & 0.13 \\
\hline HD 62779 & -0.74 & 8.72 & -0.01 & 4 & 3 & 15 & -6 & 223 & 8.91 & 8.56 & 0.19 & 0.02 \\
\hline $\mathrm{V}^{*}$ HK Pup & -1.11 & 9.19 & 0.10 & 90 & -181 & 121 & 86 & 47 & 10.20 & 1.64 & 5.49 & 0.73 \\
\hline $\mathrm{V}^{*} \mathrm{TW}$ Lyn & -0.66 & 9.96 & 0.76 & -61 & 21 & 21 & -59 & 242 & 13.62 & 9.04 & 1.25 & 0.20 \\
\hline $\mathrm{V}^{*} \mathrm{AL} \mathrm{CMi}$ & -0.85 & 9.92 & 0.46 & 76 & 15 & -68 & 53 & 241 & 13.81 & 9.24 & 2.45 & 0.20 \\
\hline BD-18 2065 & -0.76 & 8.73 & 0.03 & -58 & -145 & 34 & -61 & 72 & 9.10 & 1.83 & 0.52 & 0.67 \\
\hline
\end{tabular}


TABLE 3. (continued)

\begin{tabular}{|c|c|c|c|c|c|c|c|c|c|c|c|c|}
\hline $\begin{array}{l}\text { Star } \\
(1)\end{array}$ & $\begin{array}{c}{[\mathrm{Fe} / \mathrm{H}]} \\
(\mathrm{dex}) \\
(2)\end{array}$ & $\begin{array}{c}R \\
(\mathrm{kpc}) \\
(3)\end{array}$ & $\begin{array}{c}Z \\
(\mathrm{kpc}) \\
(4)\end{array}$ & $\begin{array}{c}U \\
(\mathrm{~km} / \mathrm{s}) \\
(5)\end{array}$ & $\begin{array}{c}V \\
(\mathrm{~km} / \mathrm{s}) \\
(6)\end{array}$ & $\begin{array}{c}W \\
(\mathrm{~km} / \mathrm{s}) \\
(7)\end{array}$ & $\begin{array}{c}V_{R} \\
(\mathrm{~km} / \mathrm{s}) \\
(8)\end{array}$ & $\begin{array}{c}V_{\phi} \\
(\mathrm{km} / \mathrm{s}) \\
(9)\end{array}$ & $\begin{array}{c}R_{a p} \\
(\mathrm{kpc}) \\
(10)\end{array}$ & $\begin{array}{c}R_{p r} \\
(\mathrm{kpc}) \\
(11)\end{array}$ & $\begin{array}{c}Z_{\max } \\
(\mathrm{kpc}) \\
(12)\end{array}$ & $\begin{array}{c}e \\
(13)\end{array}$ \\
\hline $\mathrm{V}^{*} \mathrm{SZ}$ Gem & -1.46 & 9.89 & 0.60 & 269 & -273 & -23 & 271 & -38 & 21.13 & 0.83 & 2.28 & 0.92 \\
\hline HD 63791 & -1.67 & 8.77 & 0.18 & 3 & -140 & -112 & 4 & 80 & 8.77 & 2.74 & 3.84 & 0.53 \\
\hline HD 65354 & -0.77 & 8.62 & -0.02 & 12 & -22 & 6 & 4 & 198 & 8.63 & 6.96 & 0.07 & 0.11 \\
\hline $\mathrm{V}^{*}$ AQ Pup & -0.76 & 8.77 & 0.00 & 23 & -66 & 12 & 13 & 155 & 8.80 & 4.77 & 0.14 & 0.30 \\
\hline $\mathrm{V}^{*} \mathrm{UY}$ Cam & -1.33 & 9.53 & 0.75 & 41 & -113 & -20 & 49 & 103 & 9.85 & 3.02 & 0.97 & 0.53 \\
\hline HD 66037 & -0.64 & 8.66 & -0.02 & 18 & -19 & -57 & 8 & 201 & 8.70 & 7.54 & 0.99 & 0.07 \\
\hline HD 66277 & -0.65 & 8.76 & 0.00 & 29 & -47 & 67 & 17 & 174 & 8.85 & 6.13 & 1.38 & 0.18 \\
\hline HD 66598 & -0.65 & 8.56 & 0.00 & 17 & -24 & 2 & 13 & 196 & 8.62 & 6.73 & 0.02 & 0.12 \\
\hline $\mathrm{V}^{*} \mathrm{SS}$ Cnc & -0.07 & 9.91 & 0.73 & -14 & -7 & -58 & -24 & 212 & 10.73 & 8.99 & 1.79 & 0.09 \\
\hline HD 67380 & -0.60 & 8.72 & 0.01 & 57 & 8 & 19 & 43 & 231 & 10.52 & 7.93 & 0.28 & 0.14 \\
\hline$V^{*}$ XX Pup & -1.33 & 9.22 & 0.18 & 254 & -299 & -50 & 261 & -51 & 18.92 & 1.09 & 2.30 & 0.89 \\
\hline $\mathrm{V}^{*}$ SZ Lyn & -1.16 & 8.99 & 0.31 & 41 & -54 & -5 & 42 & 166 & 9.35 & 5.27 & 0.33 & 0.28 \\
\hline HD 68298 & -0.60 & 8.64 & -0.01 & 14 & 22 & 9 & 2 & 242 & 10.22 & 8.64 & 0.11 & 0.08 \\
\hline V* DD Hya & -0.97 & 9.98 & 0.64 & 79 & -129 & 28 & 68 & 99 & 10.62 & 2.92 & 0.92 & 0.57 \\
\hline HD 68960 & -0.72 & 8.53 & 0.00 & -6 & 8 & 7 & -8 & 228 & 9.04 & 8.45 & 0.08 & 0.03 \\
\hline HD 69083 & -0.85 & 8.52 & 0.00 & 1 & -10 & 5 & 0 & 210 & 8.52 & 7.63 & 0.05 & 0.05 \\
\hline CD-44 4311 & -0.85 & 8.73 & -0.10 & 27 & 97 & -39 & -15 & 318 & 20.11 & 8.72 & 1.16 & 0.40 \\
\hline HD 70765 & -0.70 & 8.60 & -0.02 & -25 & -23 & 10 & -35 & 196 & 9.02 & 6.53 & 0.12 & 0.16 \\
\hline HD 70714 & -0.73 & 8.66 & 0.02 & 12 & 10 & 9 & 1 & 230 & 9.23 & 8.66 & 0.11 & 0.03 \\
\hline $\mathrm{V}^{*}$ BB Pup & -0.57 & 9.38 & 0.29 & 162 & -16 & -31 & 129 & 226 & 15.37 & 6.74 & 1.06 & 0.39 \\
\hline HD 71160 & -0.60 & 8.70 & 0.03 & 17 & 15 & -40 & 1 & 235 & 9.95 & 8.70 & 0.65 & 0.07 \\
\hline $\mathrm{V}^{*}$ AS Cnc & -1.89 & 10.42 & 1.21 & 52 & -120 & 342 & 46 & 102 & 34.49 & 9.98 & 32.94 & 0.55 \\
\hline HD 71584 & -0.72 & 8.68 & 0.01 & 10 & 8 & 12 & -6 & 228 & 9.15 & 8.62 & 0.14 & 0.03 \\
\hline HD 71624 & -0.67 & 8.66 & 0.01 & 26 & -9 & 19 & 12 & 212 & 8.79 & 7.84 & 0.23 & 0.06 \\
\hline HD 71670 & -0.60 & 8.67 & 0.02 & -38 & -8 & 5 & -52 & 209 & 9.81 & 6.95 & 0.07 & 0.17 \\
\hline HD 72348 & -0.60 & 8.53 & 0.00 & 26 & 0 & -16 & 22 & 221 & 9.09 & 7.94 & 0.20 & 0.07 \\
\hline BD-20 2583 & -1.66 & 8.85 & 0.14 & 17 & -251 & 107 & 19 & -29 & 8.90 & 0.94 & 3.86 & 0.81 \\
\hline $\mathrm{V}^{*} \mathrm{TT}$ Cnc & -1.57 & 9.50 & 0.62 & 125 & -159 & -268 & 121 & 69 & 19.10 & 7.89 & 18.66 & 0.42 \\
\hline HD 73075 & -0.65 & 8.52 & -0.01 & -19 & 19 & -7 & -24 & 239 & 10.15 & 8.27 & 0.09 & 0.10 \\
\hline HD 73676 & -0.70 & 8.56 & 0.00 & -3 & 10 & -26 & -12 & 230 & 9.38 & 8.42 & 0.34 & 0.05 \\
\hline HD 73700 & -0.71 & 8.55 & -0.01 & 10 & -4 & 8 & 2 & 216 & 8.55 & 8.10 & 0.08 & 0.03 \\
\hline HD 73808 & -0.68 & 8.56 & 0.00 & 48 & -16 & 23 & 41 & 205 & 9.28 & 6.92 & 0.30 & 0.15 \\
\hline V* GL Hya & -1.45 & 10.51 & 1.22 & 191 & -205 & -93 & 186 & 47 & 15.65 & 1.70 & 9.05 & 0.81 \\
\hline HD 74000 & -1.82 & 8.55 & 0.03 & -179 & -307 & 66 & -178 & -88 & 12.51 & 2.08 & 1.89 & 0.71 \\
\hline HD 74617 & -0.76 & 8.60 & 0.01 & 10 & -40 & -5 & 0 & 180 & 8.60 & 5.92 & 0.06 & 0.19 \\
\hline HD 74721 & -1.42 & 8.79 & 0.21 & -16 & -181 & -118 & -17 & 39 & 8.83 & 1.35 & 4.61 & 0.74 \\
\hline BD-12 2669 & -1.40 & 8.63 & 0.08 & 137 & -95 & -411 & 134 & 128 & 64.16 & 8.02 & 60.82 & 0.78 \\
\hline HD 74462 & -1.42 & 8.87 & 0.32 & -121 & -284 & 70 & -122 & -60 & 10.52 & 1.59 & 2.27 & 0.74 \\
\hline $\mathrm{V}^{*} \mathrm{SV}$ Vol & -2.18 & 8.18 & -0.57 & -227 & -162 & -43 & -234 & 8 & 14.43 & 0.16 & 2.20 & 0.98 \\
\hline HD 75745 & -1.37 & 8.50 & -0.02 & 29 & -115 & -27 & 27 & 106 & 8.58 & 2.80 & 0.35 & 0.51 \\
\hline
\end{tabular}


TABLE 3. (continued)

\begin{tabular}{|c|c|c|c|c|c|c|c|c|c|c|c|c|}
\hline $\begin{array}{l}\text { Star } \\
(1)\end{array}$ & $\begin{array}{c}{[\mathrm{Fe} / \mathrm{H}]} \\
(\mathrm{dex}) \\
(2)\end{array}$ & $\begin{array}{c}R \\
(\mathrm{kpc}) \\
(3)\end{array}$ & $\begin{array}{c}Z \\
(\mathrm{kpc}) \\
(4)\end{array}$ & $\begin{array}{c}U \\
(\mathrm{~km} / \mathrm{s}) \\
(5)\end{array}$ & $\begin{array}{c}V \\
(\mathrm{~km} / \mathrm{s}) \\
(6)\end{array}$ & $\begin{array}{c}W \\
(\mathrm{~km} / \mathrm{s}) \\
(7)\end{array}$ & $\begin{array}{c}V_{R} \\
(\mathrm{~km} / \mathrm{s}) \\
(8)\end{array}$ & $\begin{array}{c}V_{\phi} \\
(\mathrm{km} / \mathrm{s}) \\
(9)\end{array}$ & $\begin{array}{c}R_{a p} \\
(\mathrm{kpc}) \\
(10)\end{array}$ & $\begin{array}{c}R_{p r} \\
(\mathrm{kpc}) \\
(11)\end{array}$ & $\begin{array}{c}Z_{\max } \\
(\mathrm{kpc}) \\
(12)\end{array}$ & $\begin{array}{c}e \\
(13)\end{array}$ \\
\hline HD 75837 & -0.62 & 8.52 & -0.01 & 31 & -58 & 7 & 26 & 163 & 8.64 & 4.93 & 0.07 & 0.27 \\
\hline $\mathrm{V}^{*}$ GO Hya & -0.83 & 9.82 & 0.99 & -49 & -47 & -58 & -69 & 167 & 11.16 & 5.90 & 2.06 & 0.31 \\
\hline $\mathrm{V}^{*} \mathrm{DG}$ Hya & -1.42 & 9.66 & 0.83 & 18 & -264 & -117 & 25 & -41 & 9.73 & 1.70 & 6.26 & 0.71 \\
\hline $\mathrm{V}^{*} \mathrm{AN} \mathrm{Cnc}$ & -1.45 & 10.74 & 1.78 & -63 & -327 & -157 & -49 & -114 & 12.44 & 6.51 & 8.84 & 0.32 \\
\hline HD 77119 & -0.63 & 8.50 & -0.01 & 17 & -17 & 8 & 13 & 203 & 8.58 & 7.13 & 0.09 & 0.09 \\
\hline HD 77142 & -0.70 & 8.50 & -0.01 & 11 & -24 & -18 & 6 & 196 & 8.52 & 6.79 & 0.21 & 0.11 \\
\hline $\mathrm{V}^{*} \mathrm{DH}$ Hya & -1.55 & 9.56 & 0.75 & 271 & -296 & -50 & 280 & -32 & 21.74 & 0.73 & 5.03 & 0.94 \\
\hline HD 77489 & -0.61 & 8.50 & -0.01 & 34 & 18 & -11 & 29 & 238 & 10.24 & 8.14 & 0.14 & 0.11 \\
\hline HD 77567 & -0.90 & 8.51 & -0.02 & 109 & -54 & 20 & 100 & 171 & 10.36 & 4.78 & 0.29 & 0.37 \\
\hline $\mathrm{V}^{*} \mathrm{TT}$ Lyn & -1.56 & 9.02 & 0.47 & 135 & -125 & -242 & 136 & 95 & 17.34 & 6.69 & 15.92 & 0.45 \\
\hline HD 77927 & -0.68 & 8.50 & -0.03 & 75 & -25 & 17 & 64 & 198 & 9.73 & 6.21 & 0.22 & 0.22 \\
\hline HD 77941 & -1.03 & 8.57 & 0.03 & 0 & -56 & 2 & -11 & 164 & 8.59 & 5.05 & 0.03 & 0.26 \\
\hline HD 78747 & -0.76 & 8.50 & 0.00 & -19 & 17 & -19 & -20 & 237 & 9.94 & 8.29 & 0.25 & 0.09 \\
\hline HD 78746 & -0.76 & 8.50 & 0.00 & 6 & -77 & 14 & 6 & 143 & 8.51 & 4.12 & 0.16 & 0.35 \\
\hline HD 78737 & -1.30 & 8.53 & 0.02 & -44 & 43 & 18 & -47 & 263 & 12.85 & 8.13 & 0.29 & 0.22 \\
\hline $\mathrm{V}^{*}$ XX Hya & -1.33 & 9.24 & 0.58 & -227 & -135 & -5 & -237 & 51 & 16.67 & 1.10 & 1.02 & 0.88 \\
\hline HD 79091 & -0.85 & 8.50 & 0.00 & 35 & -19 & -8 & 33 & 201 & 8.92 & 6.76 & 0.09 & 0.14 \\
\hline HD 79134 & -0.80 & 8.51 & 0.01 & -33 & -26 & 11 & -38 & 193 & 8.96 & 6.32 & 0.13 & 0.17 \\
\hline HD 79350 & -0.66 & 8.50 & -0.01 & 66 & 16 & 16 & 59 & 238 & 11.24 & 7.55 & 0.24 & 0.20 \\
\hline HD 79349 & -0.62 & 8.53 & 0.00 & 118 & -38 & -28 & 101 & 192 & 10.95 & 5.50 & 0.43 & 0.33 \\
\hline V* SZ Hya & -1.75 & 9.15 & 0.57 & -169 & -313 & -85 & -158 & -111 & 13.12 & 3.03 & 3.15 & 0.63 \\
\hline $\mathrm{V}^{*} \mathrm{AQ} \mathrm{Cnc}$ & -1.53 & 9.70 & 1.15 & 237 & -457 & 23 & 259 & -214 & 29.42 & 5.01 & 3.25 & 0.71 \\
\hline $\mathrm{V}^{*} \mathrm{RW}$ Cnc & -1.67 & 9.73 & 1.22 & -125 & -257 & -85 & -123 & -41 & 11.99 & 1.20 & 3.79 & 0.82 \\
\hline HD 80936 & -0.87 & 8.48 & -0.01 & 22 & -125 & -15 & 20 & 95 & 8.53 & 2.46 & 0.17 & 0.55 \\
\hline HD 81223 & -0.94 & 8.42 & -0.07 & 190 & -19 & -11 & 183 & 208 & 16.13 & 4.90 & 0.24 & 0.53 \\
\hline HD 81173 & -0.67 & 8.50 & 0.00 & 25 & -35 & -13 & 21 & 185 & 8.62 & 6.06 & 0.15 & 0.17 \\
\hline HD 81290 & -0.63 & 8.50 & 0.00 & -11 & -7 & -20 & -20 & 212 & 8.79 & 7.56 & 0.25 & 0.08 \\
\hline HD 81713 & -0.54 & 8.59 & 0.11 & 31 & -10 & 35 & 25 & 210 & 9.03 & 7.53 & 0.52 & 0.09 \\
\hline HD 82004 & -0.65 & 8.47 & -0.02 & 29 & 27 & -12 & 18 & 248 & 10.73 & 8.36 & 0.17 & 0.12 \\
\hline CD-36 5714 & -1.83 & 8.52 & 0.04 & 20 & -320 & 44 & 23 & -100 & 8.58 & 2.68 & 0.70 & 0.52 \\
\hline HD 82403 & -0.60 & 8.48 & -0.01 & 24 & -11 & -22 & 17 & 210 & 8.69 & 7.49 & 0.27 & 0.07 \\
\hline HD 82456 & -0.65 & 8.49 & 0.00 & 38 & -70 & -7 & 35 & 150 & 8.69 & 4.36 & 0.08 & 0.33 \\
\hline $\mathrm{V}^{*} \mathrm{WW}$ Leo & -1.48 & 9.77 & 1.36 & -157 & -176 & -140 & -161 & 24 & 14.77 & 0.86 & 9.59 & 0.89 \\
\hline HD 82590 & -1.29 & 8.69 & 0.23 & -214 & -341 & -47 & -207 & -133 & 15.27 & 3.02 & 1.26 & 0.67 \\
\hline HD 83155 & -0.69 & 8.48 & -0.01 & 33 & -22 & 3 & 29 & 199 & 8.80 & 6.70 & 0.03 & 0.14 \\
\hline HD 83212 & -1.48 & 8.66 & 0.22 & 18 & -114 & -16 & 12 & 107 & 8.68 & 2.89 & 0.31 & 0.50 \\
\hline $\mathrm{V}^{*} \mathrm{UU}$ Hya & -1.65 & 9.63 & 1.29 & 231 & -214 & 68 & 229 & 36 & 17.49 & 0.84 & 3.24 & 0.91 \\
\hline HD 233666 & -1.53 & 8.61 & 0.12 & -58 & -23 & -29 & -57 & 197 & 9.65 & 6.37 & 0.45 & 0.20 \\
\hline HD 84903 & -2.62 & 8.51 & 0.08 & 18 & -72 & -15 & 10 & 149 & 8.53 & 4.40 & 0.20 & 0.32 \\
\hline HD 85454 & -0.63 & 8.42 & -0.03 & 9 & 20 & 0 & -8 & 240 & 9.85 & 8.38 & 0.03 & 0.08 \\
\hline
\end{tabular}


TABLE 3. (continued)

\begin{tabular}{|c|c|c|c|c|c|c|c|c|c|c|c|c|}
\hline $\begin{array}{l}\text { Star } \\
(1)\end{array}$ & $\begin{array}{c}{[\mathrm{Fe} / \mathrm{H}]} \\
(\mathrm{dex}) \\
(2)\end{array}$ & $\begin{array}{c}R \\
(\mathrm{kpc}) \\
(3)\end{array}$ & $\begin{array}{c}Z \\
(\mathrm{kpc}) \\
(4)\end{array}$ & $\begin{array}{c}U \\
(\mathrm{~km} / \mathrm{s}) \\
(5)\end{array}$ & $\begin{array}{c}V \\
(\mathrm{~km} / \mathrm{s}) \\
(6)\end{array}$ & $\begin{array}{c}W \\
(\mathrm{~km} / \mathrm{s}) \\
(7)\end{array}$ & $\begin{array}{c}V_{R} \\
(\mathrm{~km} / \mathrm{s}) \\
(8)\end{array}$ & $\begin{array}{c}V_{\phi} \\
(\mathrm{km} / \mathrm{s}) \\
(9)\end{array}$ & $\begin{array}{c}R_{a p} \\
(\mathrm{kpc}) \\
(10)\end{array}$ & $\begin{array}{c}R_{p r} \\
(\mathrm{kpc}) \\
(11)\end{array}$ & $\begin{array}{c}Z_{\max } \\
(\mathrm{kpc}) \\
(12)\end{array}$ & $\begin{array}{c}e \\
(13)\end{array}$ \\
\hline HD 237846 & -2.63 & 9.49 & 1.13 & -202 & -137 & -185 & -198 & 92 & 20.24 & 3.51 & 14.49 & 0.71 \\
\hline$V^{*} \mathrm{~T}$ Sex & -1.34 & 8.82 & 0.46 & 29 & -76 & -58 & 21 & 145 & 8.89 & 4.69 & 1.44 & 0.31 \\
\hline $\mathrm{V}^{*} \mathrm{SU}$ Leo & -1.41 & 10.44 & 2.55 & -192 & -56 & -38 & -220 & 124 & 20.30 & 3.39 & 4.66 & 0.71 \\
\hline HD 85773 & -2.27 & 8.94 & 0.68 & 13 & -213 & -130 & 11 & 9 & 8.97 & 0.41 & 6.59 & 0.92 \\
\hline HD 86268 & -0.77 & 8.44 & -0.01 & 66 & -83 & -1 & 61 & 139 & 8.95 & 3.82 & 0.01 & 0.40 \\
\hline CD-24 8642 & -1.00 & 8.55 & 0.12 & 1 & -99 & 53 & -3 & 121 & 8.55 & 3.50 & 0.96 & 0.42 \\
\hline HD 87030 & -0.64 & 8.47 & 0.00 & 32 & -2 & -4 & 28 & 219 & 9.12 & 7.64 & 0.05 & 0.09 \\
\hline HD 87103 & -0.71 & 8.41 & 0.00 & 72 & -55 & 3 & 60 & 170 & 9.09 & 4.99 & 0.04 & 0.29 \\
\hline HD 87254 & -0.60 & 8.44 & 0.00 & -105 & 2 & 46 & -115 & 217 & 12.65 & 6.06 & 0.97 & 0.35 \\
\hline HD 86986 & -1.68 & 8.65 & 0.22 & -276 & -222 & 54 & -276 & -7 & 18.96 & 0.13 & 2.58 & 0.99 \\
\hline $\mathrm{V}^{*}$ X LMi & -1.68 & 9.80 & 1.77 & -164 & -177 & 13 & -164 & 42 & 13.19 & 1.11 & 3.00 & 0.85 \\
\hline $\mathrm{V}^{*} \mathrm{RR}$ Leo & -1.60 & 9.04 & 0.81 & 88 & -72 & 23 & 83 & 151 & 10.30 & 4.51 & 1.00 & 0.39 \\
\hline HD 88226 & -0.66 & 8.44 & 0.00 & -53 & 10 & -25 & -61 & 228 & 10.70 & 7.25 & 0.36 & 0.19 \\
\hline V* WZ Hya & -1.39 & 8.77 & 0.58 & 36 & -277 & 120 & 41 & -54 & 9.08 & 1.90 & 4.90 & 0.66 \\
\hline HD 88609 & -2.82 & 9.10 & 0.78 & -41 & -138 & 28 & -39 & 83 & 9.28 & 2.30 & 1.09 & 0.60 \\
\hline $\mathrm{V}^{*} \mathrm{WY}$ Ant & -1.48 & 8.60 & 0.39 & -279 & -223 & 6 & -277 & -34 & 18.84 & 0.66 & 0.96 & 0.93 \\
\hline $\mathrm{V}^{*} \mathrm{~V}$ LMi & -1.15 & 9.28 & 1.32 & -277 & -191 & -12 & -278 & 20 & 20.86 & 0.43 & 3.05 & 0.96 \\
\hline HD 90862 & -0.62 & 8.90 & 0.78 & -23 & 23 & 0 & -32 & 242 & 11.40 & 8.58 & 0.97 & 0.14 \\
\hline BD+30 2034 & -1.52 & 9.69 & 2.14 & 223 & -188 & -49 & 222 & 42 & 16.70 & 1.23 & 7.95 & 0.86 \\
\hline HD 92205 & -0.63 & 8.47 & 0.00 & 5 & 13 & -2 & 2 & 233 & 9.28 & 8.46 & 0.02 & 0.05 \\
\hline $\mathrm{BD}+092384$ & -0.71 & 8.65 & 0.37 & -107 & -202 & -106 & -107 & 15 & 10.05 & 0.42 & 3.98 & 0.92 \\
\hline CD-30 8626 & -1.67 & 8.49 & 0.18 & -45 & -263 & 32 & -43 & -45 & 8.66 & 1.10 & 0.53 & 0.77 \\
\hline $\mathrm{V}^{*} \mathrm{FS}$ Vel & -1.17 & 8.36 & 0.36 & -357 & -184 & 161 & -358 & -29 & 39.14 & 0.69 & 25.48 & 0.96 \\
\hline$V^{*}$ RV Sex & -1.10 & 8.92 & 1.38 & 47 & -151 & 1 & 35 & 76 & 9.14 & 2.08 & 1.40 & 0.63 \\
\hline HD 93529 & -1.67 & 8.50 & 0.18 & -26 & -156 & 1 & -28 & 63 & 8.58 & 1.55 & 0.19 & 0.69 \\
\hline $\mathrm{V}^{*} \mathrm{AF} \mathrm{Vel}$ & -1.49 & 8.31 & 0.16 & -275 & -157 & 69 & -281 & 29 & 19.11 & 0.56 & 3.28 & 0.94 \\
\hline HD 94510 & -0.04 & 8.49 & 0.00 & -19 & 7 & 17 & -20 & 227 & 9.26 & 8.14 & 0.21 & 0.06 \\
\hline $\mathrm{V}^{*} \mathrm{SW}$ Leo & -1.45 & 9.17 & 2.22 & 40 & -115 & -50 & 18 & 111 & 9.40 & 3.75 & 3.04 & 0.43 \\
\hline HD 95096 & -0.76 & 8.31 & -0.03 & -25 & 57 & 5 & -45 & 274 & 13.56 & 8.02 & 0.09 & 0.26 \\
\hline HD 95338 & -0.83 & 8.49 & 0.00 & 11 & -111 & -37 & 11 & 109 & 8.50 & 2.96 & 0.53 & 0.48 \\
\hline $\mathrm{V}^{*} \mathrm{SZ}$ Leo & -1.86 & 9.08 & 1.88 & 57 & -349 & 3 & 72 & -122 & 9.99 & 3.71 & 2.04 & 0.46 \\
\hline HD 97320 & -1.18 & 8.48 & 0.00 & -83 & -9 & -31 & -84 & 210 & 10.84 & 6.34 & 0.48 & 0.26 \\
\hline $\mathrm{V}^{*} \mathrm{TV}$ Leo & -1.97 & 8.75 & 1.49 & -94 & 109 & -28 & -142 & 311 & 26.86 & 7.60 & 4.20 & 0.56 \\
\hline V* BT Leo & -0.81 & 9.34 & 2.64 & 111 & -107 & 61 & 100 & 123 & 11.70 & 3.84 & 3.57 & 0.51 \\
\hline-191105 D & -1.40 & 18.22 & 12.65 & 353 & -377 & -264 & 290 & 255 & $\ldots$ & $\ldots$ & $\cdots$ & $\ldots$ \\
\hline $\mathrm{V}^{*}$ AN Leo & -1.14 & 8.85 & 1.82 & -174 & -179 & -147 & -178 & 21 & 15.00 & 0.65 & 8.97 & 0.92 \\
\hline $\mathrm{V}^{*} \mathrm{RX}$ Leo & -1.38 & 9.02 & 1.66 & -198 & -162 & -93 & -200 & 51 & 15.27 & 1.26 & 4.46 & 0.85 \\
\hline HD 99383 & -1.65 & 8.48 & 0.02 & -7 & -194 & 124 & -7 & 26 & 8.49 & 0.89 & 4.70 & 0.81 \\
\hline $\mathrm{V}^{*} \mathrm{AE}$ Leo & -1.71 & 9.05 & 2.24 & -321 & 2 & 20 & -338 & 196 & 44.51 & 4.14 & 15.54 & 0.83 \\
\hline HD 99513 & -0.60 & 8.38 & 0.01 & 2 & -60 & -16 & -3 & 160 & 8.38 & 4.79 & 0.18 & 0.27 \\
\hline
\end{tabular}


TABLE 3. (continued)

\begin{tabular}{|c|c|c|c|c|c|c|c|c|c|c|c|}
\hline $\begin{array}{l}\text { Star } \\
(1)\end{array}$ & $\begin{array}{c}{[\mathrm{Fe} / \mathrm{H}]} \\
(\mathrm{dex}) \\
(2)\end{array}$ & $\begin{array}{c}R \\
(\mathrm{kpc}) \\
(3)\end{array}$ & $\begin{array}{c}Z \\
(\mathrm{kpc}) \\
(4)\end{array}$ & $\begin{array}{c}U \\
(\mathrm{~km} / \mathrm{s}) \\
(5)\end{array}$ & $\begin{array}{c}V \\
(\mathrm{~km} / \mathrm{s}) \\
(6)\end{array}$ & $\begin{array}{c}W \\
(\mathrm{~km} / \mathrm{s}) \\
(7)\end{array}$ & $\begin{array}{c}V_{R} \\
(\mathrm{~km} / \mathrm{s}) \\
(8)\end{array}$ & $\begin{array}{c}V_{\phi} \\
(\mathrm{km} / \mathrm{s}) \\
(9)\end{array}$ & $\begin{array}{c}R_{a p} \\
(\mathrm{kpc}) \\
(10)\end{array}$ & $\begin{array}{c}R_{p r} \\
(\mathrm{kpc}) \\
(11)\end{array}$ & $\begin{array}{c}Z_{\max } \\
(\mathrm{kpc}) \\
(12)\end{array}$ \\
\hline $\mathrm{V}^{*} \mathrm{~W}$ Crt & -0.54 & 8.45 & 0.85 & 74 & -114 & -37 & 61 & 114 & 8.91 & 3.20 & 1.37 \\
\hline $\mathrm{BD}+042466$ & -1.88 & 8.77 & 1.75 & -107 & -538 & -259 & -69 & -329 & 54.26 & 8.47 & 31.20 \\
\hline HD 99833 & -0.70 & 8.52 & 0.09 & -19 & -41 & -1 & -20 & 179 & 8.61 & 5.76 & 0.09 \\
\hline HD 99978 & -1.12 & 8.39 & 0.03 & -59 & -44 & -19 & -65 & 174 & 9.25 & 5.12 & 0.23 \\
\hline $\mathrm{V}^{*} \mathrm{TU}$ UMa & -1.51 & 8.70 & 0.65 & 150 & -239 & 25 & 150 & -18 & 11.04 & 0.40 & 0.88 \\
\hline $\mathrm{V}^{*} \mathrm{AX}$ Leo & -1.72 & 8.85 & 1.93 & 85 & -356 & 52 & 96 & -128 & 10.72 & 3.79 & 2.60 \\
\hline $\mathrm{V}^{*} \mathrm{SS}$ Leo & -1.79 & 8.58 & 1.03 & 46 & -243 & 34 & 47 & -19 & 8.87 & 0.49 & 1.31 \\
\hline HD 100906 & -0.43 & 8.48 & 0.12 & 7 & 25 & -4 & 3 & 245 & 10.33 & 8.47 & 0.15 \\
\hline HD 101063 & -1.15 & 8.46 & 0.10 & 209 & -259 & 4 & 210 & -35 & 13.23 & 0.71 & 0.16 \\
\hline $\mathrm{V}^{*}$ SU Dra & -1.80 & 8.83 & 0.52 & 10 & -331 & -7 & 6 & -111 & 8.85 & 3.10 & 0.54 \\
\hline $\mathrm{V}^{*} \mathrm{BX}$ Leo & -1.28 & 8.76 & 1.41 & 122 & -157 & -42 & 119 & 69 & 10.35 & 1.88 & 2.74 \\
\hline $\mathrm{V}^{*} \mathrm{ST}$ Leo & -1.17 & 8.68 & 1.26 & -51 & -273 & 75 & -47 & -56 & 8.91 & 1.68 & 3.26 \\
\hline $\mathrm{V}^{*}$ AA Leo & -1.47 & 8.76 & 1.87 & -136 & -280 & -67 & -130 & -72 & 11.35 & 1.90 & 2.97 \\
\hline HD 102200 & -1.20 & 8.47 & 0.02 & -101 & -133 & 17 & -101 & 86 & 9.55 & 2.08 & 0.23 \\
\hline $\mathrm{V}^{*} \mathrm{X}$ Crt & -2.00 & 8.40 & 1.16 & -145 & -229 & -109 & -143 & -26 & 11.31 & 0.69 & 4.49 \\
\hline HD 102780 & -0.63 & 8.62 & 0.77 & -5 & 11 & 84 & -10 & 230 & 10.70 & 8.65 & 2.60 \\
\hline CD-70 889 & -2.57 & 8.25 & -0.08 & 125 & -389 & -75 & 135 & -161 & 11.56 & 4.26 & 1.91 \\
\hline $\mathrm{BD}+222411$ & -1.95 & 8.91 & 2.42 & 0 & -188 & 2 & -2 & 32 & 9.19 & 0.90 & 2.42 \\
\hline $\mathrm{V}^{*} \mathrm{TY}$ Vir & -1.78 & 8.44 & 0.96 & 17 & -391 & 3 & 31 & -169 & 8.70 & 5.40 & 0.98 \\
\hline HD 103295 & -1.04 & 8.44 & 0.12 & 48 & 0 & 3 & 43 & 221 & 9.67 & 7.37 & 0.14 \\
\hline BD-01 2582 & -2.23 & 8.48 & 0.53 & -122 & -271 & -177 & -120 & -56 & 11.53 & 2.75 & 9.65 \\
\hline [OM87] 115221.21-0 & -2.21 & 8.17 & 1.09 & -299 & -171 & -89 & -303 & -7 & 22.88 & 0.12 & 4.60 \\
\hline HD 103545 & -2.14 & 8.54 & 0.79 & 99 & -271 & 81 & 101 & -47 & 9.81 & 1.25 & 2.59 \\
\hline CD-35 7576 & -1.88 & 8.30 & 0.30 & 102 & -160 & -9 & 97 & 67 & 9.21 & 1.58 & 0.37 \\
\hline HD 104053 & -1.10 & 8.66 & 1.05 & 54 & -137 & -83 & 53 & 84 & 8.97 & 2.66 & 3.28 \\
\hline HD 233891 & -1.54 & 8.62 & 0.32 & 15 & -6 & -47 & 17 & 214 & 8.96 & 8.09 & 0.92 \\
\hline $\mathrm{V}^{*} \mathrm{GK}$ Com & -1.93 & 8.58 & 0.71 & 238 & -227 & -35 & 238 & -3 & 15.39 & 0.06 & 2.41 \\
\hline $\mathrm{BD}+092574$ & -1.95 & 8.52 & 1.00 & -78 & -187 & -123 & -80 & 30 & 9.60 & 0.99 & 5.36 \\
\hline HD 104785 & -0.78 & 8.55 & 0.50 & -67 & -208 & -80 & -67 & 11 & 9.06 & 0.30 & 2.31 \\
\hline HD 104893 & -1.99 & 8.08 & 0.98 & 207 & -165 & -88 & 194 & 91 & 13.04 & 2.34 & 5.21 \\
\hline $\mathrm{V}^{*} \mathrm{IK}$ Hya & -1.24 & 8.31 & 0.40 & 30 & -358 & -25 & 39 & -136 & 8.53 & 3.81 & 0.59 \\
\hline CD-37 7677 & -1.53 & 8.28 & 0.25 & -96 & -194 & 7 & -97 & 20 & 9.13 & 0.45 & 0.30 \\
\hline $\mathrm{V}^{*} \mathrm{UU}$ Vir & -0.87 & 8.43 & 0.79 & 149 & -77 & -35 & 141 & 150 & 11.60 & 3.94 & 1.69 \\
\hline HD 105546 & -1.40 & 8.54 & 0.09 & 0 & -18 & 40 & 1 & 202 & 8.54 & 7.36 & 0.59 \\
\hline CD-28 9325 & -1.83 & 7.95 & 1.36 & -284 & -121 & -79 & -299 & 25 & 21.91 & 0.45 & 4.15 \\
\hline $\mathrm{V}^{*} \mathrm{~V}$ Com & -1.75 & 8.96 & 3.22 & 143 & -62 & 3 & 138 & 162 & 13.42 & 5.08 & 4.81 \\
\hline HD 105740 & -0.82 & 8.52 & 0.31 & -43 & -127 & -25 & -44 & 92 & 8.73 & 2.38 & 0.50 \\
\hline $\mathrm{V}^{*} \mathrm{AB} \mathrm{UMa}$ & -0.49 & 8.80 & 0.94 & 14 & -94 & -27 & 18 & 126 & 8.88 & 3.78 & 1.18 \\
\hline HD 106373 & -2.48 & 8.45 & 0.08 & 28 & -89 & 62 & 27 & 131 & 8.56 & 3.89 & 1.20 \\
\hline HD 106383 & -0.61 & 8.53 & 0.15 & 42 & -10 & -28 & 42 & 210 & 9.40 & 7.14 & 0.45 \\
\hline
\end{tabular}


TABle 3. (continued)

\begin{tabular}{|c|c|c|c|c|c|c|c|c|c|c|c|}
\hline $\begin{array}{l}\text { Star } \\
(1)\end{array}$ & $\begin{array}{c}{[\mathrm{Fe} / \mathrm{H}]} \\
(\mathrm{dex}) \\
(2)\end{array}$ & $\begin{array}{c}R \\
(\mathrm{kpc}) \\
(3)\end{array}$ & $\begin{array}{c}Z \\
(\mathrm{kpc}) \\
(4)\end{array}$ & $\begin{array}{c}U \\
(\mathrm{~km} / \mathrm{s}) \\
(5)\end{array}$ & $\begin{array}{c}V \\
(\mathrm{~km} / \mathrm{s}) \\
(6)\end{array}$ & $\begin{array}{c}W \\
(\mathrm{~km} / \mathrm{s}) \\
(7)\end{array}$ & $\begin{array}{c}V_{R} \\
(\mathrm{~km} / \mathrm{s}) \\
(8)\end{array}$ & $\begin{array}{c}V_{\phi} \\
(\mathrm{km} / \mathrm{s}) \\
(9)\end{array}$ & $\begin{array}{c}R_{a p} \\
(\mathrm{kpc}) \\
(10)\end{array}$ & $\begin{array}{c}R_{p r} \\
(\mathrm{kpc}) \\
(11)\end{array}$ & $\begin{array}{c}Z_{\max } \\
(\mathrm{kpc}) \\
(12)\end{array}$ \\
\hline CD-28 9374 & -0.79 & 8.40 & 0.17 & -62 & -126 & -114 & -64 & 92 & 9.01 & 2.95 & 3.69 \\
\hline HD 106411 & -0.75 & 8.47 & 0.02 & -55 & -64 & -32 & -56 & 155 & 9.00 & 4.53 & 0.46 \\
\hline HD 106670 & -1.06 & 8.42 & 0.04 & 59 & 13 & -18 & 54 & 234 & 10.68 & 7.50 & 0.25 \\
\hline$V^{*}$ IW Com & -1.47 & 8.63 & 1.06 & -64 & -8 & -9 & -66 & 211 & 10.61 & 6.95 & 1.26 \\
\hline $\mathrm{V}^{*} \mathrm{SW}$ Dra & -1.12 & 8.88 & 0.66 & 50 & -74 & -3 & 58 & 144 & 9.42 & 4.27 & 0.70 \\
\hline HD 107363 & -0.72 & 8.49 & 0.65 & -119 & -113 & -43 & -121 & 105 & 10.37 & 2.64 & 1.16 \\
\hline $\mathrm{V}^{*} \mathrm{UV}$ Vir & -1.19 & 8.31 & 1.47 & 31 & -252 & -8 & 33 & -30 & 8.51 & 0.75 & 1.53 \\
\hline HD 107752 & -2.74 & 8.45 & 1.28 & 134 & -398 & 120 & 142 & -171 & 14.29 & 4.89 & 5.11 \\
\hline $\mathrm{BD}+292287$ & -0.66 & 8.57 & 0.68 & -29 & -34 & -28 & -29 & 185 & 8.88 & 6.28 & 0.87 \\
\hline MA 300142 & -0.81 & 8.84 & 3.13 & 278 & -117 & 24 & 278 & 104 & 23.54 & 2.44 & 9.13 \\
\hline HD 108317 & -2.27 & 8.48 & 0.15 & 90 & -68 & -6 & 89 & 152 & 9.70 & 4.17 & 0.19 \\
\hline MA 300157 & -0.61 & 8.89 & 3.66 & -385 & -214 & 16 & -385 & 5 & 45.45 & 0.14 & 35.03 \\
\hline HD 108405 & -0.60 & 8.46 & 0.02 & 72 & -66 & -78 & 71 & 154 & 9.42 & 4.76 & 1.82 \\
\hline [MFF90] PHI 2/2 97 & -1.10 & 7.78 & 1.26 & 92 & -115 & -80 & 64 & 124 & 8.32 & 3.85 & 2.96 \\
\hline HD 108577 & -2.56 & 8.46 & 0.66 & 137 & -205 & -161 & 136 & 18 & 11.15 & 1.25 & 10.67 \\
\hline $\mathrm{BD}+422309$ & -1.24 & 8.71 & 0.99 & -25 & -196 & -95 & -24 & 24 & 8.87 & 0.75 & 3.71 \\
\hline V* IX Com & -1.08 & 8.62 & 1.24 & 50 & -111 & -20 & 50 & 109 & 8.98 & 3.12 & 1.46 \\
\hline $\mathrm{BD}+042621$ & -2.41 & 8.32 & 1.46 & 2 & -268 & -154 & 6 & -48 & 8.47 & 2.80 & 7.56 \\
\hline $\mathrm{V}^{*} \mathrm{RR}$ CVn & -1.08 & 8.82 & 2.29 & -32 & -375 & 32 & -35 & -154 & 9.23 & 5.49 & 2.68 \\
\hline $\mathrm{BD}+302282$ & -1.12 & 8.59 & 0.90 & 9 & 66 & 3 & 9 & 286 & 15.33 & 8.62 & 1.50 \\
\hline $\mathrm{V}^{*} \mathrm{UZ}$ CVn & -1.89 & 8.88 & 1.98 & -70 & -240 & 31 & -71 & -17 & 9.51 & 0.48 & 2.73 \\
\hline V* SV Hya & -1.50 & 8.21 & 0.51 & 88 & -110 & 100 & 80 & 116 & 9.27 & 3.47 & 2.92 \\
\hline HD 108976 & -1.43 & 8.51 & 0.08 & -6 & 7 & 5 & -6 & 227 & 8.91 & 8.44 & 0.10 \\
\hline MA 30210 & -1.04 & 8.55 & 0.52 & 24 & -50 & 5 & 24 & 170 & 8.68 & 5.40 & 0.53 \\
\hline [MFF90] PHI 2/2 46 & -1.34 & 8.18 & 4.41 & 82 & -410 & 35 & 200 & -53 & 14.79 & 1.43 & 7.85 \\
\hline $\mathrm{V}^{*} \mathrm{~S}$ Com & -1.91 & 8.60 & 1.64 & 40 & -157 & -58 & 40 & 63 & 8.83 & 1.87 & 2.86 \\
\hline [MFF90] PHI 2/2 40 & -0.75 & 7.73 & 1.28 & 132 & -83 & 34 & 96 & 164 & 9.63 & 4.45 & 1.62 \\
\hline CD-33 8486 & -1.87 & 8.07 & 0.53 & 65 & -207 & 31 & 63 & 20 & 8.45 & 0.47 & 0.75 \\
\hline [OM87] 123129.29-0 & -2.35 & 7.44 & 2.27 & 249 & -417 & -246 & 314 & -44 & 40.53 & 4.76 & 40.12 \\
\hline $\mathrm{BD}+302294$ & -1.09 & 8.65 & 1.80 & 4 & -185 & 64 & 4 & 35 & 8.83 & 1.02 & 3.04 \\
\hline $\mathrm{BD}+302297$ & -1.17 & 8.69 & 2.50 & 35 & -40 & 51 & 36 & 180 & 9.75 & 6.65 & 3.05 \\
\hline $\mathrm{V}^{*} \mathrm{SV}$ CVn & -2.20 & 8.88 & 2.60 & -178 & -248 & 92 & -179 & -22 & 12.93 & 1.05 & 10.89 \\
\hline $\mathrm{BD}+262368$ & -1.08 & 8.55 & 1.26 & -67 & -116 & 103 & -68 & 103 & 9.11 & 3.69 & 4.46 \\
\hline $\mathrm{V}^{*} \mathrm{BQ}$ Vir & -1.32 & 8.08 & 1.96 & -82 & -169 & 44 & -88 & 40 & 8.92 & 1.11 & 3.13 \\
\hline HD 109823 & -1.68 & 8.55 & 0.83 & -2 & -81 & 14 & -2 & 139 & 8.58 & 4.14 & 0.88 \\
\hline $\mathrm{V}^{*} \mathrm{FU}$ Vir & -1.17 & 8.32 & 2.28 & -172 & 40 & -94 & -189 & 248 & 24.21 & 5.81 & 6.79 \\
\hline HD 109995 & -1.66 & 8.54 & 0.25 & 1 & -224 & -91 & 1 & -4 & 8.54 & 0.12 & 2.74 \\
\hline $\mathrm{V}^{*} \mathrm{U}$ Com & -1.25 & 8.57 & 1.66 & 272 & -282 & -32 & 272 & -61 & 19.46 & 1.36 & 6.29 \\
\hline HD 110184 & -2.46 & 8.35 & 1.16 & 15 & -117 & 118 & 11 & 103 & 8.51 & 3.82 & 4.40 \\
\hline $\mathrm{V}^{*} \mathrm{SW} \mathrm{CVn}$ & -1.53 & 8.85 & 2.67 & -37 & -269 & 28 & -39 & -48 & 9.28 & 1.41 & 3.16 \\
\hline
\end{tabular}


TABle 3. (continued)

\begin{tabular}{|c|c|c|c|c|c|c|c|c|c|c|c|}
\hline $\begin{array}{l}\text { Star } \\
(1)\end{array}$ & $\begin{array}{c}{[\mathrm{Fe} / \mathrm{H}]} \\
(\mathrm{dex}) \\
(2)\end{array}$ & $\begin{array}{c}R \\
(\mathrm{kpc}) \\
(3)\end{array}$ & $\begin{array}{c}Z \\
(\mathrm{kpc}) \\
(4)\end{array}$ & $\begin{array}{c}U \\
(\mathrm{~km} / \mathrm{s}) \\
(5)\end{array}$ & $\begin{array}{c}V \\
(\mathrm{~km} / \mathrm{s}) \\
(6)\end{array}$ & $\begin{array}{c}W \\
(\mathrm{~km} / \mathrm{s}) \\
(7)\end{array}$ & $\begin{array}{c}V_{R} \\
(\mathrm{~km} / \mathrm{s}) \\
(8)\end{array}$ & $\begin{array}{c}V_{\phi} \\
(\mathrm{km} / \mathrm{s}) \\
(9)\end{array}$ & $\begin{array}{c}R_{a p} \\
(\mathrm{kpc}) \\
(10)\end{array}$ & $\begin{array}{c}R_{p r} \\
(\mathrm{kpc}) \\
(11)\end{array}$ & $\begin{array}{c}Z_{\max } \\
(\mathrm{kpc}) \\
(12)\end{array}$ \\
\hline [MFF90] PHI 4/3 13 & -0.72 & 7.82 & 1.07 & 150 & -43 & 150 & 117 & 201 & 14.44 & 5.79 & 6.27 \\
\hline $\mathrm{BD}+282144$ & -0.82 & 8.54 & 1.37 & 60 & -95 & 87 & 60 & 125 & 9.46 & 3.98 & 3.11 \\
\hline [MFF90] PHI 4/3 1 & -1.67 & 7.46 & 4.01 & 398 & -407 & 166 & 420 & 131 & 88.30 & 2.01 & 38.77 \\
\hline [MFF90] PHI 4/1 170 & -1.41 & 7.32 & 2.33 & 697 & -532 & 159 & 764 & 12 & $\cdots$ & $\cdots$ & $\cdots$ \\
\hline UP 25091 & -0.65 & 8.51 & 1.65 & 27 & -40 & 52 & 25 & 180 & 9.01 & 6.44 & 2.19 \\
\hline MA 34370 & -0.86 & 8.90 & 4.62 & 88 & -105 & -23 & 93 & 111 & 10.92 & 4.14 & 6.04 \\
\hline [M60b]+34 382 & -0.77 & 8.63 & 1.60 & -64 & 33 & -27 & -60 & 254 & 13.73 & 8.07 & 2.46 \\
\hline [MFF90] PHI 4/1 99 & -2.01 & 8.56 & 5.72 & -123 & -305 & -173 & 23 & -148 & 13.52 & 8.49 & 11.11 \\
\hline HD 111721 & -1.26 & 8.44 & 0.12 & 56 & -274 & -133 & 57 & -54 & 8.84 & 1.98 & 5.34 \\
\hline $\mathrm{BD}+272181$ & -0.70 & 8.49 & 1.57 & -95 & 519 & -40 & -95 & 739 & $\cdots$ & $\cdots$ & $\cdots$ \\
\hline [MFF90] PHI 4/1 7 & -1.01 & 7.23 & 2.17 & 120 & 0 & 126 & 26 & 249 & 13.95 & 7.17 & 6.02 \\
\hline $\mathrm{V}^{*}$ AS Vir & -1.49 & 7.97 & 1.38 & -241 & -175 & -122 & -245 & 19 & 17.37 & 0.40 & 6.04 \\
\hline HD 111980 & -0.99 & 8.47 & 0.06 & -280 & -195 & -102 & -280 & 23 & 20.24 & 0.49 & 6.00 \\
\hline HD 111971 & -0.55 & 8.47 & 0.01 & 13 & 19 & 30 & 12 & 239 & 9.99 & 8.40 & 0.43 \\
\hline HD 112126 & -1.52 & 8.56 & 1.30 & 50 & -214 & -39 & 50 & 6 & 8.83 & 0.15 & 1.94 \\
\hline $\mathrm{BD}+282162$ & -0.95 & 8.48 & 2.22 & -138 & -183 & 22 & -137 & 37 & 10.64 & 0.99 & 3.52 \\
\hline $\mathrm{V}^{*}$ AT Vir & -1.60 & 8.09 & 1.21 & 162 & -470 & 215 & 181 & -237 & 30.42 & 5.98 & 16.47 \\
\hline BD+27 2191 & -0.77 & 8.49 & 0.23 & 51 & -31 & 60 & 51 & 189 & 9.36 & 6.23 & 1.20 \\
\hline BD+10 2495 & -1.83 & 8.40 & 0.49 & 73 & -47 & 275 & 71 & 174 & 21.49 & 7.81 & 17.30 \\
\hline Weis 60529 & -0.77 & 8.47 & 3.43 & 213 & -291 & 91 & 212 & -75 & 16.97 & 1.78 & 6.20 \\
\hline Weis 35550 & -0.62 & 8.49 & 0.38 & 46 & -17 & 13 & 47 & 203 & 9.37 & 6.71 & 0.45 \\
\hline CD-33 8772 & -2.03 & 7.76 & 0.78 & 188 & -174 & 62 & 179 & 74 & 11.49 & 1.59 & 1.77 \\
\hline Weis 62467 & -0.99 & 8.47 & 1.31 & 41 & 8 & 5 & 43 & 228 & 10.49 & 7.84 & 1.56 \\
\hline
\end{tabular}


TABLE 3. (continued)

\begin{tabular}{|c|c|c|c|c|c|c|c|c|c|c|c|}
\hline $\begin{array}{c}\text { Star } \\
\text { (1) }\end{array}$ & $\begin{array}{c}{[\mathrm{Fe} / \mathrm{H}]} \\
(\mathrm{dex}) \\
(2)\end{array}$ & $\begin{array}{c}R \\
(\mathrm{kpc}) \\
(3)\end{array}$ & $\begin{array}{c}Z \\
(\mathrm{kpc}) \\
(4)\end{array}$ & $\begin{array}{c}U \\
(\mathrm{~km} / \mathrm{s}) \\
(5)\end{array}$ & $\begin{array}{c}V \\
(\mathrm{~km} / \mathrm{s}) \\
(6)\end{array}$ & $\begin{array}{c}W \\
(\mathrm{~km} / \mathrm{s}) \\
(7)\end{array}$ & $\begin{array}{c}V_{R} \\
(\mathrm{~km} / \mathrm{s}) \\
(8)\end{array}$ & $\begin{array}{c}V_{\phi} \\
(\mathrm{km} / \mathrm{s}) \\
(9)\end{array}$ & $\begin{array}{c}R_{a p} \\
(\mathrm{kpc}) \\
(10)\end{array}$ & $\begin{array}{c}R_{p r} \\
(\mathrm{kpc}) \\
(11)\end{array}$ & $\begin{array}{c}Z_{\max } \\
(\mathrm{kpc}) \\
(12)\end{array}$ \\
\hline $\mathrm{BD}+282176$ & -1.49 & 8.46 & 1.22 & 94 & 7 & 5 & 95 & 226 & 12.48 & 6.77 & 1.72 \\
\hline Weis 62561 & -0.63 & 8.46 & 1.32 & 151 & -88 & 116 & 152 & 131 & 13.38 & 3.65 & 4.92 \\
\hline $\mathrm{BD}+122547$ & -2.07 & 8.15 & 1.79 & -299 & -350 & -119 & -293 & -144 & 28.79 & 2.80 & 8.09 \\
\hline $\mathrm{V}^{*} \mathrm{RY}$ Com & -1.65 & 8.32 & 2.19 & -58 & -176 & -33 & -59 & 44 & 8.98 & 1.17 & 2.40 \\
\hline Basel SA 57-0789 & -1.74 & 8.47 & 1.07 & -403 & -301 & -13 & -404 & -78 & 47.95 & 1.26 & 6.37 \\
\hline Basel SA 57-0758 & -1.26 & 8.45 & 1.55 & -3 & -79 & -10 & -1 & 141 & 8.56 & 4.39 & 1.57 \\
\hline Basel SA 57-0760 & -0.70 & 8.47 & 0.78 & -38 & -30 & -6 & -37 & 191 & 8.95 & 6.38 & 0.82 \\
\hline Basel SA 57-0739 & -3.00 & 8.44 & 1.59 & -171 & -123 & 28 & -169 & 99 & 12.17 & 2.52 & 3.12 \\
\hline Weis 50158 & -0.48 & 8.48 & 0.51 & 43 & -64 & 50 & 43 & 156 & 8.88 & 4.82 & 1.13 \\
\hline $\mathrm{V}^{*} \mathrm{ST}$ Com & -1.10 & 8.29 & 1.37 & -17 & -260 & -72 & -17 & -40 & 8.46 & 1.13 & 2.72 \\
\hline HD 115772 & -0.70 & 8.38 & 0.08 & 10 & 29 & -30 & 6 & 250 & 10.74 & 8.37 & 0.47 \\
\hline $\mathrm{V}^{*}$ AV Vir & -1.25 & 8.06 & 1.55 & -259 & -207 & 54 & -259 & 3 & 16.72 & 0.08 & 6.74 \\
\hline HD 116064 & -1.91 & 8.46 & 0.03 & 95 & -215 & 120 & 95 & 5 & 9.50 & 0.16 & 4.76 \\
\hline $\mathrm{V}^{*} \mathrm{BC}$ Vir & -1.50 & 7.90 & 1.86 & -198 & -43 & -65 & -207 & 165 & 16.70 & 3.65 & 3.76 \\
\hline $\mathrm{V}^{*}$ AM Vir & -1.37 & 7.86 & 0.98 & -175 & -212 & -127 & -175 & -8 & 12.18 & 0.19 & 5.43 \\
\hline $\mathrm{V}^{*}$ AU Vir & -1.50 & 7.88 & 1.25 & -61 & -214 & 18 & -62 & 1 & 8.27 & 0.02 & 1.45 \\
\hline $\mathrm{V}^{*} \mathrm{~W}$ Vir & -0.60 & 7.55 & 2.13 & 63 & 9 & -37 & 37 & 235 & 10.39 & 7.59 & 3.03 \\
\hline $\mathrm{V}^{*} \mathrm{SX}$ UMa & -1.81 & 8.74 & 0.99 & 260 & -267 & -84 & 257 & -63 & 18.64 & 1.54 & 7.82 \\
\hline $\mathrm{V}^{*} \mathrm{FX}$ Hya & -1.08 & 7.36 & 1.31 & -79 & -41 & -29 & -110 & 161 & 9.59 & 4.05 & 1.65 \\
\hline [OM87] 132605.43-0 & -0.66 & 8.31 & 0.17 & 54 & 22 & -21 & 48 & 243 & 11.00 & 7.72 & 0.40 \\
\hline HD 117220 & -0.86 & 8.37 & 0.09 & 47 & -21 & -12 & 44 & 200 & 9.03 & 6.46 & 0.18 \\
\hline $\mathrm{V}^{*} \mathrm{RZ} \mathrm{CVn}$ & -1.84 & 8.34 & 1.56 & 299 & -246 & 80 & 298 & -37 & 23.05 & 0.70 & 4.59 \\
\hline CD-33 9314 & -2.10 & 8.02 & 0.36 & -353 & -303 & -6 & -347 & -104 & 29.97 & 1.71 & 1.26 \\
\hline $\mathrm{V}^{*} \mathrm{RV}$ Oct & -1.71 & 8.02 & -0.36 & 7 & -202 & 60 & 5 & 19 & 8.03 & 0.46 & 1.29 \\
\hline
\end{tabular}


TABLE 3. (continued)

\begin{tabular}{|c|c|c|c|c|c|c|c|c|c|c|c|c|}
\hline $\begin{array}{l}\text { Star } \\
(1)\end{array}$ & $\begin{array}{c}{[\mathrm{Fe} / \mathrm{H}]} \\
(\mathrm{dex}) \\
(2)\end{array}$ & $\begin{array}{c}R \\
(\mathrm{kpc}) \\
(3)\end{array}$ & $\begin{array}{c}Z \\
(\mathrm{kpc}) \\
(4)\end{array}$ & $\begin{array}{c}U \\
(\mathrm{~km} / \mathrm{s}) \\
(5)\end{array}$ & $\begin{array}{c}V \\
(6 \mathrm{~km} / \mathrm{s}) \\
(6)\end{array}$ & $\begin{array}{c}W \\
(\mathrm{~km} / \mathrm{s}) \\
(7)\end{array}$ & $\begin{array}{c}V_{R} \\
(\mathrm{~km} / \mathrm{s}) \\
(8)\end{array}$ & $\begin{array}{c}V_{\phi} \\
(\mathrm{km} / \mathrm{s}) \\
(9)\end{array}$ & $\begin{array}{c}R_{a p} \\
(\mathrm{kpc}) \\
(10)\end{array}$ & $\begin{array}{c}R_{p r} \\
(\mathrm{kpc}) \\
(11)\end{array}$ & $\begin{array}{c}Z_{\max } \\
(\mathrm{kpc}) \\
(12)\end{array}$ & (13) \\
\hline HD 120183 & -0.65 & 8.47 & 0.28 & -5 & -69 & -3 & -4 & 151 & 8.48 & 4.49 & 0.28 & \\
\hline $\mathrm{V}^{*} \mathrm{SS}$ CVn & -1.37 & 8.46 & 1.66 & -286 & -226 & 30 & -286 & 11 & 20.24 & 0.25 & 6.69 & \\
\hline $\mathrm{V}^{*} \mathrm{RX} \mathrm{CVn}$ & -1.31 & 8.50 & 2.29 & -55 & -4 & -155 & -36 & 220 & 14.80 & 8.02 & 7.72 & \\
\hline $\mathrm{V}^{*} \mathrm{BB}$ Vir & -1.61 & 8.01 & 1.10 & 196 & -171 & 27 & 195 & 53 & 12.35 & 1.12 & 1.60 & 0.8 \\
\hline HD 120559 & -1.13 & 8.48 & 0.00 & 20 & -35 & -30 & 20 & 185 & 8.60 & 6.14 & 0.38 & 0.17 \\
\hline HD 121135 & -1.57 & 8.31 & 0.38 & -33 & -84 & 119 & -34 & 136 & 8.51 & 4.99 & 3.61 & 0.27 \\
\hline HD 121261 & -1.52 & 7.69 & 0.54 & 65 & -206 & 39 & 63 & 20 & 8.06 & 0.47 & 0.86 & \\
\hline V* V499 Cen & -1.43 & 7.77 & 0.35 & -271 & -235 & -28 & -268 & -42 & 16.41 & 0.75 & 0.85 & \\
\hline $\mathrm{V}^{*} \mathrm{ST}$ CVn & -1.07 & 8.27 & 1.30 & 26 & -142 & -90 & 29 & 77 & 8.39 & 2.47 & 3.57 & \\
\hline $\mathrm{V}^{*} \mathrm{UY}$ Boo & -2.56 & 8.05 & 1.16 & -230 & -209 & 69 & -230 & 10 & 14.35 & 0.23 & 5.42 & 0.9 \\
\hline $\mathrm{V}^{*} \mathrm{RU}$ CVn & -1.37 & 8.22 & 1.75 & 145 & -138 & 38 & 149 & 75 & 11.10 & 1.79 & 2.27 & 0.72 \\
\hline HD 122196 & -1.89 & 8.46 & 0.02 & 98 & -66 & 12 & 97 & 155 & 9.95 & 4.17 & 0.15 & \\
\hline HD 122563 & -2.62 & 8.39 & 0.25 & 130 & -217 & 24 & 130 & 3 & 9.99 & 0.07 & 0.45 & \\
\hline V* V674 Cen & -1.53 & 7.55 & 0.60 & 144 & -373 & 36 & 161 & -135 & 11.04 & 2.98 & 1.00 & 0.57 \\
\hline HD 122956 & -1.75 & 8.33 & 0.20 & -40 & -173 & 118 & -41 & 47 & 8.50 & 1.52 & 4.19 & 0.70 \\
\hline $\mathrm{V}^{*} \mathrm{AD}$ Vir & -1.15 & 6.93 & 2.25 & 75 & -257 & 142 & 79 & -28 & 8.73 & 0.89 & 6.02 & 0.82 \\
\hline $\mathrm{V}^{*} \mathrm{~W}$ CVn & -1.22 & 8.41 & 0.90 & 1 & -79 & 82 & 6 & 141 & 8.48 & 4.78 & 2.45 & 0.28 \\
\hline $\mathrm{V}^{*}$ AL Vir & -0.70 & 7.47 & 1.21 & -13 & -20 & 21 & -28 & 198 & 7.89 & 6.46 & 1.35 & 0.10 \\
\hline BD+09 2860 & -1.67 & 8.26 & 0.48 & -80 & -56 & -54 & -81 & 164 & 9.59 & 4.76 & 1.21 & 0.34 \\
\hline HD 124358 & -1.98 & 7.95 & 0.65 & 33 & -439 & 293 & 41 & -218 & 27.77 & 7.76 & 21.12 & 0.56 \\
\hline HD 124884 & -0.84 & 8.01 & 1.09 & -26 & -70 & 9 & -26 & 150 & 8.17 & 4.47 & 1.12 & 0.2 \\
\hline BD+09 2870 & -2.39 & 7.70 & 1.54 & 377 & -273 & 55 & 378 & -49 & 36.62 & 0.76 & 6.48 & 0.9 \\
\hline $\mathrm{V}^{*} \mathrm{TV}$ Boo & -2.44 & 8.42 & 1.20 & -146 & -184 & -28 & -143 & 45 & 10.72 & 1.05 & 1.50 & \\
\hline $\mathrm{V}^{*} \mathrm{XX}$ Vir & -2.40 & 6.78 & 2.32 & 72 & -227 & -77 & 72 & 0 & 7.34 & 0.01 & 4.28 & 1.0 \\
\hline $\mathrm{BD}+012916$ & -1.82 & 7.37 & 1.73 & 85 & -232 & 14 & 85 & -9 & 8.19 & 0.20 & 1.86 & 0.95 \\
\hline $\mathrm{BD}+082856$ & -2.03 & 7.68 & 1.49 & -186 & -70 & -21 & -187 & 148 & 13.29 & 3.31 & 2.38 & 0.60 \\
\hline HD 126238 & -1.65 & 8.36 & 0.05 & -204 & -166 & -15 & -205 & 52 & 12.94 & 1.06 & 0.25 & 0.85 \\
\hline HD 126587 & -2.62 & 8.13 & 0.30 & -119 & -157 & 12 & -121 & 60 & 9.53 & 1.36 & 0.43 & 0.75 \\
\hline $\mathrm{V}^{*} \mathrm{AE}$ Vir & -1.16 & 6.80 & 2.68 & -257 & -208 & 76 & -257 & 3 & 15.27 & 0.09 & 11.98 & 0.99 \\
\hline $\mathrm{V}^{*} \mathrm{RY} \mathrm{Lib}$ & -1.48 & 6.60 & 1.66 & 103 & -186 & 64 & 95 & 50 & 7.79 & 1.15 & 2.25 & 0.75 \\
\hline $\mathrm{V}^{*}$ SW Boo & -1.12 & 8.16 & 1.96 & 310 & -285 & 156 & 303 & -92 & 30.44 & 1.84 & 12.02 & 0.89 \\
\hline $\mathrm{V}^{*} \mathrm{ST}$ Vir & -0.67 & 7.74 & 1.07 & -47 & -96 & -38 & -50 & 123 & 8.16 & 3.31 & 1.33 & 0.42 \\
\hline $\mathrm{V}^{*}$ AF Vir & -1.33 & 7.65 & 1.43 & 291 & -280 & 148 & 291 & -58 & 24.11 & 1.12 & 9.45 & 0.9 \\
\hline BD+18 2890 & -1.61 & 8.23 & 0.60 & -167 & -249 & -57 & -167 & -27 & 11.26 & 0.61 & 1.51 & 0.90 \\
\hline V* KS Hya & -1.61 & 6.16 & 1.75 & 469 & -299 & 360 & 474 & 37 & $\cdots$ & $\cdots$ & $\cdots$ & 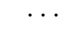 \\
\hline $\mathrm{V}^{*} \mathrm{RS}$ Boo & -0.36 & 8.31 & 0.73 & -15 & 29 & -8 & -7 & 250 & 10.89 & 8.32 & 0.92 & 0.13 \\
\hline $\mathrm{V}^{*} \mathrm{SV}$ Boo & -1.55 & 8.11 & 2.89 & -229 & -278 & -50 & -235 & -23 & 16.18 & 0.50 & 5.26 & \\
\hline HD 128188 & -1.37 & 8.01 & 0.51 & 26 & -25 & -26 & 21 & 196 & 8.18 & 6.49 & 0.68 & 0.12 \\
\hline HD 128279 & -2.20 & 8.39 & 0.07 & -8 & -62 & -234 & -9 & 158 & 13.04 & 8.37 & 10.24 & 0.23 \\
\hline $\mathrm{V}^{*} \mathrm{DO}$ Vir & -0.80 & 5.78 & 3.22 & 388 & -255 & 321 & 389 & 13 & 101.34 & 0.18 & 63.72 & 1.00 \\
\hline
\end{tabular}


TABLE 3. (continued)

\begin{tabular}{|c|c|c|c|c|c|c|c|c|c|c|c|}
\hline $\begin{array}{l}\text { Star } \\
(1)\end{array}$ & $\begin{array}{c}{[\mathrm{Fe} / \mathrm{H}]} \\
(\mathrm{dex}) \\
(2)\end{array}$ & $\begin{array}{c}R \\
(\mathrm{kpc}) \\
(3)\end{array}$ & $\begin{array}{c}Z \\
(\mathrm{kpc}) \\
(4)\end{array}$ & $\begin{array}{c}U \\
(\mathrm{~km} / \mathrm{s}) \\
(5)\end{array}$ & $\begin{array}{c}V \\
(\mathrm{~km} / \mathrm{s}) \\
(6)\end{array}$ & $\begin{array}{c}W \\
(\mathrm{~km} / \mathrm{s}) \\
(7)\end{array}$ & $\begin{array}{c}V_{R} \\
(\mathrm{~km} / \mathrm{s}) \\
(8)\end{array}$ & $\begin{array}{c}V_{\phi} \\
(\mathrm{km} / \mathrm{s}) \\
(9)\end{array}$ & $\begin{array}{c}R_{a p} \\
(\mathrm{kpc}) \\
(10)\end{array}$ & $\begin{array}{c}R_{p r} \\
(\mathrm{kpc}) \\
(11)\end{array}$ & $\begin{array}{c}Z_{\max } \\
(\mathrm{kpc}) \\
(12)\end{array}$ \\
\hline BPS CS 22871-0060 & -0.69 & 7.72 & 0.61 & 11 & -7 & 61 & 0 & 214 & 7.94 & 7.69 & 1.31 \\
\hline $\mathrm{V}^{*} \mathrm{SZ}$ Boo & -1.68 & 7.74 & 2.35 & -12 & -133 & 8 & -4 & 87 & 8.02 & 2.44 & 2.37 \\
\hline $\mathrm{V}^{*} \mathrm{TW}$ Boo & -1.46 & 8.32 & 1.20 & -254 & -253 & -17 & -256 & -15 & 16.63 & 0.30 & 2.29 \\
\hline $\mathrm{V}^{*} \mathrm{RU}$ Boo & -1.50 & 7.04 & 3.60 & 130 & -215 & 56 & 130 & -12 & 9.89 & 0.28 & 4.41 \\
\hline $\mathrm{V}^{*} \mathrm{AE} \mathrm{Boo}$ & -1.39 & 8.05 & 0.86 & -82 & -181 & 92 & -81 & 41 & 8.67 & 1.18 & 3.53 \\
\hline $\mathrm{V}^{*}$ TY Aps & -0.95 & 7.65 & -0.25 & 56 & -204 & 151 & 53 & 23 & 7.97 & 0.95 & 6.04 \\
\hline $\mathrm{V}^{*} \mathrm{BT}$ Dra & -1.75 & 8.73 & 1.28 & -241 & -183 & -18 & -235 & 64 & 16.18 & 1.38 & 2.27 \\
\hline $\mathrm{V}^{*} \mathrm{XZ}$ Aps & -1.06 & 7.57 & -0.55 & -91 & -242 & 122 & -86 & -37 & 8.52 & 1.06 & 3.92 \\
\hline HD 132475 & -1.70 & 8.43 & 0.05 & -49 & -353 & 61 & -49 & -133 & 8.77 & 3.87 & 1.16 \\
\hline BD-11 3853 & -0.81 & 8.41 & 0.08 & 21 & -65 & 47 & 20 & 155 & 8.48 & 4.75 & 0.74 \\
\hline HD 133461 & -0.61 & 8.12 & 0.61 & -104 & -47 & 13 & -102 & 175 & 10.11 & 4.78 & 0.83 \\
\hline BD-08 3901 & -1.43 & 8.13 & 0.34 & 107 & -26 & -37 & 105 & 194 & 10.77 & 5.39 & 0.90 \\
\hline BD+30 2611 & -1.32 & 8.07 & 1.12 & -10 & -59 & -284 & -1 & 161 & 19.98 & 8.04 & 16.98 \\
\hline $\mathrm{BD}+182976$ & -2.42 & 7.43 & 1.82 & 10 & -488 & -62 & -7 & -268 & 13.17 & 7.56 & 3.60 \\
\hline HD 135148 & -1.90 & 7.36 & 1.63 & 206 & -159 & 80 & 209 & 52 & 13.04 & 1.06 & 3.20 \\
\hline$V^{*}$ AP Ser & -1.58 & 7.78 & 0.96 & 19 & -338 & -23 & 17 & -119 & 7.85 & 3.22 & 1.09 \\
\hline$V^{*}$ BH Ser & -1.59 & 7.19 & 2.27 & -34 & -221 & -71 & -34 & 2 & 7.70 & 0.05 & 3.22 \\
\hline$V^{*}$ DF Ser & -0.74 & 7.38 & 1.89 & -71 & 1 & -39 & -54 & 225 & 10.23 & 6.72 & 2.57 \\
\hline HD 135449 & -1.47 & 8.08 & 0.18 & 174 & -249 & 88 & 175 & -25 & 11.44 & 0.57 & 2.65 \\
\hline $\mathrm{V}^{*} \mathrm{BF}$ Ser & -2.50 & 7.14 & 2.12 & 81 & -246 & -54 & 78 & -33 & 7.80 & 0.89 & 3.34 \\
\hline $\mathrm{V}^{*}$ UU Boo & -1.92 & 7.93 & 1.84 & -335 & -134 & -18 & -322 & 126 & 28.10 & 2.31 & 6.74 \\
\hline $\mathrm{BD}+172844$ & -0.64 & 8.05 & 0.71 & 53 & -84 & 60 & 57 & 135 & 8.62 & 3.85 & 1.48 \\
\hline $\mathrm{V}^{*} \mathrm{TV}$ Lib & -0.27 & 7.41 & 0.91 & 62 & 72 & 5 & 57 & 293 & 15.22 & 7.18 & 1.70 \\
\hline HD 136316 & -1.92 & 8.19 & 0.02 & 88 & -53 & -66 & 83 & 170 & 9.59 & 4.96 & 1.34 \\
\hline $\mathrm{V}^{*} \mathrm{TV} \mathrm{CrB}$ & -2.33 & 7.76 & 1.55 & 14 & -97 & -85 & 24 & 121 & 7.90 & 3.97 & 3.13 \\
\hline $\mathrm{V}^{*} \mathrm{FW}$ Lup & -0.20 & 8.18 & 0.09 & -5 & -12 & -11 & -9 & 208 & 8.23 & 7.22 & 0.15 \\
\hline $\mathrm{BD}+013070$ & -1.85 & 8.12 & 0.38 & 351 & 56 & -105 & 352 & 275 & 67.32 & 4.65 & 17.19 \\
\hline$V^{*}$ CS Ser & -1.57 & 6.99 & 1.55 & -297 & -37 & -266 & -292 & 192 & 52.35 & 3.67 & 32.20 \\
\hline $\mathrm{V}^{*} \mathrm{ST}$ Boo & -1.76 & 8.15 & 1.00 & -27 & -79 & 69 & -17 & 143 & 8.21 & 4.62 & 2.10 \\
\hline$V^{*}$ VY Ser & -1.79 & 7.94 & 0.55 & 266 & -284 & 112 & 266 & -66 & 18.83 & 1.35 & 5.52 \\
\hline $\mathrm{V}^{*}$ AR Ser & -1.78 & 7.28 & 1.21 & 66 & -165 & 297 & 67 & 54 & 16.08 & 6.11 & 16.12 \\
\hline CD-33 10593 & -0.85 & 8.41 & 0.03 & -18 & 7 & 47 & -19 & 227 & 9.38 & 8.15 & 0.77 \\
\hline $\mathrm{V}^{*} \mathrm{CG} \mathrm{Lib}$ & -1.19 & 7.40 & 0.54 & 311 & -273 & 77 & 313 & -40 & 21.56 & 0.66 & 3.09 \\
\hline BPS CS 30312-0062 & -2.63 & 6.73 & 1.53 & -53 & -103 & 45 & -51 & 118 & 7.09 & 3.11 & 2.03 \\
\hline HD 140283 & -2.47 & 8.45 & 0.03 & 242 & -240 & 49 & 242 & -20 & 15.46 & 0.40 & 1.33 \\
\hline HD 141531 & -1.57 & 7.62 & 0.93 & -189 & -278 & -67 & -191 & -50 & 11.83 & 1.04 & 2.05 \\
\hline $\mathrm{V}^{*} \mathrm{VY}$ Lib & -1.34 & 7.31 & 0.66 & -233 & -256 & -136 & -232 & -40 & 15.00 & 0.87 & 6.06 \\
\hline $\mathrm{BD}+053098$ & -2.40 & 7.71 & 0.74 & 53 & -368 & -61 & 49 & -149 & 8.08 & 4.41 & 1.62 \\
\hline $\mathrm{V}^{*}$ AN Ser & -0.07 & 7.89 & 0.68 & 3 & -31 & -31 & 10 & 189 & 7.93 & 6.24 & 0.88 \\
\hline $\mathrm{V}^{*}$ AT Ser & -2.03 & 7.45 & 1.02 & 22 & -48 & -40 & 29 & 171 & 7.65 & 5.22 & 1.37 \\
\hline
\end{tabular}


TABle 3. (continued)

\begin{tabular}{|c|c|c|c|c|c|c|c|c|c|c|c|}
\hline $\begin{array}{l}\text { Star } \\
(1)\end{array}$ & $\begin{array}{c}{[\mathrm{Fe} / \mathrm{H}]} \\
(\mathrm{dex}) \\
(2)\end{array}$ & $\begin{array}{c}R \\
(\mathrm{kpc}) \\
(3)\end{array}$ & $\begin{array}{c}Z \\
(\mathrm{kpc}) \\
(4)\end{array}$ & $\begin{array}{c}U \\
(\mathrm{~km} / \mathrm{s}) \\
(5)\end{array}$ & $\begin{array}{c}V \\
(\mathrm{~km} / \mathrm{s}) \\
(6)\end{array}$ & $\begin{array}{c}W \\
(\mathrm{~km} / \mathrm{s}) \\
(7)\end{array}$ & $\begin{array}{c}V_{R} \\
(\mathrm{~km} / \mathrm{s}) \\
(8)\end{array}$ & $\begin{array}{c}V_{\phi} \\
(\mathrm{km} / \mathrm{s}) \\
(9)\end{array}$ & $\begin{array}{c}R_{a p} \\
(\mathrm{kpc}) \\
(10)\end{array}$ & $\begin{array}{c}R_{p r} \\
(\mathrm{kpc}) \\
(11)\end{array}$ & $\begin{array}{c}Z_{\max } \\
(\mathrm{kpc}) \\
(12)\end{array}$ \\
\hline HD 141864 & -2.27 & 8.30 & -0.09 & -208 & -206 & -92 & -208 & 9 & 13.43 & 0.20 & 3.69 \\
\hline HD 142948 & -0.89 & 8.36 & 0.00 & -13 & -28 & -2 & -15 & 191 & 8.44 & 6.32 & 0.03 \\
\hline $\mathrm{V}^{*}$ AR Her & -1.30 & 8.30 & 0.97 & 295 & -417 & -20 & 274 & -225 & 29.05 & 4.49 & 4.28 \\
\hline $\mathrm{V}^{*} \mathrm{AV}$ Ser & -1.20 & 7.57 & 0.72 & 27 & 62 & -34 & 34 & 281 & 13.54 & 7.49 & 1.52 \\
\hline $\mathrm{V}^{*}$ AW Ser & -1.67 & 6.86 & 1.83 & -33 & -178 & -102 & -26 & 46 & 7.27 & 1.34 & 3.70 \\
\hline HD 145293 & -1.10 & 8.01 & 0.25 & -77 & -179 & 33 & -77 & 41 & 8.52 & 0.95 & 0.60 \\
\hline $\mathrm{V}^{*} \mathrm{RV} \mathrm{CrB}$ & -1.69 & 7.88 & 0.99 & -49 & -245 & 0 & -51 & -21 & 8.15 & 0.49 & 1.01 \\
\hline $\mathrm{V}^{*} \mathrm{BS}$ Aps & -1.33 & 7.17 & -0.53 & 93 & 10 & 46 & 49 & 243 & 9.97 & 6.76 & 1.17 \\
\hline $\mathrm{V}^{*} \mathrm{~V} 1023 \mathrm{Oph}$ & -1.21 & 6.64 & 1.11 & 46 & -37 & 56 & 55 & 180 & 7.64 & 4.88 & 1.60 \\
\hline $\mathrm{V}^{*} \mathrm{~V} 445 \mathrm{Oph}$ & -0.19 & 7.78 & 0.39 & 30 & 1 & 39 & 33 & 220 & 8.83 & 7.11 & 0.76 \\
\hline $\mathrm{V}^{*}$ V413 Oph & -1.00 & 7.22 & 0.62 & 13 & -122 & -17 & 14 & 98 & 7.26 & 2.36 & 0.69 \\
\hline BD+11 2998 & -1.14 & 8.41 & 0.07 & -48 & -31 & 71 & -47 & 190 & 9.15 & 6.41 & 1.46 \\
\hline V* VX Her & -1.58 & 7.89 & 0.62 & 366 & -250 & -27 & 364 & -51 & 31.31 & 0.83 & 4.09 \\
\hline $\mathrm{V}^{*} \mathrm{~V} 716 \mathrm{Oph}$ & -1.55 & 6.78 & 0.92 & 216 & -280 & 29 & 213 & -69 & 11.53 & 1.27 & 1.43 \\
\hline BPS CS 22878-0013 & -2.16 & 8.07 & 0.34 & -60 & -94 & 37 & -57 & 127 & 8.47 & 3.42 & 0.74 \\
\hline $\mathrm{V}^{*} \mathrm{UV}$ Oct & -1.74 & 8.19 & -0.22 & 212 & -264 & -102 & 214 & -34 & 13.98 & 0.77 & 4.06 \\
\hline $\mathrm{BD}+093223$ & -2.41 & 8.31 & 0.14 & -126 & -80 & 27 & -125 & 141 & 10.49 & 3.56 & 0.50 \\
\hline $\mathrm{V}^{*} \mathrm{RW}$ Dra & -1.55 & 8.54 & 1.08 & -28 & -121 & -12 & -13 & 103 & 8.62 & 2.85 & 1.11 \\
\hline V* GY Her & -1.92 & 7.75 & 1.70 & 133 & 12 & -151 & 180 & 198 & 18.47 & 5.94 & 11.00 \\
\hline $\mathrm{V}^{*}$ AF Her & -1.94 & 7.84 & 1.93 & 38 & -348 & -7 & 5 & -133 & 8.03 & 3.99 & 1.94 \\
\hline$V^{*}$ AG Her & -2.01 & 7.84 & 1.82 & -116 & -274 & 92 & -125 & -25 & 9.40 & 0.83 & 5.83 \\
\hline $\mathrm{V}^{*} \mathrm{CW}$ Her & -2.09 & 7.66 & 1.55 & 269 & -216 & -25 & 263 & -53 & 16.54 & 1.07 & 4.75 \\
\hline $\mathrm{V}^{*} \mathrm{SW}$ Her & -1.50 & 6.14 & 2.66 & 121 & -204 & 132 & 116 & -38 & 9.12 & 0.93 & 5.20 \\
\hline $\mathrm{V}^{*} \mathrm{RW} \operatorname{Tr} \mathrm{A}$ & -0.13 & 7.65 & -0.29 & 55 & 9 & 36 & 36 & 232 & 9.28 & 7.20 & 0.67 \\
\hline V* VZ Her & -1.02 & 7.99 & 0.78 & -41 & -185 & 27 & -36 & 40 & 8.12 & 0.97 & 1.01 \\
\hline $\mathrm{V}^{*} \mathrm{~V} 452 \mathrm{Oph}$ & -1.72 & 7.28 & 0.72 & 274 & -191 & -136 & 275 & -1 & 18.34 & 0.03 & 10.98 \\
\hline $\mathrm{V}^{*}$ DL Her & -1.32 & 7.21 & 0.84 & 17 & 29 & -107 & 52 & 244 & 11.64 & 7.00 & 3.58 \\
\hline V* V394 Her & -1.48 & 6.89 & 1.20 & 109 & -27 & 68 & 148 & 165 & 10.94 & 3.67 & 2.25 \\
\hline $\mathrm{BD}+173248$ & -2.03 & 8.31 & 0.13 & 71 & -127 & -15 & 73 & 91 & 8.86 & 2.25 & 0.24 \\
\hline $\mathrm{V}^{*} \mathrm{ST}$ Oph & -1.30 & 7.22 & 0.42 & -22 & 0 & 25 & -5 & 221 & 7.49 & 7.20 & 0.55 \\
\hline $\mathrm{V}^{*}$ EL Aps & -1.56 & 6.95 & -0.96 & 50 & -88 & -115 & 18 & 140 & 7.18 & 4.49 & 2.98 \\
\hline$V^{*}$ V494 Sco & -1.01 & 7.84 & -0.01 & -35 & 9 & 9 & -36 & 229 & 9.13 & 7.24 & 0.10 \\
\hline $\mathrm{V}^{*}$ V816 Oph & -0.96 & 6.99 & 0.57 & -66 & -84 & -94 & -48 & 143 & 7.49 & 4.03 & 2.01 \\
\hline HD 160617 & -1.78 & 8.44 & -0.01 & -78 & -116 & -49 & -78 & 104 & 9.14 & 2.72 & 0.86 \\
\hline $\mathrm{V}^{*} \mathrm{LU}$ Aps & -2.06 & 6.51 & -1.01 & 68 & -337 & 60 & 96 & -96 & 7.39 & 2.25 & 1.93 \\
\hline HD 161770 & -2.12 & 8.41 & 0.01 & 82 & -132 & -5 & 82 & 88 & 9.10 & 2.13 & 0.07 \\
\hline CD-68 1881 & -1.81 & 7.62 & -0.41 & -39 & -73 & -180 & -52 & 143 & 8.81 & 6.43 & 6.02 \\
\hline $\mathrm{V}^{*} \mathrm{TW}$ Her & -0.69 & 7.94 & 0.50 & -26 & 4 & -11 & -1 & 225 & 8.39 & 7.95 & 0.55 \\
\hline $\mathrm{V}^{*} \mathrm{WY}$ Pav & -0.98 & 7.03 & -0.46 & 168 & -281 & 138 & 173 & -45 & 10.53 & 1.22 & 5.92 \\
\hline $\mathrm{V}^{*} \mathrm{MR}$ Ara & -1.00 & 4.15 & -0.85 & 193 & -436 & -116 & 243 & -158 & 12.17 & 1.90 & 2.94 \\
\hline
\end{tabular}


TABLE 3. (continued)

\begin{tabular}{|c|c|c|c|c|c|c|c|c|c|c|c|}
\hline $\begin{array}{l}\text { Star } \\
(1)\end{array}$ & $\begin{array}{c}{[\mathrm{Fe} / \mathrm{H}]} \\
(\mathrm{dex}) \\
(2)\end{array}$ & $\begin{array}{c}R \\
(\mathrm{kpc}) \\
(3)\end{array}$ & $\begin{array}{c}Z \\
(\mathrm{kpc}) \\
(4)\end{array}$ & $\begin{array}{c}U \\
(\mathrm{~km} / \mathrm{s}) \\
(5)\end{array}$ & $\begin{array}{c}V \\
(\mathrm{~km} / \mathrm{s}) \\
(6)\end{array}$ & $\begin{array}{c}W \\
(\mathrm{~km} / \mathrm{s}) \\
(7)\end{array}$ & $\begin{array}{c}V_{R} \\
(\mathrm{~km} / \mathrm{s}) \\
(8)\end{array}$ & $\begin{array}{c}V_{\phi} \\
(\mathrm{km} / \mathrm{s}) \\
(9)\end{array}$ & $\begin{array}{c}R_{a p} \\
(\mathrm{kpc}) \\
(10)\end{array}$ & $\begin{array}{c}R_{p r} \\
(\mathrm{kpc}) \\
(11)\end{array}$ & $\begin{array}{c}Z_{\max } \\
(\mathrm{kpc}) \\
(12)\end{array}$ \\
\hline$V^{*}$ V690 Sco & -1.16 & 7.60 & -0.13 & 49 & 30 & 36 & 44 & 251 & 10.61 & 7.22 & 0.57 \\
\hline $\mathrm{V}^{*} \mathrm{~S}$ Ara & -0.71 & 7.70 & -0.18 & -174 & -127 & 43 & -176 & 87 & 11.09 & 1.83 & 0.77 \\
\hline $\mathrm{V}^{*} \mathrm{KQ} \mathrm{CrA}$ & -0.70 & 1.39 & -1.27 & 102 & -751 & -147 & 428 & 331 & 89.71 & 0.87 & 29.80 \\
\hline $\mathrm{V}^{*} \mathrm{MS}$ Ara & -1.48 & 7.00 & -0.42 & 16 & -271 & -124 & 20 & -50 & 7.07 & 1.45 & 3.31 \\
\hline HD 165195 & -2.14 & 8.02 & 0.12 & -137 & -188 & -27 & -136 & 37 & 9.73 & 0.80 & 0.40 \\
\hline $\mathrm{V}^{*}$ IN Ara & -1.75 & 6.94 & -0.48 & -115 & -127 & 110 & -123 & 83 & 8.78 & 1.98 & 2.78 \\
\hline HD 166161 & -1.22 & 8.38 & 0.01 & -119 & -88 & 10 & -118 & 132 & 10.22 & 3.30 & 0.13 \\
\hline$V^{*}$ V675 Sgr & -2.28 & 7.72 & -0.11 & 114 & -10 & -111 & 113 & 211 & 12.36 & 5.80 & 3.32 \\
\hline V* BC Dra & -2.00 & 9.00 & 0.73 & 158 & -237 & 54 & 154 & -39 & 11.78 & 0.95 & 1.67 \\
\hline $\mathrm{V}^{*} \mathrm{~V} 455 \mathrm{Oph}$ & -1.07 & 7.27 & 0.41 & -161 & -267 & 135 & -166 & -22 & 10.41 & 0.62 & 5.89 \\
\hline HD 166913 & -1.52 & 8.45 & -0.02 & 47 & -31 & 79 & 46 & 189 & 9.23 & 6.51 & 1.72 \\
\hline $\mathrm{V}^{*} \mathrm{BD}$ Dra & -1.74 & 9.42 & 1.16 & -114 & -355 & 183 & -141 & -107 & 14.70 & 6.06 & 12.15 \\
\hline $\mathrm{V}^{*} \mathrm{~V} 1290 \mathrm{Sgr}$ & -1.20 & 1.10 & -1.44 & 57 & -170 & -341 & -53 & -54 & 10.60 & 1.15 & 11.11 \\
\hline $\mathrm{V}^{*} \mathrm{IO} \mathrm{Lyr}$ & -1.14 & 7.88 & 0.54 & 255 & -111 & 108 & 269 & 66 & 18.91 & 1.31 & 5.16 \\
\hline $\mathrm{V}^{*}$ AE Dra & -1.54 & 8.57 & 1.06 & -30 & -249 & -16 & -37 & -20 & 8.76 & 0.51 & 1.12 \\
\hline HD 170737 & -0.91 & 8.43 & 0.03 & 53 & -85 & -87 & 55 & 134 & 8.91 & 4.18 & 2.16 \\
\hline $\mathrm{V}^{*} \mathrm{AC}$ Her & -1.20 & 7.97 & 0.22 & -9 & 7 & 22 & 11 & 227 & 8.59 & 7.84 & 0.37 \\
\hline $\mathrm{V}^{*}$ CC Lyr & -2.20 & 8.62 & 2.70 & -319 & -130 & -178 & -76 & 323 & 36.94 & 8.30 & 17.08 \\
\hline HD 171496 & -1.12 & 8.11 & -0.05 & 30 & -2 & 34 & 32 & 218 & 8.92 & 7.32 & 0.46 \\
\hline $\mathrm{V}^{*} \mathrm{Y}$ Lyr & -1.03 & 8.12 & 1.09 & -83 & -68 & -34 & -27 & 171 & 8.40 & 5.50 & 1.32 \\
\hline $\mathrm{V}^{*} \mathrm{LX}$ Lyr & -1.42 & 7.99 & 0.97 & -314 & -228 & 60 & -300 & 94 & 22.49 & 1.86 & 6.45 \\
\hline $\mathrm{V}^{*}$ CN Lyr & -0.58 & 8.02 & 0.26 & -52 & 20 & -1 & -27 & 245 & 10.12 & 7.80 & 0.32 \\
\hline$V^{*}$ V1640 Sgr & -0.54 & 6.59 & -0.46 & -50 & 9 & 4 & -50 & 229 & 8.33 & 5.97 & 0.56 \\
\hline $\mathrm{V}^{*} \mathrm{RZ}$ Lyr & -2.13 & 7.95 & 0.39 & 229 & -112 & -69 & 243 & 72 & 15.47 & 1.47 & 3.14 \\
\hline HD 175305 & -1.45 & 8.54 & 0.07 & 62 & -68 & -285 & 64 & 151 & 20.56 & 8.16 & 17.67 \\
\hline $\mathrm{V}^{*} \mathrm{EZ} \mathrm{Lyr}$ & -1.22 & 8.04 & 0.39 & 94 & -32 & 26 & 121 & 172 & 10.69 & 4.41 & 0.63 \\
\hline $\mathrm{V}^{*} \mathrm{~V} 413 \mathrm{CrA}$ & -1.26 & 7.67 & -0.23 & 60 & -129 & 107 & 60 & 91 & 8.07 & 2.63 & 2.86 \\
\hline $\mathrm{V}^{*}$ AP Her & -0.80 & 7.19 & 0.28 & -58 & -63 & -28 & -22 & 166 & 7.29 & 4.55 & 0.44 \\
\hline $\mathrm{V}^{*}$ CX Lyr & -1.79 & 7.66 & 0.45 & -24 & -226 & -46 & -24 & 0 & 7.73 & 0.01 & 0.91 \\
\hline HD 174578 & -1.69 & 7.44 & -0.22 & -2 & 118 & 87 & 6 & 338 & 21.96 & 7.44 & 3.38 \\
\hline HD 175606 & -2.45 & 8.37 & -0.05 & -100 & -117 & -88 & -101 & 102 & 9.67 & 2.83 & 2.40 \\
\hline HD 176203 & -1.61 & 8.33 & -0.04 & -84 & -68 & -61 & -83 & 153 & 9.49 & 4.37 & 1.20 \\
\hline $\mathrm{V}^{*} \mathrm{MT} \mathrm{Tel}$ & -1.85 & 8.07 & -0.17 & -79 & -338 & 184 & -78 & -118 & 10.07 & 5.31 & 7.30 \\
\hline $\mathrm{V}^{*} \mathrm{NR}$ Lyr & -2.42 & 8.03 & 0.49 & 240 & -17 & 117 & 281 & 140 & 24.23 & 2.81 & 6.86 \\
\hline $\mathrm{V}^{*} \mathrm{XZ}$ Dra & -0.79 & 8.59 & 0.28 & 16 & -6 & -34 & 32 & 212 & 9.25 & 7.53 & 0.64 \\
\hline HD 178443 & -2.07 & 8.14 & -0.14 & -322 & -112 & -129 & -322 & 107 & 29.82 & 2.16 & 11.82 \\
\hline$V^{*}$ BK Dra & -1.95 & 8.74 & 0.49 & 114 & -131 & 113 & 125 & 72 & 11.01 & 2.14 & 4.50 \\
\hline BPS CS 22947-0187 & -2.49 & 6.16 & -1.06 & 287 & -196 & 58 & 285 & 43 & 15.30 & 0.71 & 4.55 \\
\hline HD 181007 & -1.64 & 8.14 & -0.10 & -90 & -302 & -47 & -91 & -81 & 8.98 & 1.97 & 0.83 \\
\hline$V^{*}$ V1130 Sgr & -1.70 & 6.49 & -0.59 & 45 & -71 & -68 & 60 & 144 & 7.07 & 3.55 & 1.24 \\
\hline
\end{tabular}


TABle 3. (continued)

\begin{tabular}{|c|c|c|c|c|c|c|c|c|c|c|c|}
\hline $\begin{array}{l}\text { Star } \\
(1)\end{array}$ & $\begin{array}{c}{[\mathrm{Fe} / \mathrm{H}]} \\
(\mathrm{dex}) \\
(2)\end{array}$ & $\begin{array}{c}R \\
(\mathrm{kpc}) \\
(3)\end{array}$ & $\begin{array}{c}Z \\
(\mathrm{kpc}) \\
(4)\end{array}$ & $\begin{array}{c}U \\
(\mathrm{~km} / \mathrm{s}) \\
(5)\end{array}$ & $\begin{array}{c}V \\
(\mathrm{~km} / \mathrm{s}) \\
(6)\end{array}$ & $\begin{array}{c}W \\
(\mathrm{~km} / \mathrm{s}) \\
(7)\end{array}$ & $\begin{array}{c}V_{R} \\
(\mathrm{~km} / \mathrm{s}) \\
(8)\end{array}$ & $\begin{array}{c}V_{\phi} \\
(\mathrm{km} / \mathrm{s}) \\
(9)\end{array}$ & $\begin{array}{c}R_{a p} \\
(\mathrm{kpc}) \\
(10)\end{array}$ & $\begin{array}{c}R_{p r} \\
(\mathrm{kpc}) \\
(11)\end{array}$ & $\begin{array}{c}Z_{\max } \\
(\mathrm{kpc}) \\
(12)\end{array}$ \\
\hline HD 181743 & -1.79 & 8.42 & -0.04 & 36 & -336 & -61 & 36 & -116 & 8.58 & 3.29 & 1.14 \\
\hline $\mathrm{V}^{*} \mathrm{RR}$ Lyr & -1.39 & 8.44 & 0.05 & -253 & -121 & 9 & -250 & 106 & 17.21 & 2.12 & 0.25 \\
\hline $\mathrm{V}^{*} \mathrm{BN}$ Vul & -1.61 & 8.14 & 0.04 & -17 & -311 & 68 & -24 & -89 & 8.20 & 2.38 & 1.32 \\
\hline V* V440 Sgr & -1.40 & 7.79 & -0.26 & 16 & -196 & -32 & 17 & 24 & 7.82 & 0.55 & 0.50 \\
\hline $\mathrm{V}^{*} \mathrm{XZ}$ Cyg & -1.44 & 8.50 & 0.17 & 23 & -73 & -265 & 33 & 145 & 16.13 & 8.39 & 13.79 \\
\hline $\mathrm{V}^{*} \mathrm{HH}$ Tel & -1.02 & 6.82 & -0.83 & -114 & -169 & 81 & -115 & 47 & 8.22 & 1.05 & 1.96 \\
\hline $\mathrm{V}^{*} \mathrm{WY}$ Dra & -1.66 & 9.57 & 1.02 & 24 & -76 & 139 & 53 & 135 & 10.84 & 6.00 & 6.04 \\
\hline HD 184266 & -1.63 & 8.31 & -0.06 & 302 & -258 & -87 & 302 & -41 & 22.42 & 0.79 & 4.75 \\
\hline HD 184711 & -2.51 & 7.71 & -0.37 & -70 & -189 & -98 & -70 & 31 & 8.15 & 0.82 & 2.83 \\
\hline $\mathrm{V}^{*} \mathrm{BN} \mathrm{Pav}$ & -1.32 & 6.78 & -1.07 & -76 & -68 & -59 & -93 & 142 & 7.95 & 3.65 & 2.03 \\
\hline HD 232078 & -1.61 & 8.03 & -0.03 & 158 & -356 & -82 & 146 & -149 & 11.51 & 3.77 & 2.15 \\
\hline HD 186478 & -2.58 & 7.62 & -0.34 & -175 & -367 & -68 & -182 & -138 & 12.28 & 3.11 & 2.14 \\
\hline V* V796 Sgr & -1.87 & 6.81 & -0.87 & 232 & -275 & -108 & 232 & -54 & 13.63 & 1.01 & 3.62 \\
\hline BPS CS 22964-0024 & -1.76 & 7.73 & -0.39 & 129 & -100 & -1 & 130 & 120 & 9.69 & 2.75 & 0.48 \\
\hline BPS CS 22964-0019 & -2.44 & 3.14 & -2.76 & 208 & -90 & 279 & 208 & 130 & 20.93 & 3.40 & 19.95 \\
\hline V* V2194 Sgr & -1.62 & 7.04 & -0.75 & -35 & -147 & 85 & -34 & 74 & 7.24 & 1.88 & 2.03 \\
\hline HD 187111 & -1.78 & 8.02 & -0.18 & 137 & -184 & -90 & 139 & 31 & 10.05 & 0.75 & 2.49 \\
\hline BPS CS 22964-0074 & -2.45 & 7.40 & -0.57 & 418 & -327 & -65 & 418 & -107 & 51.33 & 1.50 & 5.13 \\
\hline BPS CS 22964-0092 & -0.70 & 7.18 & -0.71 & 44 & -6 & -68 & 42 & 214 & 8.69 & 6.39 & 1.58 \\
\hline BPS CS 22964-0052 & -1.15 & 7.48 & -0.54 & 120 & 2 & 34 & 121 & 221 & 11.81 & 5.52 & 1.22 \\
\hline BPS CS 22964-0064 & -0.70 & 7.89 & -0.32 & -68 & 0 & -16 & -68 & 220 & 9.91 & 6.49 & 0.48 \\
\hline $\mathrm{V}^{*} \mathrm{~V} 2202 \mathrm{Sgr}$ & -2.25 & 2.70 & -3.09 & 132 & -73 & -27 & 138 & 141 & 6.46 & 2.10 & 4.58 \\
\hline BPS CS 22964-0138 & -1.68 & 7.66 & -0.45 & 47 & -88 & -29 & 47 & 131 & 7.97 & 3.44 & 0.63 \\
\hline HD 188031 & -2.04 & 8.36 & -0.08 & 162 & -310 & 15 & 162 & -89 & 11.29 & 2.00 & 0.27 \\
\hline BPS CS 22964-0111 & -1.62 & 7.44 & -0.58 & -45 & -21 & -42 & -45 & 199 & 8.17 & 6.08 & 1.00 \\
\hline BPS CS 22964-0149 & -0.88 & 7.30 & -0.64 & -26 & -43 & 28 & -25 & 177 & 7.49 & 5.18 & 0.78 \\
\hline BPS CS 22964-0115 & -2.32 & 7.30 & -0.67 & 116 & -382 & 224 & 116 & -162 & 14.66 & 6.22 & 11.05 \\
\hline $\mathrm{V}^{*} \mathrm{BP}$ Pav & -1.48 & 6.90 & -1.17 & -243 & -480 & 72 & -205 & -291 & 25.95 & 5.17 & 4.53 \\
\hline BD-18 5550 & -2.89 & 8.13 & -0.17 & 65 & -203 & -32 & 65 & 16 & 8.50 & 0.37 & 0.47 \\
\hline BPS CS 22964-0172 & -0.13 & 7.55 & -0.55 & -6 & -48 & -2 & -6 & 172 & 7.57 & 5.04 & 0.55 \\
\hline$V^{*}$ V1711 Sgr & -1.10 & 4.06 & -2.40 & -26 & -93 & 167 & 1 & 130 & 5.81 & 3.80 & 4.83 \\
\hline BPS CS 22964-0183 & -2.60 & 7.51 & -0.57 & -69 & -232 & -79 & -69 & -12 & 7.91 & 0.29 & 2.14 \\
\hline BPS CS 22964-0157 & -1.66 & 7.28 & -0.69 & -82 & -450 & 29 & -83 & -229 & 10.47 & 6.02 & 1.04 \\
\hline BPS CS 22964-0181 & -0.82 & 5.74 & -1.60 & -193 & -102 & 88 & -193 & 117 & 10.93 & 2.09 & 3.08 \\
\hline BPS CS 22964-0176 & -2.61 & 7.06 & -0.83 & -3 & -226 & 104 & -3 & -6 & 7.11 & 0.17 & 3.02 \\
\hline BPS CS 22964-0158 & -1.90 & 7.56 & -0.53 & 193 & -65 & 85 & 194 & 154 & 13.71 & 3.52 & 3.57 \\
\hline BPS CS 22964-0161 & -2.30 & 7.67 & -0.48 & -86 & -208 & 47 & -86 & 12 & 8.34 & 0.27 & 0.95 \\
\hline HD 190287 & -1.59 & 8.35 & -0.09 & -139 & -118 & -64 & -139 & 102 & 10.69 & 2.51 & 1.55 \\
\hline BPS CS 22964-0214 & -2.47 & 7.80 & -0.42 & 8 & -344 & -118 & 8 & -124 & 7.83 & 4.22 & 3.24 \\
\hline BPS CS 22964-0232 & -0.44 & 7.93 & -0.34 & 41 & 9 & 1 & 42 & 229 & 9.50 & 7.24 & 0.39 \\
\hline
\end{tabular}


TABle 3. (continued)

\begin{tabular}{|c|c|c|c|c|c|c|c|c|c|c|c|}
\hline $\begin{array}{l}\text { Star } \\
(1)\end{array}$ & $\begin{array}{c}{[\mathrm{Fe} / \mathrm{H}]} \\
(\mathrm{dex}) \\
(2)\end{array}$ & $\begin{array}{c}R \\
(\mathrm{kpc}) \\
(3)\end{array}$ & $\begin{array}{c}Z \\
(\mathrm{kpc}) \\
(4)\end{array}$ & $\begin{array}{c}U \\
(\mathrm{~km} / \mathrm{s}) \\
(5)\end{array}$ & $\begin{array}{c}V \\
(\mathrm{~km} / \mathrm{s}) \\
(6)\end{array}$ & $\begin{array}{c}W \\
(\mathrm{~km} / \mathrm{s}) \\
(7)\end{array}$ & $\begin{array}{c}V_{R} \\
(\mathrm{~km} / \mathrm{s}) \\
(8)\end{array}$ & $\begin{array}{c}V_{\phi} \\
(\mathrm{km} / \mathrm{s}) \\
(9)\end{array}$ & $\begin{array}{c}R_{a p} \\
(\mathrm{kpc}) \\
(10)\end{array}$ & $\begin{array}{c}R_{p r} \\
(\mathrm{kpc}) \\
(11)\end{array}$ & $\begin{array}{c}Z_{\max } \\
(\mathrm{kpc}) \\
(12)\end{array}$ \\
\hline V* HY Tel & -0.17 & 6.87 & -1.11 & -6 & -78 & -44 & -17 & 141 & 6.95 & 3.87 & 1.46 \\
\hline$V^{*}$ V1644 Sgr & -2.51 & 6.84 & -0.94 & -33 & -305 & 2 & -38 & -83 & 7.02 & 1.93 & 0.95 \\
\hline $\mathrm{V}^{*} \mathrm{TW}$ Cap & -1.20 & 5.16 & -1.99 & -108 & -259 & -19 & -115 & 9 & 6.33 & 0.18 & 2.51 \\
\hline HD 192718 & -0.87 & 8.46 & -0.02 & 106 & -64 & -36 & 107 & 156 & 10.30 & 4.20 & 0.59 \\
\hline BPS CS 22885-0040 & -3.03 & 7.07 & -0.92 & -166 & -114 & 132 & -165 & 108 & 11.55 & 2.57 & 4.61 \\
\hline $\mathrm{V}^{*} \mathrm{HV} \mathrm{Pav}$ & -1.64 & 6.23 & -1.68 & 65 & -347 & 102 & 81 & -118 & 7.00 & 3.53 & 3.72 \\
\hline BPS CS 22885-0054 & -1.97 & 7.46 & -0.68 & -137 & -144 & 28 & -137 & 77 & 9.36 & 1.67 & 0.90 \\
\hline BPS CS 22885-0069 & -2.24 & 5.35 & -2.08 & -25 & -163 & 21 & -25 & 57 & 5.69 & 1.28 & 2.12 \\
\hline HD 193242 & -1.76 & 8.25 & -0.15 & 45 & -172 & 47 & 46 & 47 & 8.44 & 1.15 & 0.82 \\
\hline BPS CS 22885-0105 & -2.19 & 4.72 & -2.48 & 202 & -241 & 73 & 201 & -28 & 8.56 & 0.65 & 6.20 \\
\hline $\mathrm{V}^{*} \mathrm{~V} 1645 \mathrm{Sgr}$ & -1.74 & 7.03 & -0.98 & 431 & -302 & -56 & 431 & -81 & 52.73 & 1.07 & 6.49 \\
\hline BPS CS 22885-0096 & -3.60 & 5.19 & -2.19 & 202 & -164 & 132 & 203 & 53 & 9.90 & 1.75 & 8.65 \\
\hline BPS CS 22885-0094 & -1.19 & 8.04 & -0.31 & -56 & -67 & 16 & -56 & 153 & 8.54 & 4.22 & 0.38 \\
\hline BPS CS 22885-0106 & -2.12 & 7.35 & -0.78 & 105 & -245 & 60 & 105 & -26 & 8.29 & 0.60 & 1.90 \\
\hline BPS CS 22943-0097 & -2.20 & 7.87 & -0.45 & -38 & -56 & -62 & -40 & 163 & 8.16 & 5.00 & 1.33 \\
\hline$V^{*}$ V2279 Sgr & -1.16 & 6.59 & -1.33 & -70 & -36 & -2 & -69 & 185 & 7.83 & 4.86 & 1.52 \\
\hline BPS CS 22943-0095 & -2.17 & 8.22 & -0.20 & 181 & -65 & 42 & 180 & 156 & 13.38 & 3.62 & 1.11 \\
\hline BPS CS 22885-0143 & -2.51 & 2.72 & -4.02 & -2 & -229 & 105 & -3 & -9 & 4.42 & 0.26 & 5.03 \\
\hline BPS CS 22950-0096 & -1.62 & 7.93 & -0.35 & 14 & -97 & 23 & 19 & 123 & 7.98 & 3.25 & 0.50 \\
\hline BPS CS 22885-0203 & -2.17 & 7.64 & -0.61 & 75 & -254 & -85 & 74 & -35 & 8.26 & 0.86 & 2.16 \\
\hline BPS CS 22955-0117 & -2.02 & 7.67 & -0.55 & -255 & -163 & -66 & -253 & 67 & 15.71 & 1.34 & 3.49 \\
\hline BPS CS 22955-0101 & -0.94 & 7.97 & -0.36 & 79 & -193 & 93 & 80 & 25 & 8.56 & 0.67 & 2.79 \\
\hline CD-23 16310 & -2.44 & 7.35 & -0.77 & 92 & -134 & -69 & 97 & 80 & 8.46 & 1.91 & 1.69 \\
\hline BPS CS 22885-0175 & -0.79 & 7.11 & -1.00 & 38 & -14 & -18 & 39 & 206 & 8.00 & 6.13 & 1.12 \\
\hline $\mathrm{V}^{*} \mathrm{~V} 341 \mathrm{Aql}$ & -1.22 & 7.90 & -0.36 & 45 & -122 & -112 & 53 & 94 & 8.33 & 2.86 & 3.23 \\
\hline BPS CS 22950-0153 & -1.84 & 7.60 & -0.59 & 168 & -147 & -78 & 173 & 62 & 10.99 & 1.34 & 2.08 \\
\hline HD 195636 & -2.80 & 8.23 & -0.17 & 50 & -273 & 153 & 49 & -54 & 8.56 & 2.37 & 6.43 \\
\hline BPS CS 22955-0153 & -1.54 & 7.64 & -0.60 & 15 & -228 & -51 & 15 & -9 & 7.69 & 0.21 & 1.17 \\
\hline BPS CS 22879-0012 & -2.70 & 7.29 & -0.91 & 125 & -86 & -106 & 125 & 133 & 10.09 & 3.39 & 3.14 \\
\hline BD-17 6036 & -2.70 & 7.27 & -0.86 & -259 & -290 & 90 & -264 & -45 & 16.50 & 0.82 & 3.34 \\
\hline BPS CS 22955-0134 & -1.71 & 7.93 & -0.40 & 73 & -55 & 32 & 78 & 163 & 8.98 & 4.52 & 0.75 \\
\hline $\mathrm{V}^{*} \mathrm{AA} \mathrm{Aql}$ & -0.58 & 7.64 & -0.58 & -104 & -98 & 70 & -89 & 133 & 8.87 & 3.49 & 1.61 \\
\hline BPS CS 22879-0029 & -2.16 & 7.61 & -0.67 & 193 & -219 & -12 & 193 & -1 & 11.23 & 0.02 & 0.92 \\
\hline BPS CS 22879-0034 & -1.10 & 7.12 & -1.03 & -19 & -34 & -64 & -17 & 186 & 7.22 & 6.10 & 1.70 \\
\hline $\mathrm{V}^{*} \mathrm{FH}$ Vul & -0.85 & 7.83 & -0.49 & -116 & 5 & -36 & -51 & 248 & 11.11 & 7.36 & 1.03 \\
\hline BPS CS 22955-0169 & -0.50 & 7.57 & -0.68 & -20 & 79 & 9 & -7 & 300 & 15.24 & 7.59 & 1.26 \\
\hline HD 196892 & -1.11 & 8.45 & -0.03 & -8 & -118 & -24 & -8 & 102 & 8.46 & 2.68 & 0.30 \\
\hline $\mathrm{V}^{*} \mathrm{VX}$ Ind & -0.91 & 6.82 & -1.33 & -187 & -33 & 41 & -193 & 182 & 13.67 & 3.61 & 2.40 \\
\hline BPS CS 22955-0174 & -2.22 & 4.41 & -3.25 & -106 & -191 & 17 & -86 & 69 & 5.84 & 1.55 & 3.60 \\
\hline BPS CS 22879-0051 & -2.50 & 7.71 & -0.61 & 29 & -304 & -20 & 28 & -84 & 7.82 & 2.07 & 0.69 \\
\hline
\end{tabular}


TABle 3. (continued)

\begin{tabular}{|c|c|c|c|c|c|c|c|c|c|c|c|}
\hline $\begin{array}{l}\text { Star } \\
(1)\end{array}$ & $\begin{array}{c}{[\mathrm{Fe} / \mathrm{H}]} \\
(\mathrm{dex}) \\
(2)\end{array}$ & $\begin{array}{c}R \\
(\mathrm{kpc}) \\
(3)\end{array}$ & $\begin{array}{c}Z \\
(\mathrm{kpc}) \\
(4)\end{array}$ & $\begin{array}{c}U \\
(\mathrm{~km} / \mathrm{s}) \\
(5)\end{array}$ & $\begin{array}{c}V \\
(\mathrm{~km} / \mathrm{s}) \\
(6)\end{array}$ & $\begin{array}{c}W \\
(\mathrm{~km} / \mathrm{s}) \\
(7)\end{array}$ & $\begin{array}{c}V_{R} \\
(\mathrm{~km} / \mathrm{s}) \\
(8)\end{array}$ & $\begin{array}{c}V_{\phi} \\
(\mathrm{km} / \mathrm{s}) \\
(9)\end{array}$ & $\begin{array}{c}R_{a p} \\
(\mathrm{kpc}) \\
(10)\end{array}$ & $\begin{array}{c}R_{p r} \\
(\mathrm{kpc}) \\
(11)\end{array}$ & $\begin{array}{c}Z_{\max } \\
(\mathrm{kpc}) \\
(12)\end{array}$ \\
\hline BD-20 6008 & -2.63 & 7.48 & -0.75 & -95 & -31 & 58 & -82 & 195 & 9.53 & 5.39 & 1.48 \\
\hline BPS CS 22879-0066 & -2.06 & 7.86 & -0.50 & -276 & -227 & -104 & -276 & -7 & 18.51 & 0.15 & 7.36 \\
\hline BPS CS 22880-0058 & -2.57 & 7.48 & -0.76 & -90 & -88 & 130 & -82 & 138 & 9.16 & 4.26 & 3.99 \\
\hline CD-30 18140 & -2.07 & 8.38 & -0.09 & -75 & -215 & -12 & -75 & 5 & 8.88 & 0.12 & 0.18 \\
\hline BD-15 5781 & -2.47 & 6.53 & -1.53 & 6 & -276 & -116 & -5 & -56 & 6.69 & 1.72 & 3.89 \\
\hline BPS CS 22879-0103 & -1.96 & 4.25 & -3.38 & -25 & -234 & 71 & -26 & -11 & 5.28 & 0.28 & 3.87 \\
\hline $\mathrm{V}^{*} \mathrm{DX}$ Del & -0.39 & 8.23 & -0.18 & 54 & -14 & 6 & 65 & 202 & 9.58 & 6.16 & 0.23 \\
\hline BPS CS 22879-0097 & -2.30 & 4.28 & -3.39 & -48 & -458 & -25 & -64 & -234 & 9.25 & 4.87 & 5.50 \\
\hline BPS CS 22879-0098 & -2.38 & 4.62 & -3.13 & -78 & -335 & 141 & -85 & -110 & 7.97 & 2.52 & 5.34 \\
\hline BPS CS 22879-0094 & -2.44 & 7.19 & -1.06 & -20 & -240 & -58 & -20 & -20 & 7.26 & 0.48 & 1.81 \\
\hline $\mathrm{V}^{*} \mathrm{FK} \mathrm{Vul}$ & -0.95 & 7.92 & -0.56 & -160 & -158 & -78 & -138 & 102 & 10.21 & 2.62 & 2.70 \\
\hline BPS CS 22880-0127 & -1.58 & 8.10 & -0.32 & 51 & -75 & 56 & 54 & 144 & 8.53 & 4.16 & 1.16 \\
\hline CD-48 13714 & -0.81 & 8.38 & -0.10 & 2 & -108 & -37 & 2 & 112 & 8.38 & 3.04 & 0.55 \\
\hline BD-14 5890 & -2.07 & 7.55 & -0.79 & -345 & -430 & -163 & -363 & -178 & 51.85 & 3.42 & 28.20 \\
\hline $\mathrm{V}^{*} \mathrm{UY}$ Cyg & -0.80 & 8.30 & -0.15 & -56 & -1 & -12 & -33 & 223 & 9.30 & 7.58 & 0.23 \\
\hline CD-37 14010 & -2.55 & 7.23 & -1.08 & 256 & -321 & -40 & 254 & -107 & 16.03 & 1.94 & 2.19 \\
\hline $\mathrm{V}^{*} \mathrm{BT}$ Aqr & -0.29 & 7.41 & -0.94 & 8 & -66 & -2 & 30 & 151 & 7.61 & 4.19 & 0.96 \\
\hline $\mathrm{V}^{*} \mathrm{XZ} \mathrm{Mic}$ & -1.22 & 6.37 & -1.84 & -74 & -246 & 93 & -74 & -24 & 7.34 & 0.59 & 3.07 \\
\hline BPS CS 22879-0141 & -1.36 & 7.68 & -0.72 & -61 & -177 & -38 & -61 & 43 & 8.00 & 1.03 & 1.15 \\
\hline BPS CS 22879-0144 & -2.01 & 7.28 & -1.06 & 204 & -348 & 38 & 203 & -130 & 12.79 & 2.76 & 2.70 \\
\hline HD 199854 & -1.94 & 8.18 & -0.27 & -32 & 18 & 17 & -26 & 239 & 9.94 & 7.91 & 0.40 \\
\hline$V^{*}$ RV Cap & -1.61 & 7.73 & -0.67 & -51 & -554 & -207 & -73 & -330 & 35.23 & 7.62 & 16.76 \\
\hline BPS CS 22898-0040 & -2.06 & 7.58 & -0.82 & -248 & -215 & -90 & -247 & 20 & 15.05 & 0.43 & 5.89 \\
\hline BPS CS 22937-0006 & -2.06 & 7.43 & -0.95 & 61 & -223 & 60 & 61 & -3 & 7.75 & 0.07 & 1.93 \\
\hline BPS CS 30492-0034 & -2.01 & 4.27 & -3.80 & -27 & -474 & -100 & -48 & -251 & 12.08 & 5.23 & 8.22 \\
\hline BPS CS 30492-0016 & -2.30 & 7.85 & -0.58 & 45 & -351 & -136 & 45 & -132 & 8.48 & 4.75 & 4.20 \\
\hline BPS CS 29501-0004 & -1.83 & 7.93 & -0.51 & 68 & -31 & 18 & 69 & 188 & 9.10 & 5.49 & 0.69 \\
\hline BPS CS 29501-0012 & -1.80 & 7.99 & -0.46 & 262 & -183 & 22 & 263 & 34 & 16.26 & 0.65 & 1.37 \\
\hline BPS CS 30492-0008 & -0.17 & 8.08 & -0.38 & 10 & -10 & 6 & 10 & 210 & 8.16 & 7.30 & 0.39 \\
\hline BPS CS 22898-0027 & -2.36 & 8.18 & -0.29 & -33 & -159 & 13 & -31 & 62 & 8.28 & 1.49 & 0.33 \\
\hline BPS CS 30492-0001 & -2.10 & 7.93 & -0.51 & 129 & -161 & 53 & 129 & 59 & 9.56 & 1.38 & 1.59 \\
\hline CD-62 1346 & -1.56 & 8.29 & -0.19 & -73 & -168 & -27 & -73 & 51 & 8.78 & 1.22 & 0.46 \\
\hline BPS CS 22937-0008 & -0.44 & 8.12 & -0.34 & -68 & 5 & -2 & -68 & 225 & 10.43 & 6.80 & 0.43 \\
\hline HD 200654 & -2.93 & 8.31 & -0.17 & 226 & -312 & -108 & 226 & -91 & 16.03 & 2.09 & 4.95 \\
\hline BPS CS 22937-0004 & -1.97 & 7.53 & -0.89 & 71 & -44 & -150 & 71 & 176 & 10.37 & 5.84 & 5.02 \\
\hline BPS CS 22937-0039 & -0.87 & 7.82 & -0.62 & 1 & -50 & -17 & 1 & 170 & 7.84 & 5.16 & 0.68 \\
\hline BPS CS 29501-0032 & -2.40 & 7.61 & -0.81 & 146 & -147 & 34 & 147 & 70 & 9.77 & 1.58 & 1.56 \\
\hline BPS CS 30492-0050 & -1.48 & 7.88 & -0.57 & -144 & -167 & 37 & -144 & 53 & 9.98 & 1.16 & 0.93 \\
\hline BPS CS 22937-0034 & -1.92 & 7.85 & -0.60 & -43 & -117 & -84 & -42 & 103 & 8.04 & 2.98 & 2.31 \\
\hline BPS CS 22937-0019 & -1.50 & 7.77 & -0.68 & 55 & -10 & 14 & 57 & 209 & 9.09 & 6.37 & 0.84 \\
\hline
\end{tabular}


TABLE 3. (continued)

\begin{tabular}{|c|c|c|c|c|c|c|c|c|c|c|c|}
\hline $\begin{array}{l}\text { Star } \\
\text { (1) }\end{array}$ & $\begin{array}{c}{[\mathrm{Fe} / \mathrm{H}]} \\
(\mathrm{dex}) \\
(2)\end{array}$ & $\begin{array}{c}R \\
(\mathrm{kpc}) \\
(3)\end{array}$ & $\begin{array}{c}Z \\
(\mathrm{kpc}) \\
(4)\end{array}$ & $\begin{array}{c}U \\
(\mathrm{~km} / \mathrm{s}) \\
(5)\end{array}$ & $\begin{array}{c}V \\
(\mathrm{~km} / \mathrm{s}) \\
(6)\end{array}$ & $\begin{array}{c}W \\
(\mathrm{~km} / \mathrm{s}) \\
(7)\end{array}$ & $\begin{array}{c}V_{R} \\
(\mathrm{~km} / \mathrm{s}) \\
(8)\end{array}$ & $\begin{array}{c}V_{\phi} \\
(\mathrm{km} / \mathrm{s}) \\
(9)\end{array}$ & $\begin{array}{c}R_{a p} \\
(\mathrm{kpc}) \\
(10)\end{array}$ & $\begin{array}{c}R_{p r} \\
(\mathrm{kpc}) \\
(11)\end{array}$ & $\begin{array}{c}Z_{\max } \\
(\mathrm{kpc}) \\
(12)\end{array}$ \\
\hline BPS CS 30492-0047 & -0.97 & 7.79 & -0.66 & 70 & -5 & 26 & 71 & 215 & 9.80 & 6.34 & 1.01 \\
\hline BPS CS 29501-0028 & -1.88 & 7.95 & -0.51 & -6 & -48 & -36 & -4 & 172 & 7.97 & 5.38 & 0.80 \\
\hline $\mathrm{V}^{*} \mathrm{CP}$ Aqr & -0.90 & 7.74 & -0.76 & -120 & -57 & -13 & -99 & 177 & 9.65 & 4.73 & 1.01 \\
\hline BPS CS 22898-0043 & -2.69 & 5.21 & -3.36 & -158 & -209 & -127 & -145 & 65 & 7.63 & 3.50 & 7.69 \\
\hline BPS CS 22937-0023 & -1.38 & 7.81 & -0.65 & 224 & -165 & 109 & 224 & 54 & 14.29 & 1.31 & 6.44 \\
\hline $\mathrm{V}^{*} \mathrm{~V}$ Ind & -1.50 & 7.99 & -0.48 & -307 & -278 & 54 & -307 & -59 & 22.03 & 1.04 & 2.09 \\
\hline BPS CS 22937-0042 & -2.11 & 7.11 & -1.31 & 131 & -102 & 38 & 131 & 118 & 9.14 & 2.84 & 2.24 \\
\hline BPS CS 29501-0043 & -1.36 & 7.88 & -0.59 & -35 & -88 & -7 & -34 & 133 & 8.04 & 3.59 & 0.62 \\
\hline BPS CS 30492-0095 & -1.02 & 8.04 & -0.44 & 64 & -113 & -15 & 65 & 107 & 8.52 & 2.69 & 0.50 \\
\hline BPS CS 30492-0080 & -1.20 & 7.70 & -0.76 & 102 & -58 & 57 & 102 & 162 & 9.47 & 4.44 & 1.84 \\
\hline BPS CS 22937-0072 & -2.19 & 7.41 & -1.05 & 91 & -11 & 120 & 94 & 207 & 11.32 & 6.36 & 4.65 \\
\hline $\mathrm{V}^{*} \mathrm{SW}$ Aqr & -1.63 & 7.91 & -0.62 & -310 & -271 & 50 & -313 & -19 & 22.13 & 0.33 & 2.08 \\
\hline BPS CS 22937-0073 & -2.04 & 7.78 & -0.70 & 99 & -143 & 119 & 100 & 76 & 8.92 & 2.42 & 4.66 \\
\hline $\mathrm{V}^{*} \mathrm{RR}$ Mic & -1.40 & 2.11 & -6.18 & -181 & -336 & 120 & -167 & -135 & 12.88 & 3.02 & 12.71 \\
\hline BPS CS 29501-0056 & -1.64 & 7.24 & -1.22 & 120 & -74 & -157 & 123 & 143 & 11.65 & 4.09 & 6.02 \\
\hline BPS CS 29501-0055 & -2.19 & 8.02 & -0.46 & -58 & -73 & 81 & -57 & 147 & 8.68 & 4.44 & 1.92 \\
\hline BPS CS 22937-0054 & -1.78 & 7.57 & -0.90 & 76 & -192 & -164 & 77 & 28 & 8.81 & 1.23 & 7.35 \\
\hline $\mathrm{V}^{*} \mathrm{Z} \mathrm{Mic}$ & -1.10 & 7.54 & -0.94 & 99 & -51 & -33 & 105 & 165 & 9.57 & 4.24 & 1.25 \\
\hline $\mathrm{BD}+424040$ & -0.99 & 8.49 & -0.01 & 85 & -247 & 7 & 85 & -27 & 9.14 & 0.63 & 0.08 \\
\hline BPS CS 22937-0060 & -0.87 & 7.21 & -1.26 & -55 & -68 & -24 & -53 & 153 & 7.71 & 4.26 & 1.49 \\
\hline BPS CS 22937-0057 & -1.38 & 7.86 & -0.63 & -106 & -119 & 69 & -106 & 102 & 9.30 & 2.55 & 1.71 \\
\hline BPS CS 29501-0082 & -1.84 & 7.76 & -0.72 & -237 & -250 & -110 & -237 & -26 & 15.00 & 0.63 & 7.28 \\
\hline BPS CS 30492-0114 & -1.78 & 8.05 & -0.44 & 49 & -94 & 90 & 50 & 126 & 8.38 & 3.86 & 2.40 \\
\hline [MFF90] 95942134 & -1.32 & 7.39 & -1.44 & -46 & -328 & -117 & -74 & -90 & 7.97 & 3.16 & 4.74 \\
\hline [MFF90] 9594299 & -0.65 & 7.84 & -0.75 & -113 & -47 & 26 & -88 & 187 & 9.72 & 5.21 & 0.99 \\
\hline BPS CS 29501-0095 & -1.94 & 7.23 & -1.26 & 162 & -395 & 83 & 158 & -178 & 12.20 & 4.53 & 4.30 \\
\hline $\mathrm{V}^{*} \mathrm{YZ} \mathrm{Cap}$ & -1.06 & 7.76 & -0.77 & -39 & -135 & 98 & -33 & 87 & 7.99 & 2.56 & 2.83 \\
\hline BPS CS 29501-0075 & -2.20 & 8.06 & -0.44 & -133 & -136 & -83 & -132 & 86 & 10.05 & 2.21 & 2.75 \\
\hline [MFF90] 9594249 & -0.79 & 8.16 & -0.37 & 87 & -61 & 76 & 97 & 153 & 9.76 & 4.43 & 2.08 \\
\hline [MFF90] 9594226 & -0.62 & 8.00 & -0.57 & -21 & -7 & 25 & 0 & 214 & 8.02 & 7.76 & 0.70 \\
\hline [M93] 27036 & -0.63 & 6.05 & -2.44 & 35 & -119 & 21 & 34 & 102 & 6.48 & 2.66 & 2.60 \\
\hline $\mathrm{V}^{*} \mathrm{DM}$ Cyg & -0.14 & 8.38 & -0.21 & 33 & 24 & -31 & 60 & 238 & 11.29 & 7.46 & 0.58 \\
\hline BPS CS 30492-0116 & -2.05 & 6.18 & -2.34 & 165 & -175 & -105 & 166 & 42 & 10.22 & 0.87 & 4.08 \\
\hline [M93] 17089 & -2.35 & 6.31 & -2.20 & 135 & -184 & 115 & 133 & 41 & 8.02 & 1.54 & 6.64 \\
\hline BPS CS 30492-0110 & -2.71 & 2.16 & -6.46 & 198 & -155 & -275 & 208 & 12 & 27.70 & 0.22 & 26.71 \\
\hline [M93] 26113 & -1.21 & 6.67 & -1.84 & 23 & -80 & 29 & 22 & 140 & 6.90 & 3.95 & 2.01 \\
\hline BPS CS 30492-0113 & -1.76 & 7.79 & -0.71 & -286 & -288 & -46 & -286 & -66 & 18.99 & 1.24 & 3.58 \\
\hline BPS CS 29501-0099 & -0.19 & 8.09 & -0.42 & -46 & 32 & 32 & -45 & 252 & 11.50 & 7.67 & 0.83 \\
\hline [M93] 16090 & -2.63 & 5.53 & -3.00 & -66 & -257 & 196 & -63 & -41 & 8.89 & 1.85 & 8.47 \\
\hline [M93] 25072 & -2.24 & 5.13 & -3.41 & 225 & -403 & -109 & 232 & -175 & 18.17 & 2.81 & 8.95 \\
\hline
\end{tabular}


TABle 3. (continued)

\begin{tabular}{|c|c|c|c|c|c|c|c|c|c|c|c|}
\hline $\begin{array}{l}\text { Star } \\
(1)\end{array}$ & $\begin{array}{c}{[\mathrm{Fe} / \mathrm{H}]} \\
(\mathrm{dex}) \\
(2)\end{array}$ & $\begin{array}{c}R \\
(\mathrm{kpc}) \\
(3)\end{array}$ & $\begin{array}{c}Z \\
(\mathrm{kpc}) \\
(4)\end{array}$ & $\begin{array}{c}U \\
(\mathrm{~km} / \mathrm{s}) \\
(5)\end{array}$ & $\begin{array}{c}V \\
(\mathrm{~km} / \mathrm{s}) \\
(6)\end{array}$ & $\begin{array}{c}W \\
(\mathrm{~km} / \mathrm{s}) \\
(7)\end{array}$ & $\begin{array}{c}V_{R} \\
(\mathrm{~km} / \mathrm{s}) \\
(8)\end{array}$ & $\begin{array}{c}V_{\phi} \\
(\mathrm{km} / \mathrm{s}) \\
(9)\end{array}$ & $\begin{array}{c}R_{a p} \\
(\mathrm{kpc}) \\
(10)\end{array}$ & $\begin{array}{c}R_{p r} \\
(\mathrm{kpc}) \\
(11)\end{array}$ & $\begin{array}{c}Z_{\max } \\
(\mathrm{kpc}) \\
(12)\end{array}$ \\
\hline [MFF90] 9591136 & -0.71 & 8.02 & -0.58 & -7 & -7 & -14 & 17 & 212 & 8.32 & 7.35 & 0.64 \\
\hline [MFF90] 9591194 & -0.80 & 8.30 & -0.23 & 28 & -47 & 19 & 35 & 172 & 8.55 & 5.28 & 0.36 \\
\hline [MFF90] 9591158 & -0.87 & 8.14 & -0.43 & -35 & -4 & -1 & -17 & 218 & 8.52 & 7.63 & 0.44 \\
\hline [M93] 14080 & -1.39 & 7.54 & -0.98 & 27 & -86 & 82 & 25 & 135 & 7.63 & 4.16 & 2.32 \\
\hline [MFF90] 95931167 & -1.76 & 7.59 & -1.20 & -10 & -156 & -173 & 4 & 65 & 7.80 & 3.69 & 7.01 \\
\hline [MFF90] 9591151 & -0.61 & 8.11 & -0.47 & -34 & -47 & -67 & -19 & 176 & 8.19 & 5.93 & 1.49 \\
\hline BPS CS 22937-0090 & -2.08 & 7.41 & -1.12 & -13 & 12 & -26 & -13 & 232 & 8.89 & 7.44 & 1.42 \\
\hline [M93] 23058 & -1.91 & 3.79 & -4.85 & -365 & -272 & 151 & -358 & -87 & 38.04 & 1.24 & 30.40 \\
\hline [M93] 13101 & -1.07 & 7.72 & -0.80 & 18 & 9 & 93 & 15 & 229 & 9.91 & 7.73 & 2.67 \\
\hline [MFF90] 95931190 & -0.69 & 7.53 & -1.32 & -49 & -117 & -129 & -23 & 112 & 7.63 & 4.26 & 4.48 \\
\hline [MFF90] 9591139 & -0.66 & 8.20 & -0.37 & -38 & -12 & 14 & -24 & 210 & 8.57 & 7.19 & 0.42 \\
\hline [MFF90] 959113 & -0.62 & 8.14 & -0.45 & -21 & -57 & 5 & -7 & 165 & 8.15 & 4.98 & 0.46 \\
\hline BPS CS 30492-0133 & -2.10 & 4.78 & -3.86 & 318 & -494 & -337 & 314 & -278 & 142.75 & 3.05 & 89.89 \\
\hline $\mathrm{V}^{*}$ AO Peg & -0.92 & 8.02 & -0.92 & -255 & -4 & -126 & -191 & 274 & 27.27 & 6.38 & 10.10 \\
\hline [M93] 12085 & -1.52 & 6.11 & -2.48 & -103 & -805 & 302 & -74 & -589 & $\ldots$ & $\cdots$ & $\cdots$ \\
\hline BD-03 5215 & -1.57 & 8.33 & -0.19 & 149 & -200 & 142 & 150 & 16 & 11.30 & 0.55 & 7.62 \\
\hline V* SS Gru & -2.35 & 6.62 & -1.99 & -110 & -215 & 166 & -110 & 8 & 9.51 & 0.27 & 7.09 \\
\hline [MFF90] 9593287 & -1.20 & 7.38 & -1.77 & 289 & -120 & 168 & 306 & 1 & 25.94 & 1.17 & 26.24 \\
\hline [M93] 12029 & -2.56 & 6.76 & -1.82 & -58 & -119 & 184 & -61 & 100 & 9.06 & 3.95 & 7.05 \\
\hline HD 235518 & -2.57 & 8.66 & 0.00 & -22 & -10 & -3 & 7 & 211 & 8.69 & 7.77 & 0.03 \\
\hline [MFF90] 9593298 & -1.26 & 7.96 & -0.69 & 52 & -48 & -59 & 71 & 165 & 9.09 & 4.81 & 1.46 \\
\hline $\mathrm{BD}+463330$ & -1.93 & 8.53 & -0.03 & -8 & 3 & 3 & 6 & 223 & 8.71 & 8.37 & 0.04 \\
\hline [M93] 22013 & -2.96 & 2.27 & -6.57 & 125 & -165 & 55 & 121 & 64 & 5.90 & 4.96 & 7.02 \\
\hline $\mathrm{BD}+473427$ & -0.64 & 8.51 & -0.01 & 0 & 17 & -11 & 7 & 237 & 9.69 & 8.48 & 0.14 \\
\hline HD 204543 & -1.76 & 8.27 & -0.27 & -11 & -130 & 26 & -8 & 90 & 8.28 & 2.30 & 0.47 \\
\hline [M93] 21083 & -0.91 & 5.89 & -2.75 & 15 & -266 & -79 & 16 & -45 & 6.49 & 1.21 & 3.63 \\
\hline [M93] 11059 & -1.83 & 7.93 & -0.60 & -18 & -62 & 68 & -19 & 158 & 8.05 & 4.96 & 1.56 \\
\hline BPS CS 29506-0090 & -3.00 & 7.78 & -0.80 & -95 & -201 & 8 & -93 & 24 & 8.59 & 0.54 & 0.86 \\
\hline [M93] 44088 & -2.34 & 4.55 & -4.22 & 288 & -304 & 351 & 290 & -76 & 64.61 & 5.63 & 64.11 \\
\hline [M93] 44052 & -3.44 & 5.06 & -14.51 & -101 & -864 & 149 & 146 & 635 & $\cdots$ & $\cdots$ & $\cdots$ \\
\hline [M93] 46143 & -1.26 & 4.52 & -4.27 & -40 & -370 & -6 & -37 & -151 & 5.99 & 4.57 & 4.46 \\
\hline [M93] 44005 & -1.57 & 5.69 & -3.01 & -69 & -260 & 29 & -68 & -42 & 6.74 & 0.99 & 3.19 \\
\hline BPS CS 22948-0012 & -0.69 & 8.09 & -0.45 & -33 & -59 & 25 & -32 & 162 & 8.30 & 4.78 & 0.59 \\
\hline BPS CS 29495-0005 & -2.20 & 7.78 & -0.81 & 131 & -144 & 29 & 134 & 71 & 9.56 & 1.63 & 1.39 \\
\hline BPS CS 22948-0006 & -2.09 & 3.07 & -5.92 & -39 & -320 & -80 & -47 & -97 & 6.04 & 3.79 & 6.55 \\
\hline [M93] 33015 & -1.62 & 5.24 & -3.53 & -296 & -288 & 227 & -292 & -83 & 31.61 & 1.20 & 17.74 \\
\hline [M93] 32141 & -1.72 & 5.46 & -3.31 & 49 & -49 & 54 & 40 & 174 & 6.39 & 5.71 & 3.81 \\
\hline BPS CS 29495-0002 & -2.19 & 8.09 & -0.47 & 218 & -171 & -32 & 219 & 45 & 13.47 & 0.90 & 0.91 \\
\hline $\mathrm{BD}+433979$ & -0.74 & 8.50 & -0.02 & 18 & -34 & 21 & 22 & 186 & 8.63 & 6.12 & 0.26 \\
\hline BPS CS 22948-0024 & -2.03 & 6.93 & -1.74 & 197 & -77 & -71 & 199 & 141 & 13.48 & 2.84 & 3.28 \\
\hline
\end{tabular}


TABle 3. (continued)

\begin{tabular}{|c|c|c|c|c|c|c|c|c|c|c|c|}
\hline $\begin{array}{l}\text { Star } \\
(1)\end{array}$ & $\begin{array}{c}{[\mathrm{Fe} / \mathrm{H}]} \\
(\mathrm{dex}) \\
(2)\end{array}$ & $\begin{array}{c}R \\
(\mathrm{kpc}) \\
(3)\end{array}$ & $\begin{array}{c}Z \\
(\mathrm{kpc}) \\
(4)\end{array}$ & $\begin{array}{c}U \\
(\mathrm{~km} / \mathrm{s}) \\
(5)\end{array}$ & $\begin{array}{c}V \\
(\mathrm{~km} / \mathrm{s}) \\
(6)\end{array}$ & $\begin{array}{c}W \\
(\mathrm{~km} / \mathrm{s}) \\
(7)\end{array}$ & $\begin{array}{c}V_{R} \\
(\mathrm{~km} / \mathrm{s}) \\
(8)\end{array}$ & $\begin{array}{c}V_{\phi} \\
(\mathrm{km} / \mathrm{s}) \\
(9)\end{array}$ & $\begin{array}{c}R_{a p} \\
(\mathrm{kpc}) \\
(10)\end{array}$ & $\begin{array}{c}R_{p r} \\
(\mathrm{kpc}) \\
(11)\end{array}$ & $\begin{array}{c}Z_{\max } \\
(\mathrm{kpc}) \\
(12)\end{array}$ \\
\hline [M93] 46112 & -1.19 & 6.57 & -2.11 & 39 & -188 & -40 & 38 & 32 & 7.01 & 0.77 & 2.33 \\
\hline V* SX Aqr & -1.87 & 7.85 & -0.94 & -369 & -345 & 138 & -383 & -68 & 45.01 & 1.12 & 13.84 \\
\hline $\mathrm{V}^{*} \mathrm{RY}$ Oct & -1.83 & 7.57 & -1.00 & 12 & -177 & 114 & 6 & 44 & 7.62 & 1.40 & 4.01 \\
\hline BPS CS 29495-0041 & -2.93 & 3.85 & -5.85 & -169 & -428 & -95 & -247 & -104 & 15.97 & 5.56 & 15.92 \\
\hline HD 205547 & -1.85 & 7.76 & -0.82 & -182 & -186 & 96 & -182 & 34 & 11.79 & 0.76 & 3.18 \\
\hline BPS CS 22948-0019 & -1.96 & 7.49 & -1.13 & -43 & -152 & 18 & -42 & 68 & 7.74 & 1.66 & 1.19 \\
\hline [M93] 41114 & -2.07 & 5.05 & -3.83 & -193 & -317 & 128 & -189 & -105 & 13.10 & 1.91 & 7.41 \\
\hline [M93] 45105 & -2.02 & 5.21 & -3.64 & -177 & -301 & 131 & -174 & -87 & 11.89 & 1.68 & 6.64 \\
\hline V* RR Gru & -0.45 & 7.07 & -1.58 & -131 & -51 & 72 & -134 & 167 & 10.92 & 3.97 & 2.73 \\
\hline $\mathrm{BD}+404598$ & -0.72 & 8.50 & -0.01 & 0 & -150 & 48 & 1 & 70 & 8.50 & 1.82 & 0.79 \\
\hline BPS CS 29495-0031 & -2.12 & 7.83 & -0.80 & 130 & -313 & 68 & 126 & -98 & 9.63 & 2.53 & 2.52 \\
\hline BPS CS 22948-0057 & -2.07 & 7.28 & -1.39 & -58 & 6 & 73 & -56 & 227 & 10.46 & 6.54 & 2.60 \\
\hline $\mathrm{V}^{*}$ CG Peg & -0.50 & 8.34 & -0.38 & -24 & 0 & 1 & 2 & 221 & 8.39 & 8.31 & 0.38 \\
\hline BPS CS 22944-0011 & -2.06 & 8.28 & -0.28 & -24 & -201 & 23 & -23 & 19 & 8.33 & 0.46 & 0.43 \\
\hline [M93] 34118 & -1.92 & 6.38 & -2.41 & 315 & -456 & 10 & 322 & -226 & 35.20 & 3.62 & 14.14 \\
\hline HD 235596 & -0.72 & 8.55 & -0.01 & -2 & -11 & -2 & 7 & 209 & 8.59 & 7.56 & 0.02 \\
\hline HD 206739 & -1.56 & 8.17 & -0.43 & 82 & -107 & -64 & 86 & 110 & 9.15 & 2.91 & 1.43 \\
\hline HD 206642 & -0.75 & 8.36 & -0.17 & 75 & -168 & 0 & 75 & 52 & 8.87 & 1.23 & 0.18 \\
\hline BPS CS 22948-0066 & -3.10 & 6.48 & -2.39 & 85 & -68 & 142 & 90 & 149 & 8.41 & 5.89 & 6.05 \\
\hline BD-09 5831 & -1.83 & 7.71 & -1.13 & -85 & -90 & -40 & -69 & 139 & 8.39 & 3.90 & 1.69 \\
\hline BPS CS 22948-0045 & -0.69 & 7.53 & -1.14 & 44 & -48 & 67 & 43 & 172 & 7.94 & 5.55 & 2.11 \\
\hline BPS CS 29493-0037 & -2.13 & 7.81 & -0.85 & 87 & -80 & 59 & 90 & 138 & 8.94 & 3.85 & 1.96 \\
\hline BPS CS 22948-0075 & -2.06 & 7.41 & -1.29 & 64 & -128 & -4 & 65 & 91 & 7.94 & 2.26 & 1.36 \\
\hline BPS CS 22951-0042 & -1.57 & 8.05 & -0.53 & -148 & -34 & -197 & -149 & 186 & 17.88 & 6.22 & 11.46 \\
\hline BPS CS 22948-0070 & -0.85 & 8.05 & -0.55 & -25 & -64 & 11 & -24 & 156 & 8.16 & 4.57 & 0.57 \\
\hline BPS CS 22948-0067 & -1.45 & 7.80 & -0.84 & 47 & -40 & 3 & 49 & 180 & 8.41 & 5.40 & 0.90 \\
\hline $\mathrm{V}^{*} \mathrm{RS}$ Oct & -1.60 & 7.53 & -1.11 & -55 & -324 & 111 & -30 & -114 & 7.88 & 3.66 & 3.40 \\
\hline BPS CS 22948-0079 & -1.69 & 7.87 & -0.76 & 72 & -125 & 5 & 73 & 95 & 8.44 & 2.33 & 0.83 \\
\hline BPS CS 29493-0023 & -2.50 & 7.65 & -1.07 & 101 & -146 & -178 & 104 & 70 & 10.39 & 2.84 & 8.07 \\
\hline BPS CS 29495-0074 & -0.20 & 8.14 & -0.46 & -35 & -44 & -55 & -32 & 177 & 8.38 & 5.74 & 1.21 \\
\hline BPS CS 29495-0071 & -1.86 & 8.20 & -0.38 & 12 & -38 & 30 & 15 & 182 & 8.26 & 5.89 & 0.61 \\
\hline HD 206998 & -1.12 & 8.44 & -0.07 & 66 & -45 & 84 & 66 & 175 & 9.56 & 5.72 & 2.01 \\
\hline BPS CS 29493-0044 & -2.02 & 7.68 & -1.02 & -185 & -150 & 153 & -183 & 75 & 13.62 & 1.99 & 7.23 \\
\hline BPS CS 29493-0043 & -0.95 & 7.94 & -0.70 & 132 & 0 & -10 & 136 & 218 & 13.10 & 5.48 & 1.07 \\
\hline BPS CS 29493-0050 & -2.79 & 7.74 & -0.96 & -163 & -397 & 147 & -169 & -172 & 15.68 & 4.42 & 6.54 \\
\hline BPS CS 22948-0077 & -1.45 & 7.93 & -0.70 & -180 & -127 & -30 & -180 & 94 & 11.66 & 2.08 & 1.55 \\
\hline BPS CS 29493-0049 & -1.28 & 8.00 & -0.64 & -48 & -199 & 63 & -47 & 22 & 8.26 & 0.55 & 1.58 \\
\hline BPS CS 29495-0080 & -1.75 & 8.04 & -0.59 & 5 & -504 & -107 & -1 & -284 & 16.35 & 8.06 & 4.21 \\
\hline BPS CS 22948-0093 & -3.72 & 7.39 & -1.35 & -400 & -114 & -183 & -400 & 106 & 61.55 & 2.56 & 47.38 \\
\hline $\mathrm{BD}+434063$ & -1.05 & 8.51 & -0.02 & -13 & 30 & 1 & -9 & 250 & 10.78 & 8.48 & 0.03 \\
\hline
\end{tabular}


TABle 3. (continued)

\begin{tabular}{|c|c|c|c|c|c|c|c|c|c|c|c|}
\hline $\begin{array}{l}\text { Star } \\
(1)\end{array}$ & $\begin{array}{c}{[\mathrm{Fe} / \mathrm{H}]} \\
(\mathrm{dex}) \\
(2)\end{array}$ & $\begin{array}{c}R \\
(\mathrm{kpc}) \\
(3)\end{array}$ & $\begin{array}{c}Z \\
(\mathrm{kpc}) \\
(4)\end{array}$ & $\begin{array}{c}U \\
(\mathrm{~km} / \mathrm{s}) \\
(5)\end{array}$ & $\begin{array}{c}V \\
(\mathrm{~km} / \mathrm{s}) \\
(6)\end{array}$ & $\begin{array}{c}W \\
(\mathrm{~km} / \mathrm{s}) \\
(7)\end{array}$ & $\begin{array}{c}V_{R} \\
(\mathrm{~km} / \mathrm{s}) \\
(8)\end{array}$ & $\begin{array}{c}V_{\phi} \\
(\mathrm{km} / \mathrm{s}) \\
(9)\end{array}$ & $\begin{array}{c}R_{a p} \\
(\mathrm{kpc}) \\
(10)\end{array}$ & $\begin{array}{c}R_{p r} \\
(\mathrm{kpc}) \\
(11)\end{array}$ & $\begin{array}{c}Z_{\max } \\
(\mathrm{kpc}) \\
(12)\end{array}$ \\
\hline BPS CS 22948-0091 & -2.01 & 7.13 & -1.67 & -104 & -102 & -17 & -105 & 118 & 8.53 & 2.96 & 2.15 \\
\hline BPS CS 29493-0052 & -1.77 & 7.99 & -0.65 & 119 & -272 & -11 & 118 & -55 & 9.35 & 1.24 & 0.75 \\
\hline BPS CS 22948-0104 & -2.42 & 6.77 & -2.14 & -91 & -53 & 60 & -87 & 170 & 9.05 & 4.47 & 2.86 \\
\hline $\mathrm{V}^{*} \mathrm{RT}$ Gru & -1.49 & 6.79 & -2.06 & 403 & -394 & -205 & 408 & -160 & 82.44 & 2.19 & 31.74 \\
\hline $\mathrm{V}^{*} \mathrm{AV} \mathrm{Peg}$ & -0.08 & 8.38 & -0.31 & 19 & -59 & -19 & 32 & 159 & 8.57 & 4.76 & 0.41 \\
\hline BPS CS 29493-0058 & -1.96 & 5.79 & -3.67 & 76 & -44 & -259 & 108 & 159 & 20.66 & 4.11 & 15.98 \\
\hline BPS CS 29493-0062 & -2.24 & 8.14 & -0.47 & -16 & 0 & -10 & -13 & 220 & 8.49 & 7.86 & 0.51 \\
\hline BPS CS 29493-0059 & -2.34 & 4.68 & -5.38 & 38 & -412 & 41 & -29 & -194 & 8.56 & 5.92 & 6.63 \\
\hline $\mathrm{V}^{*} \mathrm{SS}$ Oct & -1.60 & 7.70 & -0.88 & -60 & -399 & -20 & -35 & -185 & 8.05 & 5.79 & 1.01 \\
\hline HD 208069 & -1.83 & 8.33 & -0.22 & 171 & -303 & 8 & 171 & -84 & 11.55 & 1.85 & 0.35 \\
\hline BPS CS 29493-0064 & -1.23 & 8.13 & -0.49 & -9 & 26 & -51 & -4 & 246 & 10.56 & 8.14 & 1.27 \\
\hline BPS CS 29493-0066 & -0.74 & 6.74 & -2.36 & 2 & -9 & 32 & 22 & 210 & 7.85 & 6.94 & 2.74 \\
\hline BPS CS 29493-0061 & -1.96 & 8.18 & -0.43 & -199 & -227 & 95 & -200 & -4 & 12.99 & 0.10 & 3.41 \\
\hline BPS CS 22881-0017 & -2.16 & 7.28 & -1.55 & -10 & -62 & -34 & -9 & 158 & 7.41 & 4.82 & 1.76 \\
\hline BPS CS 29493-0060 & -1.75 & 7.46 & -1.40 & 79 & -475 & -30 & 65 & -259 & 12.64 & 6.97 & 2.27 \\
\hline $\mathrm{BD}+473617$ & -1.76 & 8.53 & -0.02 & -19 & -9 & 6 & -13 & 211 & 8.66 & 7.61 & 0.07 \\
\hline HD 208360 & -1.96 & 8.43 & -0.09 & -43 & -140 & -37 & -43 & 79 & 8.61 & 2.01 & 0.58 \\
\hline BPS CS 29493-0095 & -2.19 & 7.76 & -1.00 & 117 & -280 & 79 & 115 & -64 & 9.07 & 1.71 & 3.20 \\
\hline BPS CS 29493-0094 & -2.44 & 7.84 & -0.89 & 177 & -120 & 97 & 179 & 95 & 12.01 & 2.49 & 5.03 \\
\hline BPS CS 22881-0010 & -2.04 & 7.61 & -1.14 & -97 & -458 & 84 & -98 & -238 & 13.36 & 6.32 & 3.06 \\
\hline BPS CS 22881-0006 & -1.48 & 7.61 & -1.14 & 68 & -91 & 157 & 67 & 129 & 8.51 & 5.61 & 5.81 \\
\hline BPS CS 29493-0090 & -3.09 & 3.52 & -7.21 & -183 & -54 & -209 & -92 & 229 & 24.75 & 6.41 & 23.20 \\
\hline BPS CS 22965-0035 & -1.88 & 7.83 & -1.13 & 420 & -120 & -168 & 429 & 51 & 71.70 & 0.80 & 26.66 \\
\hline BPS CS 22881-0008 & -2.47 & 1.46 & -9.07 & -129 & -450 & -134 & -118 & -236 & 22.42 & 7.45 & 22.51 \\
\hline $\mathrm{BD}+463527$ & -0.60 & 8.53 & -0.03 & 52 & -92 & -41 & 56 & 127 & 8.94 & 3.51 & 0.64 \\
\hline BPS CS 29493-0092 & -1.94 & 5.15 & -4.75 & -71 & 33 & 129 & -15 & 262 & 16.48 & 6.10 & 11.32 \\
\hline BPS CS 22951-0117 & -2.05 & 7.93 & -0.73 & 104 & -147 & 118 & 104 & 74 & 9.18 & 2.37 & 4.82 \\
\hline $\mathrm{V}^{*} \mathrm{TZ}$ Aqr & -1.24 & 7.77 & -1.31 & 30 & -84 & -45 & 49 & 131 & 8.27 & 3.69 & 1.69 \\
\hline BPS CS 22881-0028 & -1.96 & 7.78 & -0.95 & 223 & -136 & -37 & 223 & 84 & 14.02 & 1.68 & 1.66 \\
\hline $\mathrm{V}^{*} \mathrm{BV}$ Aqr & -1.42 & 7.93 & -0.86 & 76 & -165 & 181 & 78 & 52 & 8.83 & 3.76 & 8.56 \\
\hline BPS CS 22881-0032 & -2.91 & 7.30 & -1.58 & 3 & -17 & 42 & 2 & 203 & 7.48 & 7.16 & 1.86 \\
\hline $\mathrm{BD}+463563$ & -1.75 & 8.53 & -0.03 & -9 & 11 & 2 & -1 & 232 & 9.22 & 8.53 & 0.04 \\
\hline $\mathrm{BD}+473694$ & -0.77 & 8.54 & -0.03 & 18 & -105 & 13 & 22 & 114 & 8.60 & 3.07 & 0.15 \\
\hline BPS CS 22881-0036 & -2.42 & 7.97 & -0.73 & 23 & -382 & -79 & 23 & -162 & 8.19 & 5.34 & 1.97 \\
\hline BPS CS 22881-0040 & -2.27 & 6.51 & -2.75 & -78 & -145 & -111 & -77 & 76 & 7.20 & 3.03 & 5.68 \\
\hline BPS CS 22881-0039 & -3.24 & 2.88 & -7.79 & -357 & -373 & 164 & -360 & -146 & 59.47 & 4.77 & 58.43 \\
\hline HD 210295 & -1.36 & 8.23 & -0.46 & -182 & -186 & 20 & -181 & 40 & 11.64 & 0.84 & 0.66 \\
\hline $\mathrm{V}^{*}$ VV Peg & -1.88 & 8.33 & -0.86 & 12 & -50 & -130 & 41 & 165 & 9.40 & 6.38 & 4.25 \\
\hline BPS CS 29512-0030 & -2.50 & 7.93 & -1.07 & 376 & -20 & -133 & 392 & 167 & 60.98 & 2.65 & 15.78 \\
\hline BPS CS 22881-0060 & -2.12 & 7.85 & -0.94 & 115 & -358 & 34 & 115 & -138 & 9.68 & 3.58 & 1.61 \\
\hline
\end{tabular}


TABLE 3. (continued)

\begin{tabular}{|c|c|c|c|c|c|c|c|c|c|c|c|}
\hline $\begin{array}{l}\text { Star } \\
(1)\end{array}$ & $\begin{array}{c}{[\mathrm{Fe} / \mathrm{H}]} \\
(\operatorname{dex}) \\
(2)\end{array}$ & $\begin{array}{c}R \\
(\mathrm{kpc}) \\
(3)\end{array}$ & $\begin{array}{c}Z \\
(\mathrm{kpc}) \\
(4)\end{array}$ & $\begin{array}{c}U \\
(\mathrm{~km} / \mathrm{s}) \\
(5)\end{array}$ & $\begin{array}{c}V \\
(\mathrm{~km} / \mathrm{s}) \\
(6)\end{array}$ & $\begin{array}{c}W \\
(\mathrm{~km} / \mathrm{s}) \\
(7)\end{array}$ & $\begin{array}{c}V_{R} \\
(\mathrm{~km} / \mathrm{s}) \\
(8)\end{array}$ & $\begin{array}{c}V_{\phi} \\
(\mathrm{km} / \mathrm{s}) \\
(9)\end{array}$ & $\begin{array}{c}R_{a p} \\
(\mathrm{kpc}) \\
(10)\end{array}$ & $\begin{array}{c}R_{p r} \\
(\mathrm{kpc}) \\
(11)\end{array}$ & $\begin{array}{c}Z_{\max } \\
(\mathrm{kpc}) \\
(12)\end{array}$ \\
\hline HD 211075 & -0.61 & 8.42 & -0.29 & -7 & 9 & -11 & 6 & 229 & 9.02 & 8.38 & 0.34 \\
\hline $\mathrm{V}^{*} \mathrm{YZ}$ Aqr & -1.55 & 7.56 & -1.86 & -52 & -15 & 210 & -19 & 211 & 16.00 & 7.39 & 10.49 \\
\hline HD 235766 & -2.35 & 8.70 & -0.08 & -305 & -260 & -4 & -307 & -8 & 22.38 & 0.14 & 0.26 \\
\hline $\mathrm{V}^{*} \mathrm{DH}$ Peg & -1.24 & 8.36 & -0.34 & 55 & -47 & 23 & 63 & 171 & 9.13 & 5.08 & 0.54 \\
\hline BPS CS 22881-0070 & -2.70 & 7.75 & -1.09 & 103 & -17 & 76 & 102 & 203 & 11.04 & 6.01 & 3.10 \\
\hline $\mathrm{V}^{*}$ TY Gru & -2.16 & 5.59 & -4.26 & 171 & -36 & -111 & 173 & 182 & 15.78 & 3.70 & 8.93 \\
\hline BPS CS 22886-0026 & -1.78 & 7.97 & -1.08 & -145 & -100 & 96 & -134 & 132 & 11.26 & 3.48 & 3.24 \\
\hline HD 211744 & -1.03 & 8.40 & -0.15 & 22 & -26 & -13 & 22 & 194 & 8.56 & 6.46 & 0.21 \\
\hline V* UW Gru & -1.68 & 6.65 & -2.56 & -310 & -125 & 169 & -319 & 57 & 29.83 & 0.93 & 12.05 \\
\hline BPS CS 22875-0004 & -1.56 & 7.95 & -0.82 & 315 & -365 & -60 & 315 & -145 & 27.75 & 2.56 & 3.28 \\
\hline $\mathrm{V}^{*}$ CQ Lac & -2.04 & 8.87 & -0.52 & 11 & 22 & -28 & 66 & 233 & 11.93 & 7.68 & 0.88 \\
\hline BPS CS 22960-0060 & -2.30 & 7.14 & -2.04 & -9 & -333 & 97 & -7 & -113 & 7.48 & 3.65 & 3.61 \\
\hline BPS CS 22875-0012 & -2.12 & 7.80 & -1.08 & -70 & -382 & 82 & -71 & -161 & 9.17 & 4.83 & 2.40 \\
\hline BPS CS 22960-0062 & -1.76 & 8.13 & -0.55 & 10 & -34 & -48 & 10 & 186 & 8.20 & 6.31 & 1.06 \\
\hline BPS CS 22960-0064 & -2.98 & 7.19 & -1.97 & 80 & -129 & 43 & 79 & 92 & 7.95 & 2.48 & 2.80 \\
\hline HD 212516 & -0.64 & 8.38 & -0.57 & -20 & 28 & -45 & 5 & 249 & 11.13 & 8.38 & 1.26 \\
\hline V* UY Gru & -1.78 & 5.21 & -5.11 & 36 & 126 & -166 & 30 & 347 & 36.82 & 6.37 & 25.38 \\
\hline $\mathrm{V}^{*}$ AE Peg & -1.84 & 8.41 & -1.46 & -224 & -588 & -86 & -310 & -298 & 60.58 & 5.72 & 20.66 \\
\hline BPS CS 29512-0076 & -1.97 & 8.18 & -0.69 & -29 & 17 & 29 & -17 & 238 & 9.98 & 8.06 & 1.01 \\
\hline $\mathrm{V}^{*} \mathrm{BN}$ Aqr & -1.33 & 7.80 & -1.74 & -13 & -392 & -31 & -39 & -168 & 8.23 & 5.46 & 2.06 \\
\hline BPS CS 22875-0028 & -1.38 & 8.20 & -0.47 & 18 & -68 & -93 & 18 & 152 & 8.32 & 5.15 & 2.36 \\
\hline BPS CS 22875-0029 & -2.54 & 6.10 & -3.88 & 121 & -266 & -162 & 120 & -48 & 11.25 & 1.28 & 7.87 \\
\hline BPS CS 22875-0026 & -2.04 & 8.06 & -0.71 & 292 & -209 & -45 & 292 & 11 & 19.73 & 0.20 & 1.90 \\
\hline HD 213487 & -1.25 & 8.29 & -0.41 & -5 & 0 & -3 & -1 & 220 & 8.25 & 8.25 & 0.41 \\
\hline HD 213467 & -1.45 & 8.43 & -0.12 & 159 & -144 & -30 & 159 & 76 & 11.20 & 1.71 & 0.50 \\
\hline HD 213657 & -1.98 & 8.45 & -0.09 & -80 & -100 & -1 & -80 & 120 & 9.22 & 3.10 & 0.09 \\
\hline $\mathrm{V}^{*}$ AA Aqr & -2.09 & 7.82 & -1.78 & 169 & -77 & -97 & 187 & 119 & 14.04 & 2.74 & 4.13 \\
\hline HD 214161 & -2.16 & 8.18 & -0.53 & 290 & -90 & 262 & 290 & 131 & 44.26 & 4.69 & 37.32 \\
\hline HD 214362 & -1.77 & 8.29 & -0.43 & 331 & -236 & -134 & 330 & -22 & 29.65 & 0.43 & 11.48 \\
\hline BPS CS 22875-0038 & -1.28 & 8.16 & -0.58 & -32 & -94 & 119 & -31 & 126 & 8.45 & 4.42 & 3.64 \\
\hline V* RZ Cep & -1.77 & 8.64 & 0.04 & 301 & -112 & 240 & 306 & 95 & 41.69 & 3.45 & 34.84 \\
\hline HD 214925 & -2.14 & 7.68 & -1.86 & 129 & -512 & 136 & 103 & -302 & 24.64 & 7.54 & 10.75 \\
\hline V* RW Gru & -2.00 & 7.29 & -2.04 & -54 & -423 & 74 & -49 & -204 & 9.56 & 6.31 & 3.13 \\
\hline $\mathrm{V}^{*} \mathrm{DX}$ Cep & -1.83 & 9.76 & 0.88 & 181 & -94 & -13 & 202 & 88 & 15.58 & 2.19 & 1.78 \\
\hline BPS CS 22887-0048 & -1.72 & 8.32 & -0.50 & -147 & -216 & 35 & -146 & 9 & 10.45 & 0.20 & 0.86 \\
\hline HD 215601 & -1.56 & 8.38 & -0.25 & 11 & -199 & 7 & 11 & 21 & 8.39 & 0.50 & 0.27 \\
\hline HD 215801 & -2.45 & 8.42 & -0.14 & 13 & -205 & 124 & 13 & 15 & 8.44 & 0.51 & 4.71 \\
\hline HD 216143 & -2.16 & 8.32 & -0.56 & -329 & -268 & 78 & -331 & -34 & 27.08 & 0.61 & 4.19 \\
\hline $\mathrm{V}^{*} \mathrm{BH}$ Peg & -1.22 & 8.48 & -0.51 & -206 & -311 & 62 & -212 & -76 & 14.31 & 1.64 & 1.91 \\
\hline $\mathrm{V}^{*} \mathrm{BO}$ Aqr & -1.80 & 7.97 & -1.68 & -116 & -73 & 29 & -100 & 158 & 10.02 & 4.40 & 2.04 \\
\hline
\end{tabular}


TABle 3. (continued)

\begin{tabular}{|c|c|c|c|c|c|c|c|c|c|c|c|}
\hline $\begin{array}{l}\text { Star } \\
(1)\end{array}$ & $\begin{array}{c}{[\mathrm{Fe} / \mathrm{H}]} \\
(\mathrm{dex}) \\
(2)\end{array}$ & $\begin{array}{c}R \\
(\mathrm{kpc}) \\
(3)\end{array}$ & $\begin{array}{c}Z \\
(\mathrm{kpc}) \\
(4)\end{array}$ & $\begin{array}{c}U \\
(\mathrm{~km} / \mathrm{s}) \\
(5)\end{array}$ & $\begin{array}{c}V \\
(\mathrm{~km} / \mathrm{s}) \\
(6)\end{array}$ & $\begin{array}{c}W \\
(\mathrm{~km} / \mathrm{s}) \\
(7)\end{array}$ & $\begin{array}{c}V_{R} \\
(\mathrm{~km} / \mathrm{s}) \\
(8)\end{array}$ & $\begin{array}{c}V_{\phi} \\
(\mathrm{km} / \mathrm{s}) \\
(9)\end{array}$ & $\begin{array}{c}R_{a p} \\
(\mathrm{kpc}) \\
(10)\end{array}$ & $\begin{array}{c}R_{p r} \\
(\mathrm{kpc}) \\
(11)\end{array}$ & $\begin{array}{c}Z_{\max } \\
(\mathrm{kpc}) \\
(12)\end{array}$ \\
\hline BPS CS 30493-0016 & -1.26 & 6.63 & -4.10 & -7 & -96 & -9 & -2 & 125 & 7.56 & 3.99 & 4.11 \\
\hline BPS CS 30493-0014 & -2.20 & 7.91 & -1.29 & -240 & -456 & 56 & -243 & -234 & 25.33 & 4.66 & 3.97 \\
\hline HD 217515 & -0.82 & 8.34 & -0.46 & 34 & 32 & 5 & 39 & 251 & 11.47 & 8.01 & 0.62 \\
\hline BPS CS 22888-0010 & -1.97 & 8.01 & -1.10 & 48 & -228 & 89 & 48 & -9 & 8.22 & 0.26 & 3.48 \\
\hline BPS CS 30493-0001 & -1.76 & 8.29 & -0.45 & 219 & -169 & 54 & 219 & 51 & 13.90 & 1.09 & 2.32 \\
\hline CD-63 1588 & -1.36 & 8.26 & -0.37 & 60 & -211 & -64 & 60 & 11 & 8.63 & 0.27 & 1.46 \\
\hline HD 217808 & -2.18 & 8.23 & -0.53 & -32 & -96 & -143 & -33 & 123 & 8.40 & 5.18 & 4.92 \\
\hline BPS CS 22888-0014 & -2.40 & 8.00 & -1.16 & 122 & -388 & 35 & 120 & -169 & 10.69 & 4.65 & 2.08 \\
\hline BPS CS 22888-0002 & -2.20 & 7.94 & -1.23 & -85 & -311 & -12 & -85 & -90 & 8.77 & 2.29 & 1.45 \\
\hline BPS CS 22888-0001 & -1.98 & 7.94 & -1.24 & -147 & -172 & 59 & -147 & 49 & 10.48 & 1.12 & 2.02 \\
\hline HD 218367 & -0.74 & 8.56 & -1.00 & -9 & 20 & 0 & 24 & 239 & 10.66 & 8.39 & 1.21 \\
\hline BPS CS 30493-0029 & -0.64 & 8.25 & -0.60 & 43 & 16 & 23 & 44 & 236 & 10.46 & 7.69 & 0.90 \\
\hline BPS CS 30493-0031 & -0.17 & 8.26 & -0.57 & -73 & -15 & -14 & -72 & 206 & 10.03 & 6.30 & 0.76 \\
\hline $\mathrm{V}^{*} \mathrm{YY}$ Tuc & -1.82 & 7.60 & -1.56 & -31 & -222 & 47 & -31 & -5 & 7.86 & 0.13 & 2.00 \\
\hline HD 218810 & -0.65 & 8.47 & -0.08 & 16 & 10 & -11 & 16 & 230 & 9.30 & 8.25 & 0.16 \\
\hline BPS CS 22888-0039 & -1.78 & 8.02 & -1.18 & 349 & -256 & -170 & 349 & -39 & 38.41 & 0.75 & 17.65 \\
\hline HD 218857 & -1.94 & 8.42 & -0.28 & -92 & -141 & 161 & -91 & 80 & 10.06 & 3.36 & 7.13 \\
\hline BPS CS 22888-0031 & -3.08 & 7.91 & -1.40 & 326 & -337 & -10 & 325 & -120 & 27.53 & 2.13 & 5.07 \\
\hline BPS CS 30493-0049 & -0.61 & 8.03 & -1.11 & 83 & -56 & -43 & 84 & 164 & 9.55 & 4.66 & 1.54 \\
\hline BPS CS 22888-0044 & -2.24 & 7.63 & -2.19 & 146 & -85 & -183 & 149 & 131 & 15.52 & 3.87 & 9.51 \\
\hline BPS CS 30493-0050 & -1.21 & 8.13 & -0.90 & 65 & -34 & 32 & 66 & 185 & 9.26 & 5.75 & 1.33 \\
\hline BPS CS 30493-0058 & -2.30 & 7.98 & -1.31 & -29 & -125 & 82 & -28 & 95 & 8.25 & 2.83 & 2.77 \\
\hline BPS CS 29513-0002 & -1.03 & 8.00 & -1.09 & 34 & 34 & -49 & 31 & 254 & 11.95 & 7.83 & 2.00 \\
\hline BPS CS 22888-0029 & -1.48 & 8.03 & -1.14 & -54 & -195 & -145 & -54 & 26 & 8.30 & 1.39 & 7.42 \\
\hline BPS CS 29513-0014 & -2.24 & 6.86 & -3.85 & 207 & -152 & -127 & 206 & 69 & 15.85 & 1.46 & 7.63 \\
\hline BPS CS 29513-0015 & -2.02 & 8.04 & -1.09 & -190 & -98 & 30 & -190 & 122 & 13.15 & 2.70 & 1.69 \\
\hline BPS CS 30493-0060 & -0.44 & 8.22 & -0.71 & -30 & -24 & 25 & -29 & 196 & 8.61 & 6.60 & 0.86 \\
\hline BPS CS 29513-0009 & -1.14 & 8.06 & -1.00 & -189 & -92 & -5 & -190 & 127 & 13.07 & 2.85 & 1.71 \\
\hline HD 219221 & -0.67 & 8.46 & -0.08 & -35 & 13 & -12 & -35 & 233 & 10.03 & 7.90 & 0.19 \\
\hline BPS CS 29513-0003 & -2.15 & 6.85 & -3.69 & 36 & -278 & 124 & 39 & -56 & 7.61 & 2.95 & 6.99 \\
\hline BPS CS 22888-0026 & -1.78 & 6.91 & -3.84 & -407 & -345 & 75 & -408 & -124 & 58.53 & 2.05 & 31.77 \\
\hline BPS CS 30493-0065 & -1.20 & 8.28 & -0.55 & 6 & -39 & 50 & 7 & 181 & 8.30 & 6.16 & 1.13 \\
\hline BPS CS 30493-0071 & -2.20 & 8.25 & -0.63 & 38 & -35 & -10 & 39 & 185 & 8.69 & 5.92 & 0.67 \\
\hline BPS CS 29513-0010 & -1.67 & 7.99 & -1.20 & -53 & -169 & -1 & -54 & 50 & 8.33 & 1.24 & 1.25 \\
\hline BPS CS 30493-0072 & -1.77 & 8.14 & -0.90 & -14 & -11 & -13 & -14 & 209 & 8.34 & 7.53 & 0.95 \\
\hline $\mathrm{V}^{*} \mathrm{AC}$ And & -1.16 & 8.89 & -0.22 & -37 & -33 & 5 & -15 & 190 & 8.97 & 6.63 & 0.23 \\
\hline BPS CS 29513-0016 & -1.97 & 8.18 & -0.79 & 51 & -247 & -67 & 51 & -27 & 8.51 & 0.70 & 1.90 \\
\hline BPS CS 30493-0064 & -1.15 & 7.67 & -2.18 & 80 & -97 & 30 & 82 & 122 & 8.69 & 3.53 & 2.84 \\
\hline $\mathrm{V}^{*} \mathrm{DN}$ Aqr & -1.66 & 8.13 & -1.22 & 272 & -211 & 106 & 272 & 0 & 19.03 & 0.01 & 11.44 \\
\hline$V^{*}$ DZ Peg & -1.52 & 8.68 & -1.20 & -12 & -378 & 10 & -36 & -154 & 9.00 & 4.88 & 1.23 \\
\hline
\end{tabular}


TABLE 3. (continued)

\begin{tabular}{|c|c|c|c|c|c|c|c|c|c|c|c|}
\hline $\begin{array}{l}\text { Star } \\
(1)\end{array}$ & $\begin{array}{c}{[\mathrm{Fe} / \mathrm{H}]} \\
(\mathrm{dex}) \\
(2)\end{array}$ & $\begin{array}{c}R \\
(\mathrm{kpc}) \\
(3)\end{array}$ & $\begin{array}{c}Z \\
(\mathrm{kpc}) \\
(4)\end{array}$ & $\begin{array}{c}U \\
(\mathrm{~km} / \mathrm{s}) \\
(5)\end{array}$ & $\begin{array}{c}V \\
(\mathrm{~km} / \mathrm{s}) \\
(6)\end{array}$ & $\begin{array}{c}W \\
(\mathrm{~km} / \mathrm{s}) \\
(7)\end{array}$ & $\begin{array}{c}V_{R} \\
(\mathrm{~km} / \mathrm{s}) \\
(8)\end{array}$ & $\begin{array}{c}V_{\phi} \\
(\mathrm{km} / \mathrm{s}) \\
(9)\end{array}$ & $\begin{array}{c}R_{a p} \\
(\mathrm{kpc}) \\
(10)\end{array}$ & $\begin{array}{c}R_{p r} \\
(\mathrm{kpc}) \\
(11)\end{array}$ & $\begin{array}{c}Z_{\max } \\
(\mathrm{kpc}) \\
(12)\end{array}$ \\
\hline BPS CS 22888-0047 & -2.25 & 8.07 & -1.15 & 66 & -83 & 146 & 67 & 137 & 9.00 & 5.91 & 5.65 \\
\hline BPS CS 22888-0057 & -2.06 & 7.92 & -1.52 & 53 & -544 & 39 & 52 & -324 & 21.63 & 7.90 & 4.36 \\
\hline HD 220127 & -1.79 & 8.31 & -0.40 & 205 & -727 & 2 & 211 & -505 & 174.04 & 7.56 & 7.89 \\
\hline BPS CS 29513-0032 & -2.02 & 8.12 & -0.92 & 207 & -30 & 154 & 206 & 191 & 20.99 & 5.33 & 11.35 \\
\hline $\mathrm{V}^{*} \mathrm{UZ} \mathrm{Scl}$ & -1.14 & 7.83 & -1.98 & -108 & -180 & 23 & -107 & 43 & 9.24 & 1.06 & 2.23 \\
\hline BPS CS 29513-0035 & -1.90 & 8.06 & -1.11 & -127 & -183 & -42 & -127 & 36 & 9.61 & 0.88 & 2.16 \\
\hline BPS CS 29513-0033 & -1.63 & 7.98 & -1.30 & 17 & 18 & -59 & 15 & 238 & 10.58 & 7.92 & 2.28 \\
\hline BPS CS 22888-0049 & -1.84 & 8.08 & -1.17 & -57 & -153 & -5 & -56 & 67 & 8.45 & 1.69 & 1.24 \\
\hline BPS CS 29513-0031 & -2.79 & 7.81 & -1.73 & -89 & -52 & -275 & -91 & 167 & 21.72 & 7.82 & 18.50 \\
\hline BPS CS 29513-0034 & -1.76 & 8.07 & -1.08 & -250 & -232 & -116 & -250 & -14 & 16.89 & 0.38 & 10.65 \\
\hline HD 220662 & -1.59 & 8.09 & -1.48 & 2 & -170 & 47 & 4 & 49 & 8.20 & 1.32 & 2.07 \\
\hline HD 220838 & -1.74 & 8.06 & -1.48 & -27 & -76 & 22 & -23 & 145 & 8.28 & 4.41 & 1.58 \\
\hline BPS CS 22941-0011 & -2.16 & 7.84 & -1.93 & 87 & -343 & -63 & 85 & -124 & 9.36 & 3.45 & 2.68 \\
\hline $\mathrm{V}^{*} \mathrm{RV}$ Phe & -1.69 & 7.75 & -1.69 & 294 & -289 & -179 & 297 & -57 & 28.30 & 1.17 & 14.11 \\
\hline BPS CS 29513-0048 & -1.49 & 8.18 & -0.83 & 74 & -155 & -118 & 74 & 66 & 9.08 & 2.07 & 4.30 \\
\hline HD 221170 & -2.12 & 8.60 & -0.23 & -119 & -123 & -22 & -114 & 103 & 10.11 & 2.55 & 0.49 \\
\hline $\mathrm{V}^{*} \mathrm{BK}$ Tuc & -1.82 & 7.28 & -1.99 & 111 & -250 & -44 & 115 & -5 & 8.88 & 0.11 & 2.38 \\
\hline BPS CS 29513-0040 & -1.54 & 6.95 & -4.19 & 126 & -121 & -12 & 124 & 101 & 9.88 & 2.89 & 5.44 \\
\hline $\mathrm{V}^{*} \mathrm{DM}$ And & -2.32 & 9.42 & -1.12 & 19 & -441 & -290 & -36 & -219 & 32.73 & 9.47 & 25.38 \\
\hline BPS CS 22941-0014 & -1.84 & 7.77 & -2.24 & -2 & -56 & -23 & 0 & 164 & 8.04 & 5.48 & 2.33 \\
\hline BPS CS 29496-0003 & -2.20 & 8.28 & -0.70 & 34 & -279 & 3 & 34 & -59 & 8.42 & 1.46 & 0.71 \\
\hline BPS CS 22941-0017 & -2.40 & 7.41 & -3.34 & -111 & -4 & -80 & -107 & 218 & 13.03 & 7.14 & 7.00 \\
\hline BPS CS 22941-0020 & -2.17 & 8.05 & -1.34 & 132 & -262 & -98 & 131 & -43 & 10.46 & 1.10 & 3.60 \\
\hline BPS CS 22941-0027 & -2.57 & 6.76 & -5.11 & 241 & 16 & 23 & 238 & 239 & 27.98 & 6.14 & 19.29 \\
\hline BPS CS 22941-0021 & -1.48 & 8.23 & -0.82 & 92 & -95 & 60 & 92 & 125 & 9.34 & 3.53 & 2.07 \\
\hline $\mathrm{V}^{*} \mathrm{BK}$ And & -0.08 & 9.30 & -0.71 & 23 & 64 & -120 & 81 & 273 & 20.24 & 8.55 & 6.08 \\
\hline BPS CS 29513-0053 & -1.21 & 8.13 & -0.99 & 134 & -97 & 10 & 133 & 124 & 10.45 & 3.09 & 1.36 \\
\hline BPS CS 22952-0011 & -2.70 & 8.47 & -0.78 & 75 & -213 & 247 & 76 & 3 & 11.37 & 7.25 & 12.05 \\
\hline BPS CS 29496-0021 & -2.27 & 8.08 & -1.44 & -232 & -444 & 70 & -235 & -221 & 23.96 & 4.62 & 4.40 \\
\hline BPS CS 29496-0022 & -2.25 & 8.08 & -1.46 & -326 & -534 & 23 & -330 & -310 & 67.08 & 5.14 & 11.35 \\
\hline $\mathrm{V}^{*} \mathrm{BR}$ Aqr & -0.74 & 8.38 & -1.18 & 3 & -48 & -54 & 14 & 172 & 8.56 & 5.93 & 1.80 \\
\hline BPS CS 29496-0014 & -1.87 & 8.34 & -0.60 & -24 & -229 & -22 & -24 & -8 & 8.40 & 0.20 & 0.76 \\
\hline BPS CS 29499-0017 & -2.22 & 8.14 & -1.63 & -529 & -599 & -35 & -544 & -357 & $\ldots$ & $\ldots$ & $\ldots$ \\
\hline BPS CS 29499-0010 & -1.45 & 8.03 & -2.01 & 42 & -36 & -17 & 50 & 182 & 9.05 & 5.97 & 2.18 \\
\hline BPS CS 29499-0003 & -1.88 & 8.22 & -1.14 & -188 & -173 & 96 & -187 & 50 & 13.04 & 1.17 & 3.75 \\
\hline BPS CS 29496-0013 & -2.50 & 6.74 & -7.01 & -34 & -413 & -70 & -57 & -188 & 10.48 & 9.31 & 8.40 \\
\hline BPS CS 22941-0043 & -2.04 & 8.05 & -1.54 & -291 & -615 & -70 & -294 & -392 & 101.50 & 6.45 & 28.67 \\
\hline HD 222434 & -1.90 & 8.16 & -1.13 & -117 & -219 & 23 & -117 & 1 & 9.55 & 0.02 & 1.31 \\
\hline BPS CS 29499-0027 & -1.78 & 8.28 & -1.07 & -32 & -134 & -85 & -29 & 86 & 8.39 & 2.68 & 3.04 \\
\hline $\mathrm{V}^{*} \mathrm{VZ}$ Peg & -1.80 & 8.96 & -1.04 & -17 & -371 & -64 & -40 & -146 & 9.24 & 5.04 & 2.30 \\
\hline
\end{tabular}



TABLE 3. (continued)

\begin{tabular}{|c|c|c|c|c|c|c|c|c|c|c|c|}
\hline $\begin{array}{l}\text { Star } \\
\text { (1) }\end{array}$ & $\begin{array}{c}{[\mathrm{Fe} / \mathrm{H}]} \\
(\mathrm{dex}) \\
(2)\end{array}$ & $\begin{array}{c}R \\
(\mathrm{kpc}) \\
(3)\end{array}$ & $\begin{array}{c}Z \\
(\mathrm{kpc}) \\
(4)\end{array}$ & $\begin{array}{c}U \\
(\mathrm{~km} / \mathrm{s}) \\
(5)\end{array}$ & $\begin{array}{c}V \\
(\mathrm{~km} / \mathrm{s}) \\
(6)\end{array}$ & $\begin{array}{c}W \\
(\mathrm{~km} / \mathrm{s}) \\
(7)\end{array}$ & $\begin{array}{c}V_{R} \\
(\mathrm{~km} / \mathrm{s}) \\
(8)\end{array}$ & $\begin{array}{c}V_{\phi} \\
(\mathrm{km} / \mathrm{s}) \\
(9)\end{array}$ & $\begin{array}{c}R_{a p} \\
(\mathrm{kpc}) \\
(10)\end{array}$ & $\begin{array}{c}R_{p r} \\
(\mathrm{kpc}) \\
(11)\end{array}$ & $\begin{array}{c}Z_{\max } \\
(\mathrm{kpc}) \\
(12)\end{array}$ \\
\hline $\mathrm{V}^{*}$ AT And & -1.18 & 8.82 & -0.27 & -192 & -216 & -105 & -191 & 21 & 13.48 & 0.57 & 5.61 \\
\hline HD 222766 & -1.40 & 8.50 & -0.05 & 119 & -128 & 34 & 119 & 92 & 10.07 & 2.22 & 0.56 \\
\hline BPS CS 29499-0034 & -1.67 & 8.19 & -1.34 & 26 & -134 & -37 & 28 & 85 & 8.40 & 2.30 & 1.64 \\
\hline BPS CS 22941-0046 & -1.62 & 8.20 & -1.02 & 5 & -22 & 39 & 5 & 198 & 8.25 & 7.19 & 1.35 \\
\hline HD 222925 & -1.19 & 8.34 & -0.31 & 221 & -235 & 68 & 221 & -11 & 14.00 & 0.24 & 2.79 \\
\hline BPS CS 29517-0010 & -1.29 & 8.36 & -1.21 & -66 & -57 & 3 & -58 & 166 & 9.13 & 5.14 & 1.30 \\
\hline HD 223224 & -1.16 & 8.39 & -0.25 & 49 & -52 & -56 & 47 & 168 & 8.89 & 5.28 & 1.06 \\
\hline BPS CS 22876-0003 & -1.98 & 8.27 & -0.81 & -62 & -118 & -54 & -62 & 102 & 8.71 & 2.83 & 1.73 \\
\hline BPS CS 29499-0058 & -2.44 & 8.32 & -0.93 & -175 & -191 & -135 & -174 & 31 & 12.36 & 1.20 & 9.31 \\
\hline BPS CS 29499-0053 & -1.71 & 8.31 & -1.10 & 22 & -69 & -12 & 26 & 151 & 8.49 & 4.60 & 1.13 \\
\hline BPS CS 22876-0016 & -1.17 & 8.13 & -1.54 & -30 & -62 & -78 & -29 & 158 & 8.33 & 5.69 & 2.90 \\
\hline BPS CS 29499-0060 & -2.63 & 8.36 & -0.69 & 265 & -43 & 8 & 266 & 175 & 22.58 & 3.53 & 2.08 \\
\hline BPS CS 29499-0065 & -2.70 & 8.18 & -1.86 & 36 & -381 & -43 & 30 & -162 & 8.68 & 5.37 & 2.21 \\
\hline BPS CS 29499-0068 & -2.26 & 8.35 & -0.92 & -397 & -272 & 61 & -398 & -46 & 44.63 & 0.73 & 5.13 \\
\hline BPS CS 22957-0015 & -2.10 & 8.51 & -1.23 & 317 & -315 & -80 & 310 & -116 & 27.94 & 2.21 & 4.91 \\
\hline BPS CS 22876-0027 & -2.07 & 8.21 & -1.18 & -6 & -92 & 113 & -7 & 128 & 8.36 & 4.76 & 3.85 \\
\hline
\end{tabular}


This figure "fig4.gif" is available in "gif" format from: http://arxiv.org/ps/astro-ph/0003103v1 
This figure "fig6.gif" is available in "gif" format from: http://arxiv.org/ps/astro-ph/0003103v1 
This figure "fig7.gif" is available in "gif" format from: http://arxiv.org/ps/astro-ph/0003103v1 
This figure "fig8.gif" is available in "gif" format from: http://arxiv.org/ps/astro-ph/0003103v1 